\title{
Dissertation
}

\section{Totalsynthese von Nosiheptid}

zur Erlangung des akademischen Grades doctor rerum naturalium

(Dr. rer. nat.)

im Fach Chemie

an der Chemisch-Geowissenschaftlichen Fakultät

der Friedrich-Schiller-Universität Jena

eingereicht von

Dipl.-Chem. Kamil Philip Wojtas

geboren am 22. November 1984 in Kwidzyn 
Gutachter: 1. Prof. Dr. Hans-Dieter Arndt (FSU Jena)

2. Prof. Dr. Georg Pohnert (FSU Jena)

3. Prof. Dr. Christoph Schneider (Universität Leipzig)

Datum der Verteidigung: 05. April 2017 
Für meine Familie 

„Auf diesem beweglichen Erdball ist doch nur in der wahren Liebe, der Wohltätigkeit und den Wissenschaften die einzige Freude und Ruhe.“

J. W. v. Goethe im November 1781 



\section{Inhaltsverzeichnis}

1 Allgemeine Einleitung 1

1.1 Warum und wofür Totalsynthese? 2

1.2 Totalsynthese im Rahmen dieser Arbeit 5

$\begin{array}{lll}1.3 & \text { Thiopeptidantibiotika } & 6\end{array}$

$\begin{array}{lll}\text { 1.3.1 Strukturelle Merkmale } & 6\end{array}$

$\begin{array}{lll}\text { 1.3.2 Biologische Aktivität } & 8\end{array}$

1.4 Totalsynthesen von Thiopeptidantibiotika 10

1.4.1 Diskussion der Totalsynthese von Baringolin 12

$\begin{array}{lll}1.5 & \text { Nosiheptid als Syntheseziel } & 17\end{array}$

$\begin{array}{lll}\text { 1.5.1 Strukturcharakteristika und verwandte Moleküle } & 17\end{array}$

$\begin{array}{ll}\text { 1.5.2 Bisherige synthetische Ansätze } & 19\end{array}$

1.5.3 Retrosynthetische Analyse von Nosiheptid nach Lu und Riedrich 20

1.5.4 Thiazol-Synthesen durch Aza-Wittig-Ringschlüsse nach Riedrich 21

1.5.5 Synthese des Pyridinkerns nach Lu 24

1.5.6 Synthese des Indols nach Riedrich 26

1.5.7 Verknüpfung der Bausteine und Ringschluss nach Lu und Riedrich 27

1.5.8 Synthese des Nocathiacin-Pyridinkerns nach Ciufolini et al. 28

2 Ziele der Arbeit $\quad 31$

2.1 Totalsynthese von Nosiheptid 32

2.2 Regioselektive Umfunktionalisierung von 3-Hydroxypicolinaten 34

2.3 Synthese substituierter 3-Hydroxypicolinsäuren 34

2.4 Synthese tetradentater Biscarboxamid-Liganden 36

3 Totalsynthese von Nosiheptid $\quad 37$

3.1 Einleitung 38

3.2 Synthesestrategie 39

3.2.1 Rückschlüsse aus früheren Arbeiten 39

3.2.2 Retrosynthetische Analyse 41

3.2.3 Etappenziele und Herausforderungen 42

3.3 Synthese des Indol-Bausteins 45

3.3.1 Aufbau des Indols 45

3.3.2 Untersuchungen zur selektiven Dpm-Spaltung 48

3.4 Synthese der Bis-Thiazol-Fragmente 49

3.4.1 Synthese des indoltragenden Thiazols 49

3.4.2 Synthese der Peptidthiazole $\quad 52$

3.4.3 Kupplung der Fragmente $\quad 54$

3.5 Synthese des 3-Hydroxypyridin-Kerns 56 
3.5.1 Synthese des Alkinylketons 56

3.5.2 Untersuchungen zur hetero-Diels-Alder-Reaktion 57

3.5.3 Abschluss der Pyridinfragment-Synthese $\quad 62$

3.6 Untersuchungen zum B-Ring-Aufbau 64

3.7 Untersuchungen zur Dehydroalanin-Bildung 67

3.8 Untersuchungen zur Ts-Abspaltung am 3-Hydroxypyridin 69

3.9 Abschluss der Totalsynthese von Nosiheptid 73

$\begin{array}{lll}3.10 & \text { Strukturbestätigung } & 79\end{array}$

3.11 Vergleich der biologischen Aktivität 82

3.12 Fazit 83

4 Nachbargruppen-assistierte Umfunktionalisierung von 3-Hydroxypicolinaten 85

4.1 Einleitung 86

4.1.1 Substratdirigierte Reaktionen 86

4.1.2 Frühere Arbeiten zur regioselektiven Umfunktionalisierung von 3-Hydroxypicolinaten $\quad 88$

$\begin{array}{lll}4.2 & \text { Fragestellung } & 89\end{array}$

$\begin{array}{lll}4.3 & \text { Ergebnisse und Diskussion } & 90\end{array}$

4.3.1 Untersuchungen zur regioselektiven Hydrolyse 90

4.3.2 Untersuchungen zur regioselektiven Umesterung 91

4.3.3 Untersuchungen zur regioselektiven Aminolyse 94

4.3.4 Mechanistische Überlegungen 95

$\begin{array}{lll}4.4 & \text { Fazit } & 97\end{array}$

5 Synthese substituierter 3-Hydroxypicolinsäuren 99

$\begin{array}{lll}5.1 & \text { Einleitung } & 100\end{array}$

$\begin{array}{lll}\text { 5.1.1 Biologische Aktivität } & 101\end{array}$

$\begin{array}{lll}5.2 & \text { Syntheseziel } & 102\end{array}$

5.3 Substitution in Position $6 \quad 102$

5.3.1 Synthese via Palladium-katalysierter Kreuzkupplungen 102

5.3.2 Synthese via Kondensations- und Cycloadditionsreaktionen 105

$\begin{array}{ll}\text { 5.3.3 Darstellung der Picolinsäuren } & 106\end{array}$

$\begin{array}{lll}5.4 & \text { Substitution in Position } 4 & 107\end{array}$

$\begin{array}{lll}5.5 & \text { Substitution in Position 5 } & 109\end{array}$

$\begin{array}{lll}5.6 & \text { Fazit } & 111\end{array}$

6 Synthese tetradentater Biscarboxamid-Liganden 113

$\begin{array}{lll}6.1 & \text { Einleitung } & 114\end{array}$

6.2 Syntheseziel 115

6.3 Synthese der nicht fluorierten Biscarboxamid-Liganden 116

6.4 Synthese der fluorierten Biscarboxamid-Liganden 117 
$\begin{array}{lll}6.5 & \text { Fazit } & 118\end{array}$

7 Zusammenfassung $\quad 119$

$\begin{array}{ll}7.1 & \text { Totalsynthese von Nosiheptid } \\ & 120\end{array}$

7.2 Regioselektive Umfunktionalisierung von 3-Hydroxypciolinaten 122

$\begin{array}{lll}7.3 & \text { Synthese substituierter 3-Hydroxypicolinsäuren } & 123\end{array}$

$\begin{array}{lll}\text { 7.3.1 Als Bausteine zur Derivatisierung von Pyridomycin } & 123\end{array}$

7.3.2 Zur Synthese fluorierter tetradentater Bis-Carboxamid-Liganden 124

$\begin{array}{lll}7.4 & \text { Fazit und Ausblick } & 125\end{array}$

8 Experimenteller Teil $\quad 127$

$\begin{array}{lll}8.1 & \text { Chemische Methoden } & 128\end{array}$

$\begin{array}{lll}\text { 8.1.1 Geräte und Methoden } & 128\end{array}$

$\begin{array}{ll}\text { 8.1.2 Chromatographie } & 129\end{array}$

8.1.3 Reagenzien, Lösungsmittel und Reaktionsbedingungen 130

$\begin{array}{lll}\text { 8.1.4 Pufferlösungen } & 131\end{array}$

8.2 Mikrobiologische Methoden 131

8.3 Allgemeine Arbeitsvorschriften 132

8.4 Totalsynthese von Nosiheptid 133

8.5 Semisynthetische Arbeiten an Nosiheptid 184

8.6 Nachbargruppen-assistierte Umsetzungen 186

8.7 Synthese von 3-Hydroxypicolinsäurederivaten 196

8.8 Synthese von Bis-Carboxamid-Liganden 224

9 Abkürzungsverzeichnis $\quad 235$

$\begin{array}{ll}10 \text { Literaturverzeichnis } & 239\end{array}$

11 Analytische Daten $\quad 257$

11.1 Daten zu den Einkristall-Röntgenstrukturanalysen 258

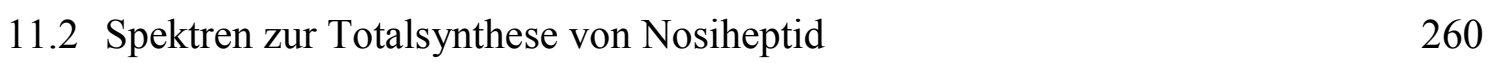

11.3 Spektren zu Nachbargruppen-assistierten Umsetzungen 303

11.4 Spektren zur Synthese substituierter 3-Hydroxypicolinate 310

11.5 Spektren zur Synthese von Bis-Carboxamid-Liganden 330

11.6 Spektren zu semisynthetischen Arbeiten an Nosiheptid 335

\begin{tabular}{ll}
12 & Danksagung \\
\hline
\end{tabular}

$\begin{array}{ll}13 \text { Selbständigkeitserklärung } & 339\end{array}$ 

1 Allgemeine Einleitung 


\subsection{Warum und wofür Totalsynthese?}

Unter einer Totalsynthese wird die chemische Darstellung einer Substanz mit komplexer molekularer Struktur verstanden, bei der von einfachen und in der Regel kommerziell erhältlichen Startmaterialien ausgegangen wird. Die Synthese von Naturstoffen bzw. Biomolekülen, von Organismen produzierte Verbindungen mit biologischer Funktion, hat in der organischen Chemie eine lange Tradition und diente $\mathrm{zu}$ Beginn vornehmlich zur Aufklärung der Struktur eines Moleküls. Sie wird oft als eine „Kunst“ angesehen, die das Aushängeschild der organischen Synthese darstellt. ${ }^{[1,2]}$ Ihr Sinn und Zweck wird dabei immer wieder kontrovers diskutiert. ${ }^{[3-8]}$

Wenn bekannt ist was in jedem einzelnen Syntheseschritt passiert, so ist das Resultat planund vorhersagbar. Herausragende frühe Beispiele dafür sind die Untersuchungen zur Struktur von Strychnin (1 in Abbildung 1.1) durch Robinson und dessen Totalsynthese von Woodward. ${ }^{[9-12]}$ Viele weitere Meilensteine aus den Anfängen der Totalsynthese ließen sich aufzählen. ${ }^{[13]}$

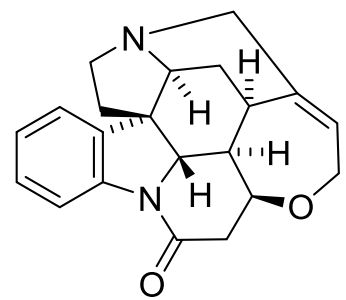

1: Strychnin<smiles>Oc1cccc(CCN2C(c3cccc(O)c3)=C[C@@]3(c4cccc(O)c4)c4c(ccc(O)c4O)C[C@@H]23)c1</smiles>

2: Haouamin B

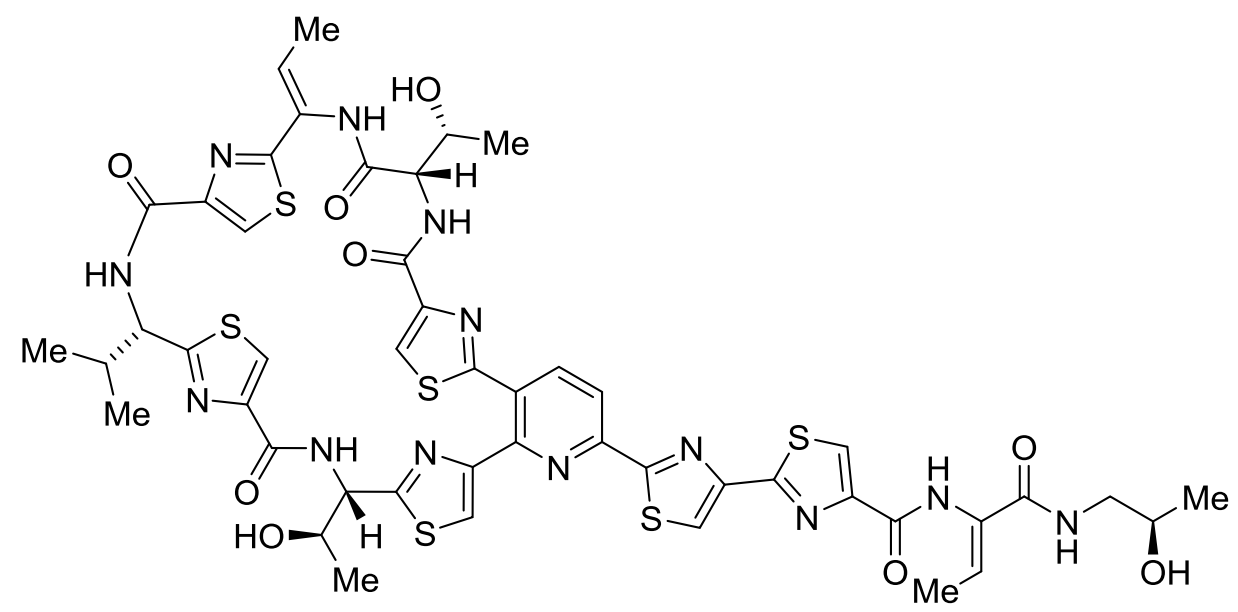

3: Micrococcin P1

Abbildung 1.1: Totalsynthetisch dargestellte Naturstoffe. 
Dank des Fortschritts in der Strukturanalyse, insbesondere durch die EinkristallRöntgenstrukturanalyse und die Kernspinresonanzspektroskopie, ist die Strukturaufklärung als Motivation jedoch stark in den Hintergrund getreten.

Trotz dieses Fortschritts wird auch heute noch erst durch eine Totalsynthese die korrekte Struktur eines Naturstoffs wirklich aufgeklärt. Als zwei von vielen Beispielen seien hier die Strukturen des Alkaloids Haouamin B (2) und des Thiopeptids Micrococcin P1 (3) genannt (Abbildung 1.1). ${ }^{[14,15]}$ In der fast 200 jährigen Geschichte der Naturstoffsynthese haben sich die Motivation und die dabei angelegten Maßstäbe immer wieder verändert. ${ }^{[16]} \mathrm{Zu}$ Beginn des 21. Jahrhunderts stellt sie ein wichtiges Element sowohl in der Entdeckung neuer Wirkstoffe als auch in der „Chemischen Biologie“ dar. ${ }^{[16,17]}$

Eine Vielzahl an neu zugelassenen Wirkstoffen sind auch heute noch ein Naturstoff, ein Derivat eines Naturstoffs oder ein synthetisches Molekül, das einem Naturstoff gleicht. ${ }^{[18]}$ Die Möglichkeiten einen vorliegenden Naturstoff strukturell $\mathrm{zu}$ modifizieren sind oft recht eingeschränkt. Mit der Entwicklung einer Totalsynthese des Zielmoleküls, die eine Diversität von modifizierten Produkten ermöglicht, eröffnen sich viele neue Optionen. Neben der gezielten Untersuchung von Struktur-Aktivitäts-Beziehungen ermöglichen die mit charakteristischen Strukturelementen erhaltenen Zwischenstufen vielleicht die Identifikation des minimalen Pharmakophors eines Wirkstoffs. ${ }^{[19]}$ Ein prominentes Beispiel dafür ist Eribulin (4, Abbildung 1.2, Handelsname: Halaven ${ }^{\circledR}$, Hersteller: Eisai). Dieses vom Naturstoff Halichondrin B abgeleitete Zytostatikum stellt derzeit den strukturell komplexesten nicht-peptidischen Wirkstoff dar, der totalsynthetisch produziert wird. ${ }^{[18]}$

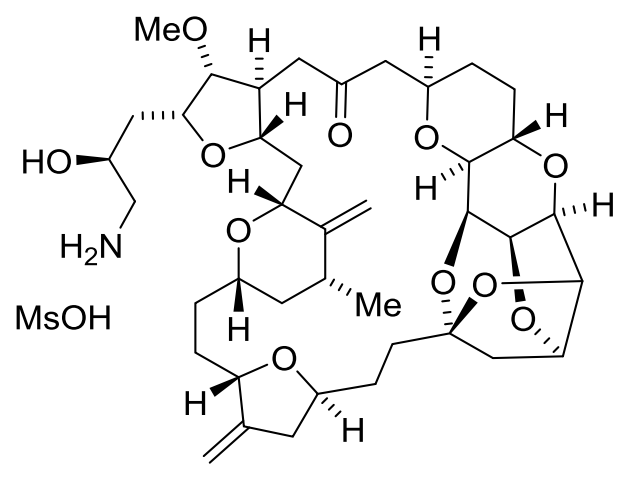

4: Eribulin

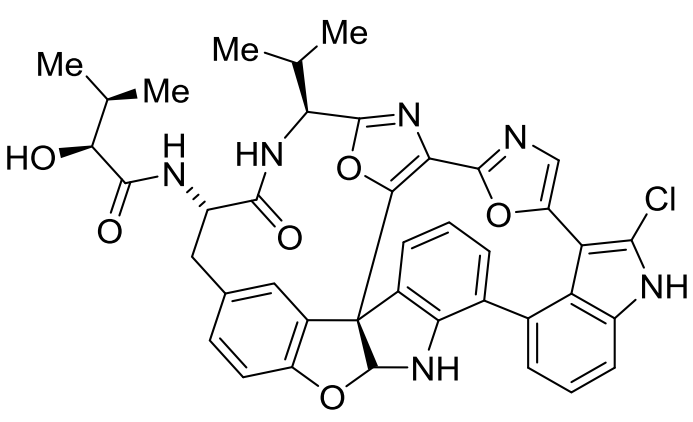

5: Diazonamid A

Abbildung 1.2: Struktur der totalsynthetisch dargestellten Wirkstoffe Eribulin (4) und Diazonamid A (5). 
Die Entwicklung der Synthese dieses Wirkstoffs beruhte maßgeblich auf der Totalsynthese von Halichondrin B durch Kishi et al.. ${ }^{[20]}$ Dies zeigt, wie wichtig die während einer Totalsynthese gewonnene Erkenntnis zur Chemie des Zielmoleküls ist.

Die Totalsynthese von Diazonamid A (5, Abbildung 1.2) durch die Gruppe von Harran und die darauf aufbauenden Studien verdeutlichen, welche entscheidende Rolle die Naturstoffsynthese für das Verständnis biologischer Systeme einnehmen kann. ${ }^{[21,22]}$ Mit Hilfe des totalsynthetischen Zugangs war es möglich, ein biotinyliertes Derivat von Diazonamid A zu synthetisieren und dessen Wirkungsort, die Ornithin- $\delta$-Aminotransferase, zu identifizieren. Dabei konnte auch die Wirkungsweise des Proteins aufgeklärt und dieses als potentielle Zielstruktur für neuartige Zytostatika ermittelt werden. ${ }^{[22]}$

Wären Glycoproteine mit einer Masse von dutzenden Kilodalton (kDa) als Zielstrukturen noch vor wenigen Jahren nur schwer vorstellbar gewesen, so konnte die Gruppe von Danishefsky vor kurzem sowohl die $\beta$-Untereinheit des humanen follikelstimulierenden Hormons $(17.9 \mathrm{kDa}){ }^{[23]}$ als auch Erythropoietin $(20.6 \mathrm{kDa})^{[24]}$ durch gezielte Synthese erfolgreich darstellen. Diese Errungenschaft markiert die Grenzen des heute Machbaren. Wobei angemerkt werden muss, dass sich diese Grenzen durch die Fortentwicklung der Synthesewissenschaft stetig weiter verschieben.

Das unabdingbare Herzstück einer jeden Totalsynthese ist jedoch, die Fähigkeiten und das chemische Verständnis des jeweiligen Chemikers weiter zu entwickeln. Wie und wieso finden Reaktionen statt? Wie steht dies im Zusammenhang mit der Struktur des Moleküls? Lassen sich neuartige Transformationen finden, oder bekannte im neuen Kontext überhaupt einsetzen? Wie können Probleme in möglichst kurzer Zeit gelöst werden und wie geht man mit den vielen Rückschlägen um, die unweigerlich auftreten? Während einer aufwendigen Totalsynthese ist die Organisation der „Nachschubwege“ von immenser Bedeutung und bedarf sorgfältiger Planung. Am Ende jeder Totalsynthese steht ein Kunstwerk, denn „künstlich“ ist das jeweilige Produkt in jedem Aspekt. Jedoch wurde durch die Erforschung von chemischen Transformationen ein Erkenntnisfortschritt erzielt, der die organische Chemie in jedem Fall weiterentwickelt.

Der Wissenschaftszweig der Naturstoffsynthese konnte in seiner über zwei Jahrhunderte langen Geschichte mit den wechselnden Anforderungen stetig wachsen. „Vor allem, wenn sich weiterhin Wissenschaftler finden, die sich in ein Molekül mit einer neuartigen Struktur verlieben und verbeißen und dann bedingungslos darum kämpfen, einen Syntheseweg zu erschließen,“ [16] wird die Naturstoffsynthese auch die Herausforderungen des 21. Jahrhunderts annehmen und bewältigen können. 


\subsection{Totalsynthese im Rahmen dieser Arbeit}

Das Ziel dieser Arbeit war die Totalsynthese des Sekundärmetaboliten Nosiheptid (6 in Abbildung 1.3). Der Naturstoff wurde zu Beginn der 1960er Jahre durch die Firma RhônePoulenc S. A. (heute Teil von Sanofi S. A.) in Frankreich aus dem Actinomyceten Streptomyces actuosus 40037 isoliert. ${ }^{[25]}$ Nosiheptid gehört zur Klasse der tetrasubstituierten Thiopeptidantibiotika ${ }^{[26-29]}$ und zeigt in vitro ein breites Wirkungsspektrum gegen Grampositive Bakterien. ${ }^{[25,30]}$ In einer späteren Wiederentdeckung aus einem marinen Produzenten, wurde gezeigt, dass Nosiheptid auch gegen klinisch relevante Methicillin-resistente Staphylococcus aureus (MRSA) Stämme antibiotisch aktiv ist. ${ }^{[31]}$ Nosiheptid inhibiert die Proteinbiosynthese durch Wechselwirkung mit dem bakteriellen Ribosom (s. Kap. 1.3.2). ${ }^{[32,33]}$

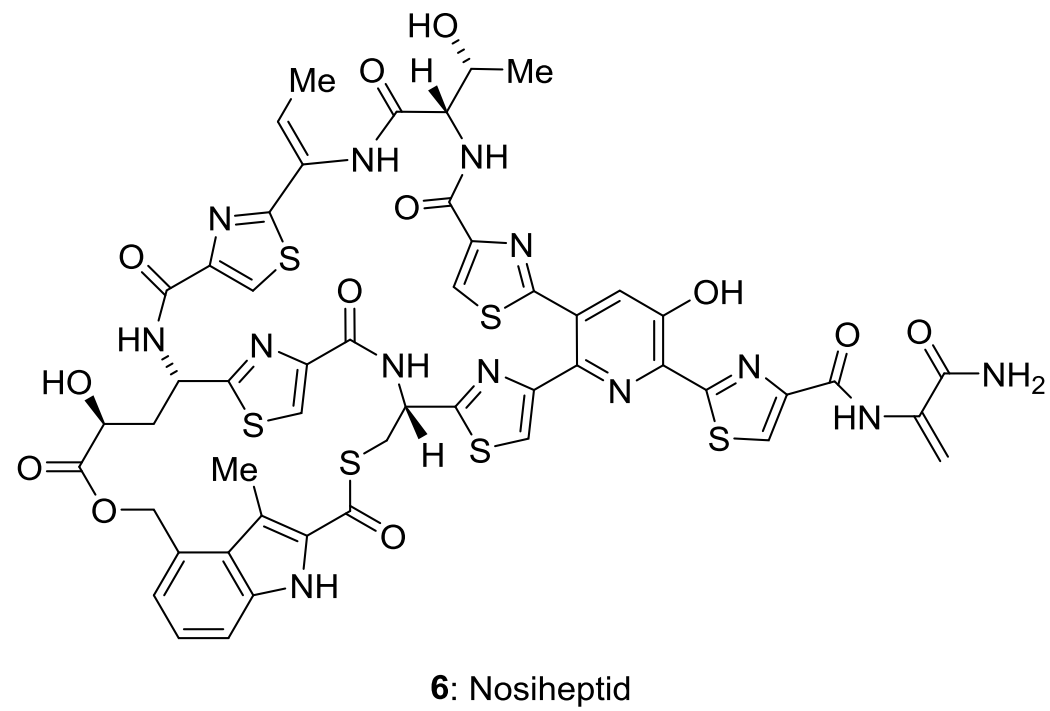

Abbildung 1.3: Struktur des Thiopeptidnaturstoffs Nosiheptid.

Trotz seiner hohen Potenz wird Nosiheptid derzeit nicht klinisch genutzt. Hauptgrund dafür ist die geringe Wasserlöslichkeit und mangelnde Bioverfügbarkeit. Bisher wurde es nur als Futterzusatzmittel in der Schweine- und Geflügelmast eingesetzt. ${ }^{[34,35]}$

Eine erfolgreiche und modulare Synthesestrategie würde die gezielte Darstellung von derzeit nicht zugänglichen Derivaten ermöglichen. Damit wäre es unter anderem möglich die physikochemischen Eigenschaften von Nosiheptid $\mathrm{zu}$ verbessern oder weitere Untersuchungen zu Wirkort(en) und Wirkungsmechanismus durchzuführen. Als Grundlage eines neuen Anlaufs zu einer Totalsynthese dienten die in der Arbeitsgruppe durchgeführten Arbeiten von Dr. Matthias Riedrich und Dr. Jin-Yong Lu. ${ }^{[36,37]}$

Die Klasse der Thiopeptidantibiotika wird einleitend im Kapitel 1.3 vorgestellt. Dabei werden 
ihre strukturellen und biologischen Eigenschaften beleuchtet. Anschließend wird die vorliegende Arbeit in den Kontext bereits abgeschlossener Totalsynthesen gestellt (Kap. 1.4).

\subsection{Thiopeptidantibiotika}

Die Naturstoffklasse der Thiopeptidantibiotika (auch als Thiazolylpeptidantibiotika oder Thiopeptide bezeichnet) besteht aus schwefelreichen, hochmodifizierten cyclischen Sekundärmetaboliten peptidischen Ursprungs. Produziert werden sie hauptsächlich von Actinomyceten aus der Gattung Streptomyces. Sie inhibieren die Proteinbiosynthese in Grampositiven Bakterien. ${ }^{[26-29]}$ Heute sind mehr als einhundert Thiopeptidantibiotika bekannt. ${ }^{[29]}$ Als erstes Mitglied dieser Gruppe wurde Micrococcin P1 (3) bereits 1948 entdeckt. ${ }^{[38]}$ Einige Jahre später wurde mit Thiostrepton (7 in Abbildung 1.4) eines der bedeutendsten Thiopeptidantibiotika aus Streptomyces azureus ATC 14921 isoliert. $^{[39-41]}$ Dieses Thiopeptidantibiotikum wird in der Veterinärmedizin topisch genutzt. ${ }^{[42]}$

\subsubsection{Strukturelle Merkmale}

Die Thiopeptidantibiotika sind peptidischen Ursprungs und grundsätzlich aus $\alpha$-Aminosäuren aufgebaut. Ein Thiopeptidvorläuferpeptid wird in einem ribosomalen Prozess aufgebaut und anschließend posttranslational modifiziert. ${ }^{[43]}$ Sie bestehen vorrangig aus Heterocyclen, die häufig in Makrocyclen eingebettet sind. Die wichtigsten Vertreter sind Thiazol, Oxazol, Indol und Pyridin, wobei wie z.B. in Thiostrepton (7, Abbildung 1.4) auch deren reduzierte Formen vorkommen. Neben einem zentralen heterocyclischen Kern, der ein tri- oder tetrasubstituiertes Pyridin und Teil eines Makrocyclus ist, enthalten Thiopeptidantibiotika als gemeinsames strukturelles Merkmal Dehydroaminosäuren, die häufig als ein „Schwanz“ angehängt sind.

Die Oxidationsstufe und das Substitutionsmuster des azolsubstituierten zentralen stickstoffhaltigen Sechsrings definieren dabei die Zugehörigkeit zu einer, der heute bekannten fünf Klassen A-E (Abbildung 1.4). 


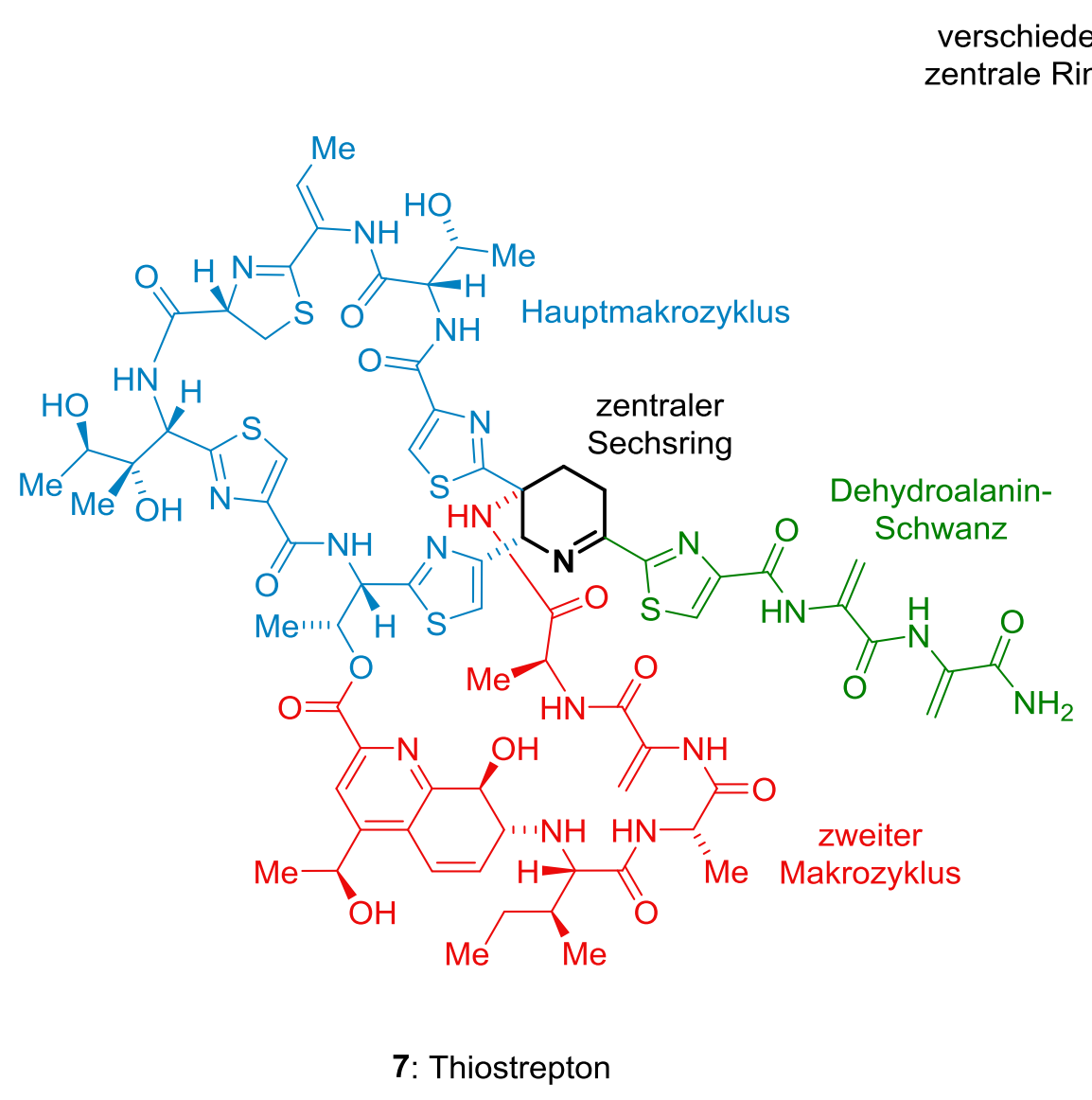

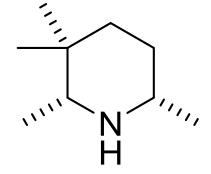

Klasse A<smiles>CC1=N[C@H](C)C(C)(C)CC1</smiles>

Klasse B<smiles>CC1=NC(C)(C)N2C1C(C)(C)CC[C@@H]2C</smiles>

Klasse C<smiles>Cc1ccc(C)c(C)n1</smiles>

Klasse D<smiles>Cc1cc(O)c(C)nc1C</smiles>

Klasse E

Abbildung 1.4: Struktur von Thiostrepton mit farblich hervorgehobenen Strukturelementen und Klassifizierung der Thiopeptidantibiotika auf Grundlage des zentralen Sechsrings. ${ }^{[26]}$ Adaptiert nach $\mathrm{X}$. Just-Baringo et al.. ${ }^{[44]}$

$\mathrm{Zu}$ den Klassen $\mathrm{A}$ und $\mathrm{B}$ werden die strukturell sehr ähnlichen Piperidine bzw. Dehydropiperidine (z. B. Thiostrepton) gezählt. Deren charakteristisches bismakrocyclisches Grundgerüst weist neben Thiazol(inen) und Dehydroaminosäuren eine Chinolinsäure auf. Der einzige Vertreter der Klasse $\mathrm{C}$ ist $\mathrm{Sch} 40832,{ }^{[45]}$ das einen einzigartigen bicyclischen Dihydroimidazopiperidin-Kern aufweist, ansonsten aber sehr eng mit den Klassen A und B verwandt ist. Die größte Klasse D umfasst die meist monomakrocyclischen (2,3,6-triazolyl) trisubstituierten Pyridine (z. B. Micrococcin P1, 3). Thiazolylpeptidantibiotika der Klasse E kennzeichnet ein zum 3-Hydroxypyridin oxidierter Kern (z. B. Nosiheptid, 6). Diese Klasse weist zusätzlich ein 2,3,4-substituiertes Indol bzw. $N$-Hydroxyindol auf, welches Bestandteil eines zweiten Makrocyclus ist. Erwähnenswert ist, dass viele Mitglieder der Klasse E auch mit Aminozuckern (Nocathiacine ${ }^{[46-48]}$ und Thiazomycine ${ }^{[49,50]}$ ) oder methylierten Desoxyzuckern (Philipimycin ${ }^{[51]}$ ) glykosyliert vorkommen. 


\subsubsection{Biologische Aktivität}

Ein Großteil der Thiopeptide besitzt eine antibiotische Wirkung. Darüber hinaus wurden aber auch antiproliferative Wirkungen gegenüber Krebszelllinien ${ }^{[52-60]}$ und dem Malariaerreger

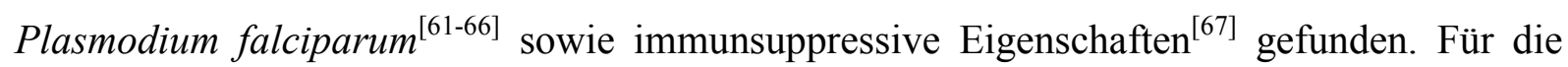
strukturell sehr ungewöhnlichen bismakrocyclischen Cyclothiazomycine ${ }^{[68-70]}$ (Klasse D) wurden die Inhibition der Endopeptidase Renin ${ }^{[68]}$ und einer RNA-Polymerase ${ }^{[69]}$ sowie eine antimykotische Wirkung ${ }^{[71]}$ gezeigt. Die selektive antibiotische Aktivität der Cyclothiazomycine B und C gegen Bakterien der Gattung Bacillus fällt besonders auf. ${ }^{[70]}$

\subsubsection{Antibakterielle Aktivität}

Die ausgiebig untersuche antibiotische Wirkung der Thiopeptidantibiotika beruht auf der Inhibition der ribosomalen Proteinsynthese. Hervorzuheben ist, dass dabei zwei von der Größe des Makrocyclus abhängige Mechanismen vorherrschen. Die Thiopeptidantibiotika mit 26-gliedrigem Ring (z. B. Thiostrepton und Nosiheptid) binden an die GTPase-assoziierte Region des Ribosoms zwischen 23S rRNA und dem Protein L11. Sie verhindern damit das Binden des Elongationsfaktors G (EF-G), wodurch es zum Erliegen der Peptidtranslokation kommt (Abbildung 1.5, a). ${ }^{[32,72-77]}$

a

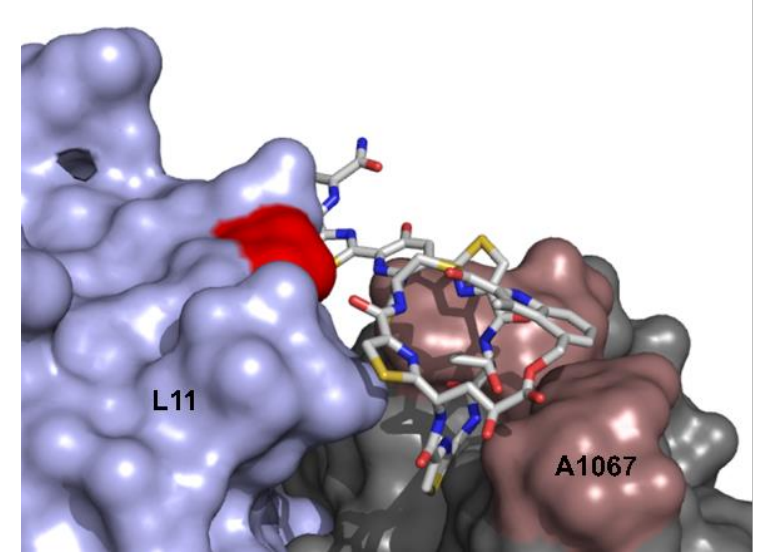

b

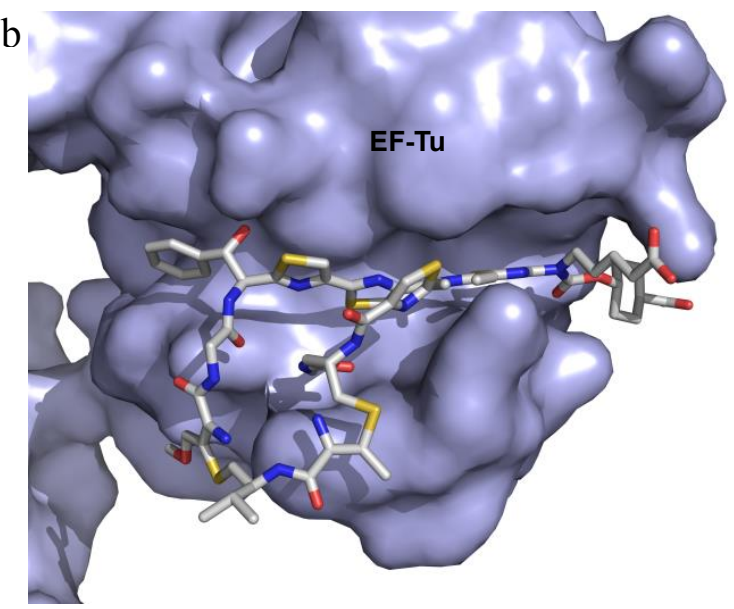

Abbildung 1.5: Bindungsmodi verschiedener Thiopeptide an der Zielstruktur. a) Komplex aus Nosiheptid mit der 23S rRNA (braun/grau) und dem ribosomalen Protein L11 (violett) bei $3.7 \AA$ Auflösung (PDB 2ZJP, ${ }^{[32]}$ entnommen aus Riedrich ${ }^{[36]}$ ). b) Komplex aus LFF571 mit dem Elongationsfaktor Tu (violett) bei $2.7 \AA$ Auflösung (PDB 3U2Q). ${ }^{[78]}$ 
Die 29-gliedrigen Thiopeptidantibiotika, wie GE2270 A und das semi-synthetische Derivat LFF571 ${ }^{[78]}$, binden hingegen an den Elongationsfaktor Tu (EF-Tu) und verhindern dort die

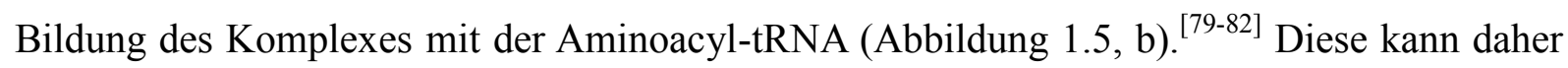
nicht zum Ribosom transportiert werden, was die Verlängerung der Peptidkette beeinträchtigt. Die Gruppe der 35-gliedrigen Thiopeptidantibiotika besitzen die größte bekannte Ringgröße (z. B. Berninamycine ${ }^{[83-86]}$ und Promoinducin ${ }^{[87]}$ ) und zeigen ebenfalls eine antibiotische Wirkung. Die Zielstruktur und der Wirkungsmechanismus sind derzeit nicht näher bekannt. Untersuchungen deuten jedoch darauf hin, dass Berninamycin A ähnlich wie Thiostrepton (7) wirkt. ${ }^{[83,88]}$ Einige Thiopeptidantibiotika unterschiedlicher Ringgröße sind in Abbildung 1.6 dargestellt. Die Bindungen des Makrocyclus sind hervorgehoben.

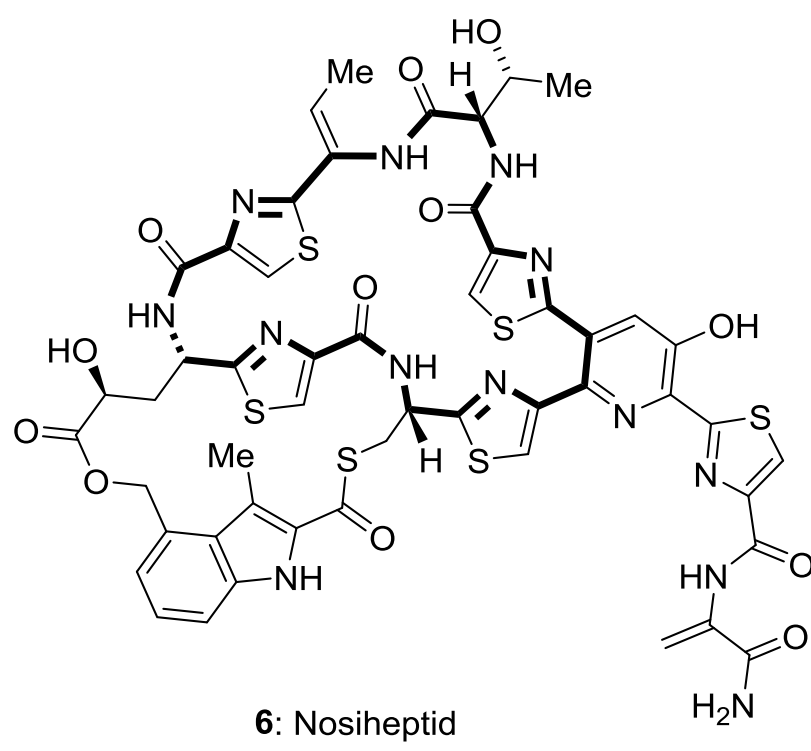

(26-gliedriger Ring)

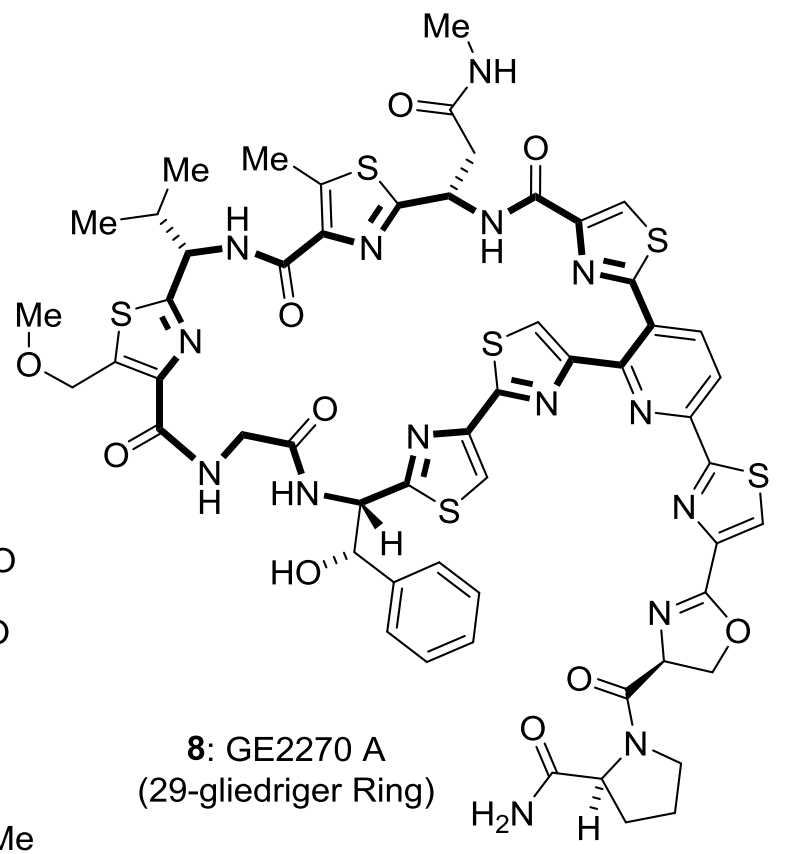

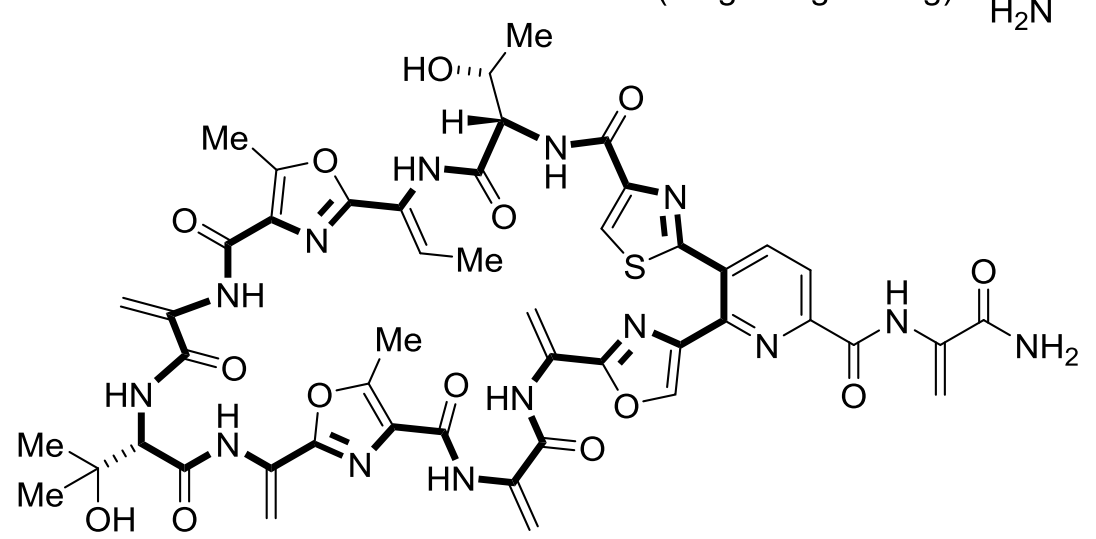

9: Berninamycin C (35-gliedriger Ring)

Abbildung 1.6: Die Ringgröße des Thiopeptids bestimmt seinen Wirkungsmechanismus. 


\subsubsection{Induktion der tipA-Gene}

Einige Streptomyceten, die nicht in der Lage sind Thiopeptidantibiotika zu produzieren, besitzen überraschenderweise einen Signalmechanismus, bei dem zwei so genannte Thiostrepton-induzierte Proteine, TipAL (L: long) und TipAS (S: short), beteiligt sind. ${ }^{[89]}$ Beide Proteine besitzen Thiopeptid-spezifische Erkennungsdomänen, TipAL wirkt als Transkriptionsfaktor. Das TipAS-Protein ist wahrscheinlich in der Lage, über eine SulfaMichael-Addition ein Thiopeptidantibiotikummolekül am Dehydroaminosäureschwanz kovalent zu binden. ${ }^{[90,91]}$ Die Funktion dieser ungewöhnlichen Proteine ist weitgehend unbekannt und Gegenstand aktueller Forschung. Nicht-kovalente Interaktionen bei der Erkennung der unterschiedlichen Thiopeptidstrukturen spielen dabei anscheinend eine entscheidende Rolle. ${ }^{[92]}$ Die Eigenschaften von Substanzen das tipA-Gen zu induzieren, wurde erfolgreich in Screenings eingesetzt, um neue Thiopeptidnaturstoffe zu finden. ${ }^{[93-96]}$

\subsection{Totalsynthesen von Thiopeptidantibiotika}

Die Strukturvielfalt der Thiopeptidantibiotika stellt die Syntheseforschung vor große Herausforderungen. Daher haben viele Gruppen verschiedene Ansätze verfolgt, um Thiopeptide zu synthetisieren. Ein Großteil der Arbeiten beschäftigt sich mit dem Aufbau des zentralen Sechsring-Heterocyclus. Dabei werden zwei grundlegende Strategien eingesetzt: zum einen die Manipulation eines Pyridins, und zum anderen der Aufbau des Heterocyclus mittels Cycloadditionen. Diese Arbeiten wurden erst kürzlich in einer Übersicht dargestellt. ${ }^{[29]}$ Die Anzahl an tatsächlich abgeschlossenen Totalsynthesen bleibt jedoch recht gering (Tabelle 1.1). Mehrere der Synthesen leiten sich dabei voneinander ab, indem für sehr ähnliche Zielmoleküle die gleichen Synthesemethoden und -strategien eingesetzt wurden. ${ }^{[29]}$ Die überwiegende Mehrheit hatte ein monomakrocyclisches Thiopeptid der Klasse D als Ziel. Nur zwei Totalsynthesen bismakrocyclischer Thiopeptidantibiotika aus zwei unterschiedlichen Klassen waren zu Beginn dieser Arbeit bekannt, wobei Thiostrepton und Siomycin A eine sehr große Ähnlichkeit aufweisen (Tabelle 1.1, unten). Synthesen von Thiopeptiden der Klasse E waren nicht bekannt. 
Tabelle 1.1: Übersicht bereits totalsynthetisch dargestellter Thiopeptidantibiotika, gegliedert nach Anzahl der Makrocyclen und Jahr der Veröffentlichung.

\begin{tabular}{ccc}
\hline Thiopeptidantibiotikum & Jahr & Gruppe \\
\hline monomakrocyclisch & & \\
Micrococcin P1/P2 & 1998 & Shin $^{[97,98]}$ \\
& 1999 & Shin $^{[99]} /$ Cuifolini $^{[100]}$ \\
& $\mathbf{2 0 0 9}$ & Ciufolini $^{[14]}$ \\
Promothiocin A & 1998 & Moody $^{[101,102]}$ \\
Amythiamicin D & 2004 & Moody $^{[103,104]}$ \\
GE2270 A/T & 2006 & Nicolaou $^{[105,106]}$ \\
GE2270 A & 2007 & Bach $^{[107,108]}$ \\
Amythiamicin A/B/C & 2008 & Nicolaou $^{[109]}$ \\
Amythiamicin C/D & 2010 & Bach $^{[110]}$ \\
Thiocillin I & 2011 & Ciufolini $^{[111]}$ \\
Baringolin & 2013 & Albericio $^{[112]}$ \\
\hline bismakrocyclisch & & \\
Thiostrepton & 2004 & Nicolaou \\
Siomycin A & 2007 & Hashimoto, Nakata $^{[113-116]}$ \\
Nosiheptid & 2016 & diese Arbeit $^{[120]}$ \\
\hline
\end{tabular}

Die ersten Totalsynthesen wurden für vorgeschlagene Strukturen der Micrococcine P1 (3) und P2 in den Jahren 1998/99 von Shin et al. und Ciufolini et al. berichtet. ${ }^{[97-100]}$ Jedoch wurde erst zehn Jahre später durch Ciufolini et al. die korrekte Konstitution und Stereochemie in einer weiteren Totalsynthese korrigiert und final aufgeklärt. ${ }^{[14]}$ Somit wäre die erste Totalsynthese eines Thiopeptidantibiotikums die des Promothiocins A aus dem Jahre 1998 durch Moody et al.. ${ }^{[101,102]}$ Erwähnenswert ist die Totalsynthese von GE2270 A (8) durch die Gruppe von Bach aus München. Die Funktionalisierung des zentralen Pyridins erfolgte dabei durch aufeinanderfolgende, regioselektive Kreuzkupplungen (vgl. Kap. 1.4.1). ${ }^{[107,108]}$

Die erste Totalsynthese eines bismakrocyclischen Thiopeptidantibiotikums (Thiostrepton, 7) gelang der Gruppe von Nicolaou im Jahre 2004. ${ }^{[113-116]}$ Die Totalsynthese des strukturell sehr ähnlichen Siomycins A folgte 2007 in den Gruppen von Hashimoto und Nakata. ${ }^{[117-119]}$ Die im Rahmen der vorliegenden Arbeit erfolgreich entwickelte Totalsynthese von Nosiheptid (6) ist die erste für ein Thiopeptidantibiotikum der tetrasubstituierten Klasse E und gleichzeitig erst die dritte für einen bismakrocyclischen Vertreter dieser Naturstofffamilie. ${ }^{[120]}$

Die Totalsynthesen von Amythiamicin D, der GE2270-Reihe, "Micrococcin P1", Promothiocin A, Siomycin A und Thiostrepton wurden in Übersichtsartikeln ${ }^{[27,28]}$ und Dissertationen $^{[36,37]}$ der Arbeitsgruppe bereits ausführlich diskutiert. Daher soll im folgenden 
Abschnitt beispielhaft die kürzlich erschienene Totalsynthese des monomakrocyclischen Baringolins analysiert werden.

\subsubsection{Diskussion der Totalsynthese von Baringolin}

Das Thiopeptidantibiotikum Baringolin (10 in Schema 1.1) wurde 2012 durch die spanische Firma Biomar SA entdeckt. ${ }^{[121]}$ Seine Struktur wurde mittels NMR-Spektroskopie und hochaufgelöster Massenspektrometrie aufgeklärt. Für Baringolin wurde eine antibakterielle Aktivität im nanomolaren Bereich gegenüber Gram-positiven Bakterien und eine moderate Zytotoxizität gegenüber Krebszelllinien nachgewiesen. ${ }^{[121]}$ Es gehört zur trisubstituierten Klasse D mit einem 29-gliedrigen Makrozyklus und besitzt einen ungewöhnlich langen Dehydroamino-säureschwanz. Strukturell ist es sehr eng mit dem bereits bekannten GE37468 $\mathrm{A}^{[122,123]}$ verwandt. Die Totalsynthese wurde 2013 durch Baringo et al. publiziert und nutze - der Präzedenz von Bach ${ }^{[107,108]}$ grob folgend - sequentielle Kreuzkupplungen zum Aufbau des zentralen Pyridinkerns. ${ }^{[120]}$ Die retrosynthetische Analyse von Baringolin ist in Schema 1.1 dargestellt.

Durch Auflösen verschiedener Amidbindungen und Einführung latenter Funktionalitäten und Schutzgruppen wurde der Makrozyklus auf den hochsubstituierten Pyridinkern 11, das Pentapeptid 12, das Thiazolin 13, das geschützte Tyrosin 14 und den Thiazolbaustein 15 zurückgeführt. Das Pentapeptid 12 sollte über eine Festphasensynthese aus den jeweiligen Aminosäurebausteinen dargestellt werden, wobei für die Dehydroaminosäuren latente $\mathrm{PhSe}$ Maskierungen nach Shirahama und van der Donk eingeplant wurden. ${ }^{[124-126]}$ 


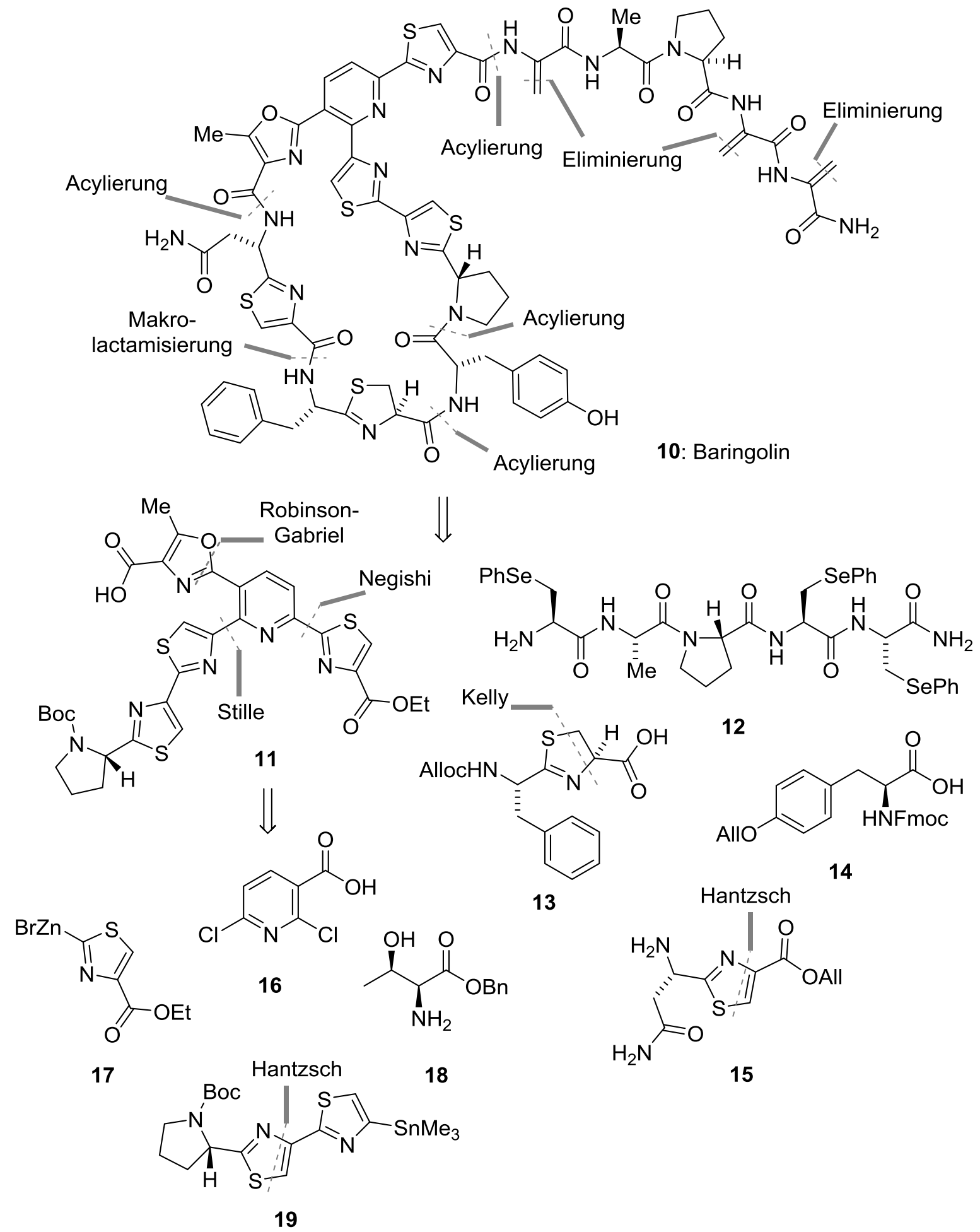

Schema 1.1: Struktur und retrosynthetische Analyse von Baringolin nach Baringo et al. ${ }^{[112]}$

Das Thiazolin 13 würde durch in situ Dehydrierung/Cyclisierung eines geeigneten $S$-Trgeschützten Dipeptids nach Kelly et al. ${ }^{[127]}$ zugänglich sein. Eine Hantzsch-Thiazolsynthese aus einem entsprechenden Thioamid und Brombrenztraubensäureester sollte das Thiazol 15 ergeben. ${ }^{[128]}$ Eine weitere Zerlegung des Pyridinkerns 11 führte auf 2,6-Dichlorpicolinsäure 
(16) zurück, dessen Carbonylrest zusammen mit $L$-Threoninbenzylester (18) als Vorläufer für das 5-Methyloxazol dienten. Das Pyridin 16 sollte über die unterschiedlichen $\alpha$-Substituenten in geeigneter Reihenfolge mit den Organometallverbindungen $17^{[108]}$ und 19 mittels Negishibzw. Stille-Kreuzkupplungen verknüpft werden. Das Bisthiazol 19 sollte ebenfalls über eine Hantzsch-Thiazolsynthese synthetisiert werden. ${ }^{[129]}$ Die Synthese der strukturell einfachen Bausteine 12, 13, 14, 15, und 19 wird nachfolgend nicht im Detail gezeigt.

In Schema 1.2 ist die Synthese des Triazolylpyridins 11 nach Baringo et al. dargestellt. Ausgehend von 2,6-Dichlornicotinsäure (16) wurde zur Vorbereitung sequentieller Kreuzkupplungen im ersten Schritt in Position 6 selektiv eine Methoxygruppe eingefügt und anschließend mit $L$-Threoninbenzylester das Amid 21 aufgebaut.<smiles>[R]c1ccc(C(=O)O)c(Cl)n1</smiles><smiles>[R10]C(=O)c1nc(-c2ccc(-c3nc(C(=O)OCC)cs3)nc2-c2csc(-c3csc([C@@H]4CCCN4[C@@H](C(=O)O)C(=O)OC(C)(C)C)n3)n2)oc1C</smiles>

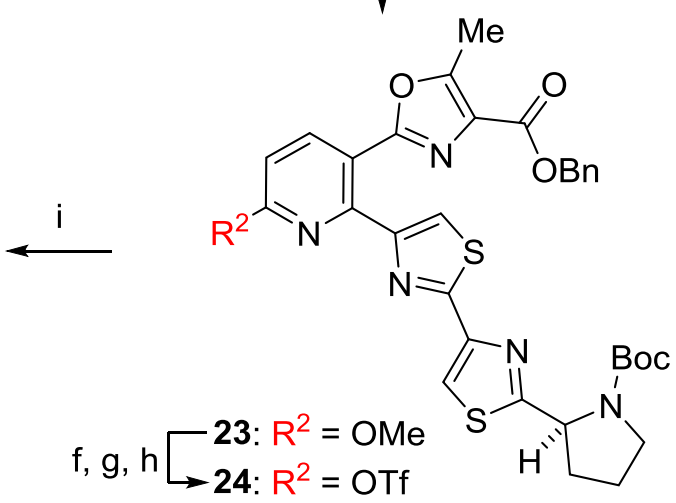

Schema 1.2: Synthese des Triazolylpyridins 11. Bedingungen: a) $t \mathrm{BuOK}, \mathrm{MeOH}, 65^{\circ} \mathrm{C}, 96 \mathrm{~h}, 85 \%$. b) 18, PyBOP, EtNiPr 2 THF, $0{ }^{\circ} \mathrm{C}, 3 \mathrm{~h}, 89 \%$. c) Dess-Martin-Periodinan, $\mathrm{CH}_{2} \mathrm{Cl}_{2}, \mathrm{RT}, 6 \mathrm{~h}, 95 \%$. d) $\mathrm{PPh}_{3}, \mathrm{I}_{2}, \mathrm{Et}_{3} \mathrm{~N}, \mathrm{CH}_{2} \mathrm{Cl}_{2}, 0{ }^{\circ} \mathrm{C} \rightarrow \mathrm{RT}, 15 \mathrm{~h}, 78 \%$. e) 19, $\mathrm{Pd}\left(\mathrm{PPh}_{3}\right)_{4}, 1,4$-Dioxan, $80{ }^{\circ} \mathrm{C}, 48 \mathrm{~h}, 88 \%$. f) $\mathrm{HBr}, \mathrm{AcOH}, \mathrm{RT}, 28 \mathrm{~h}, 73 \%$. g) (Boc) ${ }_{2} \mathrm{O}, \mathrm{Et}_{3} \mathrm{~N}, \mathrm{CH}_{2} \mathrm{Cl}_{2}, 0{ }^{\circ} \mathrm{C}, 4 \mathrm{~h}, 94 \%$. h) $\mathrm{Tf}_{2} \mathrm{O}, 2$, 6-Lutidin, DMAP, $\mathrm{CH}_{2} \mathrm{Cl}_{2}, 0{ }^{\circ} \mathrm{C} \rightarrow \mathrm{RT}, 3 \mathrm{~h}, 88 \%$. i) $17, \mathrm{Pd}\left(\mathrm{PPh}_{3}\right)_{4}, \mathrm{DMA}, 45^{\circ} \mathrm{C}, 1 \mathrm{~h}$, quant. j) $\mathrm{H}_{2}$ (1 atm), $\mathrm{Pd}$, $\mathrm{CH}_{2} \mathrm{Cl}_{2} / \mathrm{EtOH}(1: 1), \mathrm{RT}, 4 \mathrm{~h}$, quantitativ.

Nach Oxidation des Alkohols 21 zum entsprechenden Keton wurde über eine RobinsonGabriel-Cyclisierung das Oxazol 22 erhalten. ${ }^{[130]}$ Durch eine Stille-Kupplung wurde das Bisthiazol 19 eingeführt, der erhaltene Methylether 23 anschließend sauer entschützt und in 
das Triflat 24 umgewandelt. Nach Einführung des dritten Azols mit dem Zinkreagenz 17 über eine Negishi-Kupplung $(\mathbf{2 4}>\mathbf{2 5})$ wurde der Benzylester reduktiv zur Carbonsäure 11 gespalten.

Anschließend wurden sukzessiv die Bausteine 11, 13, 14 und 15 miteinander zum linearen Vorläufer 26 verknüpft, der Makrozyklus geschlossen und die Seitenkette eingeführt (Schema 1.3). Dabei wurde zuerst der Pyridinkern 11 mit dem Thiazolbaustein 15 zum Amid 26 gekuppelt. Nach anschließender $N$-Boc-Entschützung am Pyrrolidin wurde dieses mit dem geschützten Tyrosin 14 acyliert. Fmoc-Entschützung des Bis-Amids 27 und die Anknüpfung des Thiazolinbausteins 13 lieferten den Zyklisierungsvorläufer 28. Nach gleichzeitiger Pdvermittelter Alloc- und Allyl-Entschützung wurde der Makrozyklus geschlossen. Eine milde $\mathrm{Me}_{3} \mathrm{SnOH}^{[131]}$ vermittelte Verseifung des Ethylesters lieferte die Carbonsäure 29, welche anschließend mit dem Pentapeptid 12 verknüpft wurde. Abschließende oxidative Eliminierung der Phenylselenid-Gruppen lieferte den Naturstoff Baringolin (10).

Mit dem Abschluss der Totalsynthese konnten die Autoren durch Vergleich der Spektren von synthetischem und natürlichem Material die absolute Konfiguration der Stereozentren im Naturstoff eindeutig bestimmen. Dabei wurde die Annahme, dass alle Stereozentren sich von $L$-Aminosäuren ableiten, bestätigt. ${ }^{[112]}$ Dies korreliert mit dem biosynthetischen Ursprung der Thiopeptidantibiotika als ribosomale all- $L$-Peptide (vgl. Kap. 1.3.1). Eine zum isolierten Naturstoff vergleichbare antibiotische Aktivität der synthetischen Substanz wurde ebenfalls nachgewiesen. Die Modularität der Synthese erlaubt den Austausch einzelner Bausteine und somit die Synthese verschiedener Derivate, um Struktur-Aktivitäts-Beziehungen untersuchen zu können. Die Autoren konnten dies ins späteren Arbeiten erfolgreich zeigen. ${ }^{[132]}$ 

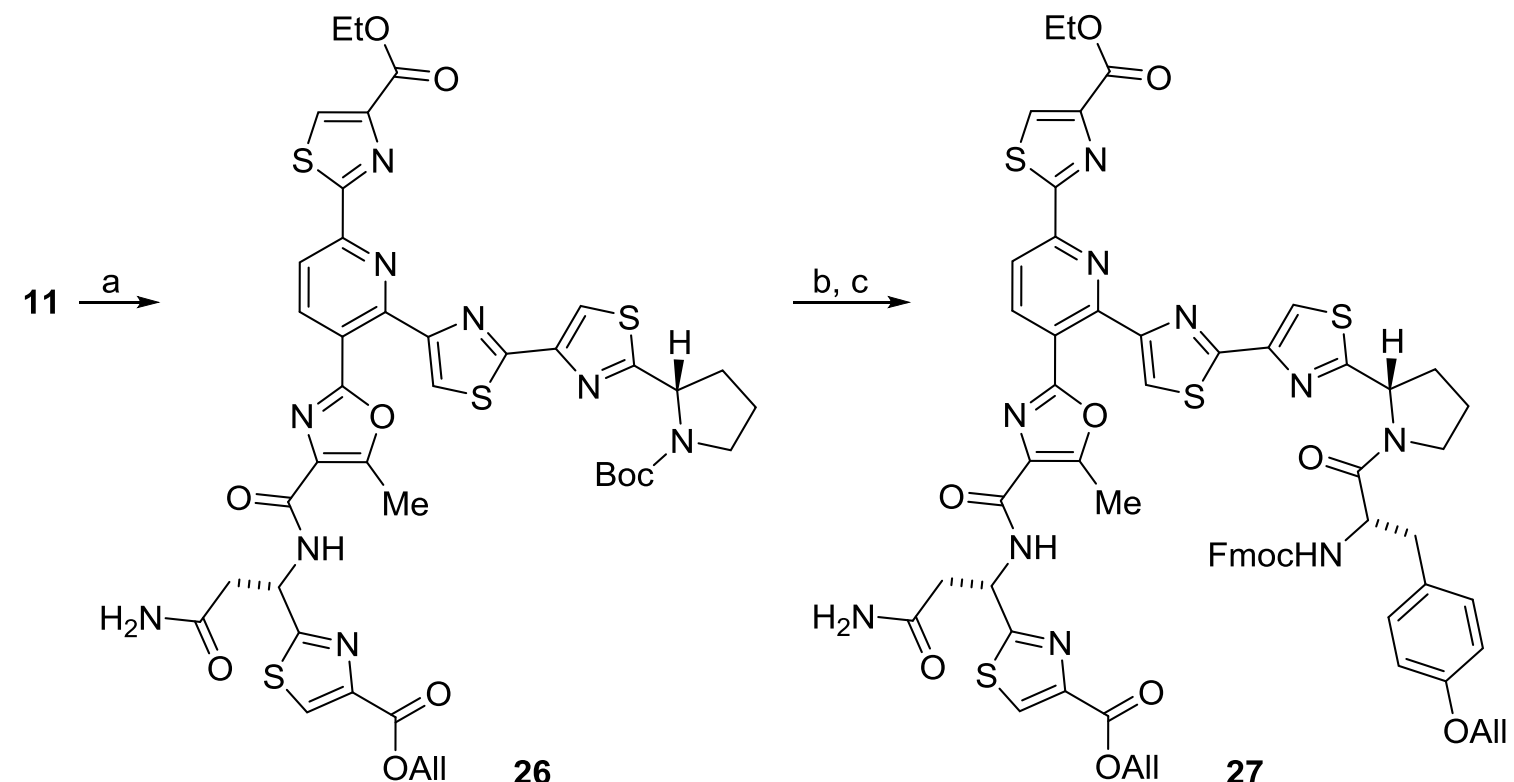

(n)

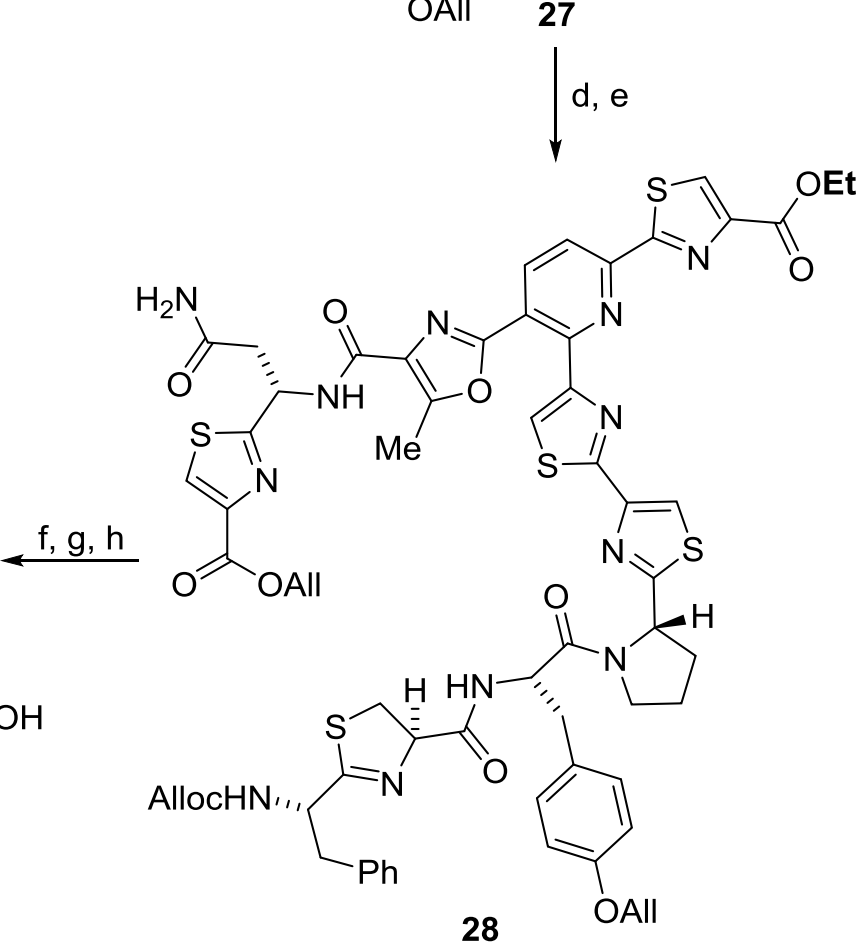

8

Schema 1.3: Abschluss der Totalsynthese von Baringolin. Bedingungen: a) 15, EDCI, HOAt, $i \mathrm{Pr}_{2} \mathrm{NEt}$, DMF, $0{ }^{\circ} \mathrm{C}, 18$ h, 82\%. b) HCl, 1,4-Dioxan, RT, 7 h. c) 14, EDCI, HOAt, EtNiPr 2 , DMF, $0{ }^{\circ} \mathrm{C}, 7 \mathrm{~h}$, $71 \%$ (ü. 2 Stufen). d) Piperidin, $\mathrm{CH}_{2} \mathrm{Cl}_{2}$, RT, 3 h, 87\%. e) 13, EDCI, HOAt, EtNiPr ${ }_{2}, \mathrm{DMF}, 0{ }^{\circ} \mathrm{C}, 3 \mathrm{~h}$, $68 \%$. f) $\mathrm{Pd}\left(\mathrm{PPh}_{3}\right)_{4}, \mathrm{PhSiH}_{3}, \mathrm{CH}_{2} \mathrm{Cl}_{2}$, RT, 7 h. g) EDCI, HOAt, EtNiPr 2 , DMF (1 mM), $0{ }^{\circ} \mathrm{C} \rightarrow \mathrm{RT}$, 21 h, $30 \%$ (ü. 2 Stufen). h) $\mathrm{Me}_{3} \mathrm{SnOH}, \mathrm{DCE}, 60^{\circ} \mathrm{C}, 19$ h. i) 12, EDCI, HOAt, EtNiPr $2, \mathrm{DMF}, 0{ }^{\circ} \mathrm{C}$, 3 h, $81 \%$ (ü. 2 Stufen). j) $t \mathrm{BuOOH}, \mathrm{CH}_{2} \mathrm{Cl}_{2}, \mathrm{RT}, 12$ h, $66 \%$. 


\subsection{Nosiheptid als Syntheseziel}

\subsubsection{Strukturcharakteristika und verwandte Moleküle}

Nosiheptid gehört mit seinem tetrasubstituierten Pyridinkern zur Klasse E der Thiopeptidantibiotika. Die Struktur von Nosiheptid (6, Abbildung 1.7) wurde in den 1970er Jahren durch chemischen Abbau, ${ }^{[133,134]}$ isotopenmarkierte Fütterungsexperimente und ausführliche NMR-Studien ${ }^{[135-137]}$ sowie Röntgenkristallstrukturanalyse ${ }^{[138,139]}$ aufgeklärt.

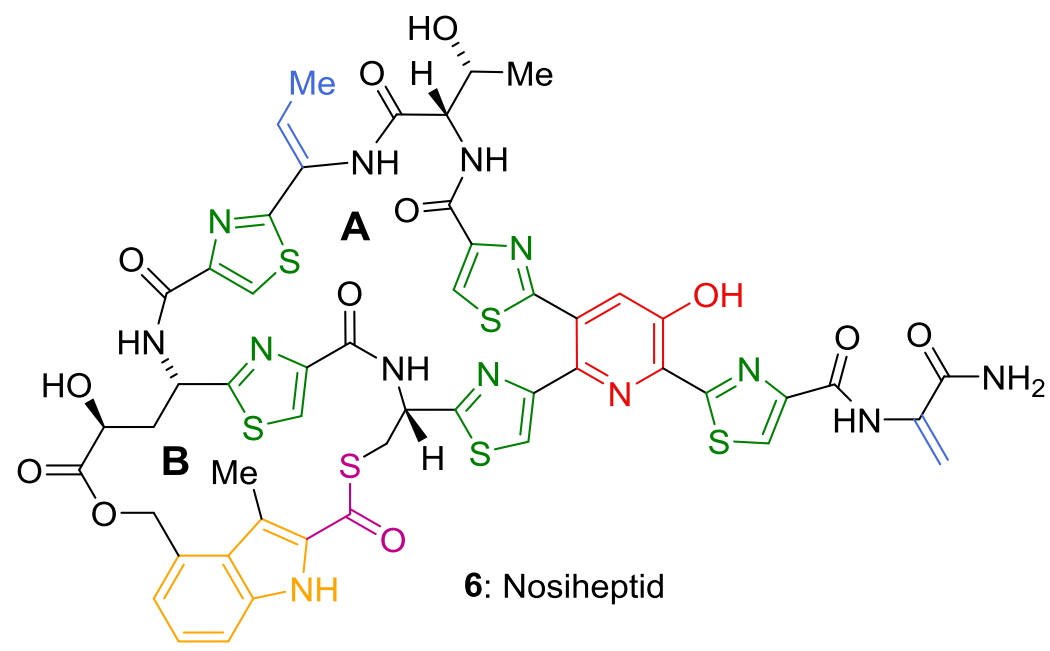

Abbildung 1.7: Struktur von Nosiheptid mit farblich hervorgehobenen strukturellen Merkmalen.

Der Bismakrocyclus besitzt als heteroaromatische Struktureinheiten neben dem zentralen 3-Hydroxypyridinkern (rot) fünf 2,4-disubstituierteThiazole (grün) und ein trisubstituiertes Indol (gelb). Daneben sind zwei Dehydroaminosäuren (blau) im Molekül enthalten. Der große 26-gliedrige Makrocyclus wird als A-Ring bezeichnet und besteht aus dem 3-Hydroxypyridin, vier Thiazolen und einem $L$-Threonin. Der kleinere 18-gliedrige B-Ring wird durch das 3-Methylindol (gelb) gebildet. Dieser B-Ring enthält ein in Naturstoffen sehr selten vorkommendes Thiolacton (magenta).

In der Biosynthese von Nosiheptid werden das Dehydroalanin und -butyrin aus Serin bzw. Threonin gebildet, ${ }^{[135]}$ während die Thiazole aus Cysteinresten abgeleitet werden. ${ }^{[140]}$ Der zentrale Pyridin-Kern scheint über eine [4+2]-Cycloaddition zweier Dehydroalaninreste unter gleichzeitigem Ringschluss des peptidischen Makrocyclus aufgebaut zu werden. ${ }^{[141,142]}$ Die Oxidation zum 3-Hydroxypyridin erfolgt anschließend durch Hydroxylierung an Position 5 des Heterocyclus mittels eines einem Cytochrom P450 ähnlichen Enzyms. ${ }^{[143]}$ Das Indol wird über eine ungewöhnliche Radikal-vermittelte Reaktion aus $L$-Tryptophan gebildet und 
höchstwahrscheinlich erst nach dem Aufbau des A-Rings angebracht. ${ }^{[144-146]}$

Es sind derzeit etwa 15 weitere bismakrocylische und drei monomakrocyclische Thiopeptidnaturstoffe aus der Klasse E bekannt. Ausgewählte Moleküle sind in Abbildung 1.8 gezeigt. Wesentliche Unterschiede zu Nosiheptid bestehen in oxidativen Modifizierungen, Methylierungen sowie Glycosidierungen. Bei den Nocathiacinen (30-33) und Thiazomycinen findet sich eine transannulare Etherbrücke über dem B-Ring. Diese ist bei Phillipimycin (34) jedoch nicht geschlossen. Das schwefelfreie Makrobislacton zeigt, dass offensichtlich ein Cystein gegen Serin ausgetauscht wurde. Bei den monomakrocyclischen Thiazomycinen $\mathrm{E}_{1}$ $\mathrm{E}_{3}$ fehlt das B-Ring-Lacton und sie besitzen keine nennenswerte antibiotische Aktivität. ${ }^{[50]}$

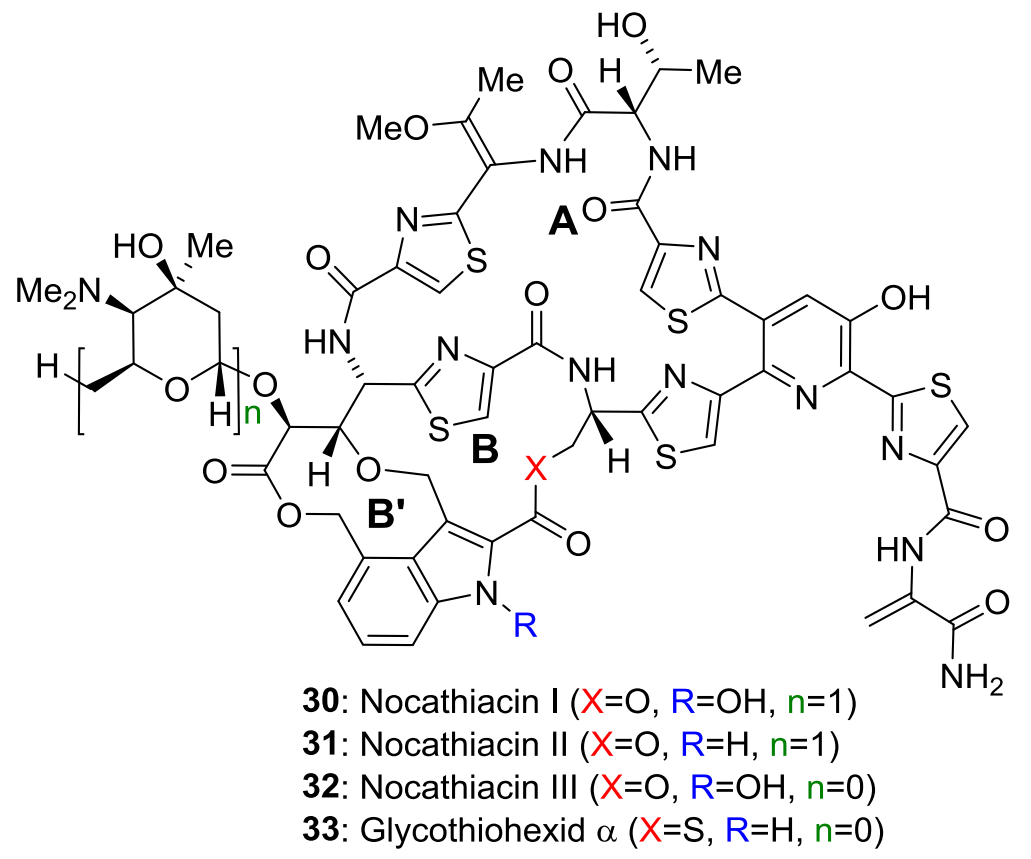

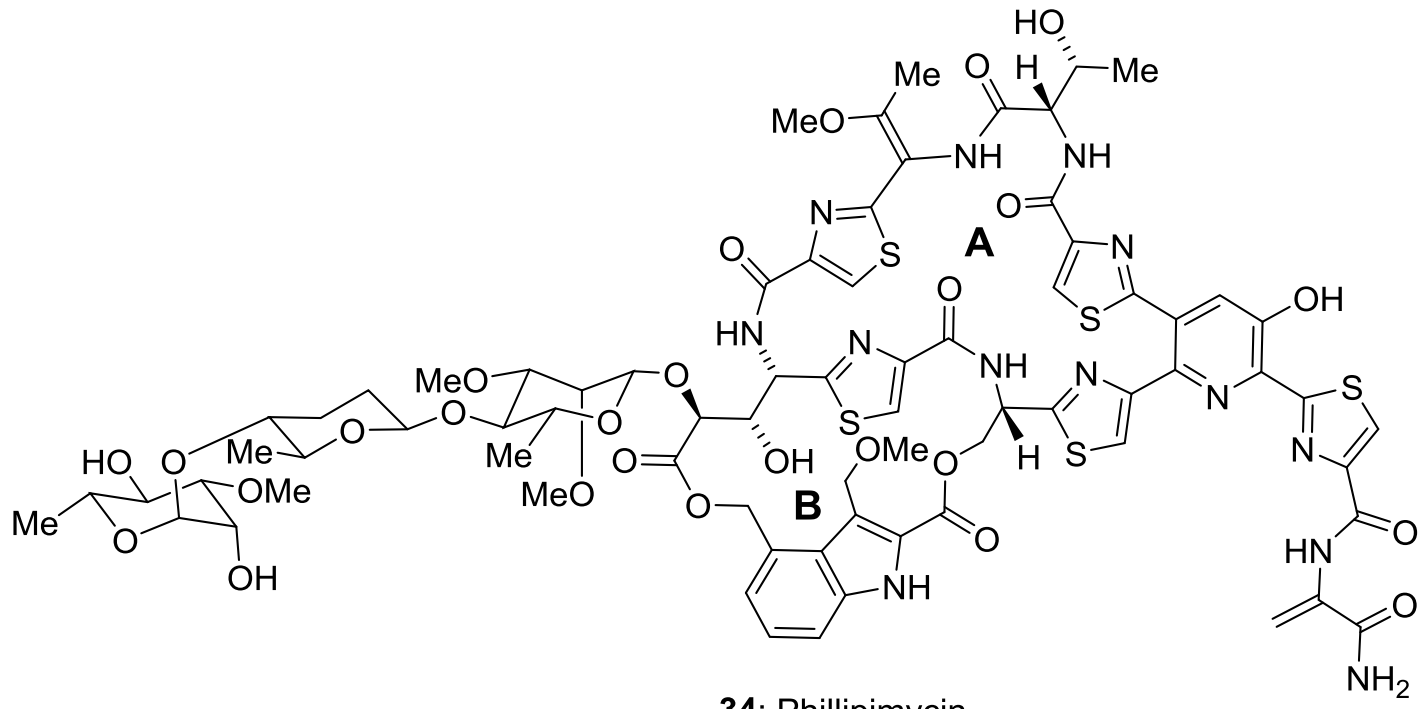

34: Phillipimycin

Abbildung 1.8: Strukturformeln weiterer Thiopeptide der Klasse E. 
Nosiheptid (6) kann somit als strukturell minimaler Prototyp der bioaktiven Thiopeptidantibiotika der Klasse E angesehen werden.

\subsubsection{Bisherige synthetische Ansätze}

Seit der Strukturaufklärung von Nosiheptid im Jahr 1977 berichteten zahlreiche Studien über Synthesen für die in Abbildung 1.9 gezeigten Fragmente. Synthetische Studien zu den Nocathiacinen beschränken sich bisher vor allem auf den Aufbau von substituierten $N$-Hydroxyindolen. ${ }^{[147,148]}$<smiles>[R1]N[C@@H](C[C@@H]([R2])C(=O)O[R2])c1nc(C(=O)O[R])cs1</smiles>

36<smiles>[R20]Cc1csc(-c2cc(O[R1])c([R20])nc2-c2csc(C(C[R5])N[R])n2)n1</smiles>

35<smiles>[R6]OCc1cccc2[nH]c(C(=O)O[R6])c(C)c12</smiles>

37

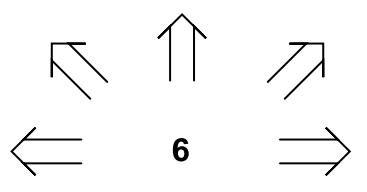<smiles>C/C=C(/NC(=O)[C@H](NC(=O)c1csc(Br)n1)C(C)[OH+])c1nc(C(=O)OC)cs1</smiles>

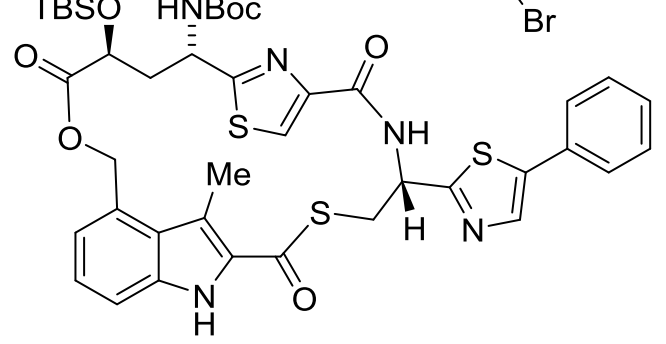

39

Abbildung 1.9: Literaturbekannte Studien zu Fragmenten von Nosiheptid: Pyridin-Kern 35, ${ }^{[149]}$ Thiazol 36, ${ }^{[150,151]}$ Indol 37, ${ }^{[152-154]}$ Bisthiazol 38, ${ }^{[155]}$ und Makrothiolacton 39. ${ }^{[156]}$ Adaptiert nach Riedrich. ${ }^{[36]}$

Bis 2009 waren eine Synthese für den Pyridin-Kern 35 ${ }^{[149]}$ und zwei für das Thiazol $\mathbf{3 6}^{[150,151]}$ bekannt. Die meisten Zugänge wurden bisher für das Indol 37 publiziert. ${ }^{[150,151]}$ Die Gruppe von Moody hatte mit der Synthese des im A-Ring enthaltenen Bisthiazols 38 $^{[155]}$ und des kompletten B-Ring-Thiolactons 39 ${ }^{[156]}$ wesentliche Beiträge geleistet. Diese Arbeiten wurden bereits in den Dissertation von J.-Y. Lu ${ }^{[37]}$ und M. Riedrich ${ }^{[36]}$ ausführlich diskutiert. Daher werden nachfolgend die gruppeneigenen Vorarbeiten und der erst kürzlich publizierte Aufbau des 3-Hydroxypyridinkerns der Nocathiacine vorgestellt. ${ }^{[157]}$ 


\subsubsection{Retrosynthetische Analyse von Nosiheptid nach Lu und Riedrich}

Die retrosynthetische Analyse von Nosiheptid nach Lu und Riedrich ist in Schema 1.4 dargestellt.

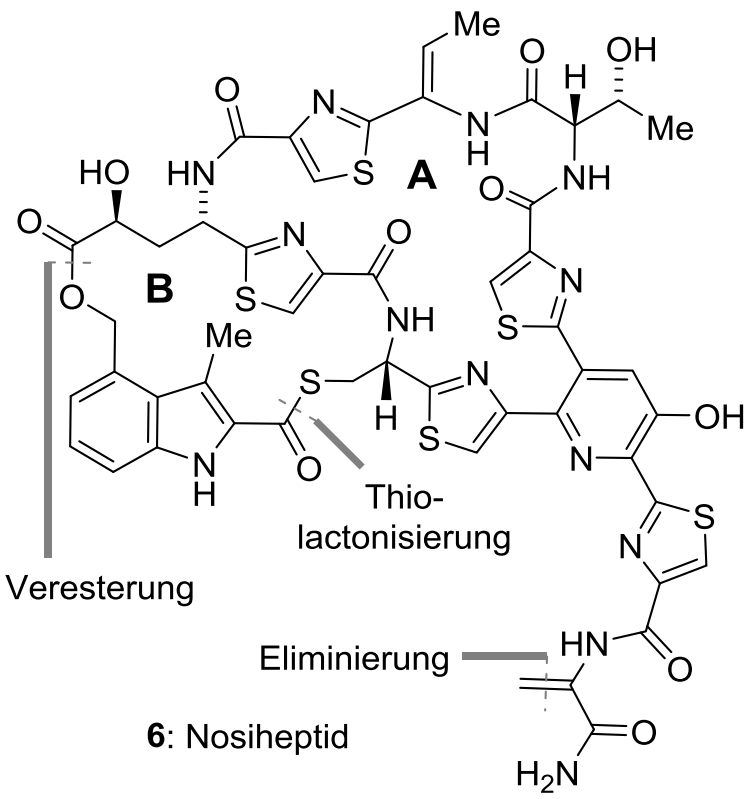

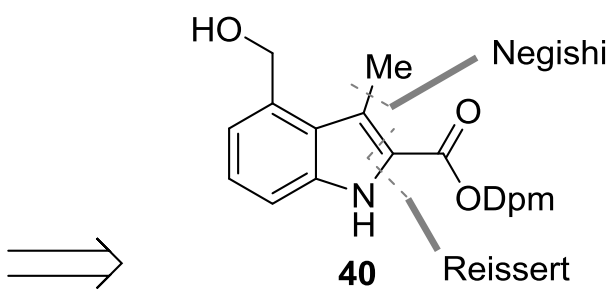

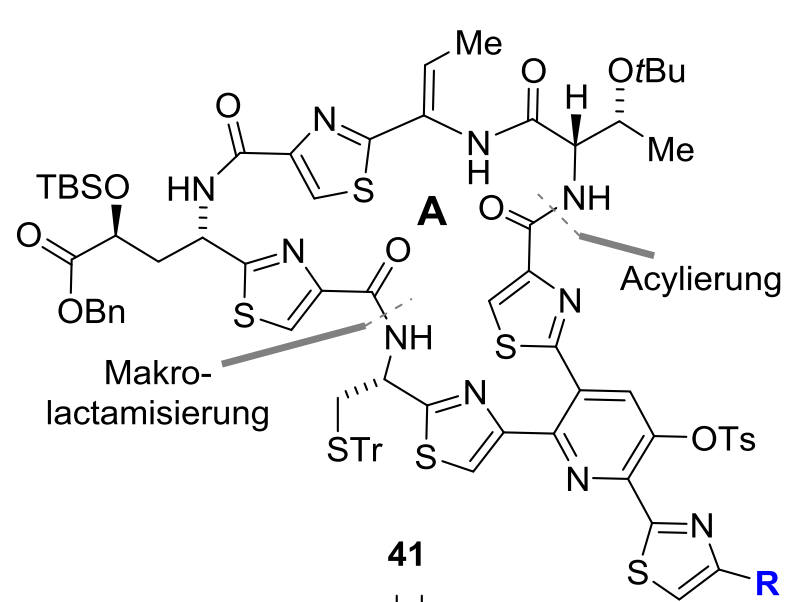

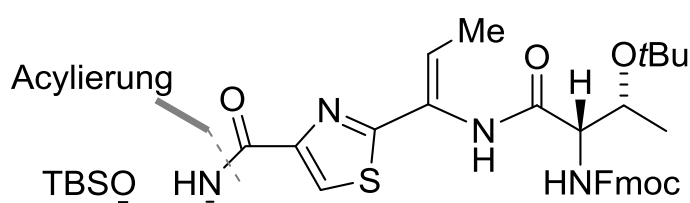<smiles>CC(C)C(=O)OCc1ccccc1</smiles>

43<smiles>C=CC(=C)CCCC</smiles>
Wittig<smiles>C=C(NC(=O)C(NC(=O)[O-])C(C)CCCCC)c1nc(C(=O)O)cs1</smiles>

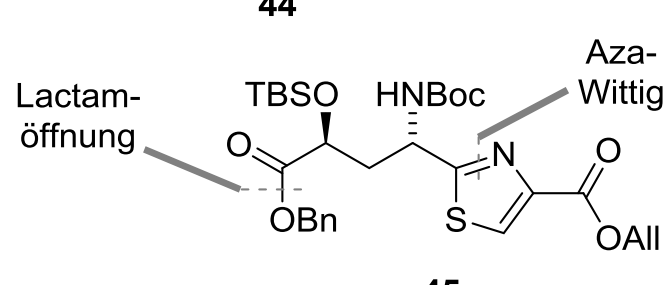

45

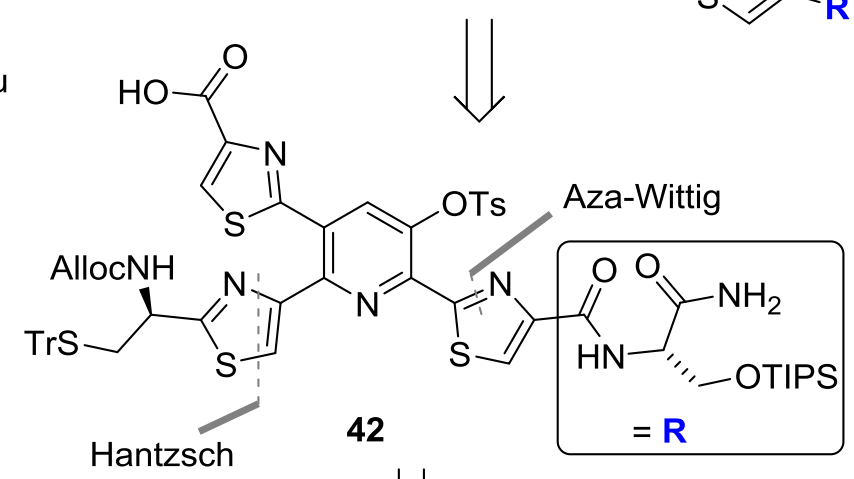

Hantzsch

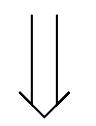<smiles>COC(=O)c1csc(-c2cc(O)c(C(=O)OC)nc2C(C)=O)n1</smiles><smiles>NC(=O)[C@H](CO[In-])NC(=O)[C@H](N)CS</smiles>

Schema. 1.4: Retrosynthetische Analyse von Nosiheptid nach Lu und Riedrich. ${ }^{[36,37]}$ 
Nach Spaltung des B-Rings, Einführung latenter Funktionalitäten und entsprechender Schutzgruppen, wurde Nosiheptid retrosynthetisch zum Indol $\mathbf{4 0}$ und dem A-Ring Makrozyklus 41 vereinfacht. Die Synthese des Indols 40 sollte über eine modifizierte Reissert-Indolsynthese ${ }^{[158]}$ und Negishi-Kupplung ${ }^{[159]}$ erfolgen. Dieses könnte über eine Veresterung mit dem A-Ring verknüpft werden. Eine gleichzeitige Spaltung des Diphenylmethylesters (Dpm) und der $S$-Tritylgruppe würden den Aufbau des B-Rings ermöglichen. Das Dehydroalanin wurde aufgrund seiner potentiellen Reaktivität als MichaelAkzeptor auf ein geschütztes Serin zurückgeführt. Die weitere Zerlegung des Makrozyklus führt zum Tristhiazolylpyridin 42 und dem peptidischen Bisthiazol 43. Letzteres könnte durch Verknüpfung der beiden Thiazole 44 und 45 erhalten werden. Deren Synthese wiederum, wäre mittels Aza-Wittig-Ringschlüssen möglich. Die Eliminierung einer Threonin-Seitenkette könnte das Enamid liefern.

Für den Aufbau des Tristhiazolylpyridins 42 sollten eine Aza-Wittig-Cyclisierung und eine Hantzsch-Thiazolsynthese eingesetzt werden. Der 3-Hydroxypyridinkern 46 sollte über eine hetero-Diels-Alder-Reaktion dargestellt werden.

Die Entwicklung der Synthesemethode zur Darstellung von peptidintegrierten Oxa- und Thiazolen ausgehend von Aminosäuren durch Aza-Wittig-Ringschlüsse und die Darstellung des Bisthiazols 44, sowie die Synthese des Indolbausteins 40 wurden von M. Riedrich durchgeführt. ${ }^{[36,160-162]}$ Die Synthese des Tristhiazolylpyridins 42 und die Ausarbeitung der hetero-Diels-Alder-Reaktion erfolgten durch J.-Y. Lu. ${ }^{[37,163]}$

\subsubsection{Thiazol-Synthesen durch Aza-Wittig-Ringschlüsse nach Riedrich}

Nachfolgend sind die Synthesen der beiden Thiazole 44 und 45, sowie deren Verknüpfung zum Bisthiazol 43 beschrieben. In Schema 1.5 ist die Synthese des Thiazols 44 dargestellt. Ausgehend von $S$-Trityl- $L$-Cystein 49 wurde die Aminfunktion mittels Diazotransfers nach Wong et al. ${ }^{[164,165]}$ in das entsprechende Azid überführtt und die Carbonsäure zum Allylester alkyliert. Saure Trityl-Entschützung lieferte das Thiol 50, welches danach mit $L$-Boc $(t \mathrm{Bu})$ Threonin zum Thioester $\mathbf{5 1}$ acyliert wurde. Dessen Aza-Wittig-Cyclisierung und anschließende Oxidation des intermediären Thiazolins lieferte das Thiazol 52. Dieses wurde sauer $N$ - und $O$-entschützt und mit $L$-Fmoc $(t \mathrm{Bu})$-Threonin zum Amid $53 N$-acyliert. Durch Einsatz von Grieco-Reagenz (55) und oxidativer syn-Eliminierung konnte der Alkohol stereoselektiv in das Enamid 54 überführt werden. ${ }^{[166,167]}$ Abschließend wurde der Allylester Pd-vermittelt gespalten, um die Acylierung des Thiazols 45 zu ermöglichen. 


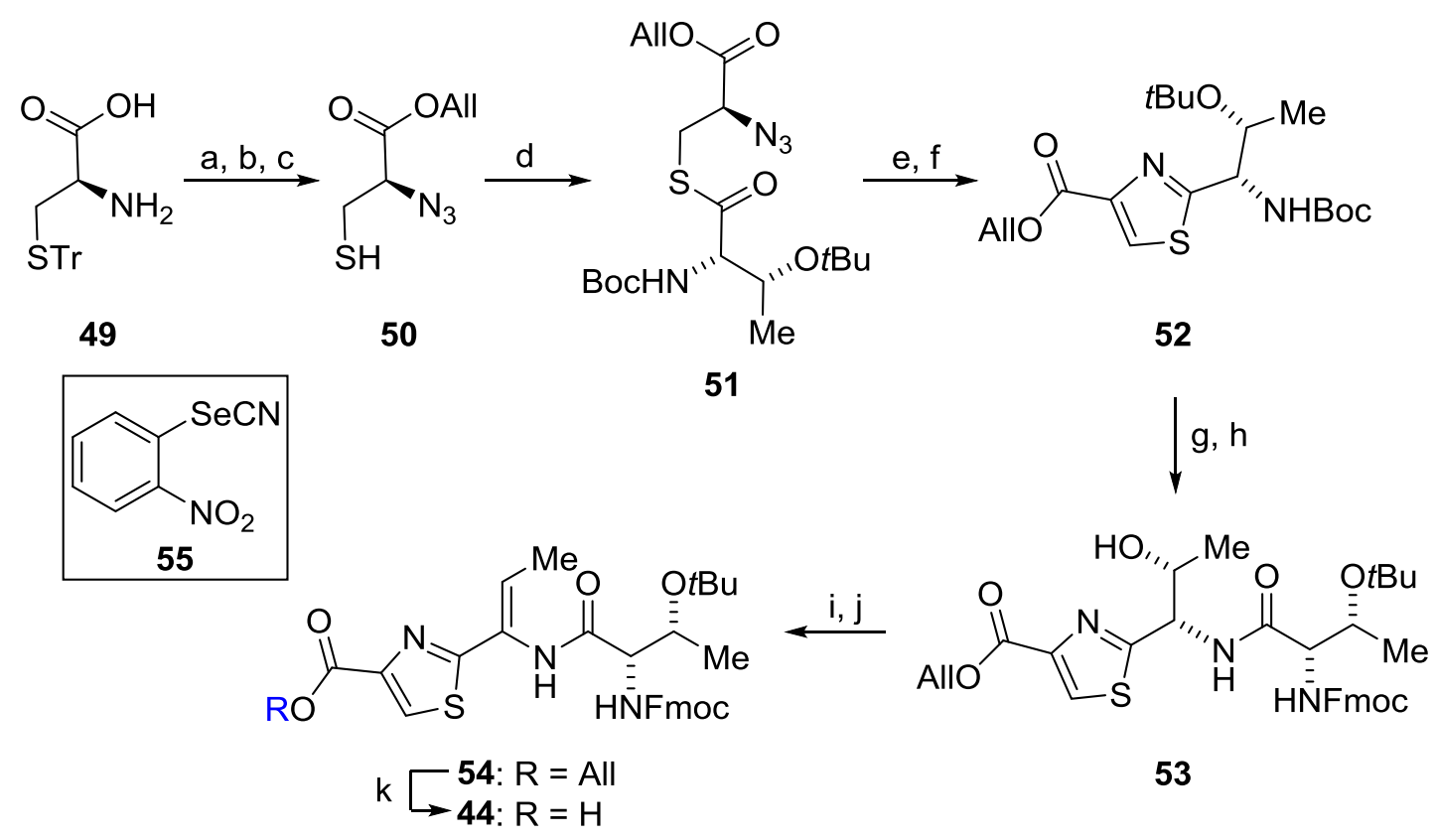

Schema 1.5: Synthese des Thiazols 44 durch Aza-Wittig-Cyclisierung nach Riedrich. Bedingungen: a) Tf- $\mathrm{N}_{3}, \mathrm{CuSO}_{4}, \mathrm{Et}_{3} \mathrm{~N}, \mathrm{H}_{2} \mathrm{O} / \mathrm{MeOH} / \mathrm{CH}_{2} \mathrm{Cl}_{2}, 0^{\circ} \mathrm{C} \rightarrow \mathrm{RT}, 12$ h. b) i. $\mathrm{Cs}_{2} \mathrm{CO}_{3}, \mathrm{MeOH}$; ii. Allylbromid, DMF, RT, 5 h, $88 \%$ (ü. 2 Stufen). c) TFA, $\mathrm{Et}_{3} \mathrm{SiH}, \mathrm{CH}_{2} \mathrm{Cl}_{2}, \mathrm{RT}, 1$ h. d) $\operatorname{BocThr}(\mathrm{Bu}) \mathrm{OH}, \mathrm{HOBt}, \mathrm{EDCI}$, $\mathrm{Et}_{3} \mathrm{~N}, \mathrm{CH}_{2} \mathrm{Cl}_{2}, 0^{\circ} \mathrm{C} \rightarrow \mathrm{RT}, 12 \mathrm{~h}, 79 \%$ (ü. 2 Stufen). e) $\mathrm{PPh}_{3}$, THF, $0{ }^{\circ} \mathrm{C} \rightarrow 40{ }^{\circ} \mathrm{C}, 6$ h. f) $\mathrm{DBU}, \mathrm{BrCCl}_{3}$, $\mathrm{CH}_{2} \mathrm{Cl}_{2}, 0^{\circ} \mathrm{C} \rightarrow$ RT. 4 h, $94 \%$ (ü. 2 Stufen). g) TFA, $\mathrm{CH}_{2} \mathrm{Cl}_{2}$, RT, 30 min. h) FmocThr( $t \mathrm{Bu}$ )OH, HOBt, EDCI, $\mathrm{Et}_{3} \mathrm{~N}, \mathrm{CH}_{2} \mathrm{Cl}_{2}, 0^{\circ} \mathrm{C} \rightarrow \mathrm{RT}, 12$ h, 97\% (ü. 2 Stufen). i) 55, $\mathrm{PBu}_{3}$, THF, RT, 16 h. j) $\mathrm{H}_{2} \mathrm{O}_{2}, \mathrm{RT}$, 30 min, 90\% (ü. 2 Stufen). k) $\mathrm{Pd}\left(\mathrm{PPh}_{3}\right)_{4}, \mathrm{PhSiH}_{3}, \mathrm{CH}_{2} \mathrm{Cl}_{2}, \mathrm{RT}, 10$ min, 98\%. ${ }^{[36]}$

Die in Schema 1.6 dargestellte Synthese des Thiazols 45 beginnt mit kommerziell erhältlichem $N$-Boc-geschützten trans- $L$-Hydroxyprolin 56. Dieses wurde im ersten Schritt unter Mitsunobu-Bedingungen in das Lacton 57 überführt und so die Konfiguration an Position 4 invertiert. Nach anschließender Öffnung des Lactons 57 mit Natriumtrichlorethanolat wurde der freigesetzte Alkohol mit einer TBS-Schutzgruppe versehen. Das Pyrrolidin 58 wurde dann $\mathrm{RuO}_{4}$-vermittelt zum Lactam 59 oxidiert. ${ }^{[168,169]}$ Die Öffnung des Lactams 59 mit Natriumbenzylalkoholat lieferte die vierfach orthogonal geschützte $\gamma$-Hydroxyglutaminsäure 60. Der Trichlorethylester 60 wurde reduktiv mit $\mathrm{Zn}$ gespalten und die erhaltene Carbonsäure mit dem Thiol 50 zum Thioesters 61 verknüpft. Dieser konnte dann mittels Aza-Wittig-Ringschluss und Oxidation in das Thiazol 45 überführt werden. 


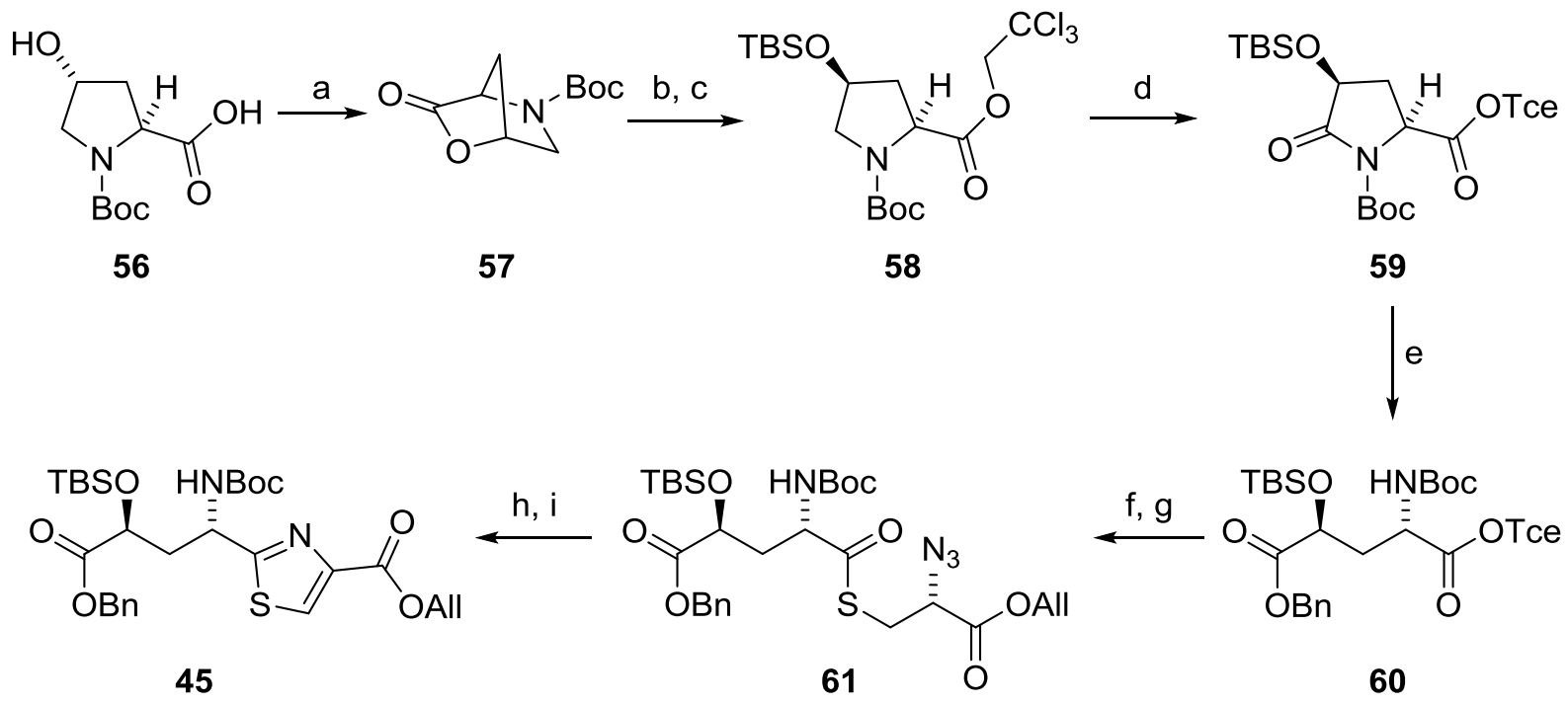

Schema 1.6: Synthese des Thiazols 45 ausgehend von trans- $L$-Hydroxyprolin: a) $\mathrm{PPh}_{3}, \mathrm{DIAD}, \mathrm{THF}$, $0{ }^{\circ} \mathrm{C} \rightarrow \mathrm{RT}, 16 \mathrm{~h}, 62 \%$ b) NaH, 2,2,2-Trichlorethanol, THF, $-78{ }^{\circ} \mathrm{C}, 30$ min. c) TBSCl, Imidazol, DMF, $0{ }^{\circ} \mathrm{C} \rightarrow$ RT, 13 h, 69\% (ü. 2 Stufen). d) $\mathrm{RuO}_{2}, \mathrm{NaIO}_{4}, \mathrm{H}_{2} \mathrm{O} / \mathrm{MeCN} / \mathrm{CCl}_{4}, \mathrm{RT}, 13$ h, 76\%. e) $\mathrm{NaH}$, Benzylalkohol, THF, $-78{ }^{\circ} \mathrm{C}, 30 \mathrm{~min}, 63 \%$ (87\% b.r.s.m.). f) $\mathrm{Zn}, \mathrm{KH}_{2} \mathrm{PO}_{4}, \mathrm{THF} / \mathrm{H}_{2} \mathrm{O}$, ))), RT, $16 \mathrm{~h}$, $85 \%$. g) Thiol 50, HOBt, EDCI, $\mathrm{Et}_{3} \mathrm{~N}, \mathrm{CH}_{2} \mathrm{Cl}_{2}, 0{ }^{\circ} \mathrm{C} \rightarrow \mathrm{RT}, 14 \mathrm{~h}, 86 \%$. h) $\mathrm{PPh}_{3}, \mathrm{THF}, 0{ }^{\circ} \mathrm{C} \rightarrow 40{ }^{\circ} \mathrm{C}$, 6 h. i) $\mathrm{DBU}, \mathrm{BrCCl}_{3}, \mathrm{CH}_{2} \mathrm{Cl}_{2}, 0{ }^{\circ} \mathrm{C} \rightarrow \mathrm{RT}, 4$ h, $92 \%$ (ü. 2 Stufen). ${ }^{[36]}$

Die Verknüpfung der beiden Thiazol-Bausteine 44 und 45 ist in Schema 1.7 gezeigt. Nach Boc-Entschützung des Thiazols 45 wurde dieses mit dem Thiazol 44 verknüpft. Basische Fmoc-Entschützung lieferte das Amin 63, bereit für die Verknüpfung mit dem Tristhiazolylpyridin $\mathbf{4 2}$.<smiles>[R1]N[C@H](C[C@H](Cc1ccccc1)O[R5])c1nc(C(=O)O[Na])cs1</smiles>

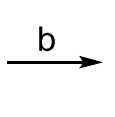

$$
\begin{aligned}
& \text { a5: } R^{1}=B o c \\
& \text { 62: } R^{1}=H
\end{aligned}
$$

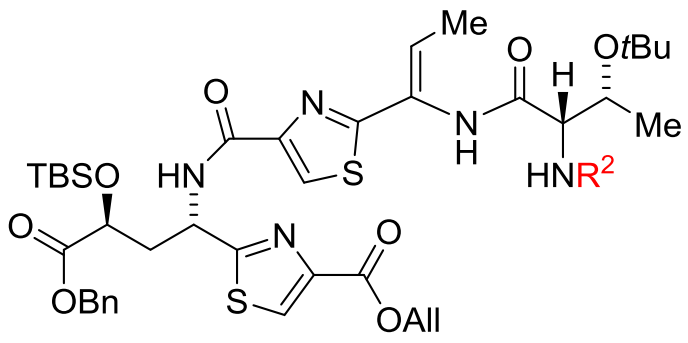

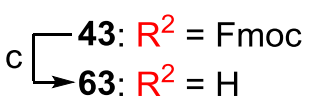

Schema 1.1: Verknüpfung der beiden Thiazole zum Peptid-Bisthiazols 43 nach Riedrich. Bedingungen: a) TFA, $\mathrm{CH}_{2} \mathrm{Cl}_{2}$, RT, 1 h. b) 44, $\mathrm{HOBt}$, EDCI, $\mathrm{Et}_{3} \mathrm{~N}, \mathrm{CH}_{2} \mathrm{Cl}_{2}, 0{ }^{\circ} \mathrm{C} \rightarrow \mathrm{RT}, 15$ h, $58 \%$ (ü. 2 Stufen). c) DBU, Piperidin, $\mathrm{CH}_{2} \mathrm{Cl}_{2}$, RT, 20 min, $96 \%$. 
Unter Anwendung der Aza-Wittig-Cyclisierung als Schlüsselmethode zum Aufbau peptidintegrierter Thiazole konnte M. Riedrich ausgehend von kommerziellen AminosäureBausteinen das Bisthiazol 43 innerhalb von 23 Stufen aufbauen.

\subsubsection{Synthese des Pyridinkerns nach Lu}

Die Synthese des zentralen 3-Hydroxypyridinkerns 42 über eine hetero-Diels-Alder Cycloaddition wurde durch Dr. Jin-Yong Lu erarbeitet (Schema 1.8). ${ }^{[37,163]}$ Die Umsetzung des Alkinylketons 64 mit dem 1-Azabutadien $\mathbf{6 5}^{[170]}$ lieferte die beiden 3-Hydroxypyridine $\mathbf{6 6}$ und 46 in einem Verhältnis von etwa 1:2 zu Gunsten des gewünschten Isomers 46. Anschließend wurde das Phenol als Triflat geschützt und das Keton mit TIPSOTf zu einem Silylenolether umgesetzt. Die Spaltung des Ethers mit $N$-Bromsuccinimid (NBS) lieferte das $\alpha$-Bromketon 67, welches in einer modifizierten Hantzsch-Thiazolsynthese ${ }^{[171-173]}$ mit dem Thioamid $47^{[98]}$ zu einem Bisthiazolylpyridin umgesetzt wurde (Schritt e). Das Triflat wurde basisch entfernt, um anschließend über eine $\mathrm{Sc}^{\mathrm{III}}$-vermittelte Umsetzung selektiv einen der beiden Methylester im Diester 68 spalten zu können (Schritt g). Das Boc-geschützte Thioaminal wurde danach umgeschützt $(69>70)$, da der Cysteinrest in späteren Stufen ansonsten nicht freigesetzt werden konnte. Zur Installation der Seitenkette wurde die Carbonsäure mit Phosgen aktiviert und mit dem Peptidthiol 48 umgesetzt. Nach sofortigem Aza-Wittig-Ringschluss und darauffolgender Oxidation wurde das Tristhiazolylpyridin 71 erhalten, das am Phenol als Sulfonat geschützt wurde (Schritt o). Die Freisetzung der Carbonsäure gelang mit $\mathrm{Me}_{3} \mathrm{SnOH}^{[131]}$ ohne Spaltung des Sulfonats. Der vollständige Tristhiazolylpyrdinkern 42 konnte durch Lu ausgehend von Alkinylketon 64 über eine lineare Sequenz von 16 Stufen und einer Gesamtausbeute von 4\% erfolgreich hergestellt werden. 
<smiles>C=C(O[Na])C(=NOC(C)(C)C)C(=O)OC</smiles>

64<smiles></smiles>

66

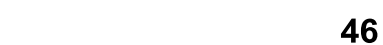

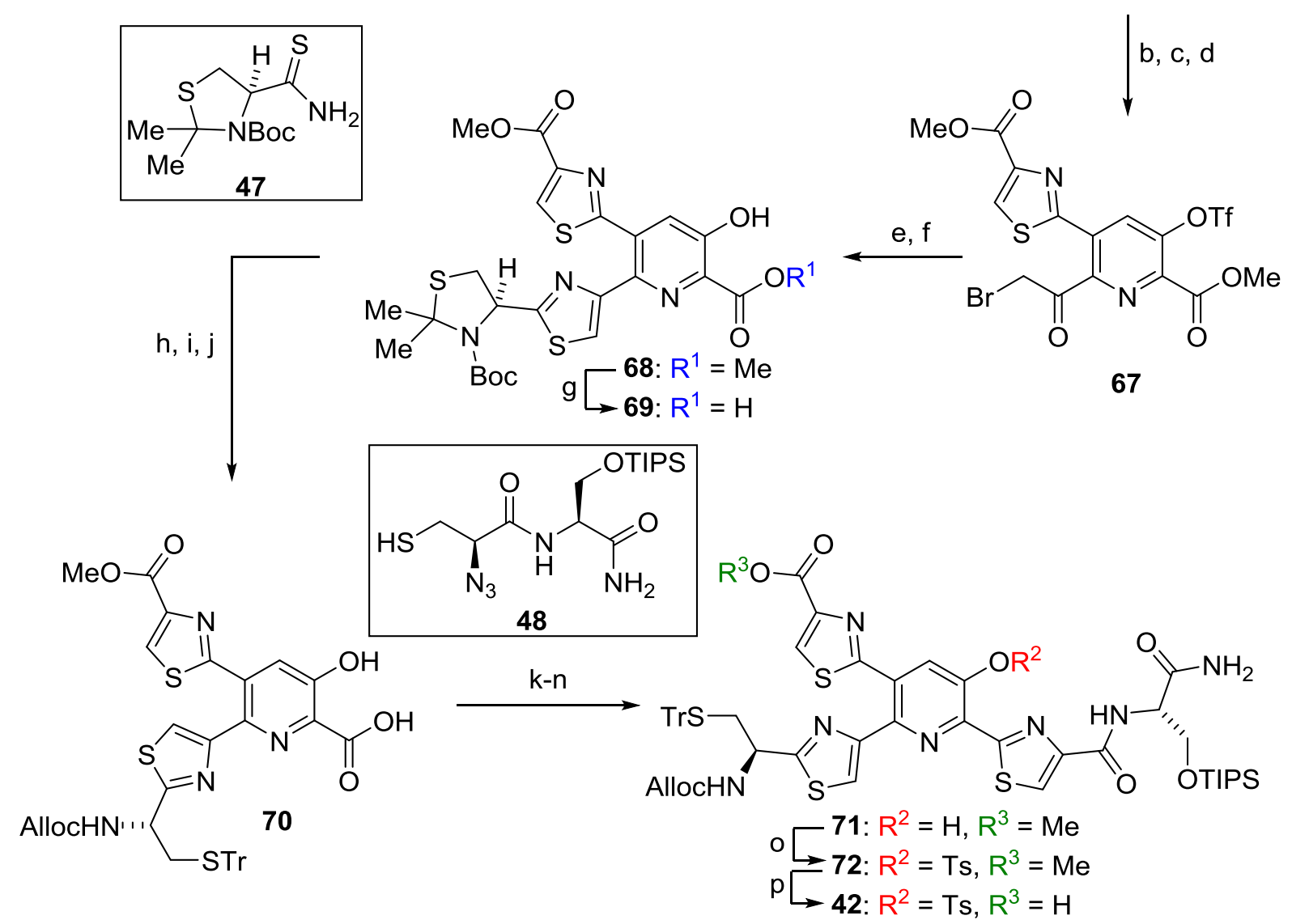

Schema 1.8: Synthese des Tristhiazolylpyridins 42 via hetero-Diels-Alder Reaktion nach Lu. Bedinungen: a) Toluol, $180{ }^{\circ} \mathrm{C}, 3 \mathrm{~h}, \mathbf{6 6}: 28 \%$; 46: 56\%. b) $\mathrm{Tf}_{2} \mathrm{O}, \mathrm{Et}_{3} \mathrm{~N}, \mathrm{CH}_{2} \mathrm{Cl}_{2}, 0{ }^{\circ} \mathrm{C} \rightarrow \mathrm{RT}, 12 \mathrm{~h}, 80 \%$. c) TIPSOTf, $\mathrm{Et}_{3} \mathrm{~N}, \mathrm{CH}_{2} \mathrm{Cl}_{2}, 0^{\circ} \mathrm{C} \rightarrow \mathrm{RT}, 12 \mathrm{~h}, 99 \%$. d) NBS, pH 7 Puffer, THF, 30 min, 97\%. e) 47, $\mathrm{KHCO}_{3}$, THF, $-40{ }^{\circ} \mathrm{C} \rightarrow \mathrm{RT}, 48 \mathrm{~h}$; 2,6-Lutidin, TFAA, $-20{ }^{\circ} \mathrm{C}, 2 \mathrm{~h}, 60 \%$. f) TBAOH, 1,4-Dioxan oder $\mathrm{NaOMe} / \mathrm{MeOH}$, RT, 5-30 min, 99\%. g) 5 mol\% Sc(OTf) , pH 8.5, 1,4-Dioxan, $60{ }^{\circ} \mathrm{C}, 8.5$ h, 90\%. h) TFA, $\mathrm{CH}_{2} \mathrm{Cl}_{2}, 30$ min. i) TrCl, DMF, 14 h. j) AllocCl, $\mathrm{NaHCO}_{3}$, THF/ $\mathrm{H}_{2} \mathrm{O}, 0^{\circ} \mathrm{C} \rightarrow \mathrm{RT}, 2$ h, 82\% (ü. 3 Stufen). k) Phosgen, $\mathrm{Et}_{3} \mathrm{~N}$, THF, $-40{ }^{\circ} \mathrm{C}, 2$ h. 1) 48, DMAP, $-40{ }^{\circ} \mathrm{C} \rightarrow \mathrm{RT}, 48$ h. m) $\mathrm{PPh}_{3}, \mathrm{THF},-20{ }^{\circ} \mathrm{C}$ $\rightarrow 40{ }^{\circ} \mathrm{C}, 21$ h. n) $\mathrm{BrCCl}_{3}$, DBU, $\mathrm{CH}_{2} \mathrm{Cl}_{2},-20^{\circ} \mathrm{C} \rightarrow \mathrm{RT}, 2$ h, $46 \%$ (ü. 4 Stufen). o) TsCl, $\mathrm{Et}_{3} \mathrm{~N}, \mathrm{DMAP}^{\circ}$ $\mathrm{CH}_{2} \mathrm{Cl}_{2}, 0{ }^{\circ} \mathrm{C}, 1.5$ h, $49 \%$. p) $\mathrm{Me}_{3} \mathrm{SnOH}, \mathrm{DCE}, 60^{\circ} \mathrm{C}, 4 \mathrm{~h}, 99 \% .{ }^{[37,161,163]}$ 


\subsubsection{Synthese des Indols nach Riedrich}

Basierend auf einer bekannten Synthese des Nosiheptid-Indolfragments von Shin et al., ${ }^{[153]}$ bei der der Indolester $\mathbf{7 6}$ als Intermediat auftrat, nutzte M. Riedrich eine Negishi-Kupplung zur Einführung der Methylgruppe (Schema 1.9).

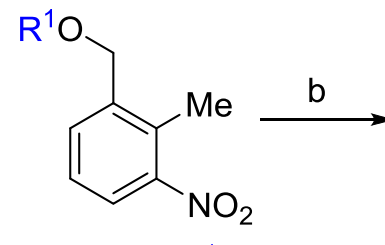

a 73: $R^{1}=H$
74: $R^{1}=$ THP<smiles>[R]OC(=O)c1[nH]c2cccc(CO)c2c1C</smiles>

$h \longrightarrow$ 79: $R^{2}=H$
$\rightarrow 40: R^{2}=D p m$
THPO<smiles>CCOC(=O)C(=O)Cc1c(CO)cccc1[N+](=O)[O-]</smiles>

75

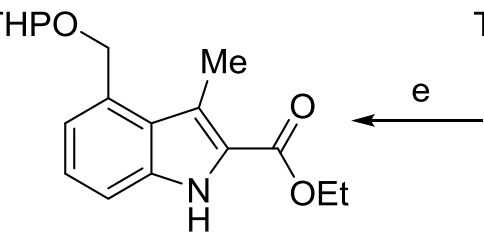

78<smiles>CCOC(=O)c1cc2c(COP)cccc2[nH]1</smiles>

76

THPO

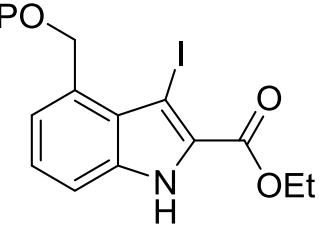

77

Schema 1.9: Synthese des Indolylalkohols 40 nach Riedrich. Bedingungen: a) 3,4-Dihydro- $2 H$-pyran, $p$-Toluolsulfonsäure, $\mathrm{CH}_{2} \mathrm{Cl}_{2}, 0{ }^{\circ} \mathrm{C} \rightarrow \mathrm{RT}, 13 \mathrm{~h}, 95 \%$. b) (COOEt) $)_{2}, \mathrm{NaH}, \mathrm{DMF}, 0{ }^{\circ} \mathrm{C} \rightarrow \mathrm{RT}, 15 \mathrm{~h}$, 85\%. c) $\mathrm{H}_{2}$, Pd/C, EtOH, RT, 1 h, 82\%. d) $\mathrm{K}_{2} \mathrm{CO}_{3}, \mathrm{I}_{2}, \mathrm{DMF}, \mathrm{RT}, 5$ h, $89 \%$. e) $\mathrm{Me}_{2} \mathrm{Zn}, \mathrm{Pd}(\mathrm{dppf}) \mathrm{Cl}_{2}$, 1,4-Dioxan, $110{ }^{\circ} \mathrm{C}, 16 \mathrm{~h}, 96 \%$. f) $10 \% \mathrm{NaOH}, \mathrm{H}_{2} \mathrm{O} / \mathrm{EtOH}, 80^{\circ} \mathrm{C}, 30 \mathrm{~min}$. g) $70 \% \mathrm{AcOH}, \mathrm{RT}, 8 \mathrm{~h}$, $73 \%$ (ü. 2 Stufen). h) $\mathrm{Ph}_{2} \mathrm{CN}_{2}$, kat. TFA, THF, $60{ }^{\circ} \mathrm{C}, 3.5$ h, $68 \% .{ }^{[36]}$

Nach Schützung des Benzylalkohols 73 als Acetal wurde dieser mit Oxalsäurediethylester zum $\alpha$-Ketoester 75 acyliert. Durch Reduktion der Nitrogruppe mittels $\mathrm{Pd} / \mathrm{C}$ unter $\mathrm{H}_{2}$ Atmosphäre wurde durch Kondensation des erhaltenen Amins mit der Ketogruppe das Indol 76 erhalten. Anschließende Iodierung lieferte selektiv das 3-Iodindol 77, das Pd-katalysiert mit $\mathrm{Me}_{2} \mathrm{Zn}$ zum 3-Methylindol 78 umgesetzt wurde. Danach wurden der Ester und die Acetalschutzgruppe gespalten. Säurekatalysierte Alkylierung der Carbonsäure mit Diphenyldiazomethan lieferte den gewünschten Dpm-Ester 40.

Der Aufbau des Indol-Bausteins 40 gelang Riedrich ausgehend von kommerziell erhältlichem Benzylalkohol 73 über acht Stufen und einer Gesamtausbeute von 28\%. 


\subsubsection{Verknüpfung der Bausteine und Ringschluss nach Lu und Riedrich}

Die Verknüpfung des Peptid-Bisthiazols 63 mit dem Tristhiazolylpyridin 42 konnte durch Lu und Riedrich nach Optimierung der Kupplungsbedingungen mit 3-(Diethoxyphosphoryloxy)1,2,3-benzotriazin-4(3H)-on (DEPBT) ${ }^{[174]}$ realisiert werden (Schema 1.10). ${ }^{[37]}$

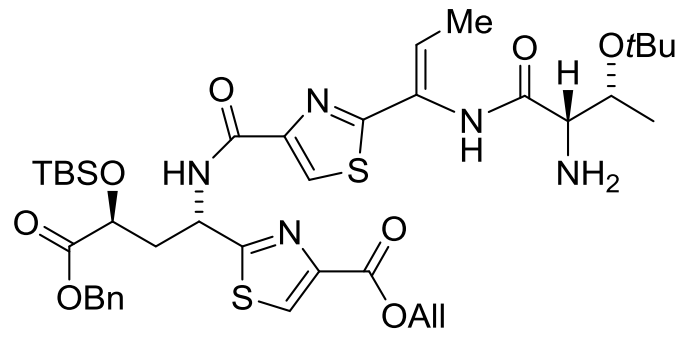

63

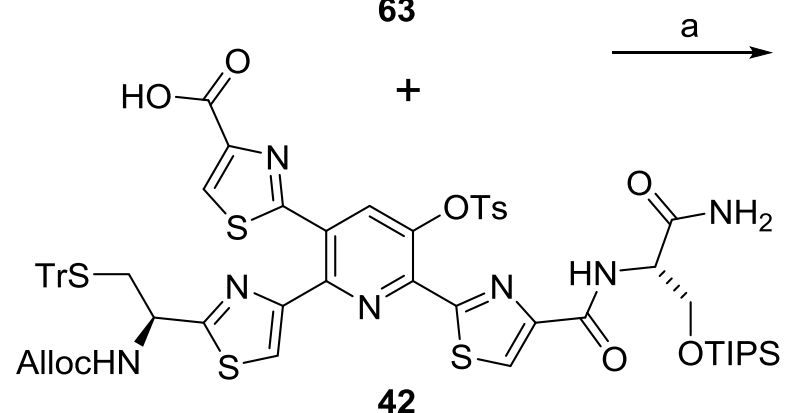

42

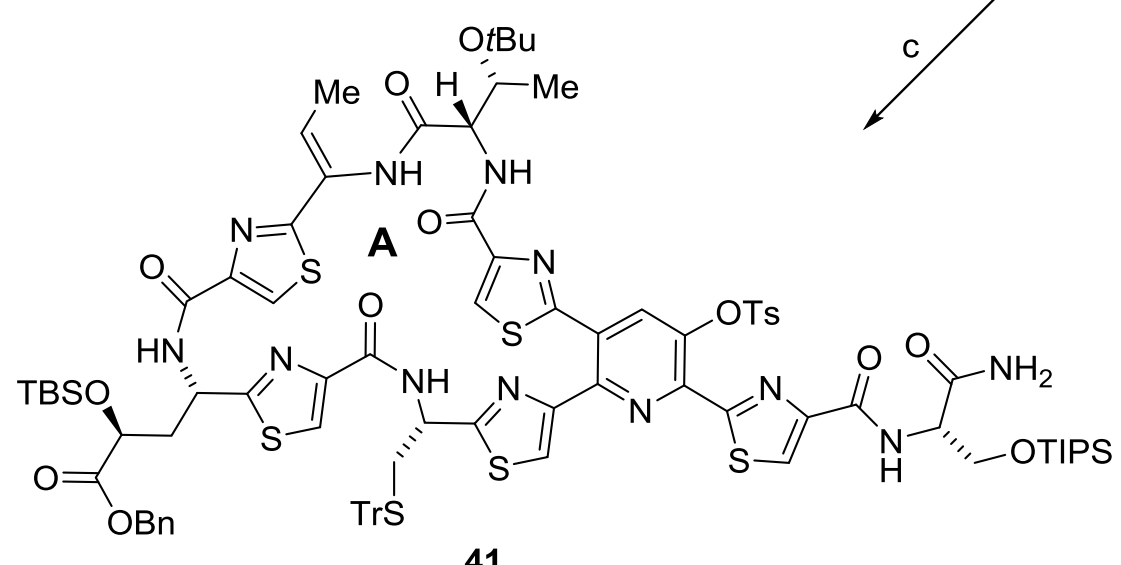

41
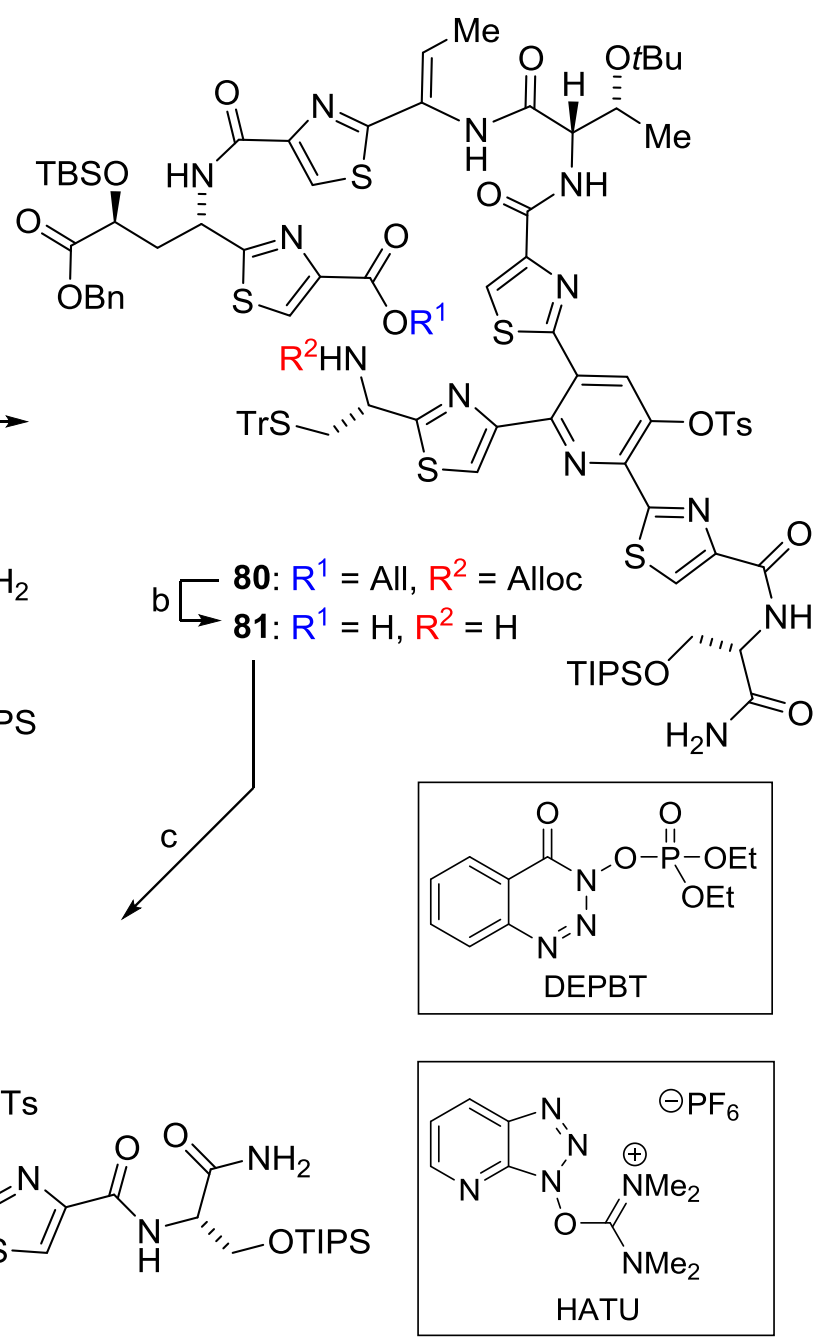

Schema 1.10: Verknüpfung der Bausteine 63 und 42 und Synthese des Makrolactams 41. Bedingungen: a) DEPBT, $\mathrm{NaHCO}_{3}$, THF, RT, 15 h, 47\%. b) $\mathrm{Pd}\left(\mathrm{PPh}_{3}\right)_{4}, \mathrm{PhSiH}_{3}, \mathrm{CH}_{2} \mathrm{Cl}_{2}, \mathrm{RT}, 20 \mathrm{~min}$, $83 \%$. c) HATU, EtNiPr $2, \mathrm{CH}_{2} \mathrm{Cl}_{2} / \mathrm{DMF}, \mathrm{RT}, 16$ h, $56 \%$.

Die gleichzeitige Pd-vermittelte Alloc- und Allyl-Entschützung lieferte anschließend die Aminosäure 81 als linearen Vorläufer. Der Ringschluss zum A-Ring-Makrolactam gelang danach mit dem uroniumbasierten Kupplungsreagenz HATU. ${ }^{[160]}$

Der erfolgreiche Aufbau des A-Ring-Makrolactams 41 legte den Grundstein für weitere Untersuchungen zur Einführung des Indolylalkohols 40. Eine selektive Spaltung des 
Benzylesters konnte jedoch nicht realisiert werden. Der Austausch gegen einen labileren p-Methoxybenzylesters war möglich, jedoch mit synthetischen Herausforderungen und großen Ausbeuteeinbußen verbunden. ${ }^{[175]}$

\subsubsection{Synthese des Nocathiacin-Pyridinkerns nach Ciufolini et al.}

Basierend auf den Erfahrungen im Aufbau des Micrococcin-Kerns ${ }^{[176]}$ wurde 2015 die Synthese des Tristhiazolylpyridins 82 über eine modifizierte Hantzsch-Pyridinsynthese durch die Gruppe von Ciufolini publiziert. ${ }^{[157]}$<smiles>CCOC(=O)c1csc(-c2cc(O)c(-c3nc(COC(C)(C)C)cs3)c(-c3csc([C@@H]4COC(C)(C)N4C(=O)OC(C)(C)C)n3)n2)n1</smiles>

82

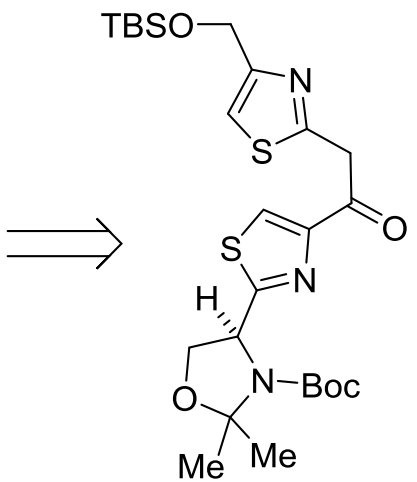

83<smiles>C=C(OC)C(=O)c1nc(C(=O)OCC)cs1</smiles>

84

Schema 1.11: Retrosynthetische Analyse des Nocathiacin-Kerns 82 nach Ciufolini et al. ${ }^{[157]}$

Der Pyridinkern 82 kann durch retrosynthetische Zerlegung auf das Bisthiazol-Keton 83 und das Thiazolyl-Enon 84 zurückgeführt werden (Schema 1.11).

Die Synthese der beiden Ketone ist in Schema 1.12 gezeigt. Die Synthese des Ketons 83 wurde durch die Umsetzung des literaturbekannten Esters $\mathbf{8 5}^{[177]}$ mit zweifachem Überschuss der Organolithiumspezies $\mathbf{8 6}^{[176]}$ realisiert. Das überschüssige Äquivalent dient dabei wahrscheinlich zur Deprotonierung des gebildeten Ketons 83.

Für die Darstellung des Enon 84 wurde im ersten Schritt der Aldehyd 87 ${ }^{[178]}$ mit dem lithiierten Methoxymethylvinylether 88 umgesetzt. Dabei wurde intermediär das Acetal 89 erhalten, welches mit TBAF zum Alkohol 90 gespalten wurde. Dieser wurde danach direkt mit $\mathrm{MnO}_{2}$ zum Enon 84 oxidiert. 
<smiles>CCOC(=O)c1csc([C@@H]2COC(C)(C)N2C(=O)OC(C)(C)C)n1</smiles>

85<smiles>[Li]Cc1nc(COS(=O)(=O)[O-])cs1</smiles>

86<smiles>[R]C(=O)c1nc(C(=O)OCC)cs1</smiles>

89

Schema 1.12: Synthese der Ketone 83 und 84. Bedingungen: a) THF, $-78^{\circ} \mathrm{C} \rightarrow \mathrm{RT}, 2 \mathrm{~h}, 74 \%$. b) Tetrahydropyran, $-78^{\circ} \mathrm{C}, 20 \mathrm{~min}$; TMSCl, 10 min. c) TBAF, THF; 3 h. d) $\mathrm{MnO}_{2}, \mathrm{CH}_{2} \mathrm{Cl}_{2}, 48 \mathrm{~h}, 25 \%$ (ü. 3 Stufen). ${ }^{[157]}$

Die beiden Bausteine wurden anschließend über eine 1,4-Addition verknüpft und das Diketon 91 umgehend unter sauren und oxidativen Bedingungen kondensierend zum Pyridin 92 umgesetzt. Dies gelang jedoch nur mit einer mäßigen Ausbeute von 28\% (Schema 1.13).

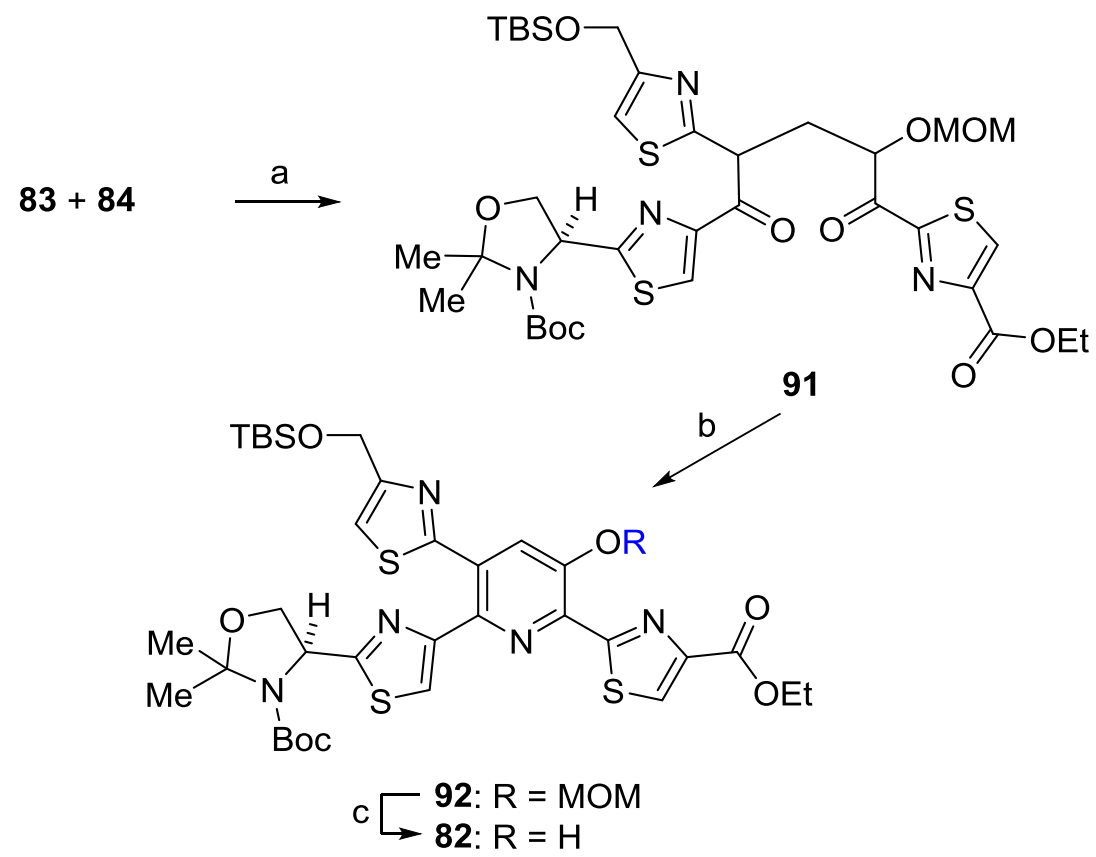

Schema 1.13: Modifizierte Hantzsch-Synthese des Pyridins 82 nach Ciufolini et al. Bedingungen: a) $\mathrm{NaH}$, THF, 90 min, RT. b) $\mathrm{NH}_{4} \mathrm{OAc}$, AcOH/TFE, PPTS, $\mathrm{O}_{2}$-Atmosphäre, $60^{\circ} \mathrm{C}, 14$ h, $28 \%$ (ü. 2 Stufen). c) $\mathrm{PPh}_{3}, \mathrm{CBr}_{4}, \mathrm{DCE}, 40{ }^{\circ} \mathrm{C}, 3 \mathrm{~h}, 71 \%$. 
Die Entschützung der MOM-Gruppe gelang selektiv mit Hilfe von $\mathrm{PPh}_{3}$ und $\mathrm{CBr}_{4}$ und lieferte das 3-Hydroxypyridin 82. ${ }^{[179]}$ Unter Einbeziehung der Schritte zur Darstellung der Startmaterialien 85, 86 und 87, ausgehend von kommerziellen Substanzen, gelang der Cuifolini-Gruppe der Aufbau des MOM-Pyridins 92 in 14 Stufen mit einer längsten linearen Sequenz von nur sechs Stufen. ${ }^{[157]}$ 
2 Ziele der Arbeit 


\subsection{Totalsynthese von Nosiheptid}

Das vor fast 55 Jahren entdeckte Thiopeptidantibiotikum Nosiheptid (6, Abbildung 2.1) stellt aufgrund seiner strukturellen Vielfalt und dem herausragenden biologischen Wirkungsprofil ein lohnendes und herausforderndes Syntheseziel dar.

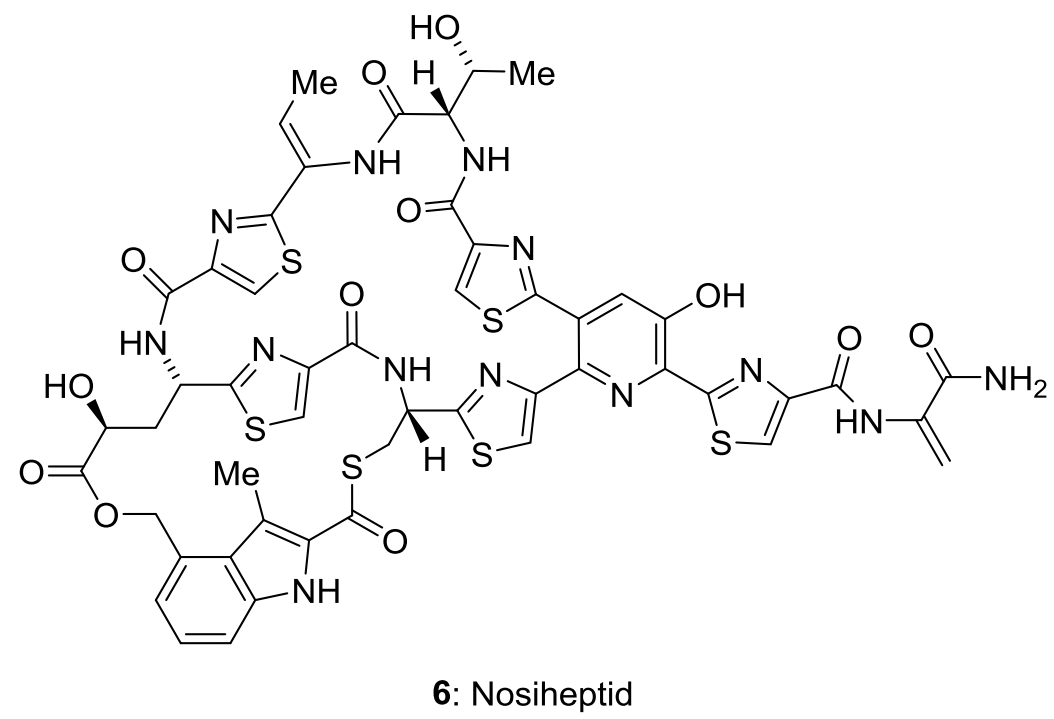

Abbildung 2.1: Struktur von Nosiheptid.

Die Entwicklung einer erfolgreichen Synthesestrategie würde neben der Darstellung des Moleküls selbst, einen Zugang zu Derivaten ermöglichen, welche über semisynthetische bzw. biotechnologische Wege nicht oder nur schwer verfügbar sein würden. ${ }^{[44]}$ Die Synthese geeigneter Derivate könnte zum besseren Verständnis des Wirkungsmechanismus und der Struktur-Wirkungsbeziehungen dieser Substanzklasse beitragen. Der Erkenntnisgewinn der Totalsynthese könnte darüber hinaus zur Synthese verwandter Verbindungen genutzt werden. Im Rahmen dieser Doktorarbeit sollte eine Totalsynthese, in Weiterentwicklung gruppeneigener Vorarbeiten von J.-Y. Lu und M. Riedrich (Kap. 1.5.3), erarbeitet werden. Dabei sollten zuerst das Bisthiazol-Fragment 93 bzw. 94, sowie der bereits bekannte Tristhiazolylpyridin-Kern 42 ${ }^{[37]}$ synthetisiert werden (Abbildung 2.2). Anschließend sollten diese reißverschlussartig (1. - 3.) miteinander verknüpften werden. 


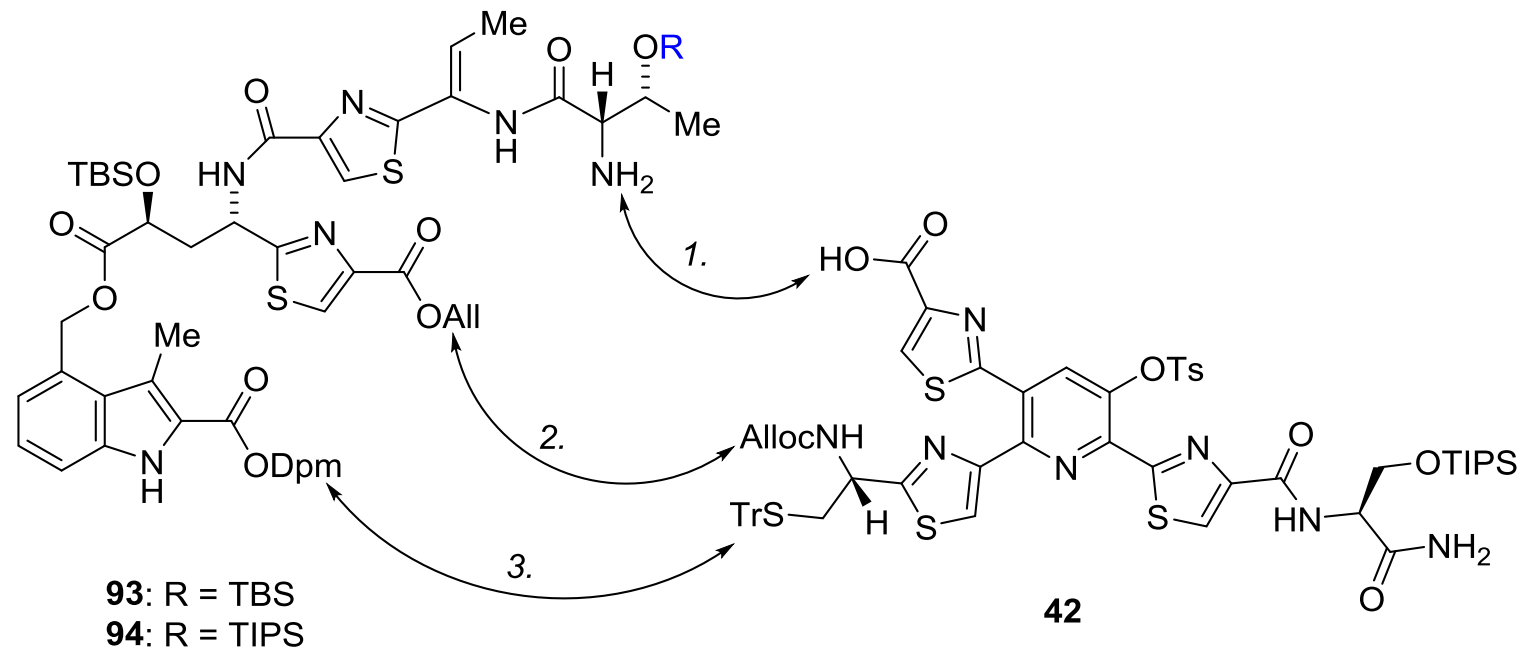

Abbildung 2.2: Geplante reißverschlussartige Verknüpfung der Fragmente 93/94 und 42.

Nach erfolgreichem Aufbau des bismakrocyclischen Grundgerüsts, sollte die SerinSeitenkette des Pyridin-Fragments $\mathbf{4 2}$ selektiv in ein Dehydroalanin überführt werden. Abschließend sollten etwaige Schutzgruppen entfernt werden.

Da sich die Einführung des Indol-Fragments in späten Synthesestufen zuvor als sehr herausfordernd erwiesen hatte, ${ }^{[37]}$ sollte dieses bereits während der Synthese des Thiazols 95 eingebracht werden (Schema 2.1).
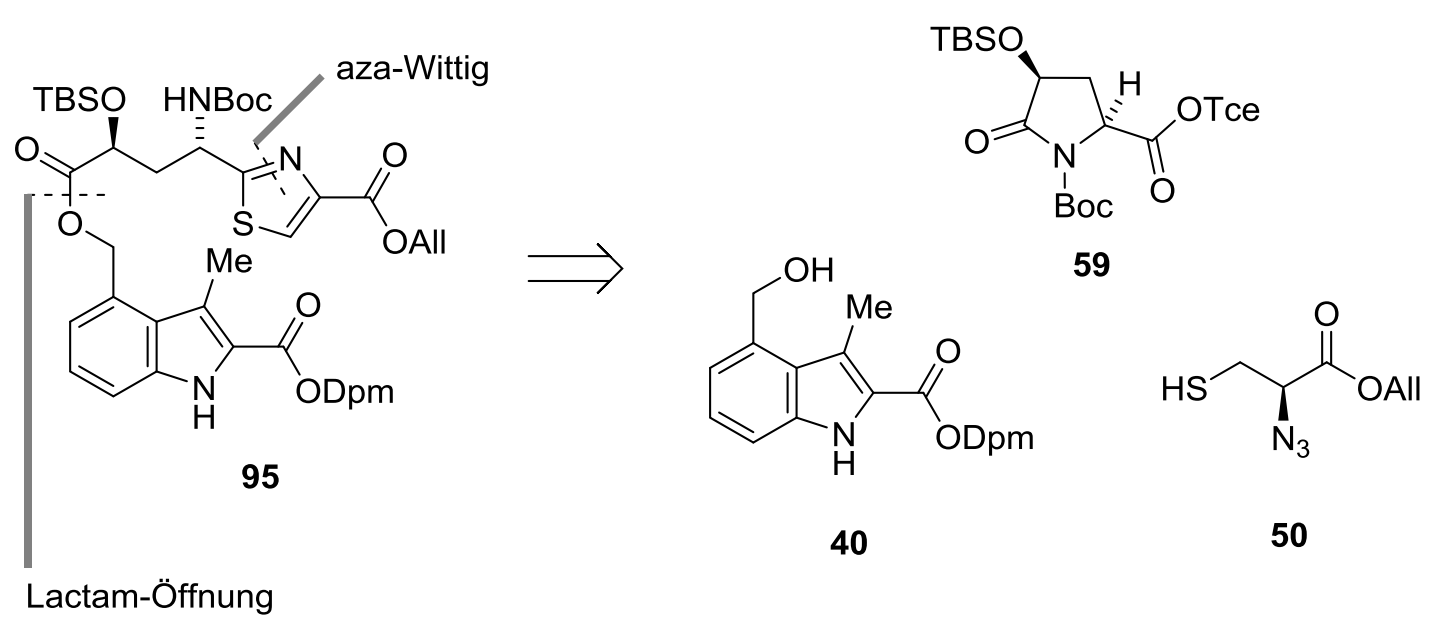

Schema 2.1: Retrosynthetische Analyse des Thiazols 95.

Die Synthese des Thiazols 95 sollte grundsätzlich der durch M. Riedrich erarbeiteten Route folgen und das Thiazol durch Aza-Wittig-Cyclisierung aufgebaut werden (s. Kapitel 1.5.3). Die Einführung des Indol-Fragments 40 sollte durch Ring-Öffnung des Lactams $\mathbf{5 9}$ erfolgen. 


\subsection{Regioselektive Umfunktionalisierung von 3-Hydroxypicolinaten}

Im Verlauf der Entwicklung einer Totalsynthese des Thiopeptidantibiotikums Nosiheptid war von J.-Y. Lu eine selektive Hydrolyse des 3-Hydroxypyridins 96 gefunden worden (Schema $2.2)^{[37]}$

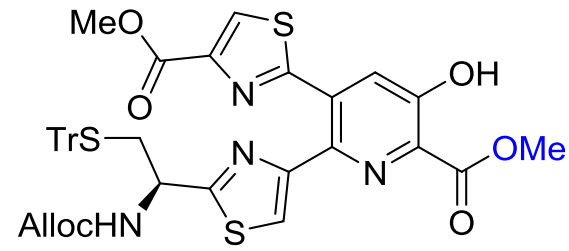

96

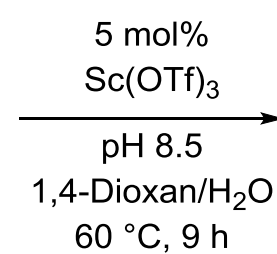

$60{ }^{\circ} \mathrm{C}, 9 \mathrm{~h}$

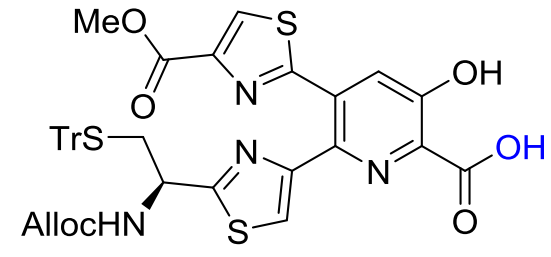

$70(93 \%)$

Schema 2.2: Regioselektive Verseifung des Bisthiazolylpyridins 96 nach Lu. ${ }^{[37]}$

Im Rahmen dieser Arbeit sollte die mechanistische Hypothese dieser selektiven Umsetzung experimentell überprüft und ggf. verfeinert werden. Dabei sollten Untersuchungen sowohl zum Einfluss des Sc(III)-Salzes als auch dem der OH-Gruppe mit den beiden Triestern 97 und 98 durchgeführt werden (Abbildung 2.3)<smiles>COC(=O)c1cc(O)c(C(=O)OC)nc1C(=O)OC</smiles>

97<smiles>COC(=O)c1cc(OC)c(C(=O)OC)nc1C(=O)OC</smiles>

98

Abbildung 2.3: Strukturen der beiden als Testsubstrate vorgesehenen Triester 97 und 98.

\subsection{Synthese substituierter 3-Hydroxypicolinsäuren}

Im Laufe der Arbeit wurden verschiedene 3-Hydroxypyridinderivate hergestellt. Für eine Zusammenarbeit mit Prof. Dr. Karl-Heinz Altmann (ETH Zürich) sollte die dafür entwickelte Chemie genutzt werden, um substituierte 3-Hydroxypicolinsäuren zur Derivatisierung des Tuberkulostatikums Pyridomycin ${ }^{[180-182]}$ (99 in Abbildung 2.4) herzustellen. 
<smiles>CC/C(C)=C(\OC(=O)C(C)[C@@H](O)[C@@H](Cc1cccnc1)NC(=O)C(NC(=O)c1ncccc1O)C(C)O)C(=O)O</smiles>

99: Pyridomycin

Abbildung 2.4: Struktur des Tuberkulostatikums Pyridomycin.

Ziel war es, ausgehend von 3-Hydroxypicolinsäuremethylester jeweils eine der aromatischen Positionen zu substituieren (Schema 2.5). Dafür sollten die Positionen 4 und 6 über eine Bromierung und anschließende Pd-katalysierte Suzuki-Miyaura Kreuzkupplung ${ }^{[183]}$ substituiert werden. Die Position 5 sollte mittels Ir-katalysierter C-H-Aktivierung boryliert werden. ${ }^{[184,185]}$ Anschließend wäre ebenfalls eine Suzuki-Miyaura-Kupplung möglich.

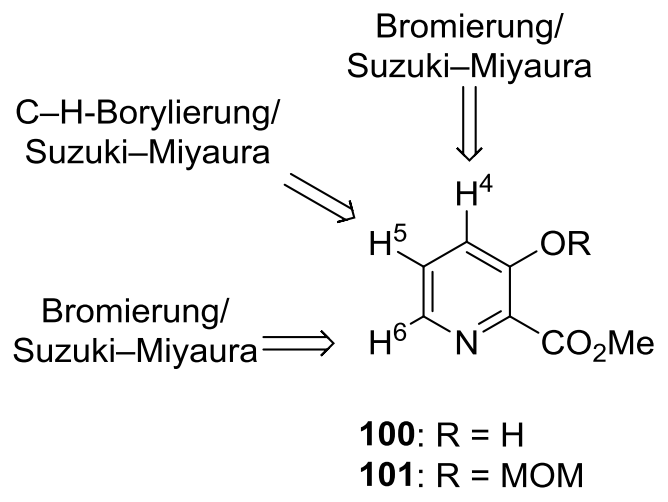

Schema 2.5: Schema zur Substitution der aromatischen Positionen in 3-Hydroxypicolinsäuremethylester.

In Zukunft sollen in Zürich Derivate des Pyridomycins hergestellt und auf veränderte pharmakologische Eigenschaften hin untersucht werden. 


\subsection{Synthese tetradentater Biscarboxamid-Liganden}

Basierend auf den Erfahrungen mit der Synthese von substituierten 3-Hydroxypicolinsäuren sollten in Zusammenarbeit mit Jun.-Prof. Dr. Alexander Schiller (Friedrich-SchillerUniversität Jena, Institut für Anorganische und Analytische Chemie) tetradentate 1,2Bis(pyridin-2-carboxamido)-benzen-Liganden synthetisiert werden, die einen 3-Hydroxybzw. 3-Methoxy-Substituenten tragen (Abbildung 2.6).

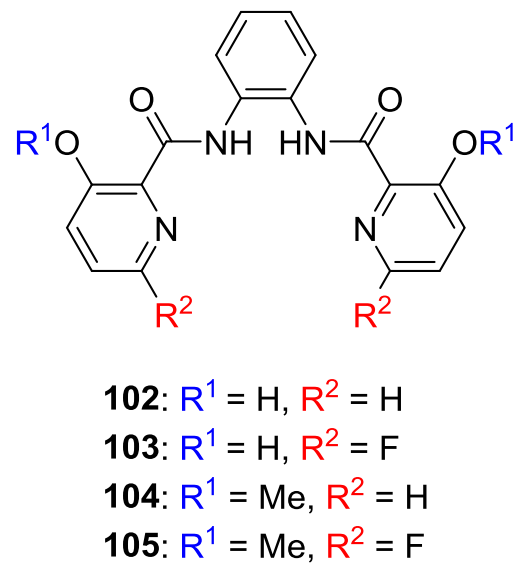

Abbildung 2.6: Strukturen der geplanten Biscarboxamid-Liganden.

Diese sollten anschließend vom Kooperationspartner zu Nitrosyl-Ruthenium-Komplexen umgesetzt werden und auf ihre Eigenschaften als NO-freisetzende Materialien hin untersucht werden. 
3 Totalsynthese von Nosiheptid 


\subsection{Einleitung}

Nosiheptid (6, Abbildung 3.1 $)^{[139]}$ gehört mit einer minimalen Hemmkonzentration (MIC) von $0.06-0.125 \mathrm{mg} / \mathrm{L}$ gegenüber verschiedenen $S$. aureus-Stämmen ${ }^{[31]} \mathrm{zu}$ den potentesten Vertretern der Thiopeptidantibiotika. ${ }^{[26]}$

„Es enthält einen ungewöhnlichen 3-Hydroxypyridin-Kern, verschieden substituierte Thiazole, ein sterisch gehindertes aromatisches B-Ring-Thiolacton und einen Indolylmethylester, die alle in ein makrobicyclisches Gerüst eingebettet sind, das eine abgehende Seitenkette mit einer Dehydroaminosäure trägt. ““[120]

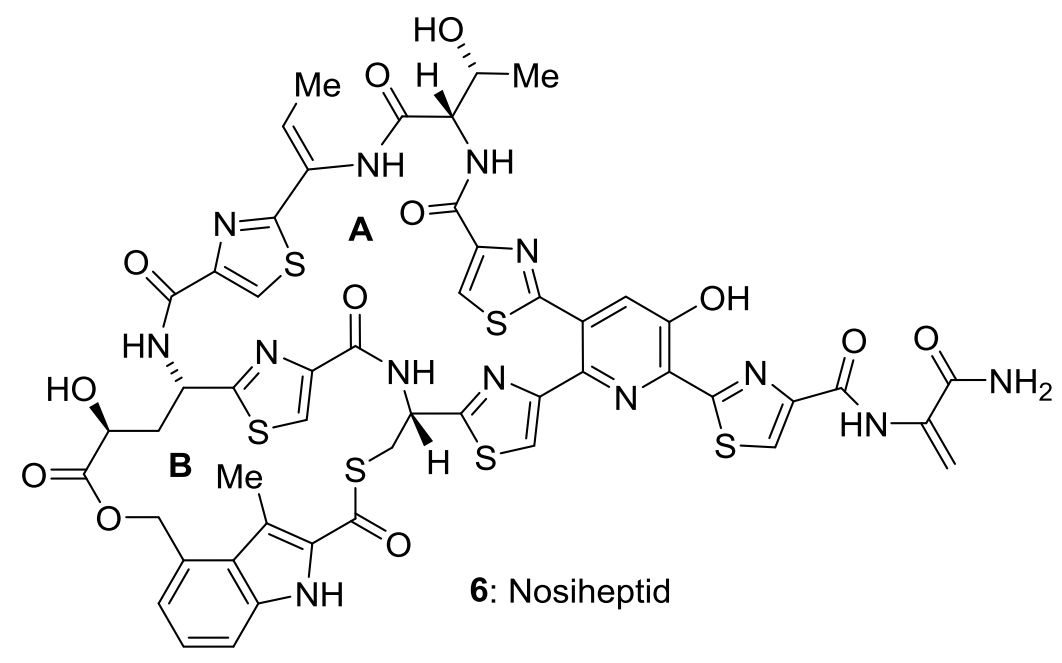

Abbildung 3.1: Struktur von Nosiheptid.

Diese einzigartigen strukturellen Eigenschaften in einer hochfunktionalisierten Architektur machen Nosiheptid zu einer lohnenden Zielstruktur für die Entwicklung neuer chemischer Methoden. Durch eine konvergente Synthese, die den Austausch von Bausteinen zulässt, könnten neue Derivate hergestellt werden, die eine weitergehende chemisch-biologische Profilierung dieses potenten und strukturell ungewöhnlichen Antibiotikums ermöglichen könnten. 


\subsection{Synthesestrategie}

\subsubsection{Rückschlüsse aus früheren Arbeiten}

Wie bereits in Kapitel 1.5.3 dargestellt, gelang es J.-Y. Lu und M. Riedrich in vorhergehenden Arbeiten den A-Ring-Makrocyclus aus den beiden Fragmenten $\mathbf{4 2}$ und $\mathbf{6 3}$ durch aufeinanderfolgende Amid-Knüpfungen aufzubauen (Schema 3.1). ${ }^{[36,37,161]}$

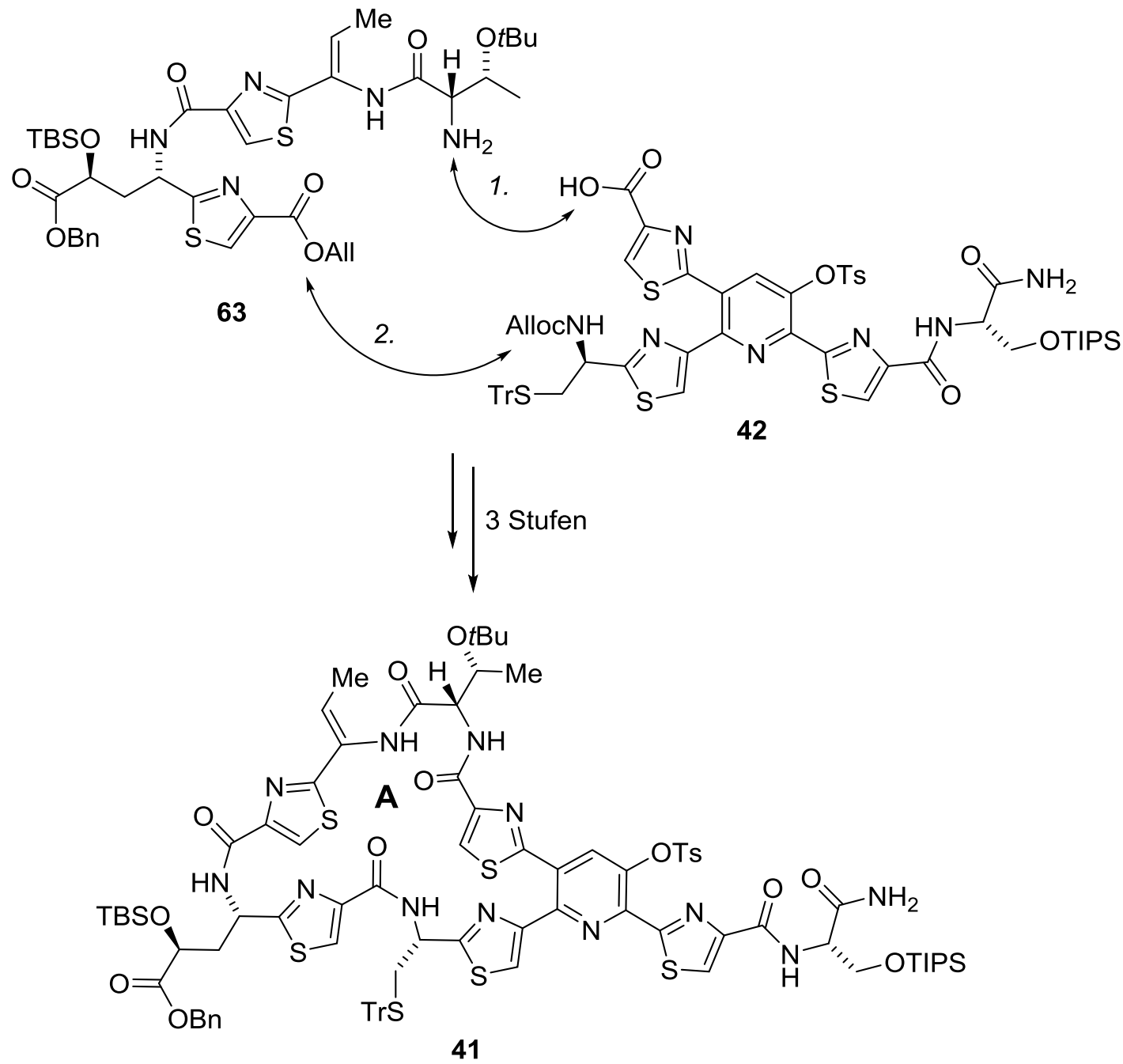

Schema 3.1: Aufbau des A-Ring-Makrocyclus von Nosiheptid nach Lu und Riedrich mit angegebener Reihenfolge der Amid-Knüpfungen. ${ }^{[36,37]}$

Diese Strategie sollte nun in modifizierter Form für eine Totalsynthese von Nosiheptid genutzt werden.

Eine Acylierung des Indolylalkohols 106 durch Öffnung des Lactams 59, zur früheren Einführung des Indolfragments, konnte zuvor von P. Winter durchgeführt werden (Schema $3.2){ }^{[186]}$ 


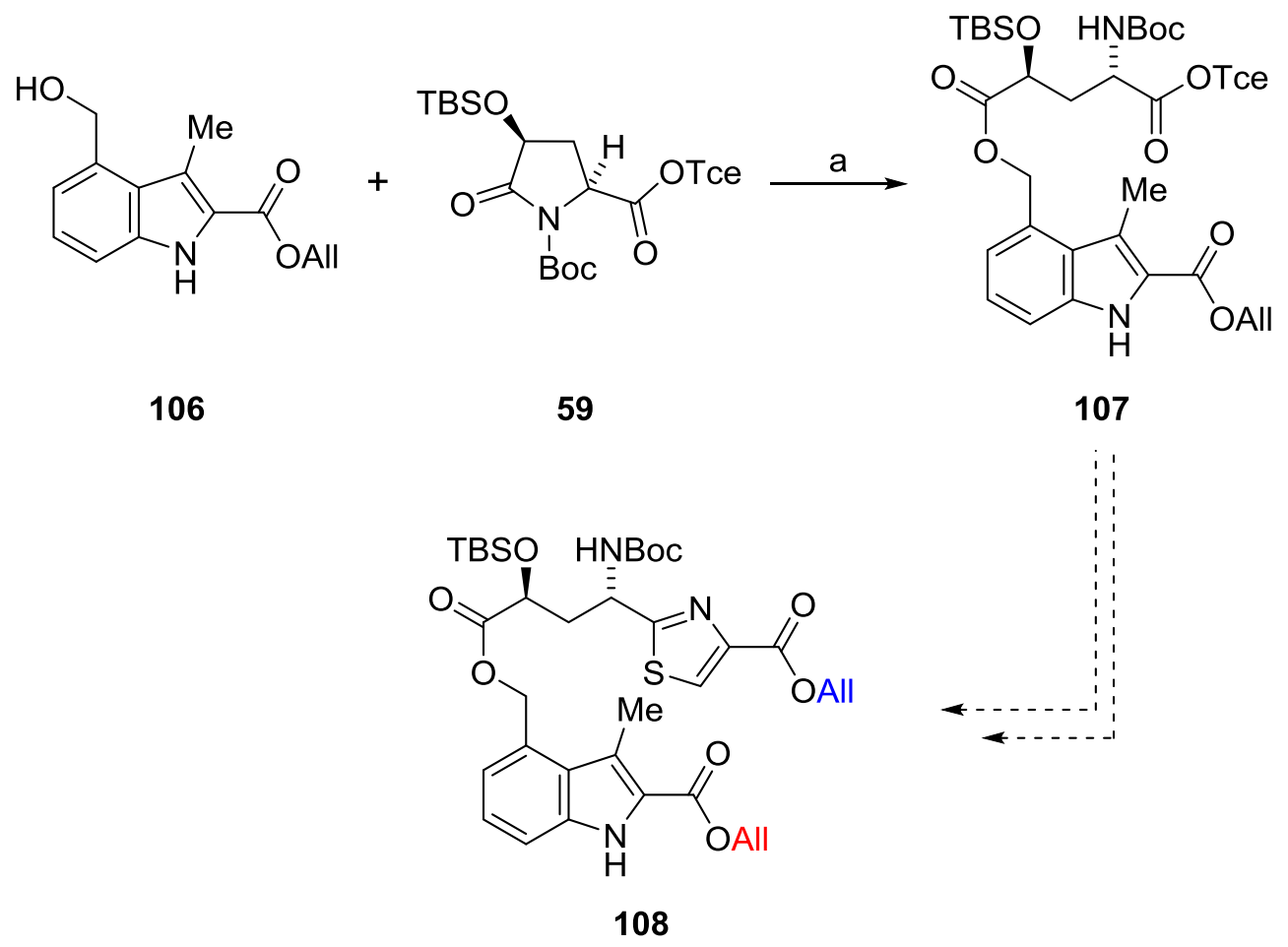

Schema 3.2: Einführung des Indols durch Lactam-Öffnung nach P. Winter. Bedingungen: a) NaH, THF, $-78{ }^{\circ} \mathrm{C}, 75 \mathrm{~min}, 41 \%$.

Der durch Winter eingesetzte Allyl-Ester (rot) wäre jedoch in späteren Stufen nicht mit dem am Thiazol angebrachten Allylester (blau) kompatibel und müsste durch eine orthogonale Esterschutzgruppe ausgetauscht werden. Dafür würde sich ein Diphenylmethylester (Dpm) anbieten, da dieser gleichzeitig mit der Trityl-Gruppe am Cystein-Rest abgespalten werden könnte. Der B-Ring könnte dann über eine Makrothiolactonisierung geschlossen werden. Diese konnte bereits durch Moody et al. mit einem vereinfachten Testsystem (109) erfolgreich demonstriert werden (Schema 3.3). ${ }^{[156]}$<smiles>Cc1c(C(=O)O)[nH]c2cccc(COC(=O)[C@@H](C[C@H](OC(C)(C)C)C(C)(C)C)NC(=O)c3csc([C@H](CS)NC(=O)c4nc(-c5ccccc5)cs4)n3)c12</smiles>

109

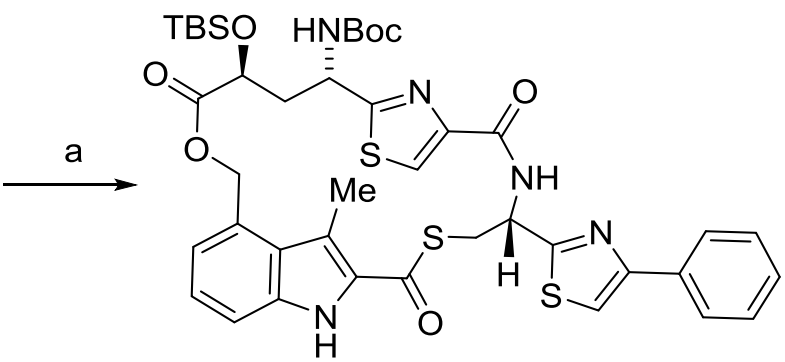

39

Schema 3.3: Aufbau des B-Ring-Makrothiolactons nach Moody et al.. Bedingungen: a) DCC, DMAP THF, 16 h, 52\% oder PyBOP, EtNiPr 2 , THF, 16 h, 47\%. ${ }^{[156]}$ 
Eine Anpassung der Schutzgruppe an der Threonin-Seitenkette sollte ebenfalls vorgenommen werden, da die zuvor eingesetzte $t \mathrm{Bu}$-Gruppe nicht orthogonal zum Dpm-Ester und der TritylSchutzgruppe am Cystein-Rest wäre.

\subsubsection{Retrosynthetische Analyse}

Basierend auf den im vorhergehenden Abschnitt dargestellten Überlegungen erfolgte die retrosynthetische Planung der Synthese von Nosiheptid (6) wie in Schema 3.4 gezeigt.

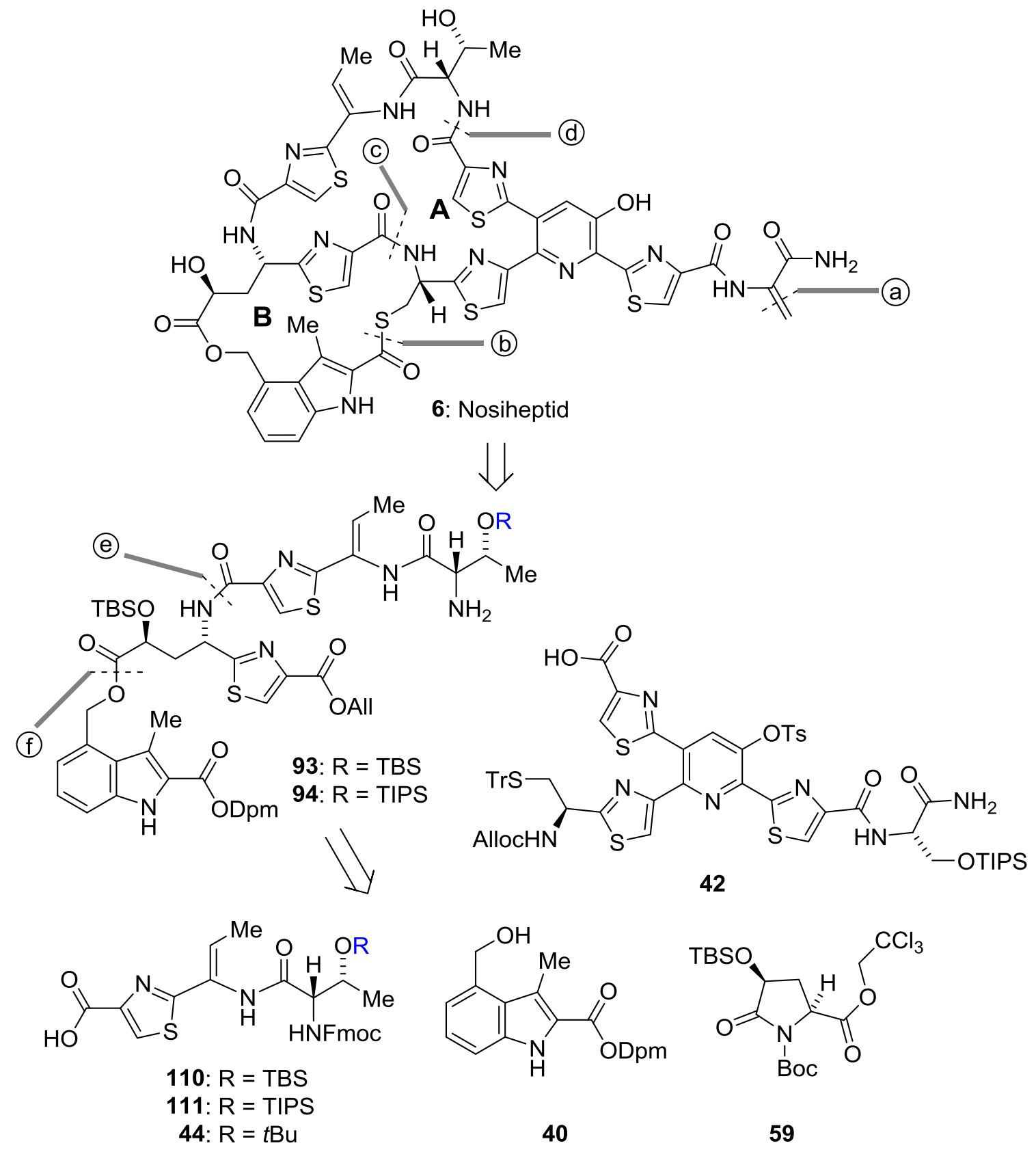

Schema 3.4: Retrosynthetische Analyse von Nosiheptid. 
Die Einführung des Dehydroalanins (DHA) sollte aufgrund seiner Reaktivität als MichaelAkzeptor, und der damit verbundenen Inkompatibilität mit Thiolvorstufen des Thioesters, erst zu einem späten Zeitpunkt der Synthese stattfinden. Dies sollte über eine Eliminierung einer Serin-Seitenkette erfolgen (a). Der Thioester wurde, wie bereits oben erläutert, auf eine $\omega$-Mercaptosäure mit säurelabilen Schutzgruppen (Dpm, Tr) zurückgeführt (b). Die parallele Spaltung dieser beiden Gruppen würde den Aufbau des B-Ring-Makrobislactons ermöglichen. Basierend auf der erfolgreichen Makrolactamisierung zum A-Ring-Modell ${ }^{[161,175]}$ (Kap. 1.5.3), wurde an zwei strategischen Amidbindungen (c/d) weiter zerlegt und somit die Bis-ThiazolFragmente 93/94 und das bereits bekannte Tristhiazolylpyridin $\mathbf{4 2}^{[37]}$ mit den entsprechenden Schutzgruppen erhalten. Als Schutzgruppe an der Threonin-Seitenkette sollte eine Silylschutzgruppe eingesetzt werden, die ggf. in Ihrer Stabilität angepasst werden könnte. Die Bis-Thiazol-Fragmente 93/93 lassen sich auf die Peptidthiazole 110/111 vereinfachen (e), deren Synthesen der bekannten Route für das Derivat 44 $^{[36]}$ folgen sollten (Kap. 1.5.3). Wie bereits zuvor diskutiert, sollte die Einführung des Indol-Bausteins 40 über die Öffnung des Lactams 59 (f) erfolgen.

\subsubsection{Etappenziele und Herausforderungen}

Neben der Darstellung bereits literaturbekannter Fragmente, war eines der ersten Etappenziele die Darstellung des Bisthiazols 93, beispielhaft mit einer TBS-Gruppe an der ThreoninSeitenkette versehen (Schema 3.5). 
<smiles>CC#CC#CCCCCCOC(=O)c1[nH]c2cccc(CO)c2c1C(=O)OCC(Cl)(Cl)Cl</smiles>

59
40

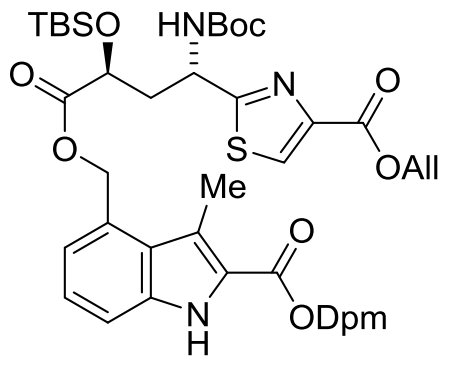

95

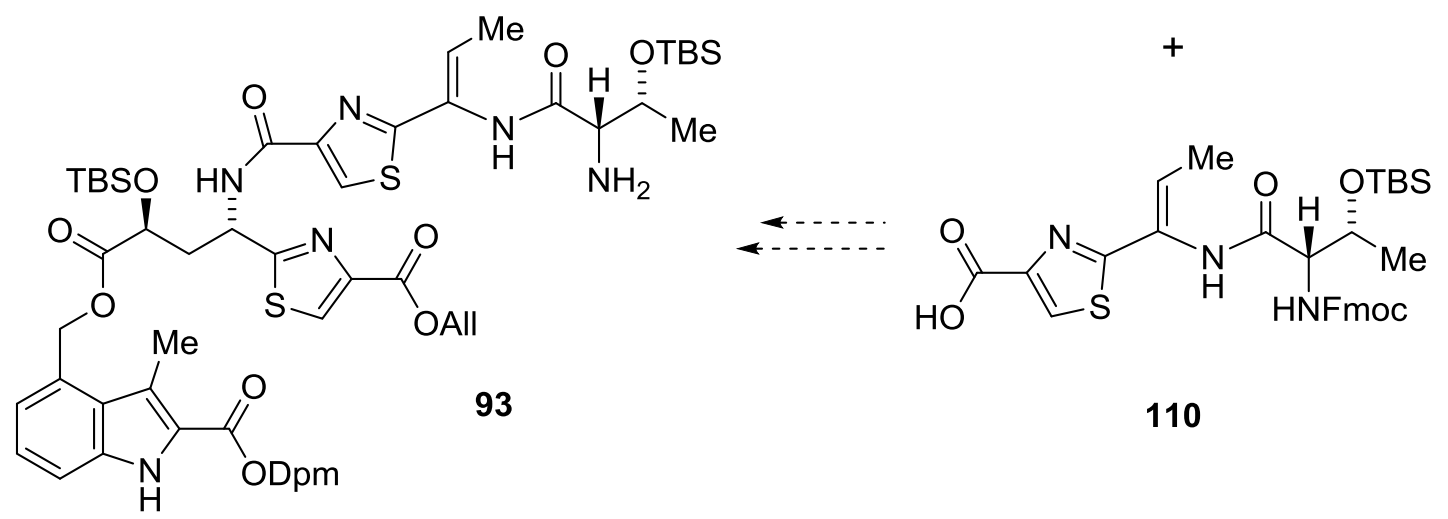

Schema 3.5: Darstellung des indoltragenden Bisthiazolfragments 93 als erstes Etappenziel.

Dieses könnte anschließend mit dem Pyridin-Kern verknüpft und die Makrocyclen aufeinanderfolgend aufgebaut werden. Dafür mussten jedoch zuerst die Bedingungen zur gleichzeitigen Entschützung des Dpm-Esters und der Trityl-Schutzgruppe und anschließenden Makrothiolactonisierung entwickelt werden. Dies sollte mit Hilfe des in Abbildung 3.2 dargestellten Testsystems erfolgen.

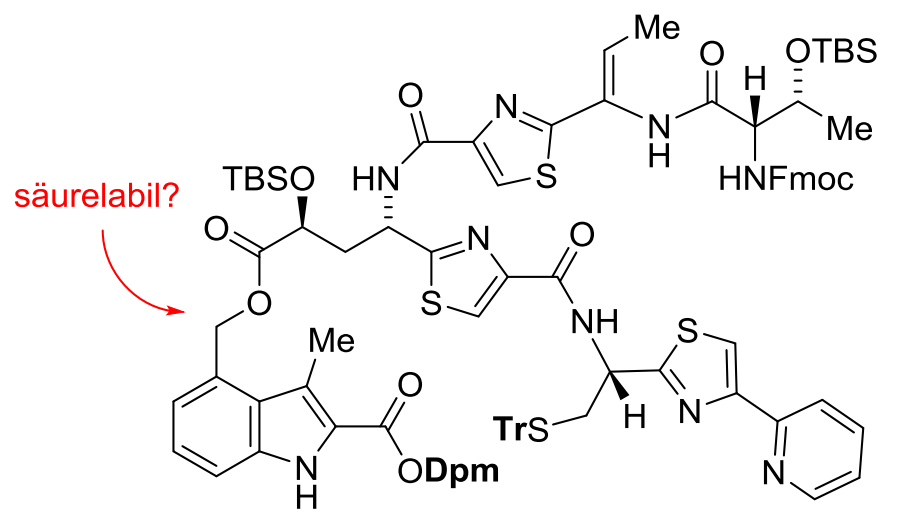

113

Abbildung 3.2: Testsystem zur gleichzeitigen Dpm- und Tr-Spaltung und anschließender Makrothiolactonisierung. 
Die unbekannte Stabilität des Indolylesters musste dafür ergründet und in der späteren Synthese berücksichtigt werden (Kap. 3.3).

Die vollständigen linearen Vorläufer 114/115 sollten dann durch aufeinander folgende Makrocyclisierungen in die Bismakrocyclen 116/117 überführt werden (Schema 3.6).

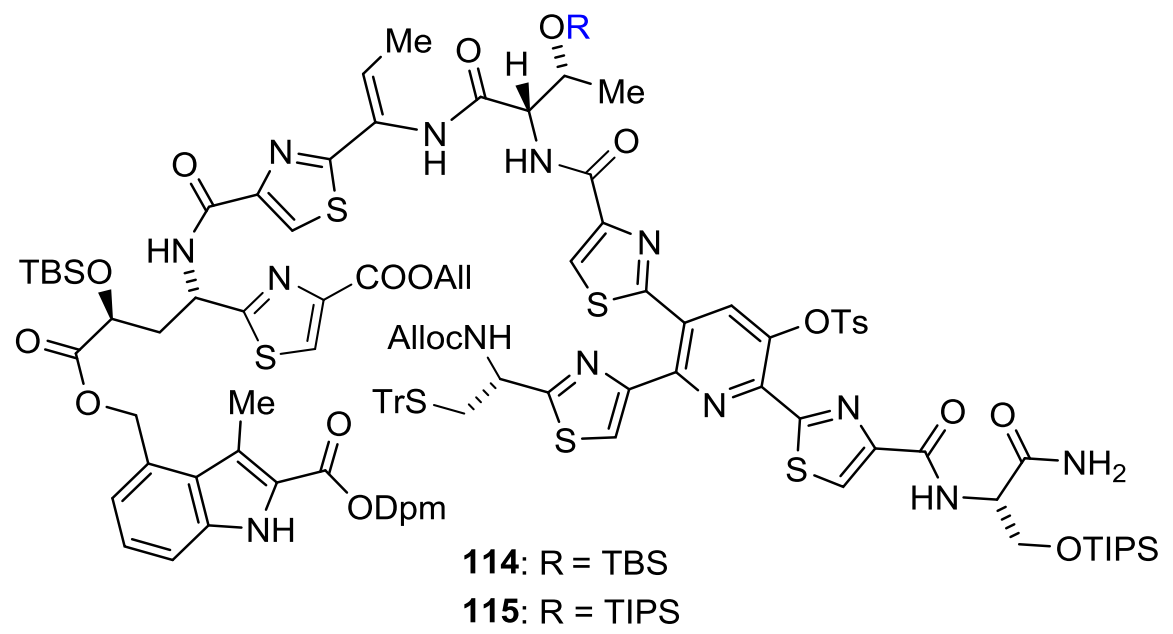

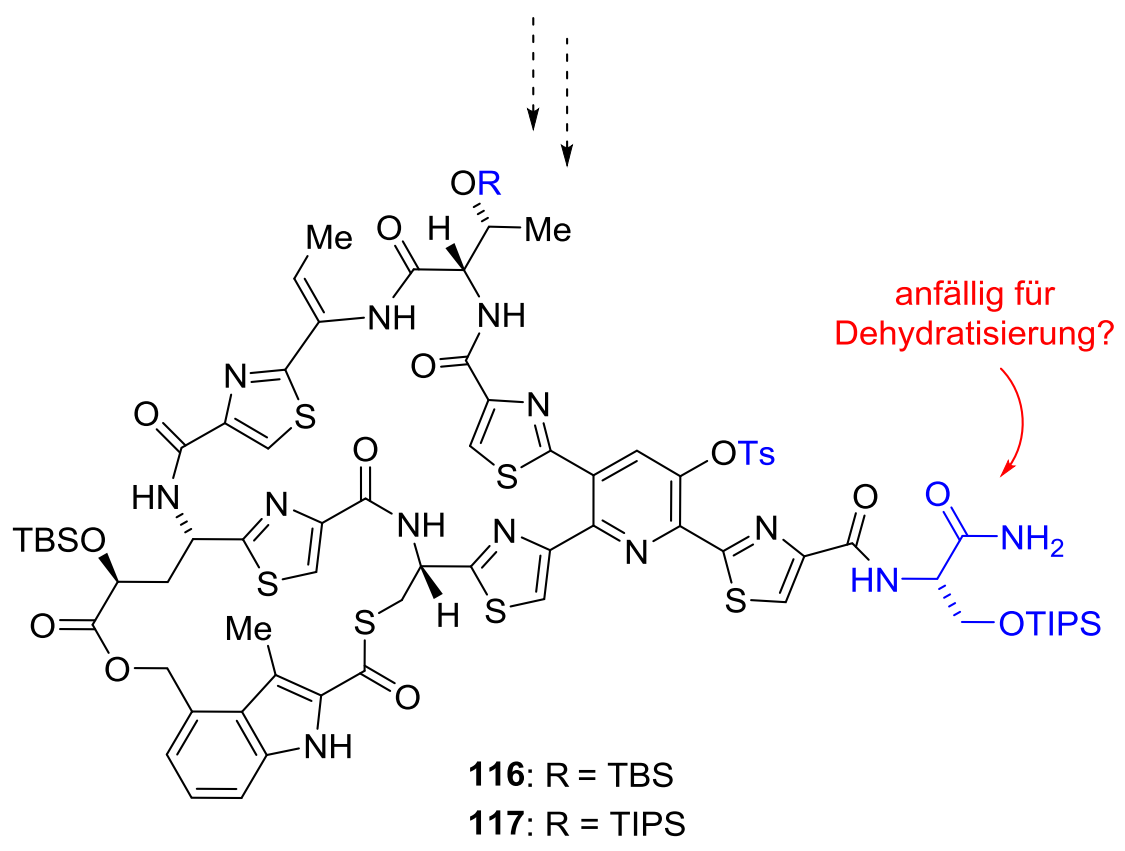

Schema 3.6: Geplante Darstellung der Bismakrocyclen 116/117 aus den linearen Vorläufern 114/115.

Nach Aufbau des B-Ring-Thiolactons wäre die Einführung des elektrophilen Dehydroalanins möglich. Dabei wurden zwei mögliche Vorgehensweisen in Betracht gezogen. Zum einen, eine vollständige Entschützung aller Silylschutzgruppen und anschließende selektive Sulfonierung der primären $\mathrm{OH}-$ Gruppe und nachfolgende Eliminierung des Sulfonats, zum anderen eine selektive Spaltung der primären Silylschutzgruppe und eine drauffolgende Umwandlung des Serinrest in ein Dehydroalanin. Die mögliche Neigung des terminalen 
Amids zur Dehydratisierung musste hierbei besonders berücksichtigt werden.

Darüber hinaus mussten Bedingungen für eine selektive Entschützung der Ts-Gruppe mit einem geeigneten Nucleophil gefunden werden. Dies sollte, sowohl über Studien am Pyridinfragment als auch an semisynthetisch dargestellten Verbindungen erfolgen.

\subsection{Synthese des Indol-Bausteins}

\subsubsection{Aufbau des Indols}

Der Synthesegang begann mit dem Aufbau des Indols. Es war ursprünglich geplant den IndolBaustein 40 nach der durch M. Riedrich erarbeiteten Route zu synthetisieren (Kapitel 1.5.3). Beim Nachvollziehen der Synthesevorschrift wurde aber in der Negishi-Kupplung mit $\mathrm{Me}_{2} \mathrm{Zn}$ immer wieder eine begleitende Hydrodehalogenierung des 3-Iodindols 77 beobachtet (Schema 3.7). ${ }^{[159]}$

THPO<smiles>CCOC(=O)c1[nH]c2cccc(CO)c2c1I</smiles>

77

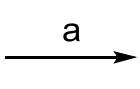

THPO

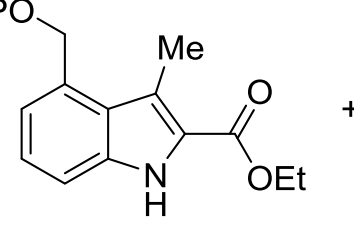

78
THPO<smiles>CCOC(=O)c1cc2c(CO)cccc2[nH]1</smiles>

76

b

Schema 3.7: Synthese des 3-Methylindols 78 über Negishi-Kreuzkupplung. Bedingungen: a) $\mathrm{Me}_{2} \mathrm{Zn}$, $\mathrm{Pd}$ (dppf)Cl $\mathrm{Cl}_{2}$, 1,4-Dioxan, 16 h, $110{ }^{\circ} \mathrm{C}, 95 \%$. b) $\mathrm{I}_{2}, \mathrm{~K}_{2} \mathrm{CO}_{3}$, DMF, RT, 16 h, 17-23\% 77, 70-75\% 78.

Das Verhältnis zwischen den nicht trennbaren Produkten schwankte je nach Versuch zwischen 3:1 und 5:1 (Me:H). Erst durch Re-Iodierung des Gemischs konnte eine chromatographische Trennung erreicht werden. Dieser Umweg erwies sich bei Ansätzen in größerem Maßstab als sehr aufwendig.

In bisher nicht publizierten Arbeiten hatte M. Riedrich alternativ gezeigt, dass eine Einführung der Methylgruppe über eine Methylenierung des $\alpha$-Ketoesters $\mathbf{7 5}$ und dessen anschließende Umsetzung in einer Reissert-Indol-Synthese zum 3-Methylindol 78 mit $\mathrm{PtO}_{2}$ möglich war (Schema 3.8). ${ }^{[187]}$ 
<smiles>CCOC(=O)C(=O)Cc1c(COP)cccc1[N+](=O)[O-]</smiles>

75<smiles>C=C(C(=O)OCC)c1c(COP)cccc1[N+](=O)[O-]</smiles>

118<smiles>CCOC(=O)c1[nH]c2cccc(COP)c2c1C</smiles>

78

Schema 3.8: Synthese des 3-Methylindols 78 nach Riedrich. ${ }^{[187]}$ Bedingungen: a) Dimethylmethylideniminiumchlorid (Böhme Salz), ${ }^{[188]} \mathrm{Et}_{3} \mathrm{~N}, \mathrm{CH}_{2} \mathrm{Cl}_{2}$, RT, 13 h, 84\%. b) $\mathrm{PtO}_{2}$, EtOAc, 1 bar $\mathrm{H}_{2}$, RT, 2 h, 70\%.

Diese Umsetzung hing jedoch sehr stark von der genutzten $\mathrm{PtO}_{2}-$ Charge ab und konnte in späteren Versuchen durch M. Riedrich nicht reproduziert werden. In der vorliegenden Arbeit wurde diese Vorgehensweise erneut aufgegriffen. Dabei flossen auch die durch die Gruppe von Nicolaou gewonnen Erkenntnisse zur Synthese von $\mathrm{N}$-Hydroxyindolen ein, die Nitroarylenone als Substrate einsetzten. ${ }^{[147,148,189]}$

Um den Gedankengang zur Optimierung der Hydrierungsbedingungen zu illustrieren, ist Schema 3.9 ein hypothetischer Mechanismus der Reissert-Indol-Synthese, ausgehend vom Nitroarylenon 119 dargestellt. Mögliche Nebenreaktionen sind ebenfalls gezeigt.

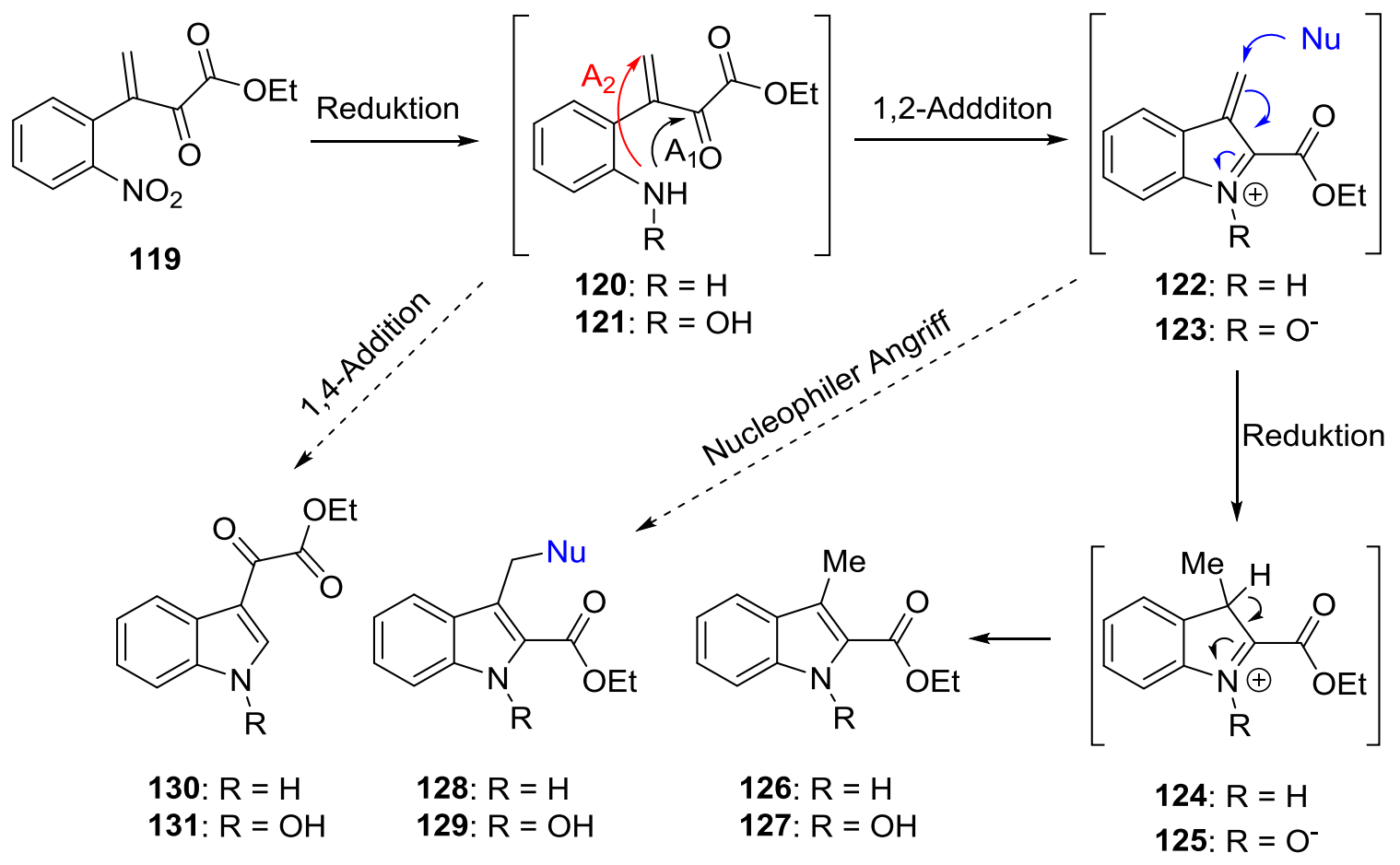

Schema 3.9: Mechanismus der Reissert-Indol-Synthese mit Nitroarylenon 119 und mögliche Nebenreaktionen. 
Im ersten Schritt findet eine Reduktion der Nitro-Gruppe zum Anilin 120 statt. Dabei ist auch eine teilweise Reduktion zum Hydroxylamin 121 möglich. Anschließend kann das Anilin 120 bzw. Hydroxylamin 121 über zwei Wege reagieren. Über den Reaktionspfad $A_{1}$ reagiert der Stickstoff über eine Kondensationsreaktion mit dem Carbonylkohlenstoff des Ketons zum $\alpha, \beta$-ungesättigten Iminium 122 bzw. Nitron 123. Der Reaktionspfad $A_{2}$ hingegen verläuft über eine intramolekulare 1,4-Adition des Stickstoffatoms am benachbarten MichaelAkzeptor und liefert einen nicht gewünschten $\alpha$-Ketoester 130/131. ${ }^{[147]}$

Das nach der Kondensation gebildete $\alpha, \beta$-ungesättigte Iminium 122 bzw. Nitron 123 kann wiederum über zwei Wege reagieren. Über eine 1,4-Addition eines Nucleophils wird direkt ein substituiertes $(N$-Hydroxy)indol erhalten (128/129). Dieser Reaktionsweg wurde durch Nicolaou et al. intensiv studiert und zur Synthese eines Nocathiacin-B-Ring-Models genutzt. $^{[147,148,189]}$ Wird indes das Alken reduziert, kann das gebildete Iminium 122 bzw. Nitron 123 zum 3-Methyl(N-hydroxy)indol 126/127 tautomerisieren. Dies führt zum gewünschten 3-Methylindol 126.

Um möglichst wenig $\mathrm{N}$-Hydroxyindol $127 \mathrm{zu}$ generieren, musste ein hoch reaktiver Katalysator eingesetzt werden, der eine schnelle und vollständige Reduktion der Nitro-Gruppe zum Anilin bewirkt. Durch den Einsatz protischer Lösungsmittel sollte die „Selbst“" Vergiftung des Katalysators durch das in der Reaktion gebildete Anilin unterdrückt werden können. ${ }^{[190]}$ Das Lösungsmittel darf wiederum nur wenig nucleophil sein, da es sonst mit dem $\alpha, \beta$-ungesättigten Iminiumion 122 reagieren könnte. Hierfür wurde 2-Propanol gewählt.

Das durch die Reduktion der Nitrogruppe und in der Kondensationsreaktion gebildete Wasser könnte alternativ addieren und muss daher aus dem Reaktionsmedium entfernt werden. Daher wurde 4 A Molekularsieb zugesetzt. ${ }^{[147,148,189]}$ Um eine ausreichende Löslichkeit der Substanz zu gewährleisten wurde des weiteren THF als Co-Solvens zugesetzt.

Mit den so gewählten Reaktionsbedingungen konnte, nach kurzer Testierung verschiedener Katalysatoren, eine reproduzierbare Umsetzung des Nitroarylenons 118 zum 3-Methylindol 78 mit zufriedenstellenden Ausbeuten von etwa 68\% erreicht werden (Schema 3.10). Die Bildung eines $\alpha$-Ketoesters (analog $\mathrm{zu}$ 130) konnte unter diesen Bedingungen nicht beobachtet werden. 


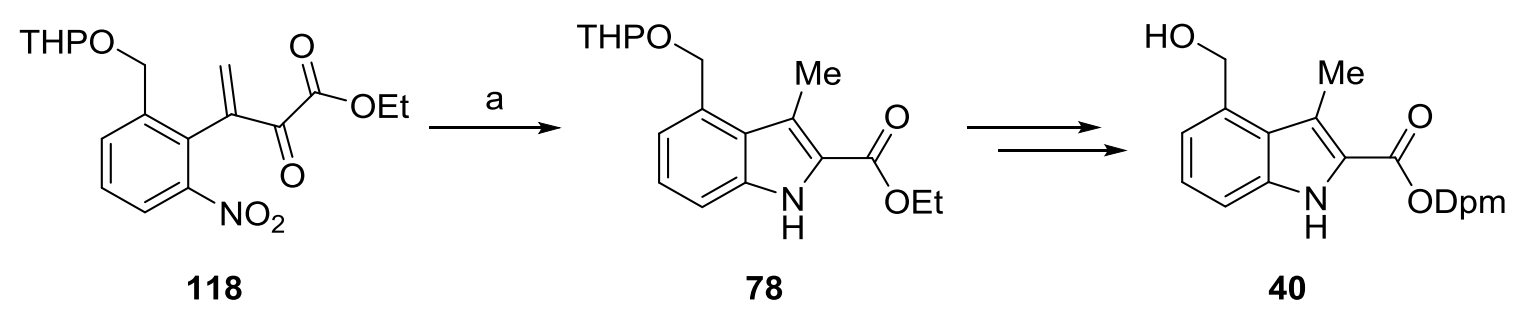

Schema 3.10: Synthese des 3-Methylindols 78 nach Optimierung. Bedingungen: a) $10 \% \mathrm{Pd} / \mathrm{C}$ (10 gew.\%, Acros 19503), 4 Å Molekularsieb (20 gew.\%), THF/IPA (1:1), 1 bar $\mathrm{H}_{2}$, RT, 24 h, 68\%.

Versuche mit höherem Wasserstoffdruck (bis 4 bar) lieferten keine verbesserten Ausbeuten. Die Reaktion konnte auch in größerem Maßstab $(>15 \mathrm{~g})$ mit nur geringen Einbußen in der Ausbeute (61\%) durchgeführt werden. Die weitere Umsetzung zum Indolylalkohol 40 erfolgte entsprechend dem von M. Riedrich beschriebenen Syntheseweg (s. Kapitel 1.5.3). ${ }^{[36]}$

\subsubsection{Untersuchungen zur selektiven Dpm-Spaltung}

Um geeignete Bedingungen für die selektive Spaltung des Diphenylmethylesters zu finden, wurde der Indolylalkohol 40 mit Buttersäureanhydrid und einer sehr guten Ausbeute von 98\% zum Buttersäureester 132 umgesetzt (Schema 3.11).

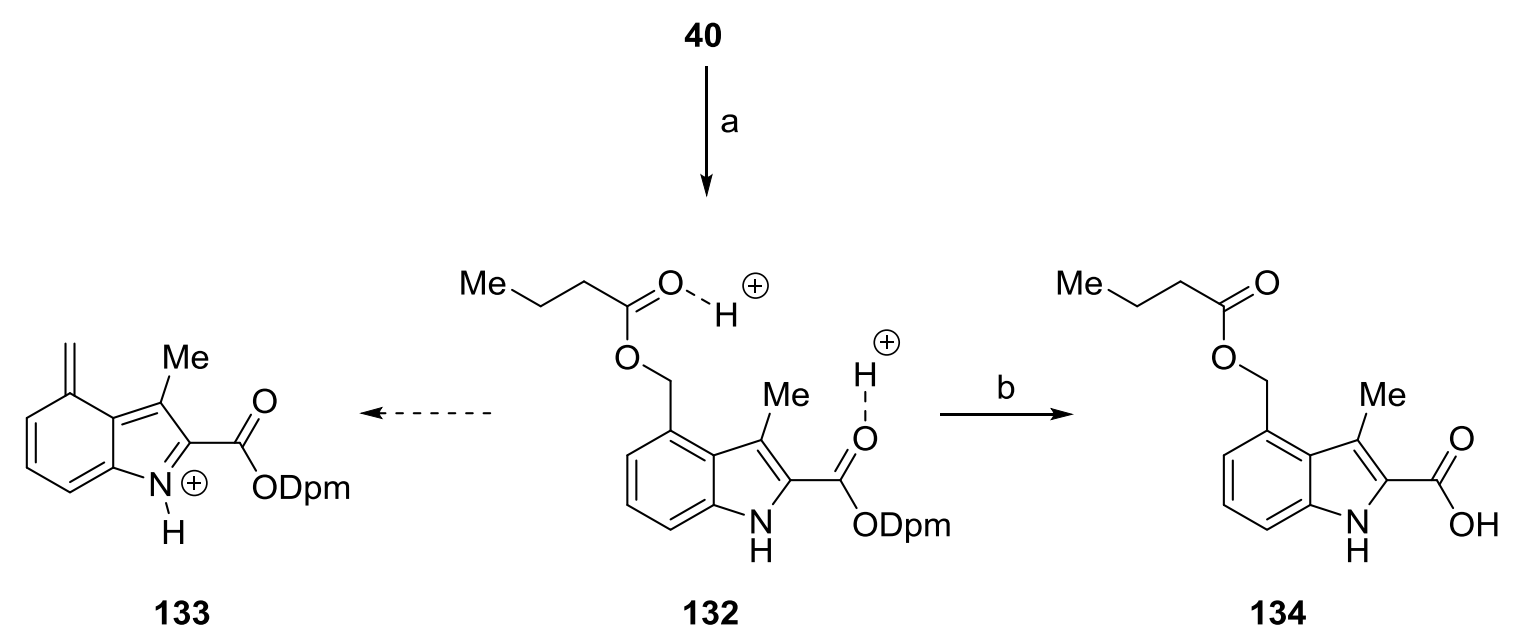

Schema 3.11: Acylierung des Indolylalkohols 40 zum Buttersäureester 132 und Spaltung des DpmEsters. Bedingungen: a) Buttersäureanhydrid, EtNiPr 2 , DMAP, THF, $0{ }^{\circ} \mathrm{C} \rightarrow \mathrm{RT}, 13 \mathrm{~h}, 98 \%$. b) TFA, $\mathrm{Et}_{3} \mathrm{SiH}$, Anisol, $0{ }^{\circ} \mathrm{C}, 8 \mathrm{~h}, 5 \mathrm{mg}-\mathrm{Ma}$ stab, saubere Umsetzung (LC-MS).

Bei Versuchen den Dpm-Ester mit TFA in $\mathrm{CH}_{2} \mathrm{Cl}_{2}$ zur Carbonsäure 134 zu spalten, wurden unspezifische Spaltungen beobachtet (LC-MS). Auch bei niedrigen Säurekonzentrationen (ca. $1 \%$ ) und verminderter Temperatur (bis $-20^{\circ} \mathrm{C}$ ) wurden in HPLC-MS-Experimenten Signale 
detektiert, die auf eine Spaltung des Buttersäureesters zu einem 4-Methylenindolinylkation (133) hindeuteten. Dieses Phänomen ist auch für elektronenreiche Anisolester bekannt und wurde ausführlich studiert. ${ }^{[191,192]}$ Die Isolierung der Zersetzungsprodukte des stabilen Ethylesters 135 gelang nicht.

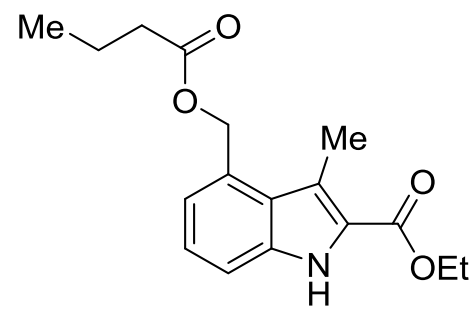

135

Abbildung 3.2: Struktur des Ethylesters 135.

Versuche mit $\mathrm{HCl}$ in 1,4-Dioxan oder Pd-vermittelter Spaltung ${ }^{[193]}$ des Dpm-Esters zeigten keine Selektivität oder einen geringen Umsatz. Durch in situ Generierung von HF aus $\mathrm{BF}_{3} \times \mathrm{Et}_{2} \mathrm{O}$ und Essigsäure konnte dann eine selektive Spaltung des Dpm-Esters erreicht werden. ${ }^{[194]}$ Dies war jedoch mit dem in der Synthese geplanten Silylschutzgruppen nicht kompatibel. Versuche mit TFA in Ansiol, ${ }^{[195,196]}$ in Gegenwart von $\mathrm{Et}_{3} \mathrm{SiH}$ als Abfangreagenz für das gebildete Diphenylmethyl-Kation, ${ }^{[197]}$ ergaben eine selektive Spaltung des Dpm-Esters ohne weitere Zersetzung des Moleküls. Die besten Ergebnisse wurden dabei bei strikt wasserfreien Bedingungen und $0{ }^{\circ} \mathrm{C}$ erhalten.

\subsection{Synthese der Bis-Thiazol-Fragmente}

\subsubsection{Synthese des indoltragenden Thiazols}

Der Aufbau des A- und B-Ring verbrückenden Thiazols 95 sollte in Analogie zu der in Kapitel 1.5.3 vorgestellten Synthese des Thiazols 45 erfolgen. Für die Synthese des benötigten Lactams 59 wurde im ersten Schritt das Lacton $\mathbf{5 7}^{[198]}$ in Gegenwart von $\mathrm{NaN}_{3}$ mit 2,2,2,-Trichlorethanol zum 4-Hydroxyprolinester 136 geöffnet (Schema 3.12). ${ }^{[199]}$ 


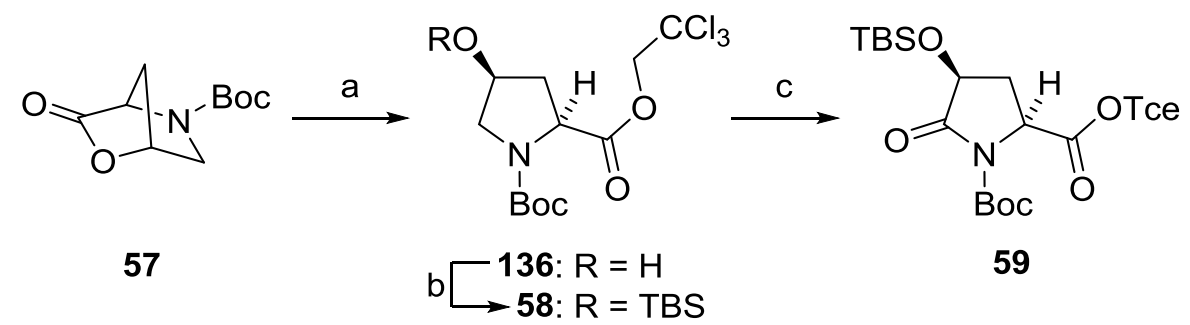

Schema 3.12: Synthese des Lactams 59 ausgehend vom Lacton 57. Bedingungen: a) $\mathrm{NaN}_{3}, 2,2,2-$ Trichlorethanol, $50{ }^{\circ} \mathrm{C}, 1 \mathrm{~h}, 83 \%$. b) TBSCl, Imidazol, DMF, $0{ }^{\circ} \mathrm{C} \rightarrow \mathrm{RT}, 18 \mathrm{~h}, 99 \%$. c) $\mathrm{RuO}_{2}, \mathrm{NaIO}_{4}$, $\mathrm{H}_{2} \mathrm{O} / \mathrm{MeCN} / \mathrm{CCl}_{4}, \mathrm{RT}, 13 \mathrm{~h}, 76 \%$.

Die $\mathrm{NaN}_{3}$-vermittelte Öffnung des Lactons 57 lieferte, im Vergleich zur bisher beschriebenen Methode mit Natriumtrichloroethanolat bei tiefer Temperatur, ${ }^{[36]}$ eine gute Ausbeute von $83 \%$. Auch die Bildung von Dimeren durch Umesterung des Trichlorethylesters, mit dem nach der Lacton-Öffnung gebildeten Alkoholats, wurde so unterbunden.

Die freigesetzte Hydroxygruppe wurde anschließend mit TBSCl und mit einer exzellenten Ausbeute von 99\% als TBS-Ether geschützt. Anschließend konnte das Pyrrolidin 58 mittels $\mathrm{RuO}_{4}-\mathrm{Katalyse}^{[200]}$ mit einer Ausbeute von 76\% regioselektiv zum $\gamma$-Lactam 59 oxidiert werden.

Die Einführung des Indolylalkohols 40 erfolgte durch dessen Acylierung mit dem aktivierten Lactam 59 unter basischen Bedingungen (Schema 3.13). 


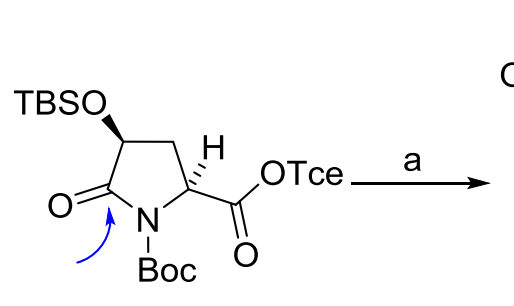

59

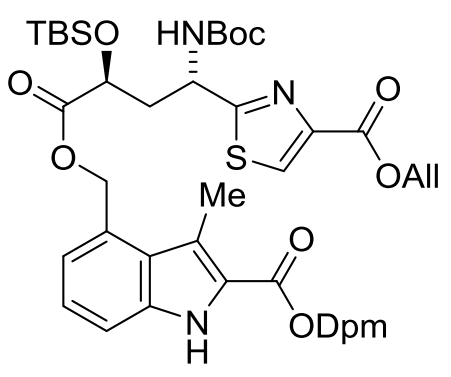

95

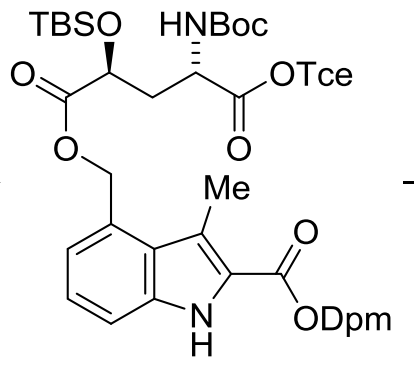

137

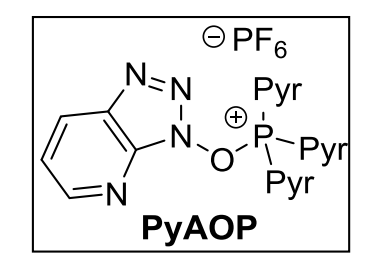

d, e

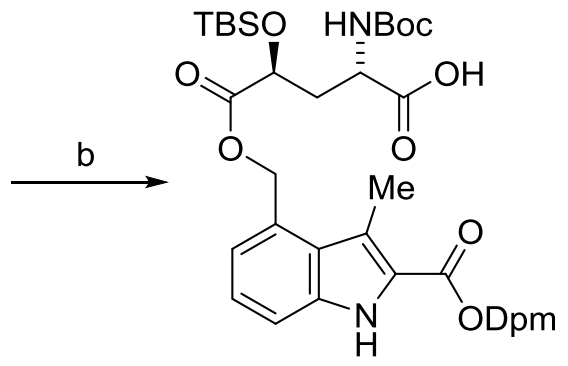

138
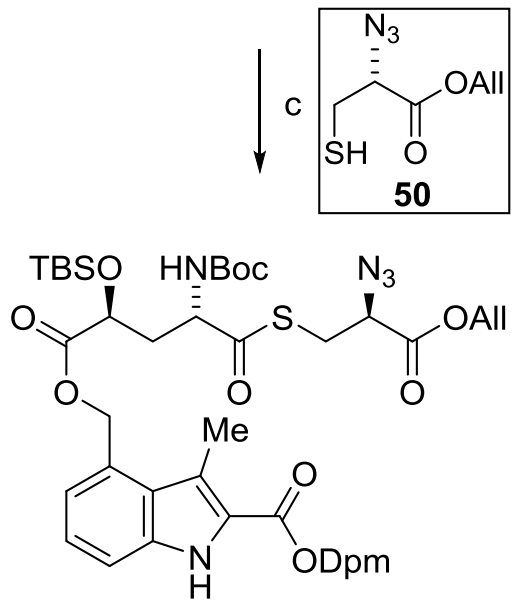

139

Schema 3.13: Synthese des indoltragenden Thiazols 95. Bedingungen: a) 40 (0.5 eq.), NaH, THF, $-78^{\circ} \mathrm{C}, 2$ h, $89 \%$ (98\% b.r.s.m.). b) $\mathrm{Zn}, 1 \mathrm{M} \mathrm{KH}_{2} \mathrm{PO}_{4}$, THF, $45^{\circ} \mathrm{C}$, ))), 10 h, $82 \%$ (95\% b.r.s.m.). c) 50, PyAOP, EtNiPr 2 , THF, $0{ }^{\circ} \mathrm{C} \rightarrow \mathrm{RT}, 3 \mathrm{~h}, 96 \%$. d) $\mathrm{PPh}_{3}$, THF, $-20{ }^{\circ} \mathrm{C} \rightarrow 40{ }^{\circ} \mathrm{C}, 16$ h. e). DBU, $\mathrm{BrCCl}_{3}, \mathrm{CH}_{2} \mathrm{Cl}_{2},-20{ }^{\circ} \mathrm{C}, 1$ h, $90 \%$ (ü. 2 Stufen). Pyr = Pyrrolidin-1-yl.

Bei tiefer Temperatur und einem Überschuss an $\gamma$-Lactam 59 konnte der Triester 137 in einer guten Ausbeute von 89\% zusammen mit unverändertem Indolylalkohol 40 (9\%) isoliert werden. Des Weiteren wurden 85\% des überschüssigen $\gamma$-Lactams 59 zurück gewonnen. Eine zum Teil beobachtete Umesterung des Trichlorethylesters mit dem Indolylalkohol 40 konnte durch eine Optimierung der Reaktionszeit minimiert werden. Anschließend wurde der TceEster reduktiv gespalten und die Carbonsäure 138 mit einer guten Ausbeute von 82\% erhalten. Diese wurde anschließend mit dem Azidothiol 50 zum Thioester 139 umgesetzt (96\%). Dabei bewies das phosphonium-basierte Kupplungsreagenz PyAOP eine überragende Effizienz im Vergleich $\mathrm{zu}$ anderen eingesetzten Kupplungsreagenzien wie PyBOP, EDCI/HOBt oder HATU. Nach anschließender Aza-Wittig-Cyclisierung und Thiazolinoxidation wurde das Thiazol 95 in einer sehr guten Ausbeute von 90\% über zwei Stufen dargestellt. 


\subsubsection{Synthese der Peptidthiazole}

Zurückgreifend auf die Synthese des bekannten Peptidthiazols 44 (Kapitel 1.5.3), wurde $L$-Boc(TBS)Threonin ${ }^{[201]}$ (140) zunächst mit dem Azidothiol 50 umgesetzt und der Thioester 141 mit einer guten Ausbeute von 78\% erhalten (Schema 3.14). Anschließende Aza-WittigCyclisierung und Thiazolinoxidation lieferte das Thiazol 142 mit einer Ausbeute von 84\% über zwei Stufen. Die gleichzeitige Entschützung der Boc- und TBS-Gruppe gelang unter stark sauren Bedingungen. $N$-selektive Acylierung des freien Amins mit L-Fmoc(TBS)Threonin ${ }^{[202]}$ (143) ergab das Amid 144 mit einer moderaten Ausbeute von 67\%. Die alternative Darstellung aus dem bekannten Thiazol $\mathbf{5 2}^{[36]}$ lieferte dabei eine wesentlich bessere Ausbeute von 96\%. Die stereoselektive Einführung des Z-konfigurierten Enamids 145 gelang über eine Carbodiimid vermittelte, $\mathrm{Cu}(\mathrm{I})$-katalysierte $\beta$-Eliminierung der Hydroxygruppe. Diese Methode wurde durch Corey et al. erstmals für die Synthese von $\alpha, \beta$-ungesätigten Ketonen aus $\beta$-Hydroxyketonen beschrieben. ${ }^{[203]}$ Miller nutze diese zur Synthese von Dehydroaminosäureestern, ${ }^{[204]}$ und die Albericio-Gruppe weitete sie erfolgreich auf Peptide aus. ${ }^{[205-208]}$ Dabei verläuft die Eliminierung über die Bildung eines iso-Harnstoffs. Dieser wird unter gleichzeitiger Ausbildung der Doppelbindung über einen sechsgliedrigen Übergangszustand abgespalten. ${ }^{[209]} \mathrm{Da}$ es sich dabei um eine syn-Eliminierung handelt, wird zuerst das nicht gewünschte $E$-Isomer gebildet. Dieses isomerisiert anschließend in das stabilere Z-Isomer. ${ }^{[209]}$ 


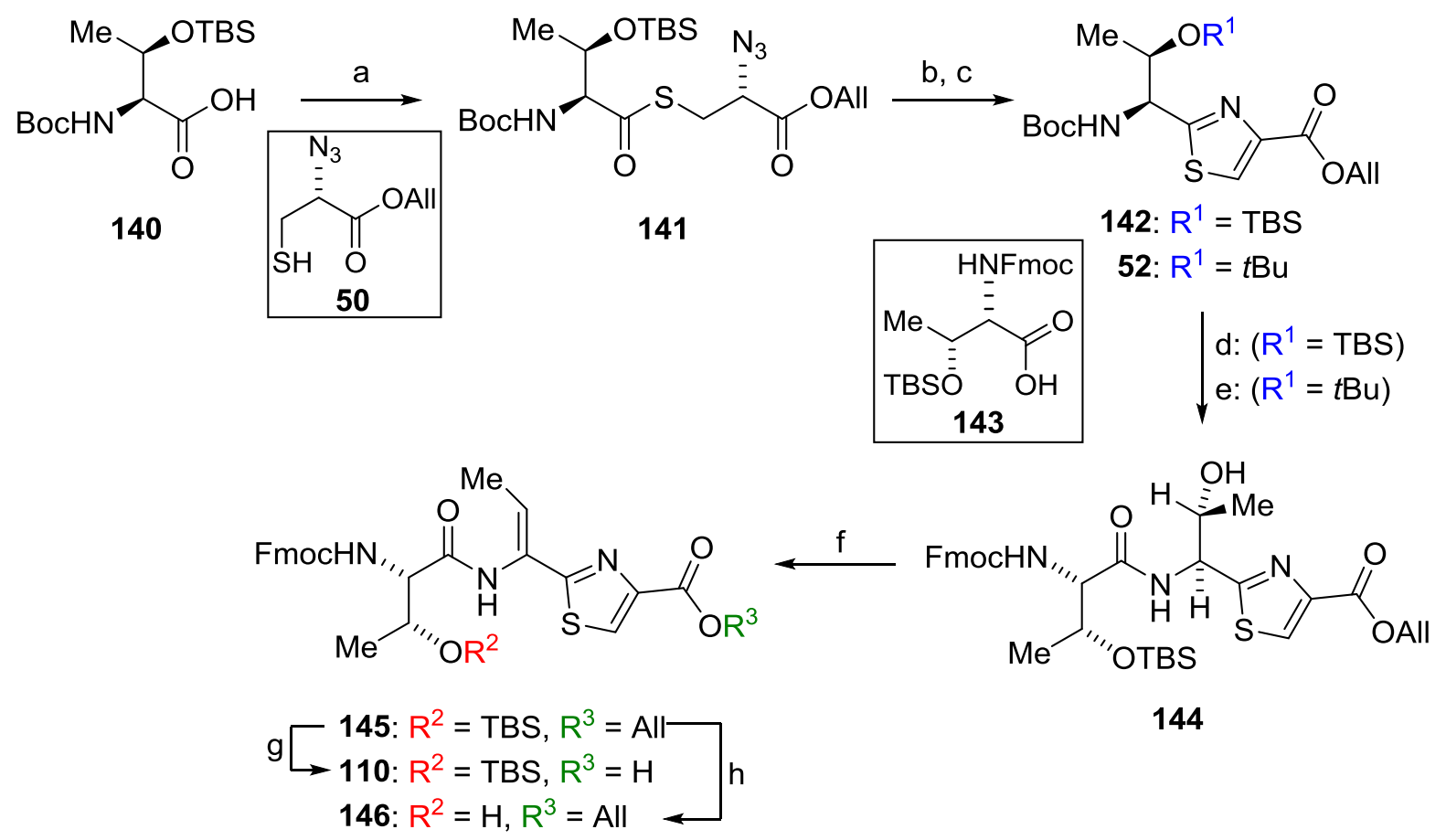

Schema 3.14: Synthese des Peptidthiazols 110. Bedingungen: a) 50, EDCI, HOBt, $\mathrm{Et}_{3} \mathrm{~N}_{,} \mathrm{CH}_{2} \mathrm{Cl}_{2}$, $0{ }^{\circ} \mathrm{C} \rightarrow \mathrm{RT}, 24$ h, 78\%. b) $\mathrm{PPh}_{3}$, THF, $-20{ }^{\circ} \mathrm{C} \rightarrow 40{ }^{\circ} \mathrm{C}, 18$ h. c) $\mathrm{DBU}, \mathrm{BrCCl}_{3}, \mathrm{CH}_{2} \mathrm{Cl}_{2},-20^{\circ} \mathrm{C} \rightarrow \mathrm{RT}$, 2 h, 84\% (ü. 2 Stufen). d) i. konz. HCl, EtOAc, RT, 30 min; ii. 143, EDCI, HOBt, Et ${ }_{3} \mathrm{~N}, \mathrm{RT}, 18$ h, 67\%. e) i. TFA, $\mathrm{Et}_{3} \mathrm{SiH}, \mathrm{CH}_{2} \mathrm{Cl}_{2}$, RT, 25 min; ii. 143, HATU, HOAt, EtNiPr 2 , DMF, $0{ }^{\circ} \mathrm{C} \rightarrow \mathrm{RT}, 14 \mathrm{~h}$,

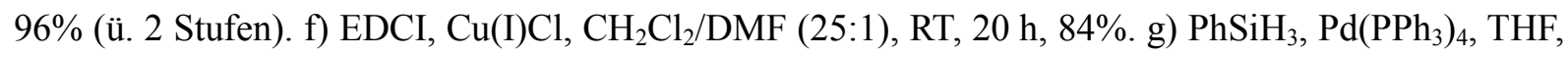
$0{ }^{\circ} \mathrm{C}, 30 \mathrm{~min}, 96 \%$. h) TBAF, AcOH, THF, $0{ }^{\circ} \mathrm{C} \rightarrow \mathrm{RT}, 5 \mathrm{~h}, 98 \%$.

Abschließend wurde der Allylester Pd-katalysiert gespalten und die Thiazolcarbonsäure 110 in einer sehr guten Ausbeute von 96\% isoliert. Für die Synthese des TIPS-geschützten Thiazols 111 (siehe unten) wurde der TBS-Ether des Allylesters 145 mit TBAF zum Alkohol 146 umgesetzt (98\%).

Ausgehend vom bekannten Amid 53 ${ }^{[36]}$ konnte die Einführung des Enamids 54 mit den oben beschriebenen Bedingungen ebenfalls stereoselektiv und mit einer etwas besseren Ausbeute von $75 \%$ durchgeführt werden (Schema 3.15). Im nächsten Schritt wurde der $t$ Bu-Ether zum Alkohol 146 sauer gespalten. Die Einführung der TIPS-Gruppe gelang mit einer sehr guten Ausbeute von $92 \%$ mit TIPSOTf. ${ }^{[210]}$ 


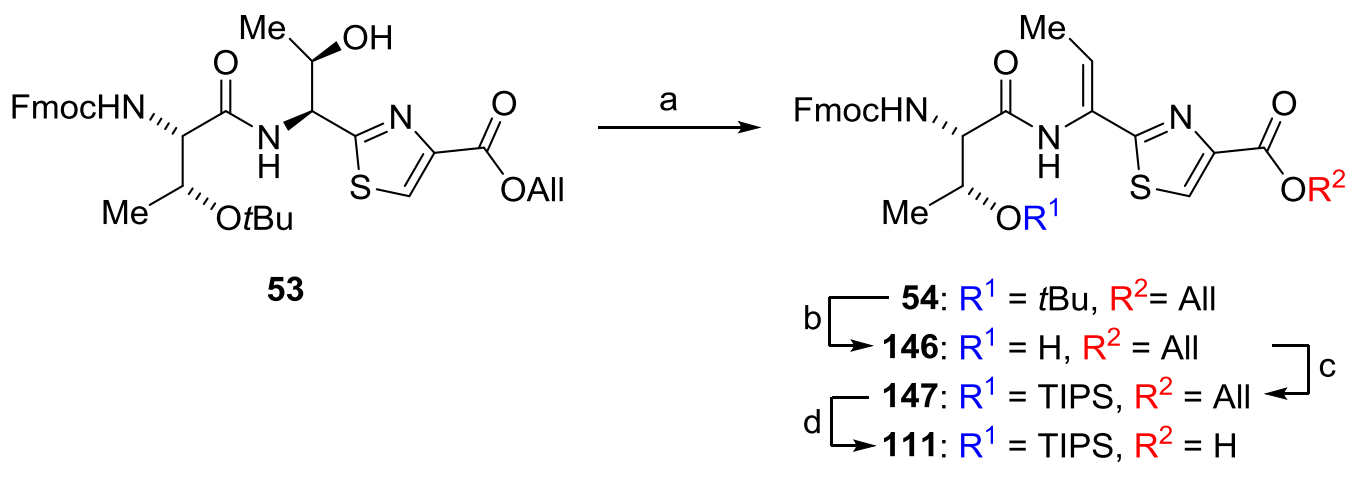

Schema 3.15: Synthese des TIPS-geschützten Peptidthiazols 111. Bedingungen: a) EDCI, Cu(I)Cl, $\mathrm{CH}_{2} \mathrm{Cl}_{2} / \mathrm{DMF}$ (25:1), RT, 20 h, 75\%. b) TFA; $\mathrm{Et}_{3} \mathrm{SiH}, \mathrm{CH}_{2} \mathrm{Cl}_{2}$, RT, 1 h, 95\%. c) TIPSOTf, 2,6-Lutidin, $0{ }^{\circ} \mathrm{C}, 1$ h, 92\%. d) $\mathrm{PhSiH}_{3}, \mathrm{Pd}\left(\mathrm{PPh}_{3}\right)_{4}$, THF, $0{ }^{\circ} \mathrm{C}, 30 \mathrm{~min}, 99 \%$.

Abschließende Pd-katalysierte Spaltung des Allylesters 147 lieferte die Thiazolcarbonsäure 111, bereit für eine Vereinigung mit dem indoltragenden Thiazol 95, in nahezu quantitativer Ausbeute.

\subsubsection{Kupplung der Fragmente}

Eine saure Freisetzung der Aminogruppe im Thiazol 95 war aufgrund der Säurelabilität des Dpm- und Indolylesters nicht möglich. Es gelang aber mit Hilfe von TBSOTf, die BocGruppe chemoselektiv in das Silylcarbamat 148 umzuwandeln (Schema 3.16). ${ }^{[211,212]}$ Die Verknüpfung mit den Thiazolsäuren 110 oder 111 gelang durch den Einsatz von HATU/HOAt und festem $\mathrm{NaHCO}_{3}$. Dabei wird offensichtlich das Amin aus dem TBS-Carbamat in situ sehr langsam freigesetzt.

Durch Optimierung der Reaktionsdauer der Umschützung der Boc-Gruppe ( $2.5 \mathrm{~h}$ statt $18 \mathrm{~h}$ ) konnte in einem späteren Stadium der Arbeit die Ausbeute der Kupplung mit der TIPSThiazolylsäure 111 signifikant von 39\% auf 71\% gesteigert werden. Diese Ausbeute konnte in mehreren Versuchen mit unterschiedlichen Ansatzgrößen erfolgreich reproduziert werden. 


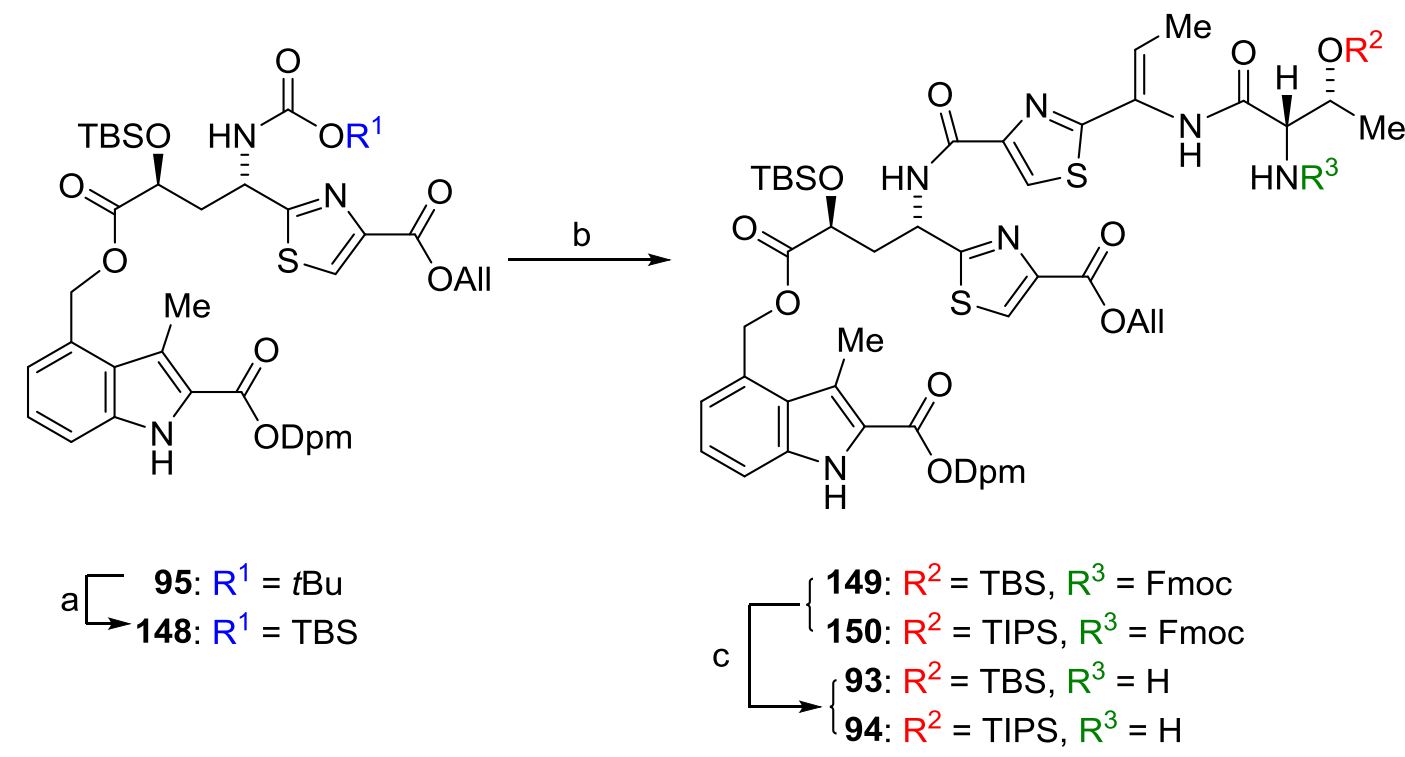

Schema 3.16: Kupplung der Thiazol-Fragmente zu den Bis-Thiazolen 93 und 94 . Bedingungen: a) TBSOTf, 2,6-Lutidin, $\mathrm{CH}_{2} \mathrm{Cl}_{2}, 0{ }^{\circ} \mathrm{C} \rightarrow \mathrm{RT}, 18 \mathrm{~h}$; optimiert: $0{ }^{\circ} \mathrm{C}, 1 \mathrm{~h} \rightarrow \mathrm{RT}, 2.5 \mathrm{~h}$; b) 110 oder 111, HATU, HOAt, $\mathrm{NaHCO}_{3}$, THF, $0{ }^{\circ} \mathrm{C} \rightarrow \mathrm{RT}, 41$ h, 39\% (149); $71 \%$ (150) (jeweils ü. 2 Stufen). c) DBU, $\mathrm{CH}_{2} \mathrm{Cl}_{2},-20^{\circ} \mathrm{C}, 5 \mathrm{~min}, 98 \%(\mathbf{9 3}), 95 \%(\mathbf{9 4})$.

In der darauf folgenden Fmoc-Entschützung der Bis-Thiazole 149 bzw. 150 wurde unter Standardbedingungen einer Fmoc-Entschützung (Piperidin/DBU, RT) eine E/ZIsomerisierung der Dehydrobutyrin-Doppelbindung beobachtet. Da eine Isomerisierung der Dhb-Doppelbindung mit einer $t$ Bu-Schutzgruppe an der Threonin-Seitenkette von $\mathrm{M}$. Riedrich nicht beobachtet wurde (Substrat 43, Kapitel 1.5.3), im Fall der TBS-Gruppe (Substrat 149) bereits eine Isomerisierung von etwa 25\% und bei einer TIPS-Gruppe (Substrat 150) sogar ein 1:1 Isomeren-Gemisch auftraten, besteht offensichtlich eine Korrelation zwischen dem Raumanspruch der $O$-Threonin-Schutzgruppe (TIPS $>$ TBS $>>t \mathrm{Bu}$ ) und der Isomerisierungsrate bzw. Anfälligkeit der Doppelbindung zur Isomerisierung. Mit DBU (5\% in $\mathrm{CH}_{2} \mathrm{Cl}_{2}$ ) bei tiefer Temperatur und einer möglichst kurzen Reaktionszeit konnte jedoch eine weitgehend isomerisierungsfreie Spaltung der Fmoc-Gruppe erreicht werden. Die BisThiazol-Amine 93 und 94 wurden dabei in sehr guten Ausbeuten von 98\% bzw. 95\% erhalten. 


\subsection{Synthese des 3-Hydroxypyridin-Kerns}

Die Synthese des zentralen Pyridinkerns folgte grundlegend der durch J.-Y. Lu erarbeiteten Route (s. Kapitel 1.5.3). ${ }^{[37]}$ In den nachfolgenden Abschnitten werden dabei durchgeführte Optimierungen und neue Ergebnisse beschrieben.

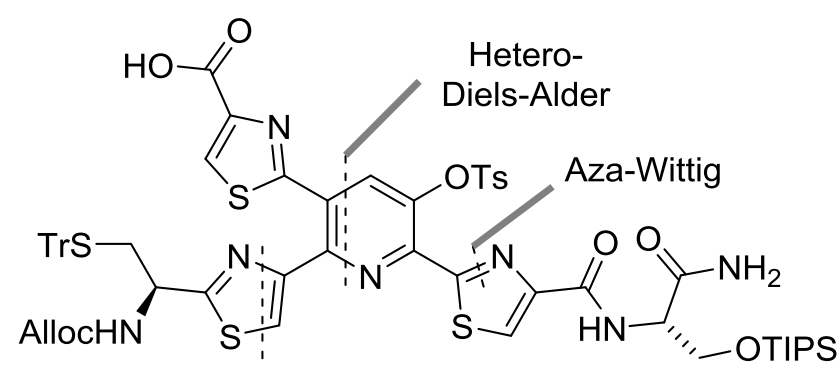<smiles>COC(=O)c1csc(C#CC(C)=O)n1</smiles>

64<smiles>CC(C)(C)N1[C@H](C(N)=S)CSC1(C)C</smiles>

47
42<smiles>C=CCC</smiles><smiles>C=C(OC)C(=NOC(C)(C)C)C(=O)OC</smiles>

65<smiles>NC(=O)[C@H](C[OH+])NC(=O)[C@@H](N)CS</smiles>

48

Schema 3.17: Retrosynthetische Analyse des Tristhiazolylpyridin-Kerns 42 nach Lu. ${ }^{[37]}$

Die Thiazole in 2- und 6-Position sollten mittels Aza-Wittig-Cyclisierung und einer HantzschThiazol-Synthese am Pyridin angebracht werden (Schema 3.17). Daraus ergeben sich das Azido-Dipeptid 48 und das cysteinabgeleitete Thioamid 47. Für den Aufbau des 3-Hydroxypyridin-Grundkörpers sollte eine hetero-Diels-Alder-Reaktion zwischen dem Alkinyketon 64 und dem 1-Azabutadien 65 genutzt werden.

\subsubsection{Synthese des Alkinylketons}

Für die Synthese des Alkinylketons 64 wurden größere Mengen des $\alpha$-Brompyruvats 152 benötigt. Dieses ließ sich nach Kruse et al. zweckmäßig durch bromierende Oxidation von 
Methyllactat (151) darstellen (Schema 3.18). ${ }^{[213]}$ Eine Hantzsch-Thiazolsynthese mit Thioharnstoff ergab dann das kristalline 2-Aminothiazol 153 in 93\% Ausbeute.

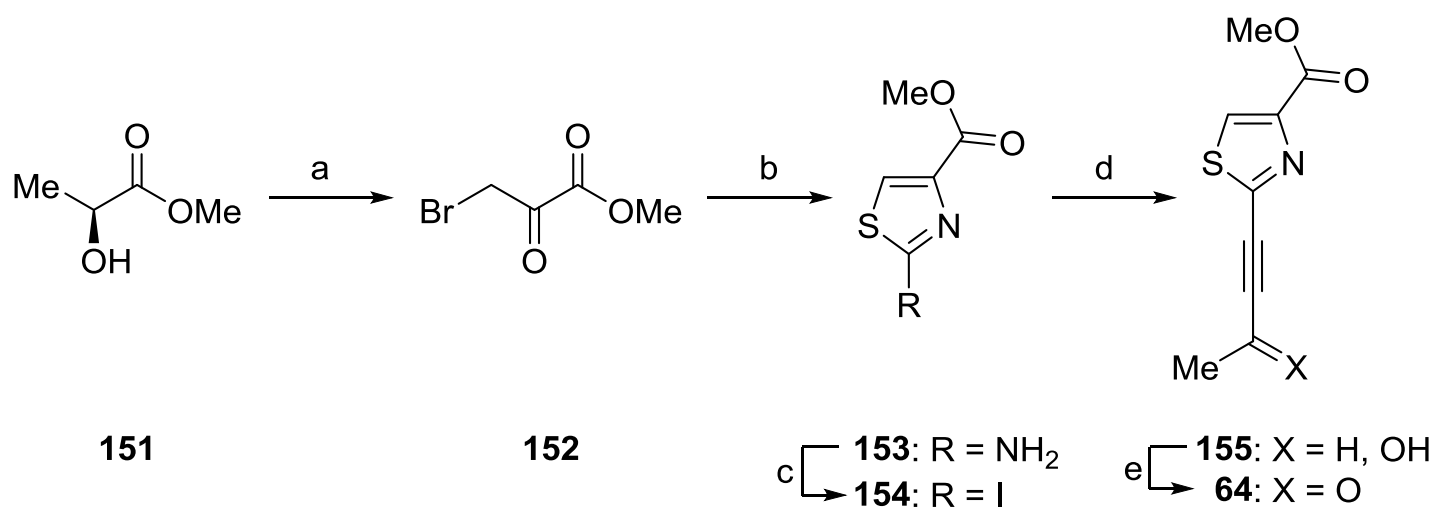

Schema 3.18: Synthese des Alkinylketons 64. Bedingungen: a) NBS, $\mathrm{CCl}_{4}, 77^{\circ} \mathrm{C}, 6 \mathrm{~h}, 61 \%$. b). Thioharnstoff, $\mathrm{MeOH}, 65^{\circ} \mathrm{C}, 2 \mathrm{~h}, 93 \%$. c) $t \mathrm{BuONO}, \mathrm{CH}_{2} \mathrm{I}_{2}$, THF, $0^{\circ} \mathrm{C}, 1.5 \mathrm{~h}, 4{ }^{\circ} \mathrm{C}, 2 \mathrm{~h}, 75 \%$. d) 3-Butin-2-ol, $\mathrm{Pd}\left(\mathrm{PPh}_{3}\right)_{2} \mathrm{Cl}_{2}, \mathrm{CuI}, \mathrm{Et}_{3} \mathrm{~N}, \mathrm{DMF}, 50{ }^{\circ} \mathrm{C}, 2 \mathrm{~h}, 88 \%$. e) Dess-Martin-Periodinan, $\mathrm{CH}_{2} \mathrm{Cl}_{2}$, $4{ }^{\circ} \mathrm{C} \rightarrow \mathrm{RT}, 1 \mathrm{~h}, 79 \%$.

Nun musste im Synthesegang das Anilin 153 in ein Arylhalogenid umgewandelt werden. In den Arbeiten von Lu wurde die Bildung von dihalogenierten Produkten in Gegenwart von Kupfer-Salzen beobachtet. ${ }^{[37,163]}$ Durch Diazotierung des Aminothiazols 153 mit tertButylnitrit gelang es, das Iodid 154 durch in situ Zersetzung des Diazoniumsalzes, in Gegenwart von Diiodmethan als Abfangreagenz, erfolgreich und in großem Maßstab mit einer Ausbeute von bis zu 75\% nebenproduktfrei darzustellen. ${ }^{[108]}$ Eine Sonogashira-Kupplung mit 3-Butin-2-ol ergab den Alkohol 155 in 88\% Ausbeute, der mit Dess-Martin-Periodinan ${ }^{[214]}$ glatt zum Alkinylketon 64 oxidiert wurde (79\%). Bei Versuchen, diese Transformation unter den durch J.-Y Lu beschriebenen Bedingungen ${ }^{[37]}$ mit 2-Iodoxybenzoesäure (IBX) als Oxidationsmittel durchzuführen, wurden nur sehr niedrige Ausbeuten erhalten.

\subsubsection{Untersuchungen zur hetero-Diels-Alder-Reaktion}

Mit dem dargestellten Alkinylketon 64 wurde anschließend die hetero-Diels-Alder-Reaktion mit dem 1-Azabutadien 65 zum Aufbau des 3-Hydroxypyridinkerns durchgeführt (Schema 3.19). 
<smiles>C=C(O[Na])C(=NOC)C(=O)OC</smiles>

Schema 3.19: hetero-Diels-Alder-Reaktion zur Synthese des 3-Hydroxypyridins 46. Bedingungen: a) Toluol, $180^{\circ} \mathrm{C}, 4 \mathrm{~h}$.

Dem Protokoll von Lu folgend (3Äq. 65, $180{ }^{\circ} \mathrm{C}, 4 \mathrm{~h}$ ) wurden jedoch schwankende Ausbeuten erhalten ( $\approx 50 \%$ ). Das Isomerenverhältnis (46:66) lag im Durchschnitt nur bei etwa 12:10, anstatt wie durch Lu beschrieben bei 2:1. ${ }^{[37]}$ Versuche, die Ausgangsmaterialien mit möglichst hoher Reinheit (Umkristallisation, mehrfache Destillation) einzusetzen, änderten an den Ergebnissen nur wenig. Das eingesetzte Toluol wurde zuvor stets von Natrium/Benzophenon destilliert.

Durch Untersuchung der polaren Chromatographiefraktionen konnte ein weiteres, bislang nicht beschriebenes 3-Hydroxypyridinisomer isoliert und seine Struktur mittels NMR, HRMS, Elementaranalyse sowie Röntgenkristallstrukturanalyse eindeutig belegt werden (Abbildung 3.3).
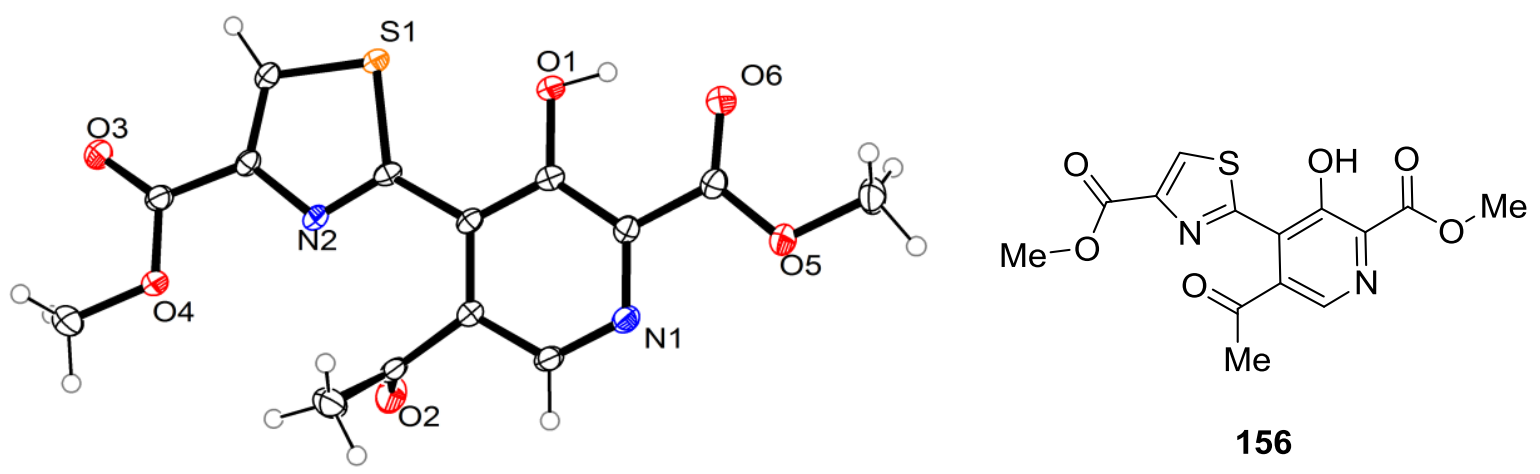

Abbildung 3.3: ORTEP-Darstellung der Röntgenkristallstrukturanalyse des gefunden 3-Hydroxypyridin-Isomers 156 und die dementsprechende Strukturformel. 
Zusätzlich gelang es eine Röntgenkristallstrukturanalyse für das bereits bekannte „Minder“Isomer 66 zu erhalten (Abbildung 3.4)

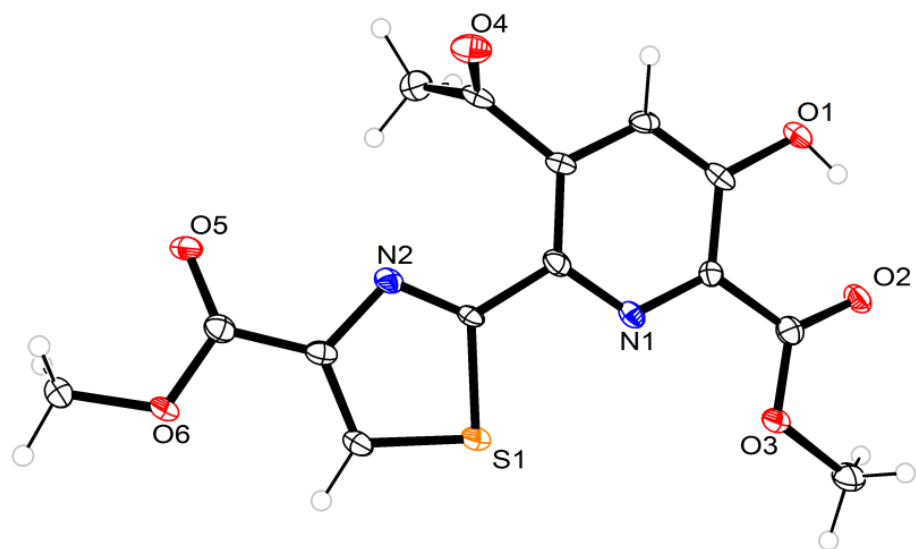

Abbildung 3.4: ORTEP-Darstellung der Röntgenkristallstrukturanalyse des „Minder“-Isomers 66.

Zusammen mit der durch J.-Y. Lu erhaltenen Röntgenkristallstrukturanalye eines Derivats des Isomers $\mathbf{4 6}^{[37]}$ konnten so alle erhaltenen Isomere strukturell eindeutig zugeordnet werden.

Da es sich bei dieser Reaktion um einen Schlüsselschritt der Synthese handelt und diese früh im Syntheseweg durchgeführt wird, wurden größere Mengen des gewünschten Isomers 46 benötigt. Da eine hohe Reinheit der Startmaterialien keinen wesentlichen Einfluss auf Ausbeute und Isomerenverhältnis zeigte, wurde der Einfluss der Reaktionstemperatur auf die Ausbeute an Isomer 46 hin untersucht (Tabelle 3.1). Untersuchungen zum Lösungsmitteleinfluss oder der Einsatz von Additiven (Säuren/Basen) wurden nicht durchgeführt. 
Tabelle 3.1: Ausbeute an Isomer 46 der hetero-Diels-Alder-Reaktion mit Alkinylketon 64 bei verschiedenen Temperaturen. Bedingungen: a) $100 \mathrm{mg}$ 64, Toluol (0.1 mL), T, $4 \mathrm{~h}$.<smiles>COC(=O)c1csc(C#CC(C)=O)n1</smiles>

64<smiles>C=C(O[Na])/C(=N/OC(C)(C)C)C(=O)OC</smiles>

65<smiles>COC(=O)c1csc(-c2cc(O)c(C(C)=O)nc2C(C)=O)n1</smiles>

46

\begin{tabular}{ccc}
\hline & Temperatur $\left({ }^{\circ} \mathrm{C}\right)$ & Ausbeute (46) \\
\hline $\mathrm{a}$ & 150 & $22 \%$ \\
$\mathrm{~b}$ & 155 & $20 \%$ \\
$\mathrm{c}$ & 160 & $28 \%$ \\
$\mathrm{~d}$ & 165 & $31 \%$ \\
$\mathrm{e}$ & 170 & $27 \%$ \\
\hline
\end{tabular}

Für die Versuche wurde das Reaktionsgemisch in ein kaltes Ölbad eingetaucht und anschließend auf die jeweilige Temperatur erwärmt. Unterhalb von $150^{\circ} \mathrm{C}$ wurde kein vollständiger Umsatz beobachtet. Bei einer Temperatur von $150{ }^{\circ} \mathrm{C}$ wurde eine Ausbeute von 22\% des gewünschten Isomers 46 erhalten (Eintrag a). Durch weitere Steigerung der Temperatur auf $160{ }^{\circ} \mathrm{C}$ konnte auch ein Anstieg der Ausbeute beobachtet werden. Das Optimum lag bei $165{ }^{\circ} \mathrm{C}$ und lieferte das Isomer 46 mit 31\% Ausbeute (Eintrag d). Weitere Temperatursteigerung führte wiederum zu niedrigeren Ausbeuten (Eintrag e). In präparativ sinnvollen Umsetzungen (Gramm-Maßstab) wurden unter den optimierten Bedingungen die drei Isomere mit einer Gesamtausbeute von $83 \%$ in nahezu gleichem Verhältnis erhalten (Schema 3.20). 


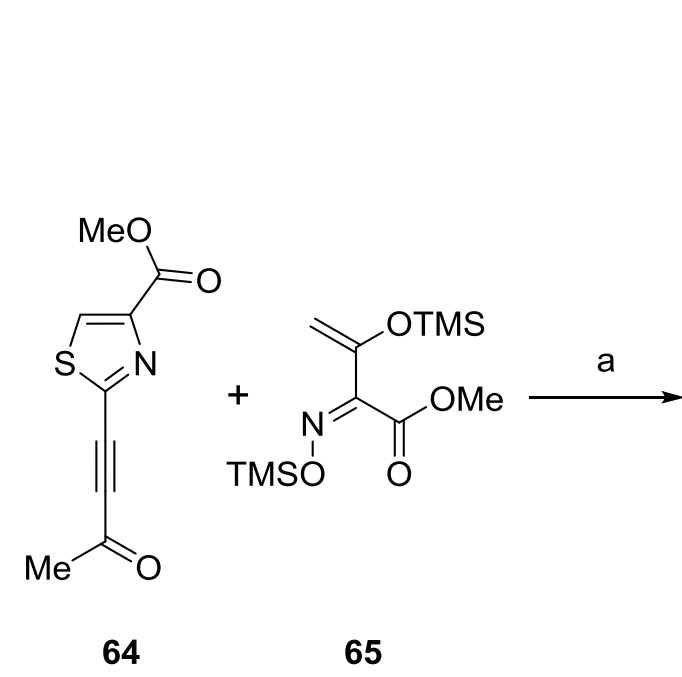<smiles>COC(=O)c1csc(-c2cc(O)c(C(=O)OC)nc2C(C)=O)n1</smiles>

46<smiles>COC(=O)c1csc(-c2nc(C(=O)OC)c(O)cc2C(C)=O)n1</smiles>

66

Schema 3.20: Synthese der 3-Hydroxypyridin-Isomere. Bedingungen: a) Toluol, $165^{\circ} \mathrm{C}, 4 \mathrm{~h}, 28 \%$ (46), 27\% (66), 28\% (156).

Falls die Bildung des 2,3,4,5-substituierten Isomers 156 ebenfalls über eine hetero-DielsAlder-Reaktion verläuft, so kann das 2-Azabutadien 157 als Zwischenstufe vermutet werden (Schema 3.21).<smiles>COC(=O)c1csc(C#CC(C)=O)n1</smiles>

64<smiles>[R]O[N+](=C)C(=COC([R6])([O-])[O-])C(=O)OC</smiles>

157<smiles>COC(=O)c1csc(-c2nc(C(=O)OC)cnc2C(C)=O)n1</smiles>

156<smiles>COC(=O)c1csc(-c2cnc(C(=O)OC)c(O)c2C(C)=O)n1</smiles>

158

nicht beobachtet

Schema 3.21: Mögliche Bildung des Isomers 157 via hetero-Diels-Alder-Chemie mit einem hypothetischen 2-Azabutadien.

Dabei wäre auch die Bildung des Isomers 158 möglich, welches aber bisher nicht beobachtet wurde. Möglicherweise verläuft diese Umsetzung hoch selektiv und das vierte Isomer 158 wird nicht oder nur in sehr geringen Mengen gebildet. Das 2-Azabutadien 157 könnte durch eine Isomerisierung aus dem 1-Azabutadien 65 gebildet werden. Dies ist derzeit Gegenstand laufender Untersuchungen in der Arbeitsgruppe. 


\subsubsection{Abschluss der Pyridinfragment-Synthese}

Die weitere Umsetzung des Ketons 46 gelang auf dem von J.-Y. Lu erarbeiteten Weg (Schema 3.22). ${ }^{[37]}$ Nach Schützung der Hydroxyfunktion als Triflat (96\%), wurde das Keton in zwei Stufen und einer Ausbeute von $72 \%$ in das $\alpha$-Bromketon 67 überführt. Eine anschließende modifizierte Hantzsch-Thiazol-Synthese mit dem Thioamid 47 ${ }^{[98]}$ lieferte das Bisthiazolylpyridin 157 in einer sehr guten Ausbeute von 98\%.

Die darauf folgende Entschützung des Triflats 157 konnte wie durch Lu beschrieben durchgeführt werden. Es zeigte sich jedoch, dass das Schwefelatom des $N$-Boc-Thiazolidins 68 dadurch sehr anfällig gegenüber Oxidation wurde (LC-MS). Daher wurde das Rohprodukt direkt weiter umgesetzt und die Schutzgruppen an $S$ - und $N$-Atom ausgetauscht. Das 3-Hydroxypyridin 96 wurde dabei mit einer guten Ausbeute von 89\% über vier Stufen erhalten. Durch Zugabe von $\mathrm{Et}_{3} \mathrm{SiH}$ konnte intermediär gebildetes Sulfoxid wieder reduziert werden. ${ }^{[215]}$ Im vorliegenden Fall war die gewählte Reihenfolge der Um- und Entschützungen von entscheidender Wichtigkeit. Wurde das Triflat erst nach Austausch der Schutzgruppen am Cystein-Rest entschützt, so wurden in späteren Synthesestufen Diastereomere beobachtet, die aufwändig abgetrennt werden mussten. Wahrscheinlich epimerisierte das Cystein-Fragment während der Triflat-Entschützung durch die stark basischen Reaktionsbedingungen sobald das Thiomainal entfernt wurde, das eine Planarisierung des benzylischen Zentrums erschwert. ${ }^{[216]}$ Dieses $N$-Boc geschützte Thioaminal wurde schon mehrfach verwendet, um Cysteinseitenketten vor Epimerisierung des stereogenen Zentrums zu schützen. ${ }^{[98,106,115,217,218]}$ 
<smiles>COC(=O)c1csc(-c2cc(O)c(C(C)=O)nc2C(C)=O)n1</smiles>

46<smiles>[R]OC(=O)c1nc(-c2csc([C@H]([AlH2])CS[3H])n2)c(-c2nc(C(=O)OC)cs2)cc1O</smiles>

$$
\begin{aligned}
& \mathrm{j} \longrightarrow \text { 96: } \mathrm{R}^{2}=\mathrm{Me} \\
& \longrightarrow 70: \mathrm{R}^{2}=\mathrm{H}
\end{aligned}
$$<smiles>CCOC[C@H](NC(=O)[C@H]([NH3+])CS)C(N)=O</smiles>

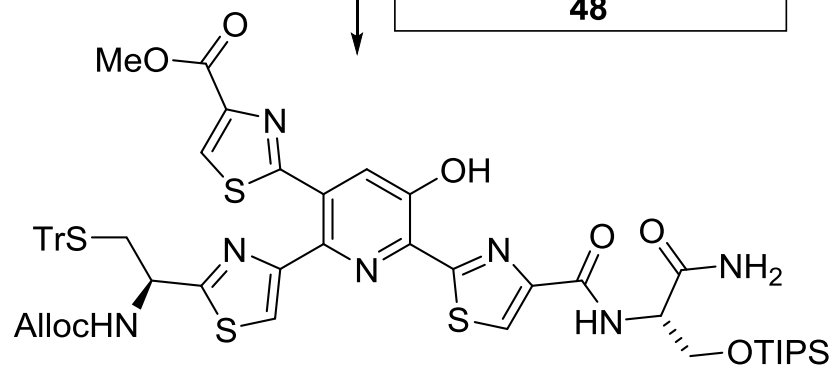

71<smiles>COC(=O)c1nc(C(=O)CBr)c(-c2nc(C(=O)O[Na])cs2)cc1[O+]</smiles>

67

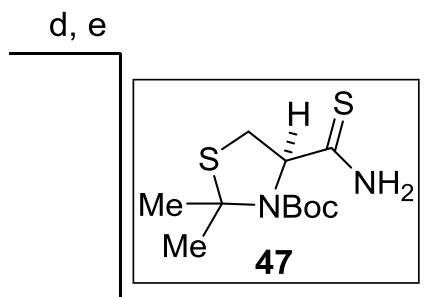

$\mathrm{g}, \mathrm{h}, \mathrm{i}$<smiles>[R7]Oc1cc(-c2nc(C(=O)OC)cs2)c(-c2csc([C@@H]3CSC(C)(C)N3C(=O)OC(C)(C)C)n2)nc1C(=O)OC</smiles>

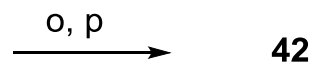

Schema 3.22: Synthese des Tristhiazolylpyridins 42 ausgehend von Keton 46. Bedingungen: a) $\mathrm{Tf}_{2} \mathrm{O}$, $\mathrm{Et}_{3} \mathrm{~N}, \mathrm{CH}_{2} \mathrm{Cl}_{2}, 0^{\circ} \rightarrow \mathrm{RT}, 12 \mathrm{~h}, 96 \%$. b) TIPSOTf, $\mathrm{Et}_{3} \mathrm{~N}, \mathrm{CH}_{2} \mathrm{Cl}_{2}, 0^{\circ} \mathrm{C} \rightarrow \mathrm{RT}, 12$ h, $96 \%$. c) NBS, pH 7 Puffer, THF, $30 \mathrm{~min}, 75 \%$ d) 47, $\mathrm{KHCO}_{3}$, THF, $-40{ }^{\circ} \mathrm{C} \rightarrow \mathrm{RT}, 48$ h. e) 2,6-Lutidin, TFAA, $-20{ }^{\circ} \mathrm{C}$, 16 h, 98\% (ü. 2 Stufen). f) TBAOH, 1,4-Dioxan oder NaOMe/MeOH, RT, 5-30 min. g) TFA, Et 3 SiH, $\mathrm{CH}_{2} \mathrm{Cl}_{2}, 30$ min. h) TrCl, DMF, 48 h. i) AllocCl, $\mathrm{NaHCO}_{3}, \mathrm{THF} / \mathrm{H}_{2} \mathrm{O}, 0{ }^{\circ} \mathrm{C} \rightarrow \mathrm{RT}, 18$ h, $89 \%$ (ü. 4 Stufen). j) $\mathrm{NaHCO}_{3}$, 1,4-Dioxan $\left./ \mathrm{H}_{2} \mathrm{O}, 6{ }^{\circ} \mathrm{C}, 9 \mathrm{~h} . \mathrm{k}\right)$ Phosgen, $\left.\mathrm{Et}_{3} \mathrm{~N}, \mathrm{THF},-40{ }^{\circ} \mathrm{C}, 2 \mathrm{~h} .1\right)$ 48, DMAP, $-40{ }^{\circ} \mathrm{C} \rightarrow \mathrm{RT}, 48$ h. m) $\mathrm{PPh}_{3}$, THF, $-20^{\circ} \mathrm{C} \rightarrow 40{ }^{\circ} \mathrm{C}, 21$ h. n) $\mathrm{BrCCl}_{3}, \mathrm{DBU}, \mathrm{CH}_{2} \mathrm{Cl}_{2},-20^{\circ} \mathrm{C} \rightarrow$ RT, 2 h, $26 \%$ (ü. 5 Stufen). o) TsCl, $\mathrm{Et}_{3} \mathrm{~N}, \mathrm{DMAP}, \mathrm{CH}_{2} \mathrm{Cl}_{2}, 0{ }^{\circ} \mathrm{C}, 3$ h, 99\%. p) $\mathrm{Me}_{3} \mathrm{SnOH}, \mathrm{DCE}, 60^{\circ} \mathrm{C}$, $15 \mathrm{~h}, 75 \%$.

Anschließend konnte der Methylester am Pyridinring selektiv unter Assistenz der benachbarten Hydroxygruppe gespalten werden (Details s. Kapitel 4). Die erhaltene Picolinsäure 70 zeigte während der Chromatographie an $\mathrm{SiO}_{2}$ jedoch eine rasche Zersetzung. 
Daher wurde das erhaltene Rohprodukt direkt mit Phosgen aktiviert, um das Peptidthiol 48 zu acylieren. Nach Aza-Wittig-Cyclisierung und Oxidation wurde das Tristhiazolylpyridin 71 erhalten. Die Ausbeute betrug dabei lediglich 26\% über fünf Stufen, wogegen Lu von wesentlich höheren Ausbeuten berichtete. ${ }^{[37]}$ Bei der Umsetzung wurden zudem viele Nebenprodukte erhalten, die sich nur schwer abtrennen ließen. Vor allem das in der AzaWittig-Reaktion gebildete Triphenylphosphanoxid konnte nur mit großem Aufwand vollständig abgetrennt werden. Durch Schützung der 3-Hydroxygruppe als Sulfonat und Verseifung des Methylester mit $\mathrm{Me}_{3} \mathrm{SnOH}^{[131]}$ unter milden Bedingungen wurde die Synthese des Pyridinfragment $\mathbf{4 2}$ abgeschlossen.

Während seiner Masterarbeit konnte I. Raztsou vergleichbare 3-Hydroxypicolinsäurethioester mit PyAOP als Kupplungsreagenz in guter Ausbeute darstellen. ${ }^{[215]}$ Bei Versuchen mit der 3-Hydroxypicolinsäure $\mathbf{7 0}$ wurde unter entsprechenden Bedingungen jedoch der $O$-phosphorylierte Thioester 159 in großen Mengen gebildet (Abbildung 3.5). Versuche das Phosphoniumsalz nach Aza-Wittig-Cyclisierung und Oxidation wieder zu entfernen waren nicht erfolgreich.

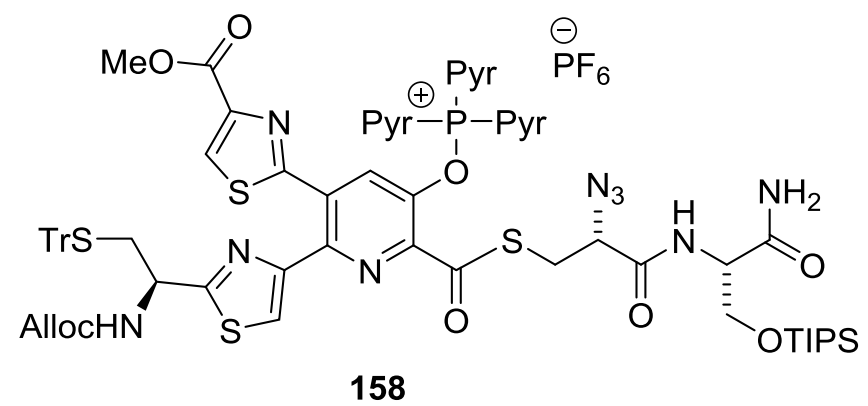

Abbildung 3.5: Phosphorylierter Thioester 158. Pyr = Pyrrolidin-1-yl.

\subsection{Untersuchungen zum B-Ring-Aufbau}

Für die weitere Synthese war es notwendig, verlässliche Bedingungen für die Entfernung der Dpm- und Tr-Schutzgruppen und die Makrothiolactonisierung zum B-Ring zu finden. Um diese experimentell $\mathrm{zu}$ ermitteln, wurde das in Schema 3.23 gezeigte Testsystem 113 aufgebaut. Dieses lässt sich aus der Carbonsäure 159 und einem Pyridylthiazol 160 erhalten. 


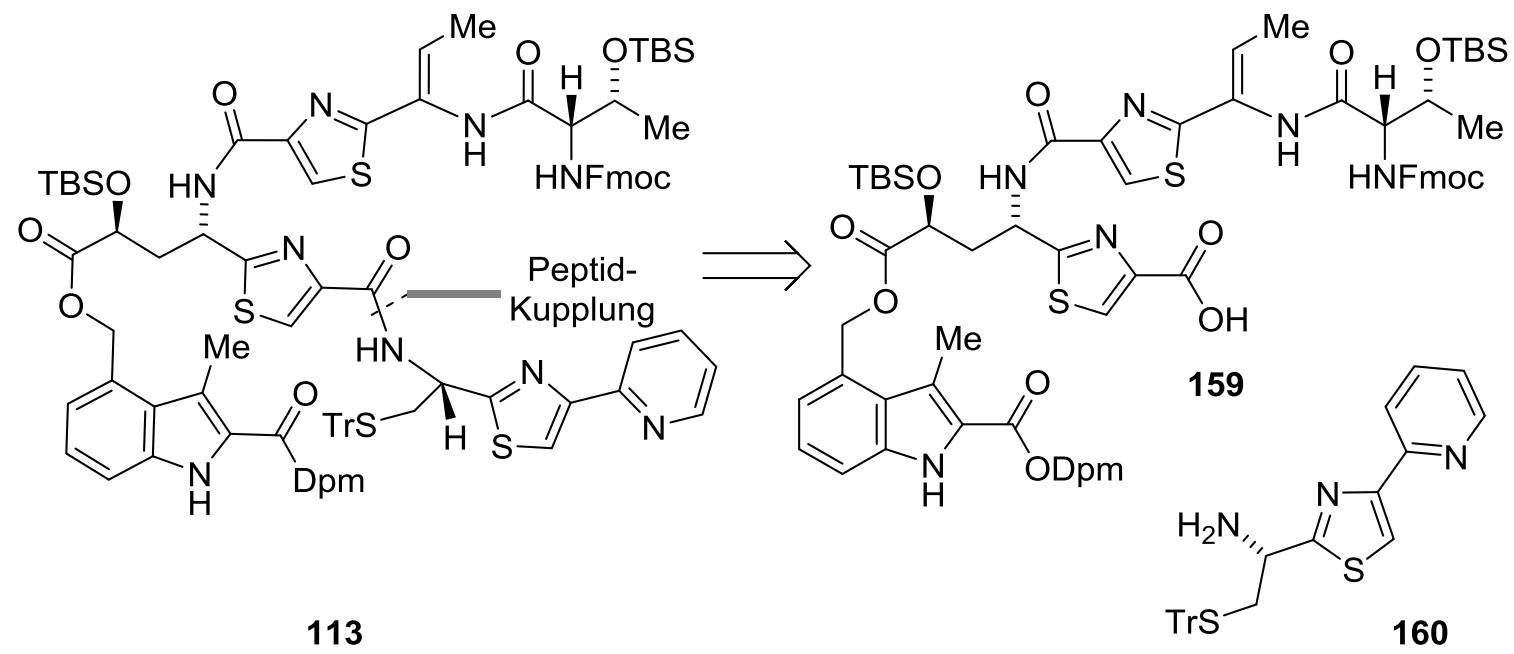

Schema 3.23: Retrosynthetische Analyse des B-Ring-Testsystem 113.

Für die Synthese des Pyridylthiazols 160 wurde das aus $L$-Cystein abgeleitete Thioamid 47 ${ }^{[98]}$ mit dem $\alpha$-Bromketon 161 ${ }^{[219]}$ zum Thiazol 162 umgesetzt (Schema 3.24), was in hervorragenden 96\% Ausbeute gelang. Anschließend wurde das $N$-Boc-geschützte Thioaminal mit TFA gespalten, das freigesetzte Thiol mit einer Trityl-Gruppe und das Amin als Allylcarbamat geschützt $\left(\mathbf{1 6 3}, 63 \%\right.$ über drei Stufen). ${ }^{[37]}$ Die Schützung des Amins erleichterte dabei die Isolierung und Reinigung. Abschließend wurde die Alloc-Gruppe Pdvermittelt wieder entfernt und das freie Amin 160 quantitativ erhalten.<smiles>CC1(C)SC[C@@H](C(N)=S)[C@@H]1C(=O)O</smiles>

47
161

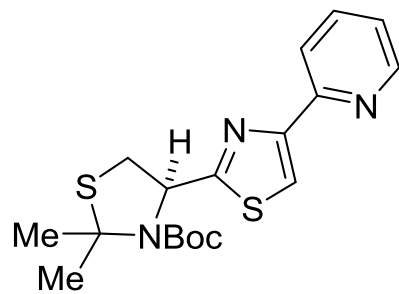

162

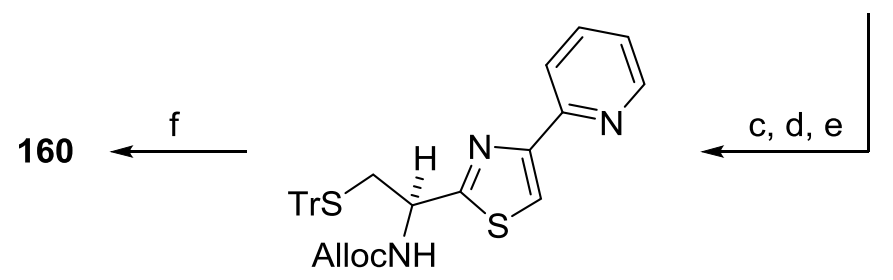

163

Schema 3.24: Synthese des Pyridylthiazols 160. Bedingungen: a) $\mathrm{KHCO}_{3}, \mathrm{THF},-40{ }^{\circ} \mathrm{C} \rightarrow \mathrm{RT}, 22 \mathrm{~h}$. b) TFAA, 2,6-Lutidin, $-40{ }^{\circ} \mathrm{C} \rightarrow$ RT, 20 h, 96\% (ü. 2 Stufen). c) TFA, $\mathrm{Et}_{3} \mathrm{SiH}, \mathrm{CH}_{2} \mathrm{Cl}_{2}, 0^{\circ} \mathrm{C} \rightarrow \mathrm{RT}$, 2 h. d) $\mathrm{TrCl}, \mathrm{DMF}, \mathrm{RT}, 48$ h. e) AllocCl, $\mathrm{NaHCO}_{3}, \mathrm{THF} / \mathrm{H}_{2} \mathrm{O}, 4$ h,63\% (ü. 3 Stufen). f) $\mathrm{Pd}\left(\mathrm{PPh}_{3}\right)_{4}$, $\mathrm{PhSiH}_{3}$, THF, $0{ }^{\circ} \mathrm{C}, 20 \mathrm{~min}$, quant. 
Für die Kupplung mit dem Thiazolamin 160 wurde zuerst der Allylester 149 mit einer Ausbeute von 93\% in die Carbonsäure 159 überführt (Schema 3.25). Die anschließende Verknüpfung mit PyBOP lieferte das lineare Testsystem 113 in einer guten Ausbeute von $77 \%$.

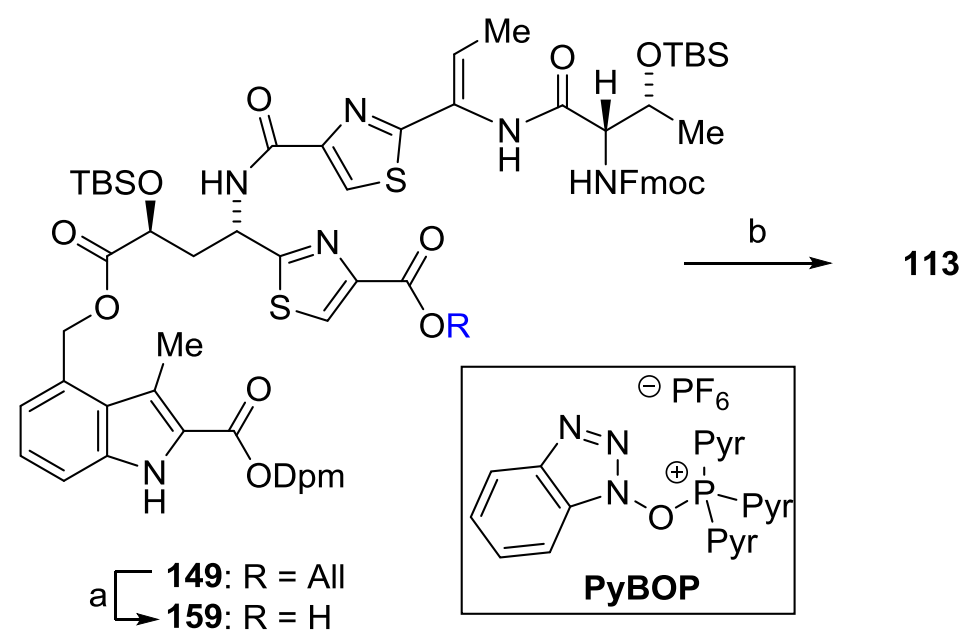

Schema 3.25: Synthese des B-Ring-Testsystems 113 für die Makrothiolactonisierung. Bedingungen: a) $\mathrm{Pd}\left(\mathrm{PPh}_{3}\right)_{4}, \mathrm{PhSiH}_{3}, \mathrm{THF}, 0{ }^{\circ} \mathrm{C}, 15 \mathrm{~min}, 93 \%$. b) 160, PyBOP, EtNiPr 2 , DMF, $0{ }^{\circ} \mathrm{C} \rightarrow \mathrm{RT}, 16 \mathrm{~h}, 77 \%$.

Mit den für die Dpm-Esterspaltung entwickelten Bedingungen (Kap. 3.3.2) konnte eine gleichzeitige Spaltung des Dpm-Esters als auch der Tr-Gruppe realisiert werden (Schema 3.26). Dabei musste die Reaktionszeit deutlich verlängert werden, um eine vollständige Spaltung beider Gruppen $\mathrm{zu}$ erreichen (HPLC-Kontrolle). Die Bedingungen wurden dahingehend optimiert, dass das Substrat zu Beginn bei sehr niedriger Temperatur zur Reaktionslösung hinzugegeben und anschließend auf die Reaktionstemperatur erwärmt wurde. Dies verringerte die Bildung von Nebenprodukten, bei denen eine der TBS-Gruppen abgespalten wurde. Eine Analyse der Fragmentierungsmuster in der LC-MS (ESI) deuteten auf eine Spaltung der TBS-Gruppe an der Threonin-Seitenkette hin.

Nach vorsichtigem und sorgfältigem Entfernen der flüchtigen Reaktionsbestandteile wurde die Makrocyclisierung direkt in THF, bei einer berechneten Konzentration von $1.1 \mathrm{mmol} / \mathrm{L}$ an $\omega$-Mercaptosäure 164, mit PyAOP als Kupplungsreagenz durchgeführt. Die Umsetzung war bereits nach $3 \mathrm{~h}$ vollständig und das Makrothiolacton 165 konnte mit einer Ausbeute von 66\% isoliert werden. Beim Aufbau des strukturell einfacheren B-Ring-Testsystems 39 berichteten Moody et al. eine Ausbeute von 52\% (Kap. 3.2). ${ }^{[156]}$ 


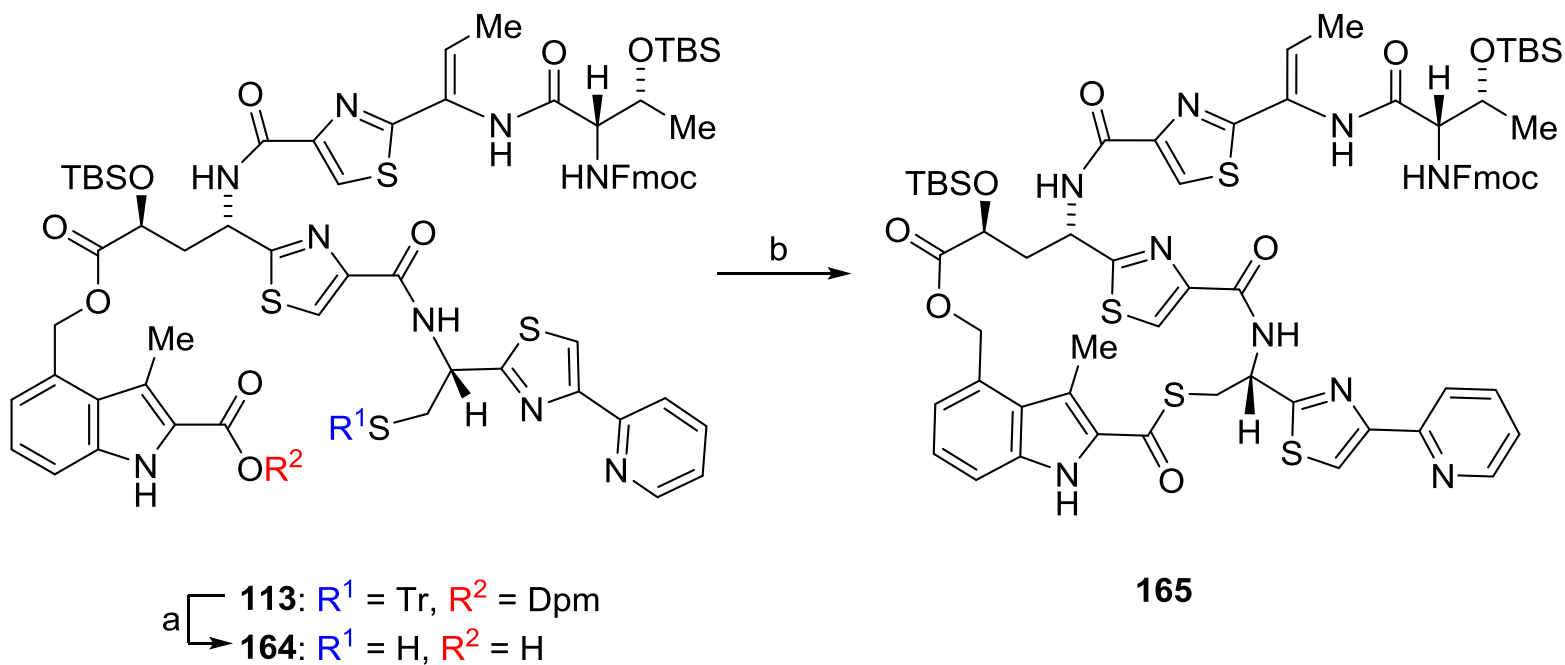

Schema 3.26: Gleichzeitige Spaltung der säurelabilen Schutzgruppen und Makrothiolactonisierung. Bedingungen: a) TFA, $\mathrm{Et}_{3} \mathrm{SiH}$, Anisol, $-25{ }^{\circ} \mathrm{C} \rightarrow 0{ }^{\circ} \mathrm{C}, 22 \mathrm{~h}$. b) PyAOP, EtNiPr 2 , THF, $0{ }^{\circ} \mathrm{C} \rightarrow \mathrm{RT}, 3 \mathrm{~h}$, $66 \%$ (ü. 2 Stufen).

Die Optimierung der Bedingungen zur gleichzeitigen Dpm- und Tr-Spaltung sowie die erfolgreiche Makrothiolactonisierung sicherten somit die gewählte Schutzgruppenstrategie ab. Da sich die an der Threonin-Seitenkette befindliche TBS-Gruppe wesentlich labiler gegenüber Säuren verhalten hatte als erwartet, wurde ein Austausch gegen eine stabilere TIPS-Gruppe in Erwägung gezogen.

\subsection{Untersuchungen zur Dehydroalanin-Bildung}

Die Einführung der Dehydroalanin-Einheit war zu einem sehr späten Zeitpunkt der Synthese geplant. In bisherigen Thiopeptid-Totalsynthesen wurde diese am häufigsten durch eine oxidative syn-Eliminierung eines Phenylselenocysteins dargestellt. ${ }^{[126]}$ Diese gegenüber Oxidation äußerst empfindliche Gruppe wurde meistens sehr spät eingeführt und direkt umgewandelt, da Selenylaminosäuren nicht sehr stabil sind.

In der vorgesehenen Synthesestrategie zur Totalsynthese von Nosiheptid wird der Dehydroalanin-Vorläufer bereits während der Pyridinkern-Synthese eingeführt und bedarf somit einer hohen chemischen Stabilität. Daher wurde als Vorläufer ein geschützter Serin-Rest gewählt, der nach Entschützung und Aktivierung über eine $\beta$-Eliminierung zum Dehydroalanin umgesetzt werden sollte. Für die Untersuchung dieser Umsetzung wurde das Tristhiazolylpyridin 71 zunächst am Serin-Rest entschützt, was chemoselektiv gelang (87\%). 
Anschließend wurden verschiedene Bedingungen zur Einführung des Dehydroalanins getestet (Schema 3.27). Ziel dabei war, mögliche Nebenreaktionen zum Nitril 168 zu unterdrücken.

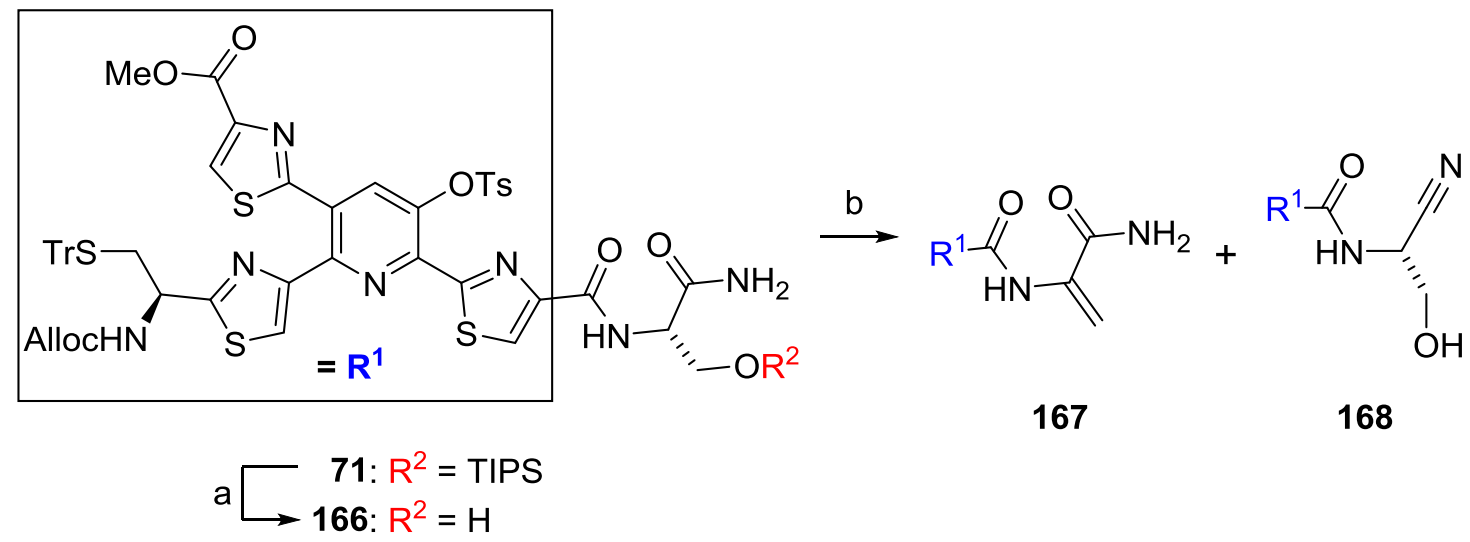

Schema 3.27: TIPS-Entschützung des Silylethers 71 und anschließende $\beta$-Eliminierung. Bedingungen: a) $\mathrm{HF}\left(48 \%\right.$ in $\left.\mathrm{H}_{2} \mathrm{O}\right)$, MeCN, RT, 16 h, $87 \%$. b) s. Tabelle.

\begin{tabular}{|c|c|c|}
\hline & Bedingungen & Ergebnis \\
\hline $\mathrm{a}$ & EDCI, $\mathrm{CuCl}, \mathrm{CH}_{2} \mathrm{Cl}_{2}, \mathrm{RT}$ & Zersetzung \\
\hline $\mathrm{b}$ & $n \mathrm{Bu}_{3} \mathrm{P}$, Grieco-Reagenz $(\mathbf{5 5}), 0{ }^{\circ} \mathrm{C}$ & Zersetzung \\
\hline $\mathrm{c}$ & $\mathrm{PPh}_{3}, \mathrm{DEAD}, \mathrm{THF}, 0^{\circ} \mathrm{C}$ & Nitril-Bildung (168) \\
\hline $\mathrm{d}$ & TsCl, DMAP, EtNiPr ${ }_{2}, \mathrm{CH}_{2} \mathrm{Cl}_{2}, \mathrm{RT}, 16 \mathrm{~h}$ & Gemisch \\
\hline e & $\begin{array}{c}\text { i., 2-MesitylSO }{ }_{2} \mathrm{Cl}, \mathrm{DMAP}, 2,6 \text {-Lutidin, } \mathrm{CH}_{2} \mathrm{Cl}_{2,}, \mathrm{RT}, 18 \mathrm{~h} \\
\text { ii. } \mathrm{DBU}, \mathrm{CH}_{2} \mathrm{Cl}_{2}, 0^{\circ} \mathrm{C}, 1 \mathrm{~h} .\end{array}$ & $65 \%(167)$ \\
\hline $\mathrm{f}$ & $\begin{array}{c}\text { i. 2-MesitylSO }{ }_{2} \mathrm{Cl} \text {, DMAP, 2,6-Lutidin, } \mathrm{CH}_{2} \mathrm{Cl}_{2}, \mathrm{RT}, 18 \mathrm{~h} \\
\text { ii. Pyridin, } 45^{\circ} \mathrm{C}, 18 \mathrm{~h}\end{array}$ & Nitril-Bildung (168) \\
\hline $\mathrm{g}$ & $\mathrm{MsCl}, \mathrm{EtNi} \mathrm{Pr}_{2}, \mathrm{CH}_{2} \mathrm{Cl}_{2}, 0^{\circ} \mathrm{C}, 3 \mathrm{~h}$ & Gemisch \\
\hline $\mathrm{h}$ & $\mathrm{Tf}_{2} \mathrm{O}, 2$,6-Lutidin, $\mathrm{CH}_{2} \mathrm{Cl}_{2}, 0^{\circ} \mathrm{C}, 1 \mathrm{~h}$ & Gemisch \\
\hline $\mathrm{i}$ & $\begin{array}{l}\text { i. } \mathrm{MsCl}, 2,6 \text {-Lutidin, } \mathrm{CH}_{2} \mathrm{Cl}_{2}, 0{ }^{\circ} \mathrm{C}, 1 \mathrm{~h} \\
\text { ii. } \mathrm{DBU}, \mathrm{CH}_{2} \mathrm{Cl}_{2},-35^{\circ} \mathrm{C}, 2 \mathrm{~h}\end{array}$ & $84 \%(167)$ \\
\hline
\end{tabular}

Beim Versuch die Dehydroalanin-Einheit unter den für die Dehydrobutyrin-Synthese bekannten Bedingungen zu erzeugen, konnte nur eine Zersetzung des Startmaterials beobachtet werden (Tabelleneinträge a, b). Eine Umsetzung unter Mitsunobu-Bedinungen ${ }^{[220]}$ lieferte erstaunlicherweise fast ausschließlich das Nitril 168 (c). Der Versuch, den Alkohol über ein $p$-Toluolsulfonat $\mathrm{zu}$ eliminieren, lieferte ein Gemisch an Produkten (d). Dabei konnten in LC-MS-Experimenten neben der Sulfonierung und Eliminierung auch 
dehydratisierte Produkte beobachtet werden. Der Einsatz des sterisch gehinderten 2-Mesitylsulfonylchlorids und 2,6-Lutidins als HCl-Fänger ermöglichte eine Sulfonierung des Serin-Alkohols mit wenigen Nebenprodukten (e). Nach Eliminierung des Sulfonats mit DBU wurde das Dehydroalanin 167 in 65\% Ausbeute isoliert. Wurde das Sulfonat in reinem Pyridin umgesetzt, so konnte nur die Bildung des Nitrils 168 beobachtet werden (f). Unter den Reaktionsbedingungen wanderte offenbar die Sulfonylgruppe auf den benachbarten Amidsauerstoff.

In der Totalsynthese von Promothiocin A nutzten Moody et al. Methansulfonsäurechlorid $(\mathrm{MsCl})$ zur Einführung eines Dehydroalanins in Nachbarschaft eines terminalen Amids. ${ }^{[102]}$ Ähnliche Bedingungen lieferten im vorliegenden Testsystem ein Gemisch (g). Der Einsatz von Trifluormethansulfonsäureanhydrid zeigte ein ähnliches Ergebnis (h). Eine saubere Sulfonierung gelang schließlich mit $\mathrm{MsCl}$ und 2,6-Lutidin als Base (i). Nach Entfernen der flüchtigen Reagenzien und anschließender Eliminierung mit DBU bei tiefer Temperatur, wurde das Dehydroalanin 167 in einer guten Ausbeute von 84\% erhalten.

In weiteren Versuchen zeigten nitrierte Sulfonylchloride oftmals eine zu hohe Reaktivität oder ermöglichten eine $\mathrm{S}_{\mathrm{N}} 2$-Substitution des intermediären Sulfonats und ergaben u.a. die entsprechenden Chloride, wenn auch nur in geringen Mengen. Beim Einsatz von $N, N^{`}$-Disuccinimidylcarbonat ${ }^{[221]}$ oder $N, N^{*}$-Carbonyldiimdazol ${ }^{[222]}$ konnte jeweils kein Umsatz beobachtet werden.

Die gefunden Bedingungen zur Einführung der Dha-Einheit ermöglichten eine selektive Sulfonierung des Serin-Rests ohne Dehydratisierung des benachbarten, terminalen Amids. Für die Eliminierung muss mit DBU jedoch eine starke Base eingesetzt werden. Dadurch werden aber eine tiefe Reaktionstemperatur und eine kurze Reaktionszeit möglich, die wiederum eine Isomerisierung des Z-Dehydrobutyrin-Rests minimieren sollten (Kap. 3.9).

\subsection{Untersuchungen zur Ts-Abspaltung am 3-Hydroxypyridin}

Die Abspaltung der Tosyl-Gruppe an der 3-Hydroxyfunktion des Pyridinkerns sollte als letzter Schritt in der Totalsynthese erfolgen. Wesentliche Herausforderung war die chemoselektive Spaltung in Gegenwart eines typischerweise sehr labilen Thioesters. Um geeignete Bedingungen $\mathrm{zu}$ finden, wurde der Naturstoff aus einem Nosiheptid-haltigen Futterzusatzmittel (,1\% Premix“) in Anlehnung an Literaturvorschriften isoliert. ${ }^{[30,136]}$ Das so erhaltene Vergleichsmaterial wurde mit TsCl selektiv an der 3-Hydroxyfunktion in einer guten 
Ausbeute von 75\% zum potentiellen Intermediat 169 umgesetzt (Schema 3.28). Das erhaltene Tosylat 169 zeigte im Gegensatz zu Nosiheptid eine wesentlich höhere Löslichkeit in hydrophoben Lösungsmitteln $\left(\mathrm{CH}_{2} \mathrm{Cl}_{2}, \mathrm{CHCl}_{3}\right)$ und konnte durch wässrige Aufarbeitung des Reaktionsgemischs isoliert werden. Die verbesserte Löslichkeit des Tosylats 169 ist auch im Hinblick auf semisynthetische Arbeiten von Nutzen, da somit eine Isolierung etwaiger Derivate wesentlich vereinfacht wird.

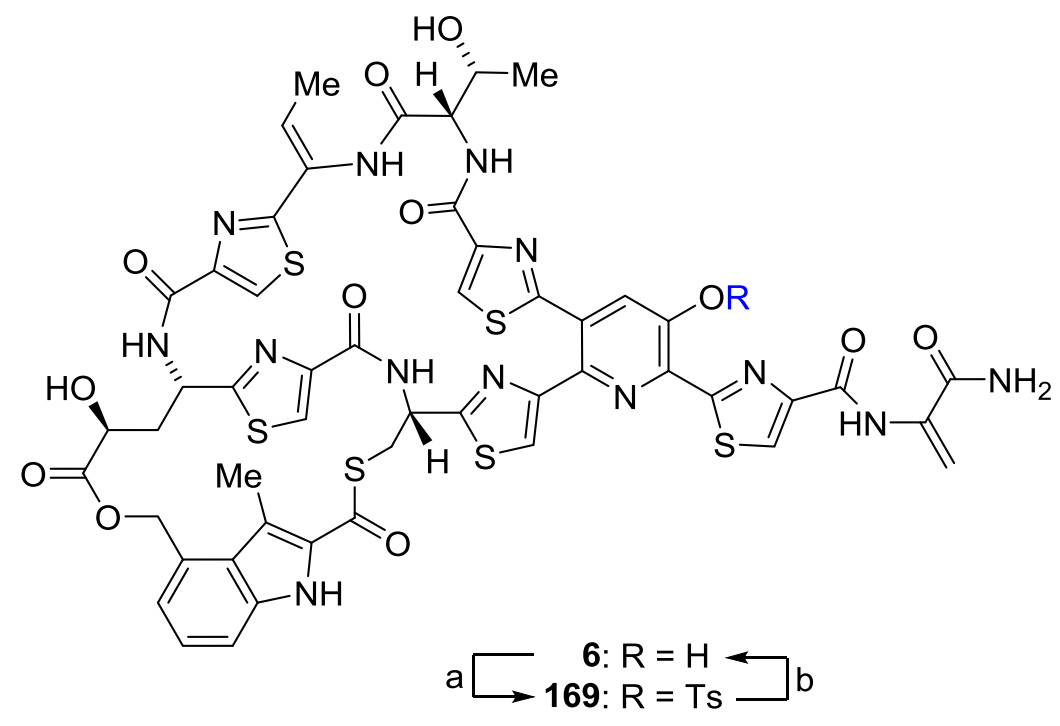

Schema 3.28 Semisynthese des Tosylats 169. Bedingungen: a) TsCl, EtNiPr 2 , DMAP, THF, $0{ }^{\circ} \mathrm{C} \rightarrow$ RT, 15 min, $75 \%$. b) s. Tabelle 3.2 .

In seiner Dissertation berichtete J.-Y. Lu von einer Abspaltung der Ts-Gruppe beim Einsatz von HATU in DMF während der Umsetzung des Pyridinfragments 42. ${ }^{[37]}$ Dabei schien das in der Reaktion frei werdende 1-Hydroxy-7-azabenzotriazol (HOAt) dafür verantwortlich zu sein. Dieser Beobachtung folgend wurde 1-Hydroxy-benzotriazol (HOBt) in Kombination mit Hünig-Base zur Abspaltung der Ts-Gruppe am Tosylat 169 getestet (Tabelle 3.2). Der Umsatz wurde mittels DC und LC-MS kontrolliert. 
Tabelle 3.2: Untersuchungen zur Ts-Abspaltung am semisynthetischen Tosylat 169.

\begin{tabular}{|c|c|c|}
\hline & Bedingungen $^{a}$ & Ergebnis \\
\hline $\mathrm{a}$ & $\mathrm{HOBt}, \mathrm{EtN} i \mathrm{Pr}_{2}$, THF, RT, $20 \mathrm{~h}$ & ca. $15 \%$ Umsatz \\
\hline $\mathrm{b}$ & HOBt, EtNiPr ${ }_{2}$, THF, $40^{\circ} \mathrm{C}, 2 \mathrm{~h}$ & ca. $40 \%$ Umsatz, Abbau Dha \\
\hline $\mathrm{c}$ & $\mathrm{HOBt}, \mathrm{EtN} i \mathrm{Pr}_{2}, \mathrm{DMF}, \mathrm{RT}, 30 \mathrm{~min}$ & sauberer Umsatz \\
\hline $\mathrm{d}$ & $\mathrm{TBAF},{ }^{b} \mathrm{THF}, 0{ }^{\circ} \mathrm{C}, 15 \mathrm{~min}$ & 100\% Umsatz, Abbau Dha \\
\hline $\mathrm{e}$ & $\mathrm{NaN}_{3},{ }^{c} \mathrm{DMF}, \mathrm{RT}, 5 \mathrm{~h}$ & $\begin{array}{c}\text { ca. } 85 \% \text { Umsatz, Isomerisierung Dhb- } \\
\text { Doppelbindung }\end{array}$ \\
\hline $\mathrm{f}$ & $\begin{array}{c}\mathrm{HOBt}, \mathrm{EtNi} \operatorname{Pr}_{2}, \mathrm{DMF}, 0^{\circ} \mathrm{C} \rightarrow \mathrm{RT}, \\
1 \mathrm{~h}\end{array}$ & $81 \%^{d}$ \\
\hline
\end{tabular}

Bei der Umsetzung in THF konnte bei RT selbst nach einer Reaktionszeit von $20 \mathrm{~h}$ nur ein geringer Umsatz festgestellt werden (Tabelleneintrag a). Durch eine Erhöhung der Temperatur konnte dieser gesteigert werden (b), jedoch wurde auch der Abbau der DehydroalaninSeitenkette (Dha) beobachtet (LC-MS). Diese könnte durch Hydrolyse des Imins 171 erfolgen, welches sich in Gegenwart einer Base aus dem $N$-Acyl-Enamid 170 bilden kann (Schema 3.29). ${ }^{[116]}$ In DMF hingegen, erfolgte eine rasche und saubere Abspaltung der Tosylgruppe innerhalb von nur 30 min. Mit dem Einsatz von TBAF ${ }^{[223]}$ wurde ebenfalls eine rasche Spaltung der Tosylgruppe beobachtet (d), eine Verkürzung der Dha-Seitenkette trat hier aber ebenfalls auf. Eine Umsetzung mit $\mathrm{NaN}_{3}$ in DMF zeigte auch eine selektive Spaltung der Ts-Gruppe (e). Im ${ }^{1} \mathrm{H}-\mathrm{NMR}$ konnte aber eine Isomerisierung der Doppelbindung der Dehydrobutyrin-Gruppe (Dhb) beobachtet werden.

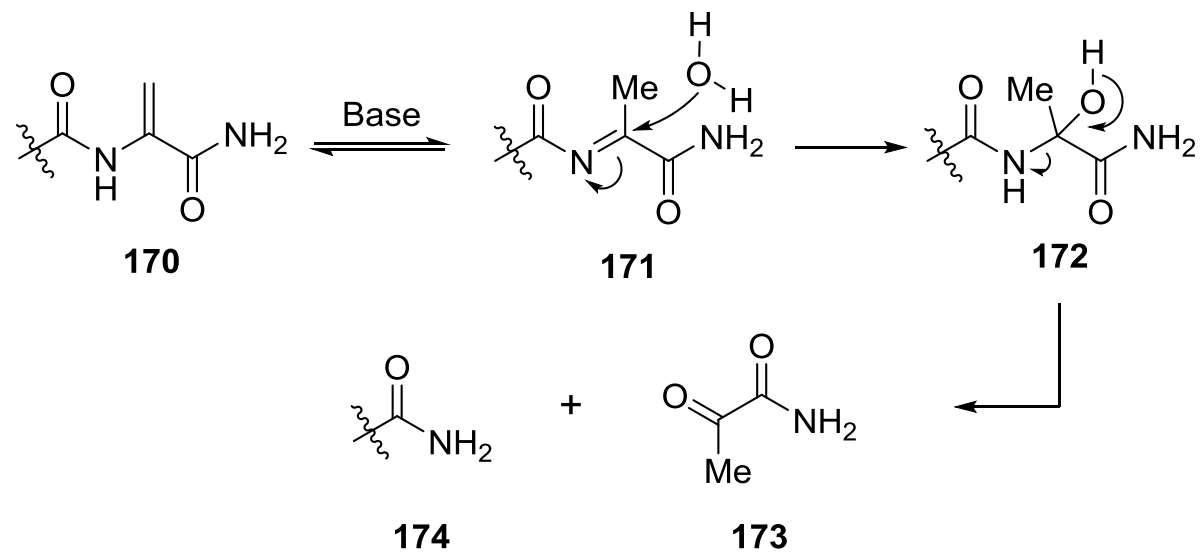

Schema 3.29: Möglicher Mechanismus zum Abbau der Dha-Seitenkette. 
Mit den in Eintrag c gezeigten Bedingungen wurde in einem vergrößerten Maßstab eine Ausbeute von $81 \%$ an entschütztem Nosiheptid (6) erhalten (f). Eine signifikante Isomerisierung der Dhb-Doppelbindung erfolgte dabei nicht.

Neben der Entwicklung einer geeigneten Methode zur Abspaltung der Ts-Gruppe ermöglichten diese Untersuchungen die Isolierung des Endprodukts aus der Reaktionslösung $\mathrm{zu}$ optimieren. Bei Versuchen, eine wässrige Aufarbeitung durchzuführen, wurde der Naturstoff nur in geringen Mengen erhalten. Die Reaktionslösung wurde daher nach vollständigem Umsatz im Vakuum eingeengt, der Rückstand mittels präparativer Dünnschichtchromatographie gereinigt und der Naturstoff erfolgreich isoliert. 


\subsection{Abschluss der Totalsynthese von Nosiheptid}

Auf dem Weg zur Totalsynthese wurden im nächsten Schritt die Bisthiazolamine 93 und 94 mit dem Tristhiazolylpyridin 42 zum jeweiligen linearen Vorläufer verbunden (Schema 3.30).

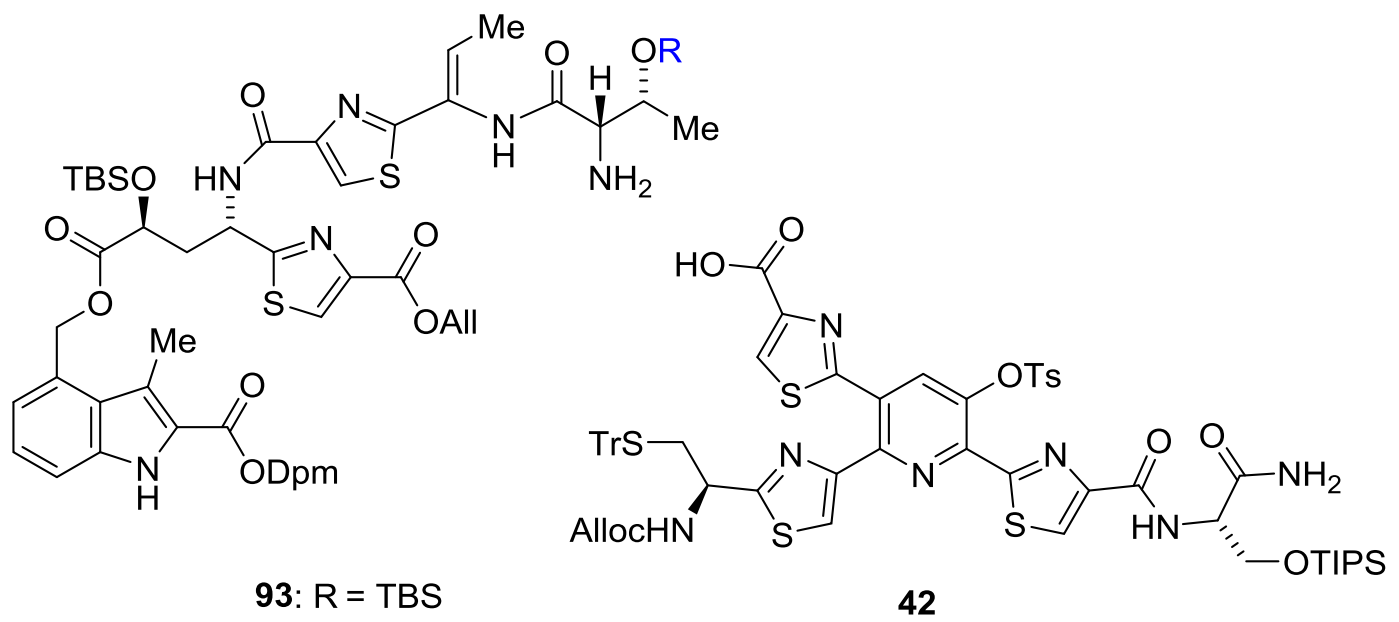

94: $R=$ TIPS

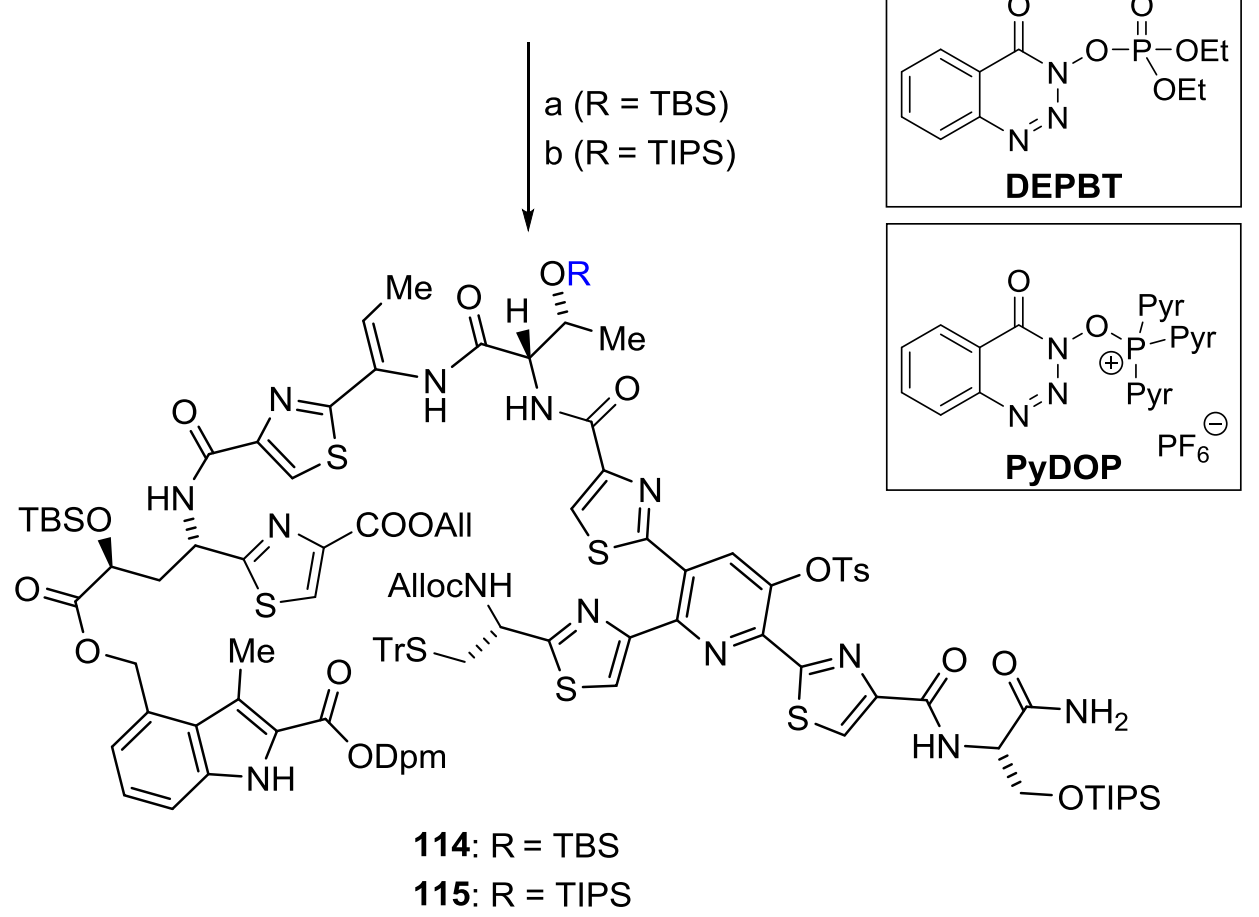

Schema 3.30: Synthese der linearen Vorläufer 114 und 115. Bedingungen: a) DEPBT, EtNiPr $2, T H F$, $0{ }^{\circ} \mathrm{C} \rightarrow \mathrm{RT}, 8$ h, 51\%. b) PyDOP, EtNiPr 2 , THF, $0{ }^{\circ} \mathrm{C} \rightarrow$ RT, 13 h, 92\%. Pyr = Pyrrolidin-1-yl.

Die Verknüpfung des Bisthiazolamins 93 mit DEPBT analog zu J.-Y. Lu ${ }^{[37]}$ lieferte den linearen Vorläufer 115 in einer Ausbeute von nur 51\%. Durch den Einsatz von PyDOP ${ }^{[224,225]}$ konnte im Fall des Thiazolamins 94 eine exzellente Ausbeute von 92\% erreicht werden. 
Für den Aufbau des A-Ring mussten sowohl der Allylester als auch die N-Alloc-Gruppe entfernt werden. Unter Verwendung der bei den Allylestern 145 und 147 bereits eingesetzten Methode gelang dies in einem Schritt (Schema 3.31).

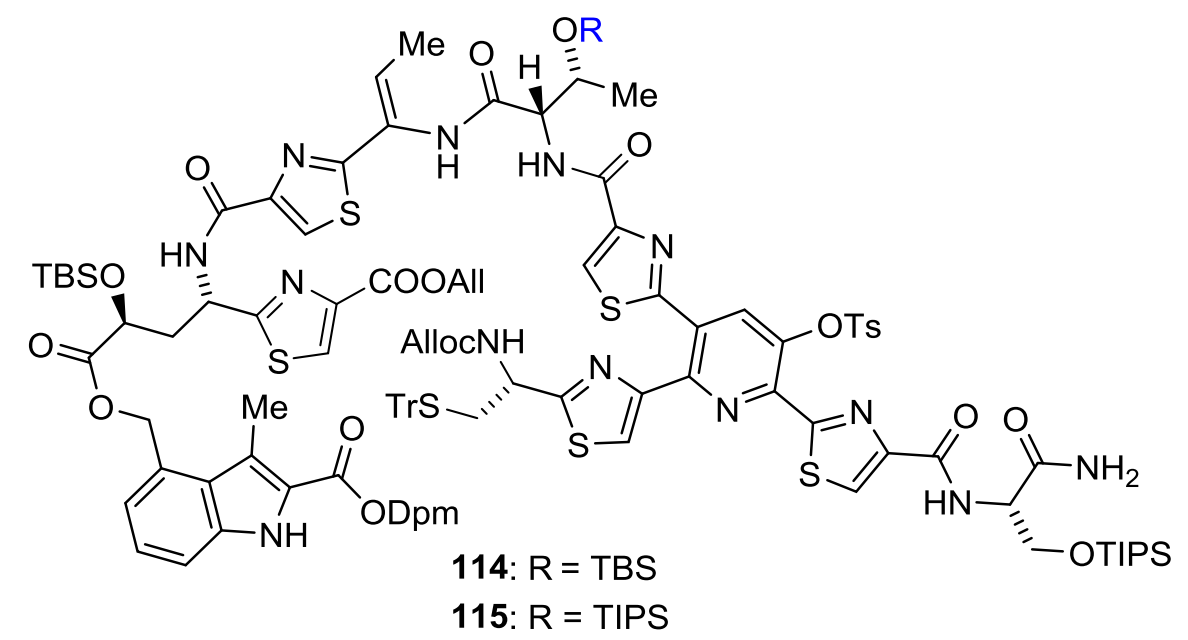

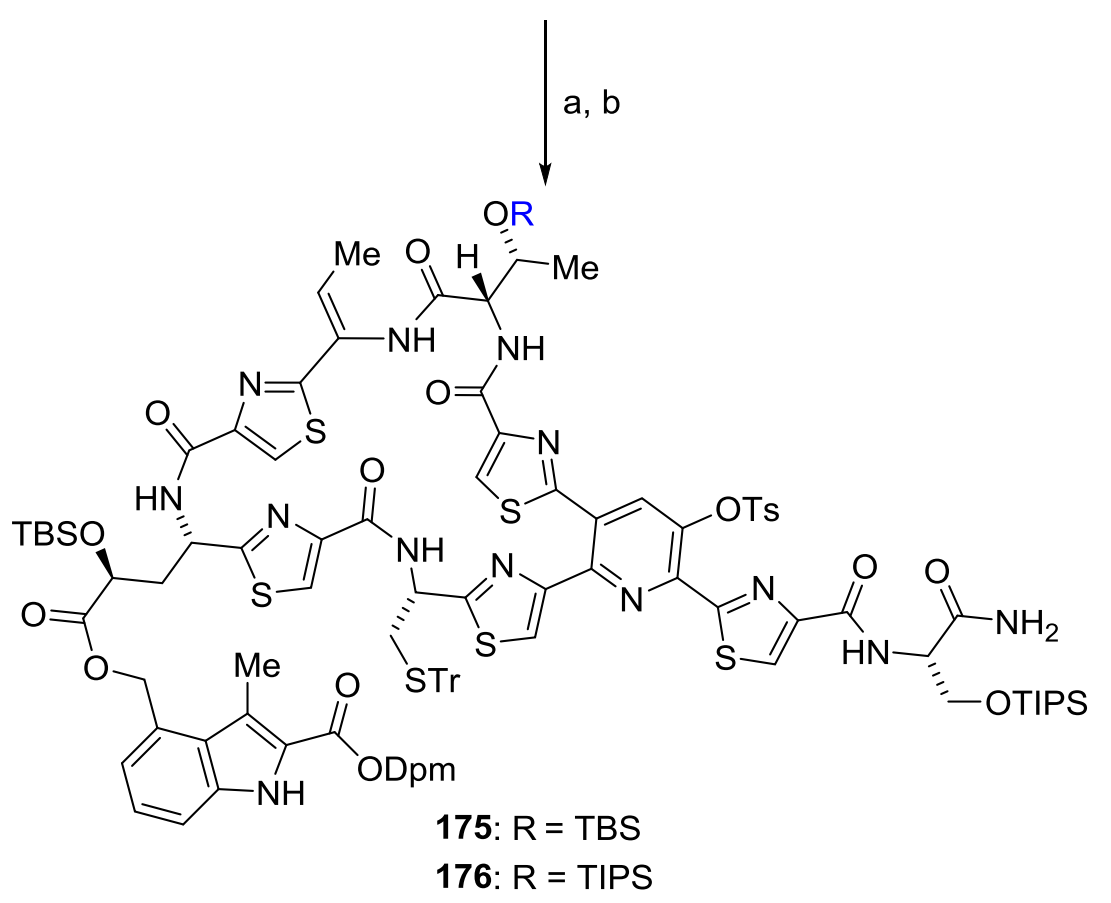

Schema 3.31: Synthese der A-Ring Makrolactame 177 und 178. Bedingungen: a) $\mathrm{Pd}\left(\mathrm{PPh}_{3}\right)_{4}, \mathrm{PhSiH}_{3}$, THF, $0{ }^{\circ} \mathrm{C}, 15-45$ min. b) HATU, EtNiPr 2 , THF (1 mMol), $0{ }^{\circ} \mathrm{C} \rightarrow$ RT, 1 h, 86\% (175), 89\% (176).

Die intermediär gebildete $\omega$-Aminosäure wurde, im Gegensatz zu den Arbeiten von Lu und Riedrich, nicht isoliert. Die Reaktionsmischung wurde nach vollständiger Entschützung der beiden Schutzgruppen mit THF verdünnt und mit Kupplungsreagenz und Base versetzt. Die A-Ring-Makrocyclen 175 und 176 wurden so in sehr guten Ausbeuten von 86\% (175) bzw. 89\% (176) nach einfacher Chromatographie isoliert. Die Reaktionszeiten waren sehr kurz und 
der Einsatz einer Spritzenpumpe war ebenfalls nicht notwendig. Versuche mit PyDOP als Kupplungsreagenz lieferten ähnliche Ausbeuten.

Die Umsetzung der erhaltenen A-Ring-Makrocyclen mit den zuvor erarbeiteten BRingschluss-Bedingungen (Kap. 3.6) ermöglichte den Aufbau des bismakrocyclischen Grundgerüsts (Schema 3.32).
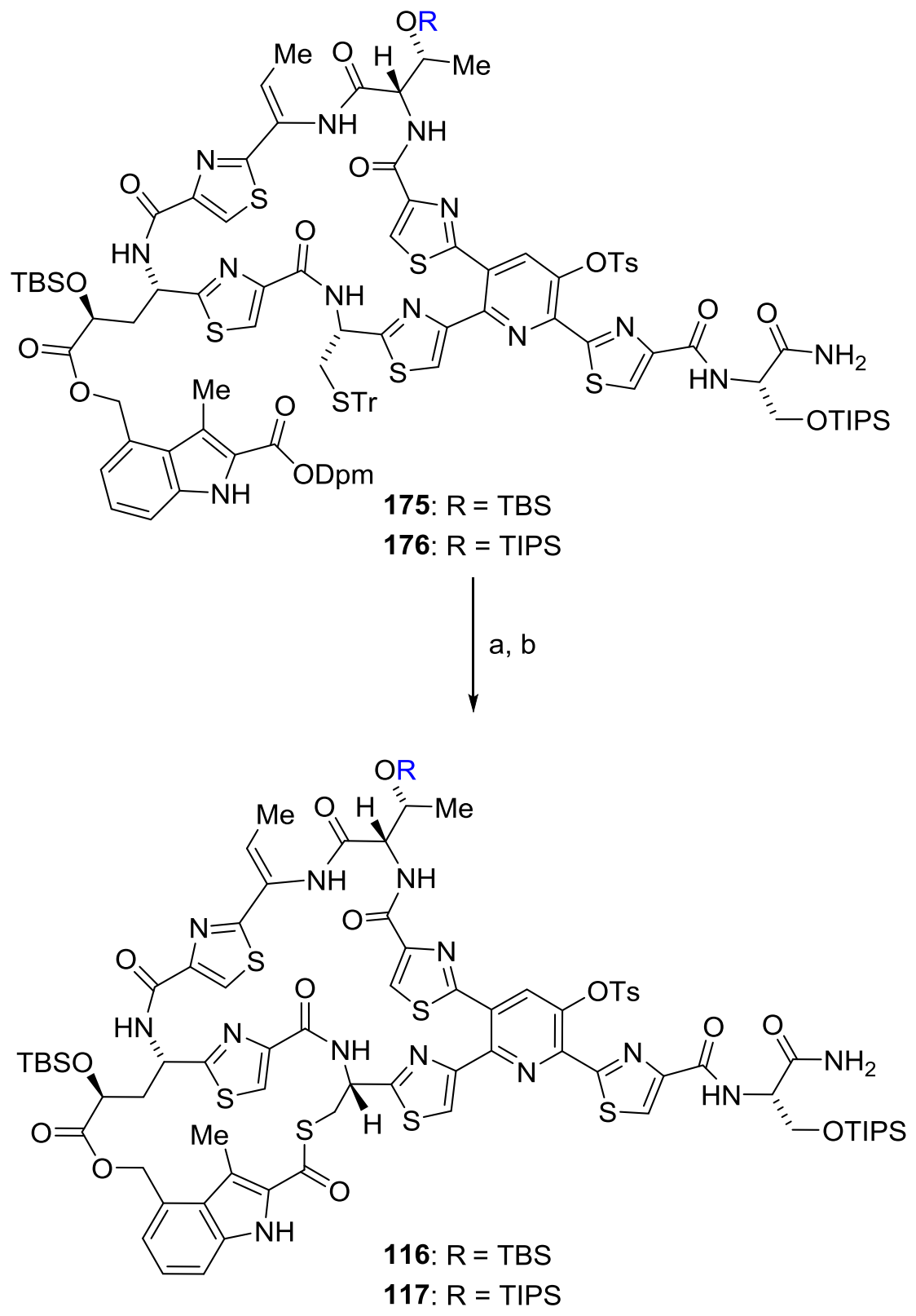

Schema 3.32: Synthese der voll geschützten Bismakrocyclen 116 und 117. Bedingungen: a) TFA, $\mathrm{Et}_{3} \mathrm{SiH}$, Anisol, $-25^{\circ} \mathrm{C} \rightarrow 0{ }^{\circ} \mathrm{C}, 24$ h. b) PyAOP, EtNiPr 2 , THF (0.5 mMol), $0{ }^{\circ} \mathrm{C} \rightarrow \mathrm{RT}, 1,5-3 \mathrm{~h}, 29 \%$ (116), $43 \%$ (117). 
Im Fall des A-Rings mit einer TBS-Gruppe an der Threonin-Seitenkette (175) wurde der Bismakrocyclus 116 mit einer geringen Ausbeute von nur 29\% erhalten. In LC-MS-Spektren wurden Nebensignale identifiziert, die auf die Abspaltung einer TBS-Gruppe hindeuteten. Im B-Ring-Testsystem wurde nur eine geringe Abspaltung der $O$-Threonin-TBS-Gruppe beobachtet und das Makrothiolacton mit einer wesentlich höheren Ausbeute erhalten (Kap. 3.6). Womöglich wird die Stabilität der TBS-Gruppe der Threonin-Seitenkette durch die Konformation des A-Ring-Makrocyclus beeinflusst. Die Nutzung einer stabileren TIPSGruppe (Substrat 176) führte zu einer akzeptablen Ausbeute von 43\% an Bismakrocyclus 117.

Für die Einführung des Dehydroalanins wurden in einem ersten Ansatz alle Silylschutzgruppen im Bismakrocyclus 116 entfernt (Schema 3.33). Anschließend sollte eine selektive Sulfonierung des primären Alkohols an der Serin-Seitenkette erfolgen, um danach das Sulfonat zum Dehydroalanin eliminieren zu können.

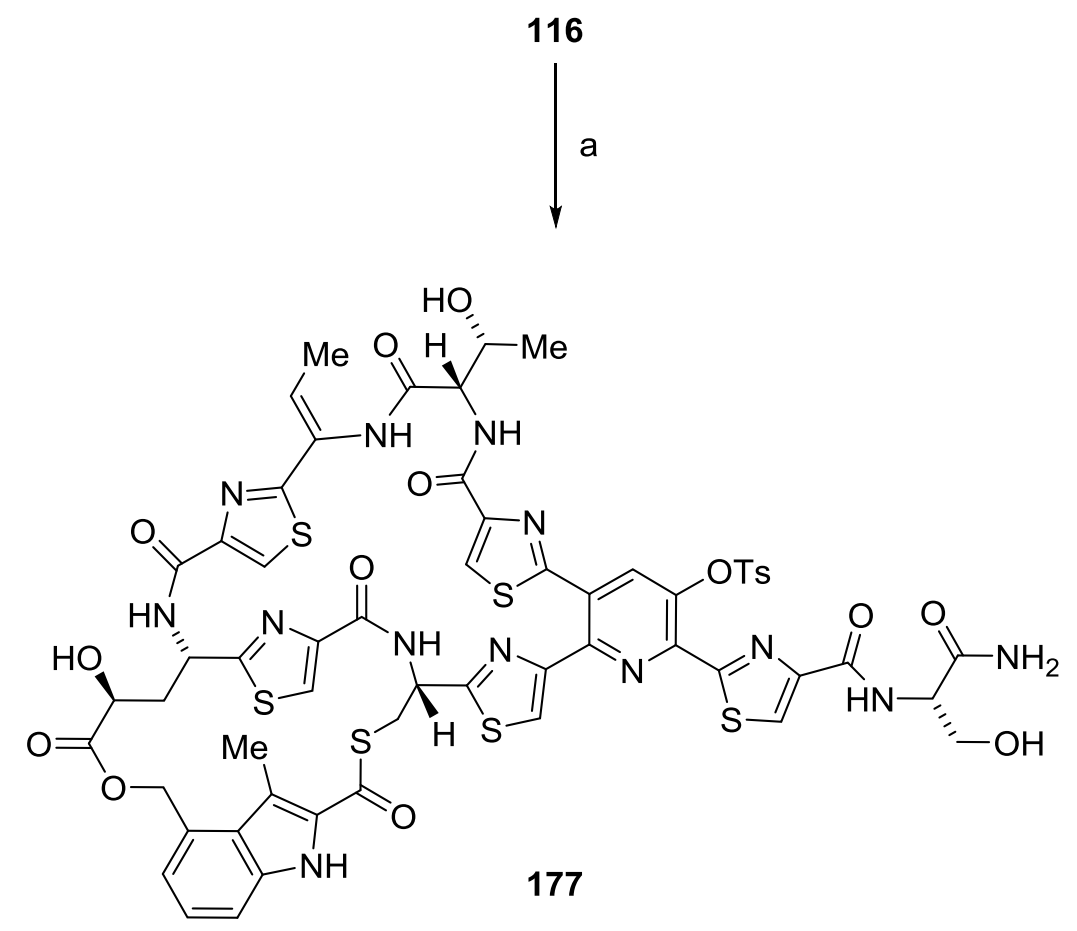

Schema 3.33: Entschützung der Silylschutzgruppen am Bismakrocyclus 116. Bedingungen: a) HF (70\% in Pyridin), THF, $0{ }^{\circ} \mathrm{C} \rightarrow \mathrm{RT}, 18 \mathrm{~h}, 96 \%$.

Es war geplant, dafür die unterschiedliche sterische Umgebung der Alkohole auszunutzen. Umsetzungen mit 2-Mesityl- oder 2,4,6-Triisopropylbenzensulfonylchlorid und DMAP in $\mathrm{CH}_{2} \mathrm{Cl}_{2}$ lieferten leider stets mehrfach sulfonierte Produkte. Beim Austausch des Lösungsmittels gegen THF konnte nur eine geringe Reaktivität der OH-Gruppen beobachtet 
werden. Eine entsprechende Umsetzung des Tristhiazolylpyridins 166 zeigte, dass die SerinSeitenkette unter diesen Bedingungen nicht sulfoniert wird.

Daher war die selektive Entschützung der Serin-Seitenkette notwendig. In der Literatur finden sich viele Beispiele für selektive mono-Entschützungen von Bis-Silylethern. ${ }^{[226-228]}$ Die selektive Entschützung der TIPS-Gruppe gelang mit $\mathrm{HF}$ in Acetonitril bei niedriger Temperatur (Schema 3.34). Beim Einsatz von MeOH oder THF als Lösungsmittel wurde erstaunlicherweise kein Umsatz beobachtet.

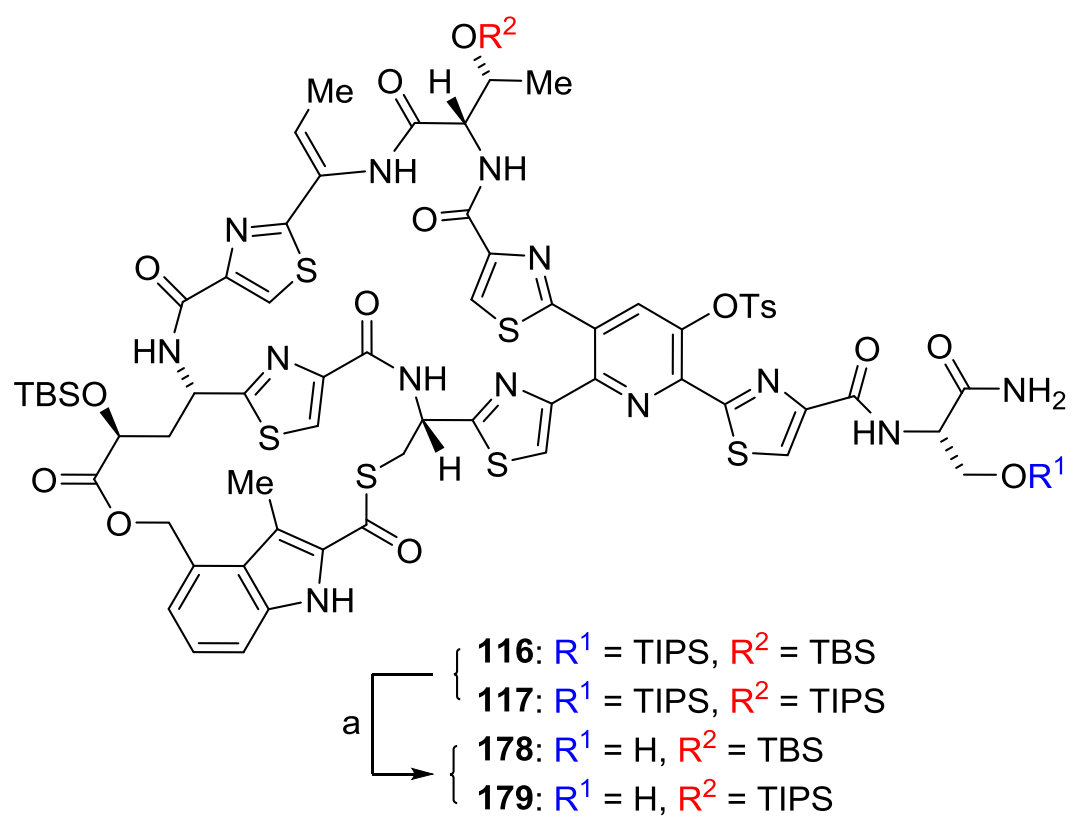

Schema 3.34: Selektive Entschützung der Serin-Seitenkette. Bedingungen: a) HF $(49 \%$ in $\left.\mathrm{H}_{2} \mathrm{O}\right) / \mathrm{MeCN}(1: 24), 0{ }^{\circ} \mathrm{C}, 15$ h, $41 \%$ (178), $75 \%$ (179, $90 \%$ b.r.s.m.).

Die mono-Entschützung des Bismakrocyclus 116 lieferte das entsprechende Produkt 178 in einer Ausbeute von 41\%, die zudem schwer zu reproduzieren war. Wie zuvor in der Makrothiolactonisierung zeigte auch hier die TBS-Schutzgruppe der Threonin-Seitenkette eine erhöhte Labilität. Mit einer TIPS-Schutzgruppe (117) wurde stattdessen eine gute Ausbeute an mono-entschütztem Bismakrocyclus 179 erreicht (75\%). Aufgrund dieser Beobachtung und der höheren Ausbeute während der Makothiolactonisierung (siehe oben) wurde die Synthese mit der TIPS-geschützten Threonin-Seitenkette fortgeführt. 
Zum Abschluss der Synthese musste nun das Dehydroalanin gebildet und die restlichen Schutzgruppen entfernt werden (Schema 3.35).

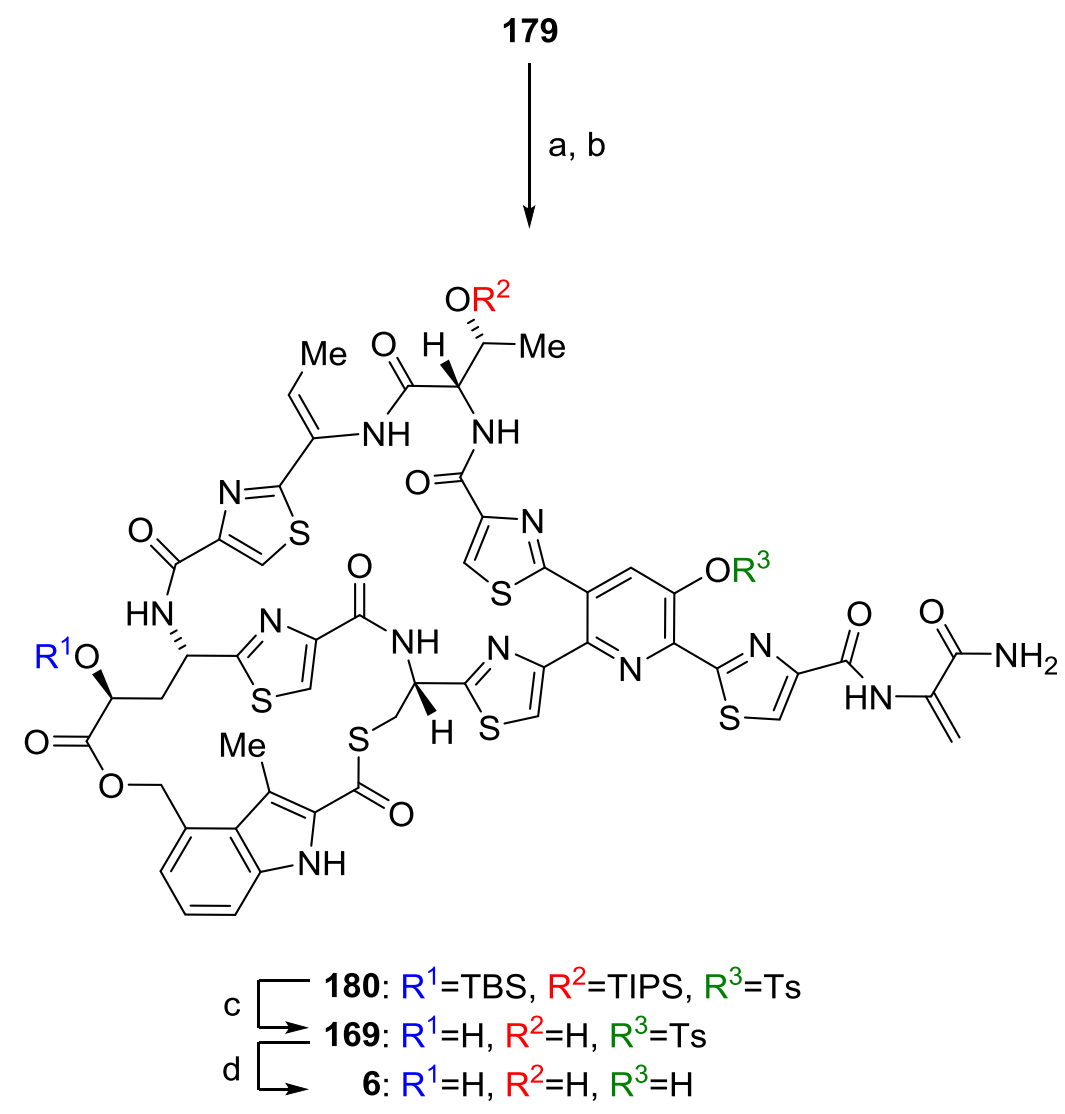

Schema 3.35: Abschluss der Totalsynthese von Nosiheptid (6). Bedingungen: a) MsCl, 2,6-Lutidin, $\mathrm{CH}_{2} \mathrm{Cl}_{2}, 0^{\circ} \mathrm{C} \rightarrow \mathrm{RT}, 0,5$ h. b) DBU ( $2 \%$ in $\left.\mathrm{CH}_{2} \mathrm{Cl}_{2}\right),-78^{\circ} \mathrm{C} \rightarrow-35^{\circ} \mathrm{C}, 4.5 \mathrm{~h}$ (35\% ü. 2 Stufen). c) $\mathrm{Et}_{3} \mathrm{~N} \times 3 \mathrm{HF} / \mathrm{THF}(1: 9), 0{ }^{\circ} \mathrm{C} \rightarrow \mathrm{RT}, 27 \mathrm{~h}$. d) $\mathrm{HOBt}, \mathrm{EtN} i \mathrm{Pr}_{2}, \mathrm{DMF}, 0{ }^{\circ} \mathrm{C} \rightarrow \mathrm{RT}, 1.5 \mathrm{~h}, 36 \%$ (ü. 2 Stufen).

Die Sulfonierung der Serin-Seitenkette verlief wie im Testsystem (Kap. 3.7) sauber und ohne Bildung an Nebenprodukten. Das nach der Eliminierung erhaltene Produkt musste jedoch nach Säulenchromatographie (ca. 70\% Ausbeute) mittels präparativer HPLC weiter gereinigt werden, da neben einem anteiligen Verlust der Ts-Gruppe weitere nicht identifizierbare Zersetzungsprodukte beobachtet wurden. Das Isomerenverhältnis der DehydrobutyrinDoppelbindung lag, basierend auf der Integration des Vinylprotons im ${ }^{1} \mathrm{H}-\mathrm{NMR}$, bei etwa 4:1 und konnte nicht weiter verbessert werden. Eine Trennung der Doppelbindungsisomere war ebenfalls nicht möglich. Die Isomerisierung trat wahrscheinlich während der präparativen HPLC-Reinigung auf (siehe weiter unten). Das Dehydroalanin 180 wurde mit einer Ausbeute von $35 \%$ über zwei Stufen erhalten.

Die Abspaltung der Silylschutzgruppen erfolgte anschließend mit $\mathrm{Et}_{3} \mathrm{~N} \times 3 \mathrm{HF}$ in THF. Bei 
Versuchen mit HF in Pyridin wurde nur eine unvollständige Abspaltung der TIPS-Gruppe erreicht. Das synthetische Tosylat 169 wurde als Rohprodukt direkt weiter umgesetzt und die Ts-Gruppe mit HOBt in Gegenwart von $\mathrm{EtNi} \mathrm{Pr}_{2}$ abgespalten (Kap. 3.8). Nach präparativer Dünschichtchromatographie (ca. 65\% Ausbeute) und anschließender präp. HPLC wurde der synthetische Naturstoff 6 in einer Ausbeute von 36\% über zwei Stufen isoliert.

\subsection{Strukturbestätigung}

Um die strukturelle Identität eindeutig zu klären wurde ein direkter Vergleich mit der zuvor isolierten Originalsubstanz vollzogen. Die analytischen Daten (DC, HRMS, $\left.[\alpha]_{\mathrm{D}}{ }^{24}\right)$ des synthetischen und natürlichen Nosiheptids stimmten gut überein. Lediglich bei der optischen Rotation gab es eine geringe Diskrepanz (syn. $[\alpha]_{\mathrm{D}}{ }^{24}=+30.5$, (Pyridin, $c=0.1$ ), nat. +31.5 (Pyridin, $c=0.1)$ ) im Bereich der Fehler für solche Messungen. Der Vergleich der HPLCChromatogramme sowie eine Ko-Injektion wiesen ebenfalls auf eine strukturelle Identität hin (Abbildung 3.6). 

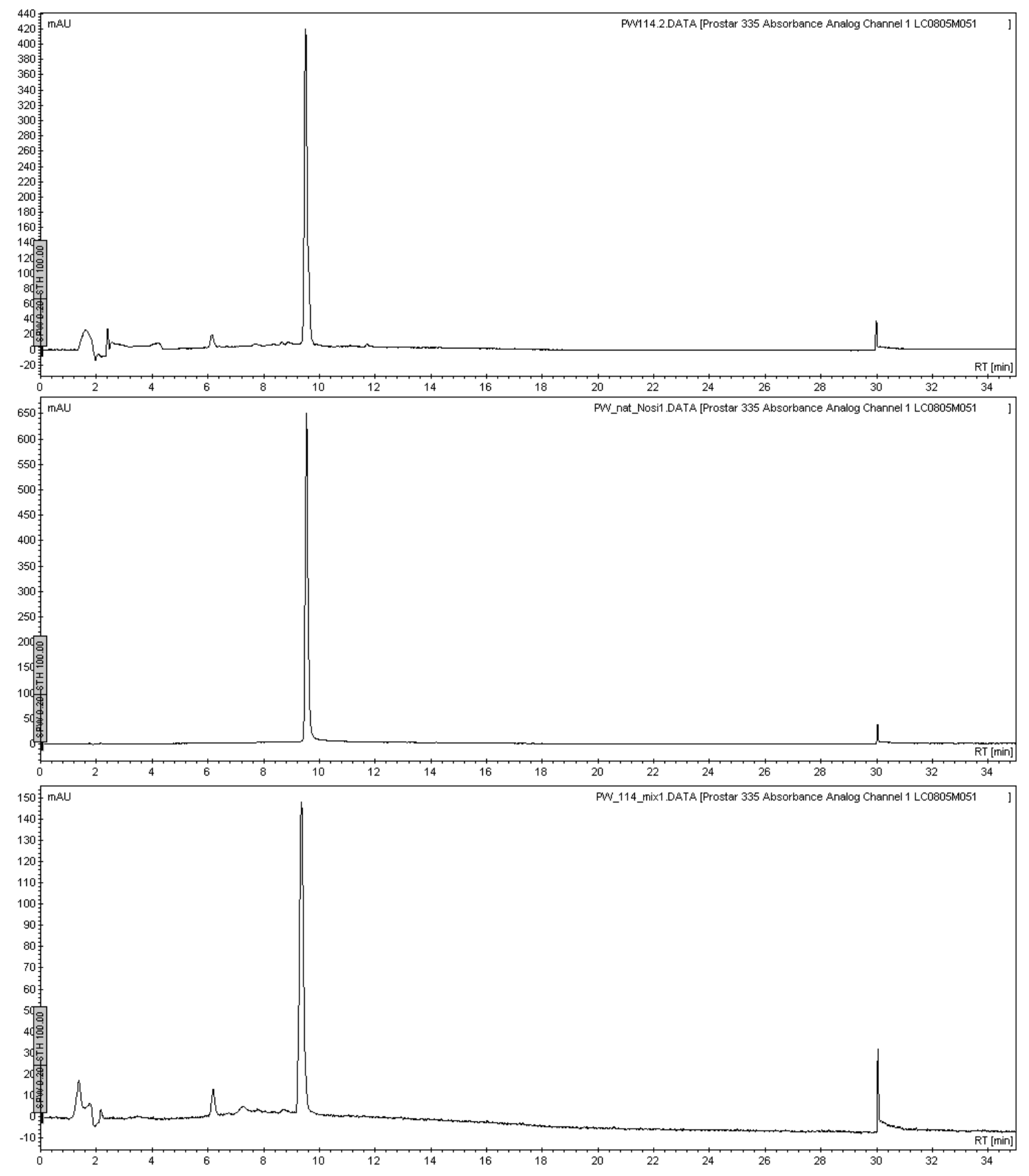

Abbildung 3.6: HPLC-Analysen des synthetischen (oben) und natürlichen Nosiheptids (mitte) sowie Ko-Injektion eines Gemisches (unten).

Der Vergleich zwischen dem ${ }^{1}$ H-NMR-Spektrum des synthetischen Nosiheptids (6) und dem des natürlichen zeigte keine signifikanten Abweichungen (Abbildung 3.7). 


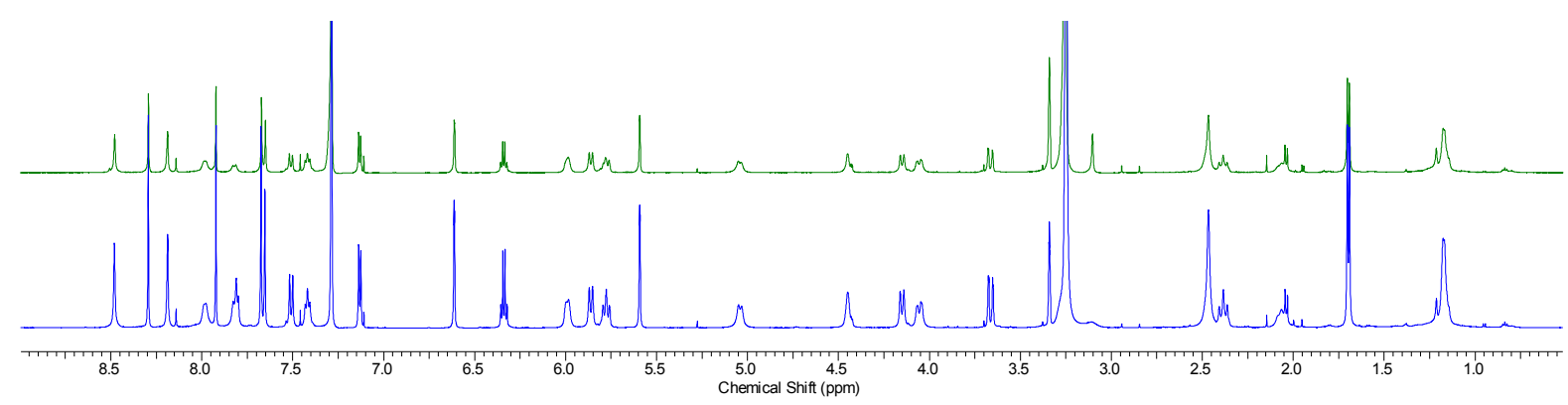

Abbildung 3.7: Vergleich der ${ }^{1} \mathrm{H}-\mathrm{NMR}-$ Spektren (je ca. $1 \mathrm{mg}, 600 \mathrm{MHz}, \mathrm{CDCl}_{3} / \mathrm{MeOH}_{-} \mathrm{d}_{4}, 9: 1$ ) zwischen synthetischem (oben) und natürlichem Nosiheptid (6) (unten).

Das isolierte natürliche Nosiheptid wurde zuvor ebenfalls mittels präparativer HPLC gereinigt und zeigte dabei auch eine Isomerisierung der Dehydrobutyrin-Doppelbindung (Z-Isomer: $d$, $1.67 \mathrm{ppm}, J=7.0 \mathrm{~Hz}, E$-Isomer: d, $2.01 \mathrm{ppm}, J=7.9 \mathrm{~Hz}$ ). Das Isomeren-Verhältnis im synthetischen Material lag bei etwa 4:1 gegenüber 5:1 im natürlichen. Natürliches Nosiheptid, das nicht mittels präparativer HPLC $\left(\mathrm{C} 18\right.$, Eluenten $\mathrm{MeCN}$ u. $\left.\mathrm{H}_{2} \mathrm{O}\right)$ gereinigt wurde, zeigte im ${ }^{1} \mathrm{H}-\mathrm{NMR}$ keine Isomerisierung der Doppelbindung.

Die Isomerisierung einer Dehydrobutyrin-Doppelbindung in wässriger Lösung nach präparativer HPLC wurde auch für das Thiopeptidantibiotikum Cyclothiazomycin B beschrieben. ${ }^{[69]}$ Für ein Dehydrobutyrin-enthaltendes Fragment des Cyclothiazomycin B wurde in theoretischen Berechnungen eine Stabilisierung des Z-Isomers gegenüber dem E-Isomer von $2.6 \mathrm{kcal} / \mathrm{mol}$ berechnet. ${ }^{[6]}$ Die Isomerisierung der Doppelbindung findet wahrscheinlich über eine Imin-Enamin-Tautomerie statt (Schema 3.36). Welche Rolle die präp. HPLC dabei spielt ( $\mathrm{pH}$, Lösungsmittel, Säulenmaterial, Druck) konnte im Rahmen dieser Arbeit nicht geklärt werden.<smiles></smiles>

181<smiles>[R]C(=O)N=C([R])CC</smiles>

182 183

Schema 3.36: Imin-Enamin-Tautomerie als Ursache der Dhb-Isomerisierung. Thz $=$ Thiazol. 


\subsection{Vergleich der biologischen Aktivität}

In einem Hemmhoftest wurde die Aktivität des isolierten und synthetischen Materials gegenüber dem Bodenbakterium Streptomyces coelicolor A3(2) getestet. Ein Bild der gebildeten Hemmhöfe nach 3-tägiger Inkubation ist in Abbildung 3.8 gezeigt.

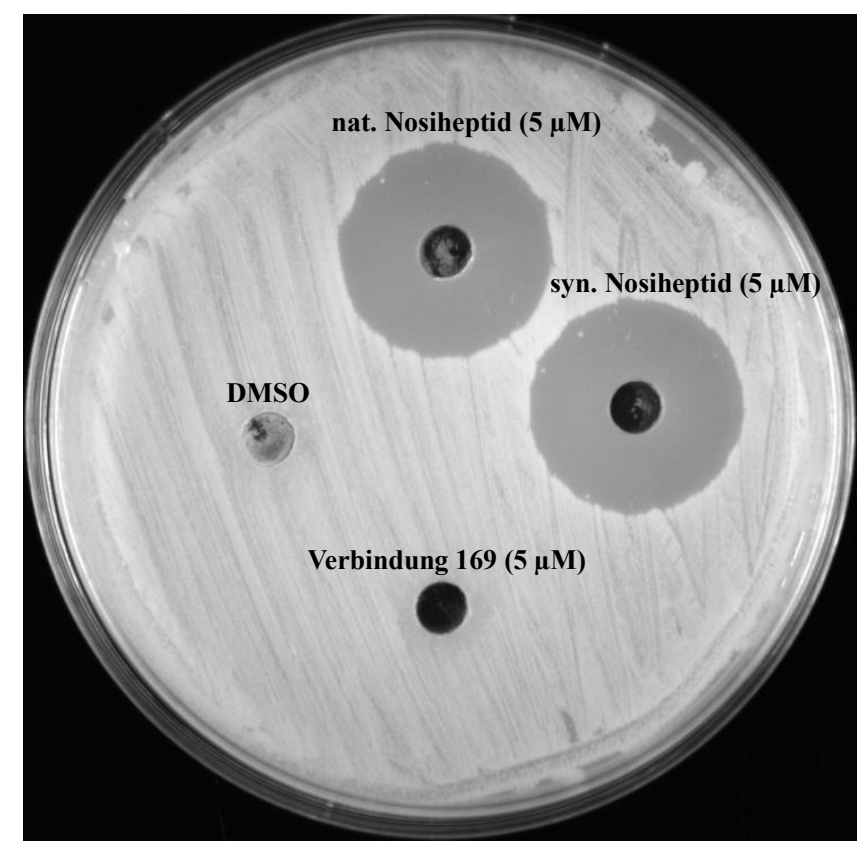

Abbildung 3.8: Hemmhoftest des synthetischen und authentischen Naturstoffs mit Streptomyces coelicolor A(3)2 nach einer Inkubationszeit von 3 Tagen, die Konzentration der Lösungen betrug $5 \mu \mathrm{mol} / \mathrm{L}$.

Wie auf dem Bild $\mathrm{zu}$ sehen, sind die von natürlichem und synthetischem Material verursachten Hemmhöfe nahezu gleich groß. Diese funktionelle Identität zeigt die antibakterielle Potenz von Nosiheptid auch gegenüber Bodenbakterien - sehr enge Verwandte und Begleiter des Produzenten S. actuosus. Überraschenderweise wurde die $O$-Tosylierung des Hydroxypyridinkerns nicht toleriert, da die Verbindung 169 keine merkliche antibakterielle Aktivität zeigte. Dies könnte sowohl durch die Beeinträchtigung der physikochemischen (Löslichkeit, Diffusionsvermögen durch das Agar-Medium) als auch der pharmako-dynamischen Eigenschaften (Zellaufnahme, Bindungsaffinität zum Ribosom) erklärt werden. 


\subsection{Fazit}

Im Rahmen der vorliegenden Arbeit konnte die erste Totalsynthese des Thiopeptidantibiotikums Nosiheptid erfolgreich abgeschlossen werden. Der Naturstoff war mit isoliertem authentischem Material im Hinblick auf spektroskopische Daten und antimikrobielle Aktivität identisch. Die Synthese von Nosiheptid gelang in 76 Stufen mit einer längsten linearen Sequenz von 32 Stufen. Schlüsselmethoden waren eine hetero-Diels-AlderReaktion zum Aufbau des 3-Hydroxypyridinkerns, Aza-Wittig-Cyclisierungen zur Synthese funktionalisierter Thiazole, eine Nachbargruppen-assistierte Verseifung sowie reißverschlussartige Makrocyclisierungen eines linearen Vorläufers. Besonders wichtig war eine fortgeschrittene Schutzgruppenstrategie, die der besonderen Labilität der Nosiheptid-Struktur angepasst wurde.

Die Totalsynthese von Nosiheptid eröffnet den ersten synthetischen Zugang zur ungewöhnlichen Klasse E der Thiopeptide. Die Modularität der entwickelten Syntheseroute erlaubt den Austausch von Bausteinen und kann somit zur Darstellung neuartiger, bisher nicht zugänglicher Derivate dienen, die eine weitergehende chemisch-biologische Profilierung dieses potenten und strukturell ungewöhnlichen Antibiotikums ermöglichen könnten. Darüber hinaus könnte die hier angewendete Synthesestrategie einen Weg zur Synthese weiterer Verbindungen aus dieser Klasse weisen, wie Glycothiohexid $\alpha$ oder Thiazomycin B. 

4 Nachbargruppen-assistierte Umfunktionalisierung von 3-Hydroxypicolinaten 


\subsection{Einleitung}

\subsubsection{Substratdirigierte Reaktionen}

Die Entwicklung von Reaktionen, bei denen eine Kontrolle der Selektivität in sämtlichen Aspekten gelingt, gehört zu den großen Herausforderungen in der organischen Synthese: „Selektivitätskontrolle kann dabei vom Reagenz oder vom Katalysator ausgehen und wird dann als Reagenz- bzw. Katalysatorkontrolle der Selektivität bezeichnet. Alternativ dazu können substratinhärente Strukturinformation genutzt werden, um die Trajektorie des angreifenden Reagenz zu steuern. Dies wird allgemein als Substratkontrolle der Selektivität bezeichnet. ““[229]

Als frühe Beispiele für substratdirigierte Reaktionen gelten die 1957 durch Henbest beschriebene hydroxydirigierte Epoxidierung von 2-Cyclohexen-1-ol, sowie die Alkoxiddirigierte Simmons-Smith-Cyclopropanierung von 3-Cyclopenten-1-ol durch Winstein aus dem Jahr 1961 (Schema 4.1). ${ }^{[230,231]}$

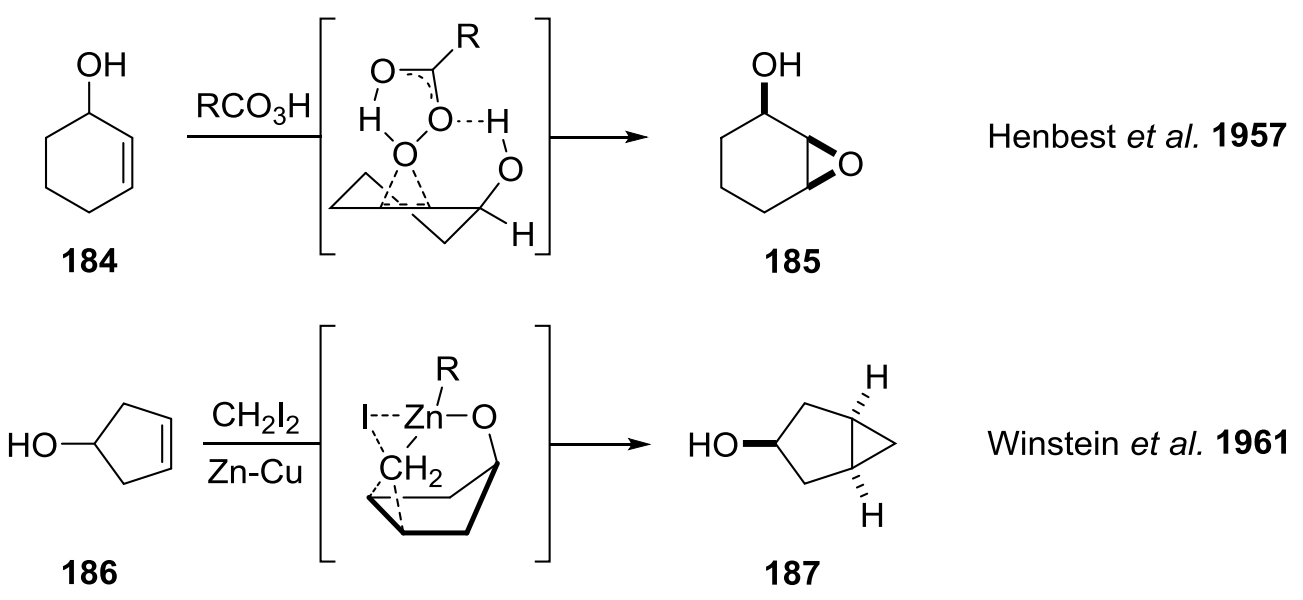

Schema 4.1: Zwei der ersten substratdirigierten Reaktionen.

Durch die aktive Einbindung der Hydroxygruppe in den Übergangszustand wird in beiden Fällen das Reagenz an die sterisch anspruchsvollere diastereofaciale Seite des Olefins dirigiert, cis zum allylischen Heteroatom, und die jeweilige Umsetzung gelingt stereoselektiv. Winstein beobachtete zudem, dass eine entsprechende Umsetzung von Cyclopentenylacetat nur einen geringen Umsatz lieferte (ca. 3\%). Die Präkoordination des Zinkreagenz über eine Zink-Alkoxid-Bindung führte somit zu einer erheblichen Reaktionsbeschleunigung. In der Enzymkatalyse sind diese auf einer „Intramolekularisierung“ durch „aktives Volumen“ basierenden Effekte ein allgemeines und bekanntes Phänomen. ${ }^{[232]}$ Auf dirigierenden Effekten 
basierende Reaktionen wurden in einem Übersichtsartikel von Hoveyda, Evans und Fu, ${ }^{[233]}$ sowie kürzlich durch Breit et al. ausführlich beschrieben. ${ }^{\text {[229] }}$

Diese Effekte werden im modernen Reaktionsdesign immer wieder genutzt. Die Gruppe von Gansäuer konnte z.B. kürzlich zeigen, dass unter Beteiligung einer benachbarten HydroxyGruppe chirale 1,4-Diole über eine Fluorid-katalysierte Epoxid-Hydrosilylierung zugänglich sind (Schema 4.2). ${ }^{[234]}$

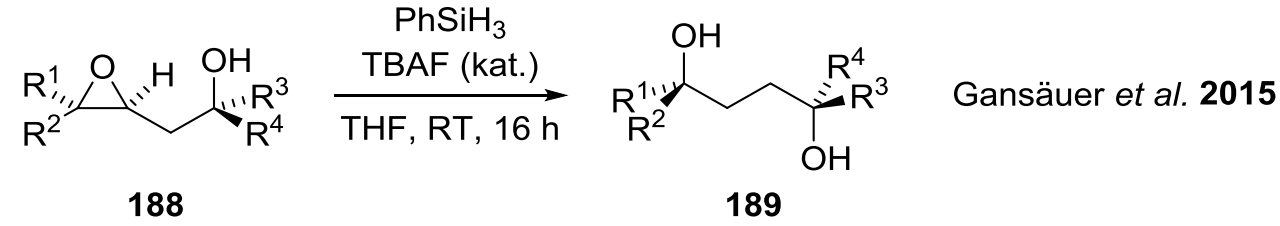

Schema 4.2: Darstellung chiraler 1,4-Diole durch Epoxid-Hydrosilylierung nach Gansäuer et al.. ${ }^{[234]}$

Die durch Gilman ${ }^{[235]}$ und Wittig ${ }^{[236]}$ unabhängig voneinander entdeckte ortho-Metallierung von Aromaten stellt ein weiteres Beispiel für eine wichtige substratdirigierte Reaktion dar. ${ }^{[237-}$ ${ }^{239]}$ Die Gruppe von Uchiyama konnte zeigen, dass durch dirigierte ortho-Cuprierung eine direkte Hydroxylierung sowie Aminierung von Aromaten möglich ist (Schema 4.3). ${ }^{[240]}$ Als dirigierende Gruppen fungierten dabei vorwiegend tertiäre Amide.

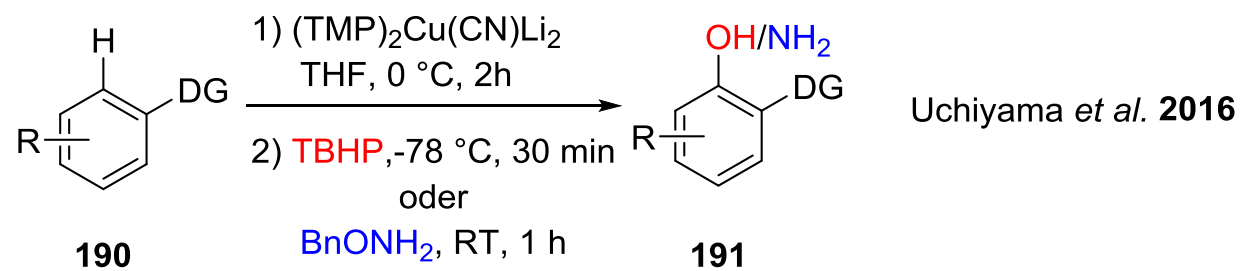

Schema 4.3: Direkte Hydroxylierung oder Aminierung von Aromaten nach Uchiyama et al.; $\mathrm{DG}=$ Dirigierende Gruppe. ${ }^{[240]}$

Diese Beispiele zeigen, dass die substratkontrollierte Selektivität ein wichtiges Werkzeug für die organische Synthese ist, indem sowohl neue Funktionalität als auch Konfiguration, unabhängig von unter Umständen aufwendig darzustellenden chiralen Reagenzien oder Katalysatoren, voraussagbar und zuverlässig aufgebaut werden können. 


\subsubsection{Frühere Arbeiten zur regioselektiven Umfunktionalisierung von}

\section{3-Hydroxypicolinaten}

Während seiner Arbeit an der Synthese des 3-Hydroxypyridin-Kerns von Nosiheptid (Kapitel 1.5.2) stand Jin-Yong Lu vor der Herausforderung selektiv nur einen der beiden Methylester im Syntheseintermediat 96 zu spalten (Schema 4.4). ${ }^{[37]}$

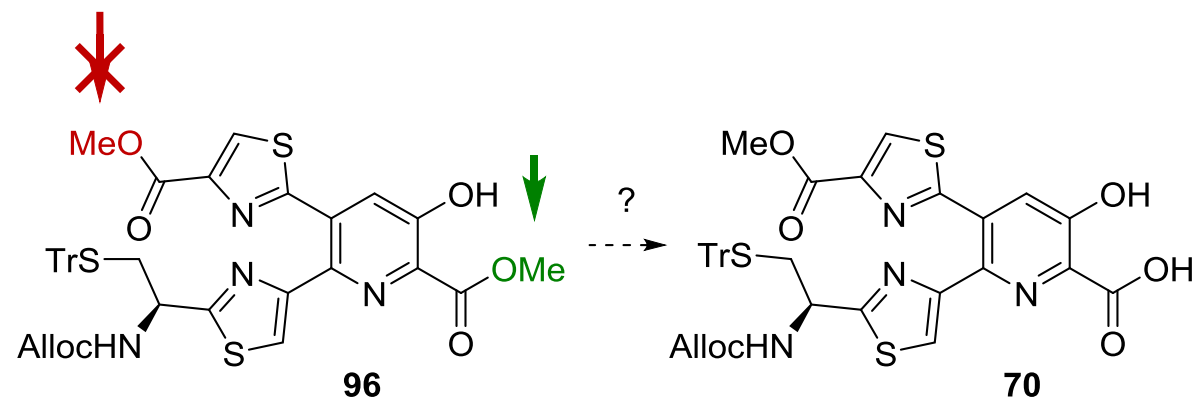

Schema 4.4: Herausforderung der regioselektiven Methylesterhydrolyse im Intermediat 96.

Nach intensiven Untersuchungen stellte sich heraus, dass die freie Hydroxygruppe von entscheidender Bedeutung ist und wahrscheinlich dirigierend wirkt. Dabei vermutete er eine Chelatisierung des in der Reaktion eingesetzten $\mathrm{Sc}(\mathrm{OTf})_{3}$ durch die OH-Gruppe, und des Carbonylsauerstoffs, was zur Aktivierung des Carbonylkohlenstoffs führen könnte (Schema 4.5). ${ }^{[37]}$ Der pH-Wert der Reaktionslösung wurde dabei durch Zugabe kleiner Mengen $\mathrm{NaHCO}_{3}$-Lösung auf 8-8.5 eingestellt. Bei einem $\mathrm{pH}$-Wert unter 8 wurde ein wesentlich geringerer Umsatz beobachtet. ${ }^{[241]}$

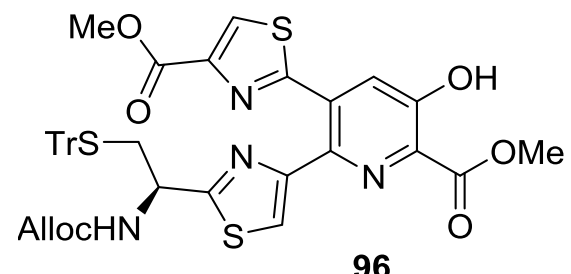

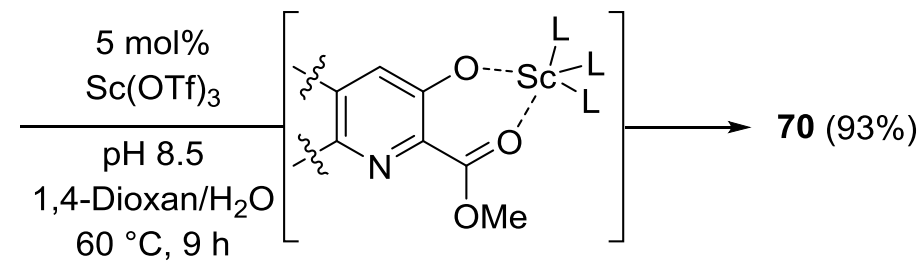

Schema 4.5: Regioselektive Verseifung des Bisthiazolylpyridins 96 und mechanistische Hypothese nach $\mathrm{Lu} ; \mathrm{L}=$ Ligand. ${ }^{[37]}$

Ausgehend von dieser Vermutung konnte Lu auch eine regioselektive Hydrolyse, Umesterung sowie Aminolyse des Triesters 97 demonstrieren (Schema 4.6). 
<smiles>COC(=O)c1cc(O)c(C(=O)OC)nc1C(=O)OC</smiles>

97<smiles>[R]C(=O)c1nc(C(=O)OC)c(C(=O)OC)cc1O</smiles>

$192(\mathrm{R}=\mathrm{OH})$

$193(\mathrm{R}=\mathrm{OAll})$

$194(\mathrm{R}=\mathrm{HNBn})$

Schema 4.6: Regioselektive Umfunktionalisierungen des Triesters 97 nach Lu. Bedingungen: a) 5 mol\% Sc(OTf $)_{3}$, pH 8.5, 1,4-Dioxan $/ \mathrm{H}_{2} \mathrm{O}, 60{ }^{\circ} \mathrm{C}, 9$ h, 98\%. b) Allylalkohol, 5 mol\% $\mathrm{Sc}(\mathrm{OTf})_{3}$, kat. $\mathrm{H}_{2} \mathrm{O}, 60^{\circ} \mathrm{C}, 18$ h, 90\%. c) Benzylamin (2 Äq.), 5mol\% Sc(OTf) 3 , 1,4-Dioxan, RT, 18 h, 99\%. ${ }^{[37]}$

Sowohl eine Röntgenkristallstruktur des Allylesters 193 ${ }^{[37]}$ als auch Untersuchungen mit isotopenmarkierten Derivaten durch Daniel Krahn im Rahmen seiner Diplomarbeit, bestätigten die Regioselektivität der Transformationen. ${ }^{[241]}$ Dabei beobachtete Krahn auch, dass die Zugabe des $\mathrm{Sc}(\mathrm{OTf})_{3}$ Katalysators den Umsatz zwar beschleunigte (93\% nach $6 \mathrm{~h}$ ), jedoch keine Auswirkungen auf die Regioselektivität der Verseifung von 97 nach 192 hatte $\left(20 \%\right.$ nach $6 \mathrm{~h}$ ohne $\left.\mathrm{Sc}(\mathrm{OTf})_{3}\right) .{ }^{[241]}$ Der Einfluss des Sc-Katalysators blieb auch im Hinblick auf das folgende Ergebnis von Lu fraglich (Schema 4.7).

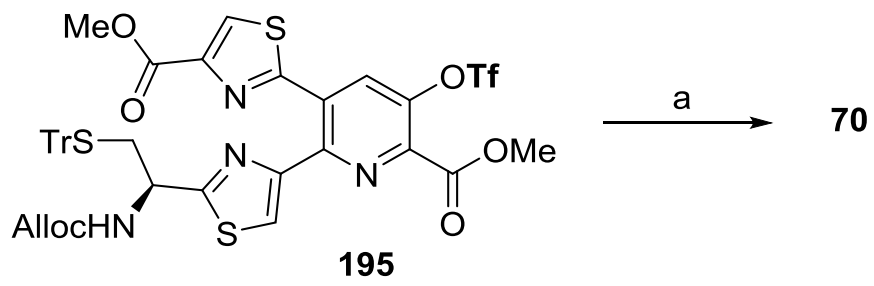

Schema 4.7: Umsetzung des Triflats 195 mit $n-\mathrm{Bu}_{4} \mathrm{OH}$. Bedingungen: a) $n$ - $\mathrm{Bu}_{4} \mathrm{OH}$ (40\%ig in $\mathrm{H}_{2} \mathrm{O}$, 4 Äq.), 1,4-Dioxan, RT, 40 min, 50\%. ${ }^{[37]}$

Die Umsetzung des Triflats 195 mit vier Äquivalenten $n$ - $\mathrm{Bu}_{4} \mathrm{OH}$ lieferte selektiv die entschützte 3-Hydroxypicolinsäure 70 nach kurzer Reaktionszeit, wenn auch mit geringer Ausbeute, bereits bei RT. Mit zwei Äquivalenten $n-\mathrm{Bu}_{4} \mathrm{OH}$ wurde hingegen selektiv nur das Triflat entfernt. ${ }^{[37]}$

\subsection{Fragestellung}

Im Hinblick auf die oben dargestellten, uneinheitlichen Ergebnisse zur katalytischen Aktivität des $\mathrm{Sc}(\mathrm{OTf})_{3}$ in der Hydrolyse, Umesterung sowie Aminolyse von 3-Hydroxypicolin- 
säureestern, sollte dessen Rolle genauer untersucht und die mechanistische Hypothese verfeinert werden.

\subsection{Ergebnisse und Diskussion}

\subsubsection{Untersuchungen zur regioselektiven Hydrolyse}

Eine Untersuchung der durch J.-Y. Lu entwickelten Methode zeigte, unter exakt kontrollierten Reaktionsbedingungen, keinen messbaren Einfluss des Sc-Salzes auf den Umsatz oder die Regioselektivität bei der Verseifung des Triesters 97 (Abbildung 4.1).<smiles>COC(=O)c1cc(O)c(C(=O)OC)nc1C(=O)OC</smiles>

97<smiles>COC(=O)c1cc(O)c(C(=O)O)nc1C(=O)O</smiles>

192

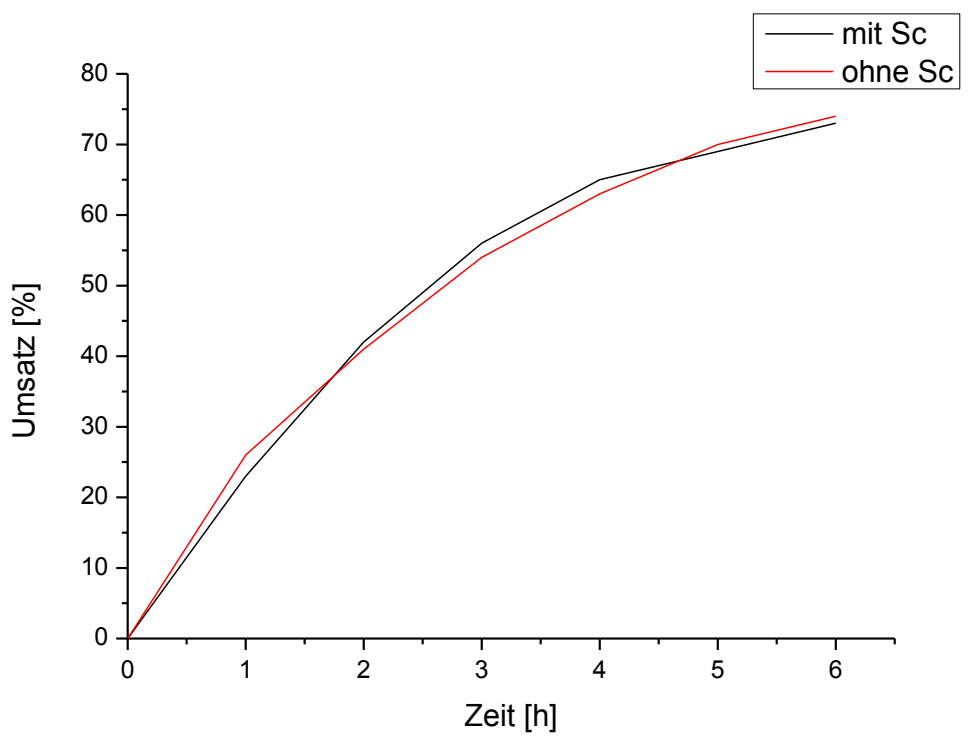

Abbildung 4.1: Graphische Darstellung des Umsatzes der Verseifung des Triesters 97 zur Säure 192 im zeitlichen Verlauf mit $\mathrm{Sc}(\mathrm{OTf})_{3}$ (schwarz) und ohne (rot). Die Kontrolle des Umsatzes erfolgte durch HPLC (C18, MeCN/ $\mathrm{H}_{2} \mathrm{O}$, Detektion bei $\left.254 \mathrm{~nm}\right)$.

Die von Krahn beobachtete Steigerung des Umsatzes in Anwesenheit des Sc-Salzes konnte nicht bestätigt werden. ${ }^{[241]}$ Sowohl für 3-Hydroxypicolinsäuremethylester als auch für das Bisthiazolylpyridin 96 wurden nahezu identische Ergebnisse erhalten. Diese Beobachtungen legen einen anderen Mechanismus für diese regioselektive Verseifung nahe, an dem das Sc- 
Salz nicht beteiligt ist. So konnte für die Umsetzung des Bisthiazolylpyridins 96 mit einer definierten Menge $\mathrm{NaHCO}_{3}$ eine sehr gute Ausbeute erzielt werden (Schema 4.8).
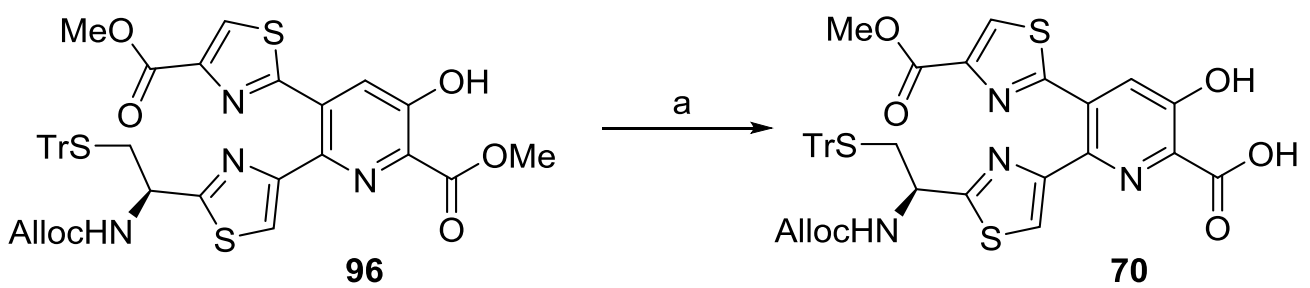

Schema 4.8: Regioselektive Hydrolyse des Bisthiazolylpyridins 96. Bedingungen: a) $\mathrm{NaHCO}_{3}$ (4 Äq.), 1,4-Dioxan $/ \mathrm{H}_{2} \mathrm{O}=2: 1,60^{\circ} \mathrm{C}, 8 \mathrm{~h}, 95 \%$ (Rohprodukt).

Es zeigte sich jedoch, dass die Picolinsäure 70 gegenüber einer Chromatographie an $\mathrm{SiO}_{2}$ nicht stabil war. Dabei wurde mehrfach eine Rotfärbung der Substanz beobachtet, die womöglich von einer Komplexierung der im Kieselgel befindlichen Fe $\mathrm{F}^{\mathrm{III}}$-Verunreinigungen stammen könnte. ${ }^{[241,242]}$ Die Bildung von stabilen Fe ${ }^{\text {III }}$-Komplexen ist für Salicylsäure bekannt und wurde als Nachweis verwendet. Dabei tritt eine Violettfärbung einer Fe ${ }^{\text {III }}$-Lösung in Gegenwart von Salicylsäure auf. ${ }^{[243]}$

\subsubsection{Untersuchungen zur regioselektiven Umesterung}

Die durch J.-Y. Lu beschriebene regioselektive Umesterung des Triesters 97 bedurfte, im Gegensatz zur Verseifung, keiner Base. Eine entsprechende Umesterung mit Ethanol in Abwesenheit von $\mathrm{Sc}(\mathrm{OTf})_{3}$ zeigte nur einen geringen Umsatz (Schema 4.9). Mit katalytischen Mengen $\mathrm{EtN} \mathrm{Pr}_{2}$ wurde jedoch eine regioselektive quantitative Ausbeute an Ethylester 196 erhalten. Unter Zugabe von Säuren (AcOH, TCE, TFA) wurde kein Umsatz festgestellt.<smiles>COC(=O)c1cc(O)c(C(=O)OC)nc1C(=O)OC</smiles>

97<smiles>CCOC(=O)c1nc(C(=O)OC)c(C(=O)OC)cc1O</smiles>

196

Schema 4.9: Versuche zur Umesterung des Triesters 97. Bedingungen: a) EtOH, kat. $\mathrm{H}_{2} \mathrm{O}, 60{ }^{\circ} \mathrm{C}$, 18 h, Umsatz ca. $30 \%$. b) EtNiPr 2 (10 mol\%), EtOH, $60{ }^{\circ} \mathrm{C}, 18$ h, quant.

Die Bandbreites dieser Basen-vermittelten Umesterung wurde durch Versuche mit weiteren Alkoholen untersucht (Tabelle 4.1). 
Tabelle 4.1: Regioselektive Umesterung des 3-Hydroxypicolinsäureesters 97.<smiles>COC(=O)c1cc(O)c(C(=O)OC)nc1C(=O)OC</smiles>

97<smiles>[R]OC(=O)c1nc(C(=O)OC)c(C(=O)OC)cc1O</smiles>

197-200

\begin{tabular}{ccc}
\hline & $\mathrm{R}$ & Ausbeute \\
\hline $\mathrm{a}$ & Allyl & $98 \%$ \\
$\mathrm{~b}$ & $i \mathrm{Pr}$ & $95 \%^{\mathrm{a}}$ \\
$\mathrm{c}$ & 2 -Hydroxyethyl & $84 \%$ \\
$\mathrm{~d}$ & Benzyl & $78 \%$ \\
$\mathrm{e}$ & $p$-Methoxybenzyl & Spuren $^{\mathrm{b}}$ \\
$\mathrm{f}$ & $o$-Nitrobenzyl & kein Umsatz \\
$\mathrm{g}$ & $t$ Bu & kein Umsatz \\
$\mathrm{h}$ & $\mathrm{Ph}$ & kein Umsatz $^{\mathrm{b}}$ \\
$\mathrm{i}$ & $D$-Glucose & kein Umsatz $^{\mathrm{c}}$ \\
\hline
\end{tabular}

[a] Durchgeführt bei $85^{\circ} \mathrm{C}$; [b] ROH/1,4-Dioxan $=1: 1$;

[c] $D$-Glucose (2 Äq.), 1,4-Dioxan $/ \mathrm{H}_{2} \mathrm{O}=1: 1$.

Bei der Umesterung mit Allylalkohol und iso-Propanol wurden sehr gute Ausbeuten erreicht (Einträge a (197) und b (198)). Die Umsetzung mit Glykol lieferte den Monoester 199 in guter Ausbeute, ohne Bildung von Dimeren mit der zweiten OH-Gruppe des Glykols (c). Bei Versuchen mit benzylischen Alkoholen (d-e) konnte nur der Benzylester 200 mit verminderter Effizienz erhalten werden. Phenol und tert-Butanol lieferten auch bei erhöhter Temperatur $\left(100{ }^{\circ} \mathrm{C}\right.$, Druckrohr) nicht die gewünschten Umesterungsprodukte (Einträge g-h). Dabei wurde, neben zurückgewonnenem Startmaterial, nur die Bildung des Hydrolyseprodukts 192 beobachtet. Mit $D$-Glucose wurde ebenfalls kein Umsatz beobachtet und somit die Einschränkungen dieser Methode deutlich (Eintrag i). Somit ist die Anwendbarkeit dieser Methode auf sehr polare Alkohole mit geringem aliphatischem Anteil anwendbar, die in der Lage sind, den 3-Hydroxypicolinsäureester in Lösung zu bringen und die nur in geringem Ausmaß sterisch gehindert sind.

Um die Regioselektivität der Basen-katalysierten Umesterung zu bestätigen wurde der Bistrideuteromethylester $\mathbf{9 7 - \boldsymbol { d } _ { \boldsymbol { \sigma } }}{ }^{[241]}$ mit Benzylalkohol umgesetzt (Schema 4.10). 
<smiles>[2H]C([2H])(C)OC(=O)c1cc(O)c(C(=O)OC)nc1C(=O)OC([2H])([2H])C</smiles>

$97-d_{6}$<smiles>CC(C)(C)OC(=O)c1cc(O)c(C(=O)OCc2ccccc2)nc1C(=O)OCc1ccccc1</smiles>

201

Schema 4.10: Regioselektive Umesterung des Bis-Trideutermethylesters 97- $\boldsymbol{d}_{\boldsymbol{6}}$. Bedingungen: a) EtNiPr 2 (10 mol\%), BnOH, $60{ }^{\circ} \mathrm{C}, 18 \mathrm{~h}, 84 \%$.

Im ${ }^{1}$ H-NMR-Spektrum des erhaltenen Produkts konnte kein Signal einer Methyl-Gruppe beobachtet werden. Die zwei Trideuteromethylgruppen waren jedoch klar im ${ }^{13} \mathrm{C}-\mathrm{NMR}$ Spektrum nachweisbar und auch HRMS-Spektren bestätigten die Summenformel des Benzylesters 201. Hieraus lässt sich schließen, dass die sechs D-Atome im Produkt vorhanden sein mussten und selektiv der an der phenolischen $\mathrm{OH}$-Gruppe benachbarte Methylester umgesetzt wurde.

Interessanterweise lieferte die Reaktion des Methylethers $\mathbf{9 8}^{[241]}$ mit $\mathrm{Sc}(\mathrm{OTf})_{3}$ den 2,6-Diethylester 203 in quantitativer Ausbeute (Schema 4.11).

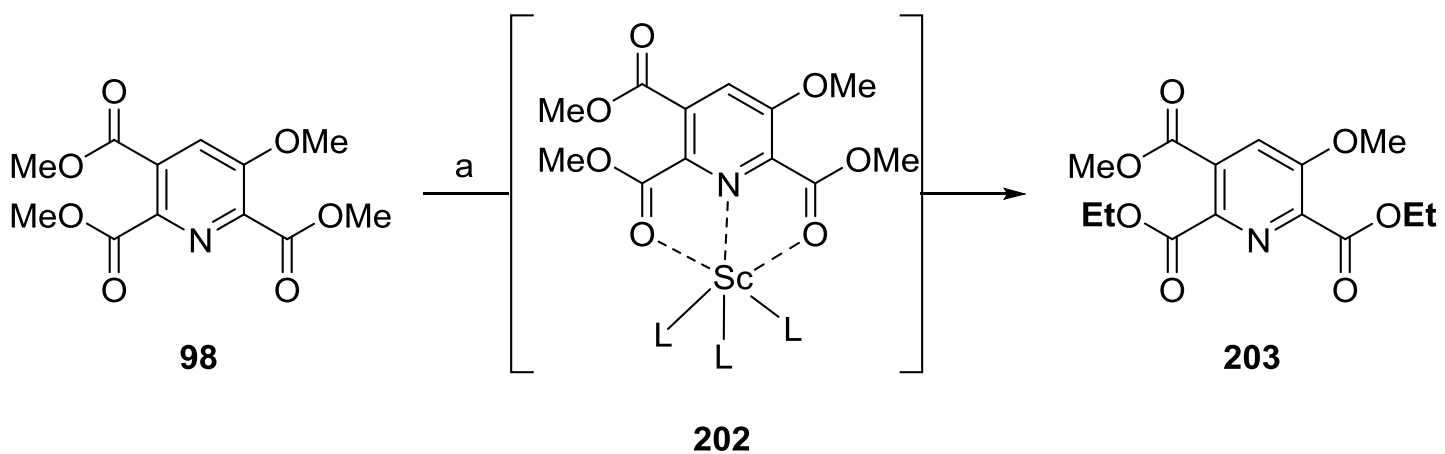

Schema 4.11: 2,6-selektive Umesterung des Methylethers 98 und mechanistische Hypothese. Bedinungen: a) $\mathrm{Sc}(\mathrm{OTf})_{3}(20 \mathrm{~mol} \%)$, EtOH, kat. $\mathrm{H}_{2} \mathrm{O}, 60^{\circ} \mathrm{C}, 18 \mathrm{~h}$, quant.; $\mathrm{L}=$ Ligand.

Die geänderte Selektivität deutet darauf hin, dass eine Beteiligung der phenolischen $\mathrm{OH}$ Gruppe in der Lewis-Säure-katalysierten Umesterung des 3-Hydroxypyridins 97 (Schema 4.6) vorliegt. Im Fall des Ethers 98 wurden womöglich durch eine tridentate Chelatisierung des Metall-Kations die beiden Estergruppen in den Positionen 2 und 6 aktiviert (202). 


\subsubsection{Untersuchungen zur regioselektiven Aminolyse}

Die Umsetzung des Brompyridins 204 mit zwei Äquivalenten $n$-Propylamin lieferte das entsprechende Amid 205 in guter Ausbeute, auch in Abwesenheit von $\operatorname{Sc}(\mathrm{OTf})_{3}$ (Schema 4.12).<smiles>COC(=O)c1nc(Br)ccc1O</smiles>

204<smiles>CCCC</smiles>

Schema 4.12: Aminolyse des Brompyridins 204. Bedingungen: a) $n$-Propylamin (2 Äq.), 1,4-Dioxan, RT, 18 h, 68\%; 66\% (zzgl. 10 mol\% EtNiPr ${ }_{2}$ ).

Eine Zugabe katalytischer Mengen an $\mathrm{EtN}_{i} \mathrm{Pr}_{2}$ führte jedoch zu keiner erhöhten Ausbeute an Amid 205.

Um die Anwendbarkeit der Methode zu untersuchen, wurde das Pyridin 210 mit einer heteroDiels-Alder-Reaktion synthetisiert und mit $n$-Propylamin umgesetzt (Schema 4.13). ${ }^{[163]}$<smiles>O=Cc1c[nH]cn1</smiles>

206<smiles>CON=C(C(=O)OC)C(=O)OC</smiles><smiles>O=Cc1cn(S)cn1</smiles>

207<smiles>CC#CC(O)c1cn(C)cn1</smiles>

208<smiles>C#CC(=O)c1cn(S)cn1</smiles>
209<smiles>CCCNC(=O)c1nc(C(=O)c2cnc[nH]2)ccc1O</smiles>

Schema 4.13: Synthese des Pyridins 211 via hetero-Diels-Alder-Chemie und anschließende Aminolyse. Bedingungen: a) $\mathrm{TsCl}, \mathrm{Et}_{3} \mathrm{~N}$, 1,4-Dioxan, THF, RT, $18 \mathrm{~h}, 96 \%$. b) Ethinylmagnesiumbromid, THF, $-20^{\circ} \mathrm{C} \rightarrow \mathrm{RT}, 18 \mathrm{~h}, 84 \%$. c) Dess-Martin-Periodinan, $\mathrm{NaHCO}_{3}, \mathrm{CH}_{2} \mathrm{Cl}_{2}, \mathrm{RT}$, 1.5 h, $91 \%$. d) 65, Mesitylen, $115^{\circ} \mathrm{C}, 15$ h, $50 \%$. e) $n$-Propylamin, 1,4-Dioxan, $60{ }^{\circ} \mathrm{C}, 5.5$ h, $72 \%$. 
Für die Synthese des Pyridins 210 wurde zuerst das Imidazol 206 mit einer guten Ausbeute von 96\% als Sulfonsäureamid geschützt. Die anschließende Umsetzung der Aldehyd-Funktion mit Ethinylmagnesiumbromid lieferte den Propargylalkohol 208 mit 84\% Ausbeute. Der Propargylalkohol 208 wurde danach mit Dess-Martin-Periodinan zum Keton 209 oxidiert (91\%). Die Umsetzung des Alkinylketons 209 mit dem 1-Azabutadien 65 in einer heteroDiels-Alder-Reaktion ergab regioselektiv das gewünschte 3-Hydroxypridin $\mathbf{2 1 0}$ in akzeptabler Ausbeute. ${ }^{[163]}$ Dabei zeigte sich, dass die Tosylgruppe unter den Reaktionbedingungen nicht stabil war. Die anschließende Aminolyse des Methylesters 210 mit $n$-Propylamin lieferte das Amid 211 in guter Ausbeute ohne eine nennenswerte IminBildung durch die Keto-Gruppe, auch bei erhöhter Temperatur. Dies konnte ebenfalls mit dem aus der Nosiheptid-Totalsynthese abgeleiteten 3-Hydroxypyridin $6 \mathbf{6}$ demonstriert werden (Schema 4.14). Dabei wurde das Amid 212 mit einer Ausbeute von 73\% erhalten.<smiles>COC(=O)c1csc(-c2nc(C(=O)OC)c(O)cc2C(C)=O)n1</smiles>

66<smiles>CCCNC(=O)c1nc(-c2nc(C(=O)OC)cs2)c(C(C)=O)cc1O</smiles>

212

Schema 4.14: Aminolyse des 3-Hydroxypyridins 66. Bedingungen: a) n-Propylamin, 1,4-Dioxan, $60^{\circ} \mathrm{C}, 5.5 \mathrm{~h}, 73 \%$.

\subsubsection{Mechanistische Überlegungen}

Bereits 1907 berichteten Goldschmidt und Scholz über eine Beschleunigung der Verseifung von Salicylsäuremethylester durch die freie phenolische OH-Gruppe. ${ }^{[244]}$ In späteren Arbeiten konnte dies für die Umesterung und Aminolyse von ungeschützten Salicylsäureestern ebenfalls gezeigt werden. ${ }^{[245-249]}$ Dabei wurden eine $\mathrm{pH}$-abhängige Beteiligung der benachbarten $\mathrm{OH}-\mathrm{Gruppe}$ am geschwindigkeitsbestimmenden Schritt bestätigt und mögliche Mechanismen durch kinetische Untersuchungen untermauert. Anwendung fand diese, für Katalysen prototypische Modellreaktion, jedoch erst in den vergangenen 15 Jahren. ${ }^{[250-254]}$ In frühen Berichten wurde für die Umsetzung von Salicylsäureestern in apolaren Medien eine intramolekulare Katalyse des Protons im nicht ionisierten Phenol A $\mathbf{A}_{\mathbf{1}}$ postuliert (Schema 4.15, Pfad A). ${ }^{[247]}$ Alternativ wurde für Transformationen in polar, protischen Lösungsmitteln die intramolekulare Assistenz des gebildeten Phenolats $\mathbf{B}_{\mathbf{1}}$ vorgeschlagen $(P f a d B) .{ }^{[248,249]}$ 


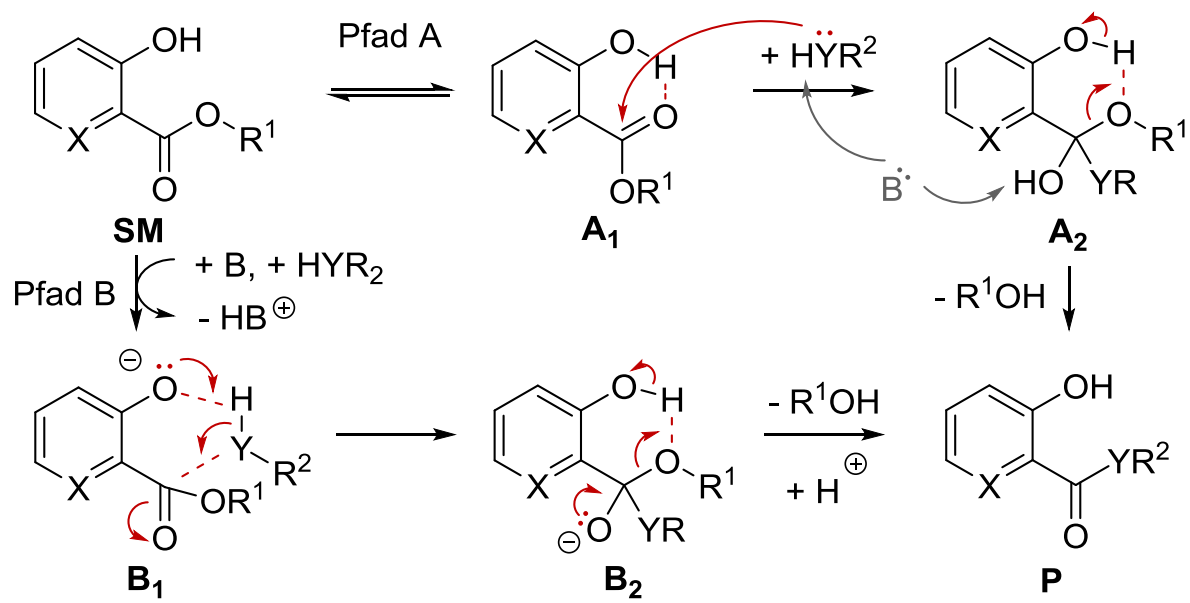

Schema 4.15: Mögliche intramolekulare Assistenz während der Transformation von Salicylsäurebzw. 3-Hydroxypicolinsäureestern (vereinfacht); $\mathbf{B}=$ externe Base, $\mathbf{S M}=$ Startmaterial, $\mathbf{P}=$ Produkt, $\mathrm{X}=\mathrm{CH}, \mathrm{N} ; \mathrm{Y}=\mathrm{O}, \mathrm{NH}$.

Während 3-Hydroxypyridin $\left(p K_{a} 8.7\right)^{[255]}$ ein wenig saurer als Salicylsäuremethylester $\left(p K_{a} 9.8\right)^{[256]}$ ist, so liegt dies im Bereich des $\mathrm{pH}-$ Werts des in der Hydrolyse verwendeten Mediums (Kap. 4.1.2). Es liegt somit nahe, dass die hier untersuchten 3-Hydroxypicolinsäureester ebenfalls zu einem phenolischen Anion $\mathbf{B}_{\mathbf{1}}$ deprotoniert werden könnten und eine ähnliche Aktivierung stattfindet. Das phenolische Anion könnte dabei während des nucleophilen Angriffs $\left(\mathbf{B}_{\mathbf{1}}\right)$ als intramolekulare Base dienen und anschließend durch Protonenübertragung den Zerfall des tetraedrischen Intermediats $\mathbf{B}_{\mathbf{2}}$ beschleunigen. Die bei einigen Substraten beobachtete Gelbfärbung während der Hydrolyse mit $\mathrm{NaHCO}_{3}$ deutet auf deren Ionisierung und den anionischen Pfad B hin. Da eine säurekatalysierte Umesterung nicht möglich war (s. oben), verlaufen die Umesterung sowie die Hydrolyse wahrscheinlich nicht über den Pfad A. Jedoch könnte auch der neutrale Pfad A von der Unterstützung einer externen Base in der Deprotonierung des Nucleophils $\left(\mathbf{A}_{\mathbf{1}}\right)$ oder in der Beschleunigung des potentiell geschwindigkeitsbestimmenden Zerfalls des neutralen tetraedrischen Intermediats A2 profitieren. ${ }^{[257]}$ Dieser Reaktionspfad kann daher für die, unter aprotischen Bedingungen stattfindende Aminolyse, derzeit nicht ausgeschlossen werden. In jedem Fall profitieren beide Reaktionspfade von einer freien phenolischen OH-Gruppe in ortho-Position als aktivierende Nachbargruppe, die die Regioselektivität der Transformation ermöglicht. 


\subsection{Fazit}

Es konnte gezeigt werden, dass sowohl die regioselektive Hydrolyse, als auch Umesterung und Aminolyse von 3-Hydroxypicolinsäureestern wahrscheinlich über eine dirigierend wirkende, intramolekulare Assistenz der in ortho-Stellung befindlichen $\mathrm{OH}$-Gruppe vermittelt werden. Dabei konnte eine Analogie zu den entsprechenden, und bereits gut untersuchten, Umsetzungen von Salicylsäureestern hergestellt und mögliche mechanistische Reaktionspfade postuliert werden. Diese Transformationen eröffnen neue Möglichkeiten sowohl für die Synthese polyfunktionaler Moleküle in der Medizinalchemie als auch für den Zugang zu neuartigen Pyridin-Donor-Liganden. In dieser Arbeit ermöglichte sie bereits die selektive Funktionalisierung des Nosiheptidkerns (Kap. 3.5). 

5 Synthese substituierter 3-Hydroxypicolinsäuren 


\subsection{Einleitung}

Im Jahre 1953 berichteten Maeda et al. über die Entdeckung einer Substanz mit signifikanter antimykobakterieller Aktivität, die aus einer Bodenprobe aus Tokio isoliert wurde. ${ }^{[180,181]}$ Die Struktur und absolute Konfiguration des als Pyridomycin (Abbildung 5.1, 99) bezeichneten Naturstoffs wurde jedoch erst 15 Jahre später durch Röntgenkristallstrukturanalyse und chemischen Abbau ermittelt. $^{[182,258]}$

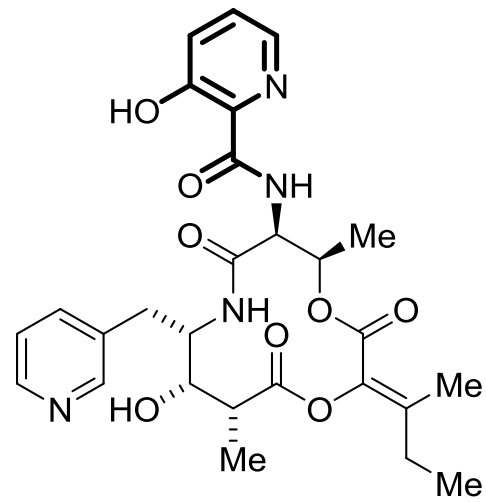

99: Pyridomycin

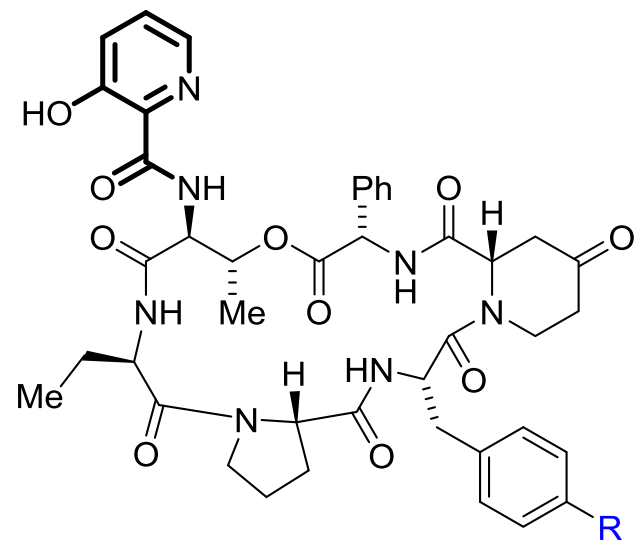

213: Virginiamycin $S_{1}(R=H)$

214: Pristinamycin $I_{A}\left(R=N\left(C_{3}\right)_{2}\right)$

Abbildung 5.1: Strukturen von Pyridomycin und der Gruppe B Streptogramine (rechts) mit hervorgehobener 3-Hydroxypicolinsäureeinheit.

Pyridomycin ist ein makrocyclisches Depsipeptid, dessen 12-gliedriger Makrocyclus einen Ester, einen Enolester sowie ein Amid enthält. Des Weiteren weist es fünf stereogene Zentren auf, von denen drei direkt benachbart sind. Neben einem über eine Methylengruppe verknüpften Pyridinring enthält Pyridomycin als charakteristisches Strukturmerkmal einen exocyclisch am $L$-Thr-Stickstoff angebrachten 3-Hydroxypicolinsäurerest (hervorgehoben). Diese ungewöhnliche Einheit findet sich auch bei den Streptogramin-Antibiotika der Gruppe B (Abbildung 5.1, rechts), ${ }^{[259]}$ ansonsten ist dieses Strukturmotiv sehr selten.

Die Biosynthese von Pyridomycin verläuft wahrscheinlich über die Kombination einer Polyketidsynthetase mit einer nichtribosomalen Peptidsynthetase. ${ }^{[260]}$ Die erste Totalsynthese von Pyridomycin wurde 1989 von Kinoshita et al. beschrieben. ${ }^{[261]}$ Die stereoselektive Darstellung der exocyclischen $(Z)$-sec-Butyliden-Einheit erwies sich dabei als eine besondere Herausforderung. Den Autoren gelang der Aufbau durch Einführung der Methylgruppe der Doppelbindung durch 1,4-Addition mit $\left(\mathrm{Me}_{2} \mathrm{Cu}\right) \mathrm{Li}$ an einen Enol-Phosphat-Ester. ${ }^{[261]}$ 


\subsubsection{Biologische Aktivität}

Pyridomycin zeigt eine hohe Spezifizität gegenüber Bakterien der Gattung Mycobacterium. Für das humanpathogene M. tuberculosis wurde eine minimale Hemm-Konzentration von $0.39 \mu \mathrm{g} / \mathrm{mL}$ bestimmt. ${ }^{[262]}$ Interessanterweise deuten zusätzliche Ergebnisse auf keine vorhandene Aktivität gegenüber anderen Gram-positiven Bakteriengattungen hin. Die biologische Zielstruktur wurde 2012 durch die Gruppe von Cole mittels Untersuchungen an resistenten Mutanten aufgeklärt. ${ }^{[262]}$ Dabei konnten die Autoren zeigen, dass Pyridomycin kompetitiv an der NADH-abhängigen Enoyl-Acyl-Carrier-Protein-Reduktase InhA bindet und somit die Synthese von Mykolsäuren stört. Diese sind wesentlicher Bestandteil der Zellwand von Mykobakterien. ${ }^{[263]}$ Bei einer Überlagerung der Strukturen des InhA-PyridomycinKomplexes mit der des InhA-Substrat-NADH-Komplexes (Abbildung 5.2) wird ersichtlich, dass Pyridomycin (gelb) sowohl in der Bindungstasche des Substrats (Fettsäure, blau) als auch in der des Coenzyms (NADH, rot) bindet. ${ }^{[264]}$
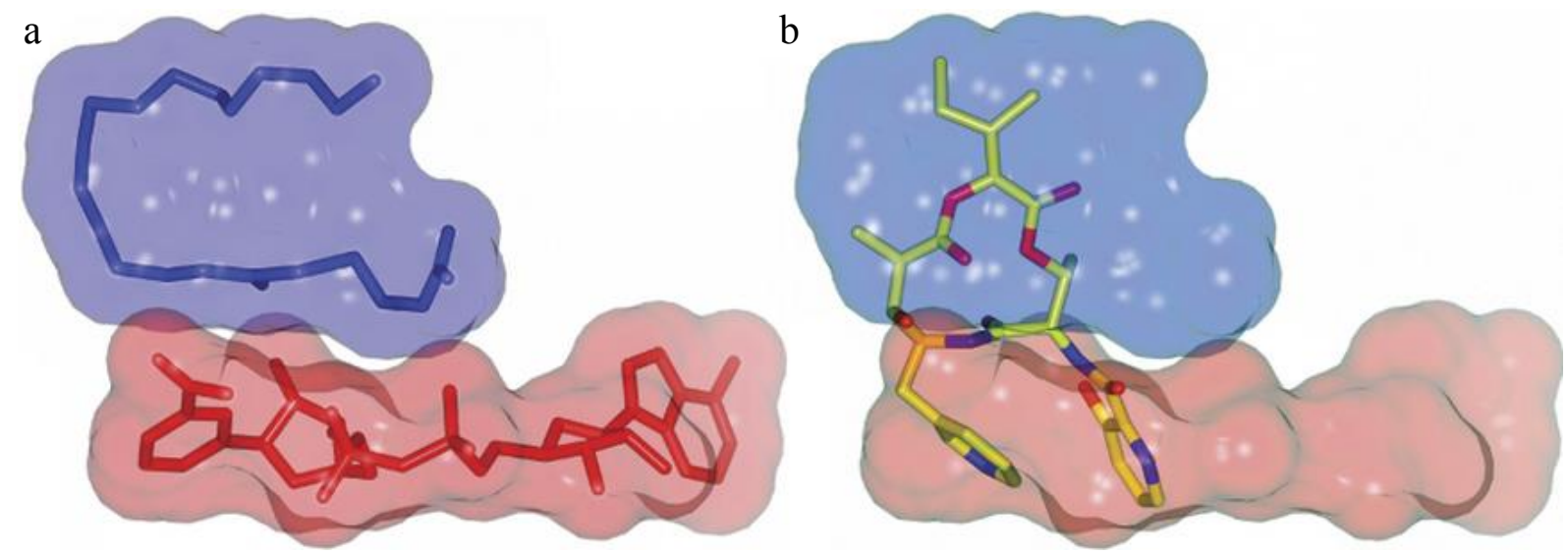

Abbildung 5.2: Vergleich der Bindungstaschen von Substrat und Coenzym mit Pyridomycin in InhA.

a) Darstellung der Oberflächen von NADH (rotes Molekül und Oberfläche) und trans-2Hexadecanoyl- $N$-acetylcysteaminthioester (blaues Molekül und Oberfläche). b) Eine Überlagerung mit Pyridomycin (gelb) zeigt, dass der Inhibitor in beiden Bindungstaschen bindet. Adaptiert nach Cole et al. ${ }^{[264]}$

Damit ist Pyridomycin mit seinem einzigartigen Bindungsmodus, das einzige Beispiel für einen Inhibitor, der effektiv die NADH-Bindung blockiert, jedoch kein NADH-Mimetikum ist. ${ }^{[264]}$ Trotz seiner hohen in vitro-Aktivität zeigte die Substanz in Tiermodellen keine Aktivität, wofür die schlechten pharmakokinetischen Eigenschaften der Substanz verantwortlich gemacht wurden. ${ }^{[265]}$ Ein wesentliches Motiv der Forschung im Feld ist daher eine Verbesserung dieser Eigenschaften durch Semisynthese. 


\subsection{Syntheseziel}

Für Untersuchungen zu Struktur-Aktivitäts-Beziehungen (SAR-Studien), die in Zusammenarbeit mit Prof. Dr. K.-H. Altmann von der ETH Zürich bearbeitet wurden, sollten substituierte 3-Hydroxypicolinsäuren (215) synthetisiert werden, um semisynthetische Derivate von Pyridomycin erhalten $\mathrm{zu}$ können. Ausgehend von 3-Hydroxypicolinsäuremethylester (100) sollte jede freie Position des Aromaten $\left(\mathrm{H}^{4}\right.$ bis $\left.\mathrm{H}^{6}\right)$ selektiv adressiert werden (Schema 5.1).<smiles>O=C(O)C1=C(O)C=C[Se]C=N1</smiles>

215<smiles>C=CCC</smiles>

Schema 5.1: Substituierte 3-Hydroxypicolinsäuren als Syntheseziel, ausgehend von 3-Hydroxypicolinsäuremethylester (100).

Das Anbringen der 3-Hydroxypicolinsäuren am Pyridomycin-Grundkörper und die anschließende Testierung der Naturstoffderivate waren an der ETH Zürich vorgesehen, wo die Gruppe von Altmann eine Methode zum Austausch der Hydroxypicolinsäure entwickelt hat. ${ }^{[266]}$ Ausgehend von den so erhobenen SAR-Daten könnte eine gezielte Verbesserung der Eigenschaften angegangen werden.

\subsection{Substitution in Position 6}

\subsubsection{Synthese via Palladium-katalysierter Kreuzkupplungen}

Literaturbekannt war 6-Brom-3-hydroxypicolinsäuremethylester (204). ${ }^{[267]}$ Aus dieser Verbindung wurden mit einer Suzuki-Miyaura-Reaktion die in Schema 5.2 gezeigten 3-Hydroxypicolinsäuremethylester-Derivate dargestellt. Als Kupplungspartner dienten die entsprechenden (hetero)aromatischen Boronsäuren bzw. deren Pinakolester, die mit wasserfreiem CsF aktiviert wurden. $\mathrm{Pd}\left(\mathrm{PPh}_{3}\right)_{4}$ wurde dabei als Katalysator eingesetzt. Die Durchführung in einem Mikrowellenreaktor ermöglichte eine erhöhte Temperatur von $150{ }^{\circ} \mathrm{C}$ und eine Reaktionszeit von nur 20 min. Das Schützen der phenolischen OH-Gruppe war dabei nicht nötig. Die Ausbeuten lagen zwischen 29\% und 87\%. Als Nebenreaktion wurde die 
Verseifung des Methylesters beobachtet, die jedoch durch strikt wasserfreie Reaktionsbedingungen minimiert werden konnte.

Für die SAR-Untersuchungen wurden vornehmlich heteroaromatische Reste eingeführt. Neben (un)substituierten Phenylresten (a-e) konnten auch benzannelierte Heterocyclen (f-i, $u$ ) gekoppelt werden. Als 6-Ring Heteroaromat wurde Pyridyl in 3'- und 4'-Position angebracht (j, k). Weiterhin wurden mit (un)substituierten Thienyl- (l-n), Furanyl- (o-q), Pyrrolyl- (r) und Pyrazolylresten $(\mathrm{s}, \mathrm{t})$ überwiegend 5-Ring Heterocyclen eingeführt. Das Vorhandensein von Acyl- (d, n) oder Formyl-Substituenten (q) im Heteroaromaten stellte kein Hindernis dar. Im Fall des 5-Pyrrolyl-Substituenten (s) wurde die Suzuki-Miyaura-Kupplung mit dem N-THPgeschützten Boronsäureester ${ }^{[268]}$ durchgeführt. Das erhaltene Produkt ließ sich erst nach saurer Abspaltung der THP-Schutzgruppe effektiv reinigen. 
<smiles>[X]OC(=O)c1nc(Br)ccc1O</smiles>

204

216a-u<smiles>[Y]c1ccccc1</smiles>

216a, $81 \%(b)$<smiles>CC(=O)c1cccc(C)c1</smiles>

216d, $81 \%$ (a)<smiles>[3H]c1cc2ccccc2o1</smiles>

$216 \mathrm{~g}, 82 \%(a)$<smiles>Cc1cccnc1</smiles>

216j, 48\% (a)<smiles>[X]c1ccsc1</smiles>

216m, 75\% (a)<smiles>Cc1ccoc1</smiles>

216p, 69\% (a)<smiles>CC(C)(C)[C]1NC=NN1</smiles>

216s, $64 \%(b)^{a}$<smiles>COc1ccc(C)cc1</smiles>

216b, $87 \%$ (a)<smiles>[Y]c1ccc(-c2ccccc2)cc1</smiles>

$216 e, 59 \%(a)$<smiles>Fc1cc2ccccc2[nH]1</smiles>

216h, $48 \%$ (b)<smiles>CC(C)(C)c1ccncc1</smiles>

216k, 55\% (b)<smiles>CC(=O)c1ccc(C(C)(C)C)s1</smiles>

216n, 76\% (a)<smiles>CC(C)(C)c1ccc(C=O)o1</smiles>

216q, $63 \%$ (a)<smiles>[X]c1cn[nH]c1</smiles>

216t, 62\% (b)<smiles>COc1ccccc1C</smiles>

216c, $52 \%$ (b)<smiles>[Y]C1(C)Cc2ccccc2S1</smiles>

216f, $87 \%$ (a)<smiles>CC(C)(C)c1c[nH]c2ccccc12</smiles>

216i, $52 \%$ (b)<smiles>CC(C)(C)c1cccs1</smiles>

216I, 61\% (b)<smiles>CC(C)(C)c1ccco1</smiles>

2160, 85\% (a)<smiles>Cc1cc[nH]c1</smiles>

$216 r, 56 \%$ (b)<smiles>CC(C)(N)c1cccc2cccnc12</smiles>

216u, 29\% (a)

Schema 5.2: Synthese 6-substituierter 3-Hydroxypicolinsäuremethylester via Suzuki-MiyauraKreuzkupplung. Bedingungen: (Het)aryl-B(OH) 2 (a) o. (Het)aryl-Bpin (b), CsF (trocken), $\mathrm{Pd}\left(\mathrm{PPh}_{3}\right)_{4}$, $\mathrm{MeOH}$, DME (!), $20 \mathrm{~min}, 150{ }^{\circ} \mathrm{C}$, Mikrowellenreaktor. ${ }^{a}$ Suzuki-Kupplung mit 1-(Tetrahydro- $2 \mathrm{H}$ pyran-2-yl)-1H-pyrazol-5-boronsäurepinakolester ${ }^{[268]}$, Ausbeute nach Abspaltung der THPSchutzgruppe mit $\mathrm{HCl}(1.25 \mathrm{M}$ in EtOH). 
Die Einführung eines Imidazol-4-yl-Substituenten sollte in einer Stille-Kupplung mit dem literaturbekannten Zinnorganyl 217 $7^{[269]}$ erfolgen (Schema 5.3).<smiles>COC(=O)c1nc(Br)ccc1O</smiles>

204<smiles>CCC[AsH2+]c1cn([In])cn1</smiles>

217

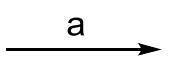

$\operatorname{Tr}^{\prime}$<smiles>COC(=O)c1nc(-c2cn(C(F)F)cn2)ccc1O</smiles>

218

Schema 5.3: Stille-Kupplung mit Zinnorganyl 217. Bedingungen: a) $\operatorname{Pd}\left(\mathrm{PPh}_{3}\right)_{4}$, Toluol, Rückfluss, $18 \mathrm{~h}, 75 \%$.

Das gewünschte Kupplungsprodukt 218 konnte in einer Ausbeute von 75\% erhalten werden. Dabei war eine mehrmalige Reinigung durch Säulenchromatographie nötig, da sich die in der Reaktion entstandenen Zinn-Nebenprodukte nur schwer abtrennen ließen. Aufgrund dieses Sachverhalts und der Giftigkeit von Zinnorganylen sollte vom Gebrauch einer StilleKupplung zur Darstellung substituierter 3-Hydroxypicolinate abgesehen werden.

\subsubsection{Synthese via Kondensations- und Cycloadditionsreaktionen}

Zwei weitere heterocyclische Substituenten wurden über mehrstufige Synthesen eingeführt. Der erste Schritt der Synthese eines 1,2,4-Oxadiazol-Substituenten war die Umsetzung des 6-Brompyridins 204 mit CuCN (Schema 5.4).<smiles>COC(=O)c1nc(Br)ccc1O</smiles>

204<smiles>[R]OC(=O)c1nc([R])ccc1O</smiles>

$219 \mathrm{R}=\mathrm{CN}$

$220 \mathrm{R}=\mathrm{C}(\mathrm{NH}) \mathrm{NHOH}$<smiles>Cc1nc(-c2ccc(O)c(C(=O)O)n2)no1</smiles>

221

Schema 5.4: Synthese der 1,2,4-Oxadiazol-substituierten Picolinsäure 221. Bedingungen: a) $\mathrm{CuCN}$, DMF, $150{ }^{\circ} \mathrm{C}, 1 \mathrm{~h}, 70 \%$. b) $\mathrm{HONH}_{3} \mathrm{Cl}, \mathrm{NaHCO}_{3}, \mathrm{MeOH}, 65^{\circ} \mathrm{C}, 2 \mathrm{~h}, 85 \%$. c) AcCl, Pyridin, $115^{\circ} \mathrm{C}$, 3 h. d) $\mathrm{LiOH} \times \mathrm{H}_{2} \mathrm{O}, \mathrm{THF} / \mathrm{H}_{2} \mathrm{O}, \mathrm{RT}, 20$ h, $80 \%$ (ü. 2 Stufen).

Das Nitril 219 wurde dabei mit einer guten Ausbeute von 70\% erhalten und anschließend mit Hydroxylamin und einer Ausbeute von $85 \%$ in das $N$-Hydroxyamidin 220 überführt. Dieses wurde mit einem Überschuss Acetylchlorid in Pyridin $\mathrm{zu}$ einem 1,2,4-Oxadiazol kondensiert. $^{[270]}$ Bei dieser Umsetzung wurde eine $O$-Acylierung der 3-Hydroxyfunktion 
beobachtet. Daher wurde das nach der Kondensation erhaltene Rohprodukt direkt mit LiOH umgesetzt und die Picolinsäure 221 mit einer Ausbeute von 80\% über zwei Stufen erhalten.

Das Nitril 219 könnte auch zur Synthese weiterer heterocyclischer Substituenten, wie Imidazolen, ${ }^{[271]} 1,2,4-$ Triazolen, ${ }^{[272]}$ Tetrazolen ${ }^{[273]}$ oder Thiazolen, ${ }^{[274]}$ genutzt werden. ${ }^{[275]}$

Die Einführung eines 1,2,3-Triazol-5-yl-Substituenten gelang über eine [3+2]-Cycloaddition (Schema 5.5). Dazu wurde das Bromid 204 in einer Sonogashira-Reaktion ${ }^{[276]}$ mit einer sehr guten Ausbeute von 95\% in das TMS-geschützte Alkin 222 überführt. Nach Entfernen der TMS-Gruppe mit KF wurde das Alkin 223 in einer $\mathrm{Cu}(\mathrm{I})$-assistierten Huisgen-[2+3]Cycloaddition mit TMS-N 3 zum $1 H-1,2,3-$ Triazol 224 umgesetzt. ${ }^{[277]}$<smiles>COC(=O)c1nc(Br)ccc1O</smiles><smiles>[R]C#Cc1ccc(O)c(C(=O)OC)n1</smiles><smiles>CC(C)(C)C</smiles><smiles>COC(=O)c1nc(-c2c[nH]nn2)ccc1O</smiles>

224

Schema 5.5: Einführung des (1H-1,2,3-triazol-5-yl)-Substituenten. Bedingungen: a) TMS-Acetylen, $\mathrm{Et}_{3} \mathrm{~N}, \mathrm{CuI}, \mathrm{Pd}\left(\mathrm{PPh}_{3}\right)_{2} \mathrm{Cl}_{2}$, RT, 24 h, 95\%. b) KF, MeOH, THF, RT, 1.5 h, 86\%. c) TMS-N 3 , CuI, MeOH, DMF, $100{ }^{\circ} \mathrm{C}, 18 \mathrm{~h}, 64 \%$.

\subsubsection{Darstellung der Picolinsäuren}

Eine Auswahl von Estern wurde anschließend über eine milde nachbargruppenassistierte Verseifung (Kap. 4) in die entsprechende Carbonsäure überführt (Schema 5.6) 


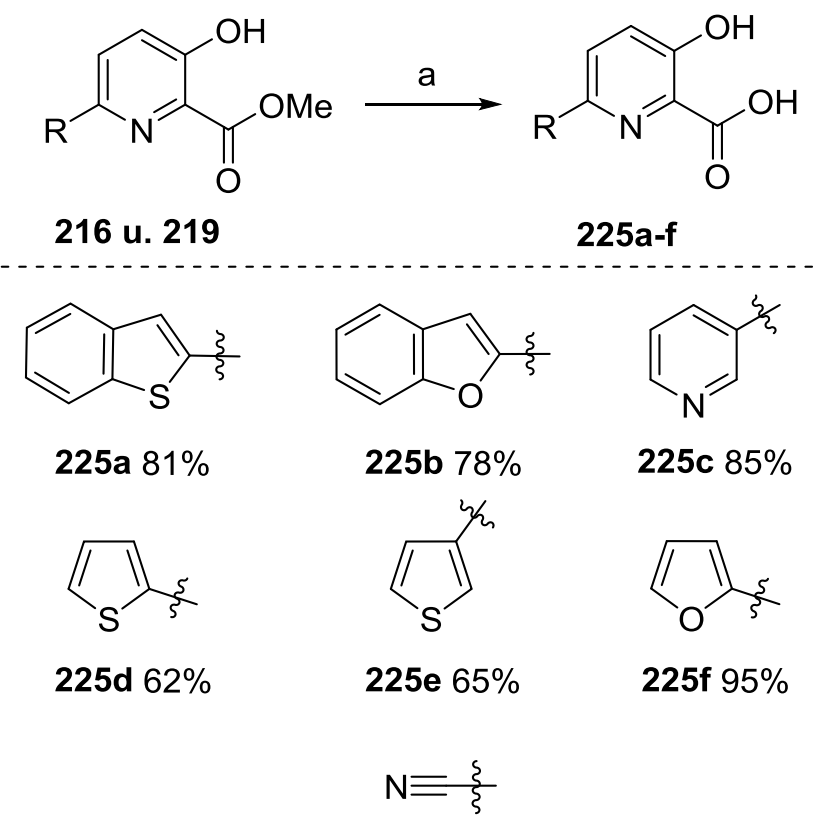

$225999 \%$

Schema 5.6: Verseifung ausgewählter Ester zu den entsprechenden Carbonsäuren. Bedingungen: a) $\mathrm{KHCO}_{3}, 1,4-$ Dioxan $/ \mathrm{H}_{2} \mathrm{O}, 60{ }^{\circ} \mathrm{C}, 18 \mathrm{~h}$.

Die 3-Hydroxypicolinsäuren konnten in guten bis sehr guten Ausbeuten isoliert werden. Aufgrund der beobachteten Instabilität von 3-Hydroxypciolinsäuren gegenüber Kieselgel (Kap. 4), erfolgte eine Reinigung der Produkte zum Teil durch Umkristallisation.

\subsection{Substitution in Position 4}

Eine Methode zur selektiven Hydrodehalogenierung des $O$-Benzyl geschützten Dibromids 226 war bereits von Renard et al. beschrieben worden (Schema 5.7). ${ }^{[278]}$<smiles>COC(=O)c1nc(Br)cc(Br)c1OCc1ccccc1</smiles>

226<smiles>COC(=O)c1nccc(Br)c1OCc1ccccc1</smiles>

227

Schema 5.7: Selektive Hydrodehalogenierung nach Renard et al. Bedingungen: a) $\mathrm{HCO}_{2} \mathrm{Na}$, $\mathrm{Pd}\left(\mathrm{PPh}_{3}\right)_{4}, \mathrm{DMF}, 80^{\circ} \mathrm{C}, 20 \mathrm{~h}, 70 \% .^{[278]}$ 
Als Hydrid-Quelle diente dabei Natriumformiat. ${ }^{[279]}$ Das erhaltene Monobromid 227 wurde durch die Autoren in weiteren Pd-katalysierten Reaktionen wie Suzuki-Miyaura, BuchwaldHartwig-Aminierung ${ }^{[280]}$ und Sonogashira ${ }^{[276]}$ erfolgreich umgesetzt. ${ }^{[278]}$ Bei einem Versuch, diese Reaktion nachzuvollziehen, wurde eine Ausbeute von 45\% an mono-bromierten Pyridin 227 erhalten. 3-Benzyl-geschützte 3-Hydroxypyridine lassen sich allerding nur schlecht entschützen.

Zurückgreifend auf diese Ergebnisse wurde daher versucht, die Benzylgruppe durch die leichter spaltbare Methoxymethyl-Gruppe zu ersetzen (Schema 5.8).<smiles>COC(=O)c1nc(Br)cc(Br)c1OCC(C)(C)C</smiles>

Schema 5.8: Synthese von 4-Brom-3-hydroxypicolinsäure (231). Bedingungen: a) MOMCl, EtNiPr${ }_{2}$, $\mathrm{CH}_{2} \mathrm{Cl}_{2}, 0^{\circ} \mathrm{C} \rightarrow \mathrm{RT}, 20 \mathrm{~h}, 87 \%$. b) $\mathrm{HCO}_{2} \mathrm{Na}, \mathrm{Pd}\left(\mathrm{PPh}_{3}\right)_{4}, \mathrm{DMF}, 80^{\circ} \mathrm{C}, 20$ h. c) TFA, $\mathrm{CH}_{2} \mathrm{Cl}_{2}$, RT, 20 min, $22 \%$ (ü. 2 Stufen). d) $\mathrm{LiOH} \times \mathrm{H}_{2} \mathrm{O}, \mathrm{THF} / \mathrm{H}_{2} \mathrm{O}, \mathrm{RT}, 24 \mathrm{~h}, 87 \%$.

Nach Einführung der MOM-Gruppe (87\%) wurde die Umsetzung des Dibromids 229 unter den publizierten Bedingungen zum Monobromid untersucht. Dabei zeigte sich jedoch eine hohe Labilität der MOM-Gruppe und es wurde ein komplexes Produktgemisch erhalten. Das gewünschte Monobromid konnte erst nach Entschützung der MOM-Gruppe in reiner Form mit einer Ausbeute von nur 22\% über zwei Stufen isoliert werden. Versuche mit anderen Reagenzien zur selektiven Hydrodehalogenierung, wie $\mathrm{Et}_{3} \mathrm{SiH} / \mathrm{Pd}_{(}\left(\mathrm{PPh}_{3}\right)_{2} \mathrm{Cl}_{2}{ }^{[281]}$ oder $\mathrm{NaBH}_{4} /$ TMEDA und verschiedenen Pd-Katalysatoren, ${ }^{[282]}$ zeigten niedrige Umsätze oder eine geringe Selektivität.

Aufgrund der unbefriedigenden Ausbeute von nur 22\% an Monobromid 230 wurde dieser Ansatz nicht weiter verfolgt. Zur Überprüfung, ob ein Substituent in 4-Position bei SARStudien generell toleriert wird, wurde der erhaltene Methylester 230 zur Picolinsäure 231 umgesetzt. 


\subsection{Substitution in Position 5}

Basierend auf Literaturbeispielen zur meta-selektiven Borylierung von Pyridinen ${ }^{\text {[283-287] }}$ mittels Ir-katalysierter C-H-Aktivierung ${ }^{[288-290]}$ wurde versucht, auf diese Weise auch die 5-Position in 3-Hydroxypicolinsäure selektiv zu substituieren (Schema 5.9).<smiles>COC(=O)c1ncccc1O</smiles>

100<smiles>COC(=O)c1ncccc1OC</smiles>

101<smiles>COC(=O)c1ncc(B2OC(C)(C)C(C)(C)O2)cc1OC</smiles>

232

Schema 5.9: Selektive Borylierung an Position 5 mittels $\mathrm{C}-\mathrm{H}-A k t i v i e r u n g$. Bedingungen: a) $\mathrm{MOMCl}$, EtNiPr $2, \mathrm{CH}_{2} \mathrm{Cl}_{2}, 0{ }^{\circ} \mathrm{C} \rightarrow \mathrm{RT}, 20 \mathrm{~h}, 98 \%$. b) $\mathrm{B}_{2} \mathrm{pin}_{2}$, $\operatorname{Ir}(\mathrm{OMe})(1,5-\mathrm{COD})_{2}$, dtbpy, THF, $50{ }^{\circ} \mathrm{C}, 20 \mathrm{~h}$.

Ausgehend vom Methylester 100 wurde die 3-Hydroxyfunktion mit der leicht spaltbaren MOM-Gruppe mit einer sehr guten Ausbeute von 98\% geschützt. Die anschließende Reaktion mit Bispinacolatodiboran, einer Ir(I)-Quelle und 4,4'-Di-tert-butyl-2,2'-dipyridyl (dtbpy) als Liganden, zeigte in der GC-MS eine saubere Umsetzung zu einem mono-borylierten Produkt. Bei anschließender Säulenchromatographie an Kieselgel wurde jedoch eine hohe Instabilität des Boronsäureesters beobachtet. ${ }^{[184]}$ Ein ${ }^{1}$ H-NMR-Spektrum des Produkts nach Chromatographie ist in Abbildung 5.3 gezeigt. 


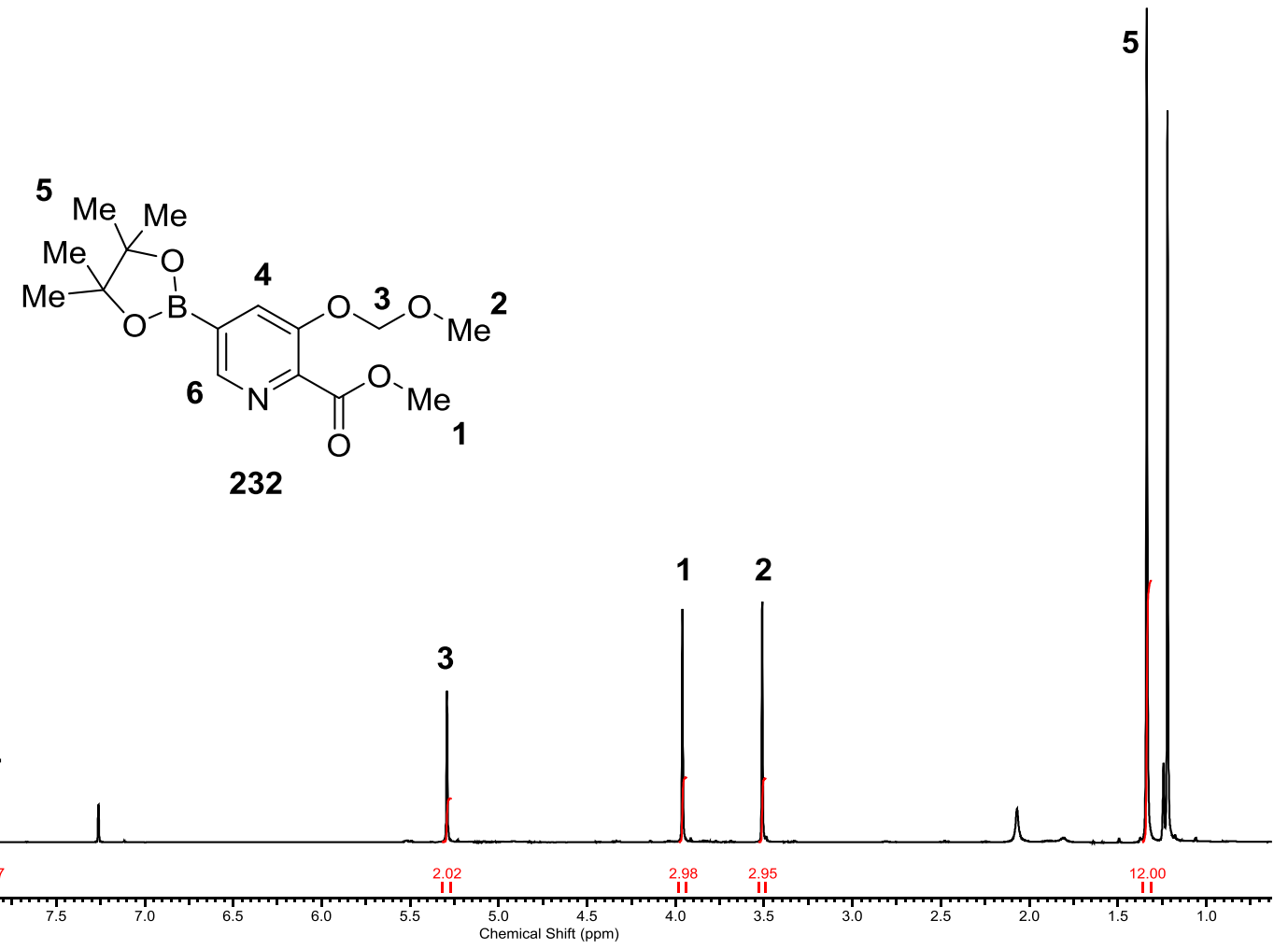

Abbildung 5.3: ${ }^{1}$ H-NMR-Spektrum des borylierten Pyridins 232 nach Säulenchromatographie.

Im ${ }^{1}$ H-NMR-Spektrum lassen sich alle Protonen des vorhergesagten Produkts eindeutig zuordnen. Die Singulets des Pyridin-Rings deuten darauf hin, dass die Protonen nicht benachbart sind und somit eine Substitution in Position 5 stattgefunden hat. Auch die Integrale der Signale stimmen mit den Erwartungen überein. Die Verunreinigung im aliphatischen Bereich ist Pinakol, das wahrscheinlich während der Chromatographie durch Umesterung des Boronsäureesters mit den $\mathrm{OH}-$ Gruppen des Silikagels freigesetzt wurde. Die Selektivität der C-H-Borylierung wurde durch Harwig et al. ausführlich untersucht. ${ }^{[184]}$ Sie basiert wahrscheinlich auf einer Kombination aus einem energetisch höherliegenden Reaktionspfad für die Borylierung in direkter Nachbarschaft zum N-Atom, in Relation zu Borylierungen an anderen Positionen sowie einer Instabilität der am basischen Stickstoff benachbarten borylierten Produkte. Im vorliegenden Anwendungsfall wird eine Borylierung in Position 4 wahrscheinlich aufgrund von sterischen Aspekten unterbunden. ${ }^{[184]}$

Der Verlust an Boronsäureester konnte mittels schneller Filtration des Rohprodukts durch eine dünne Kieselgel-Schicht minimiert werden. Über eine anschließende Suzuki-MiyauraReaktion mit Iodbenzol wurde das 5-substituierte Pyridin 233 erhalten (Schema 5.10). 
<smiles>COC(=O)c1ncccc1OC</smiles>

101<smiles>COC(=O)c1ncc(-c2ccccc2)cc1OC</smiles>

233<smiles>C#CC#CC#C</smiles>

234

Schema 5.10: Synthese des 5-Phenyl-substituierten Pyridins 233. Bedingungen: a) $B_{2} \operatorname{pin}_{2}$, $\operatorname{Ir}(\mathrm{OMe})(1,5-\mathrm{COD})_{2}$, dtbpy, THF, $50{ }^{\circ} \mathrm{C}, 20 \mathrm{~h}$. b) Iodbenzol, $\mathrm{K}_{2} \mathrm{CO}_{3}, \mathrm{Pd}\left(\mathrm{PPh}_{3}\right)_{4}, \mathrm{DMF}, 50{ }^{\circ} \mathrm{C}, 23 \mathrm{~h}$, $20 \%$ (ü. 2 Stufen).

Die Ausbeute an Pyridin 233 lag jedoch bei nur 20\% über zwei Stufen und bedarf weiterer Optimierung. Dies könnte möglicherweise durch eine Eintopfreaktion realisiert werden. ${ }^{[184]}$ Die weitere Umsetzung zur 3-Hydroxypicolinsäure 234 wurde nicht durchgeführt, sollte aber in zwei einfachen Schritten möglich sein. Versuche den Boronsäureester mittels bekannter Methoden in eine $\mathrm{OH}-$ Gruppe $^{[291]}$ oder ein Bromid $^{[284]} \mathrm{zu}$ überführen gelangen nicht. Ein Austausch der labilen MOM-Schutzgruppe sollte hierfür in Erwägung gezogen werden.

\subsection{Fazit}

Für eine Kooperation mit Prof. Dr. K.-H. Altmann wurden unterschiedlich substituierte 3-Hydroxypicolinsäurederivate dargestellt. Es gelang, ausgehend von 3-Hydroxypicolinsäuremethylester mittels Bromierung, Hydrodehalogenierung sowie Borylierung via C-H-Aktivierung, selektiv jeweils eine der freien aromatischen Positionen am Ring zu substituieren. Anschließende Reaktionen erlaubten die Einführung (hetero)aromatischer Substituenten. Der Schwerpunkt lag auf 6-substituierten 3-Hydroxypicolinsäuren, da diese einfach und ohne aufwendige Schutzgruppenchemie darstellbar sind. Eine Ausweitung der Arbeit auf weitere 4- und 5- substituierte Derivate sollte nach dem Erhalt erster SAR-Daten erfolgen.

Darüber hinaus könnte die hier gezeigte selektive Manipulation der aromatischen Positionen des 3-Hydroxpyridin-Grundkörpers ein Ansatzpunkt für eine neuartige Synthese des 3-Hydroxypyridin-Kerns der in Kapitel 1.3 gezeigten Thiopeptidantibiotika der Klasse E sein. ${ }^{[292]}$ 

6 Synthese tetradentater Biscarboxamid-Liganden 


\subsection{Einleitung}

Die Entdeckung der endogenen Freisetzung von NO und CO und der damit einhergehenden positiven Auswirkungen auf Zellen und Organismus hat zur Entwicklung von NO- und COfreisetzenden Substanzen geführt. ${ }^{[293-299]}$ Therapeutisch werden derzeit jedoch nur NOfreisetzende Verbindungen wie Nitroglycerin (235) oder Natrium-Nitroprussid (236) zur Blutgefäßerweiterung und Blutdrucksenkung genutzt (Abbildung 6.1). ${ }^{[300]}$<smiles>O=[N+]([O-])OCC(CO[N+](=O)[O-])O[N+](=O)[O-]</smiles>

235

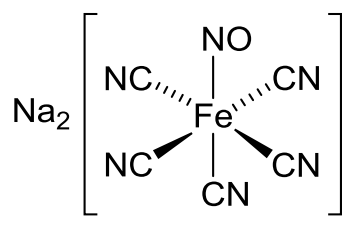

236

Abbildung 6.1: Nitroglycerin (235) und Natrium-Nitroprussid (236) als Beispiele für therapeutisch genutzte NO-freisetzende Verbindungen.

Ein Nachteil vieler NO- und CO-freisetzender Verbindungen ist jedoch, dass durch eine gleichmäßige Verteilung im Körper eine gezielte lokale Freisetzung in einem bestimmten Gewebe nicht oder nur sehr schwer möglich ist. In den vergangen Jahren wurden daher verstärkt Metallkomplexe untersucht, die nach Bestrahlung mit Licht NO oder CO freisetzen. ${ }^{[301]}$ Durch Variation der Liganden am Metallzentrum könnten die photochemischen Eigenschaften beeinflusst und eine gezielte Freisetzung der Gasmoleküle bei entsprechenden Wellenlängen ermöglicht werden. ${ }^{[301]}$ Die Freisetzung der Gasmoleküle verläuft dabei in der Regel nach photochemischer Anregung des Metallzentrums über eine Ladungsübertragung vom Metall auf den Liganden, wodurch die Metall-Ligand-Bindung geschwächt wird. ${ }^{[301]}$ Maßgebliche Arbeiten mit Nitrosyl-Ruthenium-Komplexen, die 1,2-Bis(pyridin-2carboxamido)benzol-Liganden $\left(\mathrm{H}_{2} \mathrm{bpb}\right.$, Abbildung 6.2) tragen, wurden von Mascharak et al. durchgeführt. [301-304] 


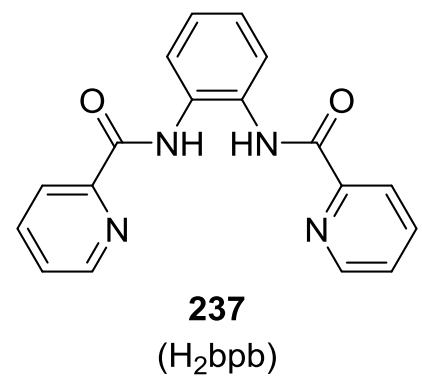

Abbildung 6.2: Strukturformel von 1,2-Bis(pyridin-2-carboxamido)benzen (237).

Dabei konnten durch Variation des Substitutionsmusters am Liganden sowohl die Quantenausbeuten erhöht als auch die Absorptionsmaxima der Komplexe in den Vis-NIRBereich verschoben werden. ${ }^{[305,306]}$ Dies ist im Hinblick auf eine medizinische Anwendung der Komplexe von Bedeutung, da hierbei Wellenlängen zwischen 400-950 nm bevorzugt werden, um durch UV-Strahlung verursachte Schäden am Gewebe zu vermindern. Das therapeutisch günstige Fenster liegt im nahen Infrarotbereich zwischen $650 \mathrm{~nm}$ und $950 \mathrm{~nm}$, da zum einem in diesem Bereich das Licht sehr tief in das Gewebe eindringen kann und diese Wellenlängen zum anderen oberhalb des Absorptionsbereich vom Häm der Erythrozyten (400-620 nm) liegen. ${ }^{[307]}$

\subsection{Syntheseziel}

Um den Einfluss von Substituenten am Picolinsäurerest auf die photochemischen Eigenschaften von Nitrosyl-Ruthenium-Komplexen mit 1,2-Bis(pyridin-2-carboxamido)benzen-Liganden untersuchen zu können, sollten Liganden mit 3-Hydroxy- bzw. 3-MethoxySubstituenten synthetisiert werden, die zudem optional ein Fluoratom in $o$-Position zum Pyridinstickstoff tragen sollten (Abbildung 6.3). 


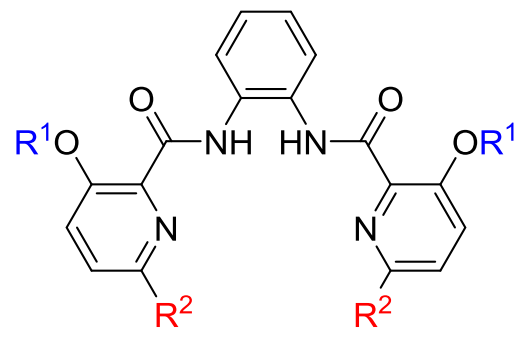

$$
\begin{aligned}
& \text { 102: } R^{1}=H, R^{2}=H \\
& \text { 103: } R^{1}=H, R^{2}=F \\
& \text { 104: } R^{1}=M e, R^{2}=H \\
& \text { 105: } R^{1}=M e, R^{2}=F
\end{aligned}
$$

Abbildung 6.3: Strukturen der geplanten Biscarboxamid-Liganden.

Die weitere Umsetzung der Liganden zu den entsprechenden Nitrosyl-Ruthenium-Komplexen und deren photochemische Evaluierung sollen in Zusammenarbeit mit der Gruppe von Jun.-Prof. Dr. Alexander Schiller (Friedrich-Schiller-Universität Jena, Institut für Anorganische und Analytische Chemie) durchgeführt werden.

\subsection{Synthese der nicht fluorierten Biscarboxamid-Liganden}

Für die Synthese der nicht fluorierten Liganden wurde zuerst 3-Hydroxypicolinsäure mit $o$-Phenyldiamin zum Liganden 102 umgesetzt (Schema 6.1).<smiles>O=C(O)c1ncccc1O</smiles>

238<smiles>Nc1ccccc1I</smiles>

239

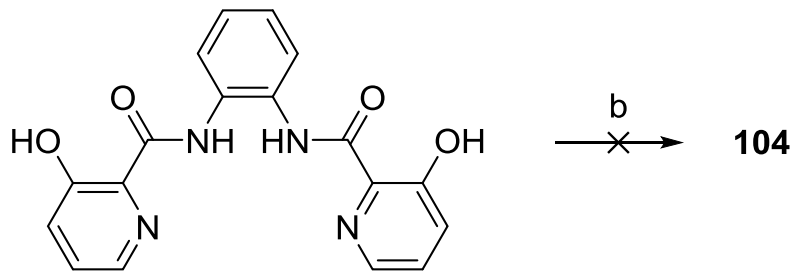

102

Schema 6.1: Synthese des Biscarboxamid-Liganden 102. Bedingungen: a) PyAOP, $E t N i \operatorname{Pr}_{2}, \mathrm{THF}$, $0{ }^{\circ} \mathrm{C} \rightarrow \mathrm{RT}, 4 \mathrm{~h}, 84 \%$. b) MeI, verschiedene Basen, DMF, RT.

Der Ligand wurde dabei in einer guten Ausbeute von $84 \%$ erhalten. Versuche mit anderen Kupplungsreagenzien wie EDCI/HOBt, HBTU, HATU oder PyBOP lieferten nur geringe Umsätze und Ausbeuten. Eine nachfolgende Alkylierung der OH-Gruppe zum MethoxyLiganden 104 gelang unter diversen Bedingungen jedoch nicht, da eine baseninduzierte Alkylierung der Amidgruppen nicht unterbunden werden konnte. 
Für die Synthese des Methoxyliganden 104 wurde daher 3-Methoxypicolinsäuremethylester (240) ${ }^{[308]}$ zu 3-Methoxypicolinsäure (241) umgesetzt (Schema 6.2).<smiles>COC(=O)c1ncccc1OC</smiles>

240<smiles>COc1cccnc1C(=O)O</smiles>

241

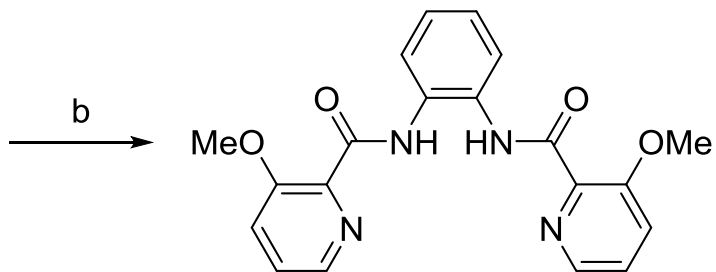

104

Schema 6.2: Synthese des Methoxy-Liganden 104. Bedingungen: a) $\mathrm{LiOH} \times \mathrm{H}_{2} \mathrm{O}, \mathrm{THF} / \mathrm{H}_{2} \mathrm{O}, \mathrm{RT}, 3 \mathrm{~h}$, 85\%. b) 239, PyAOP, EtNiPr 2 , THF, $0{ }^{\circ} \mathrm{C} \rightarrow \mathrm{RT}, 18$ h, 36\%.

Die Picolinsäure 241 konnte mittels kontinuierlicher Extraktion mit $\mathrm{CHCl}_{3}$ mit einer hohen Ausbeute von $85 \%$ isoliert werden. Bei der nachfolgenden Kupplung mit $o$-Phenyldiamin wurde der Methoxy-Ligand 104 mit einer geringen Ausbeute von nur 36\% erhalten. Zudem musste dieser nach Säulenchromatographie durch Umkristallisation weiter gereinigt werden.

\subsection{Synthese der fluorierten Biscarboxamid-Liganden}

Für die Synthese der fluorierten Biscarboxamid-Liganden 103 und 105 mussten die entsprechenden fluorierten Picolinsäuren 245 und 246 dargestellt werden (Schema 6.3). Die Einführung des Fluoratoms gelang mit einer von Hartwig et al. beschriebenen Methode zur $o$-Fluorierung von Pyridinen. ${ }^{[309]}$

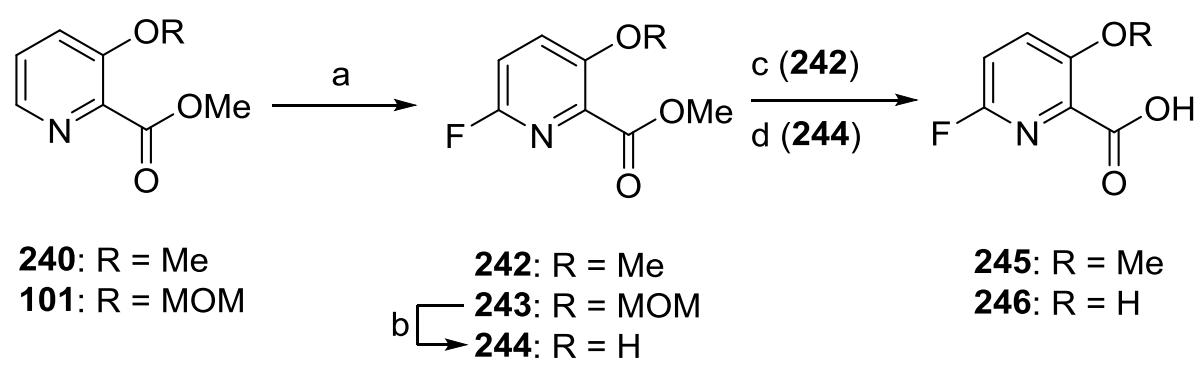

Schema 6.3: Synthese der fluorierten Picolinsäuren 245 und 246. Bedingungen: a) $\mathrm{AgF}_{2}, \mathrm{MeCN}, \mathrm{RT}$, $18 \mathrm{~h}, 68 \%$ (242, 83\% brsm); 53\% (243, 85\% brsm). b) TFA, $\mathrm{CH}_{2} \mathrm{Cl}_{2}, \mathrm{RT}, 20 \mathrm{~min}, 96 \%$. c) $\mathrm{NaOH}, \mathrm{TFE}$, $\mathrm{CH}_{2} \mathrm{Cl}_{2}, \mathrm{MeOH}, 0{ }^{\circ} \mathrm{C} \rightarrow \mathrm{RT}, 20$ h, $92 \%$. d) $\mathrm{KHCO}_{3}, 1,4-\mathrm{Dioxan} / \mathrm{H}_{2} \mathrm{O}, 60{ }^{\circ} \mathrm{C}, 5 \mathrm{~h}, 96 \%$.

Die Umsetzung der Ether 240 und 101 mit $\mathrm{AgF}_{2}$ lieferte die entsprechenden $o$-fluorierten Produkte in guter Ausbeute. Im Gegensatz zur Literatur wurden jedoch fünf statt zwei 
Äquivalente $\mathrm{AgF}_{2}$ und eine wesentlich längere Reaktionsdauer benötigt, um einen guten Umsatz erreichen zu können. ${ }^{[309]}$

Anschließend wurden die Ester unter milden Bedingungen und mit sehr guten Ausbeuten zu den entsprechenden Picolinsäuren verseift. Im Fall der 3-Hydroxypicolinsäure 246 wurde zuvor die MOM-Schutzgruppe sauer entfernt. Eine nucleophile aromatische Substitution des F-Atoms während dieser Umsetzungen wurde nicht beobachtet. Diese erfordert in der Regel wesentlich harschere Bedingungen. ${ }^{[310]}$

Die nachfolgende Umsetzung der fluorierten Picolinsäuren zu den entsprechenden Liganden mit PyAOP als Kupplungsreagenz gelang glatt (Schema 6.4). Die beiden fluorierten Biscarboxamid-Liganden $\mathbf{1 0 3}$ und $\mathbf{1 0 5}$ wurden nach Säulenchromatographie und Umkristallisation in akzeptabler Ausbeute von ca. $60 \%$ erhalten.<smiles>[R20]c1ccc(F)nc1C(=O)O</smiles><smiles>[R]Oc1ccc(F)nc1C(=O)Nc1ccccc1NC(=O)c1nc(F)ccc1[R]</smiles>

246: $R=H$

105: $R=M e$

103: $R=H$

Schema 6.4: Synthese der fluorierten Biscarboxamid-Liganden. Bedingungen: a) 239, PyAOP, $\mathrm{EtN} i \mathrm{Pr}_{2}, \mathrm{THF}, 0{ }^{\circ} \mathrm{C} \rightarrow \mathrm{RT}, 18 \mathrm{~h}, 61 \%$ (105); 58\% (103).

\subsection{Fazit}

Die Synthese substituierter 1,2-Bis(pyridin-2-carboxamido)benzen-Liganden gelang mittels PyAOP vermittelter Kupplung der entsprechenden Picolinsäuren mit $o$-Phenyldiamin in guten bis mäßigen Ausbeuten. Die Einführung eines Fluoratoms in o-Position zum PyridinStickstoff gelang mit $\mathrm{AgF}_{2}$.

Die weitere Umsetzung der vier neuartigen Liganden $\mathrm{zu}$ den entsprechenden MetallKomplexen und die Untersuchungen ihrer photochemischen Eigenschaften werden von der AG Schiller bearbeitet. 
7 Zusammenfassung 


\subsection{Totalsynthese von Nosiheptid}

Das Thiopeptidantibiotikum Nosiheptid (6) ist unter den bisher bekannten Antibiotika eines der potentesten gegen Gram-positive Bakterien und stellt aufgrund seiner ungewöhnlichen strukturellen Eigenschaften, eingebettet in einer hochfunktionalisierten Architektur, eine herausfordernde Zielstruktur für die Validierung bzw. Entwicklung neuer Synthesemethoden dar. ${ }^{[160,163,175]}$

Die Entwicklung und der erfolgreiche Abschluss der ersten Totalsynthese von Nosiheptid gelangen in dieser Arbeit durch die Synthese der beiden Fragmente 42 und 94 und eine nachfolgende ,reißverschlussartige“ (1.-3.) Verknüpfung (Schema 7.1). ${ }^{[120]}$ Mehrere AzaWittig-Ringschlüsse, unter Verwendung der beiden $\alpha$-Azidothiole 48 und 50, dienten dabei zum Aufbau verschiedener peptidintegrierter Thiazole. ${ }^{[160]}$ Der Aufbau der 4-Hydroxyglutaminsäure erfolgte durch Ringöffnung des Lactams 59 mit dem Indolylalkohol 40. Eine hetero-Diels-Alder-Reaktion zwischen dem Alkinylketon 64 und dem 1-Azabutadien $\mathbf{6 5}$ stellte den Schlüsselschritt in der Synthese des hochsubstituierten Pyridinkerns 42 dar. ${ }^{[163]}$ Der Einsatz des Thioamids 47 ermöglichte die Synthese eines mit einem Cystein-Rest substituierten Thiazols. Die Darstellung des bismakrocyclischen Grundgerüsts gelang durch Studien an einem vereinfachten B-Ring-Testsystem und der gezielten Optimierung der Entschützungs- als auch Makrocyclisierungsbedingungen. Die Entwicklung einer fortgeschrittenen Schutzgruppenstrategie, die an die besondere Labilität der NosiheptidStruktur angepasste wurde, spielte vor allem für die letzten Syntheseschritte eine entscheidende Rolle. Die Totalsynthese benötigte insgesamt 76 Reaktionsschritte, bei der eine Ausbeute von $0.03 \%$ über die längste lineare Sequenz von 32 Schritten erreicht wurde. Sie eröffnet den ersten synthetischen Zugang zur ungewöhnlichen Klasse $E$ der Thiopeptidgrundgerüste. Die Modularität der entwickelten Syntheseroute erlaubt den Austausch von Bausteinen und kann somit zur Darstellung neuartiger, bisher nicht zugänglicher Derivate dienen. 
<smiles>CC(C)(C)OC(=O)[C@H](NC(=O)OCc1ccccc1)C(=O)O</smiles>

140<smiles>N[C@@H](CS)C(=O)O[Na]</smiles>

50

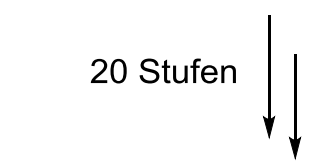<smiles>CC(C)(C)OC(=O)N1C(=O)C(OC(C)(C)C)C[C@@H]1C(=O)[O-]</smiles>

59

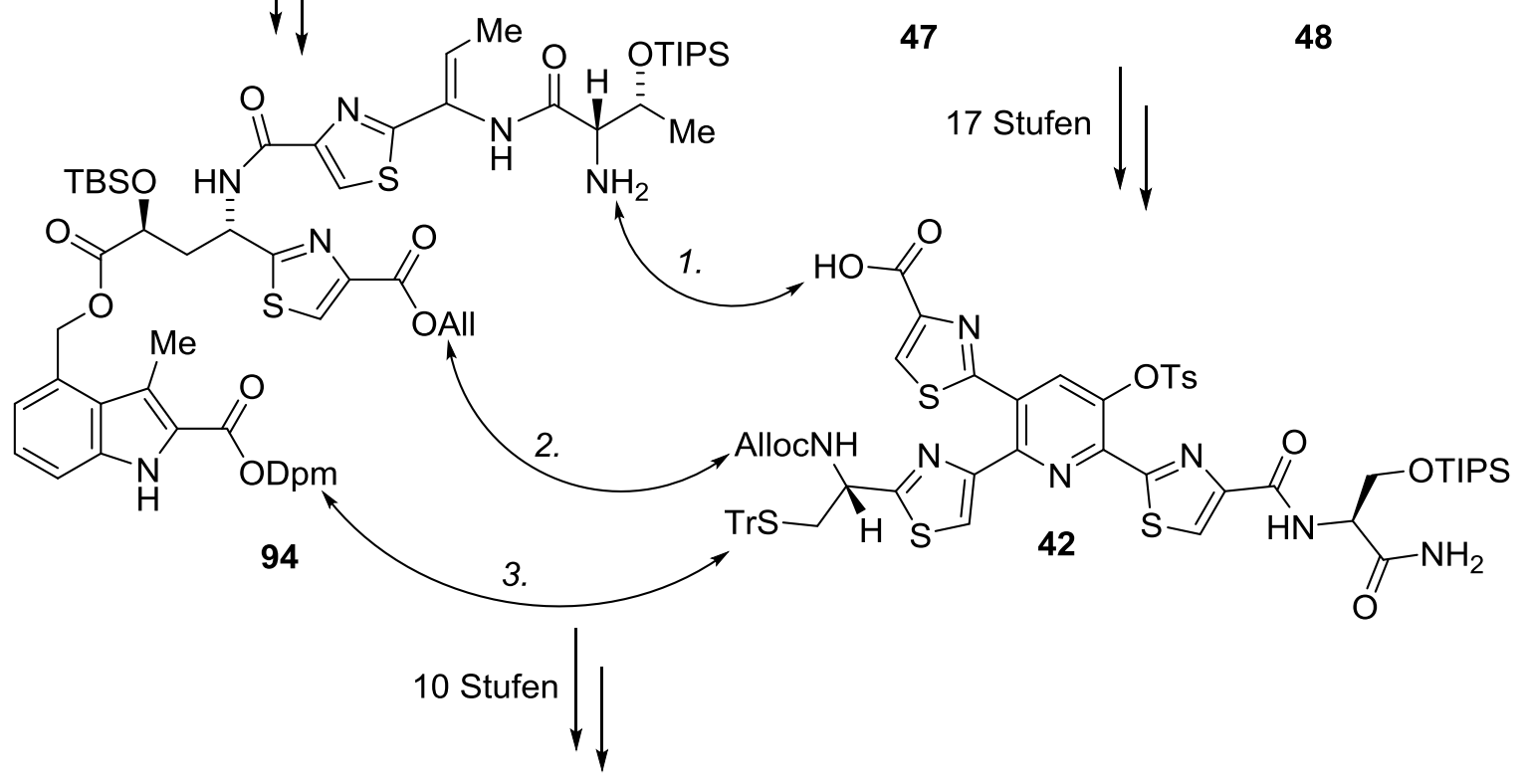

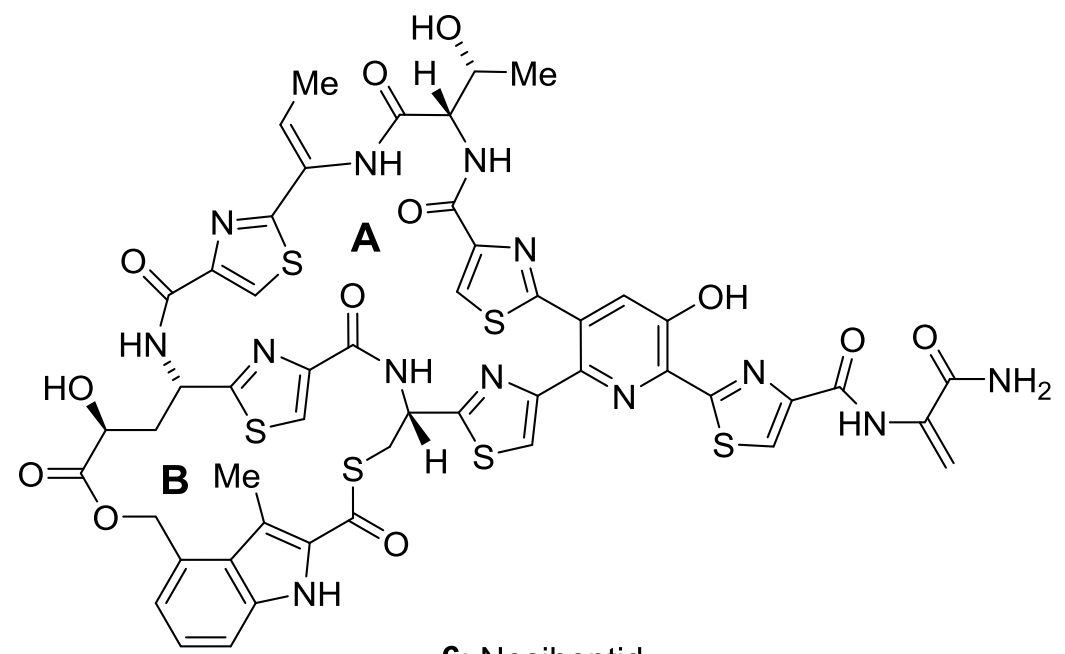

6: Nosiheptid

Schema 7.1: Übersicht zur Totalsynthese von Nosiheptid mittels des in dieser Arbeit entwickelten Reißverschlussverfahren (1.-3.). 


\subsection{Regioselektive Umfunktionalisierung von 3-Hydroxypciolinaten}

Im Verlauf der Entwicklung einer Totalsynthese des Thiopeptidantibiotikums Nosiheptid konnte durch J.-Y. Lu eine selektive Hydrolyse des 3-Hydroxypyridins 96 entwickelt werden (Schema 7.2). ${ }^{[37]}$

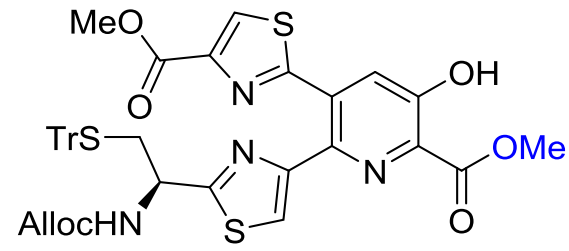

96

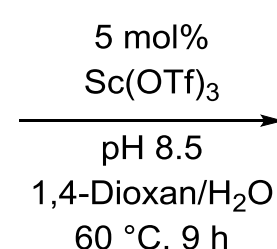

$60{ }^{\circ} \mathrm{C}, 9 \mathrm{~h}$

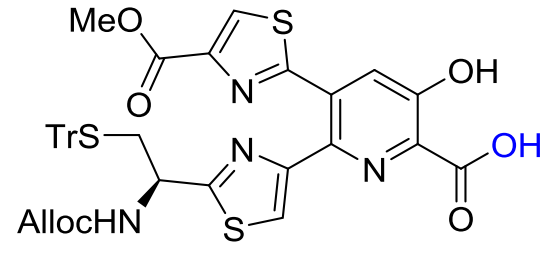

$70(93 \%)$

Schema 7.2: Regioselektive Verseifung des Bisthiazolylpyridins 96 nach Lu. ${ }^{[37]}$

Im Rahmen dieser Arbeit konnte in mechanistischen Untersuchungen gezeigt werden, dass die Selektivität der Reaktion ohne den Einsatz von $\mathrm{Sc}(\mathrm{OTf})_{3}$ erreicht werden kann. Wahrscheinlich wird diese durch eine aktive Beteiligung der benachbarten phenolischen $\mathrm{OH}-$ Gruppe ermöglicht (Schema 7.3, Mitte).<smiles>[R]c1cc(O)c(C(=O)OC)nc1[R]</smiles>

247

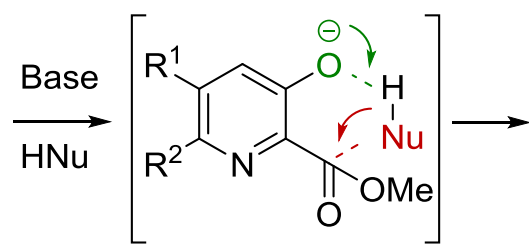<smiles>[R]c1cc(O)c(C(N)=O)nc1[R]</smiles>

248

$\mathrm{Nu}=\mathrm{NHR}^{3}, \mathrm{OR}^{3}$

Schema 7.3: Regioselektive Umfunktionalisierung von 3-Hydroxypicolinsäureestern.

Mit der Anwendung dieses Prinzips der intramolekularen Katalyse konnten die regioselektive Umesterung und Aminolyse von 3-Hydroxypicolinsäureestern unter milden Bedingungen demonstriert werden. In diesem Zusammenhang wurden auch wahrscheinliche Mechanismen unter Beteiligung der benachbarten phenolischen $\mathrm{OH}-$ Gruppe vorgestellt. ${ }^{[311]}$ 


\subsection{Synthese substituierter 3-Hydroxypicolinsäuren}

\subsubsection{Als Bausteine zur Derivatisierung von Pyridomycin}

Die in den letzten Jahrzehnten beobachtete Zunahme therapeutikaresistenter Stämme des Tuberkulose verursachenden Pathogens Mycobacterium tuberculosis erfordert die Entwicklung neuer Medikamente, vorrangig mit einem einzigartigen und bisher nicht genutzten Wirkungsmechanismus. Das bereits 1953 entdeckte Naturstoff Pyridomycin (99) könnte dabei mit seiner signifikanten in vitro Aktivität gegenüber M. tuberculosis und seines einzigartigen Wirkungsmodus als Leitstruktur dienen. ${ }^{[262,264]}$

Um einen tieferen Einblick in die Struktur-Aktivitäts-Beziehungen zu gewinnen und ggf. die Pharmakokinetik zu verbessern, wurde in einer Zusammenarbeit mit Prof. Dr. K.-H. Altmann (ETH Zürich) eine kleine Bibliothek an substituierten 3-Hydroxypicolinsäureestern (216, 230, 233) synthetisiert, die am Pyridomycin-Grundgerüst angebracht werden sollen (Schema 7.4).<smiles>CC/C(C)=C(/OC(=O)C(C)[C@@H](O)[C@@H](Cc1cccnc1)NC(=O)C(NC(=O)c1ncccc1O)C(C)O)C(=O)O</smiles>

99: Pyridomycin<smiles>COC(=O)c1ncccc1O</smiles><smiles>C#CC</smiles>

230

Schema 7.4: Allgemeine Übersicht zur Synthese substituierter 3-Hydroxypicolinsäureester zur Derivatisierung von Pyridomycin.

Durch Halogenierung, selektive Hydrodehalogenierung, Ir-katalysierte C-H-Borylierung sowie Suzuki-Miyaura-Kreuzkupplungen konnte ausgehend von 3-Hydroxypicolinsäuremethylester (100) jede der freien Positionen im Pyridinring selektiv substituiert werden. Ein Schützen der phenolischen OH-Gruppe war im Fall der 4- und 6-substituierten Derivate nicht oder nur temporär nötig. Ausgewählte 3-Hydroxypicolinsäureester wurden für die geplanten semisynthetischen Arbeiten in die entsprechenden Picolinsäuren überführt. 


\subsubsection{Zur Synthese fluorierter tetradentater Bis-Carboxamid-Liganden}

In Zusammenarbeit mit Jun.-Prof. A. Schiller (IAAC, FSU Jena) wurden vier neuartige Pyridin-Donor-Liganden für NO- oder CO- freisetzende Materialien synthetisiert die ein 3-Hydroxy- bzw. 3-Methoxypicolinamid enthalten (Schema 7.5).<smiles>O=C(O)c1ncccc1O</smiles>

238

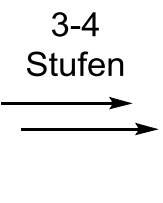<smiles>[R]Oc1ccc([R])nc1C(=O)O</smiles>

246: $R^{1}=H, R^{2}=F$

241: $R^{1}=M e, R^{2}=H$ 245: $R^{1}=M e, R^{2}=F$

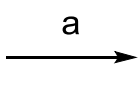<smiles>[R]Oc1ccc([R])nc1C(=O)Nc1ccccc1NC(=O)c1nc([R2])ccc1O[R1]</smiles>

102: $R^{1}=H, R^{2}=H$ 103: $R^{1}=H, R^{2}=F$ 104: $R^{1}=M e, R^{2}=H$ 105: $R^{1}=M e, R^{2}=F$

Schema 7.5: Übersicht zur Synthese der Bis-Carboxamid-Liganden 102-105. Bedingungen: a) $o$-Phenyldiamin, HATU, EtN $i \operatorname{Pr}_{2}$, THF, $0{ }^{\circ} \mathrm{C} \rightarrow \mathrm{RT}$, $4-18 \mathrm{~h}, 36-84 \%$.

Für die Synthese wurden ausgehend von 3-Hydroxypicolinsäure drei weitere Picolinsäuren dargestellt. Die Einführung eines Fluoratoms gelang durch eine selektive C-H-Fluorierung mit $\mathrm{AgF}_{2}{ }^{[309]}$ Die Bis-Carboxamid-Liganden wurden mittels PyAOP-vermittelter Amidknüpfungen aus den entsprechenden Picolinsäuren mit $o$-Phenyldiamin in guten bis mäßigen Ausbeuten erhalten.

Die weitere Umsetzung der Liganden zu den entsprechenden Metallkomplexen und deren photochemische Evaluierung werden vom Kooperationspartner bearbeitet. 


\subsection{Fazit und Ausblick}

Mit der Entwicklung einer Totalsynthese von Nosiheptid, ist erstmals ein synthetischer Zugang zur Klasse E der Thiopeptidantibiotika möglich. Die in dieser Arbeit angewendete Synthesestrategie könnte als Blaupause zur Synthese weiterer Verbindungen aus dieser Klasse, wie Glycothiohexid $\alpha$ oder Thiazomycin B, dienen. Die Zuverlässigkeit dieser Strategie konnte Ihar Raztsou bereits mit der Synthese geometrischer Isomere von Nosiheptid demonstrieren. ${ }^{[215]}$ Die Möglichkeiten zur Untersuchung der Biologie dieser Naturstoffklasse wurden somit entscheidend erweitert.

Die weitergehende Untersuchung der Chemie von 3-Hydroxypicolinsäureestern offenbarte eine Umsatzbeschleunigung bei ihrer Verseifung, Umesterung sowie Aminolyse durch intramolekulare Katalyse der benachbarten OH-Gruppe, in Analogie zu Salicylsäureestern. ${ }^{[311]}$ Des Weiteren konnten substituierte 3-Hydroxypicolinsäureester durch selektive Manipulation der aromatischen Protonen des 3-Hydroxypyridinkerns dargestellt, und zur Derivatisierung des Naturstoffs Pyridomycin als auch zur Synthese neuartiger tetradentater Liganden für funktionelle Materialien eingesetzt werden.

Neben dem Einsatz dieser Methoden zur Darstellung niedermolekularer Wirkstoffe in der pharmazeutischen und agrochemischen Industrie, ${ }^{[278]}$ könnten die substituierten 3-Hydroxypicolinsäuren auch zur Derivatisierung der Streptogramine B dienen, die ebenfalls ein 3-Hydroxypicolin tragen. 

8 Experimenteller Teil 


\subsection{Chemische Methoden}

\subsubsection{Geräte und Methoden}

NMR-Spektroskopie: ${ }^{1} \mathrm{H}$ - und ${ }^{13} \mathrm{C}-\mathrm{NMR}-S p e k t r e n$ wurden entweder mit einem Bruker Avance I (250 MHz), einem Bruker Fourier (300 MHz), einem Varian Mercury (400 MHz), einem Bruker Avance III (400 MHz), oder einem Bruker Avance III (600 MHz) Spektrometer in deuterierten Lösungsmitteln aufgenommen. Dabei dienten die Restprotonen der verwendeten Lösungsmittel als interner Standard $\left(\mathrm{CDCl}_{3}: \delta_{H}=7.26 \mathrm{ppm}, \delta_{C}=77.00 \mathrm{ppm}\right.$; $\mathrm{CD}_{3} \mathrm{OD}: \delta_{H}=3.31 \mathrm{ppm}, \delta_{C}=49.15 \mathrm{ppm} ; \mathrm{DMSO}-d_{6}: \delta_{H}=2.50 \mathrm{ppm}, \delta_{C}=39.51 \mathrm{ppm}$; 1,4-Dioxan- $\left.d_{8}: \delta_{H}=3.53 \mathrm{ppm}, \delta_{C}=66.66 \mathrm{ppm} ; \mathrm{D}_{2} \mathrm{O}: \delta_{H}=4.75 \mathrm{ppm}\right)$. Die chemischen Verschiebungen $(\delta)$ sind in parts per million (ppm) und die Kopplungskonstanten in $\mathrm{Hz}$ angegeben. Die Zuordnung der ${ }^{1} \mathrm{H}$ - Signale erfolgte nötigenfalls durch 2D Experimente (gCOSY, gHSQC, gHMBC, gTOCSY). Signalmultiplizitäten wurden folgendermaßen bezeichnet: s (Singulett), d (Dublett), t (Triplett), q (Quartett), dd (Doppeldublett), dt (Doppeltriplett), sxt (Sextett), m (Multiplett) und bs (breites Singulett). Sofern nicht anders angegeben wurden die NMR-Spektren bei $298 \mathrm{~K}$ gemessen. $\mathrm{CDCl}_{3}$ wurde durch aktiviertes basisches Aluminiumoxid geleitet, anschließend über aktiviertem Molekularsieb (3 $\AA$ ) bei $5{ }^{\circ} \mathrm{C}$ gelagert und innerhalb von 2 Wochen verbraucht.

Massenspektrometrie: HRMS (ESI-TOF)-Spektren wurden an einem maXis Impact (Bruker Daltronics, Bremen) Massenspektrometer gemessen.

IR-Spektroskopie: Die IR-Spektren wurden an einem Shimadzu IRAffinity-1 mit einer ATREinheit gemessen. Die Proben (Öle bzw. Feststoffe) wurden direkt auf die Messfläche (Diamant) aufgebracht. Feste Proben wurden mit einem Anpressstempel fixiert, Öle als Film vermessen. Die Wellenzahlen sind in $\mathrm{cm}^{-1}$ angegeben; folgende Abkürzungen geben die rel. Intensitäten der Absorptionsbanden an: s (hohe Intensität), m (mittlere Intensität), w (wenig intensiv), b (breite Intensität).

Schmelzpunktbestimmung: Die Schmelzpunkte wurden mit einem Büchi Heizmikroskop $B-450$ in offenen Kapillaren bestimmt und sind nicht korrigiert.

Spezifische optische Drehung: Optische Drehungen wurden mit einem a Jasco P-2000 Polarimeter bei $589 \mathrm{~nm}$ und $24{ }^{\circ} \mathrm{C}$ aufgenommen. Die optische Weglänge der Küvetten 
betrug $d=10 \mathrm{~mm}$. Die Konzentration $c$ in $\mathrm{g} / 100 \mathrm{~mL}$ und das verwendete Lösungsmittel (p.a.-Qualität) sind bei den jeweiligen Substanzen angegeben.

Mikrowellenunterstütze Reaktionen: Um Reaktionsgemische durch Mikrowellenstrahlung zu erhitzen wurde ein Biotage Initiator Sixty Mikrowellenreaktor mit verschlossenen Glasgefäßen eingesetzt. Die Temperaturmessung erfolgte dabei mithilfe eines IR-Sensors (Genauigkeit $\pm 2 \%$ ). Reaktionszeiten geben an, wie lange die Mischung bei der angegebenen Temperatur gerührt wurde, nicht wie lange die Mischung insgesamt bestrahlt wurde.

Ultraschallunterstütze Reaktionen: Ultraschallunterstütze Reaktionen wurden in einem $250 \mathrm{~mL}$ Doppelwandgefäß durchgeführt, das mit Wasser gekühlt wurde. Als Ultraschallquelle diente ein Branson Digital Sonifier 450 ausgestattet mit einem Branson 102-C Converter, einem $1 / 2$ " Disruptor Horn sowie einem zugespitztem Mikrotip.

\subsubsection{Chromatographie}

Dünnschichtchromatographie: Die Dünnschichtchromatographie wurde auf beschichteten Aluminium-Platten der Firma Merck (Kieselgel 60, F254) durchgeführt. Die Detektion erfolgte mittels UV-Licht (255 bzw. 366 nm) oder durch Anfärben mit einer der folgenden Mischungen und anschließendes Erwärmen.

KMnO 4 -Lösung: $2 \mathrm{~g} \mathrm{KMnO}_{4}, 13.2 \mathrm{~g} \mathrm{~K}_{2} \mathrm{CO}_{3}, 165 \mathrm{mg} \mathrm{NaOH}, 200 \mathrm{~mL} \mathrm{H} \mathrm{H}_{2} \mathrm{O}$.

Ninhydrin-Lösung: 300 mg Ninhydrin, 100 mL EtOH, 2 mL AcOH.

Phosphomolybdat: 2 Gew.-\% Molybdatophosphorsäure in EtOH.

Säulenchromatographie: Als stationäre Phase diente Kieselgel der Firma Macherey \& Nagel (MN Kieselgel $60 \mathrm{M}$, Partikelgröße 40-73 $\mu \mathrm{m}$ ). Die Trennung fand in der Regel bei 0.3-0.5 bar Überdruck statt (Geschwindigkeit der Laufmittelfront: ca. $5 \mathrm{~cm} / \mathrm{min}$ ).

Alle Lösungsmittel für die Säulenchromatographie, die nicht in geeigneter Qualität bezogen wurden, wurden zuvor mittels eines Rotationsverdampfers von nicht flüchtigen Bestandteilen befreit.

Präparative Dünnschichtchromatographie: Präparative Dünnschichtchromatographie wurde auf Silikagel beschichteten Glasplatten (Schichtdicke: $0.5 \mathrm{~mm}$ o. $1 \mathrm{~mm}$ ) der Firma Merck (60F-254) durchgeführt. 
Analytische HPLC: Die HPLC-Anlage der Firma Varian bestand aus folgenden Komponenten: Pumpe ProStar 230, Autosampler ProStar 410, Säulenwechseler Reodyne LabPRO, UV/VIS-Detektor ProStar 335. Als Trennsäulen wurden verwendet: EC250/4 Nucleodur C18 Gravity $5 \mu m$ (Macherey \& Nagel) und eine Phenomenex Luna $5 \mu$ C8(2) $100 A(250 \times 4 \mathrm{~mm})$. Es wurden lineare Gradienten bei einem Fluss von $1 \mathrm{~mL} / \mathrm{min}$ eingesetzt. Die Lösungsmittel (HPLC gradient grade) wurden von VWR bezogen.

Präparative HPLC: Für präparative Trennungen kam ein Model der Firma Varian zum Einsatz: Pumpe ProStar 215, UV/VIS-Detektor ProStar 340 und Fraktionssammler ProStar 701. Als Trennsäulen kamen zum Einsatz: VP250/21 Nucleodur C18 Gravity $5 \mu \mathrm{m}$, sowie eine Phenomenex Luna $5 \mu$ C8(2) 100A, Axia P (250×21 mm). Die Lösungsmittel (HPLC gradient grade) wurden von VWR bezogen.

LC-MS: Die HPLC-Anlage der Firma Shimadzu bestand aus folgenden Komponenten: Systemcontroller SLC-10A VP, Pumpe LC-10AT VP, Autosampler SIL-10AD VP, Säulenofen CTO-10AC VP, Splitter ICP 04-20 (Thermo Scientific), UV/VIS-Detektor SPD-10A VP. Als Trennsäule wurde verwendet: EC125/4 Nucelodur C18 Isis 3 um (Macerey Nagel). Es wurden lieneare Gradienten bei einem Fluss von $1 \mathrm{~mL} / \mathrm{min}$ eingesetzt. Als Eluenten wurden $\mathrm{H}_{2} \mathrm{O}$ und MeCN mit jeweils $1 \mathrm{Vol} \% \mathrm{AcOH}$ verwendet. Die Lösungsmittel (HPLC gradient grade) wurden von VWR bezogen. Die Aufnahme der Massenspektren erfolgte mit einem ESIIonenfallen-MS (Finnigan Mat LCQ).

\subsubsection{Reagenzien, Lösungsmittel und Reaktionsbedingungen}

Reagenzien wurden von den Firmen ABCR, Acros Chemicals, Active Scientific Alfa Aesar, Apollo Scientific, Carbolution Chemicals, Carbosynth, Grüssing, Manchester Organics, Merck, Novabiochem, Sigma-Aldrich, TCI Europe und VWR bezogen und, falls nicht anders angegeben, wie erhalten eingesetzt.

Andere Reagenzien oder fortgeschrittene Syntheseintermediate wurden der Literatur folgend dargestellt: 4-(Hydroxymethyl)-3-methyl-1H-indol-2-carbonsäurediphenylmethylester (40), ${ }^{[36]}$ Tristhiazolylpyridin $\mathbf{4 2}^{[37]}$ (4R)-N-tert-Butoxycarbonyl-2,2-dimethyl-thiazolidin-4carbothioamid (47), ${ }^{[98]} N$-Boc-5-aza-2-oxa-3-oxo-bicyclo[2.2.1] heptan (57), ${ }^{[36]} 1,2$-(Z)-Methoxycarbonyl-1,3-bis(trimethyl-silyloxy)-1,3-butadien $\quad(\mathbf{6 5}),{ }^{[37]} \quad$ 2-Nitro-6-(2‘-tetrahydropyranyloxymethyl)phenyl-brenztrauben-säureethylester $\quad(\mathbf{7 5}),{ }^{[153]} \quad 3$-Hydrox-ypyridin-5,6bis(trideutero-methoxycarbonyl)-2-carbon-säuremethylester (97-d 6 ), ${ }^{[241]}$ 
3-Hydroxypicolinsäuremethylester $\quad(\mathbf{1 0 0}),{ }^{[278]} \quad L$-Boc-Thr(TBS)-OH (140), ${ }^{[201]} \quad L$-FmocThr(TBS)-OH (143), ${ }^{[202]} 2$ '-Bromacetyl-pyridin (161), ${ }^{[219]}$ 6-Brom-3-hydroxypicolinsäuremethyl-ester (204), ${ }^{[267]}$ 4-(Tributyl-stannyl)-1-trityl-1H-imidazol (217), ${ }^{[269]}$ 4,6-Dibrom-3hydroxy-picolinsäure-diemethylester $\quad(\mathbf{2 2 8}),{ }^{[278]}$ Dess-Martin-Periodinan, ${ }^{[312]}$ 3-Hydroxypyridin-2,5,6-tricarbonsäure-trimethylester, ${ }^{[37]}$ 3-Meth-oxypicolinsäuremethyl-ester $(240)^{[308]}$ Indol-2-boron-säurepinakolester, ${ }^{[313]}$ Indol-3-boronsäurepinakol-ester, ${ }^{[314]} \quad 1 H$-Pyrrol-3boronsäure-pinakolester, ${ }^{[315]}$ 1-(Tetrahydro-2H-pyran-2-yl)-1H-pyrazol-5-boronsäurepinakolester, ${ }^{[268]}$ Pyrazol-4-boronsäurepinakolester, ${ }^{[314]}(R)$-2-Azido-3-(tritylthio)-propansäureallylester, ${ }^{[37]}$ PyDOP. ${ }^{[225]}$

Dichlormethan $\left(\mathrm{CH}_{2} \mathrm{Cl}_{2}\right)$, Triethylamin, Hünig-Base $\left(\mathrm{EtNi} \mathrm{Pr}_{2}\right)$, Pyridin und 2,6-Lutidin wurden von $\mathrm{CaH}_{2}$ destilliert und unter einer Schutzgasatmosphäre $\left(\mathrm{N}_{2}\right.$ oder Ar) gelagert. Methanol, Acetonitril und 2-Propanol wurden mittels aktiviertem Molekularsieb (3 $\AA$, 20 gew.-\%) entwässert und unter einer Schutzatmosphäre gelagert. Tetrahydrofuran, Anisol und Diethylether wurden vor dem Gebrauch von blauviolettem $\mathrm{Na}$ /Benzophenon destilliert. Methansulfonylchlorid wurde von $\mathrm{P}_{2} \mathrm{O}_{5}$ unter vermindertem Druck destilliert. Tosylchlorid und Mesitylsulfonylchlorid wurden mittels Kieselgelfiltration $\left(\mathrm{CH}_{2} \mathrm{Cl}_{2}\right)$ gereinigt.

Alle Reaktionen wurden üblicherweise unter einer Schutzgasatmosphäre $\left(\mathrm{N}_{2}\right.$ oder Ar) durchgeführt.

\subsubsection{Pufferlösungen}

Phosphatpuffer pH 3: $\mathrm{NaH}_{2} \mathrm{PO}_{4} \times 2 \mathrm{H}_{2} \mathrm{O}(246.5 \mathrm{~g}, 1.58 \mathrm{~mol})$ und $85 \% \mathrm{H}_{3} \mathrm{PO}_{4}(62.2 \mathrm{~mL}$, $0.92 \mathrm{~mol}$ ) wurden in Wasser gelöst und auf ein Volumen von $1 \mathrm{~L}$ aufgefüllt.

Phosphatpuffer pH 6.2: $\quad \mathrm{Na}_{3} \mathrm{PO}_{4} \times 12 \mathrm{H}_{2} \mathrm{O} \quad(33.8 \mathrm{~g}, 88.9 \mathrm{mmol})$ und $\mathrm{NaH}_{2} \mathrm{PO}_{4} \quad(109.3 \mathrm{~g}$, $0.91 \mathrm{~mol}$ ) wurden in Wasser gelöst und auf ein Volumen von $1 \mathrm{~L}$ aufgefüllt.

Phosphatpuffer pH 7: $\mathrm{Na}_{3} \mathrm{PO}_{4} \times 12 \mathrm{H}_{2} \mathrm{O}(54.8 \mathrm{~g}, 0.14 \mathrm{~mol})$ und $\mathrm{NaH}_{2} \mathrm{PO}_{4}(42.7 \mathrm{~g}, 0.36 \mathrm{~mol})$ wurden in Wasser gelöst und auf ein Volumen von $1 \mathrm{~L}$ aufgefüllt.

\subsection{Mikrobiologische Methoden}

\section{Bakterienkulturen}

In dieser Arbeit wurde Streptomyces coelicolor A3 (2) eingesetzt. Bakterienkulturen wurden 
auf Petrischalen $(8.5 \mathrm{~cm})$, die $30 \mathrm{~mL}$ Mannitol-Soyamehl-Agar enthielten, drei Tage lang bei $30{ }^{\circ} \mathrm{C}$ inkubiert, ${ }^{[316]}$ die Sporen wurden anschließend geerntet und in Glycerol gelagert. ${ }^{[317]}$

\section{Hemmhoftest}

Eine S. coelicolor-Sporenlösung $\left(100 \mu \mathrm{L}, 1 \times 10^{8}\right.$ aktive Sporen $\left./ \mathrm{ml}\right)$ wurde auf Petrischalen gleichmäßig verteilt $(8.5 \mathrm{~cm})$ die $30 \mathrm{~mL}$ Minimal-Medium enthielten. ${ }^{[318]}$ Anschließend wurden mit der Rückseite einer Pasteur-Pipette Löcher in das Medium gestanzt und mit $100 \mu \mathrm{L}$ der Analytlösung in DMSO gefüllt. Die Platten wurden anschließend 2-3 h stehen gelassen, damit die DMSO-Lösung in das Medium diffundieren konnte und anschließend drei Tage lang bei $30^{\circ} \mathrm{C}$ inkubiert.

\subsection{Allgemeine Arbeitsvorschriften}

\section{Mikrowellenunterstütze Suzuki-Miyaura-Kupplung (AV1):}

Das Brompyridin (0.22 mmol) wurde zusammen mit der entsprechenden Boronsäure bzw. dem Boronsäureester (0.289 mmol), wasserfreiem CsF (67 mg, $0.44 \mathrm{mmol})$ und $\mathrm{Pd}\left(\mathrm{PPh}_{3}\right)_{4}$ (12.5 mg, $5 \mathrm{~mol} \%$ ) in einer 1:1 Mischung von wasserfreiem $\mathrm{MeOH}$ und wasserfreiem 1,2-Dimethoxyethan (2 mL) gelöst und anschließend $20 \mathrm{~min}$ lang bei $150{ }^{\circ} \mathrm{C}$ im Mikrowellenreaktor gerührt. Nach Abkühlen auf RT wurde das Gemisch mit pH 6.2 Puffer $(10 \mathrm{~mL})$ versetzt, mit $\mathrm{CH}_{2} \mathrm{Cl}_{2}(3 \times 10 \mathrm{~mL})$ extrahiert und die vereinigten organischen Phasen mit $\mathrm{Na}_{2} \mathrm{SO}_{4}$ getrocknet. Nach Einengen im Vakuum wurde das Rohprodukt durch

Säulenchromatographie $\left(\mathrm{SiO}_{2}, 25 \mathrm{~g}\right.$, Petrolether/Ethylacetat, $\mathrm{CH}_{2} \mathrm{Cl}_{2} / \mathrm{MeOH}$ oder Petrolether/ $\mathrm{CH}_{2} \mathrm{Cl}_{2}$ /Aceton) gereinigt.

\section{Nachbargruppenassistierte Verseifung von 3-Hydroxypicolinsäureestern (AV2):}

Der entsprechende 3-Hydroxypicolinsäuremethylester $(0.1 \mathrm{mmol})$ wurde in 1,4-Dioxan (1.5 mL) und $\mathrm{H}_{2} \mathrm{O}(0.75 \mathrm{ml})$ gelöst, mit $\mathrm{NaHCO}_{3}$ oder $\mathrm{KHCO}_{3}(400 \mu \mathrm{mol})$ versetzt und bei $60{ }^{\circ} \mathrm{C}$ bis zum vollständigen Umsatz gerührt (9-18 h, DC-Kontrolle). Anschließend wurde die Reaktionsmischung im Vakuum eingeengt, der Rückstand in pH 3 Puffer $(5 \mathrm{~mL})$ aufgenommen und mit EtOAc $(4 \times 10 \mathrm{~mL})$ extrahiert. Die vereinigten organischen Extrakte wurden mit $\mathrm{Na}_{2} \mathrm{SO}_{4}$ entwässert und im Vakuum eingeengt. Die erhaltenen Produkte zeigten in der Regel eine ausreichende Reinheit und wurden nicht weiter gereinigt.

\section{Nachbargruppen assistierte Umesterung von 3-Hydroxypicolinsäureestern (AV3):}

Der 3-Hydroxypicolinsäureester $(52 \mu \mathrm{mol})$ wurde im entsprechenden Alkohol $(2 \mathrm{~mL})$ gelöst 
und mit $\operatorname{EtNiPr}_{2}(0.9 \mu \mathrm{L}, 5.2 \mu \mathrm{mol})$ versetzt. Das Gemisch wurde für $20 \mathrm{~h}$ bei $60^{\circ} \mathrm{C}$ gerührt. Nach Abkühlen auf RT wurde das Lösungsmittel im Vakuum entfernt und der Rückstand durch Säulenchromatographie $\left(\mathrm{SiO}_{2}, 10 \mathrm{~g}\right.$, Petrolether/EtOAc, 2:1) gereinigt.

\subsection{Totalsynthese von Nosiheptid}

\section{3-(2-Nitro-6-(((tetrahydro-2H-pyran-2-yl)oxy)methyl)phenyl)-2-oxobut-3-ensäure- ethylester (118)}<smiles>C=C(C(=O)OCC)c1c(CO[OH+])cccc1[N+](=O)[O-]</smiles>

$\mathrm{Zu}$ einer Lösung des $\alpha$-Ketoesters $\mathbf{7 5}^{[153]}$ (432 $\left.\mathrm{mg}, 1.23 \mathrm{mmol}\right)$ in wasserfreiem $\mathrm{CH}_{2} \mathrm{Cl}_{2}$ $(18 \mathrm{~mL})$ wurden $N, N$-Dimethylmethylenammoniumchlorid ${ }^{[319]}(347 \mathrm{mg}, 3.71 \mathrm{mmol})$ und $\mathrm{Et}_{3} \mathrm{~N}$ $(534 \mu \mathrm{L}, 3.83 \mathrm{mmol})$ gegeben. Die Reaktionsmischung wurde $18 \mathrm{~h}$ lang bei RT gerührt, anschließend mit ges. $\mathrm{NaHCO}_{3}$-Lsg. $(50 \mathrm{~mL})$ verdünnt und mit $\mathrm{Et}_{2} \mathrm{O}(3 \times 20 \mathrm{~mL})$ extrahiert. Die vereinigten organischen Extrakte wurden mit ges. NaCl-Lsg. (20 mL) gewaschen, mit $\mathrm{Na}_{2} \mathrm{SO}_{4}$ entwässert und am Rotationsverdampfer eingeengt. Der Rückstand wurde durch Säulenchromatographie $\left(\mathrm{SiO}_{2}, 25 \mathrm{~g}\right.$, Petrolether/EtOAc, 2:1) gereinigt. Es wurden $442 \mathrm{mg}$ (1.22 mmol, 99\%) des Enons 118 als gelbes, viskoses Öl erhalten.

DC: $R_{f}=0.30($ Heptan/EtOAc, $2: 1)$.

IR: $\tilde{v}=810(\mathrm{w}), 1026(\mathrm{~s}), 1126 \mathrm{~m}), 1242(\mathrm{~m}), 1342(\mathrm{~m}), 1450(\mathrm{w}), 1527(\mathrm{~m}), 1689(\mathrm{~m}) \mathrm{cm}^{-1}$.

${ }^{1} \mathbf{H}-\mathbf{N M R}\left(300 \mathrm{MHz}, \mathrm{CDCl}_{3}\right): \delta=1.34\left(\mathrm{t}, J=7.0 \mathrm{~Hz}, 3 \mathrm{H}, \mathrm{CH}_{3}\right), 1.68(\mathrm{~m}, 6 \mathrm{H}, \mathrm{THP}), 3.51$ (m, 1H, THP), 3.79 (t, $J=9.6 \mathrm{~Hz}, 1 \mathrm{H}, \mathrm{THP}), 4.36$ (m, 3H, $\mathrm{CH}_{2} \mathrm{Me}$, THP), 4.67 (m, 2H, THP), $6.08(\mathrm{~d}, J=10.9 \mathrm{~Hz}, 1 \mathrm{H},=\mathrm{CHH}), 6.67(\mathrm{~s}, 1 \mathrm{H},=\mathrm{CH} H), 7.53(\mathrm{t}, J=7.8 \mathrm{~Hz}, 1 \mathrm{H}, \mathrm{CH}), 7.83(\mathrm{bs}$, $1 \mathrm{H}, \mathrm{CH}), 8.02(\mathrm{~d}, J=8.2 \mathrm{~Hz}, 1 \mathrm{H}, \mathrm{CH})$.

${ }^{13}$ C-NMR $\left(75 \mathrm{MHz}, \mathrm{CDCl}_{3}\right): \delta=13.9,19.0,19.2,25.2,30.2,62.0,62.3,62.4,66.1,66.3$, 97.9, 98.5, 123.7, 123.8, 129.1, 130.5, 130.7, 132.1, 133.6, 134.1, 139.7, 139.9, 140.0, 148.3, $162.2,183.9,184.1$. 
HRMS (ESI): $m / z[\mathrm{M}+\mathrm{Na}]^{+}$für $\mathrm{C}_{18} \mathrm{H}_{21} \mathrm{NONa}$ ber.: 368.1210; gef.: 368.1210 .

\section{3-Methyl-4-((tetrahydro-2H-pyran-2-yloxy)methyl)-1H-indol-2-carbonsäureethylester} (78)<smiles>CCOC(=O)c1[nH]c2cccc(COP)c2c1C</smiles>

Aktiviertes Molekularsieb (4 A, $36 \mathrm{mg}$, Pulver) wurde zusammen mit $\mathrm{Pd} / \mathrm{C}$ (18 mg, 10 gew.\%, Acros 19503) in einer 1:1 Mischung von wasserfreiem THF und wasserfreiem 2-Propanol $(5 \mathrm{~mL})$ suspendiert. Der Kolben wurde mit $\mathrm{H}_{2}$ (1 bar) gefüllt und die Mischung 45 min lang gerührt. Das Alken 118 (178 mg, 0.49 mmol) wurde zugegeben und das Gemisch $24 \mathrm{~h}$ lang unter einer $\mathrm{H}_{2}$-Atmosphäre gerührt. Die Reaktionsmischung wurde anschließend mit EtOAc (25 ml) verdünnt, durch Kieselguhr filtriert und im Vakuum eingeengt. Reinigung des Rohprodukts durch Säulenchromatographie $\left(\mathrm{SiO}_{2}, 20 \mathrm{~g}\right.$, Petrolether/EtOAc, 2:1) ergab $105 \mathrm{mg}(0.33 \mathrm{mmol}, 68 \%)$ des Indols 78 als farblosen Feststoff.

DC: $R_{f}=0.36($ Heptan/EtOAc, $4: 1)$.

Alle relevanten analytischen Daten stimmen mit den veröffentlichten Werten überein. ${ }^{[36]}$

\section{4-((Butyryloxy)methyl)-3-methyl-1H-indol-2-carbonsäurebenzhydrylester (132)}

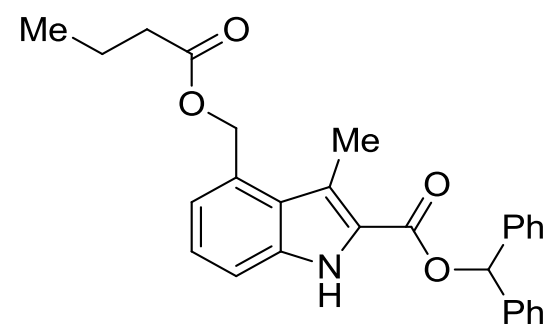

Zu einer Lösung des Indolylalkohols $\mathbf{4 0}^{[36]}$ (100 mg, $\left.0.27 \mathrm{mmol}\right)$ in wasserfreiem THF (4 mL) wurden bei $0{ }^{\circ} \mathrm{C}, \operatorname{EtNi} \operatorname{Pr}_{2}(117 \mu \mathrm{L}, 1.08 \mathrm{mmol})$, Buttersäureanhydrid (88 $\left.\mu \mathrm{L}, 0.54 \mathrm{mmol}\right)$ und DMAP (4 mg, $0.03 \mathrm{mmol}$ ) zugegeben. Das Kühlbad wurde entfernt und die Mischung $18 \mathrm{~h}$ lang bei RT gerührt. Nach Zugabe von ges. $\mathrm{NH}_{4} \mathrm{Cl}$-Lsg. $(5 \mathrm{~mL})$ und $\mathrm{H}_{2} \mathrm{O}(15 \mathrm{~mL})$ wurde mit $\mathrm{CH}_{2} \mathrm{Cl}_{2}(3 \times 10 \mathrm{~mL})$ extrahiert. Die vereinigten organischen Extrakte wurden mit halb ges. $\mathrm{NaHCO}_{3}$-Lsg. und ges. NaCl-Lsg. (je $10 \mathrm{~mL}$ ) gewaschen und mit $\mathrm{Na}_{2} \mathrm{SO}_{4}$ entwässert. Nach 
Einengen im Vakuum und Chromatographie $\left(\mathrm{SiO}_{2}, 10 \mathrm{~g}\right.$, Petrolether/EtOAc, 4:1) wurden 117 mg (0.26 mmol, 98\%) des Butersäureesters 132 als farbloser Feststoff erhalten.

Schmelzpunkt: $139.5^{\circ} \mathrm{C}$.

DC: $R_{f}=0.60($ Heptan/EtOAc, $2: 1)$.

IR: $\tilde{v}=694(\mathrm{w}), 748(\mathrm{w}), 964(\mathrm{w}), 1242(\mathrm{~m}), 1666(\mathrm{~s}), 1728(\mathrm{~m}), 2330(\mathrm{w}) \mathrm{cm}^{-1}$.

${ }^{1}$ H-NMR $\left(300 \mathrm{MHz}, \mathrm{CDCl}_{3}\right): \delta=0.95\left(\mathrm{t}, J=7.4 \mathrm{~Hz}, 3 \mathrm{H}, \mathrm{CH}_{3}\right), 1.69$ (sxt, $J=7.4 \mathrm{~Hz}, 2 \mathrm{H}$, $\left.\mathrm{CH}_{2}\right), 2.35\left(\mathrm{t}, J=7.4 \mathrm{~Hz}, 2 \mathrm{H}, \mathrm{CH}_{2}\right), 2.85\left(\mathrm{~s}, 3 \mathrm{H}, \mathrm{CH}_{3}\right), 5.50\left(\mathrm{~s}, 2 \mathrm{H}, \mathrm{OCH}_{2}\right), 7.19(\mathrm{~s}, 1 \mathrm{H}$, $\left.\mathrm{CH}(\mathrm{Ph})_{2}\right), 7.11-7.45(\mathrm{~m}, 13 \mathrm{H}, 2 \times \mathrm{Ph}$, Indol), $8.88(\mathrm{~s}, 1 \mathrm{H}, \mathrm{NH})$.

${ }^{13}$ C-NMR (75 MHz, $\left.\mathrm{CDCl}_{3}\right)$ : 11.9, 13.7, 18.4, 36.3, 64.7, 77.6, 112.6, 120.9, 122.5, 123.5, $125.4,126.4,127.2,128.1,128.6,130.3,136.6,139.9,161.5,173.4$.

Elementaranalyse: Für $\mathrm{C}_{28} \mathrm{H}_{27} \mathrm{NO}_{4}$ ber.: C, 76.17; H, 6.16; N, 3.17; gef. C, 76.37; H, 6.08; N, 3.15 .

(2S,4S)-N-Boc-4-hydroxy-pyrrolidin-2-carbonsäure-(2,2,2-trichlorethyl)ester (136)<smiles>O=C(OCC(Cl)(Cl)Cl)C1C[C@@H](O)CN1C(=O)O</smiles>

Das Lacton $\mathbf{5 7}^{[36]}(7.23 \mathrm{~g}, 33.9 \mathrm{mmol})$ wurde in 2,2,2-Trichlorethanol (35 ml) gelöst und mit $\mathrm{NaN}_{3}(6.57 \mathrm{~g}, 101.1 \mathrm{mmol})$ versetzt. Das Gemisch wurde anschließend $1 \mathrm{~h}$ lang bei $50{ }^{\circ} \mathrm{C}$ gerührt, auf RT abgekühlt und mit $\mathrm{CH}_{2} \mathrm{Cl}_{2}(500 \mathrm{~mL})$ verdünnt. Nach Filtration des entstanden Niederschlags wurde die Lösung am Rotationsverdampfer eingeengt und uberschüssiges 2,2,2-Trichlorethanol am Feinvakuum mit Hilfe einer Kugelrohrdestille entfernt. Der Rückstand wurde durch Säulenchromatographie $\left(\mathrm{SiO}_{2}, 600 \mathrm{~g}\right.$, Petrolether/EtOAc, 2:1 $\rightarrow$ 1:1) gereinigt. Es wurden $10.2 \mathrm{~g}$ (28.1 mmol, 83\%) des Pyrrolidins 136 als farbloser Feststoff erhalten.

Spez. Drehung: $[\alpha]_{\mathrm{D}}{ }^{24}=-24.3\left(\mathrm{CHCl}_{3}, c=1\right)$.

Schmelzpunkt: $107^{\circ} \mathrm{C}$. 
DC: $R_{f}=0.35($ Heptan/EtOAc, $1: 1)$

IR: $\tilde{v}=621(\mathrm{~m}), 1151(\mathrm{~s}), 1414(\mathrm{~s}), 1667(\mathrm{~s}), 1775(\mathrm{~m}), 2912(\mathrm{w}), 2936(\mathrm{w}), 3396(\mathrm{bw}) \mathrm{cm}^{-1}$.

${ }^{1} \mathbf{H}$ NMR $\left(600 \mathrm{MHz}, \mathrm{CDCl}_{3}, 323 \mathrm{~K}\right.$, Rotamere): $\delta=1.44,1.47$ (je s, 9H, Boc), 2.23 (d, $J=12.1 \mathrm{~Hz}, 1 \mathrm{H}, \mathrm{NCHH}), 2.41-2.57$ (m, 2H, CHH, $\mathrm{NCH} H), 3.59-3.64$ (m, 2H, CHH, CHOH), $4.40(\mathrm{~s}, 1 \mathrm{H}, \mathrm{OH}), 4.47-4.51(\mathrm{~m}, 1 \mathrm{H}, \mathrm{HCOO}), 4.62-4.71\left(\mathrm{~m}, 1 \mathrm{H}, \mathrm{C} H \mathrm{HCCl}_{3}\right), 4.88-5.01(\mathrm{~m}, 1 \mathrm{H}$, $\left.\mathrm{CH} \mathrm{HCCl}_{3}\right)$.

${ }^{13}$ C NMR (150 MHz, $\mathrm{CDCl}_{3}, 323 \mathrm{~K}$, Rotamere): $\delta=28.4,38.1,38.9,55.4,55.7,57.7,57.8$, 70.1, 71.2, 75.7, 80.6, 80.7, 94.6, 94.7, 153.6, 154.4, 172.6.

HRMS (ESI): $m / z[\mathrm{M}+\mathrm{Na}]^{+}$für $\mathrm{C}_{12} \mathrm{H}_{18} \mathrm{Cl}_{3} \mathrm{NNaO}_{5}$ ber.: 384.0143 , gef.: 384.0142 .

(2S,4S)-N-Boc-4-(tert-Butyldimethylsilyloxy)-pyrrolidin-2-carbonsäure-(2,2,2trichlorethyl)ester (58)

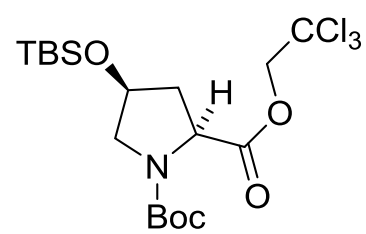

Zu einer Mischung aus 4-Hydroxypyrrolidin 136 (10,16 g, $28.0 \mathrm{mmol})$ und Imidazol (5.71 g, $84.1 \mathrm{mmol})$ in wasserfreiem DMF $(120 \mathrm{~mL})$ wurde tropfenweise eine Lösung von TBSCl $(6.21 \mathrm{~g}, 41.2 \mathrm{mmol})$ in wasserfreiem DMF $(50 \mathrm{~mL})$ zugegeben. Nach vollständiger Zugabe wurde das Eisbad entfernt und das Reaktionsgemisch $18 \mathrm{~h}$ lang bei RT gerührt. Nach Verdünnen mit pH 3 Puffer $(600 \mathrm{~mL})$ wurde die Mischung mit MTBE $(3 \times 250 \mathrm{~mL})$ extrahiert. Die vereinigten organischen Extrakte wurden mit 10\%iger Zitronensäure, $\mathrm{H}_{2} \mathrm{O}$ und ges. NaCl-Lsg (je $150 \mathrm{~mL}$ ) gewaschen und anschließend mit $\mathrm{MgSO}_{4}$ entwässert. Das Lösungsmittel wurde am Rotationsverdampfer entfernt und der Rückstand durch Säulenchromatographie $\left(\mathrm{SiO}_{2}\right.$, Petrolether/MTBE, 6:1) gereinigt. Es wurden $13.2 \mathrm{~g}$ (27.7 mmol, 99\%) des TBS-Pyrrolidins 58 als farbloser Feststoff isoliert.

DC: $R_{f}=0.47($ Heptan/MTBE, 5:1)

Alle relevanten analytischen Daten stimmen mit den veröffentlichten Werten überein. ${ }^{[36]}$ 
(4S)-5-((2'-Benzhydryloxy)carbonyl)-3'-methyl-1'H-indol-4'-yl)methyl)-4-((tert-

butyldimethylsilyl)oxy)-2- $N$-(tert-butyloxycarbonyl)- $L$-glutaminsäure-2,2,2-

trichlorethylester (137)

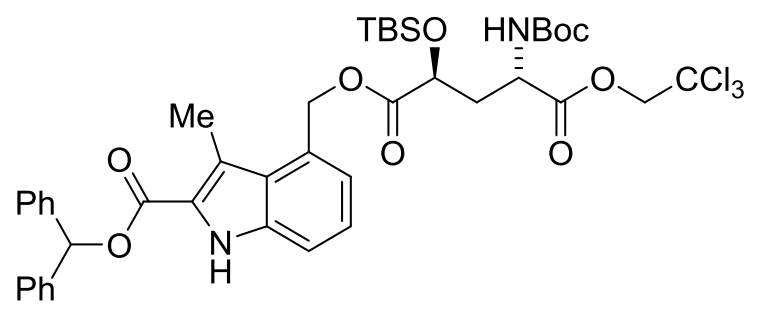

Eine Lösung des Indolylalkohols $\mathbf{4 0}^{[36]}(1.13 \mathrm{~g}, 3.03 \mathrm{mmol})$ in wasserfreiem THF $(11 \mathrm{~mL})$ wurde bei $0{ }^{\circ} \mathrm{C}$ tropfenweise $\mathrm{zu}$ einer Suspension von $\mathrm{NaH}(181 \mathrm{mg}, 7.57 \mathrm{mmol})$ in wasserfreiem THF (11 mL) gegeben. Nach vollständiger Zugabe wurde das Gemisch weitere 15 min lang gerührt und anschließend auf $-78^{\circ} \mathrm{C}$ gekühlt. Danach wurde eine Lösung des $\gamma$-Lactams $\mathbf{5 9}^{[36]}(2.97 \mathrm{~g}, 6.06 \mathrm{mmol}, 2$ Äq.) in wasserfreiem THF (45 mL) langsam zugetropft. Die Mischung wurde nach vollständiger Zugabe $2 \mathrm{~h}$ lang bei $-78^{\circ} \mathrm{C}$ gerührt und dann mit ges. $\mathrm{NH}_{4} \mathrm{Cl}$-Lsg. (50 mL) versetzt. Nach Auftauen der Mischung auf RT (ca. $30 \mathrm{~min}$ ) wurden EtOAc $(100 \mathrm{~mL})$ und $\mathrm{H}_{2} \mathrm{O}(50 \mathrm{~mL})$ zugegeben und die Phasen getrennt. Die wässrige Phase wurde mit EtOAc $(3 \times 50 \mathrm{~mL})$ extrahiert, die organischen Extrakte vereinigt und mit ges. NaCl-Lsg. (100 mL) gewaschen. Nach Entwässern mit $\mathrm{Na}_{2} \mathrm{SO}_{4}$ wurden die Lösungsmittel am Rotationsverdampfer entfernt und der Rückstand durch Säulenchromatographie $\left(\mathrm{SiO}_{2}, 300 \mathrm{~g}\right.$, Petrolether/EtOAc, 4:1) gereinigt. Es wurden $2.32 \mathrm{~g}$ (2.70 mmol, 89\%) des Triesters 137 als farbloser Schaum erhalten. Nicht umgesetzter Indolylalkohol 40 (101 mg, 9\%) und überschüssiges $\gamma$-Lactam 59 (1.26 g, 85\%) wurden unverändert zurück gewonnen.

Spez. Drehung: $[\alpha]_{\mathrm{D}}{ }^{24}=-24.5\left(\mathrm{CHCl}_{3}, c=1\right)$.

DC: $R_{f}=0.62($ Heptan/EtOAc, $2: 1)$.

IR: $\tilde{v}=702(\mathrm{~s}), 1150(\mathrm{~s}), 1250(\mathrm{~s}), 1697(\mathrm{~m}), 3340(\mathrm{w}) \mathrm{cm}^{-1}$.

${ }^{1} \mathbf{H}-\mathbf{N M R}\left(300 \mathrm{MHz}, \mathrm{CDCl}_{3}\right): \delta=0.00\left(\mathrm{~s}, 3 \mathrm{H}, \mathrm{CH}_{3}\right), 0.05\left(\mathrm{~s}, 3 \mathrm{H}, \mathrm{CH}_{3}\right), 0.89(\mathrm{~s}, 9 \mathrm{H}, t \mathrm{Bu}), 1.42$ (s, 9H, Boc), 2.11-2.35 (m, 2H, $\left.\mathrm{CH}_{2}\right), 2.84\left(\mathrm{~s}, 3 \mathrm{H}, \mathrm{CH}_{3}\right), 4.40$ (dd, J=9.3, $2.5 \mathrm{~Hz}, 1 \mathrm{H}$, CHOTBS), 4.55 (bd, $\left.J=11.7 \mathrm{~Hz}, 2 \mathrm{H}, \mathrm{CHHCCl}_{3}, \mathrm{CHNH}\right), 4.91 \quad(\mathrm{~d}, J=11.9 \mathrm{~Hz}$, 
$\left.1 \mathrm{H}, \mathrm{CH} H \mathrm{CCl}_{3}\right), 5.41(\mathrm{~d}, J=7.8 \mathrm{~Hz}, 1 \mathrm{H}, \mathrm{NH}), 5.49-5.61\left(\mathrm{~m}, 2 \mathrm{H}, \mathrm{CH}_{2} \mathrm{O}\right), 7.19(\mathrm{~s}, 1 \mathrm{H}$, $\left.\mathrm{CH}(\mathrm{Ph})_{2}\right), 7.09-7.46(\mathrm{~m}, 13 \mathrm{H}, 2 \times \mathrm{Ph}$, Indol), 8.97 (s, 1H, Indolyl-NH).

${ }^{13}$ C-NMR (75 MHz, $\left.\mathrm{CDCl}_{3}\right): \delta=-5.6,-5.0,12.1,18.0,25.6,28.2,35.9,51.1,65.4,69.6,74.2$, 77.6, 80.1, 94.5, 112.9, 120.5, 122.8, 123.6, 125.3, 126.4, 127.2, 128.1, 128.6, 129.3, 136.5, $139.8,155.3,161.4,170.8,172.4$.

HRMS (ESI): $m / z[\mathrm{M}+\mathrm{H}]^{+}$für $\mathrm{C}_{42} \mathrm{H}_{52} \mathrm{Cl}_{3} \mathrm{~N}_{2} \mathrm{O}_{9} \mathrm{Si}$ ber.: 861.2502; gef.: 861.2499.

(4S)-5-((2'-Benzhydryloxy)carbonyl)-3'-methyl-1'H-indol-4'-yl)methyl)-4-((tertbutyldimethylsilyl)oxy)-2- $N$-(tert-butyloxycarbonyl)- $L$-glutaminsäure (138)<smiles>CCCCC(CC(OC(C)(C)C)C(=O)O)C(=O)OCc1cccc2[nH]c(C(=O)OC(c3ccccc3)c3ccccc3)c(C)c12</smiles>

Der Triester $137(2 \mathrm{~g}, 2.31 \mathrm{mmol})$ wurde in THF $(40 \mathrm{~mL})$ gelöst und mit $\mathrm{KH}_{2} \mathrm{SO}_{4}$-Lsg. $(12.5 \mathrm{~mL}, 1 \mathrm{M})$ sowie aktiviertem Zinkpulver $^{1}(5.46 \mathrm{~g}, 83.5 \mathrm{mmol})$ versetzt. Die Mischung wurde $10 \mathrm{~h}$ lang bei $45^{\circ} \mathrm{C}$ gerührt und dabei ständig mit Ultraschall behandelt. Nach Filtration wurde das Gemisch im Vakuum eingeengt, der Rückstand in 5\%iger Zitronensäure $(100 \mathrm{~mL})$ aufgenommen und mit $\mathrm{CHCl}_{3}(5 \times 25 \mathrm{~mL})$ extrahiert. Die vereinigten organischen Extrakte wurden mit $\mathrm{Na}_{2} \mathrm{SO}_{4}$ entwässert und am Rotationsverdampfer eingeengt. Nach Säulenchromatographie $\left(\mathrm{SiO}_{2}, 150 \mathrm{~g}, \mathrm{CH}_{2} \mathrm{Cl}_{2} / \mathrm{MeOH}, 99: 1 \rightarrow 10: 1 \rightarrow 9: 1\right)$ wurden $1.38 \mathrm{~g}$ (1.89 mmol, 82\%) der Säure 138 als farbloses Glas erhalten, getrennt von nicht umgesetzten Ausgangsmaterial (260 mg, 13\%).

Spez. Drehung: $[\alpha]_{\mathrm{D}}{ }^{24}=-23.2\left(\mathrm{CHCl}_{3}, c=1\right)$

DC: $R_{f}=0.33\left(\mathrm{CH}_{2} \mathrm{Cl}_{2} / \mathrm{MeOH} / \mathrm{HCOOH}, 100: 5: 1\right)$.

IR: $\tilde{v}=748(\mathrm{~s}), 833(\mathrm{~m}), 1242(\mathrm{~s}), 1697(\mathrm{~m}), 2450(\mathrm{w}), 2931(\mathrm{w}) \mathrm{cm}^{-1}$.

\footnotetext{
${ }^{1}$ Das Zn-Pulver wurdezur Aktivierung über einer Glasfritte für $1 \mathrm{~min}$ mit 2\%-iger $\mathrm{HCl}$ behandelt und anschließend mit 2\%-iger $\mathrm{HCl}(3 \mathrm{x}), \mathrm{H}_{2} \mathrm{O}(3 \mathrm{x})$, EtOH $(2 \mathrm{x})$ und $\mathrm{Et}_{2} \mathrm{O}$ gewaschen.
} 
${ }^{1} \mathbf{H}-\mathbf{N M R}\left(300 \mathrm{MHz}, \mathrm{CDCl}_{3}\right): \delta=-0.04\left(\mathrm{~s}, 3 \mathrm{H}, \mathrm{CH}_{3}\right), 0.01\left(\mathrm{~s}, 3 \mathrm{H}, \mathrm{CH}_{3}\right), 0.84(\mathrm{~s}, 9 \mathrm{H}, t \mathrm{Bu})$, 1.40 (s, 9H, Boc), 2.13 (bs, 1H), 2.26 (bs, 1H), 2.83 (s, 3H, $\mathrm{CH}_{3}$ ), 4.30 (bs, 1H, CH), 4.44 (bs, $1 \mathrm{H}, \mathrm{CH}), 5.44\left(\mathrm{dd}, J=18.3,12.5 \mathrm{~Hz}, 3 \mathrm{H}, \mathrm{CH}_{2} \mathrm{O}, \mathrm{NH}\right), 7.18\left(\mathrm{~s}, 1 \mathrm{H}, \mathrm{CH}(\mathrm{Ph})_{2}\right), 7.09-7.45(\mathrm{~m}$, 13H, $2 \times \mathrm{Ph}$, Indol), 8.94 (s, 1H, Indolyl-NH).

${ }^{13}$ C-NMR (75 MHz, $\left.\mathrm{CDCl}_{3}\right): \delta=-5.7,-5.2,12.2,17.9,25.5,28.2,36.0,50.9,65.1,69.5,77.7$, $79.9,112.9,120.3,122.3,123.4,125.2,126.1,127.1,128.0,128.5,129.4,136.7,139.8,155.9$, 162.0, 172.7, 176.7.

HRMS (ESI): $m / z[\mathrm{M}+\mathrm{H}]^{+}$für $\mathrm{C}_{40} \mathrm{H}_{51} \mathrm{~N}_{2} \mathrm{O}_{9}$ Si ber.: 731.3358 ; gef.: 731.3365 .

3-[5'-((2"-Benzhydryloxy)carbonyl)-3"'-methyl-1"'H-indol-4"'-yl)methyl)-(S)-4'-((tertbutyldimethylsilyl)oxy)-2'-N'-(tert-butyloxycarbonyl)- $L$-glutaminyl)-propionylthio]-(R)2-Azidopropionsäureallylester (139)

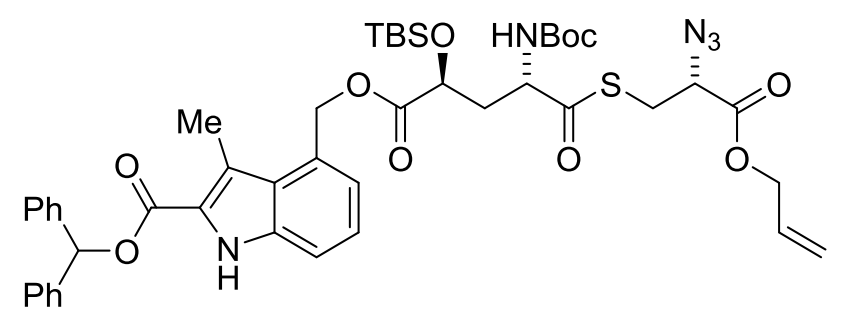

(R)-2-Azido-3-(tritylthio)propansäureallylester ${ }^{[37]}$ (880 mg, $2.05 \mathrm{mmol}$ ) wurde in $\mathrm{CH}_{2} \mathrm{Cl}_{2} / \mathrm{TFA}$ (16 mL, 9:1) gelöst, mit $\mathrm{Et}_{3} \mathrm{SiH}(600 \mu \mathrm{L}, 3.72 \mathrm{mmol})$ versetzt und $1 \mathrm{~h}$ lang bei RT gerührt. Die Mischung wurde mit Toluol $(20 \mathrm{ml})$ verdünnt und am Hochvakuum eingeengt. Der Rückstand wurde in wasserfreiem THF $(16 \mathrm{~mL})$ gelöst und auf $0{ }^{\circ} \mathrm{C}$ gekühlt. Danach wurden die Carbonsäure 138 (1.0 g, $1.37 \mathrm{mmol}), \mathrm{EtNi} \operatorname{Pr}_{2}(465 \mu \mathrm{L}, 3.42 \mathrm{mmol})$ und PyAOP (856 mg, $1.64 \mathrm{mmol}$ ) zugegeben. Das Kühlbad wurde entfernt und die Mischung $3 \mathrm{~h}$ lang bei RT gerührt, anschließend mit EtOAc $(200 \mathrm{~mL})$ verdünnt und mit pH 3 Puffer $(2.5 \mathrm{M}, 50 \mathrm{~mL})$, halb-ges. $\mathrm{NaHCO}_{3}$-Lsg $(3 \times 50 \mathrm{~mL})$ und ges. NaCl-Lsg. $(100 \mathrm{~mL})$ gewaschen. Die organische Phase wurde mit $\mathrm{MgSO}_{4}$ entwässert und im Vakuum eingeengt. Reinigung des Rückstands durch Säulenchromatographie $\left(\mathrm{SiO}_{2}, 250 \mathrm{~g}\right.$, Petrolether/EtOAc, 3:1) ergab 1.18 g (1.32 mmol, 96\%) des Thioesters 139 als farblosen Schaum.

Spez. Drehung: $[\alpha]_{\mathrm{D}}{ }^{24}=-57.2\left(\mathrm{CHCl}_{3}, c=1\right)$.

DC: $R_{f}=0.57($ Heptan/EtOAc, 2:1). 
IR: $\tilde{v}=694(\mathrm{~m}), 748(\mathrm{~m}), 964(\mathrm{w}), 1157$ (s), 1242 (s), $1697(\mathrm{~s}), 2113(\mathrm{w}), 2932(\mathrm{w}) \mathrm{cm}^{-1}$.

${ }^{1}$ H-NMR $\left(300 \mathrm{MHz}, \mathrm{CDCl}_{3}\right): \delta=-0.02\left(\mathrm{~s}, 3 \mathrm{H}, \mathrm{CH}_{3}\right), 0.03\left(\mathrm{~s}, 3 \mathrm{H}, \mathrm{CH}_{3}\right), 0.88(\mathrm{~s}, 9 \mathrm{H}, t \mathrm{Bu})$, 1.44 (s, 9H, Boc), 2.05-2.27 (m, 2H, $\left.\mathrm{CH}_{2}\right), 2.85$ (s, 3H, $\left.\mathrm{CH}_{3}\right), 3.14$ (dd, J=14.1, $7.9 \mathrm{~Hz}$, 1H,CHHS), 3.33 (dd, $J=13.9,5.5 \mathrm{~Hz}, 1 \mathrm{H}, \mathrm{CH} H \mathrm{~S}), 4.05$ (dd, $J=7.8,5.5 \mathrm{~Hz}, 1 \mathrm{H}, \mathrm{CHN}_{3}$ ), 4.38 (dd, $J=8.8,2.4 \mathrm{~Hz}, 1 \mathrm{H}, \mathrm{CHOTBS}), 4.46$ (bs, 1H, CHNHBoc), 4.65 (d, $J=5.3 \mathrm{~Hz}, 2 \mathrm{H}$, $\left.\mathrm{CH}_{2} \mathrm{CH}=\mathrm{CH}_{2}\right), 5.25-5.37\left(\mathrm{~m}, 2 \mathrm{H}, \mathrm{CH}_{2} \mathrm{CH}=\mathrm{CH}_{2}\right), 5.48-5.62\left(\mathrm{~m}, 3 \mathrm{H}, \mathrm{CH}_{2} \mathrm{O}, \mathrm{NH}\right), 5.83-5.96$ (m, $\left.1 \mathrm{H}, \mathrm{CH}_{2} \mathrm{CH}=\mathrm{CH}_{2}\right), 7.19\left(\mathrm{~s}, 1 \mathrm{H}, \mathrm{CH}(\mathrm{Ph})_{2}\right), 7.10-7.45$ (m, 13H, $2 \times \mathrm{Ph}$, Indol), $8.91(\mathrm{~s}, 1 \mathrm{H}$, Indolyl-NH).

${ }^{13}$ C-NMR (75 MHz, $\left.\mathrm{CDCl}_{3}\right): \delta=-5.6,-5.1,12.1,18.0,25.6,28.3,30.0,36.3,58.1,61.3,65.3$, 66.7, 69.4, 77.6, 80.4, 112.9, 119.4, 120.5, 122.7, 123.6, 125.4, 126.4, 127.2, 128.1, 128.6, $129.4,131.0,136.5,139.9,155.1,161.4,168.2,172.3,200.5$.

HRMS (ESI): $m / z[\mathrm{M}+\mathrm{Na}]^{+}$für $\mathrm{C}_{46} \mathrm{H}_{57} \mathrm{~N}_{5} \mathrm{O}_{9}$ SSiNa ber.: 922.3488 ; gef.: 922.3487 .

\section{(1'S,3'S)-2-(3'-(2“"-((Benzhydryloxy)carbonyl)-3“"-methyl-1 “ $H$-indol-4"6-}

yl)methoxycarbonyl))-3'-tert-butyldimethylsilyloxy-1'-tert-butoxycarbonylaminoprop1'-yl)thiazol-4-carbonsäureallylester (95)

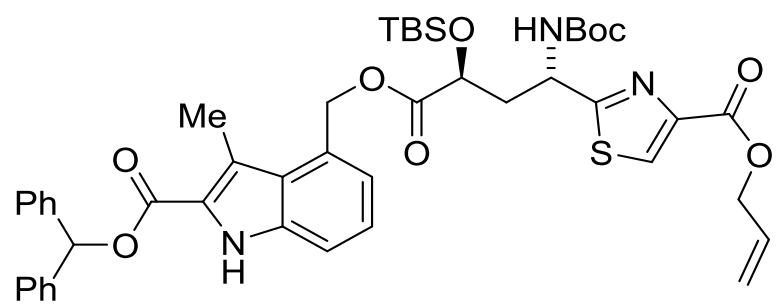

Eine Lösung des Thioesters 139 (1.17 g, $1.3 \mathrm{mmol})$ in wasserfreiem THF (26 mL) wurde auf $-20^{\circ} \mathrm{C}$ gekühlt und eine Lösung von $\mathrm{PPh}_{3}(515 \mathrm{mg}, 1.96 \mathrm{mmol})$ in THF (4 mL) langsam zugetropft. Nach vollständiger Zugabe wurde das Gemisch innerhalb von $2 \mathrm{~h}$ auf RT erwärmt und anschließend $14 \mathrm{~h}$ lang bei $40^{\circ} \mathrm{C}$ gerührt. Die Mischung wurde danach im Vakuum eingeengt und der Rückstand in wasserfreiem $\mathrm{CH}_{2} \mathrm{Cl}_{2}(27 \mathrm{~mL})$ gelöst. Die Lösung wurde auf $-20{ }^{\circ} \mathrm{C}$ gekühlt und mit $\mathrm{DBU}(410 \mu \mathrm{L}, 2.73 \mathrm{mmol})$ und $\mathrm{BrCCl}_{3}(136 \mu \mathrm{L}, 1.36 \mathrm{mmol})$ versetzt. Die Mischung wurde $1 \mathrm{~h}$ lang bei $-20{ }^{\circ} \mathrm{C}$ gerührt, mit pH 7 Puffer $(50 \mathrm{~mL})$ verdünnt und mit $\mathrm{CH}_{2} \mathrm{Cl}_{2}(3 \times 20 \mathrm{~mL})$ extrahiert. Die vereinigten organischen Extrakte wurden mit $\mathrm{MgSO}_{4}$ entwässert und am Rotationsverdampfer eingeengt. Reinigung des Rückstands durch Säulenchromatographie $\left(\mathrm{SiO}_{2}, 80 \mathrm{~g}\right.$, Petrolether/EtOAc, 3:1) lieferte $1.01 \mathrm{~g}$ (1.17 mmol, 90\%) des Thiazols 95 als farblosen Schaum. 
Spez. Drehung: $[\alpha]_{\mathrm{D}}{ }^{24}=-37.3\left(\mathrm{CHCl}_{3}, c=1\right)$.

DC: $R_{f}=0.48($ Heptan/EtOAc, $2: 1)$.

IR: $\tilde{v}=748$ (s), 1172 (b), $1226(\mathrm{~s}), 1342(\mathrm{w}), 1705(\mathrm{~s}), 2931(\mathrm{w}) \mathrm{cm}^{-1}$.

${ }^{1} \mathbf{H}-\mathbf{N M R}\left(300 \mathrm{MHz}, \mathrm{CDCl}_{3}\right): \delta=-0.02\left(\mathrm{~s}, 3 \mathrm{H}, \mathrm{SiCH}_{3}\right), 0.00\left(\mathrm{~s}, 3 \mathrm{H}, \mathrm{SiCH}_{3}\right), 0.90(\mathrm{~s}, 9 \mathrm{H}, t \mathrm{Bu})$, 1.44 (s, 9H, Boc), 2.45-2.49 (m, 2H, $\mathrm{CH}_{2}$ ), 2.87 (s, 3H, $\mathrm{CH}_{3}$ ), 4.47 (dd, J=8.1, $4.5 \mathrm{~Hz}$, 1H,CHOTBS), 4.84 (d, $\left.J=5.8 \mathrm{~Hz}, 2 \mathrm{H}, \mathrm{CH}_{2} \mathrm{CH}=\mathrm{CH}_{2}\right), 5.21(\mathrm{~m}, 1 \mathrm{H}, \mathrm{CHNHBoc}), 5.29$ (d, $\left.J=10.3 \mathrm{~Hz}, 1 \mathrm{H}, \mathrm{CH}_{2} \mathrm{CH}=\mathrm{CHH}\right), 5.40\left(\mathrm{dd}, J=17.2,1.3 \mathrm{~Hz}, 1 \mathrm{H}, \mathrm{CH}_{2} \mathrm{CH}=\mathrm{CH} H\right), 5.57$ (q, $\left.J=12.2 \mathrm{~Hz}, 2 \mathrm{H}, \mathrm{CH}_{2} \mathrm{O}\right), 5.79(\mathrm{~d}, J=7.9 \mathrm{~Hz}, 1 \mathrm{H}, \mathrm{N} H \mathrm{Boc}), 6.02\left(\mathrm{~m}, 1 \mathrm{H}, \mathrm{CH}_{2} \mathrm{CH}=\mathrm{CH}_{2}\right), 7.21$ (s, 1H, $\left.\mathrm{CH}(\mathrm{Ph})_{2}\right), 7.14-7.48$ (m, 13H, $2 \times \mathrm{Ph}$, Indol), 8.10 (s, 1H, S-CH), 9.13 (s, 1H, Indolyl$\mathrm{NH})$.

${ }^{13}$ C-NMR (75 MHz, $\left.\mathrm{CDCl}_{3}\right): \delta=-5.6,-5.1,12.1,18.0,25.6,28.2,38.8,50.1,65.1,69.5,77.6$, $80.1,112.8,118.7,120.5,122.6,123.5,125.3,126.3,127.2,127.6,128.0,128.6,129.4,131.8$, $136.6,139.9,146.9,155.1,160.9,161.5,172.5,173.8$.

HRMS (ESI): $m / z[\mathrm{M}+\mathrm{H}]^{+}$für $\mathrm{C}_{46} \mathrm{H}_{56} \mathrm{~N}_{3} \mathrm{O}_{9} \mathrm{SSi}$ ber.: 854.3501 ; gef.: 854.3508 .

(2R,2'S,3'R)-2-Azido-3-( $N$-Boc-O-(tert-butyldimethylsilyl)threonylthio)propansäureallylester (141)

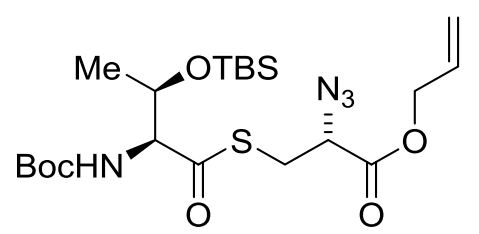

(R)-2-Azido-3-(tritylthio)propansäureallylester ${ }^{[37]}$ (10.74 g, $25.0 \mathrm{mmol}$ ) wurde in $\mathrm{CH}_{2} \mathrm{Cl}_{2} / \mathrm{TFA}$ $(100 \mathrm{~mL}, 1: 1)$ gelöst, mit $\mathrm{Et}_{3} \mathrm{SiH}(8.0 \mathrm{~mL}, 50.0 \mathrm{mmol})$ versetzt und $30 \mathrm{~min}$ lang bei $0{ }^{\circ} \mathrm{C}$ gerührt. Die Mischung wurde mit Toluol $(100 \mathrm{ml})$ verdünnt und im Vakuum eingeengt. Der Rückstand wurde in wasserfreiem $\mathrm{CH}_{2} \mathrm{Cl}_{2}$ (305 mL) gelöst, $L$-Boc-Thr(TBS)-OH $(\mathbf{1 4 0})^{[201]}(9.17 \mathrm{~g}, 27.5 \mathrm{mmol})$ zugegeben, die Lösung auf $0{ }^{\circ} \mathrm{C}$ gekühlt und nacheinander HOBt (3.80 g, $27.5 \mathrm{mmol})$ und $\mathrm{Et}_{3} \mathrm{~N}(3.84 \mathrm{~mL}, 27.5 \mathrm{mmol})$ zugefügt. Das Gemisch wurde $15 \mathrm{~min}$ lang bei $0{ }^{\circ} \mathrm{C}$ gerührt und danach EDCI (5.27 g, $\left.27.5 \mathrm{mmol}\right)$ zugegeben. Nach Entfernen des Kühlbads wurde das Gemisch $24 \mathrm{~h}$ lang bei RT gerührt und anschließend mit EtOAc (1 L) verdünnt. Die Lösung wurde mit $\mathrm{KHSO}_{4}$-Lsg. (1 M, $\left.250 \mathrm{~mL}\right)$ und ges. 
NaCl-Lsg. (250 mL) gewaschen und mit $\mathrm{MgSO}_{4}$ entwässert. Nach Einengen im Vakuum wurde der Rückstand durch Säulenchromatographie $\left(\mathrm{SiO}_{2}, 750 \mathrm{~g}\right.$, Petrolether/MTBE, 10:1 $\rightarrow 6: 1)$ gereinigt. Es wurden $9.81 \mathrm{~g}$ (19.5 mmol, 78\%) des Thioesters 141 als farbloses Öl erhalten.

Spez. Drehung: $[\alpha]_{\mathrm{D}}{ }^{24}=-89.2\left(\mathrm{CHCl}_{3}, c=1\right)$.

DC: $R_{f}=0.33($ Heptan/MTBE, 5:1).

IR: $\tilde{v}=757(\mathrm{~m}), 837(\mathrm{~m}), 1161(\mathrm{~s}), 1484(\mathrm{~m}), 1693(\mathrm{~m}), 1720(\mathrm{~s}), 1745(\mathrm{~m}), 2116(\mathrm{~m})$, $2856(\mathrm{w}), 2929(\mathrm{w}) \mathrm{cm}^{-1}$.

${ }^{1} \mathbf{H}-\mathbf{N M R}\left(250 \mathrm{MHz}, \mathrm{CDCl}_{3}\right): \delta=-0.03\left(\mathrm{~s}, 3 \mathrm{H}, \mathrm{CH}_{3}\right), 0.03\left(\mathrm{~s}, 3 \mathrm{H}, \mathrm{CH}_{3}\right), 0.83\left(\mathrm{~s}, 9 \mathrm{H},{ }^{t} \mathrm{Bu}\right), 1.18$ (d, $J=6.2 \mathrm{~Hz}, 3 \mathrm{H}, \mathrm{CH}_{3}$ ), 1.49 (s, 9H, Boc), 3.04 (dd, $\left.J=13.8,8.3 \mathrm{~Hz}, 1 \mathrm{H}, \mathrm{CHHS}\right), 3.36$ (dd, $J=13.7,5.5 \mathrm{~Hz}, 1 \mathrm{H}, \mathrm{CH} H \mathrm{~S}), 4.01\left(\mathrm{dd}, J=8.1,5.5 \mathrm{~Hz}, 1 \mathrm{H}, \mathrm{CHN}_{3}\right), 4.19(\mathrm{~d}, J=9.3 \mathrm{~Hz}, 1 \mathrm{H}$, $\mathrm{C} H \mathrm{NHBoc}), 4.48\left(\mathrm{~m}, 1 \mathrm{H}, \mathrm{CHCH}_{3}\right), 4.69\left(\mathrm{dd}, J=5.7,0.9 \mathrm{~Hz}, 2 \mathrm{H}, \mathrm{CH}_{2} \mathrm{CH}=\mathrm{CH}_{2}\right), 5.27-5.33$ $(\mathrm{m}, 2 \mathrm{H}, \mathrm{CH}=\mathrm{CH} H, \mathrm{NH}), 5.40(\mathrm{~d}, J=1.1 \mathrm{~Hz}, \mathrm{CH}=\mathrm{CH} H), 5.85-6.00\left(\mathrm{~m}, 1 \mathrm{H}, \mathrm{CH}=\mathrm{CH}_{2}\right)$.

${ }^{13}$ C-NMR (63 MHz, $\left.\mathrm{CDCl}_{3}\right): \delta=-5.3,-4.5,17.9,20.9,25.7,28.3,30.1,61.3,66.1,66.7,68.4$, $80.6,119.4,131.0,155.8,168.3,201.2$.

HRMS (ESI): $m / z[\mathrm{M}+\mathrm{Na}]^{+}$für $\mathrm{C}_{21} \mathrm{H}_{38} \mathrm{~N}_{4} \mathrm{NaO}_{6} \mathrm{SSi}$ ber.: 525.2174; gef.: 525.2177.

(1'S,2'R)-2-(2-tert-Butyldimethylsilyl-1-(tert-butoxycarbonylamino)propyl)thiazol-4carbonsäureallylester (142)

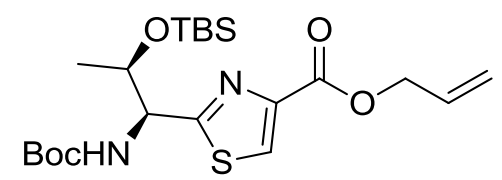

Eine Lösung des Thioesters 141 (1.56 g, $3.10 \mathrm{mmol})$ in wasserfreiem THF (62 mL) wurde auf $-20{ }^{\circ} \mathrm{C}$ gekühlt und eine Lösung von $\mathrm{PPh}_{3}(1.22 \mathrm{~g}, 4.66 \mathrm{mmol})$ in wasserfreiem THF $(9.5 \mathrm{~mL})$ langsam zugetropft. Nach vollständiger Zugabe wurde das Gemisch langsam auf RT erwärmt und anschließend $18 \mathrm{~h}$ lang bei $40^{\circ} \mathrm{C}$ gerührt. Nach Einengen im Vakuum wurde der Rückstand in wasserfreiem $\mathrm{CH}_{2} \mathrm{Cl}_{2}(62 \mathrm{~mL})$ gelöst, auf $-20^{\circ} \mathrm{C}$ gekühlt, mit DBU (974 $\mu \mathrm{mol}$, $6.51 \mathrm{mmol})$ versetzt und $5 \mathrm{~min}$ lang gerührt. Danach wurde $\mathrm{BrCCl}_{3}(321 \mu \mathrm{L}, 3.26 \mathrm{mmol})$ tropfenweise zugegeben und die Mischung langsam auf RT erwärmt (1-2 h). Nach Verdünnen 
mit EtOAc $(200 \mathrm{~mL})$ wurde mit $\mathrm{KHSO}_{4}$-Lsg. (100 mL, $\left.1 \mathrm{M}\right)$ und ges. NaCl-Lsg. (100 mL) gewaschen und mit $\mathrm{MgSO}_{4}$ entwässert. Die Lösungsmittel wurden im Vakuum entfernt und das Rohprodukt durch Säulenchromatographie $\left(\mathrm{SiO}_{2}, 250 \mathrm{~g}\right.$, Petrolether/EtOAc, 4:1) gereinigt. Es wurden $1.19 \mathrm{~g}(2.61 \mathrm{mmol}, 84 \%)$ des Thiazols 142 als farbloses, zähes Öl erhalten.

Spez. Drehung: $[\alpha]_{\mathrm{D}}{ }^{24}=-21.7\left(\mathrm{CHCl}_{3}, c=1\right)$.

DC: $R_{f}=0.41($ Heptan/EtOAc, $4: 1)$.

IR: $\tilde{v}=777(\mathrm{~m}), 835(\mathrm{~m}), 1163(\mathrm{~m}), 1482(\mathrm{~m}), 1716(\mathrm{~s}), 2856(\mathrm{w}), 2929(\mathrm{w}), 2953(\mathrm{w}) \mathrm{cm}^{-1}$.

${ }^{1} \mathbf{H}-\mathbf{N M R}\left(400 \mathrm{MHz}, \mathrm{CDCl}_{3}, 323 \mathrm{~K}\right): \delta=-0.29\left(\mathrm{~s}, 3 \mathrm{H}, \mathrm{CH}_{3}\right),-0.03\left(\mathrm{~s}, 3 \mathrm{H}, \mathrm{CH}_{3}\right), 0.76(\mathrm{~s}, 9 \mathrm{H}$, $\mathrm{Si} t \mathrm{Bu}), 1.23$ (d, $J=6.1 \mathrm{~Hz}, 3 \mathrm{H}, \mathrm{CH}_{3}$ ), 1.47 (s, 9H, Boc), 4.63 (bd, $J=4.1 \mathrm{~Hz}, 1 \mathrm{H}, \mathrm{CHOTBS}$ ), 4.78-4.92 (m, 3H, $\left.\mathrm{CH}_{2} \mathrm{CH}=\mathrm{CH}_{2}, \mathrm{CHNH}\right), 5.26\left(\mathrm{~d}, J=10.2 \mathrm{~Hz}, 1 \mathrm{H}, \mathrm{CH}_{2} \mathrm{CH}=\mathrm{CHH}\right), 5.38(\mathrm{~d}$, $\left.J=17.2 \mathrm{~Hz}, 1 \mathrm{H}, \mathrm{CH}_{2} \mathrm{CH}=\mathrm{CH} H\right), 5.57(\mathrm{bs}, 1 \mathrm{H}, \mathrm{NH}), 5.97-6.06\left(\mathrm{~m}, 1 \mathrm{H}, \mathrm{CH}_{2} \mathrm{CH}=\mathrm{CH}_{2}\right), 8.06$ (s, 1H, Thiazol-CH).

${ }^{13}$ C-NMR $\left(100 \mathrm{MHz}, \mathrm{CDCl}_{3}\right): \delta=-5.6,-4.9,17.8,20.4,25.6,28.3,58.7,65.8,70.4,80.3$, $118.6,127.3,131.9,147.1,155.7,160.9,174.8$.

HRMS (ESI): $m / z[\mathrm{M}+\mathrm{H}]^{+}$für $\mathrm{C}_{21} \mathrm{H}_{37} \mathrm{~N}_{2} \mathrm{O}_{5} \mathrm{SSi}$ ber.: 457.2187; gef.: 457.2192 .

(1'S,2'R,2"S,3"R)-2-(1-(2-(((9H-Fluoren-9-yl)methoxy)carbonylamino)-3-tertbutyldimethylsilylbutanamido)-2-hydroxypropyl)thiazol-4-carbonsäureallylester (144)

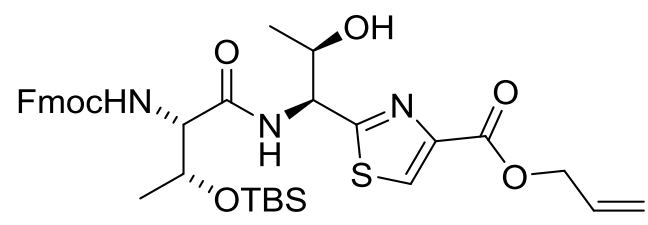

Das Thiazol 142 (983 mg, $2.15 \mathrm{mmol})$ wurde in EtOAc $(10 \mathrm{ml})$ gelöst und mit konz. $\mathrm{HCl}$ $(10 \mathrm{~mL})$ versetzt. Die Mischung wurde $30 \mathrm{~min}$ lang bei RT gerührt, mit Toluol $(50 \mathrm{~mL})$ verdünnt und anschließend im Vakuum eingeengt. Der Rückstand wurde in $\mathrm{MeOH}(10 \mathrm{~mL})$ aufgenommen und erneut eingeengt. Der erhaltene Rückstand wurde in wasserfreiem $\mathrm{CH}_{2} \mathrm{Cl}_{2}$ (45 mL) gelöst, L-Fmoc-Thr(TBS)-OH (143) $)^{[202]}(1.08 \mathrm{~g}, 2.37 \mathrm{mmol})$ zugegeben, das Gemisch auf $0{ }^{\circ} \mathrm{C}$ gekühlt und nach Zugabe von $\mathrm{HOBt}\left(445 \mathrm{mg}, 3.23 \mathrm{mmol}\right.$ ) und $\mathrm{Et}_{3} \mathrm{~N}$ $(330 \mu \mathrm{L}, 2.47 \mathrm{mmol}) 15 \mathrm{~min}$ lang bei $0^{\circ} \mathrm{C}$ gerührt. Anschließend wurde EDCI (515 mg, 
$2.69 \mathrm{mmol}$ ) zugegeben und die Reaktion $18 \mathrm{~h}$ lang bei RT gerührt. Danach wurde mit EtOAc (135 mL) verdünnt, mit pH 3 Puffer und ges. NaCl-Lsg. (je $50 \mathrm{~mL}$ ) gewaschen und mit $\mathrm{MgSO}_{4}$ entwässert. Nach Einengen im Vakuum wurde der Rückstand durch Säulenchromatographie $\left(\mathrm{SiO}_{2}, 250 \mathrm{~g}\right.$, Petrolether/EtOAc, 2:1 $\left.\rightarrow 3: 2\right)$ gereinigt. Es wurden $982 \mathrm{mg}$ (1.44 mmol, 67\%) des Peptidthiazols 144 als farbloser Schaum erhalten.

Alternative Synthese aus Thiazol 52:

Eine Lösung des Thiazol 52 ${ }^{[36]}(117 \mathrm{mg}, 0.29 \mathrm{mmol})$ in wasserfreiem $\mathrm{CH}_{2} \mathrm{Cl}_{2}(5 \mathrm{~mL})$ wurde mit $\mathrm{Et}_{3} \mathrm{SiH}(94 \mu \mathrm{L}, 0.58 \mathrm{mmol})$ und TFA $(5 \mathrm{~mL})$ versetzt und $25 \mathrm{~min}$ lang bei RT gerührt. Anschließend wurde die Mischung mit Toluol $(10 \mathrm{~mL})$ verdünnt und im Vakuum eingeengt. Der Rückstand wurde in wasserfreiem DMF $(3 \mathrm{~mL})$ gelöst und auf $0{ }^{\circ} \mathrm{C}$ gekühlt. Danach wurden aufeinander folgend $\mathrm{EtNiPr}_{2}(144 \mu \mathrm{L}, 0.87 \mathrm{mmol}), \mathrm{L}$-Fmoc-Thr(TBS)-OH (143) ${ }^{[202]}$ (148 mg, $0.32 \mathrm{mmol}$ ), HATU (132 mg, $0.34 \mathrm{mmol}$ ) und HOAt (47 mg, $0.34 \mathrm{mmol}$ ) zugegeben und die Mischung weitere $30 \mathrm{~min}$ bei $0{ }^{\circ} \mathrm{C}$ gerührt. Das Kühlbad wurde entfernt und $14 \mathrm{~h}$ lang bei RT gerührt. Die Reaktionsmischung wurde mit pH 3 Puffer $(15 \mathrm{~mL})$ verdünnt und mit $\mathrm{CH}_{2} \mathrm{Cl}_{2}(3 \times 15 \mathrm{~mL})$ extrahiert. Die vereinigten organischen Extrakte wurden mit $\mathrm{H}_{2} \mathrm{O}$ $(15 \mathrm{~mL})$ und ges. NaCl-Lsg. $(20 \mathrm{~mL})$ gewaschen und mit $\mathrm{Na}_{2} \mathrm{SO}_{4}$ entwässert. Die Lösungsmittel wurden am Rotationsverdampfer entfernt und der Rückstand durch Säulenchromatographie $\left(\mathrm{SiO}_{2}, 40 \mathrm{~g}\right.$, Petrolether/Aceton, 3:1) gereinigt. Es wurden $187 \mathrm{mg}$ (0.28 mmol, 96\%) des Peptidothiazols 144 als farbloser Schaum erhalten.

Die analytischen Daten stimmten für beide Synthesewege überein.

Spez. Drehung: $[\alpha]_{\mathrm{D}}{ }^{24}=-47.5\left(\mathrm{CHCl}_{3}, c=1\right)$.

DC: $R_{f}=0.64($ Heptan/EtOAc, $1: 1)$.

IR: $\tilde{v}=740(\mathrm{~s}), 1095(\mathrm{~m}), 1211(\mathrm{~s}), 1489(\mathrm{~m}), 1720(\mathrm{~m}), 2931(\mathrm{w}), 3332(\mathrm{~b}), 3402$ (b) $\mathrm{cm}^{-1}$.

${ }^{1} \mathbf{H}-\mathbf{N M R}\left(250 \mathrm{MHz}, \mathrm{CDCl}_{3}\right): \delta=0.11\left(\mathrm{~s}, 3 \mathrm{H}, \mathrm{SiCH}_{3}\right), 0.15\left(\mathrm{~s}, 3 \mathrm{H}, \mathrm{SiCH}_{3}\right), 0.89(\mathrm{~s}, 9 \mathrm{H}, t \mathrm{Bu})$, $0.98\left(\mathrm{~d}, J=6.2 \mathrm{~Hz}, 3 \mathrm{H}, \mathrm{CH}_{3}\right), 1.29$ (d, $\left.J=6.4 \mathrm{~Hz}, 3 \mathrm{H}, \mathrm{CH}_{3}\right), 3.47$ (bs, $\left.1 \mathrm{H}, \mathrm{OH}\right), 4.20-4.43$ (m, 5H, CHOTBS, CHNH, Fmoc), 4.65 (q, $J=5.9 \mathrm{~Hz}, 1 \mathrm{H}, \mathrm{CHOH}), 4.83$ (d, J=5.6 Hz, 2H, $\left.\mathrm{CH}_{2} \mathrm{CH}=\mathrm{CH}_{2}\right), 5.22(\mathrm{~d}, J=8.9 \mathrm{~Hz}, 1 \mathrm{H}, \mathrm{ThCHNH}), 5.30\left(\mathrm{~d}, J=10.5 \mathrm{~Hz}, 1 \mathrm{H}, \mathrm{CH}_{2} \mathrm{CH}=\mathrm{CHH}\right)$, $5.41\left(\mathrm{~d}, J=16.9 \mathrm{~Hz}, 1 \mathrm{H}, \mathrm{CH}_{2} \mathrm{CH}=\mathrm{CH} H\right), 5.85(\mathrm{~d}, J=5.8 \mathrm{~Hz}, 1 \mathrm{H}, \mathrm{NH}), 6.00(\mathrm{~m}, 1 \mathrm{H}$, $\mathrm{CH}_{2} \mathrm{CH}=\mathrm{CH}_{2}$ ), 7.35 (dt, $\left.J=27.9,7.4 \mathrm{~Hz}, 4 \mathrm{H}, \mathrm{Fmoc}\right), 7.60$ (d, J=7.4 Hz, 2H, Fmoc), 7.69 (d, $J=8.8 \mathrm{~Hz}, 1 \mathrm{H}, \mathrm{NH}), 7.76$ (d, $J=7.5 \mathrm{~Hz}, 2 \mathrm{H}, \mathrm{Fmoc}), 8.15$ (s, 1H, CH-S). 
${ }^{13}$ C-NMR (63 MHz, $\left.\mathrm{CDCl}_{3}\right): \delta=-4.97,-4.85,17.9,18.0,19.4,25.7,47.1,54.9,59.2,65.9$, $67.1,68.0,68.1,118.9,120.0,125.1,127.0,128.0,131.7,141.3,143.6,143.8,146.3,156.1$, $160.6,169.9,170.7$.

HRMS (ESI): $m / z[\mathrm{M}+\mathrm{H}]^{+}$für $\mathrm{C}_{35} \mathrm{H}_{46} \mathrm{~N}_{3} \mathrm{O}_{7} \mathrm{SSi}$ ber.: 680.2820; gef.: 680.2826.

\section{(2"S,3"R)-2-((Z)-1-(2-(((9H-Fluoren-9-yl)methoxy)carbonylamino)-3-tert-}

butyldimethylsilylbutanamido)prop-1-enyl)thiazol-4-carbonsäureallylester (145)

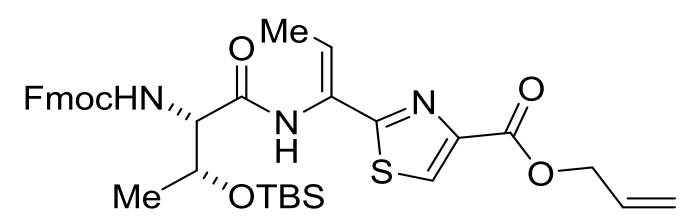

$\mathrm{Zu}$ einer Lösung des Alkohols $144(50 \mathrm{mg}, 73.6 \mu \mathrm{mol})$ in wasserfreiem $\mathrm{CH}_{2} \mathrm{Cl}_{2}(500 \mu \mathrm{L})$ und DMF $(20 \mu \mathrm{L})$ wurde zuerst EDCI $(28 \mathrm{mg}, 147 \mu \mathrm{mol})$ und anschließend $\mathrm{Cu}(\mathrm{I}) \mathrm{Cl}(8.7 \mathrm{mg}$, $88 \mu \mathrm{mol})$ zugegeben. Die Mischung wurde $20 \mathrm{~h}$ lang bei RT gerührt, im Vakuum eingeengt und der Rückstand in pH 3 Puffer $(15 \mathrm{~mL})$ aufgenommen. Es wurde mit EtOAc $(3 \times 15 \mathrm{~mL})$ extrahiert und die vereinigten organischen Extrakte mit $\mathrm{Na}_{2} \mathrm{SO}_{4}$ entwässert. Nach Einengen im Vakuum wurde der Rückstand durch Säulenchromatographie $\left(\mathrm{SiO}_{2}, 10 \mathrm{~g}\right.$, Petrolether/EtOAc, 2:1) gereinigt. Es wurden $41 \mathrm{mg}(61.8 \mu \mathrm{mol}, 84 \%)$ des Thiazols 145 als farbloser Schaum erhalten.

Spez. Drehung: $[\alpha]_{\mathrm{D}}{ }^{24}=+3.7\left(\mathrm{CHCl}_{3}, c=1.0\right)$.

DC: $R_{f}=0.38($ Heptan/EtOAc, $2: 1)$.

IR: $\tilde{v}=740(\mathrm{~s}), 833(\mathrm{~s}), 1095(\mathrm{~m}), 1226(\mathrm{~m}), 1496(\mathrm{~m}), 1712(\mathrm{~m}), 2854(\mathrm{w}), 2931(\mathrm{w}) \mathrm{cm}^{-1}$.

${ }^{1} \mathbf{H}-\mathbf{N M R}\left(300 \mathrm{MHz}, \mathrm{CDCl}_{3}\right): \delta=0.16\left(\mathrm{~s}, 3 \mathrm{H}, \mathrm{CH}_{3}\right), 0.19\left(\mathrm{~s}, 3 \mathrm{H}, \mathrm{CH}_{3}\right), 0.89(\mathrm{~s}, 9 \mathrm{H}, t \mathrm{Bu}), 1.27$ (d, $\left.J=6.4 \mathrm{~Hz}, 3 \mathrm{H}, \mathrm{CH}_{3}\right), 1.85\left(\mathrm{~d}, J=7.2 \mathrm{~Hz}, 3 \mathrm{H}, \mathrm{CH}_{3}\right), 4.24$ (t, $\left.J=7.0 \mathrm{~Hz}, 1 \mathrm{H}, \mathrm{CHCONH}\right)$, 4.39-4.48 (m, 4H, CHOTBS, Fmoc), 4.82 (d, J=5.6 Hz, 2H, $\left.\mathrm{CH}_{2} \mathrm{CH}=\mathrm{CH}_{2}\right), 5.28$ (d, $\left.J=10.4 \mathrm{~Hz}, \quad 1 \mathrm{H}, \quad \mathrm{CH}_{2} \mathrm{CH}=\mathrm{CHH}\right), 5.39\left(\mathrm{~d}, J=17.2 \mathrm{~Hz}, 1 \mathrm{H}, \mathrm{CH}_{2} \mathrm{CH}=\mathrm{CH} H\right), 5.93$ (d, $J=17.2 \mathrm{~Hz}, 1 \mathrm{H}, \mathrm{NH}), 6.01\left(\mathrm{~m}, 1 \mathrm{H}, \mathrm{CH}_{2} \mathrm{CH}=\mathrm{CH}_{2}\right), 6.66\left(\mathrm{q}, J=7.1 \mathrm{~Hz}, 1 \mathrm{H},=\mathrm{CH}-\mathrm{CH}_{3}\right), 7.34$ (dt, $J=28.4,7.4 \mathrm{~Hz}, 4 \mathrm{H}$, Fmoc), 7.61 (d, $J=7.4 \mathrm{~Hz}, 2 \mathrm{H}$, Fmoc), 7.75 (d, $J=7.5 \mathrm{~Hz}, 2 \mathrm{H}$, Fmoc), 8.08 (s, 1H, S-CH), 8.41 (bs, 1H, NH). 
${ }^{13}$ C-NMR (75 MHz, $\left.\mathrm{CDCl}_{3}\right): \delta=-5.0,-4.8,14.3,17.8,18.1,25.7,47.1,59.5,65.9,67.1,68.1$, $118.8,119.9,125.1,127.96,127.01,127.1,127.7,127.9,131.9,141.3,143.6,143.8,147.1$, $156.2,160.9,167.0,168.1$.

HRMS (ESI): $m / z[\mathrm{M}+\mathrm{H}]^{+}$ber. für $\mathrm{C}_{35} \mathrm{H}_{44} \mathrm{~N}_{3} \mathrm{O}_{6} \mathrm{SSi}$ : 662.2715; gef.: 662.2722 .

\section{(2"S,3"R)-2-((Z)-1-(2-(((9H-Fluoren-9-yl)methoxy)carbonylamino)-3-tert-}

butyldimthylsilylbutanamido)prop-1-enyl)thiazol-4-carbonsäure (110)

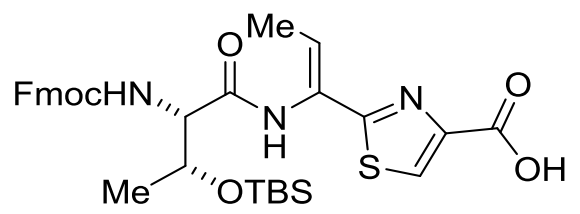

Eine Lösung des Allylesters 145 (342 mg, $0.52 \mathrm{mmol})$ in entgastem, wasserfreiem THF $(9 \mathrm{~mL})$, wurde auf $0{ }^{\circ} \mathrm{C}$ gekühlt, $\mathrm{PhSiH}_{3}(122 \mu \mathrm{L}, 0.98 \mathrm{mmol})$ und eine Lösung von $\mathrm{Pd}\left(\mathrm{PPh}_{3}\right)_{4}$ (60 mg, $0.05 \mathrm{mmol})$ in THF (1 mL) zugegeben. Die Mischung wurde $30 \mathrm{~min}$ lang gerührt und anschließend im Vakuum eingeengt. Der Rückstand wurde durch Säulenchromatographie $\left(\mathrm{SiO}_{2}, \quad 35 \mathrm{~g}, \quad \mathrm{CH}_{2} \mathrm{Cl}_{2} \rightarrow \mathrm{CH}_{2} \mathrm{Cl}_{2} / \mathrm{MeOH} \quad 30: 1 \rightarrow 20: 1\right)$ gereinigt. Es wurden $307 \mathrm{mg}$ (0.50 mmol, 96\%) der Thiazolcarbonsäure 110 als leicht gelbes Glas erhalten.

Spez. Drehung: $[\alpha]_{\mathrm{D}}{ }^{24}=+9.4\left(\mathrm{CHCl}_{3}, c=1\right)$;

DC: $R_{f}=0.23\left(\mathrm{CH}_{2} \mathrm{Cl}_{2} / \mathrm{MeOH} / \mathrm{HCOOH}, 100: 5: 1\right)$.

IR: $\tilde{v}=740$ (s), 833 (s), $1242(\mathrm{~m}), 1496$ (s), 1674 (s), 1975 (b), 2931 (w) cm ${ }^{-1}$.

${ }^{1}$ H-NMR $\left(300 \mathrm{MHz}, \mathrm{MeOH}-d_{4}\right): \delta=0.06\left(\mathrm{~s}, 3 \mathrm{H}, \mathrm{SiCH}_{3}\right), 0.11\left(\mathrm{~s}, 3 \mathrm{H}, \mathrm{SiCH}_{3}\right), 0.88(\mathrm{~s}, 9 \mathrm{H}$, $t \mathrm{Bu}$ ), 1.23 (bs, 3H, $\mathrm{CH}_{3}$ ), 1.68 (d, $\left.J=7.0 \mathrm{~Hz}, 3 \mathrm{H}, \mathrm{CH}_{3}\right), 4.13$ (t, $J=6.1 \mathrm{~Hz}, 1 \mathrm{H}, \mathrm{CHCONH}$ ), $4.33(\mathrm{~m}, 4 \mathrm{H}, \mathrm{CHOTBS}, \mathrm{Fmoc}), 6.50\left(\mathrm{bd}, J=7.2 \mathrm{~Hz}, 1 \mathrm{H},=\mathrm{CH}-\mathrm{CH}_{3}\right), 7.23(\mathrm{t}, J=6.3 \mathrm{~Hz}, 2 \mathrm{H}$, Fmoc), 7.33 (t, $J=7.4 \mathrm{~Hz}, 2 \mathrm{H}, \mathrm{Fmoc}), 7.58$ (m, 2H, Fmoc), 7.75 (d, $J=7.5 \mathrm{~Hz}, 2 \mathrm{H}, \mathrm{Fmoc}$ ), $7.92(\mathrm{~s}, 1 \mathrm{H}, \mathrm{S}-\mathrm{CH})$.

${ }^{13}$ C-NMR (75 MHz, MeOH- $\left.d_{4}\right): \delta=-4.4,-4.2,14.3,18.9,21.3,26.4,32.1,62.4,68.3,69.9$, $120.9,126.2,128.1,128.8,142.6,145.0,145.3,158.6,168.6,171.9$.

HRMS (ESI): $m / z[\mathrm{M}+\mathrm{H}]^{+}$für $\mathrm{C}_{32} \mathrm{H}_{40} \mathrm{~N}_{3} \mathrm{O}_{6} \mathrm{SSi}$ ber.: 622.2402; gef.: 662.2412 . 
(2"S,3"R)-2-((Z)-1-(2-(((9H-Fluoren-9-yl)methoxy)carbonylamino)-3-tert-

butoxybutanamido)prop-1-enyl)thiazol-4-carbonsäureallylester (54)

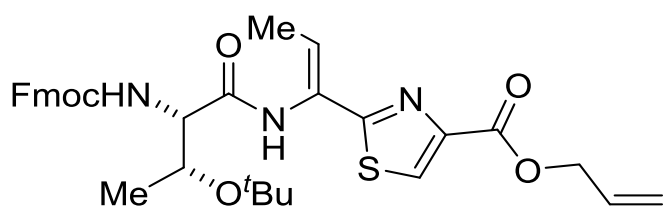

Analog der Darstellung des Thiazols 145 wurde das Thiazol 53 ${ }^{[36]}$ (50 mg, $\left.80.4 \mu \mathrm{mol}\right)$ mit EDCI $(31 \mathrm{mg}, 160.8 \mu \mathrm{mol})$ und $\mathrm{Cu}(\mathrm{I}) \mathrm{Cl}(9.6 \mathrm{mg}, 96.5 \mu \mathrm{mol})$ in $\mathrm{CH}_{2} \mathrm{Cl}_{2}(500 \mu \mathrm{L})$ und $\mathrm{DMF}$ $(20 \mu \mathrm{L})$ umgesetzt. Es wurden $36 \mathrm{mg}(60.3 \mu \mathrm{mol}, 75 \%)$ des Thiazols 54 als farbloser Schaum erhalten.

Alle relevanten analytischen Daten stimmen mit den veröffentlichten Werten überein. ${ }^{[36]}$

(2"S,3"R)-2-((Z)-1-(2-(((9H-Fluoren-9-yl)methoxy)carbonylamino)-3-

hydroxybutanamido)prop-1-enyl)thiazol-4-carbonsäureallylester (146)<smiles>C=CCOC(=O)c1csc(/C(=C/C)NC(=O)[C@@H](NC(F)F)[C@H](C)O)n1</smiles>

$\mathrm{Zu}$ einer Lösung des TBS-Ethers $145(250 \mathrm{mg}, 0.38 \mathrm{mmol})$ in wasserfreiem THF (2 mL) wurde bei $0{ }^{\circ} \mathrm{C}$ tropfenweise eine Mischung aus TBAF ( $1 \mathrm{M}$ in THF, $830 \mu \mathrm{L}, 0.83 \mathrm{mmol}$ ) und $\mathrm{AcOH}(95 \mu \mathrm{L}, 1.66 \mathrm{mmol})$ in wasserfreiem THF $(2 \mathrm{~mL})$ zugegeben. Die Mischung wurde $2 \mathrm{~h}$ lang bei $0{ }^{\circ} \mathrm{C}$ und anschließend $3 \mathrm{~h}$ lang bei RT gerührt. Nach Verdünnen mit pH 7 Puffer $(10 \mathrm{~mL})$ wurde mit EtOAc $(3 \times 15 \mathrm{~mL})$ extrahiert und die vereinigten organischen Extrakte mit $\mathrm{H}_{2} \mathrm{O}$ und ges. NaCl-Lsg. (je $20 \mathrm{~mL}$ ) gewaschen. Nach Entwässern mit $\mathrm{Na}_{2} \mathrm{SO}_{4}$ und Einengen im Vakuum wurde der Rückstand durch Säulenchromatographie $\left(\mathrm{SiO}_{2}, 25 \mathrm{~g}\right.$, Petrolether - EtOAc, 1:2) gereinigt. Es wurden 203 mg (0.37 mmol, 98\%) des Alkohols 146 als farbloser Feststoff erhalten.

Alternativ, wurde der entsprechende $t$-Bu-Ether $53(500 \mathrm{mg}, 0.83 \mathrm{mmol})$ in wasserfreiem $\mathrm{CH}_{2} \mathrm{Cl}_{2}(5 \mathrm{ml})$ gelöst, mit Et 3 SiH $(267 \mu \mathrm{L}, 1.66 \mathrm{mmol})$ und TFA $(3 \mathrm{~mL})$ versetzt und bei RT bis zum vollständigen Umsatz gerührt (30-60 min, DC-Kontrolle). Die Mischung wurde mit pH 7 Puffer $(20 \mathrm{~mL})$ verdünnt, mit $\mathrm{CH}_{2} \mathrm{Cl}_{2}(3 \times 15 \mathrm{~mL})$ extrahiert, und die vereinigten organischen Extrakte mit $\mathrm{Na}_{2} \mathrm{SO}_{4}$ entwässert. Nach Entfernen des Lösungsmittels und 
Reinigung des Rückstands durch Säulenchromatographie $\left(\mathrm{SiO}_{2}, 50 \mathrm{~g}\right.$, Petrolether/EtOAc, 1:2) wurden $430 \mathrm{mg}$ (0.79 mmol, 95\%) des Alkohols 146 als farbloser Feststoff erhalten.

Die analytischen Daten stimmten für beide Synthesewege überein.

Spez. Drehung: $[\alpha]_{\mathrm{D}}{ }^{24}=-31.5\left(\mathrm{CHCl}_{3}, c=0.3\right)$.

Schmelzpunkt: $132-133{ }^{\circ} \mathrm{C}$.

DC: $R_{f}=0.37($ Heptan/EtOAc, 1:2).

IR: $\tilde{v}=732(\mathrm{~s}), 1087(\mathrm{~m}), 1234(\mathrm{~s}), 1658(\mathrm{~s}), 2330(\mathrm{w}), 3271(\mathrm{w}) \mathrm{cm}^{-1}$.

${ }^{1}$ H-NMR $\left(300 \mathrm{MHz}, \mathrm{CDCl}_{3}\right): \delta=1.27\left(\mathrm{~d}, J=6.3 \mathrm{~Hz}, 3 \mathrm{H}, \mathrm{CH}_{3}\right), 1.85(\mathrm{~d}, J=6.9 \mathrm{~Hz}, 3 \mathrm{H}$, $\left.\mathrm{CH}_{3}\right), 4.24$ (t, $\left.J=7.0 \mathrm{~Hz}, 1 \mathrm{H}, \mathrm{Fmoc}\right), 4.36-4.49(\mathrm{~m}, 4 \mathrm{H}$, Fmoc, $2 \times \mathrm{CH}), 4.8(\mathrm{~d}, J=5.8 \mathrm{~Hz}$, $\left.2 \mathrm{H}, \mathrm{CH}_{2} \mathrm{CH}=\mathrm{CH}_{2}\right), 5.29\left(\mathrm{~d}, J=10.3 \mathrm{~Hz}, 1 \mathrm{H}, \mathrm{CH}_{2} \mathrm{CH}=\mathrm{CHH}\right), 5.38(\mathrm{~d}, J=17.1 \mathrm{~Hz}, 1 \mathrm{H}$, $\left.\mathrm{CH}_{2} \mathrm{CH}=\mathrm{CH} H\right), 5.93-6.08\left(\mathrm{~m}, 2 \mathrm{H}, \mathrm{CH}_{2} \mathrm{CH}=\mathrm{CH}_{2}, \mathrm{NH}\right), 6.56(\mathrm{q}, J=6.9 \mathrm{~Hz}, 1 \mathrm{H}, \mathrm{CH}), 7.28-$ 7.42 (m, 4H, Fmoc), 7.61 (dd, $J=6.6,3.6 \mathrm{~Hz}, 2 \mathrm{H}, \mathrm{Fmoc}$ ), 7.76 (d, J=7.2 Hz, 2H, Fmoc), $8.01(\mathrm{~s}, 1 \mathrm{H}, \mathrm{S}-\mathrm{CH})$.

${ }^{13}$ C-NMR (75 MHz, $\left.\mathrm{CDCl}_{3}\right): \delta=14.1,18.3,47.1,60.0,66.1,67.1,67.7,119.1,119.9,124.9$, $125.1,126.8,127.0,127.7,128.7,130.1,131.6,141.3,143.7,146.3,156.5,160.5,167.1$, 170.4 .

HRMS (ESI): $m / z[\mathrm{M}+\mathrm{Na}]^{+}$für $\mathrm{C}_{29} \mathrm{H}_{29} \mathrm{~N}_{3} \mathrm{O}_{6} \mathrm{SNa}$ ber.: 570.1669 ; gef.: 570.1683 .

\section{(2"S,3"R)-2-((Z)-1-(2-(((9H-Fluoren-9-yl)methoxy)carbonylamino)-3-}

\section{triisopropylsilylbutanamido)prop-1-enyl)thiazol-4-carbonsäureallylester (147)}

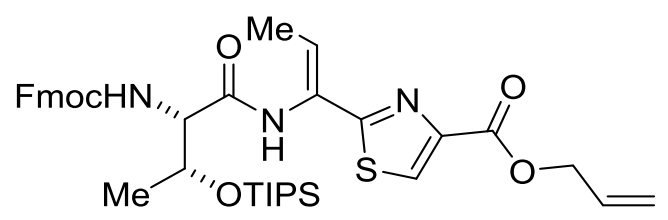

Eine Lösung des Alkohols 146 (260 mg, $0.47 \mathrm{mmol})$ in wasserfreiem $\mathrm{CH}_{2} \mathrm{Cl}_{2}(10 \mathrm{~mL})$ wurde auf $0{ }^{\circ} \mathrm{C}$ gekühlt, mit 2,6-Lutidin $(243 \mu \mathrm{L}, 2.09 \mathrm{mmol})$ und TIPSOTf $(282 \mu \mathrm{L}, 1.05 \mathrm{mmol})$ tropfenweise versetzt. Die Mischung wurde $1 \mathrm{~h}$ lang bei $0^{\circ} \mathrm{C}$ gerührt, anschließend mit EtOAc $(50 \mathrm{~mL})$ verdünnt und mit $0.5 \mathrm{M} \mathrm{HCl}, \mathrm{pH} 3$ Puffer, $\mathrm{H}_{2} \mathrm{O}$ und ges. NaCl-Lsg. (je $25 \mathrm{~mL}$ ) gewaschen. Nach Entwässern mit $\mathrm{Na}_{2} \mathrm{SO}_{4}$ wurden die Lösungsmittel im Vakuum 
entfernt und der Rückstand durch Säulenchromatographie $\left(\mathrm{SiO}_{2}, 40 \mathrm{~g}\right.$, Petrolether/EtOAc, 2:1) gereinigt. Es wurden $309 \mathrm{mg}(0.44 \mathrm{mmol}, 92 \%)$ des TIPS-Ethers 147 als farbloser Schaum erhalten.

Spez. Drehung: $[\alpha]_{\mathrm{D}}^{24}=+2.4\left(\mathrm{CHCl}_{3}, c=1\right)$.

DC: $R_{f}=0.41($ Heptan/EtOAc, 2:1).

IR: $\tilde{v}=741$ (s), 1203 (s), 1489 (s), 1697 (s), 1975 (m), $2021(\mathrm{~m}), 2939(\mathrm{w}) \mathrm{cm}^{-1}$.

${ }^{1} \mathbf{H}-\mathbf{N M R}\left(300 \mathrm{MHz}, \mathrm{CDCl}_{3}\right): \delta=1.10$ (bs, $\left.18 \mathrm{H}, \mathrm{TIPS}\right), 1.36\left(\mathrm{~d}, J=6.2 \mathrm{~Hz}, 3 \mathrm{H}, \mathrm{CH}_{3}\right), 1.85$ (d, $\left.J=7.2 \mathrm{~Hz}, 3 \mathrm{H}, \mathrm{CH}_{3}\right), 4.23$ (t, $\left.J=6.8 \mathrm{~Hz}, 1 \mathrm{H}, \mathrm{Fmoc}\right), 4.43$ (d, $\left.J=7.1 \mathrm{~Hz}, 2 \mathrm{H}, \mathrm{Fmoc}\right), 4.50$ (bs, 1H, CH), $4.58(\mathrm{~m}, 1 \mathrm{H}, \mathrm{CH}) 4.82\left(\mathrm{~d}, J=5.6 \mathrm{~Hz}, 2 \mathrm{H}, \mathrm{CH}_{2} \mathrm{CH}=\mathrm{CH}_{2}\right), 5.28(\mathrm{~d}, J=10.3 \mathrm{~Hz}$, $\left.1 \mathrm{H}, \mathrm{CH}_{2} \mathrm{CH}=\mathrm{CHH}\right), 5.40\left(\mathrm{~d}, J=18 \mathrm{~Hz}, 1 \mathrm{H}, \mathrm{CH}_{2} \mathrm{CH}=\mathrm{CH} H\right), 5.96-6.09\left(\mathrm{~m}, 2 \mathrm{H}, \mathrm{CH}_{2} \mathrm{CH}=\mathrm{CH}_{2}\right.$, $\mathrm{OH}), 6.72(\mathrm{q}, J=6.9 \mathrm{~Hz}, 1 \mathrm{H}, \mathrm{CH}), 7.26-7.42$ (m, 4H, Fmoc, NH), 7.61 (d, J=7.1 Hz, 2H, Fmoc), 7.76 (d, $J=7.5 \mathrm{~Hz}, 2 \mathrm{H}, \mathrm{Fmoc}), 8.08$ (s, 1H, S-CH).

${ }^{13}$ C-NMR (75 MHz, $\left.\mathrm{CDCl}_{3}\right): \delta=12.1,14.2,18.0,47.1,59.2,65.9,67.1,68.5,118.8,119.9$, $125.1,126.9,127.0,127.6,127.7,127.8,131.9,141.29,141.31,143.6,143.8,147.2,156.1$, $160.1,167.1,168.1$.

HRMS (ESI): $m / z[\mathrm{M}+\mathrm{Na}]^{+}$für $\mathrm{C}_{38} \mathrm{H}_{49} \mathrm{~N}_{3} \mathrm{O}_{6} \mathrm{SSiNa}$ ber.: 726.3004 ; gef.: 726.3006 .

(2"S,3"R)-2-((Z)-1-(2-(((9H-Fluoren-9-yl)methoxy)carbonylamino)-3triisopropylsilylbutanamido)prop-1-enyl)thiazol-4-carbonsäure (111)<smiles>C/C=C(\NC(=O)[C@@H](NC(F)F)C(C)C)c1nc(C(=O)O)cs1</smiles>

Eine Lösung des Allylesters 147 (300 mg, $0.43 \mathrm{mmol})$ in entgastem, wasserfreiem THF (5 mL) wurde auf $0{ }^{\circ} \mathrm{C}$ gekühlt, $\mathrm{PhSiH}_{3}(150 \mu \mathrm{L}, 1.2 \mathrm{mmol})$ und eine Lösung von $\mathrm{Pd}\left(\mathrm{PPh}_{3}\right)_{4}$ (60 mg, $0.05 \mathrm{mmol})$ in THF (1 mL) zugegeben. Die Mischung wurde 15 min lang gerührt und anschließend im Vakuum eingeengt. Der Rückstand wurde durch Säulenchromatographie $\left(\mathrm{SiO}_{2}, \quad 40 \mathrm{~g}, \mathrm{CH}_{2} \mathrm{Cl}_{2} \rightarrow \mathrm{CH}_{2} \mathrm{Cl}_{2} / \mathrm{MeOH}, \quad 30: 1 \rightarrow 20: 1\right)$ gereinigt. Es wurden $280 \mathrm{mg}$ (0.42 mmol, 99\%) der Thiazolcarbonsäure 111 als leicht gelbes Glas erhalten. 
Spez. Drehung: $[\alpha]_{\mathrm{D}}{ }^{24}=+5.3(c=1)$.

DC: $R_{f}=0.26\left(\mathrm{CH}_{2} \mathrm{Cl}_{2} / \mathrm{MeOH} / \mathrm{HCOOH}, 100: 5: 1\right)$.

IR: $\tilde{v}=741(\mathrm{~s}), 1095(\mathrm{w}), 1234(\mathrm{~m}), 1496(\mathrm{~m}), 1689(\mathrm{~m}), 1975(\mathrm{w}), 2014(\mathrm{w}), 2160(\mathrm{w})$, $2939(\mathrm{w}) \mathrm{cm}^{-1}$.

${ }^{1}$ H-NMR (300 MHz, MeOH-d $\left.)_{4}\right): \delta=1.06$ (bs, 18H, TIPS), 1.28 (d, $J=5.5 \mathrm{~Hz}, 3 \mathrm{H}, \mathrm{CH}_{3}$ ), $1.80\left(\mathrm{~d}, J=6.8 \mathrm{~Hz}, 3 \mathrm{H}, \mathrm{CH}_{3}\right), 4.24(\mathrm{~m}, 1 \mathrm{H}, \mathrm{Fmoc}), 4.34(\mathrm{~m}, 1 \mathrm{H}, \mathrm{CH}), 4.41-4.55(\mathrm{~m}, 3 \mathrm{H}$, Fmoc, CH), 6.69 (q, $J=6.9 \mathrm{~Hz}, 1 \mathrm{H}, \mathrm{CH}), 6.84(\mathrm{~d}, J=8.6 \mathrm{~Hz}, 1 \mathrm{H}, \mathrm{NH}), 7.25-7.40(\mathrm{~m}, 5 \mathrm{H}$, Fmoc, NH), 7.66 (m, 2H, Fmoc), 7.78 (d, J=7.5 Hz, 2H, Fmoc), 8.21 (s, 1H, S-CH).

${ }^{13}$ C-NMR (75 MHz, $\left.\mathrm{CDCl}_{3}\right): \delta=13.8,14.5,18.6,18.65,21.1,62.2,67.9,70.3,79.5,120.9$, $126.1,128.1,128.3,128.8,129.1,129.5,142.7,145.1,145.3,158.5,168.9,171.3$.

HRMS (ESI): $m / z[\mathrm{M}+\mathrm{Na}]^{+}$für $\mathrm{C}_{35} \mathrm{H}_{45} \mathrm{~N}_{3} \mathrm{O}_{6} \mathrm{SSiNa}$ ber.: 686.2691; gef.: 686.2695 .

\section{Bis-Thiazol 149}

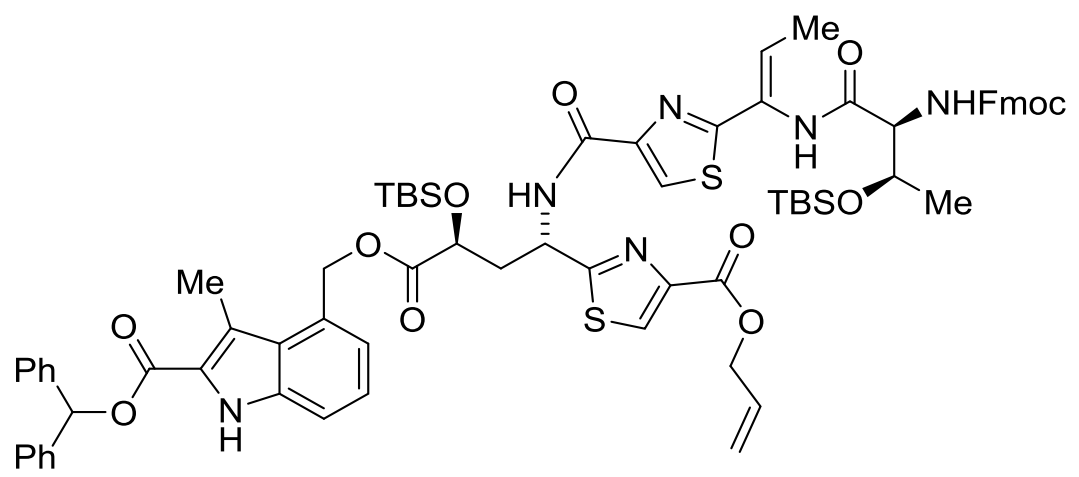

Eine Lösung des Boc-geschützten Amins $95(200 \mathrm{mg}, 0.23 \mathrm{mmol})$ in wasserfreiem $\mathrm{CH}_{2} \mathrm{Cl}_{2}$ (20 mL) wurde auf $0{ }^{\circ} \mathrm{C}$ gekühlt, mit 2,6-Lutidin $(585 \mu \mathrm{L}, 5.02 \mathrm{mmol})$ und anschließend mit einer Lösung von TBSOTf $(585 \mu \mathrm{L}, 2.54 \mathrm{mmol})$ in $\mathrm{CH}_{2} \mathrm{Cl}_{2}(5 \mathrm{~mL})$ versetzt. Die Mischung wurde langsam auf RT erwärmt und $18 \mathrm{~h}$ lang gerührt. Nach Einengen im Hochvakuum wurde der Rückstand in wasserfreiem THF $(16 \mathrm{~mL})$ gelöst und auf $0{ }^{\circ} \mathrm{C}$ gekühlt. Die Thiazolyl-Säure 110 (146 mg, $0.23 \mathrm{mmol}$ ), HATU (116 mg, $0.31 \mathrm{mmol}$ ) und HOAt (200 mg, $1.50 \mathrm{mmol})$ wurden nacheinander zugegeben. Nach $15 \mathrm{~min}$ bei $0^{\circ} \mathrm{C}$ wurde $\mathrm{NaHCO}_{3}(58 \mathrm{mg}$, $0.69 \mathrm{mmol}$ ) zugefügt und die Mischung langsam auf RT erwärmt. Nach $41 \mathrm{~h}$ Rühren bei RT (DC-Kontrolle) wurde das Gemisch mit EtOAc $(50 \mathrm{~mL})$ verdünnt, mit pH 3 Puffer $(15 \mathrm{~mL})$, halb-ges. $\mathrm{NaHCO}_{3}$-Lsg. $(3 \times 15 \mathrm{~mL}), \mathrm{H}_{2} \mathrm{O}(15 \mathrm{~mL})$ und ges. NaCl-Lsg. $(25 \mathrm{~mL})$ gewaschen. 
Die organische Phase wurde mit $\mathrm{Na}_{2} \mathrm{SO}_{4}$ entwässert und am Rotationsverdampfer eingeengt. Nach Säulenchromatographie $\left(\mathrm{SiO}_{2}, 50 \mathrm{~g}\right.$, Petrolether/EtOAc, 2:1) wurden $125 \mathrm{mg}$ (0.91 mmol, 39\%) des Bis-Thiazols 149 als farbloses Glas erhalten.

Spez. Drehung: $[\alpha]_{\mathrm{D}}{ }^{24}=-9.4(c=0.75)$.

DC: $R_{f}=0.29($ Heptan/EtOAc, $2: 1)$.

IR: $\tilde{v}=741(\mathrm{~m}), 1095(\mathrm{~m}), 1489(\mathrm{~s}), 1674(\mathrm{~m}), 1712(\mathrm{~m}), 1975(\mathrm{~b}), 2160(\mathrm{w}), 2931(\mathrm{w}) \mathrm{cm}^{-1}$.

${ }^{1} \mathbf{H}-\mathbf{N M R}\left(300 \mathrm{MHz}, \mathrm{CDCl}_{3}\right): \delta=-0.09$ (s, 3H, $\left.\mathrm{SiCH}_{3}\right),-0.03\left(\mathrm{~s}, 3 \mathrm{H}, \mathrm{SiCH}_{3}\right), 0.15$ (s, 3H, $\mathrm{SiCH}_{3}$ ), 0.18 (s, 3H, $\mathrm{SiCH}_{3}$ ), 0.83 (s, 9H, SitBu), 0.89 (s, 9H, SitBu), 1.23 (d, J=6.3 Hz, 3H, $\left.\mathrm{CH}_{3}\right), 1.82\left(\mathrm{~d}, J=6.3 \mathrm{~Hz}, 3 \mathrm{H}, \mathrm{CH}_{3}\right), 2.68$ (bs, $\left.2 \mathrm{H}, \mathrm{CH}_{2}\right), 2.82$ (s, 3H, $\left.\mathrm{CH}_{3}\right), 4.20$ (t, $J=6.7 \mathrm{~Hz}, 1 \mathrm{H}, \mathrm{Fmoc}), 4.35$ (bs, 1H, CHCONH), 4.47 (m, 4H, Fmoc, $2 \times$ CHOTBS), 4.80 (d, $\left.J=5.7 \mathrm{~Hz}, 2 \mathrm{H}, \mathrm{CH}_{2} \mathrm{CH}=\mathrm{CH}_{2}\right), 5.26\left(\mathrm{~d}, J=10.6 \mathrm{~Hz}, 1 \mathrm{H}, \mathrm{CH}_{2} \mathrm{CH}=\mathrm{CHH}\right), 5.37(\mathrm{~d}, J=17.0 \mathrm{~Hz}$, $\left.1 \mathrm{H}, \mathrm{CH}_{2} \mathrm{CH}=\mathrm{CHH}\right), 5.51$ (q, $J=12.3 \mathrm{~Hz}, 2 \mathrm{H}, \mathrm{CH}_{2} \mathrm{O}$ ), 5.69 (q, $J=7.5 \mathrm{~Hz}, 1 \mathrm{H}, \mathrm{CH}_{2} \mathrm{CHNH}$ ), $5.86(\mathrm{bs}, 1 \mathrm{H}, \mathrm{CONH}), 5.99\left(\mathrm{~m}, 1 \mathrm{H}, \mathrm{CH}_{2} \mathrm{CH}=\mathrm{CH}_{2}\right), 6.70\left(\mathrm{~d}, J=6.9 \mathrm{~Hz},=\mathrm{CHCH}_{3}\right), 7.17(\mathrm{~s}$, 1H, $\left.\mathrm{CH}(\mathrm{Ph})_{2}\right), 7.11-7.44(\mathrm{~m}, 17 \mathrm{H}$, Fmoc, Indol, $2 \times \mathrm{Ph}), 7.57$ (d, J=7.0 Hz, 2H, Fmoc), 7.74 (d, J=7.4 Hz, 2H, Fmoc), 7.96 (m, 2H, S-CH, NH), 8.06 (s, 1H, S-CH), 8.22 (s, 1H, NH), 8.84 (s, 1H, Indolyl-NH).

${ }^{13}$ C-NMR (75 MHz, $\left.\mathrm{CDCl}_{3}\right): \delta=-5.5,-5.1,-5.0,-4.7,12.2,14.1,17.9,18.1,25.6,25.8,39.1$, $47.1,47.8,59.4,65.1,65.9,67.1$, 68.0, 69.4, 77.5, 112.6, 118.8, 120.0, 120.7, 122.5, 123.5, $125.0,123.5,125.0,125.4,126.3,127.0,127.20,127.22,127.5,127.7,128.1,128.6,129.7$, $130.5,131.9,136.5,139 ., 141.3,143.6,143.7,146.7,149.6,156.2,160.6,160.8,161.4,166.8$, $168.4,172.7$.

HRMS (ESI): $m / z[\mathrm{M}+\mathrm{Na}]^{+}$für $\mathrm{C}_{73} \mathrm{H}_{84} \mathrm{~N}_{6} \mathrm{NaO}_{12} \mathrm{~S}_{2} \mathrm{Si}_{2}$ ber.: 1379.5019; gef.: 1379.5007 . 


\section{Bis-Thiazol 150}

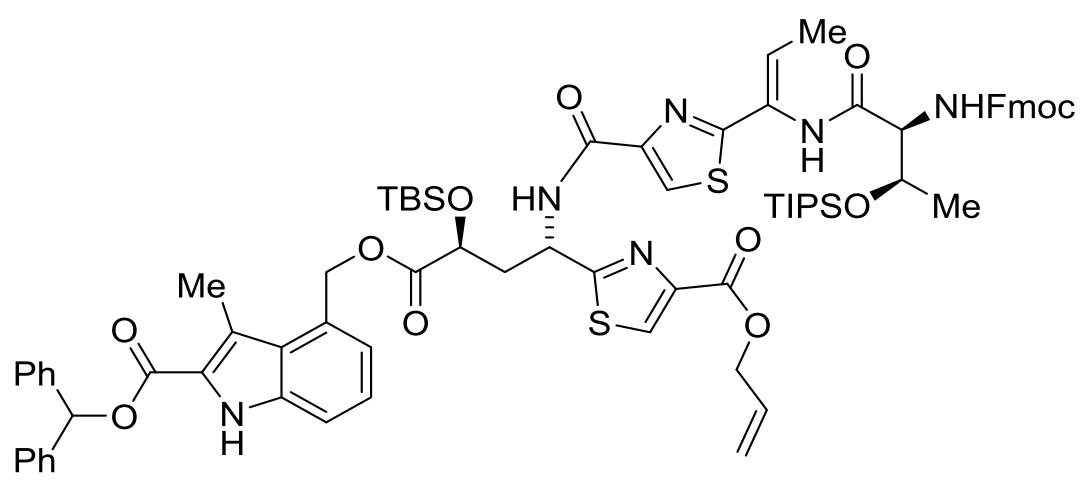

Eine Lösung des Boc-geschützten Amins 95 (100 mg, $0.12 \mathrm{mmol})$ in wasserfreiem $\mathrm{CH}_{2} \mathrm{Cl}_{2}$ (5 mL) wurde auf $0{ }^{\circ} \mathrm{C}$ gekühlt, mit 2,6-Lutidin $(68 \mu \mathrm{L}, 0.58 \mathrm{mmol})$ und anschließend mit einer Lösung von TBSOTf $(67 \mu \mathrm{L}, 0.29 \mathrm{mmol})$ in $\mathrm{CH}_{2} \mathrm{Cl}_{2}(1.5 \mathrm{~mL})$ versetzt. Die Mischung wurde $1 \mathrm{~h}$ lang bei $0{ }^{\circ} \mathrm{C}$ und anschließend $2.5 \mathrm{~h}$ bei RT gerührt. Nach Einengen im Hochvakuum (45 min) wurde der Rückstand in wasserfreiem THF (6 mL) gelöst und auf $0{ }^{\circ} \mathrm{C}$ gekühlt. Die TIPS-Thiazolyl-Säure 111 (82 mg, $0.12 \mathrm{mmol})$, HATU (100 mg, $0.26 \mathrm{mmol}$ ) und HOAt (58 mg, $0.43 \mathrm{mmol}$ ) wurden zugegeben. Nach $15 \mathrm{~min}$ bei $0{ }^{\circ} \mathrm{C}$ wurde $\mathrm{NaHCO}_{3}(30 \mathrm{mg}$, $0.36 \mathrm{mmol}$ ) zugefügt und die Mischung langsam auf RT erwärmt. Nach $41 \mathrm{~h}$ Rühren bei RT (DC-Kontrolle) wurde das Gemisch wie für die Verbindung 149 angegeben aufgearbeitet und gereinigt. Es wurden $116 \mathrm{mg}(0.08 \mathrm{mmol}, 71 \%)$ des Bis-Thiazols 150 als farbloser Schaum erhalten.

Spez. Drehung: $[\alpha]_{\mathrm{D}}{ }^{24}=-14.2(c=1.0)$.

DC: $R_{f}=0.51($ Heptan/EtOAc, $2: 1)$.

IR: $\tilde{v}=741(\mathrm{~s}), 1095$ (m), 1226 (s), 1481 (s), 1689 (s), 1975 (s), 2013 (s), $2931(\mathrm{w}) \mathrm{cm}^{-1}$.

${ }^{1} \mathbf{H}-\mathbf{N M R}\left(300 \mathrm{MHz}, \mathrm{CDCl}_{3}\right):=-0.09\left(\mathrm{~s}, 3 \mathrm{H}, \mathrm{SiCH}_{3}\right),-0.03\left(\mathrm{~s}, 3 \mathrm{H}, \mathrm{SiCH}_{3}\right), 0.83(\mathrm{~s}, 9 \mathrm{H}$, $\mathrm{Si} t \mathrm{Bu}), 1.01-1.20\left(\mathrm{~m}, 21 \mathrm{H}, \mathrm{Si}(i \mathrm{Pr})_{3}\right), 1.31$ (d, $\left.J=6.2 \mathrm{~Hz}, 3 \mathrm{H}, \mathrm{CH}_{3}\right), 1.83(\mathrm{~d}, J=7.2+\mathrm{Hz}, 3 \mathrm{H}$, $\left.\mathrm{CH}_{3}\right), 2.65-2.70\left(\mathrm{~m}, 2 \mathrm{H}, \mathrm{CH}_{2}\right), 2.82\left(\mathrm{~s}, 3 \mathrm{H}, \mathrm{CH}_{3}\right), 4.20$ (t, $\left.J=6.9 \mathrm{~Hz}, 1 \mathrm{H}, \mathrm{C} H \mathrm{NHFmoc}\right), 4.41-$ 4.51 (m, 4H, Fmoc, CHOTBS), 4.56 (m, 1H, CHOTIPS), 4.80 (d, J=5.8 Hz, 2H, $\left.\mathrm{CH}_{2} \mathrm{CH}=\mathrm{CH}_{2}\right), 5.26\left(\mathrm{dd}, J=10.6,1 \mathrm{~Hz}, 1 \mathrm{H}, \mathrm{CH}_{2} \mathrm{CH}=\mathrm{CHH}\right), 5.37(\mathrm{dd}, J=17.2,1.4 \mathrm{~Hz}, 1 \mathrm{H}$, $\left.\mathrm{CH}_{2} \mathrm{CH}=\mathrm{CHH}\right), 5.52\left(\mathrm{q}, J=12.4 \mathrm{~Hz}, 2 \mathrm{H}, \mathrm{CH}_{2} \mathrm{O}\right), 5.70\left(\mathrm{~m}, 1 \mathrm{H}, \mathrm{CH}_{2} \mathrm{CHNH}\right), 5.97(\mathrm{~m}, 2 \mathrm{H}$, $\left.\mathrm{CONH}, \mathrm{CH}_{2} \mathrm{CH}=\mathrm{CH}_{2}\right), 6.75\left(\mathrm{q}, J=7.3 \mathrm{~Hz},=\mathrm{CHCH}_{3}\right), 7.17\left(\mathrm{~s}, 1 \mathrm{H}, \mathrm{CH}(\mathrm{Ph})_{2}\right), 7.11-7.44(\mathrm{~m}$, 17H, Fmoc, Indol, $2 \times \mathrm{Ph}), 7.58(\mathrm{~d}, J=6.8 \mathrm{~Hz}, 2 \mathrm{H}, \mathrm{Fmoc}), 7.75$ (d, $J=7.5 \mathrm{~Hz}, 2 \mathrm{H}, \mathrm{Fmoc})$, 
7.94 (d, $J=8.7 \mathrm{~Hz}, 1 \mathrm{H}, \mathrm{NH}), 7.98$ (s, 1H, S-CH), 8.07 (s, 1H, S-CH), 8.88 (s, 1H, Indolyl$\mathrm{NH})$.

${ }^{13}$ C-NMR $\left(100 \mathrm{MHz}, \mathrm{CDCl}_{3}\right): \delta=-5.5,-5.2,12.09,12.13,13.9,14.2,17.98,17.99,18.04$, 18.2, 21.0, 24.3, 25.6, 39.0, 47.1, 47.8, 59.2, 60.3, 65.1, 65.8, 67.1, 68.3, 69.4, 77.5, 112.7, $118.7,119.9,120.2,120.6,122.5,123.5,125.3,126.3,127.0,127.16 ., 127.19,127.5,127.7$, 128.0, 128.6, 129.6, 131.8, 136.5, 136.6, 139.9, 141.25., 141.27, 143.6, 143.7, 146.7, 149.6, $156.1,157.5,160.6,160.8,161.4,166.9,168.4,171.1,171.6,172.6$.

HRMS (ESI): $m / z[\mathrm{M}+\mathrm{Na}]^{+}$ber. für $\mathrm{C}_{76} \mathrm{H}_{90} \mathrm{~N}_{6} \mathrm{NaO}_{12} \mathrm{~S}_{2} \mathrm{Si}_{2}: 1421.5489$; gef.: 1421.5485 .

\section{TBS-Bis-Thiazol-Amin 93}

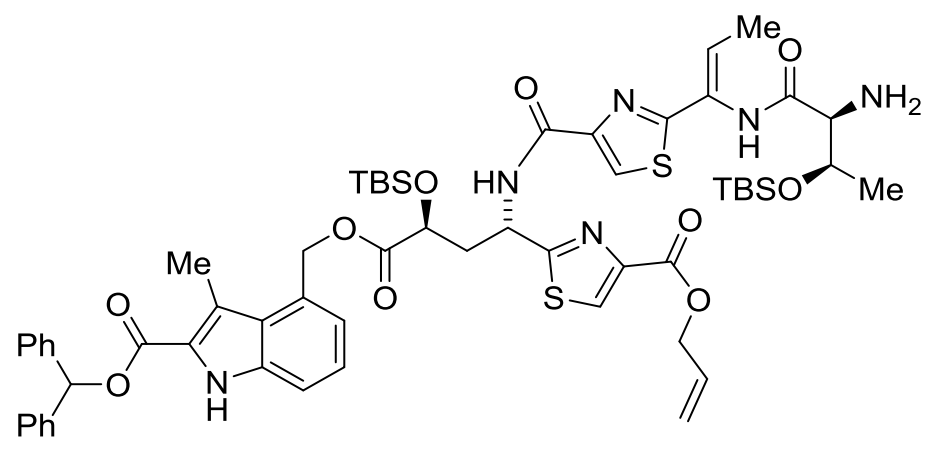

Das Fmoc-geschützte Amin 149 (61 mg, $44.9 \mu \mathrm{mol})$ wurde in wasserfreiem $\mathrm{CH}_{2} \mathrm{Cl}_{2}(14 \mathrm{~mL})$ gelöst, auf $-20^{\circ} \mathrm{C}$ gekühlt und eine Lösung von DBU $(1.04 \mathrm{~mL}, 6.70 \mathrm{mmol})$ in $\mathrm{CH}_{2} \mathrm{Cl}_{2}$ $(8 \mathrm{~mL})$ langsam zugetropft. Nach vollständiger Zugabe wurde 5 min lang gerührt und dann mit ges. $\mathrm{NaHCO}_{3}$-Lsg. und $\mathrm{H}_{2} \mathrm{O}$ (je $20 \mathrm{~mL}$ ) verdünnt. Die Mischung wurde mit $\mathrm{CH}_{2} \mathrm{Cl}_{2}$ $(3 \times 20 \mathrm{~mL})$ extrahiert und die vereinigten organischen Extrakte mit $\mathrm{Na}_{2} \mathrm{SO}_{4}$ entwässert. Nach Einengen im Vakuum wurde der Rückstand durch Säulenchromatographie $\left(\mathrm{SiO}_{2}, 15 \mathrm{~g}\right.$, Petrolether/EtOAc, 1:1, dann $\left.\mathrm{CH}_{2} \mathrm{Cl}_{2} / \mathrm{MeOH}, 20: 1\right)$ gereinigt. Es wurden $50 \mathrm{mg}$ (44.0 $\mu \mathrm{mol}$, 98\%) des Amins 93 als farbloses Glas erhalten.

Spez. Drehung: $[\alpha]_{\mathrm{D}}{ }^{24}=-28.7\left(\mathrm{CHCl}_{3}, c=1\right)$.

DC: $R_{f}=0.38\left(\mathrm{CH}_{2} \mathrm{Cl}_{2} / \mathrm{MeOH}, 20: 1\right)$.

IR: $\tilde{v}=748$ (s), 1095 (s), 1195 (s), 1226 (s), 1465 (s), $1681(\mathrm{~s}), 1975(\mathrm{~m}), 2931(\mathrm{w}) \mathrm{cm}^{-1}$.

${ }^{1} \mathbf{H}-\mathbf{N M R}\left(300 \mathrm{MHz}, \mathrm{CDCl}_{3}\right): \delta=-0.08\left(\mathrm{~s}, 3 \mathrm{H}, \mathrm{CH}_{3}\right),-0.03\left(\mathrm{~s}, 3 \mathrm{H}, \mathrm{CH}_{3}\right), 0.03\left(\mathrm{~s}, 3 \mathrm{H}, \mathrm{CH}_{3}\right)$, 0.09 (s, 3H, $\left.\mathrm{CH}_{3}\right), 0.83(\mathrm{~s}, 9 \mathrm{H}, t \mathrm{Bu}), 0.85(\mathrm{~s}, 9 \mathrm{H}, t \mathrm{Bu}), 1.24$ (d, J=6.3 Hz, 3H, $\left.\mathrm{CH}_{3}\right), 1.82$ (d, 
$\left.J=7.2 \mathrm{~Hz}, 3 \mathrm{H}, \mathrm{CH}_{3}\right), 2.65\left(\mathrm{t}, J=6.5 \mathrm{~Hz}, 2 \mathrm{H}, \mathrm{CH}_{2}\right), 2.82\left(\mathrm{~s}, 3 \mathrm{H}, \mathrm{CH}_{3}\right), 3.28(\mathrm{~d}, J=1.6 \mathrm{~Hz}$, 1H, CH), 4.49 (t, $J=6.2 \mathrm{~Hz}, 1 \mathrm{H}, \mathrm{CHOTIPS}), 4.66$ (m, 1H, CHOTBS), 4.80 (d, J=5.7 Hz, $\left.2 \mathrm{H}, \mathrm{CH}_{2} \mathrm{CH}=\mathrm{CH}_{2}\right), 5.26\left(\mathrm{~d}, J=10.2 \mathrm{~Hz}, 1 \mathrm{H}, \mathrm{CH}_{2} \mathrm{CH}=\mathrm{CHH}\right), 5.38(\mathrm{~d}, J=18.3 \mathrm{~Hz}, 1 \mathrm{H}$, $\mathrm{CH}_{2} \mathrm{CH}=\mathrm{CHH}$ ), 5.52 (q, $J=12.2 \mathrm{~Hz}, 2 \mathrm{H}, \mathrm{CH}_{2} \mathrm{O}$ ), 5.67 (q, $J=7.8 \mathrm{~Hz}, 1 \mathrm{H}, \mathrm{CH}_{2} \mathrm{CHNH}$ ), 5.93$6.06\left(\mathrm{~m}, 1 \mathrm{H}, \mathrm{CH}_{2} \mathrm{CH}=\mathrm{CH}_{2}\right), 6.58\left(\mathrm{q}, J=7.2 \mathrm{~Hz}, 1 \mathrm{H},=\mathrm{CHCH}_{3}\right), 7.17\left(\mathrm{~s}, 1 \mathrm{H}, \mathrm{CH}(\mathrm{Ph})_{2}\right), 7.11-$ 7.44 (m, 13H, Indol, $2 \times \mathrm{Ph}$ ), 7.93 (s, 1H, S-CH), 7.96 (bs, 1H, NH,), 8.07 (s, 1H, S-CH), 8.86 (s, 1H, NH), $9.35(\mathrm{~s}, 1 \mathrm{H}, \mathrm{NH})$.

${ }^{13}$ C-NMR (75 MHz, $\left.\mathrm{CDCl}_{3}\right): \delta=-5.5,-5.1,-4.8,-4.3,12.2,14.4,17.9,18.1,20.8,25.7,25.8$, $39.4,47.7,60.6,65.1,65.9,68.0,69.4,77.5,112.6,118.8,120.7,122.4,123.2,123.5,125.4$, $125.9,126.3,127.2$, 128.0, 128.1, 128.12, 128.6, 129.7, 131.9, 136.5, 139.9, 146.7, 149.3, $160.7,160.8,161.4,167.3,171.5,172.0,172.7$.

HRMS (ESI): $m / z[\mathrm{M}+\mathrm{H}]^{+}$für $\mathrm{C}_{61} \mathrm{H}_{81} \mathrm{~N}_{6} \mathrm{O}_{10} \mathrm{~S}_{2} \mathrm{Si}_{2}$ ber.: 1177.4989; gef.: 1177.4994 .

\section{TIPS-Bis-Thiazol-Amin 94}

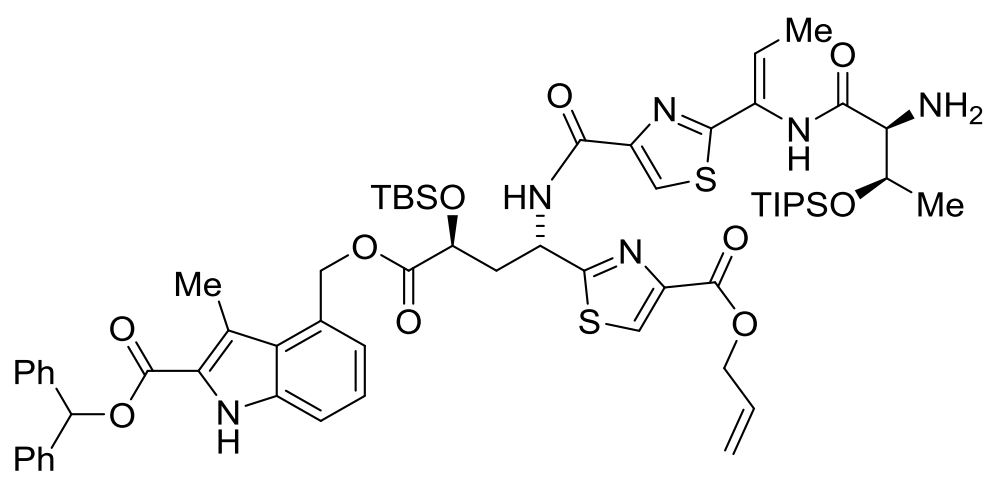

Eine Lösung des Fmoc-geschützten Amins 150 (68 mg, $48.6 \mu \mathrm{mol})$ in wasserfreiem $\mathrm{CH}_{2} \mathrm{Cl}_{2}$ $(15 \mathrm{~mL})$, wurde auf $-20^{\circ} \mathrm{C}$ gekühlt und mit einer Lösung von DBU $(1.13 \mathrm{~mL}, 7.25 \mathrm{mmol})$ in $\mathrm{CH}_{2} \mathrm{Cl}_{2}(8.5 \mathrm{~mL})$ tropfenweise versetzt. Nach erfolgter Zugabe wurde noch für 5 min gerührt, dann mit ges. $\mathrm{NaHCO}_{3}$-Lsg. und $\mathrm{H}_{2} \mathrm{O}$ (je $\left.20 \mathrm{~mL}\right)$ verdünnt und mit $\mathrm{CH}_{2} \mathrm{Cl}_{2}(3 \times 20 \mathrm{~mL})$ extrahiert. Die vereinigten organischen Extrakte wurden mit $\mathrm{Na}_{2} \mathrm{SO}_{4}$ entwässert und im Vakuum eingeengt. Reinigung des Rückstands durch Säulenchromatographie $\left(\mathrm{SiO}_{2}, 15 \mathrm{~g}\right.$, Petrolether/EtOAc 1:1, dann $\left.\mathrm{CH}_{2} \mathrm{Cl}_{2} / \mathrm{MeOH} 20: 1\right)$ lieferte $54 \mathrm{mg}(45.9 \mu \mathrm{mol}, 95 \%)$ des Amins 94 als farbloses Glas.

Spez. Drehung: $[\alpha]_{\mathrm{D}}{ }^{24}=-28.7\left(\mathrm{CHCl}_{3}, c=1\right)$.

DC: $R_{f}=0.38\left(\mathrm{CH}_{2} \mathrm{Cl}_{2} / \mathrm{MeOH}, 20: 1\right)$. 
IR: $\tilde{v}=748$ (s), 1095 (s), 1195 (s), 1226 (s), 1465 (s), $1681(\mathrm{~s}), 1975(\mathrm{~m}), 2931(\mathrm{w}) \mathrm{cm}^{-1}$.

${ }^{1}$ H-NMR $\left(300 \mathrm{MHz}, \mathrm{CDCl}_{3}\right): \delta=-0.09\left(\mathrm{~s}, 3 \mathrm{H}, \mathrm{CH}_{3}\right),-0.03\left(\mathrm{~s}, 3 \mathrm{H}, \mathrm{CH}_{3}\right), 0.83(\mathrm{~s}, 9 \mathrm{H}, t \mathrm{Bu})$, 1.05 (bs, 21H, TIPS), $1.31\left(\mathrm{~d}, J=6.3 \mathrm{~Hz}, 3 \mathrm{H}, \mathrm{CH}_{3}\right), 1.82$ (d, $\left.J=7.1 \mathrm{~Hz}, 3 \mathrm{H}, \mathrm{CH}_{3}\right), 2.64$ (t, $\left.J=6.7 \mathrm{~Hz}, 2 \mathrm{H}, \mathrm{CH}_{2}\right), 2.82\left(\mathrm{~s}, 3 \mathrm{H}, \mathrm{CH}_{3}\right), 3.35(\mathrm{~d}, J=2.4 \mathrm{~Hz}, 1 \mathrm{H}, \mathrm{CH}), 4.48(\mathrm{t}, J=6.1 \mathrm{~Hz}, 1 \mathrm{H}$, CHOTIPS), 4.66 (dd, $J=6.3,2.2 \mathrm{~Hz}, 1 \mathrm{H}, \mathrm{CHOTBS}), 4.81$ (d, $J=5.7 \mathrm{~Hz}, 2 \mathrm{H}, \mathrm{CH}_{2} \mathrm{CH}=\mathrm{CH}_{2}$ ), $5.26\left(\mathrm{~d}, J=10.4 \mathrm{~Hz}, 1 \mathrm{H}, \mathrm{CH}_{2} \mathrm{CH}=\mathrm{CHH}\right), 5.38\left(\mathrm{dd}, J=17.2,1.3 \mathrm{~Hz}, 1 \mathrm{H}, \mathrm{CH}_{2} \mathrm{CH}=\mathrm{CHH}\right), 5.52$ $\left(\mathrm{q}, J=12.4 \mathrm{~Hz}, 2 \mathrm{H}, \mathrm{CH}_{2} \mathrm{O}\right), 5.67\left(\mathrm{q}, J=7.4 \mathrm{~Hz}, 1 \mathrm{H}, \mathrm{CH}_{2} \mathrm{CHNH}\right), 5.98(\mathrm{~m}, 1 \mathrm{H}$, $\left.\mathrm{CH}_{2} \mathrm{CH}=\mathrm{CH}_{2}\right), 6.58\left(\mathrm{q}, J=7.1 \mathrm{~Hz}, 1 \mathrm{H},=\mathrm{CHCH}_{3}\right), 7.17\left(\mathrm{~s}, 1 \mathrm{H}, \mathrm{CH}(\mathrm{Ph})_{2}\right), 7.11-7.44(\mathrm{~m}, 13 \mathrm{H}$, indole, $2 \times \mathrm{Ph}), 7.91-7.93(\mathrm{~m}, 2 \mathrm{H}, \mathrm{NH}, \mathrm{S}-\mathrm{CH}), 8.07$ (s, 1H, S-CH), 8.84 (s, 1H, NH), 9.28 (s, $1 \mathrm{H}, \mathrm{NH})$.

${ }^{13}$ C-NMR $\left(100 \mathrm{MHz}, \mathrm{CDCl}_{3}\right): \delta=-5.5,-5.1,12.2,12.5,14.3,18.09,18.14,20.6,39.3,47.7$, $60.6,65.1,65.9,68.8,69.4,77.5,112.6,118.8,120.7,122.5,123.2,123.5,125.4,126.3$, 127.2 , 128.0, 128.09, 128.12, 128.6, 129.7, 131.9, 136.5, 139.9, 146.7, 149.3, 160.7, 160.8, $161.4,167.3,171.5,172.0,172.7$.

HRMS (ESI): $m / z[\mathrm{M}+\mathrm{H}]^{+}$für $\mathrm{C}_{61} \mathrm{H}_{81} \mathrm{~N}_{6} \mathrm{O}_{10} \mathrm{~S}_{2} \mathrm{Si}_{2}$ ber.: 1177.4989; gef.: 1177.4994 .

\section{Brombrenztraubensäuremethylester (152)}<smiles>COC(=O)C(=O)CBr</smiles>

Nach Kruse et al. ${ }^{[213]}$ wurde $L$-Milchsäuremethylester $(\mathbf{1 5 1}, 15 \mathrm{~g}, 0.144 \mathrm{~mol})$ in $\mathrm{CCl}_{4}$ (260 mL) gelöst, mit NBS (48.7 g, $0.27 \mathrm{~mol})$ versetzt und $6 \mathrm{~h}$ lang zumr Rückfluss erhitz. Nach Abkühlen auf RT wurde der ausgefallene Feststoff abfiltriert und mit wenig $\mathrm{CCl}_{4}$ gewaschen. Die Lösung wurde am Rotationsverdampfer eingeengt und der Rückstand durch fraktionierte Destillation im Vakuum (4 mbar) gereinigt. Es wurden $15.9 \mathrm{~g}$ (87.9 mmol, 61\%) $\alpha$-Brombrenztraubensäuremethylester (152) als gelbes Öl erhalten.

Siedepunkt: $65^{\circ} \mathrm{C}(4 \mathrm{mbar})$.

IR: $\tilde{v}=671(\mathrm{w}), 1049(\mathrm{~s}), 1226(\mathrm{~m}), 1435(\mathrm{w}), 1736(\mathrm{~s}), 3008(\mathrm{w}) \mathrm{cm}^{-1}$.

${ }^{1} \mathbf{H}$ NMR (250 MHz, $\left.\mathrm{CDCl}_{3}\right): \delta=3.93\left(\mathrm{~s}, 3 \mathrm{H}, \mathrm{CH}_{3}\right), 4.33\left(\mathrm{~s}, 2 \mathrm{H}, \mathrm{CH}_{2} \mathrm{Br}\right)$. 
${ }^{13}$ C NMR (63 MHz, $\left.\mathrm{CDCl}_{3}\right): \delta=30.56,53.52,159.72,184.24$.

\section{2-Aminothiazol-4-carbonsäuremethylester (153) ${ }^{[320]}$}<smiles>COC(=O)c1csc(N)n1</smiles>

Thioharnstoff $(4.42 \mathrm{~g}, 58 \mathrm{mmol})$ wurde $\mathrm{zu}$ einer Lösung von Brombrenztraubensäuremethylester $(10 \mathrm{~g}, 55.3 \mathrm{mmol})$ in wasserfreiem $\mathrm{MeOH}(100 \mathrm{~mL})$ gegeben und die Mischung $2 \mathrm{~h}$ lang zum Rückfluss erhitzt. Nach Abkühlen auf RT wurde das Lösungsmittel am Rotationsverdampfer entfernt, der Rückstand in Eiswasser $(150 \mathrm{~mL})$ gelöst und durch Zugabe von $\mathrm{K}_{2} \mathrm{CO}_{3} \mathrm{pH} 8$ eingestellt. Der ausgefallene Feststoff wurde abfiltriert, mehrmals mit wenig Wasser gewaschen und im Vakuum getrocknet. Es wurden 8.13 g (51.4 mmol, 93\%) des 2-Aminothiazols 153 als gelber Feststoff erhalten.

Schmelzpunkt: $174^{\circ} \mathrm{C}$.

IR: $\tilde{v}=732$ (m), $987(\mathrm{~m}), 1234$ (s), 1346 (m), 1539 (m), 1624 (w), 1693 (m), $3105(\mathrm{w})$, $3402(\mathrm{w}) \mathrm{cm}^{-1}$.

${ }^{1} \mathbf{H}$ NMR $\left(250 \mathrm{MHz}, \mathrm{DMSO}-d_{6}\right): \delta=3.73\left(\mathrm{~s}, 3 \mathrm{H}, \mathrm{CH}_{3}\right), 7.24\left(\mathrm{bs}, 2 \mathrm{H}, \mathrm{NH}_{2}\right), 7.48(\mathrm{~s}, 1 \mathrm{H}, \mathrm{CH})$.

${ }^{13}$ C NMR (63 MHz, DMSO- $\left.d_{6}\right): \delta=51.4,117.1,141.9,161.5,168.2$.

\section{2-Iodthiazol-4-carbonsäuremthylester (154) ${ }^{[110]}$}<smiles>COC(=O)c1csc(I)n1</smiles>

Nach der Methode von Delgado et al. ${ }^{[108]}$ wurde das 2-Aminothiazol 153 (7.48 g, 47.3 mmol) in wasserfreiem THF (95 mL) gelöst, die Lösung auf $0{ }^{\circ} \mathrm{C}$ gekühlt und mit $\mathrm{CH}_{2} \mathrm{I}_{2}(19.1 \mathrm{~mL}$, $237 \mathrm{mmol})$ versetzt. Anschließend wurde tert-Butylnitrit $(23.7 \mathrm{~mL}, 199 \mathrm{mmol})$ über einen Zeitraum von 75 min langsam zugetropft. Nach vollständiger Zugabe wurde die zähe Suspension weitere $15 \mathrm{~min}$ lang bei $0{ }^{\circ} \mathrm{C}$ gerührt und das Kühlbad anschließend entfernt. Nach 5-10 min wurde die Lösung klar und eine Gasentwicklung trat ein. Die Mischung wurde sofort mit kaltem Wasser $\left(5-10^{\circ} \mathrm{C}\right)$ gekühlt und weitere $2 \mathrm{~h}$ lang gerührt. Die 
Reaktionsmischung wurde mit EtOAc $(500 \mathrm{~mL})$ verdünnt, mit ges. $\mathrm{NaHCO}_{3}$-Lsg. $(2 \times 150 \mathrm{~mL})$ und anschließend ges. NaCl-Lsg. $(150 \mathrm{~mL})$ gewaschen. Nach Entwässern der organischen Lösung mit $\mathrm{Na}_{2} \mathrm{SO}_{4}$ wurde die Lösung am Rotationsverdampfer eingeengt. Reinigung des Rohprodukts durch Säulenchromatographie $\left(\mathrm{SiO}_{2}, 1000\right.$ g, Petrolether/Aceton, 3:1) ergab 9.54 g (35.5 mmol, 75\%) des Iodthiazols 154 als leicht gelben Feststoff.

DC: $R_{f}=0.51($ Heptan/Aceton, $2: 1)$.

Schmelzpunkt: $133^{\circ} \mathrm{C}$.

IR: $\tilde{v}=756$ (s), 960 (s), 983 (s), 1238 (s), 1300 (m), 1400 (m), 1432 (m), 1489 (m), 1693 (s), $3116(\mathrm{w}) \mathrm{cm}^{-1}$.

${ }^{1} \mathbf{H}$ NMR $\left(250 \mathrm{MHz}, \mathrm{CDCl}_{3}\right): \delta=3.96\left(\mathrm{~s}, 3 \mathrm{H}, \mathrm{CH}_{3}\right), 8.16(\mathrm{~s}, 1 \mathrm{H}, \mathrm{CH})$

${ }^{13}$ C NMR (63 MHz, $\left.\mathrm{CDCl}_{3}\right): \delta=52.7,101.3,133.5,148.9,160.3$.

2-(3-Hydroxybut-1-yn-1-yl)thiazol-4-carbonsäuremethylester (155)<smiles>COC(=O)c1csc(C#CC(C)O)n1</smiles>

$\mathrm{Pd}\left(\mathrm{PPh}_{3}\right)_{2} \mathrm{Cl}_{2} \quad(240 \mathrm{mg}, \quad 0.34 \mathrm{mmol})$ und $\mathrm{CuI}(130 \mathrm{mg}, 0.68 \mathrm{mmol})$ wurden in frisch destilliertem DMF (87 mL) suspendiert. Anschließend wurden das 2-Iodthiazol 154 (9.52 g, $34.15 \mathrm{mmol})$, 3-Butin-2-ol (3.7 mL, $50.9 \mathrm{mmol})$ und $\mathrm{Et}_{3} \mathrm{~N}$ (9.7 mL, $\left.69.4 \mathrm{mmol}\right)$ nacheinander zugegeben. Die Mischung wurde danach $2 \mathrm{~h}$ lang bei $50^{\circ} \mathrm{C}$ gerührt. Während dieser Zeit färbte sich die Lösung dunkelbraun. Nach Abkühlen auf RT wurde die Reaktionsmischung mit $\mathrm{CH}_{2} \mathrm{Cl}_{2}(250 \mathrm{~mL})$ verdünnt und über eine Schicht Kieselguhr filtriert. Der Filterkuchen wurde mit $\mathrm{CH}_{2} \mathrm{Cl}_{2}(3 \times 75 \mathrm{~mL})$ nachgewaschen und das Filtrat im Vakuum eingeengt. Reinigung des Rückstands durch Säulenchromatographie $\left(\mathrm{SiO}_{2}, 500\right.$ g, Petrolether/Aceton, 2:1) lieferte 6.35 g (30.1 mmol, 88\%) des Propargylalkohols 155 als leicht gelben Feststoff.

Schmelzpunkt: $89^{\circ} \mathrm{C}$.

Alle relevanten analytischen Daten stimmen mit den veröffentlichten Werten überein. ${ }^{[37]}$ 


\section{2-(3-Oxobut-1-yn-1-yl)thiazol-4- carbonsäuremethylester (64)}<smiles>COC(=O)c1csc(C#CC(C)=O)n1</smiles>

Der Propargylalkohol 155 (1.06 g, $5.0 \mathrm{mmol})$ wurde in wasserfreiem $\mathrm{CH}_{2} \mathrm{Cl}_{2}(50 \mathrm{~mL})$ gelöst, und bei 5-10 ${ }^{\circ} \mathrm{C}$ portionsweise (5-10 Portionen) mit Dess-Martin-Periodinan ${ }^{[312]}$ (2.33 g, $5.5 \mathrm{mmol}$ ) versetzt. Nach Entfernen des Kühlbades wurde das Gemisch für weitere $60 \mathrm{~min}$ gerührt und dann mit EtOAc (150 mL) verdünnt. Die Lösung wurde mit einer 1:1 Mischung aus ges. $\mathrm{NaHCO}_{3}$-Lsg. und ges. $\mathrm{Na}_{2} \mathrm{SO}_{3}$-Lsg. $(2 \times 50 \mathrm{~mL}), \mathrm{H}_{2} \mathrm{O}(2 \times 50 \mathrm{~mL}), 10 \%$ iger Zitronensäure $(50 \mathrm{~mL})$, erneut mit $\mathrm{H}_{2} \mathrm{O}(50 \mathrm{~mL})$ und abschließend mit ges. NaCl-Lsg. (50 mL) gewaschen. Die Lösung wurde mit $\mathrm{Na}_{2} \mathrm{SO}_{4}$ entwässert und am Rotationsverdampfer eingeengt. Der Rückstand wurde durch Säulenchromatographie $\left(\mathrm{SiO}_{2}, 100 \mathrm{~g}\right.$, Petrolether $/ \mathrm{CH}_{2} \mathrm{Cl}_{2} /$ Aceton, 5:5:1) gereinigt. Es wurden $826 \mathrm{mg}$ (3.95 mmol, 79\%) des Alkinylketons 64 als farbloser Feststoff erhalten.

Schmelzpunkt: $162-163^{\circ} \mathrm{C}$.

Alle relevanten analytischen Daten stimmen mit den veröffentlichten Werten überein. ${ }^{[37]}$

\section{5-Acetyl-3-hydroxy-4-(5`-(methoxycarbonyl)thiazol-2`-yl)picolinsäuremethylester (156)}<smiles>COC(=O)c1csc(-c2c(C(C)=O)cnc(C(=O)OC)c2O)n1</smiles>

Lu et al. ${ }^{[37]}$ folgend wurde eine Lösung des Alkinylketons 64 (1 g, $\left.4.78 \mathrm{mmol}\right)$ und des 1-Azabutadiens $\mathbf{6 5}^{[37]}(4.15 \mathrm{~g}, 14.4 \mathrm{mmol})$ in Toluol $(1 \mathrm{~mL})$ in einem Bombenrohr $4 \mathrm{~h}$ lang bei $165^{\circ} \mathrm{C}$ gerührt, anschließend auf RT abgekühlt und das Reaktionsgemisch auf Kieselgel (50 g) adsorbiert. Mehrmalige Säulenchromatographie $\left(\mathrm{SiO}_{2}, \quad 500 \mathrm{~g}\right.$, $\mathrm{CH}_{2} \mathrm{Cl}_{2} /$ Petrolether/Aceton/HCOOH， 50:50:10:1) lieferte $451 \mathrm{mg}(0.96 \mathrm{mmol}, 28 \%)$ des 3-Hydroxypyridins 156 als leicht gelben Feststoff. Daneben wurden die beiden Regioisomere $46(28 \%)$ und $66(27 \%)$ in etwa gleicher Menge erhalten. 
Alle relevanten analytischen Daten für die Isomere 46 und 66 stimmten mit den publizierten überein. $^{[37]}$

DC: $R_{f}=0.29$ (66), 0.42 (46), 0.48 (156), $\left(\mathrm{CH}_{2} \mathrm{Cl}_{2} / \mathrm{Heptan} /\right.$ Aceton/HCOOH, 50/50/10/1).

Schmelzpunkt: $193^{\circ} \mathrm{C}$.

IR: $\tilde{v}=682(\mathrm{~m}), 754(\mathrm{~s}), 813(\mathrm{~m}), 985(\mathrm{~m}), 1147(\mathrm{~s}), 1205(\mathrm{~s}), 1296(\mathrm{~m}), 1670(\mathrm{~m}), 1699(\mathrm{~m})$, $1743(\mathrm{~m}) \mathrm{cm}^{-1}$.

${ }^{1} \mathbf{H}$ NMR (400 MHz, $\left.\mathrm{CDCl}_{3}\right): \delta=2.59\left(\mathrm{~s}, 3 \mathrm{H}, \mathrm{CH}_{3}\right), 3.95\left(\mathrm{~s}, 3 \mathrm{H}, \mathrm{CH}_{3}\right), 4.12\left(\mathrm{~s}, 3 \mathrm{H}, \mathrm{CH}_{3}\right)$, $8.23(\mathrm{~s}, 1 \mathrm{H}, \mathrm{CH}), 8.40(\mathrm{~s}, 1 \mathrm{H}, \mathrm{CH}), 12.01(\mathrm{~s}, 1 \mathrm{H}, \mathrm{OH})$.

${ }^{13} \mathrm{C}$ NMR $\left(100 \mathrm{MHz}, \mathrm{CDCl}_{3}\right): \delta=31.1,52.5,53.8,125.2,130.8,131.1,138.3,141.1,146.5$, $154.9,157.0,161.5,169.7,201.1$.

\section{(4S)-3-tert-Butyloxycarbonyl-2,2-dimethyl-4-(5'-(pyridin-2"'-yl)thiazol-2'-yl)thiazolidin} (162)

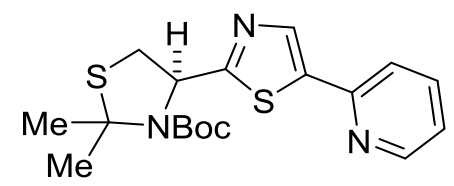

Eine Mischung aus Thioamid 47 $7^{[98]}(500 \mathrm{mg}, 1.81 \mathrm{mmol})$ und festem $\mathrm{KHCO}_{3}$ (465 mg, $4.64 \mathrm{mmol})$ wurde in wasserfreiem THF $(7.5 \mathrm{~mL})$ suspendiert und auf $-40{ }^{\circ} \mathrm{C}$ gekühlt. Anschließend wurde eine Lösung von 2‘-Bromacetylpyridin ${ }^{[219]}$ (161, $\left.250 \mathrm{mg}, 1.25 \mathrm{mmol}\right)$ in wasserfreiem THF (5 mL) tropfenweise zugegeben. Nach vollständiger Zugabe wurde die Reaktionsmischung langsam aufgetaut und $22 \mathrm{~h}$ lang bei RT gerührt. Nach Filtration unter inerten Bedingungen wurde die erhaltene Lösung auf $-40{ }^{\circ} \mathrm{C}$ gekühlt und mit Trifluoressigsäureanhydrid $(810 \mu \mathrm{L}, 5.82 \mathrm{mmol})$ und 2,6-Lutidin (1.45 mL, $12.4 \mathrm{mmol})$ versetzt. Die Mischung wurde langsam aufgetaut und $20 \mathrm{~h}$ lang bei RT gerührt. Die Reaktionsmischung wurde mit ges. NaCl-Lsg. $(20 \mathrm{~mL})$ verdünnt und mit EtOAc $(3 \times 15 \mathrm{~mL})$ extrahiert. Die vereinigten organischen Extrakte wurden mit 10\%iger Zitronensäure, ges. $\mathrm{NaHCO}_{3}$-Lsg. und ges. NaCl-Lsg. (je $20 \mathrm{~mL}$ ) gewaschen. Nach Entwässern mit $\mathrm{MgSO}_{4}$ wurden die Lösungsmittel im Vakuum entfernt und der Rückstand durch Säulenchromatographie $\left(\mathrm{SiO}_{2}, 50 \mathrm{~g}\right.$, Petrolether/EtOAc, 3:1) gereinigt. Es wurden $451 \mathrm{mg}$ (1.2 mmol, 96\%) des Pyridyl-Thiazols 162 als cremefarbener Feststoff erhalten. 
Spez. Drehung: $[\alpha]_{\mathrm{D}}{ }^{24}=-95.6\left(\mathrm{CHCl}_{3}, c=1\right)$.

Schmelzpunkt: $124-126^{\circ} \mathrm{C}$.

DC: $R_{f}=0.44($ Heptan/EtOAc, $3: 1)$.

IR: $\tilde{v}=756$ (s), 1064 (s), 1165 (s), 1342 (s), 1689 (s), 1975 (b), 2978 (w) cm $\mathrm{cm}^{-1}$.

${ }^{1}$ H-NMR $\left(400 \mathrm{MHz}, \mathrm{MeCN}^{-} d_{3}, 343 \mathrm{~K}\right): \delta=1.40(\mathrm{~s}, 9 \mathrm{H}, t \mathrm{Bu}), 1.86(\mathrm{~s}, 3 \mathrm{H}, \mathrm{Me}), 1.97(\mathrm{~s}, 3 \mathrm{H}$, Me), $3.23(\mathrm{~d}, J=12.2 \mathrm{~Hz}, 1 \mathrm{H}, \mathrm{S}-\mathrm{CHH}), 3.64$ (dd, $J=12.2,6.4 \mathrm{~Hz}, 1 \mathrm{H}, \mathrm{S}-\mathrm{CH} H), 5.74$ (d, $J=6.4 \mathrm{~Hz}, 1 \mathrm{H}, \mathrm{CH}-\mathrm{NBoc}), 7.28(\mathrm{~m}, 1 \mathrm{H}, \mathrm{CH}), 7.82(\mathrm{dt}, J=7.8,1.7 \mathrm{~Hz}, 1 \mathrm{H}, \mathrm{CH}), 8.04(\mathrm{~s}, 1 \mathrm{H}$, $=\mathrm{N}-\mathrm{CH}), 8.08(\mathrm{~d}, J=7.9 \mathrm{~Hz}, 1 \mathrm{H}, \mathrm{CH}), 8.60(\mathrm{~d}, J=4.7 \mathrm{~Hz}, 1 \mathrm{H}, \mathrm{CH})$.

${ }^{13}$ C-NMR $\left(125 \mathrm{MHz}, \mathrm{MeCN}-d_{3}, 343 \mathrm{~K}\right): \delta=29.3,29.7,30.8,35.3,67.8,73.1,82.5,122.4$, $124.5,138.7,151.3,154.2,154.3,156.6,176.23$.

HRMS (ESI): $m / z[\mathrm{M}+\mathrm{H}]^{+}$für $\mathrm{C}_{18} \mathrm{H}_{24} \mathrm{~N}_{3} \mathrm{O}_{2} \mathrm{~S}_{2}$ ber.: 378.1304; gef.: 378.1305 .

\section{2-(1'(S)-Allyloxycarbonylamino-2'-tritylthioethyl)-4-(2'-pyridyl)thiazol (163)}

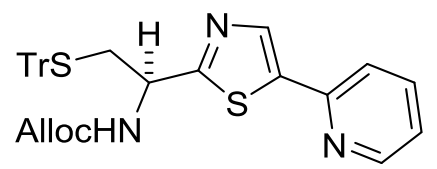

Eine Lösung des $\mathrm{N}$-Boc-Thiaminals $162(378 \mathrm{mg}, 1.0 \mathrm{mmol})$ in wasserfreiem $\mathrm{CH}_{2} \mathrm{Cl}_{2}(16 \mathrm{~mL})$ wurde auf $0{ }^{\circ} \mathrm{C}$ gekühlt, mit $\mathrm{Et}_{3} \mathrm{SiH}(1.25 \mathrm{~mL}, 7.74 \mathrm{mmol})$ und TFA $(16 \mathrm{~mL})$ versetzt, $30 \mathrm{~min}$ lang bei $0{ }^{\circ} \mathrm{C}$ und $1.5 \mathrm{~h}$ lang bei RT gerührt. Die Mischung wurde anschließend im Vakuum eingeengt und der Rückstand in wasserfreiem DMF $(15 \mathrm{~mL})$ gelöst. Triphenylmethylchlorid (463 mg, $1.66 \mathrm{mmol}$ ) wurde zugegeben und die Mischung $2 \mathrm{~d}$ lang bei RT gerührt. Das Lösungsmittel wurde am Rotationsverdampfer entfernt und der Rückstand in THF (25 mL) und $\mathrm{H}_{2} \mathrm{O}(5 \mathrm{~mL})$ gelöst. Die Mischung wurde auf $0{ }^{\circ} \mathrm{C}$ gekühlt, mit $\mathrm{NaHCO}_{3}(175 \mathrm{mg}$, $2.08 \mathrm{mmol})$ und Chlorameisensäureallylester $(112 \mu \mathrm{L}, 1.05 \mathrm{mmol})$ versetzt. Nach $2 \mathrm{~h}$ bei $0{ }^{\circ} \mathrm{C}$ wurde ein weiteres Äquivalent Chlorameisensäureallylester $(107 \mu \mathrm{L}, 1.0 \mathrm{mmol})$ zugegeben und die Mischung langsam auf RT erwärmt. Nach 4 h bei RT wurde mit pH 3 Puffer $(50 \mathrm{~mL})$ verdünnt und mit EtOAc $(3 \times 25 \mathrm{~mL})$ extrahiert. Die vereinigten organischen Extrakte wurden mit $\mathrm{H}_{2} \mathrm{O}$ und ges. NaCl-Lsg. (je $25 \mathrm{~mL}$ ) gewaschen, mit $\mathrm{Na}_{2} \mathrm{SO}_{4}$ entwässert und im Vakuum eingeengt. Reinigung des Rückstands durch Säulenchromatographie $\left(\mathrm{SiO}_{2}, 40 \mathrm{~g}\right.$, 
Petrolether/EtOAc, 2:1) lieferte $353 \mathrm{mg}(0.63 \mathrm{mmol}, 63 \%)$ des Pyridyl-Thiazols 163 als farblosen Schaum.

Spez. Drehung $[\alpha]_{\mathrm{D}}{ }^{24}=-4.7\left(\mathrm{CHCl}_{3}, c=1\right)$.

DC: $R_{f}=0.30($ Heptan/EtOAc, $2: 1)$.

IR: $\tilde{v}=694(\mathrm{~s}), 741(\mathrm{~s}), 1033(\mathrm{~m}), 1242(\mathrm{~m}), 1489(\mathrm{~m}), 1712(\mathrm{~s}), 1975$ (b), 3278 (b) cm $\mathrm{cm}^{-1}$.

${ }^{1} \mathbf{H}-\mathbf{N M R}\left(300 \mathrm{MHz}, \mathrm{CDCl}_{3}\right): \delta=2.82-2.88(\mathrm{~m}, 1 \mathrm{H}, \mathrm{S}-\mathrm{CHH}), 2.98-3.04(\mathrm{~m}, 1 \mathrm{H}, \mathrm{S}-\mathrm{CH} H)$, $4.58\left(\mathrm{~d}, J=5.4 \mathrm{~Hz}, 2 \mathrm{H}, \mathrm{CH}_{2} \mathrm{CH}=\mathrm{CH}_{2}\right), 4.98(\mathrm{~d}, J=5.9 \mathrm{~Hz}, 1 \mathrm{H}, \mathrm{C} H \mathrm{NH}), 5.22-5.45(\mathrm{~m}, 3 \mathrm{H}$, $\mathrm{NH}, \mathrm{CH}_{2} \mathrm{CH}=\mathrm{CH}_{2}$ ), 5.87-6.00 (m, $1 \mathrm{H}, \mathrm{CH}_{2} \mathrm{CH}=\mathrm{CH}_{2}$ ), 7.20-7.32 (m, 10H, Tr), 7.42-7.44 (m, $5 \mathrm{H}, \mathrm{Tr}), 7.76(\mathrm{dt}, J=7.5,1.6 \mathrm{~Hz}, 1 \mathrm{H}, \mathrm{CH}), 7.93(\mathrm{~s}, 1 \mathrm{H}$, Thiazol-CH), 8.07 (d, $J=7.8 \mathrm{~Hz}, 1 \mathrm{H}$, $\mathrm{CH}), 8.61(\mathrm{~d}, J=4.2 \mathrm{~Hz}, 1 \mathrm{H}, \mathrm{CH})$.

${ }^{13}$ C-NMR $\left(75 \mathrm{MHz}, \mathrm{CDCl}_{3}\right): \delta=37.2,52.3,65.9,67.3,117.1,117.8,121.3,122.8,126.8$, $128.0,129.5,132.5,136.9,144.4,149.4,152.4,155.2,170.4$.

HRMS (ESI): $m / z[\mathrm{M}+\mathrm{Na}]^{+}$für $\mathrm{C}_{33} \mathrm{H}_{29} \mathrm{~N}_{3} \mathrm{NaO}_{2} \mathrm{~S}_{2}$ ber.: 586.1593 ; gef.: 586.1605 .

(1'S)-2-(1'-Amino-2'-tritylthioethyl)-4-(2"'-pyridyl)thiazol (160)

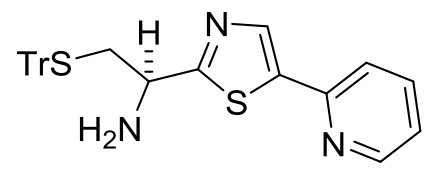

Eine Lösung des Carbamats $163(100 \mathrm{mg}, 0.18 \mathrm{mmol})$ in entgastem, wasserfreiem THF $(5 \mathrm{~mL})$ wurde auf $0{ }^{\circ} \mathrm{C}$ gekühlt, $\mathrm{PhSiH}_{3}(110 \mu \mathrm{L}, 0.88 \mathrm{mmol})$ und eine Lösung von $\mathrm{Pd}\left(\mathrm{PPh}_{3}\right)_{4}$ (46 mg, $40 \mu \mathrm{mol})$ in THF $(2.5 \mathrm{~mL})$ zugegeben. Die Mischung wurde $20 \mathrm{~min}$ lang bei $0{ }^{\circ} \mathrm{C}$ und $1.5 \mathrm{~h}$ bei RT gerührt. Die Mischung wurde im Vakuum eingeengt und der Rückstand durch Säulenchromatographie $\left(\mathrm{SiO}_{2}, 25 \mathrm{~g}\right.$, Petrolether/EtOAc/Et 3 N, 100:100:1) gereinigt. Es wurden 85 mg (0.18 mmol, quant.) des Amins 160 als leicht gelber Schaum erhalten.

Spez. Drehung: $[\alpha]_{\mathrm{D}}{ }^{24}=+19.1\left(\mathrm{CHCl}_{3}, c=1\right)$.

DC: $R_{f}=0.23($ Heptan/EtOAc/Et 3 N, 100:100:1).

IR: $\tilde{v}=694(\mathrm{~s}), 741(\mathrm{~s}), 1489(\mathrm{~m}), 1589(\mathrm{~m}), 1674(\mathrm{~m}), 1959(\mathrm{w}), 2916(\mathrm{w}), 3356(\mathrm{w}) \mathrm{cm}^{-1}$. 
${ }^{1} \mathbf{H}-\mathbf{N M R}\left(300 \mathrm{MHz}, \mathrm{CDCl}_{3}\right.$ ): $\delta=1.83$ (bs, 2H, $\mathrm{NH}_{2}$ ), 2.71-2.92 (m, 2H, $\mathrm{CH}_{2}$ ), 3.89 (dd, $J=8.1,4.5 \mathrm{~Hz}, 1 \mathrm{H}, \mathrm{CH}), 7.19-7.32$ (m, 10H, Tr, Pyridin), 7.46-7.48 (m, 6H, Tr), 7.75 (dt, $J=7.7,1.7 \mathrm{~Hz}, 1 \mathrm{H}, \mathrm{CH}), 7.94(\mathrm{~s}, 1 \mathrm{H}$, Thiazol-CH), 8.05 (d, $J=7.9 \mathrm{~Hz}, 1 \mathrm{H}, \mathrm{CH}), 8.60$ (d, $J=4.1 \mathrm{~Hz}, 1 \mathrm{H}, \mathrm{CH})$.

${ }^{13}$ C-NMR (63 MHz, $\left.\mathrm{CDCl}_{3}\right)$ : 40.5, 53.3, 67.2, 116.9, 121.1, 122.6, 126.8., 128.0, 129.6, $136.8,144.7,149.4,152.7,155.1,175.1$.

HRMS (ESI): $m / z[\mathrm{M}+\mathrm{H}]^{+}$für $\mathrm{C}_{29} \mathrm{H}_{26} \mathrm{~N}_{3} \mathrm{~S}_{2}$ ber.: 480.1563 ; gef.: 480.1566 .

\section{Bis-Thiazolcarbonsäure 159}

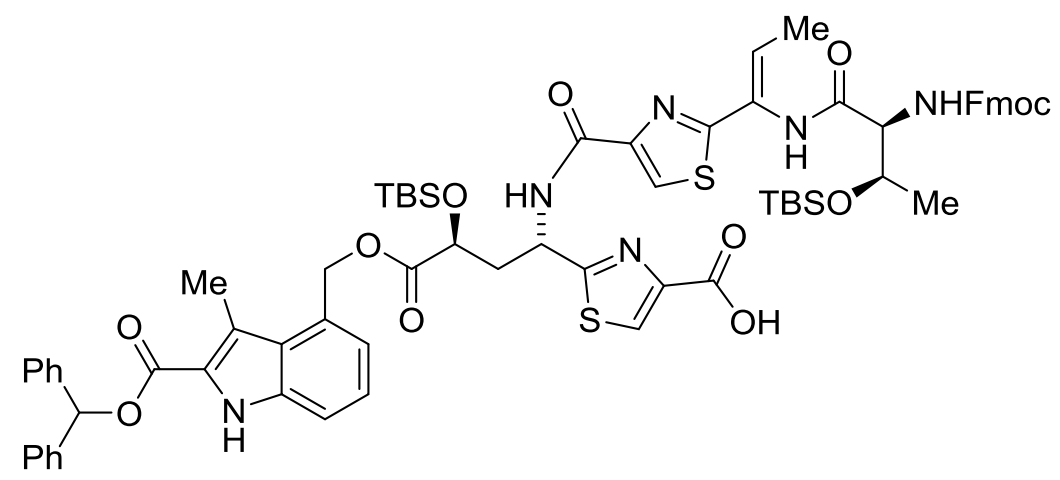

Eine Lösung des Allylesters 149 (30 mg, $22 \mu \mathrm{mol})$ in entgastem, wasserfreiem THF ( $2 \mathrm{~mL})$ wurde auf $0{ }^{\circ} \mathrm{C}$ gekühlt, mit $\mathrm{PhSiH}_{3}(24 \mu \mathrm{L}, 0.19 \mathrm{mmol})$ und einer Lösung von $\mathrm{Pd}\left(\mathrm{PPh}_{3}\right)_{4}$ $(5 \mathrm{mg}, 4.3 \mu \mathrm{mol})$ in THF $(0.5 \mathrm{~mL})$ versetzt. Die Mischung wurde $15 \mathrm{~min}$ lang gerührt und anschließend im Vakuum eingeengt. Der Rückstand wurde durch Säulenchromatographie $\left(\mathrm{SiO}_{2}, 10 \mathrm{~g}, \quad\right.$ Petrolether/EtOAc, 1:1, anschließend $\mathrm{CH}_{2} \mathrm{Cl}_{2} / \mathrm{MeOH} / \mathrm{HCOOH}, \quad 200: 8: 1$ ) gereinigt. Es wurden $27 \mathrm{mg}(20 \mu \mathrm{mol}, 93 \%)$ der Bis-Thiazolcarbonsäure 159 als leicht braunes Glas erhalten.

Spez. Drehung: $[\alpha]_{\mathrm{D}}{ }^{24}=-20.8\left(\mathrm{CHCl}_{3}, c=1\right)$.

DC: $R_{f}=0.26\left(\mathrm{CH}_{2} \mathrm{Cl}_{2} / \mathrm{MeOH} / \mathrm{HCOOH}, 100: 5: 1\right)$.

IR: $\tilde{v}=741(\mathrm{~s}), 1103(\mathrm{~m}), 1226(\mathrm{~s}), 1489(\mathrm{~m}), 1689(\mathrm{~s}), 1975(\mathrm{w}), 2931(\mathrm{w}) \mathrm{cm}^{-1}$.

${ }^{1} \mathbf{H}-\mathrm{NMR}\left(300 \mathrm{MHz}, \mathrm{CDCl}_{3}\right): \delta=-0.07\left(\mathrm{~s}, 3 \mathrm{H}, \mathrm{CH}_{3}\right),-0.02\left(\mathrm{~s}, 3 \mathrm{H}, \mathrm{CH}_{3}\right), 0.14\left(\mathrm{~s}, 3 \mathrm{H}, \mathrm{CH}_{3}\right)$, $0.17\left(\mathrm{~s}, 3 \mathrm{H}, \mathrm{CH}_{3}\right), 0.84(\mathrm{~s}, 9 \mathrm{H}, t \mathrm{Bu}), 0.88$ (s, 9h, $\left.t \mathrm{Bu}\right), 1.22$ (d, $\left.J=6.2 \mathrm{~Hz}, 3 \mathrm{H}, \mathrm{CH}_{3}\right), 1.83$ (d, $\left.J=7.1 \mathrm{~Hz}, 3 \mathrm{H}, \mathrm{CH}_{3}\right), 2.62\left(\mathrm{bs}, 2 \mathrm{H}, \mathrm{CH}_{2}\right), 2.80\left(\mathrm{~s}, 3 \mathrm{H}, \mathrm{CH}_{3}\right), 4.17$ (t, $J=6.6 \mathrm{~Hz}, 1 \mathrm{H}$, 
CHNHFmoc), 4.37-4.47 (bm, 5H, CHCONH, Fmoc, $2 \times$ CHOTBS), 5.48 (q, $J=12.7$ Hz, 2H, $\left.\mathrm{CH}_{2} \mathrm{O}\right), 5.68\left(\mathrm{q}, J=7.5 \mathrm{~Hz}, 1 \mathrm{H}, \mathrm{CH}_{2} \mathrm{CHNH}\right), 5.90(\mathrm{~d}, J=5.8 \mathrm{~Hz}, 1 \mathrm{H}, \mathrm{CON} H), 6.65$ (d, $\left.J=6.7 \mathrm{~Hz}, 1 \mathrm{H},=\mathrm{CHCH}_{3}\right), 7.17\left(\mathrm{~s}, 1 \mathrm{H}, \mathrm{CH}(\mathrm{Ph})_{2}\right), 7.07-7.44(\mathrm{~m}, 17 \mathrm{H}$, Fmoc, Indol, $2 \times \mathrm{Ph})$, 7.54 (m, 2H, Fmoc), 7.73 (d, J=7.5 Hz, 2H, Fmoc), 7.98-8.01 (m, 2H, NH, S-CH), 8.10 (s, $\mathrm{S}-\mathrm{CH}), 8.18$ (s, 1H, OH), 9.02 (s, 1H, Indolyl-NH).

${ }^{13}$ C-NMR (75 MHz, $\left.\mathrm{CDCl}_{3}\right): \delta=-5.5,-5.1,-5.0,-4.7,12.1,14.2,17.8,18.1,18.5,25.6,25.7$, 39.2 , 47.1, 47.7, 53.4, 59.6, 65.2, 67.1, 67.9, 69.3, 77.6, 112.8, 119.9, 120.6, 122.6, 123.5, $123.7,124.9,125.3,126.3,127.0,127.16,127.20,128.1,129.5,136.5,139.9,141.3,143.6$, $146.0,149.3,156.4,160.6,161.5,162.5,166.8,168.4,171.7,172.6$.

HRMS (ESI): $m / z[\mathrm{M}+\mathrm{H}]^{+}$für $\mathrm{C}_{70} \mathrm{H}_{81} \mathrm{~N}_{6} \mathrm{O}_{12} \mathrm{~S}_{2} \mathrm{Si}_{2}$ ber.: 1317.4887; gef.: 1317.4895 .

\section{B-Ring-Testsystem 113}

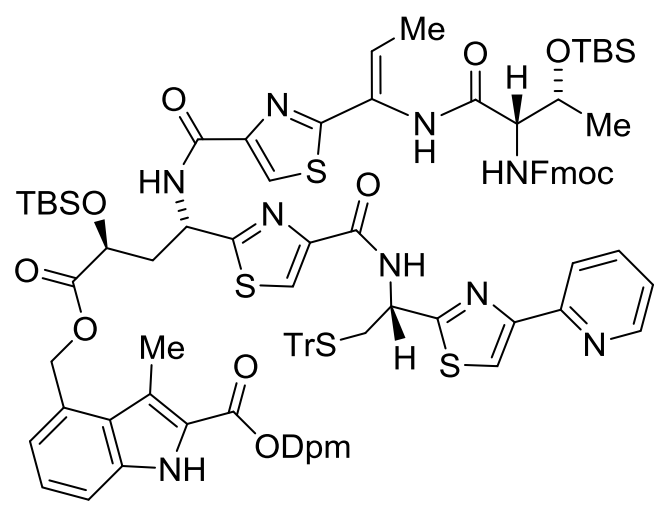

Eine Lösung der Thiazolcarbonsäure 159 (86 mg, $0.65 \mathrm{mmol})$ in wasserfreiem DMF (2 mL) wurde auf $0^{\circ} \mathrm{C}$ gekühlt, mit EtNiPr $2(26 \mu \mathrm{L}, 0.16 \mathrm{mmol})$ und PyBOP (71 mg, $\left.0.14 \mathrm{mmol}\right)$ versetzt und $5 \mathrm{~min}$ lang bei $0^{\circ} \mathrm{C}$ gerührt. Eine Lösung des Amins 160 (47 mg, $0.98 \mathrm{mmol}$ ) in wasserfreiem DMF (2 mL) wurde zugegeben, das Kühlbad entfernt und das Gemisch $16 \mathrm{~h}$ lang bei RT gerührt. Nach Verdünnen mit pH 3 Puffer $(10 \mathrm{~mL})$ wurde mit $\mathrm{CH}_{2} \mathrm{Cl}_{2}(3 \times 10 \mathrm{~mL})$ extrahiert. Die vereinigten organischen Extrakte wurden mit halb-ges. $\mathrm{NaHCO}_{3}$-Lsg. $(3 \times 10 \mathrm{~mL}), \mathrm{H}_{2} \mathrm{O}(10 \mathrm{~mL})$ und ges. NaCl-Lsg. $(10 \mathrm{ml})$ gewaschen, mit $\mathrm{Na}_{2} \mathrm{SO}_{4}$ entwässert und im Vakuum eingeengt. Säulenchromatographie $\left(\mathrm{SiO}_{2}, 25 \mathrm{~g}\right.$, Petrolether/EtOAc, 1:1) des Rückstand lieferte 89 mg (0.50 mmol, 77\%) des Tris-Thiazols 113 als leicht gelbes Glas.

Spez. Drehung: $[\alpha]_{\mathrm{D}}{ }^{24}=-31.2\left(\mathrm{CHCl}_{3}, c=1\right)$.

DC: $R_{f}=0.30($ Heptan/EtOAc, 1:1). 
IR: $\tilde{v}=694(\mathrm{~s}), 741(\mathrm{~s}), 1103(\mathrm{w}), 1242(\mathrm{~m}), 1489(\mathrm{~m}), 1535(\mathrm{~m}), 1674(\mathrm{~m}), 2931(\mathrm{w}) \mathrm{cm}^{-1}$.

${ }^{1}$ H-NMR $\left(300 \mathrm{MHz}, \mathrm{CDCl}_{3}\right): \delta=-0.10\left(\mathrm{~s}, 3 \mathrm{H}, \mathrm{CH}_{3}\right),-0.05\left(\mathrm{~s}, 3 \mathrm{H}, \mathrm{CH}_{3}\right), 0.13\left(\mathrm{~s}, 3 \mathrm{H}, \mathrm{CH}_{3}\right)$, $0.17\left(\mathrm{~s}, 3 \mathrm{H}, \mathrm{CH}_{3}\right), 0.80(\mathrm{~s}, 9 \mathrm{H}, t \mathrm{Bu}), 0.87(\mathrm{~s}, 9 \mathrm{H}, t \mathrm{Bu}), 1.19$ (d, $\left.J=6.2 \mathrm{~Hz}, 3 \mathrm{H}, \mathrm{CH}_{3}\right), 1.77$ (d, $\left.J=7.1 \mathrm{~Hz}, 3 \mathrm{H}, \mathrm{CH}_{3}\right), 2.59-2.70\left(\mathrm{~m}, 2 \mathrm{H}, \mathrm{SCH}_{2}\right), 2.77\left(\mathrm{~s}, 3 \mathrm{H}, \mathrm{CH}_{3}\right), 3.03\left(\mathrm{~m}, 2 \mathrm{H}, \mathrm{S}-\mathrm{CH}_{2}\right), 4.17$ (m, 1H, CHNHFmoc), 4.33 (bs, 1H, Fmoc), 4.41-4.49 (m, 5H, Fmoc, CHOTBS, $\mathrm{CH}_{2} \mathrm{CHN}$ ), $5.30\left(\mathrm{~m}, 1 \mathrm{H}, \mathrm{SCH}_{2} \mathrm{CHNH}\right), 5.48$ (q, $\left.J=12.2 \mathrm{~Hz}, 2 \mathrm{H}, \mathrm{CH}_{2} \mathrm{O}\right), 5.65-5.72$ (m, 1H, CHOTBS), $5.83(\mathrm{bs}, 1 \mathrm{H}, \mathrm{FmocN} H), 6.63\left(\mathrm{bs}, 1 \mathrm{H},=\mathrm{CHCH}_{3}\right), 7.01-7.43(\mathrm{~m}, 29 \mathrm{H}, \mathrm{Tr}, \mathrm{Fmoc}$, Indol, $2 \times \mathrm{Ph}), 7.54-7.56(\mathrm{~m}, 2 \mathrm{H}, \mathrm{Fmoc}), 7.72-7.74(\mathrm{~m}, 3 \mathrm{H}), 7.89-7.95$ (m, 3H), 7.99 (bs, 2H), 8.08 $(\mathrm{d}, J=7.8 \mathrm{~Hz}, 1 \mathrm{H}, \mathrm{CH}), 8.18(\mathrm{~s}, 1 \mathrm{H}, \mathrm{CH}), 8.56$ (d, J=4.4 Hz, 1H, CH), 8.89 (s, 1H, Indolyl$\mathrm{NH})$.

${ }^{13}$ C-NMR $\left(100 \mathrm{MHz}, \mathrm{CDCl}_{3}\right): \delta=-5.4,-5.1,-5.0,-4.7,12.1,14.0,17.8,18.1,18.3,25.6$, 25.8, 29.7, 36.7, 39.2, 47.1, 48.3, 50.5, 59.5, 65.1, 67.0, 67.3, 67.9, 69.5, 77.5, 112.7, 117.3, $120.0,120.6,121.4,122.2,122.7,124.3,125.0,125.3,126.2,126.7,127.0,127.2,127.6$, $127.7,127.87,127.90,128.0,128.1,128.6,129.5,129.6,136.4,137.0,139.9,141.3,143.5$, $143.6,144.5,149.2,149.4,152.4,155.0,156.2,160.1,160.7,161.3,166.9,168.4,169.3$, 172.1, 172.7 .

HRMS (ESI): $m / z[\mathrm{M}+\mathrm{H}]^{+}$für $\mathrm{C}_{99} \mathrm{H}_{104} \mathrm{~N}_{9} \mathrm{O}_{11} \mathrm{~S}_{4} \mathrm{Si}_{2}$ ber.: 1778.6271; gef.: 1778.6287.

\section{Erweiterter Nosiheptid B-Ring (165)}

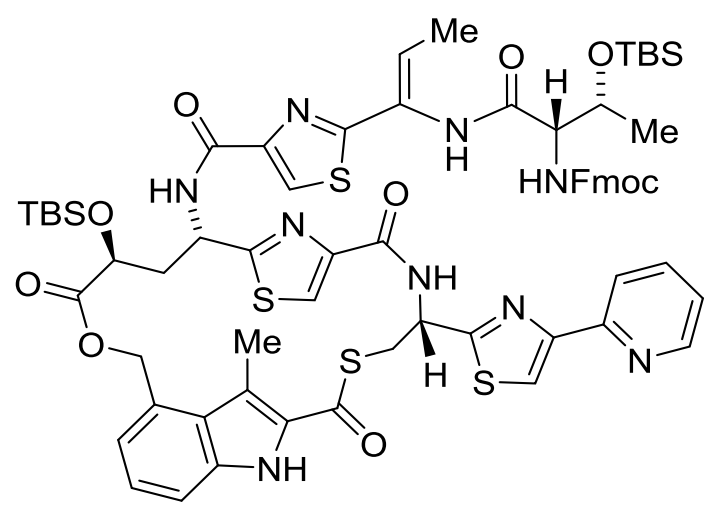

Eine Mischung aus wasserfreiem Anisol $(1 \mathrm{~mL})$, wasserfreier TFA $(1.25 \mathrm{~mL})$ und $\mathrm{Et}_{3} \mathrm{SiH}$ $(1 \mathrm{~mL})$ wurde auf $-25^{\circ} \mathrm{C}$ gekühlt und mit einer Lösung des Tris-Thiazols 113 (10 mg, $5.6 \mu \mathrm{mol})$ in Anisol $(1 \mathrm{~mL})$ tropfenweise versetzt. Es wurde $15 \mathrm{~min}$ lang bei $-25^{\circ} \mathrm{C}$ gerührt und anschließend auf $0{ }^{\circ} \mathrm{C}$ erwärmt. Die Mischung wurde bei $0^{\circ} \mathrm{C}$ bis zur vollständigen Spaltung der Dpm- und Tr- Gruppen (18-24 h, DC-Kontrolle) gerührt. Die Mischung wurde 
auf $-15^{\circ} \mathrm{C}$ gekühlt und Hochvakuum angelegt. Nach 30 min bei $-15{ }^{\circ} \mathrm{C}$ wurde für 30 min auf $0{ }^{\circ} \mathrm{C}$ erwärmt. Restliches Lösungsmittel wurde bei RT entfernt.

Der erhaltene Rückstand wurde in wasserfreiem THF ( $5 \mathrm{~mL})$ gelöst, auf $0{ }^{\circ} \mathrm{C}$ gekühlt und mit PyAOP $(3.5 \mathrm{mg}, 6.7 \mu \mathrm{mol})$ und $\mathrm{EtNiPr}_{2}(2 \mu \mathrm{L}, 12.3 \mu \mathrm{mol})$ versetzt. Die Mischung wurde 15 min lang bei $0{ }^{\circ} \mathrm{C}$ und dann $3 \mathrm{~h}$ lang bei RT gerührt. Nach Verdünnen mit pH 3 Puffer $(20 \mathrm{~mL})$ wurde mit $\mathrm{CH}_{2} \mathrm{Cl}_{2}(3 \times 10 \mathrm{~mL})$ extrahiert. Die vereinigten organischen Extrakte wurden mit halb ges. $\mathrm{NaHCO}_{3}$-Lsg und ges. $\mathrm{NaCl}$-Lsg. (je $10 \mathrm{ml}$ ) gewaschen und mit $\mathrm{Na}_{2} \mathrm{SO}_{4}$ entwässert. Nach Einengen im Vakuum und Säulenchromatographie $\left(\mathrm{SiO}_{2}, 5 \mathrm{~g}\right.$, $\left.\mathrm{CH}_{2} \mathrm{Cl}_{2} / \mathrm{MeOH}, 40: 1\right)$ wurden $5 \mathrm{mg}(3.7 \mu \mathrm{mol}, 66 \%)$ des Makrothiolactons 165 als farbloses Glas erhalten.

Spez. Drehung: $[\alpha]_{\mathrm{D}}{ }^{24}=-46.7\left(\mathrm{CHCl}_{3}, c=1\right)$.

DC: $R_{f}=0.53\left(\mathrm{CH}_{2} \mathrm{Cl}_{2} / \mathrm{MeOH}, 20: 1\right)$.

IR: $\tilde{v}=748(\mathrm{~s}), 941(\mathrm{~m}), 1134(\mathrm{~s}), 1242(\mathrm{~m}), 1481(\mathrm{~s}), 1535$ (s), $1674(\mathrm{~s}), 2854(\mathrm{w}) \mathrm{cm}^{-1}$.

${ }^{1} \mathbf{H}-\mathbf{N M R}\left(600 \mathrm{MHz}, \mathrm{CDCl}_{3}\right): \delta=0.08\left(\mathrm{~s}, 3 \mathrm{H}, \mathrm{CH}_{3}\right), 0.11\left(\mathrm{~s}, 3 \mathrm{H}, \mathrm{CH}_{3}\right), 0.13\left(\mathrm{~s}, 3 \mathrm{H}, \mathrm{CH}_{3}\right)$, $0.17\left(\mathrm{~s}, 3 \mathrm{H}, \mathrm{CH}_{3}\right), 0.87(\mathrm{bs}, 9 \mathrm{H}, t \mathrm{Bu}), 0.93(\mathrm{~s}, 9 \mathrm{H}, t \mathrm{Bu}), 1.19\left(\mathrm{~d}, J=5.7 \mathrm{~Hz}, 3 \mathrm{H}, \mathrm{CH}_{3}\right), 1.72$ $\left(\mathrm{d}, J=6.8 \mathrm{~Hz}, 3 \mathrm{H}, \mathrm{CH}_{3}\right), 2.95\left(\mathrm{~s}, 3 \mathrm{H}, \mathrm{CH}_{3}\right), 4.06(\mathrm{dd}, J=14.4,4.5 \mathrm{~Hz}, 1 \mathrm{H}, \mathrm{CH}), 4.20$ (t, $J=6.7 \mathrm{~Hz}, 1 \mathrm{H}, \mathrm{CHNHFmoc}), 4.25$ (dd, $J=14.2,7.8 \mathrm{~Hz}, 1 \mathrm{H}, \mathrm{CH}), 4.35$ (bs, 1H, CH), 4.41 $4.49\left(\mathrm{~m}, 4 \mathrm{H}, \mathrm{CH}_{2}, \mathrm{NH}, \mathrm{CH}\right), 5.36(\mathrm{~d}, J=11.9 \mathrm{~Hz}, 1 \mathrm{H}, \mathrm{O}=\mathrm{COCHH}), 5.48(\mathrm{t}, J=10.2 \mathrm{~Hz}, 1 \mathrm{H}$, $\mathrm{CH}), 5.61(\mathrm{~d}, J=11.9 \mathrm{~Hz}, 1 \mathrm{H}, \mathrm{O}=\mathrm{COCH} H), 5.81-5.87$ (m, 2H, FmocNH, CH), 7.04 (bs, 1H, $=\mathrm{CHCH} 3), 7.13-7.29$ (m, 11H, Fmoc, Indol), 7.36-7.39 (m, 2H, Fmoc), 7.58 (d, $J=7.3 \mathrm{~Hz}$, 2H), 7.69 (t, $J=6.9 \mathrm{~Hz}, 1 \mathrm{H}, \mathrm{CH}), 7.74(\mathrm{~d}, J=7.3 \mathrm{~Hz}, 2 \mathrm{H}), 8.00-8.06(\mathrm{~m}, 5 \mathrm{H}, 4 \times \mathrm{CH}, \mathrm{NH})$, 8.27 (s, 1H, CH), Indolyl-NH nicht beobachtet.

${ }^{13}$ C-NMR $\left(100 \mathrm{MHz}, \mathrm{CDCl}_{3}\right): \delta=21.2,21.5,21.7,37.1,37.2,37.4,57.3,66.4,69.3,71.8$, $118.9,121.3,121.8,121.9,122.6,122.7,122.8,123.7,123.9,124.9,125.9,126.0,126.9$, $127.1,128.7,129.7,129.8,132.7,136.2,136.7,136.9,137.1,139.6,142.8,144.6,145.1$, $147.2,148.5,148.8,149.0,149.1,152.3,154.5,154.9,155.2,156.3,157.3,173.4, C(\mathrm{O}) \mathrm{S}$ nicht beobachtet.

HRMS (ESI): $m / z[\mathrm{M}+\mathrm{H}]^{+}$ber. für $\mathrm{C}_{67} \mathrm{H}_{78} \mathrm{~N}_{9} \mathrm{O}_{10} \mathrm{~S}_{4} \mathrm{Si}_{2}$ : 1352.4288; gef.: 1352.4297 . 


\section{Serinyl-Alkohol 166}<smiles>COC(=O)c1csc(-c2cc([O-])c(-c3nc(C(=O)N[C@@H](CO)C(N)=O)cs3)nc2-c2csc(C3CCCCC3)n2)n1</smiles>

Der Silylether 71 ${ }^{[37]}(58 \mathrm{mg}, 46.6 \mu \mathrm{mol})$ wurde in einem PTFE-Gefäß in MeCN $(20 \mathrm{~mL})$ gelöst und mit $\mathrm{HF}\left(840 \mu \mathrm{L}, 49 \%\right.$ in $\left.\mathrm{H}_{2} \mathrm{O}\right)$ versetzt. Das Gemisch wurde $18 \mathrm{~h}$ lang bei RT gerührt, anschließend vorsichtig mit ges. $\mathrm{KHCO}_{3}$-Lsg. $(20 \mathrm{~mL})$ versetzt und mit $\mathrm{CH}_{2} \mathrm{Cl}_{2}$ $(3 \times 15 \mathrm{~mL})$ extrahiert. Die vereinigten organischen Extrakte wurden mit $\mathrm{Na}_{2} \mathrm{SO}_{4}$ entwässert und im Vakuum eingeengt. Nach Säulenchromatographie $\left(\mathrm{SiO}_{2}, 15 \mathrm{~g}, \mathrm{CH}_{2} \mathrm{Cl}_{2} / \mathrm{MeOH}, 20: 1\right)$ wurden $44 \mathrm{mg}(40.4 \mu \mathrm{mol}, 87 \%)$ des Alkohols 166 als leicht gelbes Glas erhalten.

Spez. Drehung: $[\alpha]_{\mathrm{D}}{ }^{24}=-0.5\left(\mathrm{CHCl}_{3}, c=1\right)$.

DC: $R_{f}=0.22\left(\mathrm{CH}_{2} \mathrm{Cl}_{2} / \mathrm{MeOH}, 20: 1\right)$.

IR: $\tilde{v}=546(\mathrm{~s}), 699(\mathrm{~s}), 740$ (s), 990 (m), $1479(\mathrm{~m}), 1239(\mathrm{~m}), 1693(\mathrm{~m}), 2927(\mathrm{w}), 3115(\mathrm{w})$, 3349 (b) $\mathrm{cm}^{-1}$.

${ }^{1}$ H-NMR $\left(300 \mathrm{MHz}, \mathrm{CDCl}_{3}\right): \delta=2.44\left(\mathrm{~s}, 3 \mathrm{H}, \mathrm{CH}_{3}\right), 2.58(\mathrm{dd}, J=13.0,5.7 \mathrm{~Hz}, 1 \mathrm{H}, \mathrm{S}-\mathrm{CHH})$, 2.74-2.80 (m, 1H, S-CHH), $3.47(\mathrm{t}, J=6.5 \mathrm{~Hz}, 1 \mathrm{H}, \mathrm{OH}), 3.93\left(\mathrm{~s}, 3 \mathrm{H}, \mathrm{CH}_{3}\right), 4.03(\mathrm{dt}, J=11.5$, $5.9 \mathrm{~Hz}, 1 \mathrm{H}, \mathrm{CH}), 4.16(\mathrm{dt}, J=11.3,5.5 \mathrm{~Hz}, 1 \mathrm{H}, \mathrm{CH}), 4.52\left(\mathrm{~d}, J=5.3 \mathrm{~Hz}, 2 \mathrm{H}, \mathrm{CH}_{2}\right), 4.65(\mathrm{bd}$, $J=6.9 \mathrm{~Hz}, 1 \mathrm{H}, \mathrm{CH}), 4.78(\mathrm{dt}, J=7.8,5.2 \mathrm{~Hz}, 1 \mathrm{H}, \mathrm{CH}), 5.16-5.53(\mathrm{~m}, 3 \mathrm{H}, 3 \times \mathrm{CH}), 5.86-5.97$ (m, 2H, CH, NH), 6.84 (s, 1H, NH), 7.19-7.37 (m, 17H, Trt, Ts), 7.68 (s, 1H, CH), 7.80 (d, $J=8.3 \mathrm{~Hz}, 2 \mathrm{H}, 2 \times \mathrm{CH}), 7.90(\mathrm{~s}, 1 \mathrm{H}, \mathrm{S}-\mathrm{CH}), 8.06(\mathrm{~s}, 1 \mathrm{H}, \mathrm{S}-\mathrm{CH}), 8.27$ (s, 1H, S-CH), 8.58 (d, $J=8.1 \mathrm{~Hz}, 1 \mathrm{H}, \mathrm{NH})$.

${ }^{13}$ C-NMR $\left(75 \mathrm{MHz}, \mathrm{CDCl}_{3}\right): \delta=21.7,52.0,54.4,65.9,67.4,117.9,122.5,126.9,128.1$, $128.2,129.5,130.2,132.5,132.9,134.1,141.5,144.0,144.3,146.2,146.6,148.9,150.9$, $151.6,161.4,161.6,163.5,164.7,170.3,173.1$.

HRMS (ESI): $m / z[\mathrm{M}+\mathrm{H}]^{+}$für $\mathrm{C}_{52} \mathrm{H}_{46} \mathrm{~N}_{7} \mathrm{O}_{10} \mathrm{~S}_{5}$ ber.: 1088.1904; gef.: 1088.1917. 


\section{Dehydroalanin-Derivat 167}

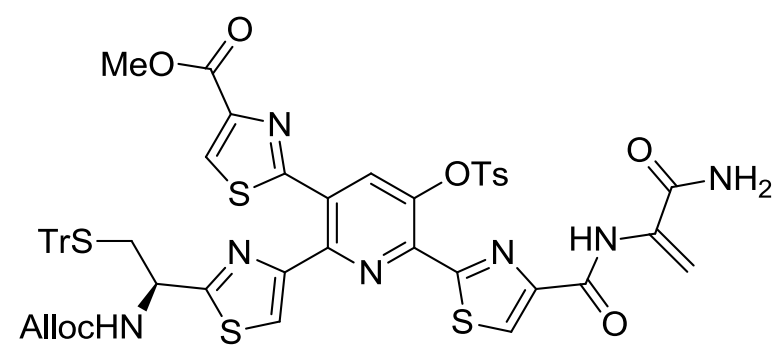

Eine Lösung des Alkohols $166(17 \mathrm{mg}, 15.6 \mu \mathrm{mol})$ wurde in wasserfreiem $\mathrm{CH}_{2} \mathrm{Cl}_{2}(9 \mathrm{~mL})$ wurde auf $0{ }^{\circ} \mathrm{C}$ gekühlt und mit 2,6-Lutidin $(900 \mu \mathrm{L})$ und Methansulfonylchlorid $(121 \mu \mathrm{L}$, $1.56 \mathrm{mmol}$ ) versetzt. Nach $5 \mathrm{~min}$ wurde das Kühlbad entfernt und das Gemisch $30 \mathrm{~min}$ lang bei RT gerührt. Die Reaktionsmischung wurde mit EtOAc $(30 \mathrm{~mL})$ verdünnt und mit halb ges. $\mathrm{NaHCO}_{3}$-Lsg., pH 3 Puffer und ges. NaCl-Lsg. (je 15 mL) gewaschen. Nach Entwässern mit $\mathrm{Na}_{2} \mathrm{SO}_{4}$ wurden die Lösungsmittel im Vakuum entfernt und der Rückstand ohne weitere Reinigung umgesetzt.

Eine Lösung des Rückstands in wasserfreiem $\mathrm{CH}_{2} \mathrm{Cl}_{2}(7.5 \mathrm{~mL})$ wurde auf $-78^{\circ} \mathrm{C}$ gekühlt und eine Lösung von DBU $(196 \mu \mathrm{L}, 1.31 \mathrm{mmol})$ in $\mathrm{CH}_{2} \mathrm{Cl}_{2}(3 \mathrm{~mL})$ wurde tropfenweise zugegeben. Nach vollständiger Zugabe wurde das Gemisch auf $-35{ }^{\circ} \mathrm{C}$ erwärmt und $2 \mathrm{~h}$ lang gerührt. Das Reaktionsgemisch wurde danach mit AcOH $(255 \mu \mathrm{L})$ versetzt und mit EtOAc (30 mL) verdünnt. Die Lösung wurde mit $\mathrm{H}_{2} \mathrm{O}$ und ges. NaCl-Lsg. (je $15 \mathrm{~mL}$ ) gewaschen und mit $\mathrm{Na}_{2} \mathrm{SO}_{4}$ entwässert. Nach Entfernen der Lösungsmittel im Vakuum wurde der Rückstand durch Säulenchromatographie $\left(\mathrm{SiO}_{2}, 15 \mathrm{~g}, \mathrm{CH}_{2} \mathrm{Cl}_{2} / \mathrm{MeOH}, 30: 1\right)$ gereinigt. Es wurden $14 \mathrm{mg}$ (13.1 $\mu \mathrm{mol}, 84 \%)$ des Dehydroalanins 167 als farbloses Glas erhalten.

Spez. Drehung: $[\alpha]_{\mathrm{D}}^{24}=+6.4\left(\mathrm{CHCl}_{3}, c=1\right)$.

DC: $R_{f}=0.37\left(\mathrm{CH}_{2} \mathrm{Cl}_{2} / \mathrm{MeOH}, 20: 1\right)$.

IR: $\tilde{v}=752(\mathrm{~s}), 1242(\mathrm{~m}), 1384(\mathrm{~m}), 1525(\mathrm{~s}), 1666(\mathrm{~s}), 1722(\mathrm{~s}), 2925(\mathrm{w}), 3337(\mathrm{w}) \mathrm{cm}^{-1}$.

${ }^{1} \mathbf{H}-\mathbf{N M R}\left(300 \mathrm{MHz}, \mathrm{CDCl}_{3}\right): \delta=2.33\left(\mathrm{~s}, 3 \mathrm{H}, \mathrm{CH}_{3}\right), 2.58(\mathrm{dd}, J=13.0,5.7 \mathrm{~Hz}, 1 \mathrm{H}, \mathrm{SCHH})$, 2.73-2.79 (m, 1H, SCHH), $3.96\left(\mathrm{~s}, 3 \mathrm{H}, \mathrm{CH}_{3}\right), 4.52$ (d, $\left.5.3 \mathrm{~Hz}, 2 \mathrm{H}, \mathrm{CH}_{2}\right), 4.64(\mathrm{~d}, 1 \mathrm{H}, \mathrm{CH})$, $5.08\left(\mathrm{~d}, J=7.9 \mathrm{~Hz}, 1 \mathrm{H},=\mathrm{CH}_{2}\right), 5.22-5.34\left(\mathrm{~m}, 2 \mathrm{H},=\mathrm{CH}_{2}, \mathrm{NH}\right), 5.58(\mathrm{~s}, 1 \mathrm{H},=\mathrm{CHH}), 5.85-$ $5.98\left(\mathrm{~m}, 1 \mathrm{H}, \mathrm{CH}=\mathrm{CH}_{2}\right), 6.18\left(\mathrm{bs}, 2 \mathrm{H}, \mathrm{NH}_{2}\right), 6.59(\mathrm{~d}, J=1.3 \mathrm{~Hz}, 1 \mathrm{H},=\mathrm{CH} H), 7.15-7.38$ 
(m, 17H, Tr, Ts), 7.75 (d, J=8.3 Hz, 2H, $2 \times \mathrm{CH}), 7.90$ (s, 1H, CH), 8.09 (s, 1H, S-CH), 8.11 (s, 1H, S-CH), 8.22 (s, 1H, S-CH), 9.82 (s, 1H, NH).

${ }^{13}$ C-NMR $\left(75 \mathrm{MHz}, \mathrm{CDCl}_{3}\right): \delta=21.6,29.7,36.8,52.0,52.5,66.0,67.5,117.9,122.4,127.0$, $128.1,128.5,129.5,129.6,129.7,129.8,132.6,134.1,135.6,141.5,143.2,144.3,145.9$, $146.8,148.8,151.5,151.9,159.4,161.5,163.7,165.7,169.9$.

HRMS (ESI): $m / z[\mathrm{M}+\mathrm{H}]^{+}$für $\mathrm{C}_{52} \mathrm{H}_{44} \mathrm{~N}_{7} \mathrm{O}_{9} \mathrm{~S}_{5}$ ber.: 1070.1799; gef.: 1070.1802 .

\section{Linearer TBS-Vorläufer 114}

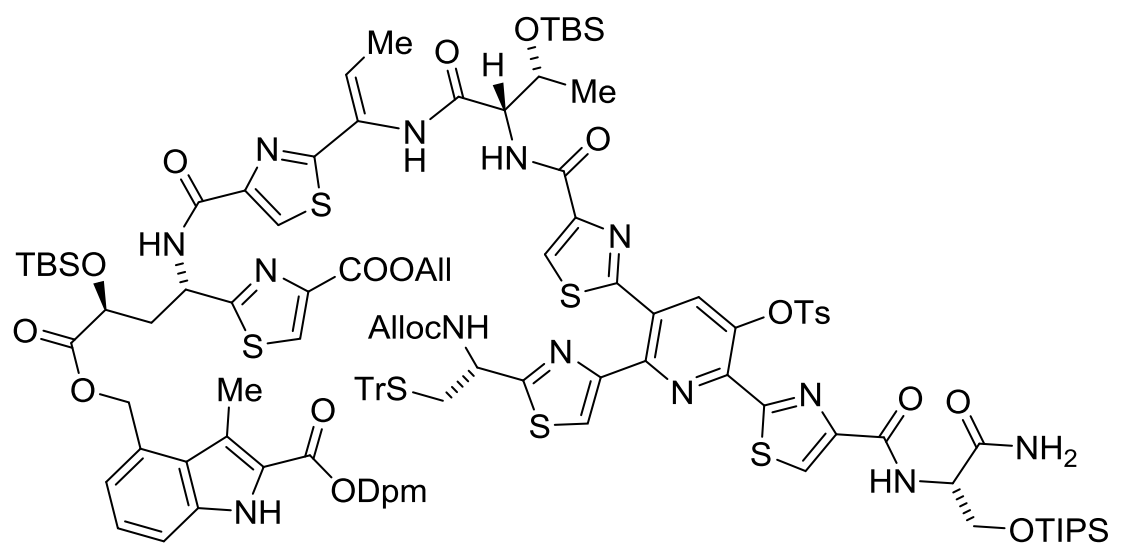

Das Amin $93(137 \mathrm{mg}, 103 \mu \mathrm{mol})$ wurde zusammen mit der Carbonsäure 42 ${ }^{[37]}(137 \mathrm{mg}$, $121 \mu \mathrm{mol})$ in wasserfreiem THF $(8 \mathrm{~mL})$ gelöst und auf $0{ }^{\circ} \mathrm{C}$ gekühlt. Nach Zugabe von DEPBT $(62 \mathrm{mg}, 207 \mu \mathrm{mol})$ und $\operatorname{EtNiPr}_{2}(35 \mu \mathrm{L}, 212 \mu \mathrm{mol})$ wurde das Gemisch langsam auf RT erwärmt und $7 \mathrm{~h}$ lang gerührt. Das Gemisch wurde mit EtOAc $(50 \mathrm{~mL})$ verdünnt und mit pH 3 Puffer, halb ges. $\mathrm{NaHCO}_{3}$-Lsg. und ges. NaCl-Lsg. (je $20 \mathrm{~mL}$ ) gewaschen. Die organische Phase wurde mit $\mathrm{Na}_{2} \mathrm{SO}_{4}$ entwässert und am Rotationsverdampfer eingeengt. Chromatographie $\left(\mathrm{SiO}_{2}, \mathrm{CH}_{2} \mathrm{Cl}_{2}\right.$ /Petrolether/Aceton, 2:2:2 $\left.\rightarrow 1: 1: 1\right)$ des Rückstands lieferte $127 \mathrm{mg}(53.1 \mu \mathrm{mol}, 51 \%)$ des Pentathiazols 114 als farbloses Glas.

Spez. Drehung: $[\alpha]_{\mathrm{D}}{ }^{24}=-4.3\left(\mathrm{CHCl}_{3}, c=0.5\right)$.

DC: $R_{f}=0.27\left(\right.$ Heptan $/ \mathrm{CH}_{2} \mathrm{Cl}_{2} /$ Aceton, $\left.2: 2: 1\right)$.

IR: $\tilde{v}=694(\mathrm{~s}), 748(\mathrm{~s}), 1095(\mathrm{~m}), 1195$ (m) $1234(\mathrm{~m}), 1666(\mathrm{~m}), 2862(\mathrm{w}), 2931(\mathrm{w}) \mathrm{cm}^{-1}$.

${ }^{1} \mathbf{H}-\mathrm{NMR}\left(600 \mathrm{MHz}, \mathrm{CDCl}_{3}\right): \delta=-0.11\left(\mathrm{~s}, 3 \mathrm{H}, \mathrm{CH}_{3}\right),-0.05\left(\mathrm{~s}, 3 \mathrm{H}, \mathrm{CH}_{3}\right), 0.18\left(\mathrm{~s}, 3 \mathrm{H}, \mathrm{CH}_{3}\right)$, 0.25 (s, 3H, CH $\left.\mathrm{CH}_{3}\right), 0.82$ (s, 9H, SitBu), 0.92 (s, 9H, SitBu), 1.07-1.19 (m, 21H, $\operatorname{Si}_{2} \operatorname{Pr}_{3}$ ), 1.25- 
$1.27\left(\mathrm{~m}, 3 \mathrm{H}, \mathrm{CH}_{3}\right), 1.84\left(\mathrm{~d}, J=7.2 \mathrm{~Hz}, 3 \mathrm{H}, \mathrm{CH}_{3}\right), 2.26\left(\mathrm{~s}, 3 \mathrm{H}, \mathrm{CH}_{3}\right), 2.60(\mathrm{dd}, J=13.0$, $\left.5.7 \mathrm{~Hz}, 1 \mathrm{H}, \mathrm{CH}_{2}\right), 2.68$ (t, $\left.J=6.9 \mathrm{~Hz}, 1 \mathrm{H}, \mathrm{CH}_{2}\right), 2.81\left(\mathrm{~s}, 3 \mathrm{H}, \mathrm{CH}_{3}\right), 4.00$ (dd, $J=9.6,6.9 \mathrm{~Hz}$, 1H, CHHOTIPS), 4.29 (dd, $J=9.9,4.4$ Hz, 1H, CHHOTIPS), 4.46-4.50 (m, 3H, CHOTBS, $\mathrm{CH}_{2} \mathrm{CH}=\mathrm{CH}_{2}$ ), 4.60-4.66 (m, 2H, CHNHAlloc, CHOTBS), 4.73 (dd, $J=6.1,3.7 \mathrm{~Hz}, 1 \mathrm{H}$, CHCHOTIPS), 4.78-4.79 (m, 2H, $\left.\mathrm{CH}_{2} \mathrm{CH}=\mathrm{CH}_{2}\right), 4.82-4.85$ (m, 1H, $\left.\mathrm{CHCONH}_{2}\right), 5.13$ (d, $J=8.3 \mathrm{~Hz}, \mathrm{NHAlloc}), 5.21\left(\mathrm{~d}, J=9.9 \mathrm{~Hz}, 1 \mathrm{H}, \mathrm{CH}_{2} \mathrm{CH}=\mathrm{CHH}\right), 5.24(\mathrm{dd}, J=10.4,1.2 \mathrm{~Hz}, 1 \mathrm{H}$, $\left.\mathrm{CH}_{2} \mathrm{CH}=\mathrm{CH} H\right), 5.27-5.30\left(\mathrm{~m}, 1 \mathrm{H}, \mathrm{CH}_{2} \mathrm{CH}=\mathrm{CHH}\right), 5.34-5.37\left(\mathrm{~m}, 1 \mathrm{H}, \mathrm{CH}_{2} \mathrm{CH}=\mathrm{CH} H\right), 5.43(\mathrm{~d}$, $J=12.3 \mathrm{~Hz}$, OCHHInd), 5.56-5.58 (m, 2H, OCHHInd, CONHH), 5.68-5.72 (m, $1 \mathrm{H}$, $\mathrm{CH}_{2} \mathrm{CHNH}$ ), 5.84-5.92 (m, $\left.1 \mathrm{H}, \mathrm{CH}_{2} \mathrm{CH}=\mathrm{CH}_{2}\right), 5.94-6.01$ (m, $\left.1 \mathrm{H}, \mathrm{CH}_{2} \mathrm{CH}=\mathrm{CH}_{2}\right), 6.76$ (q, $\left.J=7.3 \mathrm{~Hz}, 1 \mathrm{H},=\mathrm{CHCH}_{3}\right), 6.79$ (bs, $\left.1 \mathrm{H} \mathrm{CONH} H\right), 7.09$ (d, $J=7.0 \mathrm{~Hz}, 1 \mathrm{H}$, Ind-CH), 7.15 (s, 1H, $\left.\mathrm{CH}(\mathrm{Ph})_{2}\right), 7.18-7.42(\mathrm{~m}, 30 \mathrm{H}, \mathrm{Ts}, \mathrm{Tr}$, Indol, $2 \times \mathrm{Ph}), 7.74-7.76(\mathrm{~m}, 2 \mathrm{H}, \mathrm{CH}, \mathrm{NH}), 7.81(\mathrm{~s}$, $1 \mathrm{H}, \mathrm{CH}), 7.83(\mathrm{~s}, 1 \mathrm{H}, \mathrm{CH}), 7.97(\mathrm{~s}, 1 \mathrm{H}, \mathrm{CH}), 8.01$ (d, J=8.8 Hz, NH), 8.06 (s, 1H, CH), 8.14 (bs, 2H, CH, NH), 8.26 (s, 1H, CH), 8.36 (bs, 1H, NH), 8.46 (d, J=8.3 Hz, 1H, NH), 9.05 (s, $1 \mathrm{H}$, Indol-NH).

${ }^{13}$ C-NMR $\left(150 \mathrm{MHz}, \mathrm{CDCl}_{3}\right): \delta=-5.5,-5.1,-4.9,-4.7,11.8,12.2,14.1,17.86,17.91,17.94$, $18.1,18.6,21.5,23.8,25.6,25.7,25.8,29.7,36.8,39.0,47.9,52.1,54.6,54.9,58.3,63.4$, $65.1,65.85,65.9,67.5,69.4,77.5,112.8,117.9,120.6,122.2,122.5,123.4,123.5,125.3$, $126.3,126.5,126.91,126.93,127.19,127.20,127.7,128.06 .128 .09$, 128.2, 128.6, 129.49, $129.54,129.7,130.1,131.8,132.5,133.0,133.8,136.6,139.9,141.9,144.0,144.2,145.9$, $146.7,149.0,149.4,149.6,151.4,151.8,155.0,160.6,160.8,160.9,161.1,161.5,163.0$, $164.6,166.9,168.2,170.6,171.7,172.5,172.7$.

HRMS (ESI): $m / z[\mathrm{M}+2 \mathrm{Na}]^{2+}$ für $\mathrm{C}_{118} \mathrm{H}_{135} \mathrm{~N}_{13} \mathrm{Na}_{2} \mathrm{O}_{19} \mathrm{~S}_{7} \mathrm{Si}_{3}$ ber.: 1196.8582; gef.: 1196.8574 . 


\section{Linearer TIPS-Vorläufer 115}

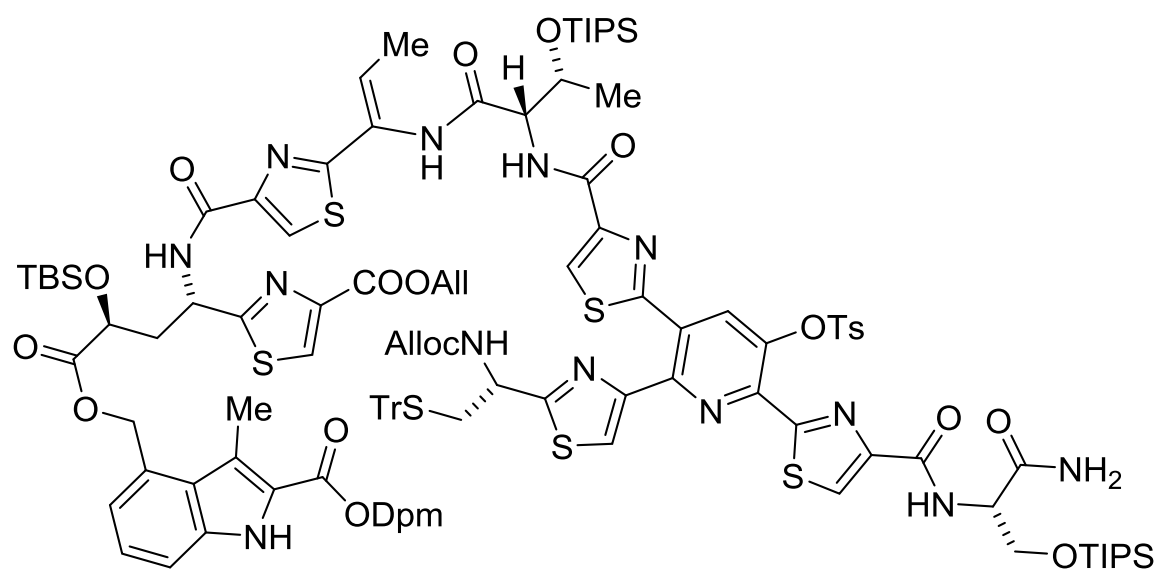

Eine Lösung des Amins 94 (99 mg, $84 \mu \mathrm{mol}, 1.2$ eq.) und der Carbonsäure 42 ${ }^{[37]}$ (86 mg, $70 \mu \mathrm{mol})$ in wasserfreiem THF $(8 \mathrm{~mL})$ wurde auf $0{ }^{\circ} \mathrm{C}$ gekühlt und nacheinander mit PyDOP (78 mg, $141 \mu \mathrm{mol})$ und EtNiPr $2(26 \mu \mathrm{L}, 157 \mu \mathrm{mol})$ versetzt. Nach 15 min wurde das Kühlbad entfernt und das Gemisch 13 h lang bei RT gerührt. Die Reaktionsmischung wurde mit EtOAc $(50 \mathrm{ml})$ verdünnt, mit pH 3 Puffer, halb-ges. $\mathrm{NaHCO}_{3}$-Lsg und ges. NaCl-Lsg. (je $20 \mathrm{~mL}$ ) gewaschen. Nach Entwässern mit $\mathrm{Na}_{2} \mathrm{SO}_{4}$ wurde die organische Phase im Vakuum eingeengt und der Rückstand durch Säulenchromatographie $\left(\mathrm{SiO}_{2}, 35 \mathrm{~g}\right.$, Petrolether $/ \mathrm{CH}_{2} \mathrm{Cl}_{2} /$ Aceton, 2:2:1) gereinigt. Es wurden $153 \mathrm{mg}(64.4 \mu \mathrm{mol}, 92 \%)$ des linearen Vorläufers 115 als farbloses Glas erhalten.

Spez. Drehung: $[\alpha]_{\mathrm{D}}{ }^{24}=-17.1\left(\mathrm{CHCl}_{3}, c=1\right)$.

DC: $R_{f}=0.42\left(\mathrm{Heptan} / \mathrm{CH}_{2} \mathrm{Cl}_{2} /\right.$ Aceton, $\left.2: 2: 1\right)$.

IR: $\tilde{v}=745(\mathrm{~m}), 1100(\mathrm{~m}), 1230(\mathrm{~m}), 1384(\mathrm{w}), 1470(\mathrm{~m}), 1532(\mathrm{~m}), 1673(\mathrm{~s}), 2865(\mathrm{w})$, $2943(\mathrm{w}) \mathrm{cm}^{-1}$.

${ }^{1} \mathbf{H}-\mathbf{N M R}\left(400 \mathrm{MHz}, \mathrm{CDCl}_{3}\right): \delta=-0.11\left(\mathrm{~s}, 3 \mathrm{H}, \mathrm{CH}_{3}\right),-0.05\left(\mathrm{~s}, 3 \mathrm{H}, \mathrm{CH}_{3}\right), 0.82(\mathrm{~s}, 9 \mathrm{H}, t \mathrm{Bu})$, 1.06-1.26 (m, 42H, $6 \times i \operatorname{Pr}), 1.35\left(\mathrm{~d}, J=6.3 \mathrm{~Hz}, 3 \mathrm{H}, \mathrm{CH}_{3}\right), 1.86\left(\mathrm{~d}, J=7.1 \mathrm{~Hz}, 3 \mathrm{H}, \mathrm{CH}_{3}\right), 2.26$ (s, 3H, $\left.\mathrm{CH}_{3}\right), 2.60\left(\mathrm{dd}, J=13.0,5.7 \mathrm{~Hz}, 1 \mathrm{H}, \mathrm{CH}_{2}\right), 2.68\left(\mathrm{t}, J=6.7 \mathrm{~Hz}, 2 \mathrm{H}, \mathrm{CH}_{2}\right), 2.78(\mathrm{bs}, 1 \mathrm{H}$, $\mathrm{CH}_{2}$ ), 2.81 (s, 3H, $\mathrm{CH}_{3}$ ), 4.01 (dd, $\left.J=9.6,6.8 \mathrm{~Hz}, 1 \mathrm{H}, \mathrm{CHHOTIPS}\right), 4.29$ (dd, $J=9.7,4.4 \mathrm{~Hz}$, 1H, CHHOTIPS), 4.46-4.54 (m, 3H, CHOTBS, $\mathrm{CH}_{2} \mathrm{CH}=\mathrm{CH}_{2}$ ), 4.63 (bs, 1H, CHNHAlloc), 4.71-4.86 (m, 5H, $\left.\mathrm{CH}_{2} \mathrm{CH}=\mathrm{CH}_{2}, \mathrm{CHCHOTIPS,} \mathrm{CHCONH}_{2}\right), 5.13(\mathrm{~d}, J=7.1 \mathrm{~Hz}, 1 \mathrm{H}$, NHAlloc), 5.19-5.30 (m, $\left.3 \mathrm{H}, 3 \times \mathrm{CH}_{2} \mathrm{CH}=\mathrm{CHH}\right), \quad 5.36 \quad(\mathrm{dd}, \quad J=17.2,1.3 \mathrm{~Hz}, \quad 1 \mathrm{H}$, $\left.\mathrm{CH}_{2} \mathrm{CH}=\mathrm{CH} H\right), 5.43(\mathrm{~d}, J=12.4 \mathrm{~Hz}, 1 \mathrm{H}, \mathrm{CHHO}), 5.55-5.58(\mathrm{~m}, 2 \mathrm{H}, \mathrm{CH} H \mathrm{O}, \mathrm{CONH}), 5.70$ 
(m, $\left.1 \mathrm{H}, \mathrm{CH}_{2} \mathrm{CHNH}\right), 5.82-6.03\left(\mathrm{~m}, 2 \mathrm{H}, 2 \times \mathrm{CH}_{2} \mathrm{CH}=\mathrm{CH}_{2}\right), 6.81\left(\mathrm{~m}, 2 \mathrm{H},=\mathrm{CHCH}_{3}, \mathrm{CON} H \mathrm{H}\right)$, $7.16\left(\mathrm{~s}, 1 \mathrm{H}, \mathrm{CH}(\mathrm{Ph})_{2}\right), 7.08-7.43(\mathrm{~m}, 31 \mathrm{H}, \mathrm{Ts}, \mathrm{Tr}, \mathrm{Indol}, 2 \times \mathrm{Ph}), 7.74-7.81(\mathrm{~m}, 4 \mathrm{H}, 4 \times \mathrm{CH})$, 7.97 (s, 1H, S-CH), 7.99 (d, $J=8.8$ Hz, 1H, Glu-NH), 8,06 (s, 1H, S-CH), 8.12 (s, 1H, S-CH), 8.17 (d, $J=6.8 \mathrm{~Hz}, 1 \mathrm{H}$, Thr-NH), 8.27 (s, 1H, S-CH), 8.47 (d, $J=8.3 \mathrm{~Hz}, 1 \mathrm{H}$, Ser-NH), 8.53 (s, 1H, Dhb-NH), 9.11 (s, 1H, Indolyl-NH).

${ }^{13}$ C-NMR $\left(100 \mathrm{MHz}, \mathrm{CDCl}_{3}\right): \delta=-5.5,-5.1,11.9,12.2,14.0,17.9,18.1,21.5,25.7,36.8$, $39.1,47.9,54.7,58.2,63.5,65.1,65.85,65.93,67.6,67.9,69.5,77.5,112.8,117.9,120.6$, $122.2,122.5,123.4,123.6,125.3,126.4,126.9,127.2,128.1,128.6,130.1,131.9,132.5$, 133.1, 133.8, 136.6, 139.9, 141.9, 144.3, 146.0, 146.7, 149.0, 149.5, 149.7, 151.5, 151.8, $160.6,160.8,161.1,161.5,163.1,164.6,167.1,168.3,170.6,171.7,172.5,172.7,175.5$.

HRMS (ESI): $m / z$ [M $+2 \mathrm{H}]^{+}$für $\mathrm{C}_{121} \mathrm{H}_{143} \mathrm{~N}_{13} \mathrm{O}_{19} \mathrm{~S}_{7} \mathrm{Si}_{3}$ ber.: 1194.8983; gef.: 1194.8990 .

\section{Makrolactam 175}

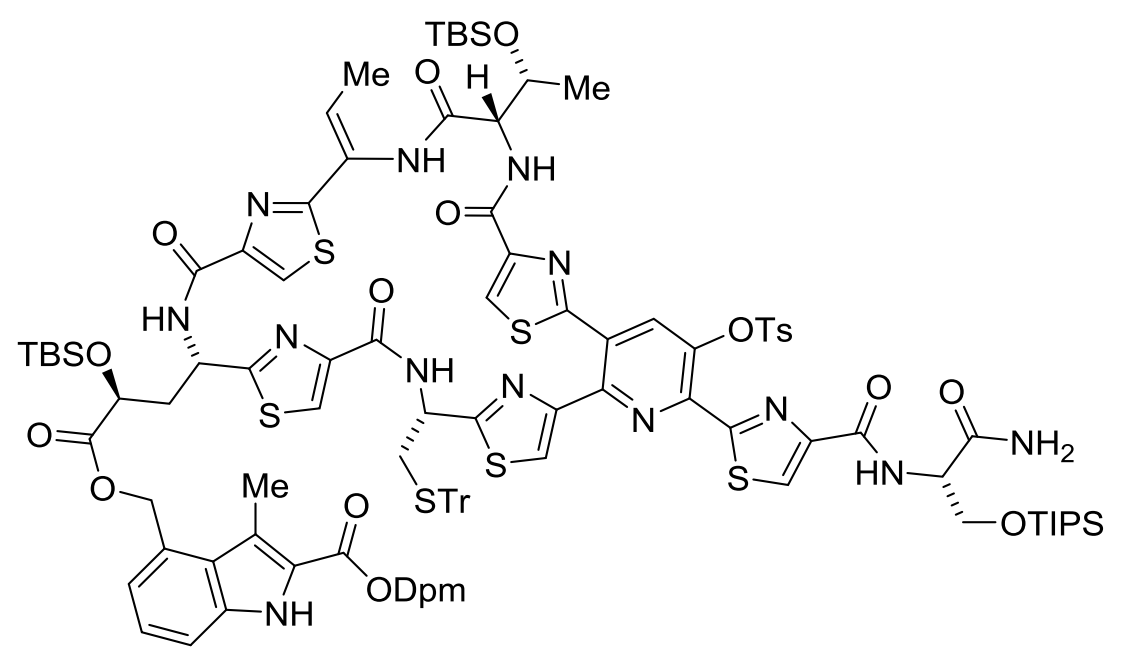

Eine Lösung des linearen Vorläufers $114(127 \mathrm{mg}, 54.1 \mu \mathrm{mol})$ in entgastem, wasserfreiem THF (26 mL) wurde mit $\mathrm{PhSiH}_{3}(80 \mu \mathrm{L}, 0.64 \mathrm{mmol})$ versetzt und auf $0{ }^{\circ} \mathrm{C}$ gekühlt. Danach wurde eine Lösung von $\mathrm{Pd}\left(\mathrm{PPh}_{3}\right)_{4}(12.7 \mathrm{mg}, 11.0 \mu \mathrm{mol})$ in THF $(1.3 \mathrm{~mL})$ zugegeben und die Mischung 15 min lang gerührt. Anschließend wurde die Mischung mit wasserfreiem THF $(28 \mathrm{~mL})$ verdünnt und HATU (102 mg, $0.27 \mathrm{mmol})$ und $\operatorname{EtNi}_{2} \operatorname{Pr}_{2}(254 \mu \mathrm{L}, 1.54 \mathrm{mmol})$ zugegeben. Nach 15 min bei $0{ }^{\circ} \mathrm{C}$ wurde das Kühlbad entfernt und die Reaktionsmischung $1 \mathrm{~h}$ lang bei RT gerührt. Nach Verdünnen mit pH 3 Puffer $(100 \mathrm{~mL})$ wurde das Gemisch mit $\mathrm{CH}_{2} \mathrm{Cl}_{2}(3 \times 50 \mathrm{~mL})$ extrahiert, die organischen Extrakte vereinigt, mit halb ges. $\mathrm{NaHCO}_{3}$ und $\mathrm{H}_{2} \mathrm{O}$ (je $50 \mathrm{~mL}$ ) gewaschen und mit $\mathrm{Na}_{2} \mathrm{SO}_{4}$ entwässert. Die Lösungsmittel wurden am Rotationsverdampfer entfernt und der Rückstand durch Säulenchromatographie $\left(\mathrm{SiO}_{2}\right.$, 
$\mathrm{CH}_{2} \mathrm{Cl}_{2} /$ Petrolether/Aceton, 2:2:2 $\left.\rightarrow 1: 1: 1\right)$ gereinigt. Es wurden $103 \mathrm{mg}(46.7 \mu \mathrm{mol}, 86 \%)$ des Makrolactams 175 als leicht gelbes Glas erhalten.

Spez. Drehung: $[\alpha]_{\mathrm{D}}{ }^{24}=-26.0\left(\mathrm{CHCl}_{3}, c=1\right)$.

DC: $R_{f}=0.36\left(\mathrm{CH}_{2} \mathrm{Cl}_{2} / \mathrm{MeOH} / \mathrm{HCOOH}, 100: 5: 1\right)$.

IR: $\tilde{v}=694(\mathrm{~m}), 748(\mathrm{~s}), 1095(\mathrm{~m}), 1226(\mathrm{~m}), 1473(\mathrm{~m}), 1527(\mathrm{~m}), 1674(\mathrm{~s}), 2931(\mathrm{w}) \mathrm{cm}^{-1}$.

${ }^{1} \mathbf{H}-\mathbf{N M R}\left(600 \mathrm{MHz}, \mathrm{CDCl}_{3}\right): \delta=-0.11\left(\mathrm{~s}, 3 \mathrm{H}, \mathrm{CH}_{3}\right), 0.03\left(\mathrm{~s}, 3 \mathrm{H}, \mathrm{CH}_{3}\right), 0.10\left(\mathrm{~s}, 3 \mathrm{H} \mathrm{CH}_{3}\right)$, 0.26 (bs, 3H, $\mathrm{CH}_{3}$ ), 0.82 (s, 9H, SitBu), 0.87 (s, 9H, SitBu), 1.09-1.12 (m, 18H, $\left.6 \times \mathrm{SiCH}\left(\mathrm{CH}_{3}\right)_{2}\right), 1.15-1.20(\mathrm{~m}, 3 \mathrm{H}, 3 \times \mathrm{SiCH}), 1.43\left(\mathrm{bs}, 3 \mathrm{H}, \mathrm{CH}_{3}\right), 1.83(\mathrm{~d}, J=6.8 \mathrm{~Hz}, 3 \mathrm{H}$, $\mathrm{CH}_{3}$ ), 1.98 (bs, 1H, $\mathrm{CH}_{2}$ ), 2.37 (s, $\left.3 \mathrm{H}, \mathrm{CH}_{3}\right), 2.53-2.63$ (m, 2H, $\left.\mathrm{CH}_{2}, \mathrm{CHHSTr}\right), 2.82$ (m, 4H, $\mathrm{CH}_{3}, \mathrm{CH} H \mathrm{STr}$ ), 4.00 (dd, $J=9.6,7.1 \mathrm{~Hz}, 1 \mathrm{H}, \mathrm{CH} H$ OTIPS), 4.31 (dd, $J=9.8,4.3 \mathrm{~Hz}, 1 \mathrm{H}$, CHHOTIPS), 4.40 (bs, 1H, CHOTBS), 4.51 (bs, 1H, CH), 4.70 (bs, 1H, CHOTBS), 4.83 (dt, $\left.J=7.4,4.6 \mathrm{~Hz}, 1 \mathrm{H}, \mathrm{CHCH}_{2} \mathrm{OTIPS}\right), 4.96$ (bs, $\left.1 \mathrm{H}, \mathrm{NH}\right), 5.48-5.53\left(\mathrm{~m}, 2 \mathrm{H}, \mathrm{OCH}_{2}\right), 5.62$ (bs, $1 \mathrm{H}, \mathrm{NH}), 5.86(\mathrm{bs}, 1 \mathrm{H}, \mathrm{OCN} H \mathrm{H}), 6.36\left(\mathrm{q}, J=7.0 \mathrm{~Hz}, 1 \mathrm{H},=\mathrm{CHCH}_{3}\right), 6.84(\mathrm{~s}, 1 \mathrm{H}, \mathrm{OCNH} H)$, $7.06(\mathrm{~d}, J=7.2 \mathrm{~Hz}, 1 \mathrm{H}$, Ind-CH), 7.13-7.23 (m, 14H), 7.25-7.32 (m, 9H), 7.34-7.37 (m, 4H), 7.43-7.44 (m, 4H), 7.73 (s, 1H, CH), 7.75 (s, 1H, CH), 7.82 (s, 1H, S-CH), 7.89 (bs, 1H, CH), 7.99 (bs, 1H, NH), 8.00 (s, 1H, S-CH), 8.06 (bs, 1H, NH) 8.23 (s, 1H, S-CH), 8.38 (d, 7.9 Hz, $1 \mathrm{H}, \mathrm{NH}), 9.15$ (s, 1H, Indol-NH).

${ }^{13}$ C-NMR (150 MHz, $\left.\mathrm{CDCl}_{3}\right): \delta=-5.4,-5.2,-5.0,-4.8,11.8,12.1,14.6,17.6,17.89,17.90$, $18.0,21.6,25.58,25.62,29.2,31.7,35.2,49.6,53.8,54.5,63.3 ., 65.1,66.9,67.5,69.5,77.5$, $112.7,120.6,122.4,123.5,124.7,125.2$, 126.2, 126.8, 26.9, 127.1, 127.97, 128.04, 128.4, $128.6,129.39,129.45,129.49,129.8,131.9,132.0,132.1,132.6,133.9,136.5,139.85$, $139.86,141.6,143.6,144.2$, 145.9, 148.8, 151.2, 152.6, 159.8, 160.2, 160.9, 161.4, 163.0, $164.5,166.1,172.3,172.5$.

HRMS (ESI): $m / z[\mathrm{M}+2 \mathrm{Na}]^{2+}$ ber. für $\mathrm{C}_{111} \mathrm{H}_{125} \mathrm{~N}_{13} \mathrm{Na}_{2} \mathrm{O}_{16} \mathrm{~S}_{7} \mathrm{Si}_{3}: 1125.3269$; gef.: 1125.3276 . 


\section{Makrolactam 176}

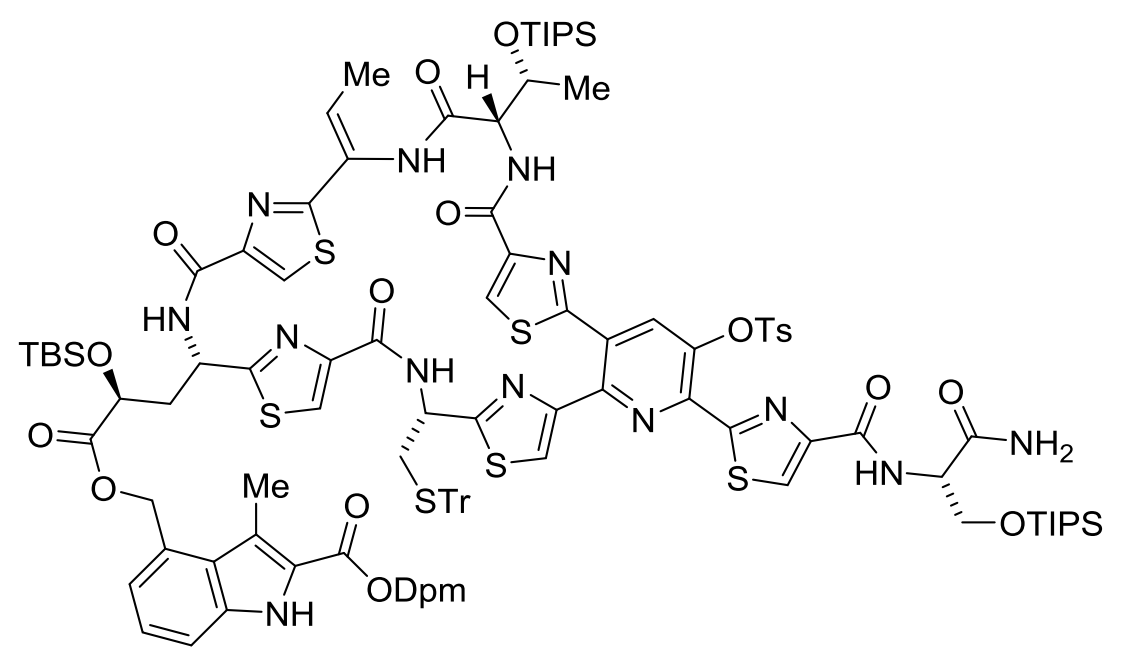

Eine Lösung des linearen Vorläufers 115 (98 mg, $41 \mu \mathrm{mol})$ in entgastem, wasserfreiem THF $(21 \mathrm{~mL})$ wurde auf $0{ }^{\circ} \mathrm{C}$ gekühlt, $\mathrm{Et}_{3} \mathrm{SiH}(133 \mu \mathrm{L}, 0.82 \mathrm{mmol})$ und eine Lösung von $\mathrm{Pd}\left(\mathrm{PPh}_{3}\right)_{4}$ $(10.4 \mathrm{mg}, 9 \mu \mathrm{mol})$ in THF $(1 \mathrm{~mL})$ zugegeben. Die Mischung wurde $45 \mathrm{~min}$ lang bei $0{ }^{\circ} \mathrm{C}$ gerührt, mit wasserfreiem THF (23 mL) verdünnt und dann mit HATU (85 mg, $0.22 \mathrm{mmol}$ ) und $\mathrm{EtNiPr}_{2}(208 \mu \mathrm{L}, 125 \mu \mathrm{mol})$ nacheinander versetzt. Nach 15 min wurde das Kühlbad entfernt und das Gemisch $1 \mathrm{~h}$ lang bei RT gerührt. Nach Verdünnen mit EtOAc $(300 \mathrm{~mL})$ wurde die Lösung mit pH 3 Puffer $(100 \mathrm{~mL})$, halb ges. $\mathrm{NaHCO}_{3}$-Lsg. $(2 \times 100 \mathrm{~mL})$ und ges. NaCl-Lsg. (150 mL) gewaschen und mit $\mathrm{Na}_{2} \mathrm{SO}_{4}$ entwässert. Die Lösungsmittel wurden im Vakuum entfernt und der Rückstand durch Säulenchromatographie $\left(\mathrm{SiO}_{2}, 35 \mathrm{~g}\right.$, Petrolether/ $\mathrm{CH}_{2} \mathrm{Cl}_{2} /$ Aceton, $\left.2: 2: 1 \rightarrow 1: 1: 1\right)$ gereinigt. Es wurden $82 \mathrm{mg}(36.5 \mu \mathrm{mol}, 89 \%)$ des Makrolactams 176 als leicht gelbes Glas erhalten.

Spez. Drehung: $[\alpha]_{\mathrm{D}}^{24}=+21.6\left(\mathrm{CHCl}_{3}, c=0.25\right)$.

DC: $R_{f}=0.38\left(\mathrm{CH}_{2} \mathrm{Cl}_{2} / \mathrm{MeOH} / \mathrm{HCOOH}, 100: 5: 1\right)$.

IR: $\tilde{v}=700(\mathrm{~m}), 743(\mathrm{~m}), 1101(\mathrm{w}), 1248(\mathrm{w}), 1470(\mathrm{~m}), 1536(\mathrm{~s}), 1668(\mathrm{~s}), 2864(\mathrm{w})$, $2943(\mathrm{w}) \mathrm{cm}^{-1}$.

${ }^{1} \mathbf{H}-\mathrm{NMR}\left(600 \mathrm{MHz}, \mathrm{CDCl}_{3}\right): \delta=-0.13\left(\mathrm{~s}, 3 \mathrm{H}, \mathrm{CH}_{3}\right), 0.02\left(\mathrm{~s}, 3 \mathrm{H}, \mathrm{CH}_{3}\right), 0.80(\mathrm{~s}, 9 \mathrm{H}, t \mathrm{Bu})$, 1.07-1.20 (m, 42H, $6 \times i \operatorname{Pr}), 1.25\left(\mathrm{bs}, 3 \mathrm{H}, \mathrm{CH}_{3}\right), 1.82\left(\mathrm{~d}, J=7.0 \mathrm{~Hz}, 3 \mathrm{H}, \mathrm{CH}_{3}\right), 2.35(\mathrm{~s}, 3 \mathrm{H}$, $\mathrm{CH}_{3}$ ), 2-60-2.65 (m, 2H, $\left.\mathrm{CH}_{2}, \mathrm{CHHSTr}\right), 2.74-2.77$ (m, 1H, CHHSTr) 2.81 (s, 3H, $\mathrm{CH}_{3}$ ), 3.98 (dd, $J=9.7,7.0 \mathrm{~Hz}, 1 \mathrm{H}$, CHHOTIPS), 4.29 (dd, $J=9.7,4.4 \mathrm{~Hz}, 1 \mathrm{H}$, CHHOTIPS), 4.37 (bs, 1H, CHOTBS), 4.54 (bs, 1H, CH), 4.74-4.83 (m, 2H, NH, $\left.\mathrm{CHCH}_{2} \mathrm{OTIPS}\right), 4.90$ (m, 1H, CH), 
$5.49\left(\mathrm{~s}, 2 \mathrm{H}, \mathrm{OCH}_{2}\right), 5.63(\mathrm{bs}, 1 \mathrm{H}, \mathrm{NH}), 5.80(\mathrm{~s}, 1 \mathrm{H}, \mathrm{NH}), 6.36\left(\mathrm{q}, J=7.0 \mathrm{~Hz}, 1 \mathrm{H},=\mathrm{CHCH}_{3}\right)$, 6.85 (s, 1H, NH), 7.06 (d, J=7.0 Hz, 1H, CH, 7.13-7.31 (m, 19H, Ts, Tr, Indol, $2 \times \mathrm{Ph}, \mathrm{NH})$, 7.33-7.36 (m, 4H), 7.42-7.47 (m, 8H), 7.53-7.55 (m, 2H), 7.65-7.68 (m, 4H), $7.72(\mathrm{~d}$, $J=8.4 \mathrm{~Hz}, 2 \mathrm{H}), 7.83(\mathrm{~s}, 1 \mathrm{H}, \mathrm{S}-\mathrm{CH}), 7.89$ (bs, 1H, CH), 7.98 (s, 1H, S-CH), 8.00 (bs, 1H, $\mathrm{CH}), 8.08$ (bs, 1H, NH), 8.22 (s, 1H, S-CH), 8.36 (d, J=7.7 Hz, 1H, Ser-NH), 9.12 (s, 1H, Indolyl-NH).

${ }^{13}$ C-NMR $\left(150 \mathrm{MHz}, \mathrm{CDCl}_{3}\right): \delta=-5.3,-5.1,11.8,12.11,12.14,12.3,12.4,14.4,17.92$, $17.93,18.1,18.2,21.6,25.7,35.2,54.5,56.1,63.4,65.1,67.4,67.6,69.5,77.5,112.6,120.8$, $121.7,122.4,123.4,124.7,125.3,126.1,126.3,126.8,126.9,127.19,127.20,127.95,128.01$, $128.1,128.4$, 128.6, 129.35, 129.40, 129.5, 129.6, 129.7, 129.8, 132.6, 134.2, 136.4, 139.9, $141.6,143.7,144.15,144.20,145.9,148.9,149.8,151.2,159.8,160.2,161.0,161.4,163.2$, 164.6, 172.3 .

HRMS (ESI): $m / z[\mathrm{M}+2 \mathrm{H}]^{2+}$ für $\mathrm{C}_{114} \mathrm{H}_{133} \mathrm{~N}_{13} \mathrm{O}_{16} \mathrm{~S}_{7} \mathrm{Si}_{3}$ ber.: 1123.8668; gef.: 1123.8660 .

\section{Makrothiolacton 116}

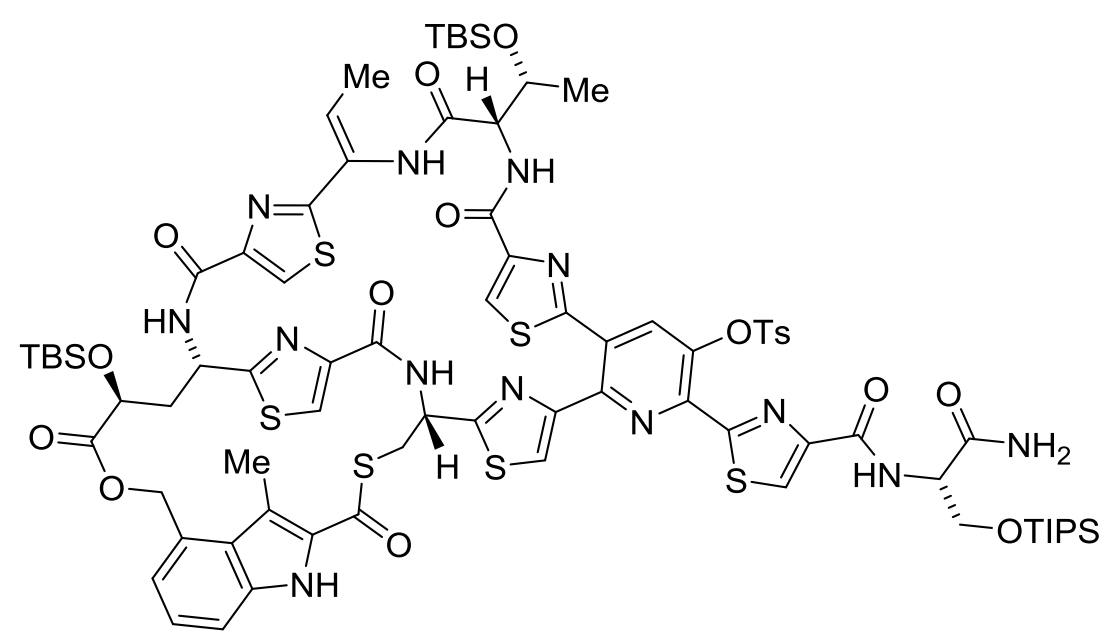

Eine Mischung aus wasserfreiem Anisol $(13 \mathrm{~mL})$, wasserfreier TFA $(6.6 \mathrm{~mL})$ und $\mathrm{Et}_{3} \mathrm{SiH}$ $(5.3 \mathrm{~mL})$ wurde auf $-20^{\circ} \mathrm{C}$ gekühlt und mit einer Lösung des Makrolactams 175 (55 mg, $24.9 \mu \mathrm{mol})$ in Anisol $(4 \mathrm{~mL})$ tropfenweise versetzt. Das Gemisch wurde $20 \mathrm{~min}$ lang bei $-20{ }^{\circ} \mathrm{C}$ gerührt und anschließend auf $0{ }^{\circ} \mathrm{C}$ erwärmt und $24 \mathrm{~h}$ lang gerührt. Die Mischung wurde dann auf $-15^{\circ} \mathrm{C}$ gekühlt und Hochvakuum angelegt. Nach $60 \mathrm{~min}$ bei $-15^{\circ} \mathrm{C}$ wurde für 60 min auf $0{ }^{\circ} \mathrm{C}$ erwärmt. Restliches Lösungsmittels wurde bei RT entfernt. 
Der erhaltene Rückstand wurde in wasserfreiem THF $(23 \mathrm{~mL})$ gelöst, auf $0{ }^{\circ} \mathrm{C}$ gekühlt und mit PyAOP (14.0 mg, $26.8 \mu \mathrm{mol})$ und $\mathrm{EtNiPr}_{2}(8 \mu \mathrm{L}, 49.2 \mu \mathrm{mol})$ versetzt. Nach 15 min wurde das Kühlbad entfernt und $1.5 \mathrm{~h}$ lang bei RT gerührt. Die Mischung wurde mit pH 7 Puffer $(25 \mathrm{~mL})$ verdünnt und mit $\mathrm{CHCl}_{3}(4 \times 10 \mathrm{~mL})$ extrahiert. Die vereinigten organischen Extrakte wurden mit halb ges. $\mathrm{NaHCO}_{3}$-Lsg und ges. NaCl-Lsg. (je $15 \mathrm{ml}$ ) gewaschen und mit $\mathrm{Na}_{2} \mathrm{SO}_{4}$ entwässert. Die Lösungsmittel wurden am Rotationsverdampfer entfernt und der Rückstand durch Säulenchromatographie $\left(\mathrm{SiO}_{2}, 25 \mathrm{~g}, \mathrm{CH}_{2} \mathrm{Cl}_{2} / \mathrm{MeOH}, 50: 1 \rightarrow 30: 1\right)$ gereinigt. Es wurden $13 \mathrm{mg}$ (7.30 $\mu \mathrm{mol}, 29 \%)$ des Makrothiolactons 116 als farbloses Glas erhalten.

Spez. Drehung: $[\alpha]_{\mathrm{D}}{ }^{24}=+40.2\left(\mathrm{CHCl}_{3}, c=0.25\right)$.

DC: $R_{f}=0.20\left(\mathrm{CH}_{2} \mathrm{Cl}_{2} / \mathrm{MeOH}, 20: 1\right)$.

IR: $\tilde{v}=578(\mathrm{~m}), 776(\mathrm{~m}), 1085(\mathrm{~s}), 1462(\mathrm{~m}), 1530(\mathrm{~m}), 1666(\mathrm{~m}), 2859(\mathrm{w}), 2927(\mathrm{w}) \mathrm{cm}^{-1}$.

${ }^{1} \mathbf{H}-\mathbf{N M R}\left(600 \mathrm{MHz}, \mathrm{CDCl}_{3}\right): \delta=0.08\left(\mathrm{~s}, 3 \mathrm{H}, \mathrm{CH}_{3}\right), 0.10\left(\mathrm{~s}, 3 \mathrm{H}, \mathrm{CH}_{3}\right), 0.64$ (bs, 6H, $\left.2 \times \mathrm{CH}_{3}\right), 0.98(\mathrm{~s}, 9 \mathrm{H}, t \mathrm{Bu}), 1.09-1.11\left(\mathrm{~m}, 18 \mathrm{H}, 6 \times \mathrm{SiCH}\left(\mathrm{CH}_{3}\right)_{2}\right), 1.15-1.21(\mathrm{~m}, 3 \mathrm{H}$, $3 \times \mathrm{SiCH}), 1.25\left(\mathrm{bs}, 3 \mathrm{H}, \mathrm{CH}_{3}\right), 1.73\left(\mathrm{~d}, J=7.0 \mathrm{~Hz}, 3 \mathrm{H}, \mathrm{CH}_{3}\right), 2.00$ (s, 3H, $\left.\mathrm{CH}_{2}, \mathrm{CH}_{3}\right), 2.17-$ $2.25\left(\mathrm{~m}, 1 \mathrm{H}, \mathrm{CH}_{2}\right), 2.29-2.36\left(\mathrm{~m}, 1 \mathrm{H}, \mathrm{CH}_{2}\right), 2.43$ (bs, 4H, $\left.\mathrm{CH}_{3}, \mathrm{CH}_{2}\right), 3.65$ (d, J=11.4 Hz, 1H, S-CHH), 3.99 (dd, J=9.7, 7.2 Hz, 1H, CHHOTIPS), 4.03 (bs, 1H, S-CHH), 4.22 (bs, 1H, $\mathrm{CH}), 4.28$ (dd, $J=9.7,4.4 \mathrm{~Hz}, 1 \mathrm{H}, \mathrm{CH} H \mathrm{OTIPS}), 4.69$ (bs, 1H, CH), 4.80 (dt, 7.5, $4.4 \mathrm{~Hz}, 1 \mathrm{H}$, $\mathrm{CHCONH}_{2}$ ), 5.13 (bs, 1H, NH), 5.55 (bs, 1H CONHH), 5.76 (t, J=12.1 Hz, 1H, CH), 5.84 (bs, 1H, NH), $6.00(\mathrm{bs}, 1 \mathrm{H}, \mathrm{CH}), 6.32\left(\mathrm{q}, J=6.7 \mathrm{~Hz}, 1 \mathrm{H},=\mathrm{CHCH}_{3}\right), 6.81(\mathrm{~s}, 1 \mathrm{H}, \mathrm{CONH} H)$, $7.13(\mathrm{~d}, J=7.0 \mathrm{~Hz}, 1 \mathrm{H}$, Ind-CH), $7.29(\mathrm{~d}, J=8.1 \mathrm{~Hz}, 2 \mathrm{H}, 2 \times \mathrm{CH}), 7.34-7.36(\mathrm{~m}, 1 \mathrm{H}, \mathrm{CH})$, $7.43(\mathrm{~d}, J=8.1 \mathrm{~Hz}, 1 \mathrm{H}, \mathrm{CH}), 7.61(\mathrm{~s}, 1 \mathrm{H}, \mathrm{S}-\mathrm{CH}), 7.79(\mathrm{~d}, J=8.3 \mathrm{~Hz}, 2 \mathrm{H}, 2 \times \mathrm{CH}), 7.88(\mathrm{~d}$, $J=8.3 \mathrm{~Hz}, 1 \mathrm{H}, \mathrm{CH}), 7.90(\mathrm{~s}, 1 \mathrm{H}, \mathrm{S}-\mathrm{CH}), 7.96(\mathrm{~s}, 1 \mathrm{H}, \mathrm{S}-\mathrm{CH}), 8.12(\mathrm{bs}, 1 \mathrm{H}, \mathrm{CH}), 8.24(\mathrm{~s}, 1 \mathrm{H}$, S-CH), 8.36-8.37 (m, 2H, S-CH, NH), 8.97 (bs, 1H, NH), 9.54 (bs, 1H, NH), C(O)S nicht beobachtet.

${ }^{13}$ C-NMR $\left(150 \mathrm{MHz}, \mathrm{CDCl}_{3}\right): \delta=-5.6,-5.2,-5.0,-4.8,1.9,11.8,14.1,14.7,17.4,17.93$, $17.95,18.3,21.7,22.9,22.7,25.3,25.7,30.0,29.3,29.7,31.2,31.9,45.8,49.4,49.8,54.5$, $58.2,63.4,66.5,68.5,72.8,116.3,120.2,121.2,121.7,124.2,124.7,125.8,126.2,127.0$, $128.5,129.4$, 129.9, 129.96, 130.03, 130.4, 132.8, 133.6, 134.4, 141.9, 143.7, 146.1, 149.1, $151.3,152.3,160.5,161.0,164.4,166.7,170.4,172.1,172.4$.

HRMS (ESI): $m / z[\mathrm{M}+2 \mathrm{H}]^{2+}$ für $\mathrm{C}_{79} \mathrm{H}_{101} \mathrm{~N}_{13} \mathrm{O}_{15} \mathrm{~S}_{7} \mathrm{Si}_{3}$ ber.: 889.7441 , gef,: 889.7449 . 


\section{Makrothiolcaton 117}

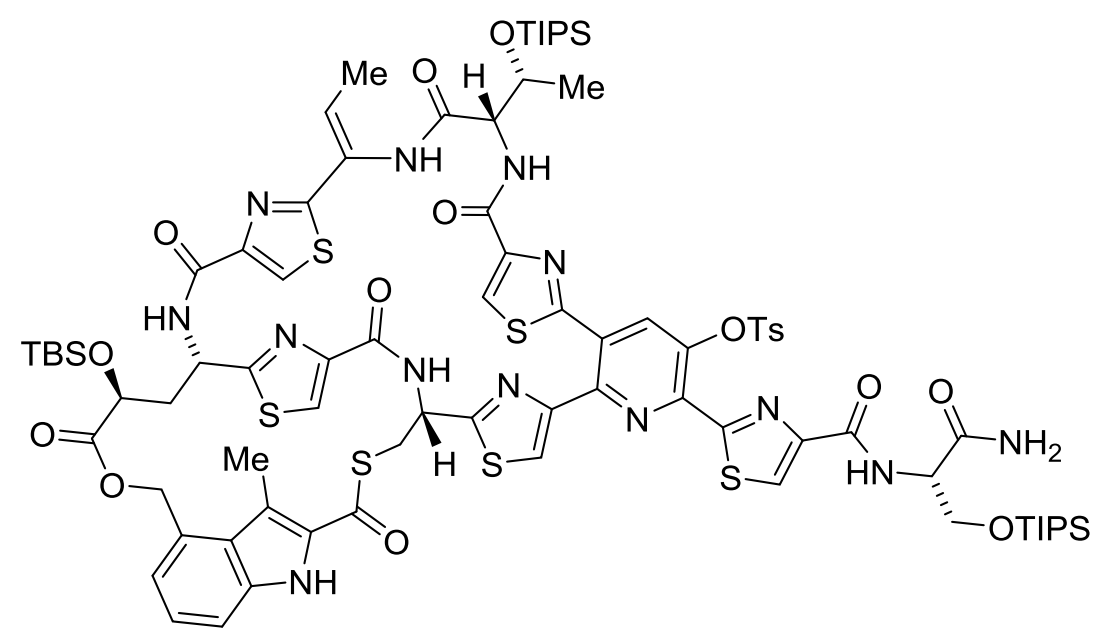

Eine Mischung aus wasserfreiem Anisol $(17 \mathrm{~mL})$, wasserfreier TFA $(8.1 \mathrm{~mL})$ und $\mathrm{Et}_{3} \mathrm{SiH}$ (6.6 mL) wurde auf $-20^{\circ} \mathrm{C}$ gekühlt und mit einer Lösung des Makrolactams 176 (66 mg, $29.4 \mu \mathrm{mol})$ in Anisol $(4 \mathrm{~mL})$ tropfenweise versetzt. Das Gemisch wurde $15 \mathrm{~min}$ lang bei $-20{ }^{\circ} \mathrm{C}$ gerührt und anschließend auf $0{ }^{\circ} \mathrm{C}$ erwärmt und $24 \mathrm{~h}$ lang gerührt. Die Mischung wurde auf $-15^{\circ} \mathrm{C}$ gekühlt und Hochvakuum angelegt. Nach 60 min bei $-15^{\circ} \mathrm{C}$ wurde für 60 min auf $0{ }^{\circ} \mathrm{C}$ erwärmt. Restliches Lösungsmittels wurde bei RT entfernt.

Der verbliebene Rückstand wurde in wasserfreiem THF $(54 \mathrm{~mL})$ gelöst, auf $0{ }^{\circ} \mathrm{C}$ gekühlt und mit PyAOP (18.4 mg, $35.3 \mu \mathrm{mol})$ und $\operatorname{EtNi}_{2} \operatorname{Pr}_{2}(10 \mu \mathrm{L}, 61.5 \mu \mathrm{mol})$ versetzt. Die Mischung wurde 15 min lang bei $0{ }^{\circ} \mathrm{C}$ und dann $3 \mathrm{~h}$ lang bei RT gerührt. Nach Verdünnen mit EtOAc $(300 \mathrm{~mL})$ wurde das Gemisch mit pH 3 Puffer $(100 \mathrm{ml})$, halb ges. $\mathrm{NaHCO}_{3}$-Lsg. $(2 \times 100 \mathrm{~mL})$ und ges. NaCl-Lsg. $(150 \mathrm{~mL})$ gewaschen. Die organische Phase wurde mit $\mathrm{Na}_{2} \mathrm{SO}_{4}$ entwässert und am Rotationsverdampfer eingeengt. Der Rückstand wurde durch Säulenchromatographie $\left(\mathrm{SiO}_{2}, 25 \mathrm{~g}, \mathrm{CH}_{2} \mathrm{Cl}_{2} / \mathrm{MeOH}, 50: 1 \rightarrow 30: 1\right)$ gereinigt. Es wurden $23 \mathrm{mg}(12.6 \mu \mathrm{mol}, 43 \%)$ des Makrothiolactons 117 als farbloses Glas erhalten.

Spez. Drehung: $[\alpha]_{\mathrm{D}}{ }^{24}=+11.3\left(\mathrm{CHCl}_{3}, c=1\right)$.

DC: $R_{f}=0.47\left(\mathrm{CH}_{2} \mathrm{Cl}_{2} / \mathrm{MeOH}, 20: 1\right)$.

IR: $\tilde{v}=735(\mathrm{~m}), 1002(\mathrm{w}), 1126(\mathrm{~m}), 1249(\mathrm{w}), 1470(\mathrm{~m}), 1530(\mathrm{~s}), 1667(\mathrm{~s}), 2864(\mathrm{w})$, 2943 (w) $\mathrm{cm}^{-1}$. 
${ }^{1} \mathbf{H}-\mathbf{N M R}\left(600 \mathrm{MHz}, \mathrm{CDCl}_{3}\right): \delta=0.08\left(\mathrm{~s}, 3 \mathrm{H}, \mathrm{CH}_{3}\right), 0.10\left(\mathrm{~s}, 3 \mathrm{H}, \mathrm{CH}_{3}\right), 0.93-1.03(\mathrm{~m}, 28 \mathrm{H}$, $t \mathrm{Bu}, i \operatorname{Pr}), 1.08-1.11(\mathrm{~m}, 20 \mathrm{H}, i \operatorname{Pr}), 1.16-1.20$ (m, 3H, $i \operatorname{Pr}), 1.25$ (bs, 3H, $\left.\mathrm{CH}_{3}\right), 1.74$ (d, $\left.J=6.4 \mathrm{~Hz}, 3 \mathrm{H}, \mathrm{CH}_{3}\right), 2.09$ (bs, $\left.1 \mathrm{H}, \mathrm{CH}_{2}\right), 2.25$ (bs, $\left.1 \mathrm{H}, \mathrm{CH}_{2}\right), 2.42\left(\mathrm{~s}, 3 \mathrm{H}, \mathrm{CH}_{3}\right), 2.49$ (bs, 3H, $\left.\mathrm{CH}_{3}\right), 3.63(\mathrm{~d}, J=13.2 \mathrm{~Hz}, 1 \mathrm{H}, \mathrm{S}-\mathrm{CHH}), 3.99$ (dd, $J=9.7,7.0 \mathrm{~Hz}, 2 \mathrm{H}, \mathrm{CHHOTIPS}, \mathrm{S}-\mathrm{CH} H$ ), 4.29 (dd, $J=9.7,4.4 \mathrm{~Hz}, 1 \mathrm{H}$, CHHOTIPS), 4.32-4.34 (bm, $2 \mathrm{H}, 2 \times \mathrm{CH}), 4.81$ (dt, $J=7.5$, $\left.4.4 \mathrm{~Hz}, 1 \mathrm{H}, \mathrm{CHCONH}_{2}\right), 4.91$ (bs, 1H, CH), 5.09 (bs, 1H, CHHO) 5.48 (s, 1H, CONHH), $5.74(\mathrm{t}, J=10.3 \mathrm{~Hz}, 1 \mathrm{H}, \mathrm{CH}), 5.85$ (bs, 1H, CHHO), 5.98 (bs, 1H, CH), 6.36 (d, J=5.9 Hz, $\left.1 \mathrm{H},=\mathrm{CHCH}_{3}\right), 6.81(\mathrm{~s}, 1 \mathrm{H}, \mathrm{CONHH}), 7.13(\mathrm{~d}, J=7.0 \mathrm{~Hz}, 1 \mathrm{H}, \mathrm{CH}), 7.29(\mathrm{~d}, J=8.1 \mathrm{~Hz}, 2 \mathrm{H}$, $2 \times \mathrm{CH}), 7.34(\mathrm{t}, J=7.3 \mathrm{~Hz}, 1 \mathrm{H}, \mathrm{CH}), 7.47$ (bs, 1H, NH), 7.57 (s, 1H, S-CH), 7.69 (bs, 1H, $\mathrm{NH}), 7.78(\mathrm{~d}, J=8.4 \mathrm{~Hz}, 2 \times \mathrm{CH}), 7.93(\mathrm{~s}, 1 \mathrm{H}, \mathrm{S}-\mathrm{CH}), 7.96(\mathrm{~s}, 1 \mathrm{H}, \mathrm{S}-\mathrm{CH}), 8.08(\mathrm{~s}, 1 \mathrm{H}, \mathrm{CH})$, 8.25 (s, 1H, S-CH), 8.36-8.39 (m, 2H, CH, NH), 8.83 (s, 1H, NH), 9.41 (bs, 1H, Indolyl-NH).

${ }^{13}$ C-NMR $\left(100 \mathrm{MHz}, \mathrm{CDCl}_{3}\right): \delta=-5.5,-4.9,11.8,11.9,12.14,14.1,14.5,17.85,17.93$, $17.95,18.0,18.3,19.1,21.7,22.7,25.7,29.3,29.7,31.2,45.8,49.4,54.6,58.9,63.4,67.1$, $68.4,119.9,122.0,124.1,124.6,125.8,127.0,128.4,128.9,129.9,130.0,130.4,132.8,133.9$, 137.2 , 141.7, 143.8, 146.1, 149.2, 151.3, 160.4, 161.0, 163.3, 164.4, 166.9, 170.4, 172.3, $172.5, C(\mathrm{O}) \mathrm{S}$ nicht beobachtet.

HRMS (ESI): $m / z[\mathrm{M}+\mathrm{Na}]^{+}$für $\mathrm{C}_{82} \mathrm{H}_{105} \mathrm{~N}_{13} \mathrm{NaO}_{15} \mathrm{~S}_{7} \mathrm{Si}_{3}$ ber.: 1842.5098; gef.: 1842.5103 .

\section{Ts-Hydrato-Nosiheptid (177)}

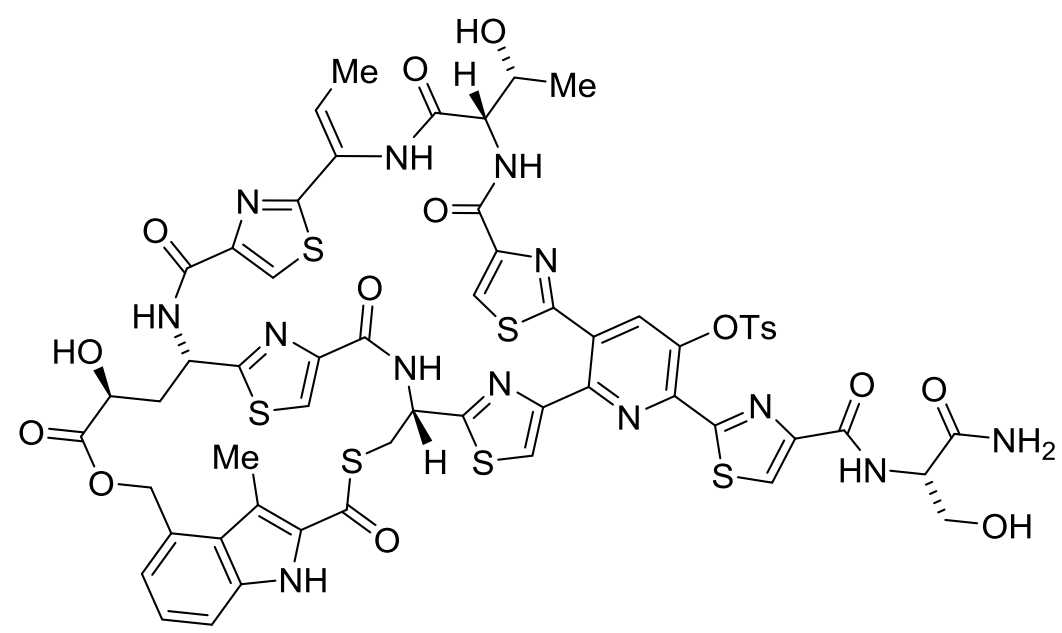

Eine Lösung des Makrolactams 116 (6 mg, $0.34 \mu \mathrm{mol})$ in wasserfreiem THF (3 mL) wurde auf $0{ }^{\circ} \mathrm{C}$ gekühlt und mit HF (1.5 mL, 70\% in Pyridin) versetzt. Die Mischung wurde langsam aufgetaut und $18 \mathrm{~h}$ lang gerührt. Nach vorsichtiger Zugabe von ges. $\mathrm{NaHCO}_{3}$-Lsg. (15 ml) wurde das Gemisch mit $\mathrm{CHCl}_{3}(4 \times 10 \mathrm{~mL})$ extrahiert, die vereinigten organischen Extrakte 
mit $\mathrm{H}_{2} \mathrm{O}(10 \mathrm{~mL})$ gewaschen und mit $\mathrm{Na}_{2} \mathrm{SO}_{4}$ entwässert. Das Lösungsmittels wurde am Rotationsverdampfer entfernt und der Rückstand durch Säulenchromatographie $\left(\mathrm{SiO}_{2}\right.$, $\left.\mathrm{CH}_{2} \mathrm{Cl}_{2} / \mathrm{MeOH}, 10: 1\right)$ gereinigt. Es wurden $4.5 \mathrm{mg}(0.32 \mu \mathrm{mol}, 96 \%)$ des Triols 177 als farbloses Glas erhalten.

Spez. Drehung: $[\alpha]_{\mathrm{D}}{ }^{24}=-13.5\left(\mathrm{CHCl}_{3} / \mathrm{MeOH}, 9: 1 ; \mathrm{c}=0.1\right)$.

DC: $R_{f}=0.36\left(\mathrm{CH}_{2} \mathrm{Cl}_{2} / \mathrm{MeOH}, 10: 1\right)$.

IR: $\tilde{v}=\left(590(\mathrm{w}), 1021(\mathrm{~m}), 1096(\mathrm{~s}), 1538(\mathrm{~m}), 1660(\mathrm{~s}), 2924(\mathrm{w}), 3365(\mathrm{~b}) \mathrm{cm}^{-1}\right.$.

${ }^{1}$ H-NMR $\left(600 \mathrm{MHz}, \mathrm{CDCl}_{3} / \mathrm{MeOH}_{-} d_{4}, 9: 1\right): \delta=1.18\left(\mathrm{~m}, 3 \mathrm{H}, \mathrm{CH}_{3}\right), 1.66(\mathrm{~d}, J=7.0 \mathrm{~Hz}, 3 \mathrm{H}$, $\left.\mathrm{CH}_{3}\right), 2.04$ (bs, $\left.1 \mathrm{H}, \mathrm{CHH}\right), 2.32-2.35(\mathrm{~m}, 1 \mathrm{H}, \mathrm{CH} H), 2.37\left(\mathrm{~s}, 3 \mathrm{H}, \mathrm{CH}_{3}\right), 3.14\left(\mathrm{~s}, 3 \mathrm{H}, \mathrm{CH}_{3}\right)$, 3.30 (verdeckt, $1 \mathrm{H}, \mathrm{CHOH}), 3.60$ (dd, $J=14.1,2.2 \mathrm{HZ}, 1 \mathrm{H}, \mathrm{S}-\mathrm{CHH}), 3.87-3.89(\mathrm{~m}, 2 \mathrm{H}$, $\left.2 \times \mathrm{CH}_{2}\right), 3.91(\mathrm{t}, J=11.4 \mathrm{~Hz}, 1 \mathrm{H}, \mathrm{CHHOH}), 4.01(\mathrm{dd}, J=11.5,5.0 \mathrm{~Hz}, \mathrm{CH} H \mathrm{OH}), 4.04(\mathrm{bs}$, $1 \mathrm{H}, \mathrm{CH}), 4.11$ (bd, $J=13.4 \mathrm{~Hz}, \mathrm{~S}-\mathrm{CH} H), 4.41$ (bs, $1 \mathrm{H}, \mathrm{C} H \mathrm{NH}), 4.64(\mathrm{t}, J=5.4 \mathrm{~Hz}, 1 \mathrm{H}$, $\mathrm{CHCONH}_{2}$ ), 5.02 (d, $\left.J=7.5 \mathrm{~Hz}, 1 \mathrm{H}, \mathrm{NH}\right), 5.47$ (dd, $\left.J=6.1,2.0 \mathrm{~Hz}, 1 \mathrm{H}, \mathrm{CHOH}\right), 5.74$ (t, $J=5.74 \mathrm{~Hz}, 1 \mathrm{H}, \mathrm{CH}), 5.82(\mathrm{~d}, J=11.6 \mathrm{~Hz}, 1 \mathrm{H}, \mathrm{CH}), 5.96(\mathrm{bs}, 1 \mathrm{H}, \mathrm{CH}), 5.96(\mathrm{q}, J=6.9 \mathrm{~Hz}$, $\left.1 \mathrm{H},=\mathrm{CHCH}_{3}\right), 7.10(\mathrm{~d}, J=6.8 \mathrm{~Hz}$, Ind-CH), $7.27(\mathrm{~s}, 1 \mathrm{H}, \mathrm{CH}), 7.37-7.40(\mathrm{~m}, 1 \mathrm{H}, \mathrm{CH}), 7.48$ (d, $J=10.3 \mathrm{~Hz}, 1 \mathrm{H}, \mathrm{NH}), 7.54(\mathrm{~s}, 1 \mathrm{H}, \mathrm{S}-\mathrm{CH}), 7.73-7.80(\mathrm{~m}, 5 \mathrm{H}, \mathrm{NH}, 4 \times \mathrm{CH}), 7.89(\mathrm{~s}, 1 \mathrm{H}, \mathrm{S}-$ $\mathrm{CH}), 7.94$ (bs, 1H, NH), 8.16 (s, 1H, S-CH), 8.20 (s, 1H, S-CH), 8.46 (bs, 1H, S-CH). Mehrere $\mathrm{OH}$ und $\mathrm{NH}-$ Gruppen aufgrund von H/D-Austausch nicht beobachtet.

HRMS (ESI): $m / z[\mathrm{M}+\mathrm{H}]^{+}$für $\mathrm{C}_{58} \mathrm{H}_{52} \mathrm{~N}_{13} \mathrm{O}_{15} \mathrm{~S}_{7}$ ber.: 1394.1745; gef.: 1394.1725. 


\section{Serinyl-Alkohol 178}

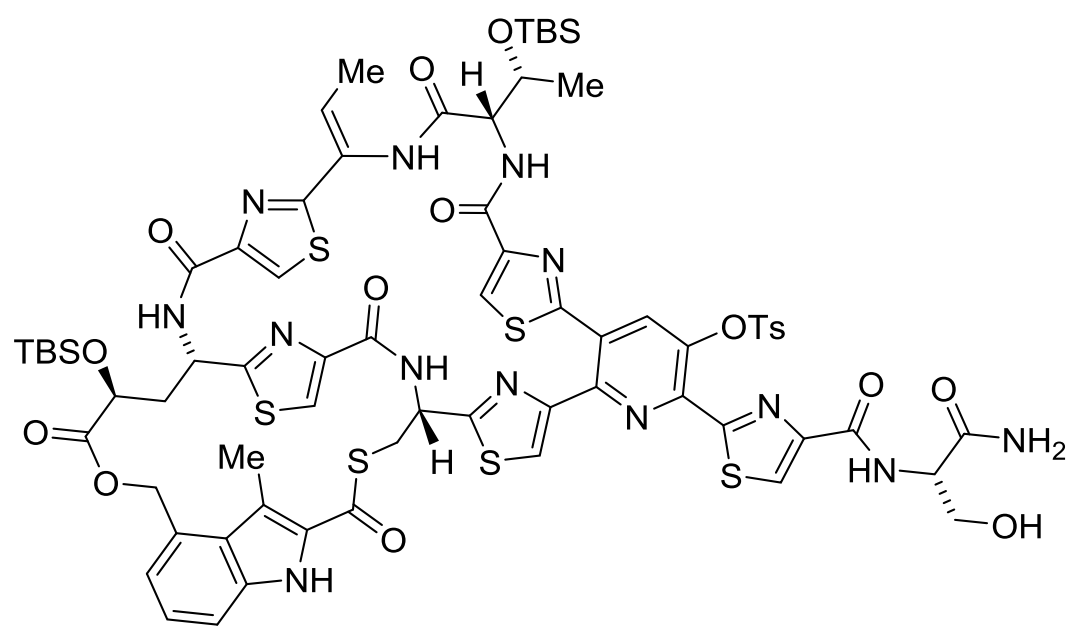

Eine Lösung des Makrothiolactons 116 (4 mg, $0.23 \mathrm{mmol})$ in $\mathrm{MeCN}(3.2 \mathrm{~mL})$ wurde auf $0{ }^{\circ} \mathrm{C}$ gekühlt und mit $\mathrm{HF}\left(200 \mu \mathrm{L}, 49 \%\right.$ in $\left.\mathrm{H}_{2} \mathrm{O}\right)$ versetzt. Das Gemisch wurde $15 \mathrm{~h}$ lang bei $0{ }^{\circ} \mathrm{C}$ gerührt, danach vorsichtig mit ges. $\mathrm{KHCO}_{3}$-Lsg. $(2 \mathrm{~mL})$ und $\mathrm{H}_{2} \mathrm{O}(10 \mathrm{~mL})$ versetzt und mit $\mathrm{CHCl}_{3}(4 \times 10 \mathrm{~mL})$ extrahiert. Die vereinigten organischen Extrakte wurden mit $\mathrm{Na}_{2} \mathrm{SO}_{4}$ entwässert und im Vakuum eingeengt. Reinigung des Rückstands durch Säulenchromatographie $\left(\mathrm{SiO}_{2}, 5 \mathrm{~g}, \mathrm{CH}_{2} \mathrm{Cl}_{2} / \mathrm{MeOH}, 20: 1\right)$ und anschließender präparativer HPLC (C18, $20 \mathrm{ml} / \mathrm{min}, \mathrm{B}: 90 \stackrel{5 \mathrm{~min}}{\longrightarrow} 90 \stackrel{10 \mathrm{~min}}{\longrightarrow} 100 \%, 60 \mathrm{~min})$ lieferte $1.5 \mathrm{mg}(0.92 \mu \mathrm{mol}, 41 \%)$ des Alkohols 178 als farbloses Glas.

DC: $R_{f}=0.30\left(\mathrm{CH}_{2} \mathrm{Cl}_{2} / \mathrm{MeOH}, 20: 1\right)$.

RP-HPLC: $t_{R}=12.2 \mathrm{~min}(\mathrm{C} 18, \mathrm{~B}: 90 \stackrel{1 \mathrm{~min}}{\longrightarrow} 90 \stackrel{10 \mathrm{~min}}{\longrightarrow} 100 \%, 25 \mathrm{~min})$.

HRMS (ESI): $m / z[\mathrm{M}+\mathrm{H}]^{+}$ber. für $\mathrm{C}_{70} \mathrm{H}_{80} \mathrm{~N}_{13} \mathrm{O}_{15} \mathrm{~S}_{7} \mathrm{Si}_{2}$ : 1622.3475; gef.: 1622.3480 . 


\section{Serinyl-Alkohol 179}

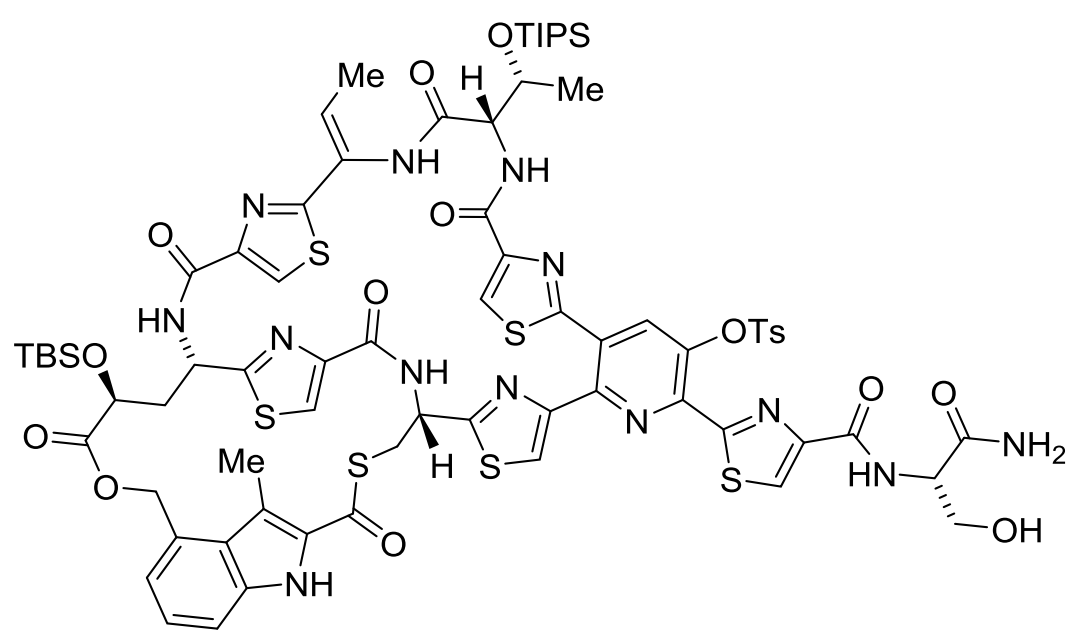

Eine Lösung des Makrothiolactons $117(8 \mathrm{mg}, 4.39 \mu \mathrm{mol})$ in $\mathrm{MeCN}(6 \mathrm{~mL})$, wurde auf $0{ }^{\circ} \mathrm{C}$ gekühlt und mit $\mathrm{HF}\left(250 \mu \mathrm{L}, 49 \%\right.$ in $\left.\mathrm{H}_{2} \mathrm{O}\right)$ tropfenweise versetzt. Die Mischung wurde $24 \mathrm{~h}$ lang bei $0{ }^{\circ} \mathrm{C}$ gerührt, danach mit $\mathrm{CHCl}_{3}(150 \mathrm{~mL})$ verdünnt und mit $\mathrm{H}_{2} \mathrm{O}$, ges. $\mathrm{NaHCO}_{3}$-Lsg. und ges. NaCl-Lsg. (je $50 \mathrm{~mL}$ ) gewaschen. Die organische Phase wurde mit $\mathrm{Na}_{2} \mathrm{SO}_{4}$ entwässert und im Vakuum eingeengt. Reinigung des Rückstands durch Säulenchromatographie $\left(\mathrm{SiO}_{2}, 15 \mathrm{~g}, \mathrm{CH}_{2} \mathrm{Cl}_{2} / \mathrm{MeOH}, 20: 1 \rightarrow 15: 1\right)$ lieferte $5.5 \mathrm{mg}(75 \%$, $3.29 \mu \mathrm{mol}$ ) des Alkohols 179 als farbloses Glas, zusammen mit nicht umgesetzten Ausgangsmaterial (117, $1.5 \mathrm{mg}, 15 \%)$.

Spez. Drehung: $[\alpha]_{\mathrm{D}}^{24}=+12.4\left(\mathrm{CHCl}_{3}, c=0.1\right)$.

DC: $R_{f}=0.27\left(\mathrm{CH}_{2} \mathrm{Cl}_{2} / \mathrm{MeOH}, 20: 1\right)$.

IR: $\tilde{v}=735(\mathrm{~m}), 1132(\mathrm{~m}), 1247(\mathrm{w}), 1469(\mathrm{~m}), 1530(\mathrm{~s}), 1660(\mathrm{~s}), 2855(\mathrm{w}), 2924(\mathrm{w}) \mathrm{cm}^{-1}$.

${ }^{1}$ H-NMR (600 MHz, $\left.\mathrm{CDCl}_{3}\right): \delta=0.08\left(\mathrm{~s}, 3 \mathrm{H}, \mathrm{CH}_{3}\right), 0.10\left(\mathrm{~s}, 3 \mathrm{H}, \mathrm{CH}_{3}\right), 0.82-1.00(\mathrm{~m}, 30 \mathrm{H}$, $t \mathrm{Bu}, 3 \times i \mathrm{Pr}), 1.25\left(\mathrm{bs}, 3 \mathrm{H}, \mathrm{CH}_{3}\right), 1.72\left(\mathrm{~d}, J=6.2 \mathrm{~Hz}, 3 \mathrm{H}, \mathrm{CH}_{3}\right), 2.09$ (bs, $\left.1 \mathrm{H}, \mathrm{CH}_{2}\right), 2.24$ (bs, $\left.1 \mathrm{H}, \mathrm{CH}_{2}\right), 2.49\left(\mathrm{~m}, 6 \mathrm{H}, 2 \times \mathrm{CH}_{3}\right), 3.11(\mathrm{t}, J=6.4 \mathrm{~Hz}, 1 \mathrm{H}, \mathrm{OH}), 3.62(\mathrm{~d}, J=13.0 \mathrm{~Hz}, 1 \mathrm{H}, \mathrm{S}-$ $\mathrm{CHH}), 3.99$ (bs, 1H, S-CHH), 4.06 (dt, $J=11.6,6.0 \mathrm{~Hz}, 1 \mathrm{H}, \mathrm{CHHOH}), 4.21$ (m, 1H, $\mathrm{CH} H \mathrm{OH}), 4.33(\mathrm{bs}, 2 \mathrm{H}, 2 \times \mathrm{CH}), 4.78\left(\mathrm{dt}, J=7.6,5.0 \mathrm{~Hz}, 1 \mathrm{H}, \mathrm{CHCONH}_{2}\right), 4.92(\mathrm{bs}, 1 \mathrm{H}$, $\mathrm{CH}), 5.09$ (bs, 1H, CHHO) $5.50(\mathrm{~s}, 1 \mathrm{H}, \mathrm{CONHH}), 5.74$ (t, J=10.3 Hz, 1H, CH), 5.86 (bs, $\mathrm{CH} H \mathrm{O}), 5.99(\mathrm{bs}, 1 \mathrm{H}, \mathrm{CH}), 6.35\left(\mathrm{bd}, J=6.2 \mathrm{~Hz},=\mathrm{CHCH}_{3}\right), 6.75(\mathrm{~s}, 1 \mathrm{H}, \mathrm{CONHH}), 7.13(\mathrm{~d}$, $J=6.8 \mathrm{~Hz}, 1 \mathrm{H}, \mathrm{CH}), 7.34(\mathrm{t}, J=7.4 \mathrm{~Hz}, 1 \mathrm{H}, \mathrm{CH}), 7.38(\mathrm{~m}, 2 \mathrm{H}, 2 \times \mathrm{CH}), 7.47(\mathrm{bs}, 1 \mathrm{H}, \mathrm{NH})$, 7.69 (bs, 1H, NH), 7.85 (d, J=8.4 Hz, 2H, $2 \times \mathrm{CH}), 7.94$ (s, 1H, S-CH), $7.96(\mathrm{~s}, 1 \mathrm{H}, \mathrm{S}-\mathrm{CH})$, 
$8.09(\mathrm{~s}, 1 \mathrm{H}, \mathrm{CH}), 8.31(\mathrm{~s}, 1 \mathrm{H}, \mathrm{S}-\mathrm{CH}), 8.35(\mathrm{~s}, 1 \mathrm{H}, \mathrm{S}-\mathrm{CH}), 8.39$ (bs, 1H, NH), 8.55 (d, $J=7.9 \mathrm{~Hz}, 1 \mathrm{H}, \mathrm{NH}), 8.85$ (s, 1H, NH), 9.38 (bs, 1H, Indolyl-NH).

${ }^{13}$ C-NMR $\left(100 \mathrm{MHz}, \mathrm{CDCl}_{3}\right): \delta=-5.6,-4.9,12.1,13.0,14.5,17.8,18.0,18.3,21.8,25.7$, 29.7, 31.1, 40.1, 45.7, 49.4, 54.1, 58.9, 62.3, 67.1, 68.4, 114.2, 119.9, 122.1, 123.6, 124.1, $124.6,125.7,127.4,128.4,128.9,129.9,130.2,132.9,133.5,137.2,141.6,143.7,146.4$, $149.2,150.8,151.4,152.9,153.0,160.4,161.1,161.6,161.7,163.1,164.7,166.8,170.3$, 172.3, 173.1, $C(\mathrm{O}) \mathrm{S}$ nicht beobachtet.

HRMS (ESI): $m / z$ [M + H] für $\mathrm{C}_{73} \mathrm{H}_{86} \mathrm{~N}_{13} \mathrm{O}_{15} \mathrm{~S}_{7} \mathrm{Si}_{2}$ ber.: 1664.3944; gef.: 1664.3946 .

\section{Dehydroalanin 180}

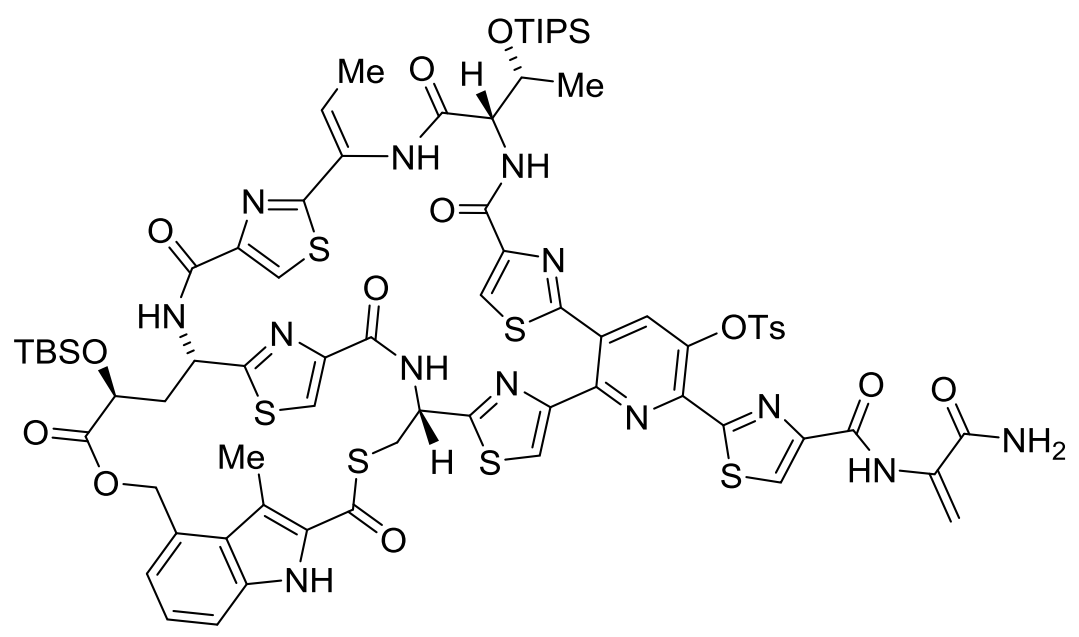

Eine Lösung des Alkohols $179(10 \mathrm{mg}, 6.0 \mu \mathrm{mol})$ in wasserfreiem $\mathrm{CH}_{2} \mathrm{Cl}_{2}(5 \mathrm{~mL})$ wurde auf $0{ }^{\circ} \mathrm{C}$ gekühlt und nacheinander mit 2,6-Lutidin $(500 \mu \mathrm{L})$ und Methansulfonylchlorid $(45 \mu \mathrm{L}$, $58 \mathrm{mmol}$ ) versetzt. Das Kühlbad wurde entfernt und die Reaktionsmischung bis zum vollständigen Umsatz gerührt (30-45 min, DC-Kontrolle). Die Mischung wurde mit EtOAc (75 mL) verdünnt, nacheinander mit halb ges. $\mathrm{NaHCO}_{3}$-Lsg., pH 3 Puffer und ges. NaCl-Lsg. (je $20 \mathrm{~mL}$ ) gewaschen. Nach Entwässern mit $\mathrm{Na}_{2} \mathrm{SO}_{4}$ wurde die Lösung im Vakuum eingeengt und der Rückstand direkt weiter umgesetzt.

Eine Lösung des Rückstands in wasserfreiem $\mathrm{CH}_{2} \mathrm{Cl}_{2}(4.5 \mathrm{~mL})$ wurde auf $-78^{\circ} \mathrm{C}$ gekühlt und tropfenweise mit einer Lösung von DBU $(115 \mu \mathrm{L})$ in $\mathrm{CH}_{2} \mathrm{Cl}_{2}(1.5 \mathrm{~mL})$ versetzt. Nach $5 \mathrm{~min}$ wurde das Gemisch auf $-35^{\circ} \mathrm{C}$ erwärmt und bis zum vollständigen Umsatz gerührt $(4.5 \mathrm{~h}$, DC-Kontrolle). Danach wurde eine Lösung von Essigsäure $(150 \mu \mathrm{L})$ in $\mathrm{CH}_{2} \mathrm{Cl}_{2}(1 \mathrm{~mL})$ zugegeben und das Reaktionsgemisch mit $\mathrm{CHCl}_{3}(50 \mathrm{~mL})$ verdünnt. Die Lösung wurde mit 
$\mathrm{H}_{2} \mathrm{O}$ und ges. NaCl-Lsg. (je $15 \mathrm{~mL}$ ) gewaschen, mit $\mathrm{Na}_{2} \mathrm{SO}_{4}$ entwässert und im Vakuum eingeengt. Reinigung des Rückstand durch präparative Dünnschichtchromatographie (1 mm, $\left.\mathrm{CH}_{2} \mathrm{Cl}_{2} / \mathrm{MeOH}, 20: 1\right)$ lieferte $7.5 \mathrm{mg}$ Substanz welche anschließend durch präparative HPLC $(\mathrm{C} 8,20 \mathrm{ml} / \mathrm{min}, \mathrm{B}: 90 \stackrel{5 \mathrm{~min}}{\longrightarrow} 90 \stackrel{5 \mathrm{~min}}{\longrightarrow} 100 \%, 60 \mathrm{~min})$ weiter gereinigt wurde. Es wurden $3.5 \mathrm{mg}$ $(2.1 \mu \mathrm{mol}, 35 \%)$ des Dehydroalanins $\mathbf{1 8 0}$ als farbloses Glas erhalten.

Spez. Drehung: $[\alpha]_{\mathrm{D}}{ }^{24}=+10.2\left(\mathrm{CHCl}_{3}, c=0.1\right)$.

DC: $R_{f}=0.41\left(\mathrm{CH}_{2} \mathrm{Cl}_{2} / \mathrm{MeOH}, 20: 1\right)$.

RP-HPLC: $t_{R}=15.5 \mathrm{~min}(\mathrm{C} 8, \mathrm{~B}: 90 \stackrel{1 \mathrm{~min}}{\longrightarrow} 90 \stackrel{10 \mathrm{~min}}{\longrightarrow} 100 \%, 25 \mathrm{~min})$.

IR: $\tilde{v}=756(\mathrm{w}), 1002(\mathrm{w}), 1141(\mathrm{w}), 1481(\mathrm{~m}), 1527(\mathrm{~s}), 1666(\mathrm{~s}), 2684(\mathrm{w}), 2916(\mathrm{w}) \mathrm{cm}^{-1}$.

${ }^{1}$ H-NMR $\left(600 \mathrm{MHz}, \mathrm{CDCl}_{3}\right): \delta=0.08\left(\mathrm{~s}, 3 \mathrm{H}, \mathrm{CH}_{3}\right), 0.10\left(\mathrm{~s}, 3 \mathrm{H}, \mathrm{CH}_{3}\right), 0.82-1.00(\mathrm{~m}, 30 \mathrm{H}$, $t \mathrm{Bu}, 3 \times i \mathrm{Pr}), 1.25$ (bs, 3H, $\left.\mathrm{CH}_{3}\right), 1.72\left(\mathrm{~d}, J=5.9 \mathrm{~Hz}, 3 \mathrm{H}, \mathrm{CH}_{3}\right), 2.11$ (bs, $\left.1 \mathrm{H}, \mathrm{CH}_{2}\right), 2.25$ (bs, 1H, $\left.\mathrm{CH}_{2}\right), 2.33\left(\mathrm{~s}, 3 \mathrm{H}, \mathrm{CH}_{3}\right), 2.46\left(\mathrm{bs}, 3 \mathrm{H}, \mathrm{CH}_{3}\right), 3.66$ (d, J=13.0 Hz, 1H, S-CHH), 4.02 (d, $J=11.7 \mathrm{~Hz}, 1 \mathrm{H}, \mathrm{S}-\mathrm{CH} H), 4.34$ (bs, 2H, $2 \times \mathrm{CH}), 4.92(\mathrm{~s}, 1 \mathrm{H}, \mathrm{CH}), 5.05$ (bs, 1H, CHHO), $5.55(\mathrm{~s}, 1 \mathrm{H},=\mathrm{CHH}), 5.75(\mathrm{t}, J=10.9 \mathrm{~Hz}, 1 \mathrm{H}, \mathrm{CH}), 5.85(\mathrm{bs}, 1 \mathrm{H}, \mathrm{CH} H \mathrm{O}), 6.00(\mathrm{~s}, 1 \mathrm{H}$, $\left.\mathrm{SCH}_{2} \mathrm{CH}\right), 6.15(\mathrm{bs}, 1 \mathrm{H}, \mathrm{NH}), 6.35\left(\mathrm{~d}, J=5.5 \mathrm{~Hz}, 1 \mathrm{H},=\mathrm{CHCH}_{3}\right), 6.64(\mathrm{~s}, 1 \mathrm{H},=\mathrm{CH} H), 7.13$ $(\mathrm{d}, J=7.0 \mathrm{~Hz}, 1 \mathrm{H}, \mathrm{CH}), 7.15(\mathrm{~d}, J=8.1 \mathrm{~Hz}, 2 \mathrm{H}, 2 \times \mathrm{CH}), 7.35(\mathrm{t}, J=7.3 \mathrm{~Hz}, 1 \mathrm{H}, \mathrm{CH}), 7.48$ (s, 1H, NH), $7.77(\mathrm{~d}, J=8.4 \mathrm{~Hz}, 2 \times \mathrm{CH}), 7.91(\mathrm{~s}, 1 \mathrm{H}, \mathrm{S}-\mathrm{CH}), 7.94(\mathrm{~s}, 1 \mathrm{H}, \mathrm{CH}), 7.96(\mathrm{~s}, 1 \mathrm{H}$, S-CH), 8.09 (s, 1H, CH), 8.19 (s, 1H, CH), 8.40 (bs, 2H, CH, NH), 8.88 (s, 1H, NH), 9.80 (s, $1 \mathrm{H}$, Indolyl-NH).

HRMS (ESI): $m / z[\mathrm{M}+\mathrm{H}]^{+}$für $\mathrm{C}_{73} \mathrm{H}_{84} \mathrm{~N}_{13} \mathrm{O}_{14} \mathrm{~S}_{7} \mathrm{Si}_{2}$ ber.: 1646.3839; gef.: 1646.3851 . 


\section{Nosiheptid (6)}

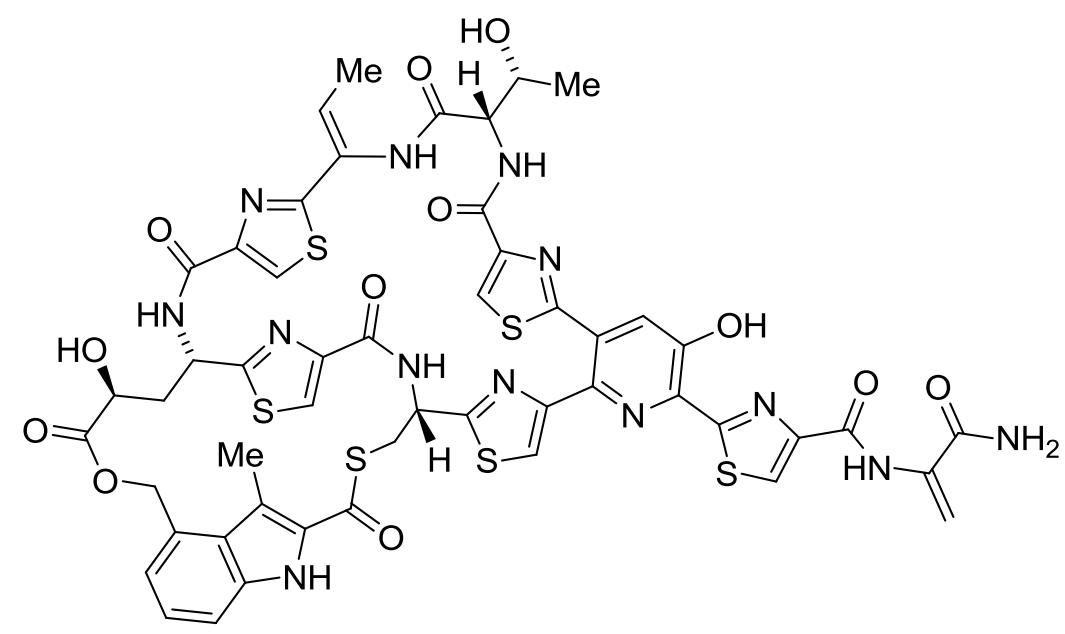

Eine Lösung des Bis-Silylethers 180 (4.5 mg, $2.5 \mu \mathrm{mol})$ in wasserfreiem THF (3 mL) wurde auf $0{ }^{\circ} \mathrm{C}$ gekühlt und tropfenweise mit einer Lösung von $\mathrm{Et}_{3} \mathrm{~N} \times 3 \mathrm{HF}(438 \mu \mathrm{L})$ in $\mathrm{THF}$ $(0.5 \mathrm{~mL})$ versetzt. Nach vollständiger Zugabe wurde das Kühlbad entfernt und die Mischung $27 \mathrm{~h}$ lang bei RT gerührt. Nach Verdünnen mit $\mathrm{CHCl}_{3}(30 \mathrm{~mL})$ wurde das Gemisch mit $\mathrm{H}_{2} \mathrm{O}$ und halb ges. $\mathrm{NaHCO}_{3}$-Lsg. gewaschen und mit $\mathrm{Na}_{2} \mathrm{SO}_{4}$ entwässert. Die Lösungsmittel wurden im Vakuum entfernt und der Rückstand direkt weiter umgesetzt.

Eine Lösung von HOBt $(3.5 \mathrm{mg}, 25.9 \mu \mathrm{mol})$ und $\mathrm{EtNiPr}_{2}(3 \mu \mathrm{L}, 18 \mu \mathrm{mol})$ in wasserfreiem DMF $(150 \mu \mathrm{L})$ wurde tropfenweise zu einer auf $0{ }^{\circ} \mathrm{C}$ gekühlten Lösung des Rückstands in wasserfreiem DMF (1 mL) zugegeben. Nach 5 min wurde das Kühlbad entfernt und das Gemisch 1.5 h lang bei RT gerührt. Das Lösungsmittel wurde im Vakuum entfernt und der Rückstand durch präparative Dünnschichtchromatographie $\left(0.5 \mathrm{~mm}, \mathrm{CHCl}_{3} / \mathrm{MeOH} / \mathrm{HCOOH}\right.$, 100:5:0.2) gereinigt. Die erhaltenen $2.3 \mathrm{mg}$ wurden danach durch präparative HPLC (C18, $25 \mathrm{ml} / \mathrm{min}, \mathrm{B}: 30 \stackrel{2 \mathrm{~min}}{\longrightarrow} 30 \stackrel{30 \mathrm{~min}}{\longrightarrow} 100 \%, 45 \mathrm{~min})$ weiter gereinigt. Es wurden $1.1 \mathrm{mg}(0.9 \mu \mathrm{mol}$, $36 \%$ ) des synthetischen Naturstoffs Nosiheptid (6) als leuchtend gelber Feststoffen erhalten.

Spez. Drehung: $[\alpha]_{\mathrm{D}}{ }^{24}=$ syn. +30.5 (Pyridin, $\left.c=0.1\right)$, nat. $+31.5($ Pyridin, $c=0.1)$.

Schmelzpunkt: $300^{\circ} \mathrm{C}$ (Zers.).

DC: $R_{f}=0.27\left(\mathrm{CHCl}_{3} / \mathrm{MeOH}, 20: 1\right)$.

RP-HPLC: $t_{R}=9.6 \mathrm{~min}(\mathrm{C} 18, \mathrm{~B}: 30 \stackrel{1 \mathrm{~min}}{\longrightarrow} 30 \stackrel{14 \mathrm{~min}}{\longrightarrow} 100 \%, 27 \mathrm{~min})$ 
IR: $\tilde{v}=748(\mathrm{~m}), 1203(\mathrm{w}), 1481(\mathrm{~m}), 1527$ (s), 1658 (s), 1975 (m), 2021 (m), 2160 (m), 3240 (b) $\mathrm{cm}^{-1}$.

${ }^{1}$ H-NMR $\left(600 \mathrm{MHz}, \mathrm{CDCl}_{3} / \mathrm{MeOH}_{-} d_{4}, 9: 1\right): \delta=1.15$ (bd, $\left.J=4.6 \mathrm{~Hz}, \mathrm{CH}_{3}\right), 1.67$ (d, $\left.J=7.0 \mathrm{~Hz}, \mathrm{CH}_{3}\right), 2.04(\mathrm{bs}, 1 \mathrm{H}, \mathrm{CHH}), 2.36$ (t, $\left.J=12.9 \mathrm{~Hz}, \mathrm{CH} H\right), 2.44\left(\mathrm{~s}, 3 \mathrm{H}, \mathrm{CH}_{3}\right), 3.24$ (verdeckt, 1H, CHOH), $3.64(\mathrm{~m}, 1 \mathrm{H}, \mathrm{S}-\mathrm{CHH}), 4.02(\mathrm{~m}, 1 \mathrm{H}, \mathrm{S}-\mathrm{CH} H), 4.12(\mathrm{~d}, J=11.7 \mathrm{~Hz}$, $1 \mathrm{H}, \mathrm{CHOH}), 4.42(\mathrm{~s}, 1 \mathrm{H}, \mathrm{CH}), 5.02(\mathrm{~d}, J=10.1 \mathrm{~Hz}, \mathrm{CHHO}), 5.57(\mathrm{~d}, J=1.8 \mathrm{~Hz}, 1 \mathrm{H},=\mathrm{CHH})$, 5.73-5.75 (m, 1H, CH), $5.83(\mathrm{~d}, J=12.2 \mathrm{~Hz}, \mathrm{CH} H \mathrm{O}), 5.96$ (bs, $\left.1 \mathrm{H}, \mathrm{SCH}_{2} \mathrm{CH}\right), 6.31$ (q, $\left.J=7.0 \mathrm{~Hz}, 1 \mathrm{H},=\mathrm{CHCH}_{3}\right), 6.58(\mathrm{~d}, J=1.8 \mathrm{~Hz}, 1 \mathrm{H},=\mathrm{CH} H), 7.11(\mathrm{~d}, J=7.0 \mathrm{~Hz}, 1 \mathrm{H}, \mathrm{CH})$, 7.39 (t, $J=7.6 \mathrm{~Hz}, 1 \mathrm{H}, \mathrm{CH}), 7.48$ (d, $J=10.1 \mathrm{~Hz}, 1 \mathrm{H}, \mathrm{NH}), 7.62$ (s, 1H, S-CH), 7.65 (s, 1H, py-CH), 7.79 (d, $J=9.5 \mathrm{~Hz}, 1 \mathrm{H}, \mathrm{NH}), 7.90$ (s, 1H, S-CH), 7.96 (d, $J=6.8 \mathrm{~Hz}, 1 \mathrm{H}, \mathrm{CH}), 8.16$ (s, 1H, S-CH), 8.27 (s, 1H, S-CH), 8.45 (s, 1H, S-CH). Alle HO- und mehrere NH-Gruppen wurden aufgrund des H/D-Austauschs nicht beobachtet.

HRMS (ESI): $m / z[\mathrm{M}+\mathrm{H}]^{+}$ber. für $\mathrm{C}_{51} \mathrm{H}_{44} \mathrm{~N}_{13} \mathrm{O}_{12} \mathrm{~S}_{6}$ : 1222.1551; gef.: 1222.1553 .

\subsection{Semisynthetische Arbeiten an Nosiheptid}

\section{Isolierung von nat. Nosiheptid}

Nosiheptid-Premix (100 g, ca. 1\%-Gehalt, BocSciences USA) wurde in einer $\mathrm{CHCl}_{3} / \mathrm{MeOH}$ Mischung (9:1, $500 \mathrm{ml})$ suspendiert und $30 \mathrm{~min}$ lang gerührt. Die Suspension wurde filtriert und der Filterkuchen mit einer $\mathrm{CHCl}_{3} / \mathrm{MeOH}$-Mischung $(3 \times 150 \mathrm{ml})$ gewaschen. Die Lösung wurde im Vakuum eingeengt, der Rückstand in Petrolether/MTBE (1:1, $500 \mathrm{~mL})$ suspendiert, filtriert und der Feststoff gewaschen $(3 \times 150 \mathrm{~mL}$, PE/MTBE, 1:1). Das erhaltene Rohprodukt (ca. $1.3 \mathrm{~g})$ wurde in THF $(100 \mathrm{~mL})$ gelöst und auf Kieselgel $(20 \mathrm{~g})$ adsorbiert. Anschließende Säulenchromatographie an einem Teledyne Isco, CombiFlash $R_{f}\left(\mathrm{SiO}_{2}, 120 \mathrm{~g}, 45 \mathrm{ml} / \mathrm{min}, \mathrm{A}\right.$ : $\mathrm{CHCl}_{3}$; B: $\mathrm{CHCl}_{3} / \mathrm{MeOH}, 9: 1$; B: $\left.0 \stackrel{60 \mathrm{~min}}{\longrightarrow} 100 \%\right)$ lieferte nach Trocknen am Hochvakuum bei $60{ }^{\circ} \mathrm{C}, 530 \mathrm{mg}(0.43 \mathrm{mmol}, 53 \%$, Reinheit $\geq 99 \%)$ sowie $160 \mathrm{mg}(0.13 \mathrm{mmol}, 16 \%$, Reinheit ca. 98\%) des Naturstoffs 6 als gelben Feststoff.

Alle relevanten analytischen Daten stimmen mit den veröffentlichten Werten überein. ${ }^{\text {[135-137] }}$ 


\section{Tosyl-Nosiheptid (169)}

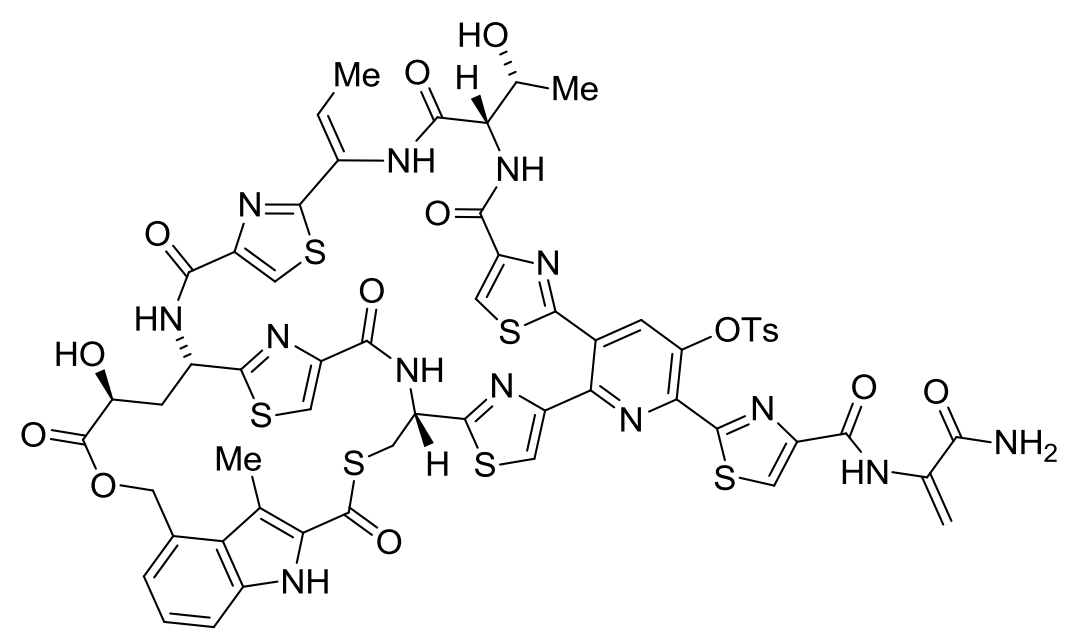

Eine Lösung von Nosiheptid (6, $250 \mathrm{mg}, 204 \mu \mathrm{mol})$ in wasserfreiem THF (37 ml) wurde bei $0{ }^{\circ} \mathrm{C}$ nacheinander mit EtNiPr $2(340 \mu \mathrm{L}, 2.04 \mathrm{mmol})$, TsCl $(194 \mathrm{mg}, 1.02 \mathrm{mmol})$ und DMAP (25 mg, $204 \mu \mathrm{mol}$ ) versetzt. Nach $5 \mathrm{~min}$ wurde das Kühlbad entfernt und weitere $10 \mathrm{~min}$ lang bei RT gerührt. Das Gemisch wurde anschließend mit pH 7 Puffer $(100 \mathrm{~mL})$ verdünnt und mit $\mathrm{CH}_{2} \mathrm{Cl}_{2}(3 \times 60 \mathrm{~mL})$ extrahiert. Die vereinigten organischen Extrakte wurden mit halb ges. $\mathrm{NaHCO}_{3}$-Lsg. (60 mL) gewaschen und mit $\mathrm{Na}_{2} \mathrm{SO}_{4}$ entwässert. Nach Entfernen der Lösungsmittel im Vakuum, wurde der Rückstand durch Säulenchromatographie (CombiFlash, $\mathrm{SiO}_{2}, 80$ g, A: $\mathrm{CH}_{2} \mathrm{Cl}_{2}$; B: $\mathrm{CH}_{2} \mathrm{Cl}_{2} / \mathrm{MeOH}, 9: 1 ; 0 \stackrel{45 \mathrm{~min}}{\longrightarrow} 100 \%$ ) gereinigt. Es wurden $211 \mathrm{mg}$ (153 $\mu \mathrm{mol}, \mathbf{7 5 \%}$ ) des Tosylats 169 als leicht gelber Feststoff erhalten.

Spez. Drehung: $[\alpha]_{\mathrm{D}}{ }^{24}=-17.7\left(\mathrm{CHCl}_{3} / \mathrm{MeOH}, 9: 1 ; \mathrm{c}=1\right)$.

Schmelzpunkt: $270{ }^{\circ} \mathrm{C}$ (Zers.).

DC: $R_{f}=0.56\left(\mathrm{CHCl}_{3} / \mathrm{MeOH}, 9: 1\right)$.

IR: $\tilde{v}=548(\mathrm{~s}), 747(\mathrm{~s}), 1177(\mathrm{~m}), 1193(\mathrm{~m}), 1479(\mathrm{~m}), 1524(\mathrm{~s}), 1651(\mathrm{~s}), 3115(\mathrm{w})$, 3338 (b) $\mathrm{cm}^{-1}$.

${ }^{1} \mathbf{H}-\mathbf{N M R}\left(300 \mathrm{MHz}, \mathrm{CDCl}_{3} / \mathrm{MeOH}-d_{4}, 9: 1\right): \delta=1.11$ (bd, $\left.J=3.7 \mathrm{~Hz}, 3 \mathrm{H}, \mathrm{CH}_{3}\right), 1.66$ (d, $\left.J=6.9 \mathrm{~Hz}, 3 \mathrm{H}, \mathrm{CH}_{3}\right), 1.97-2.12\left(\mathrm{~m}, 1 \mathrm{H}, \mathrm{CH}_{2}\right), 2.25\left(\mathrm{~s}, 3 \mathrm{H}, \mathrm{CH}_{3}\right), 2.30-2.39\left(\mathrm{~m}, 1 \mathrm{H}, \mathrm{CH}_{2}\right)$, $2.43\left(\mathrm{~s}, 3 \mathrm{H}, \mathrm{CH}_{3}\right), 3.25-3.31(\mathrm{~m}, 1 \mathrm{H}, \mathrm{S}-\mathrm{CHH}), 3.61(\mathrm{~d}, J=13.0 \mathrm{~Hz}, 1 \mathrm{H}, \mathrm{S}-\mathrm{CH} H), 4.02$ (d, $J=10.6 \mathrm{~Hz}, 1 \mathrm{H}, \mathrm{CH}), 4.13(\mathrm{~d}, J=11.5 \mathrm{~Hz}, 1 \mathrm{H}, \mathrm{CH}), 4.40$ (bs, $1 \mathrm{H}, \mathrm{CH}), 5.00$ (d, $J=10.6 \mathrm{~Hz}$, $1 \mathrm{H}, \mathrm{CH}), 5.62(\mathrm{~s}, 1 \mathrm{H},=\mathrm{CHH}), 5.72-5.83(\mathrm{~m}, 2 \mathrm{H}, 2 \times \mathrm{CH}), 5.96(\mathrm{~d}, J=8.8 \mathrm{~Hz}, 1 \mathrm{H}, \mathrm{CH}), 6.29$ 
$\left(\mathrm{q}, J=6.8 \mathrm{~Hz}, 1 \mathrm{H},=\mathrm{CH}(\mathrm{CH})_{3}\right), 6.54(\mathrm{~s}, 1 \mathrm{H},=\mathrm{CH} H), 7.06-7.10(\mathrm{~m}, 3 \mathrm{H}, 3 \times \mathrm{CH}), 7.38(\mathrm{t}$, $J=7.3 \mathrm{~Hz}, 1 \mathrm{H}, \mathrm{CH}), 7.49(\mathrm{~d}, J=10.1 \mathrm{~Hz}, 1 \mathrm{H}, \mathrm{NH}), 7.63(\mathrm{~s}, 1 \mathrm{H}, \mathrm{CH}), 7.65(\mathrm{~s}, 1 \mathrm{H}, \mathrm{CH}), 7.75-$ $7.80(\mathrm{~m}, 2 \mathrm{H}, \mathrm{CH}, \mathrm{NH}), 7.90-7.98(\mathrm{~m}, 3 \mathrm{H}, 3 \times \mathrm{CH}), 8.13(\mathrm{~s}, 1 \mathrm{H}, \mathrm{CH}), 8.16(\mathrm{~s}, 1 \mathrm{H}, \mathrm{CH}), 8.45$ $(\mathrm{s}, 1 \mathrm{H}, \mathrm{NH}), 8.52(\mathrm{~s}, 1 \mathrm{H}, \mathrm{NH}), 9.78(\mathrm{~s}, 1 \mathrm{H}, \mathrm{NH}), 9.96$ (s, 1H, Indolyl-NH). Alle HO- und einige NH-Gruppen wurden aufgrund von H/D-Austausch nicht oder mit verminderter Integrations beobachtet.

${ }^{13}$ C-NMR (75 MHz, $\left.\mathrm{CDCl}_{3} / \mathrm{MeOH}_{-} d_{4}, 9: 1\right): \delta=11.9,14.4,17.2,21.4,29.1,40.1,45.5,49.9$, $56.9,66.5,66.9,67.2,140.7,115.2,118.8,122.0,123.7,124.2,125.1,125.5,125.7,126.0$, 127.2 , 128.0, 128.1, 128.4, 128.7, 129.2, 129.5, 130.2, 130.3, 132.0, 133.6 135.2, 137.3, $137.4,141.3,142.6,146.0,147.7,149.5,149.8,150.3,151.2,153.8,159.36,159.43,160.4$, $160.6,161.0,163.3,164.3,165.99,166.04,166.3,166.6,169.4,170.6,173.3,180.4$. C(O)S nicht beobachtet.

HRMS (ESI): $m / z[\mathrm{M}+\mathrm{H}]^{+}$ber. für $\mathrm{C}_{58} \mathrm{H}_{50} \mathrm{~N}_{13} \mathrm{O}_{14} \mathrm{~S}_{7}$ : 1376.1640; gef.: 1376.1636 .

\subsection{Nachbargruppen-assistierte Umsetzungen}

\section{5,6-Bis(methoxycarbonyl)-3-hydroxypicolinsäureethylester (196)}<smiles>CCOC(=O)c1nc(C(=O)OC)c(C(=O)OC)cc1O</smiles>

Der Triester 97 ${ }^{[37]}(14 \mathrm{mg}, 52.0 \mu \mathrm{mol})$ wurde nach AV3 mit EtOH umgesetzt. Es wurden $14 \mathrm{mg}(52.0 \mu \mathrm{mol}$, quant.) des Ethylesters 196 als farbloses Glas erhalten.

DC: $R_{f}=0.56($ Heptan/EtOAc, $1: 1)$.

IR: $\tilde{v}=1118(\mathrm{w}), 1203(\mathrm{~m}), 1319(\mathrm{~m}), 1427(\mathrm{~m}), 1681(\mathrm{~s}), 1735(\mathrm{~s}) \mathrm{cm}^{-1}$.

${ }^{1}$ H-NMR $\left(400 \mathrm{MHz}, \mathrm{CDCl}_{3}\right): \delta=1.48\left(\mathrm{t}, J=7.2 \mathrm{~Hz}, 3 \mathrm{H}, \mathrm{CH}_{3}\right), 3.94\left(\mathrm{~s}, 3 \mathrm{H}, \mathrm{CH}_{3}\right), 3.96(\mathrm{~s}$, $\left.3 \mathrm{H}, \mathrm{CH}_{3}\right), 4.55$ (q, J=7.0 Hz, 2H, $\left.\mathrm{CH}_{2}\right), 7.66$ (s, 1H, Pyridyl-CH), 11.15 (s, 1H, OH).

${ }^{13}$ C-NMR $\left(100 \mathrm{MHz}, \mathrm{CDCl}_{3}\right): \delta=14.1,53.1,53.2,63.4,126.9,130.8,133.7,139.9,159.5$, 165.0, 165.4, 168.6. 
HRMS (ESI): $m / z[\mathrm{M}+\mathrm{H}]^{+}$für $\mathrm{C}_{12} \mathrm{H}_{14} \mathrm{NO}_{7}$ ber.: 284.0764, gef.: 284.0765 .

\section{5,6-Bis(methoxycarbonyl)-3-hydroxypicolinsäureallylester (197)}<smiles>C=CCOC(=O)c1nc(C(=O)OC)c(C(=O)OC)cc1O</smiles>

Der Triester 97 ${ }^{[37]}(14 \mathrm{mg}, 52.0 \mu \mathrm{mol})$ wurde nach AV3 mit Allylalkohol umgesetzt. Es wurden $15 \mathrm{mg}(50.9 \mu \mathrm{mol}, 78 \%)$ des Allylesters 197 als farbloser Feststoff erhalten.

DC: $R_{f}=0.40($ Heptan/EtOAc, $2: 1)$.

Alle relevanten analytischen Daten stimmen mit den veröffentlichten Werten überein. ${ }^{\text {[37] }}$

\section{5,6-Bis(methoxycarbonyl)-3-hydroxypicolinsäure-2-propylester (198)}<smiles>COC(=O)c1cc(O)c(C(=O)OC(C)C)nc1C(=O)OC</smiles>

Der Triester 97 ${ }^{[37]}(14 \mathrm{mg}, 52.0 \mu \mathrm{mol})$ wurde nach AV3 mit 2-Propanol umgesetzt. Es wurden $14.7 \mathrm{mg}(49.4 \mu \mathrm{mol}, 95 \%)$ des 2-Propylesters 198 als farbloser Feststoff erhalten.

Alle relevanten analytischen Daten stimmen mit den veröffentlichten Werten überein. ${ }^{[37]}$

\section{5,6-Bis(methoxycarbonyl)-3-hydroxypicolinsäure-2'-hydroxyethylester (199)}<smiles>COC(=O)c1cc(O)c(C(=O)OCCO)nc1C(=O)OC</smiles>

Der Triester 97 ${ }^{[37]}(14 \mathrm{mg}, 52.0 \mu \mathrm{mol})$ wurde nach AV3 mit Glycol umgesetzt. Es wurden $13 \mathrm{mg}(43.7 \mu \mathrm{mol}, 84 \%)$ des Glycolmonoesters 199 als farbloses Glas erhalten.

Alle relevanten analytischen Daten stimmen mit den veröffentlichten Werten überein. ${ }^{[37]}$ 


\section{5,6-Bis(methoxycarbonyl)-3-hydroxypicolinsäurebenzylester (200)}<smiles>COC(=O)c1cc(O)c(C(=O)OCc2ccccc2)nc1C(=O)OC</smiles>

Der Triester 97 ${ }^{[37]}(14 \mathrm{mg}, 52.0 \mu \mathrm{mol})$ wurde nach AV3 mit BnOH umgesetzt. Es wurden $14 \mathrm{mg}$ (40.4 $\mu \mathrm{mol}, 78 \%$ ) des Benzylesters 200 als farbloser Feststoff erhalten.

Schmelzpunkt: $109^{\circ} \mathrm{C}$.

DC: $R_{f}=0.60($ Heptan/EtOAc, $1: 1)$.

IR: $\tilde{v}=1118(\mathrm{w}), 1203(\mathrm{~m}), 1311(\mathrm{~m}), 1427(\mathrm{~m}), 1681(\mathrm{~s}), 1735(\mathrm{~s}) \mathrm{cm}^{-1}$.

${ }^{1}$ H-NMR (400 MHz, $\left.\mathrm{CDCl}_{3}\right): \delta=3.94\left(\mathrm{~s}, 3 \mathrm{H}, \mathrm{CH}_{3}\right), 3.95\left(\mathrm{~s}, 3 \mathrm{H}, \mathrm{CH}_{3}\right), 5.52\left(\mathrm{~s}, 2 \mathrm{H}, \mathrm{CH}_{2}\right)$, 7.35-7.41 (m, 3H, $3 \times \mathrm{CH}), 7.48-7.51(\mathrm{~m}, 2 \mathrm{H}, 2 \times \mathrm{CH}), 7.67(\mathrm{~s}, 1 \mathrm{H}$, Pyridyl-CH), $11.01(\mathrm{~s}$, $1 \mathrm{H}, \mathrm{OH})$.

${ }^{13} \mathbf{C}-\mathrm{NMR}\left(100 \mathrm{MHz}, \mathrm{CDCl}_{3}\right): \delta=53.1,53.2,68.4,127.0,128.7,128.8,130.8,133.5,134.5$, $140.3,159.4,165.15,165.24,168.4$.

HRMS (ESI): $m / z[\mathrm{M}+\mathrm{H}]^{+}$für $\mathrm{C}_{17} \mathrm{H}_{16} \mathrm{NO}_{7}$ ber.: 346.0927 , gef.: 346.0925 .

\section{5,6-Bis(trideuteromethoxycarbonyl)-3-hydroxypicolinsäurebenzylester (201)}<smiles>O=C([O-])OC(=O)c1nc(C(=O)OCc2ccccc2)c(O)cc1C(=O)OCc1ccccc1</smiles>

Der Triester $97-\boldsymbol{d}_{\boldsymbol{6}}{ }^{[241]}(15 \mathrm{mg}, 54.5 \mu \mathrm{mol})$ wurde nach AV3 mit BnOH umgesetzt. Es wurden $16 \mathrm{mg}$ (45.8 $\mu \mathrm{mol}, 84 \%)$ des Benzylesters 201 als farbloser Feststoff erhalten.

Schmelzpunkt: $109.5^{\circ} \mathrm{C}$.

DC: $R_{f}=0.60($ Heptan/EtOAc, $1: 1)$; 
IR: $\tilde{v}=694(\mathrm{~s}), 748(\mathrm{~s}), 1118(\mathrm{~s}), 1419(\mathrm{~m}), 1566(\mathrm{~m}), 1681(\mathrm{~s}), 1728(\mathrm{~s}) \mathrm{cm}^{-1}$.

${ }^{1} \mathbf{H}-\mathbf{N M R}\left(250 \mathrm{MHz}, \mathrm{CDCl}_{3}\right): \delta=5.52\left(\mathrm{~s}, 2 \mathrm{H}, \mathrm{CH}_{2}\right), 7.32-7.51(\mathrm{~m}, 5 \mathrm{H}, 5 \times \mathrm{CH}), 7.67(\mathrm{~s}, 1 \mathrm{H}$, Pyridyl-CH), $11.00(\mathrm{~s}, 1 \mathrm{H}, \mathrm{OH})$.

${ }^{13}$ C-NMR $\left(100 \mathrm{MHz}, \mathrm{CDCl}_{3}\right): \delta=52.0-52.9\left(\mathrm{~m}, 2 \times \mathrm{CD}_{3}\right), 68.4,127.0,128.7,128.8,130.8$, $133.5,134.5,140.3,159.4,165.2,165.3,168.4$.

HRMS (ESI): $m / z[\mathrm{M}+\mathrm{Na}]^{+}$für $\mathrm{C}_{17} \mathrm{H}_{9} \mathrm{D}_{6} \mathrm{NNaO}_{7}$ ber.: 374.1123 , gef.: 374.1120 .

\section{5,6-Bis(methoxycarbonyl)-3-methoxy-picolinsäuremethylester (98) $)^{[241]}$}<smiles>COC(=O)c1cc(OC)c(C(=O)OC)nc1C(=O)OC</smiles>

$\mathrm{Zu}$ einer Lösung des Phenols $97^{[37]}(50 \mathrm{mg}, 0.19 \mathrm{mmol})$ in wasserfreiem DMF $(0.5 \mathrm{~mL})$, wurden $\mathrm{K}_{2} \mathrm{CO}_{3}$ (39 mg, $0.28 \mathrm{mmol}$ ) und Methyliodid (17 $\left.\mu \mathrm{L}, 0.28 \mathrm{mmol}\right)$ zugegeben. Die Mischung wurde $19 \mathrm{~h}$ lang bei RT gerührt, dann mit $\mathrm{CH}_{2} \mathrm{Cl}_{2}(10 \mathrm{~mL})$ verdünnt, filtriert und am Rotationsverdampfer eingeengt. Reinigung des Rückstands durch Säulenchromatographie $\left(\mathrm{SiO}_{2}, 15 \mathrm{~g}\right.$, Petrolether/EtOAc, 2:1) lieferte $52 \mathrm{mg}$ (0.18 mmol, 99\%) des Methylethers 98 als farbloses Glas.

DC: $R_{f}=0.33($ EtOAc/Heptan, 2:1).

IR: $\tilde{v}=1118(\mathrm{~m}), 1226(\mathrm{~m}), 1327(\mathrm{~m}), 1442(\mathrm{w}), 1782(\mathrm{~s}) \mathrm{cm}^{-1}$.

${ }^{1} \mathbf{H}-\mathbf{N M R}\left(250 \mathrm{MHz}, \mathrm{CDCl}_{3}\right): \delta=3.91-3.93\left(\mathrm{~m}, 9 \mathrm{H}, 3 \times \mathrm{CH}_{3}\right), 3.96\left(\mathrm{~s}, 3 \mathrm{H}, \mathrm{CH}_{3}\right), 7.61(\mathrm{~s}, 1 \mathrm{H}$, $\mathrm{CH})$.

${ }^{13}$ C-NMR (63 MHz, $\left.\mathrm{CDCl}_{3}\right): \delta=52.9,53.0,53.2,56.5,119.8,131.3,139.9,140.7,163.9$, $165.1,165.6$.

HRMS (ESI): $m / z[\mathrm{M}+\mathrm{H}]^{+}$für $\mathrm{C}_{12} \mathrm{H}_{13} \mathrm{NO}_{7} \mathrm{Na}$ ber.: 306.0590 , gef.: 306.0587 . 
6-(Ethoxycarbonyl)-5-(methoxycarbonyl)-3-methoxypicolinsäureethylester (203)<smiles>CCOC(=O)c1nc(C(=O)OCC)c(C(=O)OC)cc1OC</smiles>

Eine Lösung des 3-Methoxypyridins $98(27 \mathrm{mg}, 95.3 \mu \mathrm{mol})$ in EtOH $(2.5 \mathrm{~mL})$ und $\mathrm{H}_{2} \mathrm{O}$ $(13 \mu \mathrm{L})$ wurde mit $\mathrm{Sc}(\mathrm{OTf})_{3}(9.4 \mathrm{mg}, 19.1 \mu \mathrm{mol})$ versetzt und $20 \mathrm{~h}$ lang bei $60{ }^{\circ} \mathrm{C}$ gerührt. Nach Abkühlen auf RT wurde mit EtOAc $(25 \mathrm{~mL})$ verdünnt und die organische Phase mit $\mathrm{HCl}(0.1 \mathrm{M}), \mathrm{H}_{2} \mathrm{O}$ und ges. NaCl-Lsg. (je $15 \mathrm{ml}$ ) gewaschen. Die Lösung wurde mit $\mathrm{Na}_{2} \mathrm{SO}_{4}$ entwässert und im Vakuum eingeengt. Es wurden $29.7 \mathrm{mg}$ (95.3 $\mu \mathrm{mol}$, quant.) des Diethylesters 203 als farbloses Glas erhalten.

DC: $R_{f}=0.35($ Heptan/EtOAc, $2: 1)$.

IR: $\tilde{v}=1118(\mathrm{~m}), 1226(\mathrm{~m}), 1327(\mathrm{~m}), 1442(\mathrm{w}), 1782(\mathrm{~s}) \mathrm{cm}^{-1}$.

${ }^{1} \mathbf{H}-\mathbf{N M R}\left(300 \mathrm{MHz}, \mathrm{CDCl}_{3}\right): \delta=1.38\left(\mathrm{q}, J=7.0 \mathrm{~Hz}, 6 \mathrm{H}, 2 \times \mathrm{CH}_{3}\right), 3.93\left(\mathrm{~s}, 3 \mathrm{H}, \mathrm{CH}_{3}\right), 3.97$ (s, 3H, $\left.\mathrm{CH}_{3}\right), 4.39-4.45\left(\mathrm{~m}, 4 \mathrm{H}, 2 \times \mathrm{CH}_{2}\right), 7.63(\mathrm{~s}, 1 \mathrm{H}, \mathrm{CH})$.

${ }^{13}$ C-NMR $\left(100 \mathrm{MHz}, \mathrm{CDCl}_{3}\right): \delta=14.0,14.1,53.1,56.5,62.2,62.3,119.8,130.4,140.9$, $141.6,155.1,163.9,165.0,165.6$.

HRMS (ESI): $m / z[\mathrm{M}+\mathrm{H}]^{+}$für $\mathrm{C}_{14} \mathrm{H}_{18} \mathrm{NO}_{7}$ ber.: 312.1078 , gef.: 312.1081 .

\section{6-Brom-3-hydroxypicolinsäure- $n$-propylamid (205)}<smiles>CCCNC(=O)c1nc(Br)ccc1O</smiles>

Eine Lösung von 6-Brom-3-hydroxypicolinsäuremethylester ${ }^{[267]}(\mathbf{2 0 4}, 100 \mathrm{mg}, 0.43 \mathrm{mmol})$ in 1,4-Dioxan $(6 \mathrm{~mL})$ wurde mit $n$-Propylamin $(73 \mu \mathrm{L}, 0.86 \mathrm{mmol})$ und $18 \mathrm{~h}$ lang bei RT gerührt. Das Gemisch wurde im Vakuum eingeengt, der Rückstand in pH 3 Puffer (15 mL) aufgenommen und mit EtOAc $(3 \times 10 \mathrm{~mL})$ extrahiert. Die vereinigten organischen Extrakte wurden mit ges. NaCl-Lsg. $(10 \mathrm{~mL})$ gewaschen und mit $\mathrm{Na}_{2} \mathrm{SO}_{4}$ getrocknet. Die Lösungsmittel wurden im Vakuum entfernt und der Rückstand durch Säulenchromatographie 
$\left(\mathrm{SiO}_{2}, 40 \mathrm{~g}\right.$, Petrolether/EtOAc, 4:1) gereinigt. Es wurden $76 \mathrm{mg}(0.29 \mathrm{mmol}, 68 \%)$ des Amids 205 als farbloser Feststoff erhalten.

Schmelzpunkt: $77^{\circ} \mathrm{C}$.

DC: $R_{f}=0.86($ Heptan/EtOAc, $2: 1)$.

IR: $\tilde{v}=623$ (s), 817 (s), 1210 (s), 1433 (s), 1528 (m), 1577 (m), 1642 (M9, 2873 (w), 2932 (w), $2967(\mathrm{~m}), 3291(\mathrm{~m}) \mathrm{cm}^{-1}$.

${ }^{1} \mathbf{H}-\mathbf{N M R}\left(300 \mathrm{MHz}, \mathrm{CDCl}_{3}\right): \delta=0.99$ (t, $J=7.4 \mathrm{~Hz}, 3 \mathrm{H}, \mathrm{CH}_{3}$ ), 1.67 (sxt, $J=7.3 \mathrm{~Hz}, 2 \mathrm{H}$, $\left.\mathrm{CH}_{2}\right), 3.40\left(\mathrm{~m}, 2 \mathrm{H}, \mathrm{CH}_{2}\right), 7.20(\mathrm{~d}, J=8.8 \mathrm{~Hz}, 1 \mathrm{H}, \mathrm{CH}), 7.45(\mathrm{~d}, J=8.7 \mathrm{~Hz}, 1 \mathrm{H}, \mathrm{CH}), 7.79$ (bs, $1 \mathrm{H}, \mathrm{NH}), 12.30(\mathrm{~s}, 1 \mathrm{H}, \mathrm{OH})$.

${ }^{13}$ C-NMR (75 MHz, $\left.\mathrm{CDCl}_{3}\right): \delta=11.4,22.7,40.8,128.7,129.4,131.8,132.9,157.4,167.6$.

HRMS (ESI): $m / z[\mathrm{M}+\mathrm{H}]^{+}$für $\mathrm{C}_{9} \mathrm{H}_{12} \mathrm{BrN}_{2} \mathrm{O}_{2}$ ber.: 259.0077, gef.: 259.0076 .

\section{1-Tosyl-1H-imidazol-4-carbaldehyd (207)}

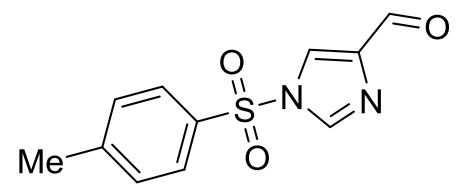

$\mathrm{Zu}$ einer Lösung von $1 H$-Imidazol-4-carbaldehyd (206, $3 \mathrm{~g}, 31 \mathrm{mmol})$ in wasserfreiem 1,4-Dioxan $(93 \mathrm{~mL})$ und wasserfreiem THF $(37 \mathrm{~mL})$ wurden nacheinander Tosylchlorid (7.14 g, $37.5 \mathrm{mmol})$ und $\mathrm{Et}_{3} \mathrm{~N}(5.2 \mathrm{~mL}, 37.5 \mathrm{mmol}) \mathrm{zu}$ gegeben und das Gemisch $18 \mathrm{~h}$ lang bei RT gerührt. Die Reaktionsmischung wurde mit pH 7 Puffer $(100 \mathrm{ml})$ versetzt und mit $\mathrm{CH}_{2} \mathrm{Cl}_{2}(3 \times 100 \mathrm{~mL})$ extrahiert. Die vereinigten organischen Extrakte wurden mit ges. NaClLsg. (50 mL) gewaschen und mit $\mathrm{Na}_{2} \mathrm{SO}_{4}$ entwässert. Die Lösungsmittel wurden im Vakuum entfernt und der Rückstand durch Säulenchromatographie gereinigt $\left(\mathrm{SiO}_{2}, 400 \mathrm{~g}\right.$, Petrolether/EtOAc, 2:3). Es wurden $7.45 \mathrm{~g}$ (29.8 mmol, 96\%) des Sulfonamids 207 als farbloser Feststoff erhalten.

Schmelzpunkt: $96{ }^{\circ} \mathrm{C}$.

DC: $R_{f}=0.41($ Heptan/EtOAc, $1: 1)$. 
IR: $\tilde{v}=1697(\mathrm{~s}), 1535(\mathrm{w}), 1381(\mathrm{~m}), 1165(\mathrm{~s}), 1072(\mathrm{~s}), 764(\mathrm{~s}), 679(\mathrm{~s}) \mathrm{cm}^{-1}$.

${ }^{1} \mathbf{H}-\mathbf{N M R}\left(250 \mathrm{MHz}, \mathrm{CDCl}_{3}\right): \delta=2.45\left(\mathrm{~s}, 1 \mathrm{H}, \mathrm{CH}_{3}\right), 7.39(\mathrm{~d}, J=8.0 \mathrm{~Hz}, 2 \mathrm{H}, 2 \times \mathrm{CH}), 7.87$ $(\mathrm{d}, J=8.3 \mathrm{~Hz}, 2 \mathrm{H}, 2 \times \mathrm{CH}), 7.91(\mathrm{~s}, 1 \mathrm{H}, \mathrm{CH}), 8.04(\mathrm{~s}, 1 \mathrm{H}, \mathrm{CH}), 9.87(\mathrm{~s}, 1 \mathrm{H}, \mathrm{O}=\mathrm{CH})$.

${ }^{13}$ C-NMR (63 MHz, $\left.\mathrm{CDCl}_{3}\right): \delta=21.8,121.7,127.7,130.8,133.8,137.2,142.9,147.4,185.6$.

HRMS (ESI): $m / z[\mathrm{M}+\mathrm{H}]^{+}$für $\mathrm{C}_{11} \mathrm{H}_{11} \mathrm{~N}_{2} \mathrm{O}_{3} \mathrm{~S}$ ber.: 251.0485, gef: 251.0487 .

\section{1-(1-Tosyl-1H-imidazol-4-yl)prop-2-yn-1-ol (208)}

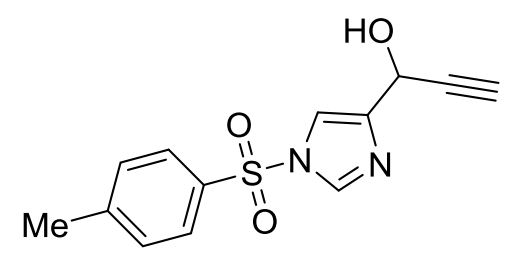

Ethinylmagnesiumbromid $(0.5 \mathrm{~N}$ in THF, $6 \mathrm{~mL}, 13 \mathrm{mmol})$ wurde bei $-20{ }^{\circ} \mathrm{C}$ tropfenweise zu einer Lösung des Aldehyds 207 ( $1 \mathrm{~g}, 4 \mathrm{mmol})$ in wasserfreiem THF (10 mL) gegeben. Nach vollständiger Zugabe wurde das Gemisch auf RT erwärmt und weitere $18 \mathrm{~h}$ lang gerührt. Das Gemisch wurde mit ges. $\mathrm{NH}_{4} \mathrm{Cl}$-Lsg. $(25 \mathrm{~mL})$ und $\mathrm{Et}_{2} \mathrm{O}(75 \mathrm{~mL})$ verdünnt und die Phasen getrennt. Die org. Phase wurde mit $\mathrm{H}_{2} \mathrm{O}(25 \mathrm{ml})$ und ges. NaCl-Lsg $(25 \mathrm{~mL})$ gewaschen, anschließend mit $\mathrm{Na}_{2} \mathrm{SO}_{4}$ entwässert und im Vakuum eingeengt. Reinigung des Rückstands durch Säulenchromatographie $\left(\mathrm{SiO}_{2}, 200 \mathrm{~g}\right.$, Petroleter/ EtOAc, 1:1) ergab $0.93 \mathrm{~g}$ (3.4 mmol, 84\%) des Propargylalkohols 208 als farblosen Feststoff.

Schmelzpunkt: $113.5^{\circ} \mathrm{C}$.

DC: $R_{f}=0.19($ Heptan/EtOAc, $1: 1)$.

IR: $\tilde{v}=3113(\mathrm{w}), 1377(\mathrm{~m}), 1173(\mathrm{~m}), 1076(\mathrm{~s}), 1003(\mathrm{w}), 818(\mathrm{w}) \mathrm{cm}^{-1}$.

${ }^{1}$ H-NMR $\left(250 \mathrm{MHz}, \mathrm{CDCl}_{3}\right): \delta=2.42\left(\mathrm{~s}, 3 \mathrm{H}, \mathrm{CH}_{3}\right), 2.55(\mathrm{~d}, J=1.9 \mathrm{~Hz}, 1 \mathrm{H},(\mathrm{HO}) \mathrm{CH}), 5.02$ (bs, 1H, OH), $5.42(\mathrm{~s}, 1 \mathrm{H}, \mathrm{CH}) 7.34$ (d, J=8.3 Hz, 2H, 2x CH), 7.37 (s, 1H, CH), 7.81 (d, $J=8.3 \mathrm{~Hz}, 2 \mathrm{H}, 2 \times \mathrm{CH}), 7.98(\mathrm{~s}, 1 \mathrm{H}, \mathrm{CH})$.

${ }^{13}$ C-NMR $\left(63 \mathrm{MHz}, \mathrm{CDCl}_{3}\right): \delta=21.6,57.7,73.6,76.5,81.9,114.6,127.5,130.5,134.4$, $136.9,144.1,146.6$. 
HRMS (ESI): $m / z[\mathrm{M}+\mathrm{H}]^{+}$für $\mathrm{C}_{13} \mathrm{H}_{13} \mathrm{~N}_{2} \mathrm{O}_{3} \mathrm{~S}$ ber.: 277.0641, gef.: 277.0642.

\section{1-(1-Tosyl-1H-imidazol-4-yl)prop-2-yn-1-on (209)}

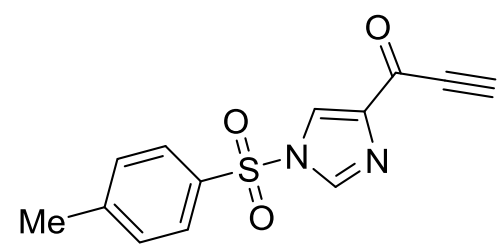

$\mathrm{Zu}$ einer Mischung aus Propargylalkohol 208 (500 mg, $1.8 \mathrm{mmol})$ und $\mathrm{NaHCO}_{3}(760 \mathrm{mg}$, $9.1 \mathrm{mmol})$ in wasserfreiem $\mathrm{CH}_{2} \mathrm{Cl}_{2}(15 \mathrm{~mL})$ wurde portionsweise Dess-Martin-Periodinan ${ }^{[312]}$ (844 mg, 2 mmol) gegeben. Nach vollständiger Zugabe wurde das Gemisch $1.5 \mathrm{~h}$ lang bei RT gerührt, mit $\mathrm{CH}_{2} \mathrm{Cl}_{2}(50 \mathrm{~mL})$ verdünnt und mit einer 1:1 Mischung aus ges. $\mathrm{Na}_{2} \mathrm{~S}_{2} \mathrm{O}_{3}$-Lsg. und ges. $\mathrm{NaHCO}_{3}$-Lsg. $(3 \times 50 \mathrm{~mL})$ gewaschen. Die organische Phase wurde mit $\mathrm{Na}_{2} \mathrm{SO}_{4}$ entwässert und im Vakuum eingeengt. Reinigung des Rohprodukt durch Säulenchromatographie $\left(\mathrm{SiO}_{2}, 100 \mathrm{~g}\right.$, Petrolether/EtOAc, $2: 1 \rightarrow$ 1:1) lieferte $450 \mathrm{mg}$ (1.64 mmol, 91\%) des Alkinylketons 209 als leicht gelben Feststoff, der sich an Licht und Luft rasch zersetzte.

Schmelzpunkt: $150-153{ }^{\circ} \mathrm{C}$ (Zers.).

DC: $R_{f}=0.43($ Heptan/EtOAc, $1: 1)$.

IR: $\tilde{v}=3190(\mathrm{w}), 2095(\mathrm{~m}), 1643(\mathrm{~m}), 1373(\mathrm{~m}), 1258(\mathrm{~m}), 1107(\mathrm{~s}), 926(\mathrm{~m}), 675(\mathrm{~s}) \mathrm{cm}^{-1}$.

${ }^{1} \mathbf{H}-\mathbf{N M R}\left(250 \mathrm{MHz}, \mathrm{CDCl}_{3}\right): \delta=2.45\left(\mathrm{~s}, 3 \mathrm{H}, \mathrm{CH}_{3}\right), 3.40(\mathrm{~s}, 1 \mathrm{H}, \mathrm{CH}), 7.40(\mathrm{~d}, J=8.3 \mathrm{~Hz}$, $2 \mathrm{H}, 2 \times \mathrm{CH}), 7.87(\mathrm{~d}, J=8.5 \mathrm{~Hz}, 2 \mathrm{H}, 2 \times \mathrm{CH}), 8.01(\mathrm{~s}, 1 \mathrm{H}, \mathrm{CH}), 8.02(\mathrm{~s}, 1 \mathrm{H}, \mathrm{CH})$.

${ }^{13}$ C-NMR (63 MHz, $\left.\mathrm{CDCl}_{3}\right): \delta=21.8,80.2,80.4,123.7,127.7,130.8,133.7,137.2,142.8$, $147.5,169.8$.

HRMS (ESI): $m / z[\mathrm{M}+\mathrm{H}]^{+}$für $\mathrm{C}_{13} \mathrm{H}_{11} \mathrm{~N}_{2} \mathrm{O}_{3} \mathrm{~S}$ ber.: 275.0485, gef.: 275.0483 . 
3-Hydroxy-6-(1H-imidazol-4/5-carbonyl)picolinsäuremethylester (210)<smiles>COC(=O)c1nc(C(=O)c2cnc[nH]2)ccc1O</smiles>

Das Alkinylketon 209 (2.5 g, $9.1 \mathrm{mmol})$ wurde zusammen mit dem 1-Azabutadien $\mathbf{6 5}^{[37]}$ $(12.3 \mathrm{~g}, 31.9 \mathrm{mmol})$ in wasserfreiem Mesitylen $(15 \mathrm{~mL}) 15 \mathrm{~h}$ lang in einem auf $115^{\circ} \mathrm{C}$ erhitzten Ölbad gerührt. Nach Abkühlen auf RT wurde die Reaktionsmischung mit EtOAc $(50 \mathrm{~mL})$ verdünnt, der ausgefallene Feststoff abfiltriert und mit $\mathrm{Et}_{2} \mathrm{O}(2 \times 25 \mathrm{~mL})$ gewaschen. Nach Trocknen im Vakuum wurden $1.1 \mathrm{~g}$ (4.6 mmol, 50\%) des 3-Hydroxypyridins 210 als leicht brauner Feststoff erhalten.

Schmelzpunkt: $230^{\circ} \mathrm{C}$ (Zers.).

DC: $R_{f}=0.1\left(\mathrm{CHCl}_{3} / \mathrm{MeOH} / \mathrm{HCOOH}, 90: 10: 1\right)$.

IR: $\tilde{v}=3147(\mathrm{w}), 633(\mathrm{~s}), 2361(\mathrm{w}), 1682(\mathrm{~s}), 1450(\mathrm{w}), 1304(\mathrm{~s}), 856(\mathrm{w}), 725(\mathrm{~m}) \mathrm{cm}^{-1}$.

${ }^{1}$ H-NMR (400 MHz, DMSO- $\left.d_{6}, 343 \mathrm{~K}\right): \delta=3.99\left(\mathrm{~s}, 3 \mathrm{H}, \mathrm{CH}_{3}\right), 7.59$ (d, $J=8.68 \mathrm{~Hz}, 1 \mathrm{H}$, $\mathrm{CH}), 7.90(\mathrm{~s}, 1 \mathrm{H}, \mathrm{CH}), 8.13(\mathrm{~s}, 1 \mathrm{H}, \mathrm{NH}), 8.18$ (d, J=8.7 Hz, 1H, CH), 8.51 (s, 1H, CH), 11.85 (bs, 1H, OH).

${ }^{13}$ C-NMR (100.6 MHz, DMSO- $\left.d_{6}, 343 \mathrm{~K}\right): \delta=52.2,125.9,127.7,132.0,135.4,138.6,144.7$, $157.4,162.0,166.3,179.2$.

HRMS (ESI): $m / z[\mathrm{M}+\mathrm{H}]^{+}$für $\mathrm{C}_{11} \mathrm{H}_{10} \mathrm{~N}_{3} \mathrm{O}_{4}$ ber.: 248.0666, gef.: 248.0666. 
<smiles>CCCNC(=O)c1nc(C(=O)c2c[nH]cn2)ccc1O</smiles>

Der Methylester 210 (50 mg, $20.2 \mu \mathrm{mol})$ wurde analog zum Vorgehen für das Amid 212 umgesetzt. Säulenchromatographie $\left(\mathrm{SiO}_{2}, 10 \mathrm{~g}, \mathrm{CHCl}_{3} / \mathrm{MeOH} / \mathrm{HCOOH}, 90: 10: 1\right)$ ergab $40 \mathrm{mg}$ (14.6 $\mu \mathrm{mol}, 72 \%)$ des Amids 211 als rot-braunes Harz.

DC: $R_{f}=0.18\left(\mathrm{CHCl}_{3} / \mathrm{MeOH} / \mathrm{HCOOH}, 90: 10: 1\right)$.

IR: $\tilde{v}=648(\mathrm{~s}), 848(\mathrm{~m}), 1111(\mathrm{~m}), 1203(\mathrm{~m}), 1350$ (s), $1543(\mathrm{~s}), 1620(\mathrm{~s}), 2960(\mathrm{w})$, $2932(\mathrm{w}), 2962(\mathrm{w}) \mathrm{cm}^{-1}$.

${ }^{1} \mathbf{H}-\mathrm{NMR}\left(300 \mathrm{MHz}, \mathrm{AcOH}-d_{4}\right): \delta=1.38\left(\mathrm{t}, J=6.9 \mathrm{~Hz}, 3 \mathrm{H}, \mathrm{CH}_{2} \mathrm{CH}_{3}\right), 2.08$ (bs, $2 \mathrm{H}$, $\left.\mathrm{CH}_{2} \mathrm{CH}_{3}\right), 3.86$ (bs, 2H, $\left.\mathrm{CH}_{2} \mathrm{CH}_{2}\right), 7.94(\mathrm{~d}, J=7.8 \mathrm{~Hz}, 1 \mathrm{H}, \mathrm{CH}), 8.66(\mathrm{~d}, J=7.5 \mathrm{~Hz}, 1 \mathrm{H}, \mathrm{CH})$, 8.94 (bs, 1H, CH), 9.20 (bs, 1H, CH).

${ }^{13}$ C-NMR $\left(75 \mathrm{MHz}, \mathrm{AcOH}-d_{4}\right): \delta=11.6,23.4,41.9,128.1,130.4,131.3,132.1,138.8,144.0$, $162.3,169.2,180.4,181.24$.

HRMS (ESI): $m / z[\mathrm{M}+\mathrm{H}]^{+}$ber. für $\mathrm{C}_{13} \mathrm{H}_{15} \mathrm{~N}_{4} \mathrm{O}_{3}: 275.1144$, gef.: 275.1154 .

\section{5-Acetyl-6-(4-(methoxycarbonyl)-thiazol-2-yl)-3-hydroxypicolinsäure-n-propylamid} (212)<smiles>CCCNC(=O)c1nc(-c2nc(C(=O)OC)cs2)c(C(C)=O)cc1O</smiles>

Eine Lösung des Methylesters 66 $^{[37]}(500 \mathrm{mg}, 2.38 \mathrm{mmol})$ in wasserfreiem 1,4-Dioxan $(30 \mathrm{~mL})$ wurde mit $n$-Propylamin $(390 \mu \mathrm{L}, 4.76 \mathrm{mmol})$ versetzt und $5.5 \mathrm{~h}$ lang bei $60{ }^{\circ} \mathrm{C}$ gerührt. Nach Zugabe von pH 3 Puffer $(75 \mathrm{~mL})$ wurde mit EtOAc $(3 \times 50 \mathrm{~mL})$ extrahiert, die vereinigten organischen Extrakte mit ges. $\mathrm{NaCl}$-Lsg. gewaschen und mit $\mathrm{Na}_{2} \mathrm{SO}_{4}$ entwässert. Die Lösungsmittel wurden im Vakuum entfernt und der Rückstand durch 
Säulenchromatographie $\left(\mathrm{SiO}_{2}, 40 \mathrm{~g}, \mathrm{CH}_{2} \mathrm{Cl}_{2} /\right.$ Petrolether/Aceton, 4:4:1) gereinigt. Es wurden $631 \mathrm{mg}$ (1.74 mmol, 73\%) des Amids 212 als farbloser Feststoff erhalten.

Schmelzpunkt: $161-163{ }^{\circ} \mathrm{C}$.

DC: $R_{f}=0.37\left(\mathrm{CH}_{2} \mathrm{Cl}_{2} /\right.$ Heptan/Aceton, $\left.4: 4: 1\right)$;

IR: $\tilde{v}=632(\mathrm{~m}), 756(\mathrm{~m}), 1234$ (s), 1643 (m), 1728 (s), $2877(\mathrm{w}), 2939$ (w), 2960 (w), $3110(\mathrm{w}), 3363(\mathrm{w}) \mathrm{cm}^{-1}$.

${ }^{1}$ H-NMR (300 MHz, $\mathrm{CDCl}_{3}$ ): $\delta=1.04$ (t, $J=7.4 \mathrm{~Hz}, 3 \mathrm{H}, \mathrm{CH}_{2} \mathrm{CH}_{3}$ ), 1.71 (sext, $J=7.3 \mathrm{~Hz}$, $\left.2 \mathrm{H}, \mathrm{CH}_{2} \mathrm{CH}_{3}\right), 2.63\left(\mathrm{~s}, 3 \mathrm{H}, \mathrm{C}(\mathrm{O}) \mathrm{CH}_{3}\right), 3.47$ (q, $\left.\mathrm{J}=6.7 \mathrm{~Hz}, 2 \mathrm{H}, \mathrm{CH}_{2} \mathrm{CH}_{2}\right), 3.93\left(\mathrm{~s}, 3 \mathrm{H}, \mathrm{CH}_{3}\right)$, 7.23 (s, 1H, CH), 7.85 (bs, 1H, NH), 8.20 (s, 1H, S-CH), 12.64 (s, 1H, OH).

${ }^{13}$ C-NMR (75 MHz, $\left.\mathrm{CDCl}_{3}\right): \delta=11.3,22.7,30.9,40.9,52.4,124.4,28.7,131.0,136.1,141.6$, $147.7,158.6,161.5,166.3,167.3,201.2$.

HRMS (ESI): $m / z$ [M $+\mathrm{Na}]^{+}$für $\mathrm{C}_{16} \mathrm{H}_{17} \mathrm{~N}_{3} \mathrm{O}_{5} \mathrm{SNa}$ ber.: 386.0781 , gef.: 386.0785 .

\subsection{Synthese von 3-Hydroxypicolinsäurederivaten}

\section{3-Hydroxy-6-phenylpicolinsäuremethylester (216a)}<smiles>COC(=O)c1nc(-c2ccccc2)ccc1O</smiles>

Phenylboronsäurepinakolester (55 mg, $0.28 \mathrm{mmol}$ ) wurde nach AV1 umgesetzt. Es wurden $40 \mathrm{mg}(17.5 \mu \mathrm{mol}, 81 \%)$ des Produkts 216a als farbloser Feststoff erhalten.

Schmelzpunkt: $107^{\circ} \mathrm{C}$.

DC: $R_{f}=0.70($ Heptan/EtOAc, $2: 1)$.

IR: $\tilde{v}=686(\mathrm{~s}), 1103(\mathrm{~m}), 1203(\mathrm{~s}), 1288(\mathrm{~m}), 1458(\mathrm{~s}), 1674(\mathrm{~m}), 2954(\mathrm{w}) \mathrm{cm}^{-1}$.

${ }^{1}$ H-NMR $\left(300 \mathrm{MHz}, \mathrm{CDCl}_{3}\right): \delta=4.07\left(\mathrm{~s}, 3 \mathrm{H}, \mathrm{CH}_{3}\right), 7.43(\mathrm{~m}, 4 \mathrm{H}, \mathrm{Ph}), 7.86(\mathrm{~d}, J=8.8 \mathrm{~Hz}$, 1H, CH), $7.95(\mathrm{~m}, 2 \mathrm{H}, 2 \times \mathrm{CH}), 10.71(\mathrm{~s}, 1 \mathrm{H}, \mathrm{OH})$. 
${ }^{13}$ C-NMR $\left(100 \mathrm{MHz}, \mathrm{CDCl}_{3}\right): \delta=53.1,126.6,126.9,128.7,128.8,129.4,138.3,149.5$, $157.8,170.2$.

HRMS (ESI): $m / z[\mathrm{M}+\mathrm{H}]^{+}$für $\mathrm{C}_{13} \mathrm{H}_{12} \mathrm{NO}_{3}$ ber.: 230.0812, gef: 230.0812 .

\section{3-Hydroxy-6-(4-methoxyphenyl)picolinsäuremethylester (216b)}<smiles>COC(=O)c1nc(-c2ccc(OC)cc2)ccc1O</smiles>

p-Anisylboronsäure (42 mg, $0.28 \mathrm{mmol}$ ) wurde nach AV1 umgesetzt. Es wurden $48.5 \mathrm{mg}$ (0.18 mmol, 87\%) des Produkts 216b als leicht gelber Feststoff erhalten.

Schmelzpunkt: $81^{\circ} \mathrm{C}$.

DC: $R_{f}=0.30($ Heptan/EtOAc, 4:1).

IR: $\tilde{v}=748(\mathrm{~s}), 852(\mathrm{~m}), 1026(\mathrm{~m}), 1172(\mathrm{~s}), 1450(\mathrm{~s}), 2954(\mathrm{w}) \mathrm{cm}^{-1}$.

${ }^{1}$ H-NMR $\left(300 \mathrm{MHz}, \mathrm{CDCl}_{3}\right): \delta=3.85\left(\mathrm{~s}, 3 \mathrm{H}, \mathrm{CH}_{3}\right), 4.06\left(\mathrm{~s}, 3 \mathrm{H}, \mathrm{CH}_{3}\right), 6.98(\mathrm{~d}, J=8.7 \mathrm{~Hz}$, $2 \mathrm{H}, 2 \times \mathrm{CH}), 7.41(\mathrm{~d}, J=8.8 \mathrm{~Hz}, 1 \mathrm{H}, \mathrm{CH}), 7.80(\mathrm{~d}, J=8.8 \mathrm{~Hz}, 1 \mathrm{H}, \mathrm{CH}), 7.89(\mathrm{~d}, J=8.7 \mathrm{~Hz}$, $2 \mathrm{H}, 2 \times \mathrm{CH}), 10.65(\mathrm{~s}, 1 \mathrm{H}, \mathrm{OH})$.

${ }^{13}$ C-NMR (75 MHz, $\left.\mathrm{CDCl}_{3}\right): \delta=53.0,55.3,114.2,126.3,127.0,127.9,129.1,131.0,149.3$, $157.4,160.3,170.2$.

HRMS (ESI): $m / z[\mathrm{M}+\mathrm{H}]^{+}$für $\mathrm{C}_{14} \mathrm{H}_{14} \mathrm{NO}_{4}$ ber.: 260.0917, gef.: 260.0918 .

\section{3-Hydroxy-6-(2-methoxyphenyl)picolinsäuremethylester (216c)}<smiles>COC(=O)c1nc(-c2ccccc2OC)ccc1O</smiles>

$o$-Anisylboronsäurepinakolester (57 mg, $0.28 \mathrm{mmol}$ ) wurde nach AV1 umgesetzt. Es wurden $29 \mathrm{mg}(0.17 \mathrm{mmol}, 52 \%)$ des Produkts 216c als leicht gelber Feststoff erhalten. 
Schmelzpunkt: $85^{\circ} \mathrm{C}$.

DC: $R_{f}=0.32($ Heptan/EtOAc, 4:1).

IR: $\tilde{v}=714(\mathrm{~m}), 733(\mathrm{~m}), 1026(\mathrm{w}), 1099(\mathrm{~m}), 1203(\mathrm{~s}), 1442(\mathrm{~s}), 1674(\mathrm{~m}), 2341(\mathrm{w})$, $2360(\mathrm{w}), 2954(\mathrm{w}) \mathrm{cm}^{-1}$.

${ }^{1} \mathbf{H}-\mathbf{N M R}\left(400 \mathrm{MHz}, \mathrm{CDCl}_{3}\right): \delta=3.83\left(\mathrm{~s}, 3 \mathrm{H}, \mathrm{CH}_{3}\right), 4.03\left(\mathrm{~s}, 3 \mathrm{H}, \mathrm{CH}_{3}\right), 6.96(\mathrm{~d}, J=8.2 \mathrm{~Hz}$, $1 \mathrm{H}, \mathrm{CH}), 7.07(\mathrm{dt}, J=7.5,0.9 \mathrm{~Hz}, 1 \mathrm{H}, \mathrm{CH}), 7.32-7.38(\mathrm{~m}, 2 \mathrm{H}, 2 \times \mathrm{CH}), 7.74(\mathrm{dd}, J=7.6$, $1.8 \mathrm{~Hz}, 1 \mathrm{H}, \mathrm{CH}), 7.95(\mathrm{~d}, J=8.8 \mathrm{~Hz}, 1 \mathrm{H}, \mathrm{CH}), 10.71(\mathrm{~s}, 1 \mathrm{H}, \mathrm{OH})$.

${ }^{13}$ C-NMR $\left(100 \mathrm{MHz} \mathrm{CDCl}_{3}\right): \delta=53.0,55.5,111.3,121.2,125.6,128.1,129.2,129.9,131.0$, $131.6,148.3,156.9,157.5,170.2$.

HRMS (ESI): $m / z[\mathrm{M}+\mathrm{H}]^{+}$für $\mathrm{C}_{14} \mathrm{H}_{14} \mathrm{NO}_{4}$ ber.: 260.0917, gef.: 260.0920 .

\section{6-(3-Acetylphenyl)-3-hydroxypicolinsäuremethylester (216d)}<smiles>COC(=O)c1nc(-c2cccc(C(C)=O)c2)ccc1O</smiles>

3-Acetylphenylboronsäure (46 mg, $0.28 \mathrm{mmol}$ ) wurde nach AV1 umgesetzt. Es wurden $31.5 \mathrm{mg}(0.12 \mathrm{mmol}, 54 \%)$ des Produkts 216d als farbloser Feststoff erhalten.

Schmelzpunkt: $160^{\circ} \mathrm{C}$.

DC: $R_{f}=0.20($ Heptan/EtOAc, $4: 1)$.

IR: $\tilde{v}=687(\mathrm{~m}), 802(\mathrm{w}), 1103(\mathrm{w}), 1203(\mathrm{~s}), 1298(\mathrm{~m}) 1450(\mathrm{~s}), 1674(\mathrm{~s}), 1735(\mathrm{w})$, $2982(\mathrm{w}) \mathrm{cm}^{-1}$.

${ }^{1} \mathbf{H}-\mathbf{N M R}\left(300 \mathrm{MHz}, \mathrm{CDCl}_{3}\right): \delta=2.68\left(\mathrm{~s}, 3 \mathrm{H}, \mathrm{CH}_{3}\right), 4.08\left(\mathrm{~s}, 3 \mathrm{H}, \mathrm{CH}_{3}\right), 7.49(\mathrm{~d}, J=8.8 \mathrm{~Hz}$, 1H, CH), 7.57 (t, $J=7.7 \mathrm{~Hz}, 1 \mathrm{H}, \mathrm{CH}), 7.93$ (d, $J=8.8 \mathrm{~Hz}, 1 \mathrm{H}, \mathrm{CH}), 7.98$ (d, $J=7.5 \mathrm{~Hz}, 1 \mathrm{H}$, $\mathrm{CH}), 8.18(\mathrm{~d}, J=7.7 \mathrm{~Hz}, 1 \mathrm{H}, \mathrm{CH}), 8.50(\mathrm{~s}, 1 \mathrm{H}, \mathrm{CH}), 10.76(\mathrm{~s}, 1 \mathrm{H}, \mathrm{OH})$.

${ }^{13}$ C-NMR (75 MHz, $\left.\mathrm{CDCl}_{3}\right): \delta=26.8,53.1,126.3,127.0,127.2,128.6,129.2,129.6,131.2$, $137.7,138.9,148.4,158.2,170.0,197.9$. 
HRMS (ESI): $m / z[\mathrm{M}+\mathrm{H}]^{+}$für $\mathrm{C}_{15} \mathrm{H}_{14} \mathrm{NO}_{4}$ ber.: 272.0917, gef.: 272.0918 .

\section{6-([1,1'-Biphenyl]-4-yl)-3-hydroxypicolinsäuremethylester (216e)}<smiles>COC(=O)c1nc(-c2ccc(-c3ccccc3)cc2)ccc1O</smiles>

4-Biphenylboronsäure (55 mg, $0.28 \mathrm{mmol}$ ) wurde nach AV1 umgesetzt. Es wurden $38.5 \mathrm{mg}$ (0.12 mmol, 59\%) des Produkts 216e als farbloser Feststoff erhalten.

Schmelzpunkt: $182^{\circ} \mathrm{C}$.

DC: $R_{f}=0.42($ Heptan/EtOAc, $4: 1)$.

IR: $\tilde{v}=740(\mathrm{~m}), 1103(\mathrm{w}), 1203(\mathrm{~s}), 1365(\mathrm{~m}), 1450(\mathrm{~m}), 1666(\mathrm{w}), 1743(\mathrm{~s}), 2237(\mathrm{w})$, $3016(\mathrm{w}) \mathrm{cm}^{-1}$.

${ }^{1} \mathbf{H}-\mathbf{N M R}\left(300 \mathrm{MHz}, \mathrm{CDCl}_{3}\right): \delta=4.09\left(\mathrm{~s}, 3 \mathrm{H}, \mathrm{CH}_{3}\right), 7.35-7.49(\mathrm{~m}, 4 \mathrm{H}, 4 \times \mathrm{CH}), 7.64-7.72$ $(\mathrm{m}, 4 \mathrm{H}, 4 \times \mathrm{CH}), 7.91(\mathrm{~d}, J=8.8 \mathrm{~Hz}, 1 \mathrm{H}, \mathrm{CH}), 8.03(\mathrm{~d}, J=8.3 \mathrm{~Hz}, 2 \mathrm{H}, 2 \times \mathrm{CH}), 10.74(\mathrm{~s}, 1 \mathrm{H}$, $\mathrm{OH})$.

${ }^{13}$ C-NMR $\left(75 \mathrm{MHz}, \mathrm{CDCl}_{3}\right): \delta=53.1,126.8,126.96,127.04,127.5,128.8,129.4,137.2$, $140.5,141.5,149.1,157.8,170.2$.

HRMS (ESI): $m / z[\mathrm{M}+\mathrm{H}]^{+}$für $\mathrm{C}_{19} \mathrm{H}_{16} \mathrm{NO}_{3}$ ber.: 306.1125 , gef.: 306.1129 .

6-(Benzo[b]thiophen-2-yl)-3-hydroxypicolinsäuremethylester (216f)<smiles>COC(=O)c1nc(-c2cc3ccccc3s2)ccc1O</smiles>

2-Benzothiophenylboronsäure (50 mg, $0.28 \mathrm{mmol}$ ) wurde nach AV1 umgesetzt. Es wurden $53 \mathrm{mg}(0.12 \mathrm{mmol}, 87 \%)$ des Produkts 216f als farbloser Feststoff erhalten.

Schmelzpunkt: $179.5^{\circ} \mathrm{C}$. 
DC: $R_{f}=0.67($ Heptan/EtOAc, $2: 1)$.

IR: $\tilde{v}=725(\mathrm{~s}), 817(\mathrm{~m}), 1099(\mathrm{~m}), 1211(\mathrm{~s}), 1288(\mathrm{w}), 1450(\mathrm{~m}), 1666(\mathrm{~m}) \mathrm{cm}^{-1}$.

${ }^{1}$ H-NMR $\left(300 \mathrm{MHz}, \mathrm{CDCl}_{3}\right): \delta=4.08\left(\mathrm{~s}, 3 \mathrm{H}, \mathrm{CH}_{3}\right), 7.31-7.38(\mathrm{~m}, 2 \mathrm{H}, 2 \times \mathrm{CH}), 7.40(\mathrm{~d}$, $J=8.8 \mathrm{~Hz}, 1 \mathrm{H}, \mathrm{CH}), 7.71(\mathrm{~s}, 1 \mathrm{H}, \mathrm{CH}), 7.76-7.79(\mathrm{~m}, 1 \mathrm{H}, \mathrm{CH}), 7.84(\mathrm{~d}, J=3.9 \mathrm{~Hz}, 1 \mathrm{H}, \mathrm{CH})$, $7.88(\mathrm{~d}, J=8.8 \mathrm{~Hz}, 1 \mathrm{H}, \mathrm{CH}), 10.80(\mathrm{~s}, 1 \mathrm{H}, \mathrm{OH})$.

${ }^{13}$ C-NMR (75 MHz, $\left.\mathrm{CDCl}_{3}\right): \delta=53.2,120.6,122.4,124.0,124.5,124.9,125.9,126.9,129.2$, $140.4,140.5,143.6,144.7,158.1,169.8$.

HRMS (ESI): $m / z[\mathrm{M}+\mathrm{H}]^{+}$für $\mathrm{C}_{15} \mathrm{H}_{12} \mathrm{NO}_{3} \mathrm{~S}$ ber.: 286.0532, gef.: 286.0534.

\section{6-(Benzofuran-2-yl)-3-hydroxypicolinsäuremethylester (216g)}

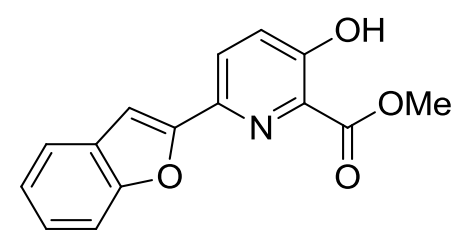

2-Benzofuranylboronsäure (45 mg, $0.28 \mathrm{mmol}$ ) wurde nach AV1 umgesetzt. Es wurden $47.5 \mathrm{mg}(0.18 \mathrm{mmol}, 82 \%)$ des Produkts $216 \mathrm{~g}$ als farbloser Feststoff erhalten.

Schmelzpunkt: $160^{\circ} \mathrm{C}$.

DC: $R_{f}=0.65($ Heptan/EtOAc, $2: 1)$.

IR: $\tilde{v}=740$ (s), 1095 (m), 1165 (m), 1296 (m), 1361 (w), 1446 (s), 1681 (m), 1739 (m), $2322(\mathrm{w}), 3174(\mathrm{w}) \mathrm{cm}^{-1}$.

${ }^{1} \mathbf{H}-\mathbf{N M R}\left(300 \mathrm{MHz}, \mathrm{CDCl}_{3}\right): \delta=4.10\left(\mathrm{~s}, 3 \mathrm{H}, \mathrm{CH}_{3}\right), 7.23-7.33(\mathrm{~m}, 2 \mathrm{H}, 2 \times \mathrm{CH}), 7.36(\mathrm{~s}, 1 \mathrm{H}$, $\mathrm{CH}), 7.48(\mathrm{~d}, J=8.8 \mathrm{~Hz}, 1 \mathrm{H}, \mathrm{CH}), 7.56(\mathrm{~d}, J=8.1 \mathrm{~Hz}, 1 \mathrm{H}, \mathrm{CH}), 7.63(\mathrm{~d}, J=7.5 \mathrm{~Hz}, 1 \mathrm{H}$, $\mathrm{CH}), 8.03(\mathrm{~d}, J=8.8 \mathrm{~Hz}, 1 \mathrm{H}, \mathrm{CH}), 10.82(\mathrm{~s}, 1 \mathrm{H}, \mathrm{OH})$.

${ }^{13}$ C-NMR (75 MHz, $\left.\mathrm{CDCl}_{3}\right): \delta=53.2,104.2,111.6,121.5,123.2,125.1,126.3,127.0,128.8$, $129.7,141.5,154.0,155.2,158.2,169.8$.

HRMS (ESI): $m / z[\mathrm{M}+\mathrm{H}]^{+}$für $\mathrm{C}_{15} \mathrm{H}_{12} \mathrm{NO}_{4}$ ber.: 270.0761, gef.: 270.0764 . 


\section{3-Hydroxy-6-(1H-indol-2-yl)picolinsäuremethylester (216h)}

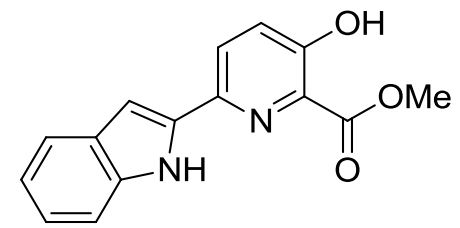

Indol-2-boronsäurepinakolester ${ }^{[313]}$ (68 mg, $\left.0.28 \mathrm{mmol}\right)$ wurde nach AV1 umgesetzt. Es wurden $27.5 \mathrm{mg}(0.10 \mathrm{mmol}, 48 \%)$ des Produkts 216h als leicht braunen Feststoff erhalten.

Schmelzpunkt: $142.5^{\circ} \mathrm{C}$.

DC: $R_{f}=0.31($ Heptan/EtOAc, $4: 1)$.

IR: $\tilde{v}=732(\mathrm{~m}), 794(\mathrm{~m}), 1095(\mathrm{~m}), 1211(\mathrm{~m}), 1288(\mathrm{~m}), 1365$ (s), 1435 (s), $1658(\mathrm{~s})$, $3402(\mathrm{w}) \mathrm{cm}^{-1}$.

${ }^{1} \mathbf{H}-\mathbf{N M R}\left(250 \mathrm{MHz}, \mathrm{CDCl}_{3}\right): \delta=4.07\left(\mathrm{~s}, 3 \mathrm{H}, \mathrm{CH}_{3}\right), 6.91(\mathrm{~d}, J=1.1 \mathrm{~Hz}, 1 \mathrm{H}, \mathrm{CH}), 7.09-7.26$ $(\mathrm{m}, 2 \mathrm{H}, 2 \times \mathrm{CH}), 7.40(\mathrm{~d}, J=8.8 \mathrm{~Hz}, 2 \mathrm{H}, 2 \times \mathrm{CH}), 7.64(\mathrm{~d}, J=7.9 \mathrm{~Hz}, 1 \mathrm{H}, \mathrm{CH}), 7.88(\mathrm{~d}$, $J=8.8 \mathrm{~Hz}, 1 \mathrm{H}, \mathrm{CH}), 9.42(\mathrm{bs}, 1 \mathrm{H}, \mathrm{NH}), 10.72(\mathrm{~s}, 1 \mathrm{H}, \mathrm{OH})$.

${ }^{13}$ C-NMR (63 MHz, $\left.\mathrm{CDCl}_{3}\right): \delta=52.9,100.3,111.3,120.2,121.1,123.2,126.4,127.1,128.6$, $129.1,135.8,136.6,142.7,157.9,169.9$.

HRMS (ESI): $m / z[\mathrm{M}+\mathrm{H}]^{+}$für $\mathrm{C}_{15} \mathrm{H}_{13} \mathrm{~N}_{2} \mathrm{O}_{3}$ ber.: 269.0921, gef.: 269.0921 .

\section{3-Hydroxy-6-(1H-indol-3-yl)picolinsäuremethylester (216i)}<smiles>COC(=O)c1nc(-c2c[nH]c3ccccc23)ccc1O</smiles>

Indol-3-boronsäurepinakolester ${ }^{[314]}$ (68 mg, $\left.0.28 \mathrm{mmol}\right)$ wurde nach AV1 umgesetzt. Es wurden $30 \mathrm{mg}(0.11 \mathrm{mmol}, 52 \%)$ des Produkts 216i als beiger Feststoff erhalten.

Schmelzpunkt: $183.5^{\circ} \mathrm{C}$.

DC: $R_{f}=0.27($ Heptan/EtOAc, 2:1). 
IR: $\tilde{v}=748(\mathrm{~m}), 1095(\mathrm{w}), 1203(\mathrm{w}), 1458(\mathrm{~s}), 1543(\mathrm{~m}), 1658(\mathrm{~s}), 1975(\mathrm{w}) \mathrm{cm}^{-1}$.

${ }^{1}$ H-NMR $\left(250 \mathrm{MHz}, \mathrm{DMSO}-d_{6}\right): \delta=4.00\left(\mathrm{~s}, 3 \mathrm{H}, \mathrm{CH}_{3}\right), 7.09-7.19(\mathrm{~m}, 2 \mathrm{H}, 2 \times \mathrm{CH}), 7.42-7.46$ $(\mathrm{m}, 2 \mathrm{H}, 2 \times \mathrm{CH}), 7.99-8.05(\mathrm{~m}, 2 \mathrm{H}, 2 \times \mathrm{CH}), 8.60(\mathrm{~d}, J=7.9 \mathrm{~Hz}, 1 \mathrm{H}, \mathrm{CH}), 10.31(\mathrm{~s}, 1 \mathrm{H}, \mathrm{OH})$, 11.42 (bs, 1H, NH).

${ }^{13}$ C-NMR (63 MHz, DMSO- $\left.d_{6}\right): \delta=52.6,111.6,114.5,119.9,121.8,121.9,125.2,125.5$, 126.4, $130.3136 .9,147.3,153.6,168.6$.

HRMS (ESI): $m / z[\mathrm{M}+\mathrm{Na}]^{+}$für $\mathrm{C}_{15} \mathrm{H}_{12} \mathrm{~N}_{2} \mathrm{NaO}_{3}$ ber.: 291.0746, gef.: 291.0743.

3-Hydroxy-6-(3'-pyridyl)picolinsäuremethylester (216j)<smiles>COC(=O)c1nc(-c2cccnc2)ccc1O</smiles>

Pyridin-3-boronsäure (34 mg, $0.28 \mathrm{mmol}$ ) wurde nach AV1 umgesetzt. Es wurden $24 \mathrm{mg}$ (0.10 mmol, 48\%) des Produkts 216j als farbloser Feststoff erhalten.

Schmelzpunkt: $138.5^{\circ} \mathrm{C}$.

DC: $R_{f}=0.50\left(\mathrm{CH}_{2} \mathrm{Cl}_{2} /\right.$ Heptan/Aceton, $\left.1: 1: 1\right)$.

IR: $\tilde{v}=702(\mathrm{~s}), 810(\mathrm{~m}), 1103(\mathrm{~m}), 1207(\mathrm{~s}), 1662(\mathrm{~s}), 1739(\mathrm{~m}), 2322(\mathrm{w}), 2357(\mathrm{w}) \mathrm{cm}^{-1}$.

${ }^{1} \mathbf{H}-\mathbf{N M R}\left(300 \mathrm{MHz}, \mathrm{CDCl}_{3}\right): \delta=4.06\left(\mathrm{~s}, 3 \mathrm{H}, \mathrm{CH}_{3}\right), 7.38(\mathrm{dd}, J=8.0,4.8 \mathrm{~Hz}, 1 \mathrm{H}, \mathrm{CH}), 7.47$ $(\mathrm{d}, J=8.8 \mathrm{~Hz}, 1 \mathrm{H}, \mathrm{CH}), 7.86(\mathrm{~d}, J=8.8 \mathrm{~Hz}, 1 \mathrm{H}, \mathrm{CH}), 8.25(\mathrm{~d}, J=7.9 \mathrm{~Hz}, 1 \mathrm{H}, \mathrm{CH}), 8.62(\mathrm{~d}$, $J=4.6 \mathrm{~Hz}, 1 \mathrm{H}, \mathrm{CH}), 9.13(\mathrm{~d}, J=1.6 \mathrm{~Hz}, 1 \mathrm{H}, \mathrm{CH}), 10.75$ (s, 1H, OH).

${ }^{13}$ C-NMR $\left(100 \mathrm{MHz}, \mathrm{CDCl}_{3}\right): \delta=53.1,123.6,126.7,127.2,129.9,133.9,134.0,146.3$, $147.9,149.7,158.2,169.9$.

HRMS (ESI): $m / z[\mathrm{M}+\mathrm{H}]^{+}$für $\mathrm{C}_{12} \mathrm{H}_{11} \mathrm{~N}_{2} \mathrm{O}_{3}$ ber.: 231.0764, gef.: 231.0765. 


\section{3-Hydroxy-6-(4'-pyridyl)picolinsäuremethylester (216k)}<smiles>COC(=O)c1nc(-c2ccncc2)ccc1O</smiles>

Pyridin-4-boronsäurepinakolester (57 mg, $0.28 \mathrm{mmol}$ ) wurde nach AV1 umgesetzt. Es wurden $27 \mathrm{mg}(0.12 \mathrm{mmol}, 55 \%)$ des Produkts 216k als farbloser Feststoff erhalten.

Schmelzpunkt: $127.5^{\circ} \mathrm{C}$.

DC: $R_{f}=0.55\left(\mathrm{CH}_{2} \mathrm{Cl}_{2} /\right.$ Heptan/Aceton, $\left.1: 1: 1\right)$.

IR: $\tilde{v}=825(\mathrm{~m}), 1103(\mathrm{~m}), 1180(\mathrm{~s}), 1296(\mathrm{~m}), 1458(\mathrm{~s}), 1604(\mathrm{w}), 1681(\mathrm{~m}), 3201(\mathrm{~b}) \mathrm{cm}^{-1}$.

${ }^{1}$ H-NMR $\left(250 \mathrm{MHz}, \mathrm{CDCl}_{3}\right): \delta=4.09\left(\mathrm{~s}, 3 \mathrm{H}, \mathrm{CH}_{3}\right), 7.50(\mathrm{~d}, J=8.8 \mathrm{~Hz}, 1 \mathrm{H}, \mathrm{CH}), 7.86(\mathrm{~m}$, $2 \mathrm{H}, 2 \times \mathrm{CH}), 7.93(\mathrm{~d}, J=8.8 \mathrm{~Hz}, 1 \mathrm{H}, \mathrm{CH}), 8.71(\mathrm{~d}, J=6.0 \mathrm{~Hz}, 2 \mathrm{H}, 2 \times \mathrm{CH}), 10.83(\mathrm{~s}, 1 \mathrm{H}$, $\mathrm{OH})$.

${ }^{13}$ C-NMR (63 MHz, $\left.\mathrm{CDCl}_{3}\right): \delta=53.2,120.7,126.8,127.2,130.0,145.3,146.3,150.4,158.9$, 169.9 .

HRMS (ESI): $m / z[\mathrm{M}+\mathrm{H}]^{+}$für $\mathrm{C}_{12} \mathrm{H}_{11} \mathrm{~N}_{2} \mathrm{O}_{3}$ ber.: 231.0764, gef.: 231.0767.

\section{3-Hydroxy-6-(thiophen-2-yl)picolinsäuremethylester (216l)}<smiles>COC(=O)c1nc(-c2cccs2)ccc1O</smiles>

2-Thiophenboronsäurepinakolester (59 mg, $0.28 \mathrm{mmol}$ ) wurde nach AV1 umgesetzt. Es wurden $31 \mathrm{mg}(0.13 \mathrm{mmol}, 61 \%)$ des Produkts 2161 als farbloser Feststoff erhalten.

Schmelzpunkt: $135^{\circ} \mathrm{C}$.

DC: $R_{f}=0.44($ Heptan/EtOAc, 4:1).

IR: $\tilde{v}=694(\mathrm{~s}), 840(\mathrm{~m}), 1095(\mathrm{~m}), 1203(\mathrm{~s}), 1296(\mathrm{~m}), 1458(\mathrm{~s}), 1658(\mathrm{~s}), 3109(\mathrm{w}) \mathrm{cm}^{-1}$. 
${ }^{1} \mathbf{H}-\mathbf{N M R}\left(400 \mathrm{MHz}, \mathrm{CDCl}_{3}\right): \delta=4.05\left(\mathrm{~s}, 3 \mathrm{H}, \mathrm{CH}_{3}\right), 7.08(\mathrm{dd}, J=5.1,3.7 \mathrm{~Hz}, 1 \mathrm{H}, \mathrm{CH}), 7.35-$ $7.38(\mathrm{~m}, 2 \mathrm{H}, 2 \times \mathrm{CH}), 7.48(\mathrm{dd}, J=3.7,1.0 \mathrm{~Hz}, 1 \mathrm{H}, \mathrm{CH}), 7.76(\mathrm{~d}, J=8.8 \mathrm{~Hz}, 1 \mathrm{H}, \mathrm{CH}), 10.71$ (s, 1H, OH).

${ }^{13}$ C-NMR (63 MHz, $\left.\mathrm{CDCl}_{3}\right): \delta=53.1,125.6,127.0,127.1,127.9,129.0,143.6,145.0,157.6$, 169.9 .

HRMS (ESI): $m / z[\mathrm{M}+\mathrm{H}]^{+}$für $\mathrm{C}_{11} \mathrm{H}_{10} \mathrm{NO}_{3} \mathrm{~S}$ ber.: 236.0376, gef.: 236.0378.

\section{3-Hydroxy-6-(thiophen-3-yl)picolinsäuremethylester (216m)}<smiles>COC(=O)c1nc(-c2ccsc2)ccc1O</smiles>

3-Thiophenboronsäure (36 mg, $0.28 \mathrm{mmol}$ ) wurde nach AV1 umgesetzt. Es wurden $38 \mathrm{mg}$ (0.16 mmol, 75\%) des Produkts $\mathbf{2 1 6 m}$ als farbloser Feststoff erhalten.

Schmelzpunkt: $114.5^{\circ} \mathrm{C}$.

DC: $R_{f}=0.49($ Heptan/EtOAc, $4: 1)$.

IR: $\tilde{v}=732(\mathrm{~s}), 786(\mathrm{~m}), 1103(\mathrm{~m}), 1180$ (s), $1280(\mathrm{~m}), 1357(\mathrm{~s}), 1458(\mathrm{~s}), 1666(\mathrm{~m}), 1743$ (m), $2954(\mathrm{w}), 3024(\mathrm{w}) \mathrm{cm}^{-1}$.

${ }^{1} \mathbf{H}-\mathbf{N M R}\left(250 \mathrm{MHz}, \mathrm{CDCl}_{3}\right): \delta=4.05\left(\mathrm{~s}, 3 \mathrm{H}, \mathrm{CH}_{3}\right), 7.36-7.40(\mathrm{~m}, 2 \mathrm{H}, 2 \times \mathrm{CH}), 7.61-7.63$ (m, 1H, CH), $7.73(\mathrm{~d}, J=8.8 \mathrm{~Hz}, 1 \mathrm{H}, \mathrm{CH}), 7.75-7.79(\mathrm{~m}, 1 \mathrm{H}, \mathrm{CH}), 10.70$ (s, 1H, OH).

${ }^{13}$ C-NMR (63 MHz, $\left.\mathrm{CDCl}_{3}\right): \delta=53.0,122.8,126.1,126.4,126.8,126.9,129.1,141.1,145.9$, $157.5,170.1$.

HRMS (ESI): $m / z[\mathrm{M}+\mathrm{H}]^{+}$für $\mathrm{C}_{11} \mathrm{H}_{10} \mathrm{NO}_{3} \mathrm{~S}$ ber.: 236.0376, gef: 236.0377. 


\section{6-(5-Acetylthiophen-2-yl)-3-hydroxypicolinsäuremethylester (216n)}

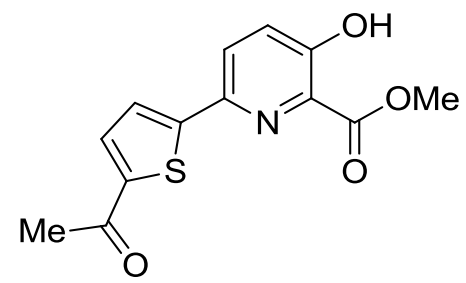

5-Acetyl-2-thiophenboronsäure (48 mg, $0.28 \mathrm{mmol}$ ) wurde nach AV1 umgesetzt. Es wurden $45 \mathrm{mg}(0.16 \mathrm{mmol}, 76 \%)$ des Produkts 216n als farbloser Feststoff erhalten.

Schmelzpunkt: $197^{\circ} \mathrm{C}$.

DC: $R_{f}=0.42($ Heptan/EtOAc, $2: 1)$.

IR: $\tilde{v}=632(\mathrm{~m}), 725(\mathrm{~m}), 1211(\mathrm{~m}), 1273(\mathrm{~s}), 1435(\mathrm{~s}), 1643(\mathrm{~m}), 2954(\mathrm{w}), 3055(\mathrm{w}) \mathrm{cm}^{-1}$.

${ }^{1} \mathbf{H}-\mathbf{N M R}\left(300 \mathrm{MHz}, \mathrm{CDCl}_{3}\right): \delta=2.56\left(\mathrm{~s}, 3 \mathrm{H}, \mathrm{CH}_{3}\right), 4.05\left(\mathrm{~s}, 3 \mathrm{H}, \mathrm{CH}_{3}\right), 7.40(\mathrm{~d}, J=8.8 \mathrm{~Hz}$, 1H, CH), 7.48 (d, $J=4.0 \mathrm{~Hz}, 1 \mathrm{H}, \mathrm{CH}), 7.65$ (d, $J=4.0 \mathrm{~Hz}, 1 \mathrm{H}, \mathrm{CH}), 7.79$ (d, $J=8.8 \mathrm{~Hz}, 1 \mathrm{H}$, $\mathrm{CH}), 10.80(\mathrm{~s}, 1 \mathrm{H}, \mathrm{OH})$.

${ }^{13}$ C-NMR (100 MHz, $\left.\mathrm{CDCl}_{3}\right): \delta=26.7,53.2,124.6,125.9,127.0,129.5,133.0,143.5,144.5$, $151.1,158.3,169.6,190.6$.

HRMS (ESI): $m / z[\mathrm{M}+\mathrm{H}]^{+}$für $\mathrm{C}_{13} \mathrm{H}_{12} \mathrm{NO}_{4} \mathrm{~S}$ ber.: 278.0482 , gef.: 278.0481 .

\section{6-(Furan-2-yl)-3-hydroxypicolinsäuremethylester (2160)}<smiles>COC(=O)c1nc(-c2ccco2)ccc1O</smiles>

Furan-2-boronsäure (31 mg, $0.28 \mathrm{mmol}$ ) wurde nach AV1 umgesetzt. Es wurden $40 \mathrm{mg}$ (0.18 mmol, 85\%) des Produkts 2160 als farbloser Feststoff erhalten.

Schmelzpunkt: $129^{\circ} \mathrm{C}$.

DC: $R_{f}=0.42($ Heptan/EtOAc, 4:1).

IR: $\tilde{v}=702(\mathrm{~s}), 1087(\mathrm{~m}), 1203(\mathrm{~m}), 1296(\mathrm{~m}), 1450(\mathrm{~s}), 1674(\mathrm{~s}), 2924(\mathrm{w}) \mathrm{cm}^{-1}$. 
${ }^{1} \mathbf{H}-\mathbf{N M R}\left(300 \mathrm{MHz}, \mathrm{CDCl}_{3}\right): \delta=4.06\left(\mathrm{~s}, 3 \mathrm{H}, \mathrm{CH}_{3}\right), 6.51(\mathrm{dd}, J=3.4,1.8 \mathrm{~Hz}, 1 \mathrm{H}, \mathrm{CH}), 6.95$ $(\mathrm{d}, J=3.4 \mathrm{~Hz}, 1 \mathrm{H}, \mathrm{CH}), 7.41(\mathrm{~d}, J=8.8 \mathrm{~Hz}, 1 \mathrm{H}, \mathrm{CH}), 7.51(\mathrm{~m}, 1 \mathrm{H}, \mathrm{CH}), 7.81(\mathrm{~d}, J=8.8 \mathrm{~Hz}$, $1 \mathrm{H}, \mathrm{CH}), 10.72(\mathrm{~s}, 1 \mathrm{H}, \mathrm{OH})$.

${ }^{13}$ C-NMR $\left(100 \mathrm{MHz}, \mathrm{CDCl}_{3}\right): \delta=53.1,118.0,111.9,125.4,127.0,129.1,141.9,143.2$, $152.5,169.9$.

HRMS (ESI): $m / z[\mathrm{M}+\mathrm{H}]^{+}$für $\mathrm{C}_{11} \mathrm{H}_{10} \mathrm{NO}_{4}$ ber.: 220.0604, gef.: 220.0608 .

\section{6-(Furan-3-yl)- 3-hydroxypicolinsäuremethylester (216p)}<smiles>COC(=O)c1nc(-c2ccoc2)ccc1O</smiles>

Furan-3-boronsäure (31 mg, $0.28 \mathrm{mmol}$ ) wurde nach AV1 umgesetzt. Es wurden $32.5 \mathrm{mg}$ (0.15 mmol, 69\%) des Produkts 216p als beiger Feststoff erhalten.

Schmelzpunkt: $94.5^{\circ} \mathrm{C}$.

DC: $R_{f}=0.30($ Heptan/EtOAc, $4: 1)$.

IR: $\tilde{v}=702(\mathrm{~m}), 802(\mathrm{~m}), 1056(\mathrm{~m}), 1157(\mathrm{~s}), 1195(\mathrm{~s}), 1365(\mathrm{~m}), 1450(\mathrm{~s}), 1666(\mathrm{~s}) \mathrm{cm}^{-1}$.

${ }^{1}$ H-NMR (400 MHz, $\left.\mathrm{CDCl}_{3}\right): \delta=4.04\left(\mathrm{~s}, 3 \mathrm{H}, \mathrm{CH}_{3}\right), 6.87(\mathrm{~d}, J=0.9 \mathrm{~Hz}, 1 \mathrm{H}, \mathrm{CH}), 7.36(\mathrm{~d}$, $J=8.8 \mathrm{~Hz}, 1 \mathrm{H}, \mathrm{CH}), 7.47(\mathrm{t}, J=1.8 \mathrm{~Hz}, 1 \mathrm{H}, \mathrm{CH}), 7.57$ (d, $J=8.8 \mathrm{~Hz}, 1 \mathrm{H}, \mathrm{CH}), 7.94(\mathrm{~s}, 1 \mathrm{H}$, $\mathrm{CH}), 10.67$ (s, 1H, OH).

${ }^{13}$ C-NMR (63 MHz, $\left.\mathrm{CDCl}_{3}\right): \delta=53.0,108.7,126.2,126.7,126.9,129.2,140.6,143.8,143.9$, $157.4,170.0$.

HRMS (ESI): $m / z[\mathrm{M}+\mathrm{H}]^{+}$für $\mathrm{C}_{11} \mathrm{H}_{10} \mathrm{NO}_{4}$ ber.: 220.0604, gef.: 220.0605 . 


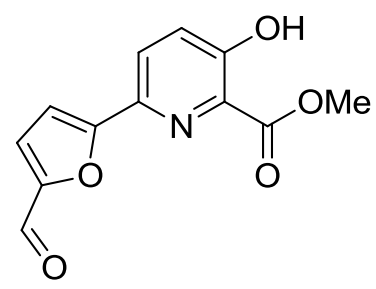

(5-Formyl-2-furanyl)-boronsäure (39 mg, $0.28 \mathrm{mmol}$ ) wurde nach AV1 umgesetzt. Es wurden $33.5 \mathrm{mg}(0.14 \mathrm{mmol}, 63 \%)$ des Produkts $216 \mathrm{q}$ als farbloser Feststoff erhalten.

Schmelzpunkt: $199.5^{\circ} \mathrm{C}$.

DC: $R_{f}=0.22($ Heptan/EtOAc, $2: 1)$.

IR: $\tilde{v}=756(\mathrm{~m}), 1180(\mathrm{~s}), 1296(\mathrm{~m}), 1365(\mathrm{~m}), 1450(\mathrm{~m}), 1681(\mathrm{~s}), 1743(\mathrm{w}) \mathrm{cm}^{-1}$.

${ }^{1} \mathbf{H}-\mathbf{N M R}\left(400 \mathrm{MHz}, \mathrm{CDCl}_{3}\right): \delta=4.07\left(\mathrm{~s}, 3 \mathrm{H}, \mathrm{CH}_{3}\right), 7.19(\mathrm{~d}, J=3.7 \mathrm{~Hz}, 1 \mathrm{H}, \mathrm{CH}), 7.34$ (d, $J=3.7 \mathrm{~Hz}, 1 \mathrm{H}, \mathrm{CH}), 7.47(\mathrm{~d}, J=8.8 \mathrm{~Hz}, 1 \mathrm{H}, \mathrm{CH}), 8.05$ (d, $J=8.8 \mathrm{~Hz}, 1 \mathrm{H}, \mathrm{CH}), 9.69$ (s, 1H, $\mathrm{O}=\mathrm{CH}), 10.86(\mathrm{~s}, 1 \mathrm{H}, \mathrm{OH})$.

${ }^{13}$ C-NMR $\left(100 \mathrm{MHz}, \mathrm{CDCl}_{3}\right): \delta=53.3,110.1,123.1,126.5,127.1,130.0,140.0,152.5$, $157.3,158.8,169.6,177.6$.

HRMS (ESI): $m / z[\mathrm{M}+\mathrm{H}]^{+}$für $\mathrm{C}_{12} \mathrm{H}_{10} \mathrm{NO}_{5}$ ber.: 248.0554, gef.: 248.0554.

\section{3-Hydroxy-6-(1H-pyrrol-3-yl)picolinsäuremethylester (216r)}<smiles>COC(=O)c1nc(-c2cc[nH]c2)ccc1O</smiles>

1H-Pyrrol-3-boronsäurepinakolester ${ }^{[315]}$ (54 mg, $0.28 \mathrm{mmol}$ ) wurde nach AV1 umgesetzt. Es wurden $26 \mathrm{mg}(0.12 \mathrm{mmol}, 56 \%)$ des Produkts 216r als beiger Feststoff erhalten.

Schmelzpunkt: $171.5^{\circ} \mathrm{C}$.

DC: $R_{f}=0.20($ Heptan/EtOAc, $2: 1)$.

IR: $\tilde{v}=678(\mathrm{~m}), 1095(\mathrm{~m}), 1195(\mathrm{~m}), 1296(\mathrm{w}), 1442(\mathrm{~s}), 1666(\mathrm{~s}), 3232(\mathrm{w}) \mathrm{cm}^{-1}$. 
${ }^{1}$ H-NMR (400 MHz, DMSO- $\left.d_{6}, 318 \mathrm{~K}\right): \delta=3.92\left(\mathrm{~s}, 3 \mathrm{H}, \mathrm{CH}_{3}\right), 6.54(\mathrm{q}, J=2.3 \mathrm{~Hz}, 1 \mathrm{H}, \mathrm{CH})$, $6.78(\mathrm{q}, J=2.3 \mathrm{~Hz}, 1 \mathrm{H}, \mathrm{CH}), 7.32(\mathrm{dt}, J=2.6,1.8 \mathrm{~Hz}, 1 \mathrm{H}, \mathrm{CH}), 7.35(\mathrm{~d}, J=8.8 \mathrm{~Hz}, 1 \mathrm{H}, \mathrm{CH})$, $7.71(\mathrm{~d}, J=8.8 \mathrm{~Hz}, 1 \mathrm{H}, \mathrm{CH}), 10.19$ (s, 1H, OH), 10.93 (bs, 1H, NH).

${ }^{13}$ C-NMR (63 MHz, DMSO- $\left.d_{6}\right): \delta=52.3,105.9,116.8,118.9,123.2,124.5,126.1,131.2$, $146.7,153.3,168.4$.

HRMS (ESI): $m / z[\mathrm{M}+\mathrm{H}]^{+}$für $\mathrm{C}_{11} \mathrm{H}_{11} \mathrm{~N}_{2} \mathrm{O}_{3}$ ber.: 219.0764, gef.: 219.0768.

\section{3-Hydroxy-6-(1H-pyrazol-5-yl)picolinsäuremethylester (216s)}<smiles>COC(=O)c1nc(-c2ccn[nH]2)ccc1O</smiles>

1-(Tetrahydro-2H-pyran-2-yl)-1H-pyrazol-5-boronsäurepinakolester ${ }^{[268]}$ (78 mg, 0.28 mmol) wurde nach AV1 umgesetzt. Das so erhaltene Rohprodukt wurde in wasserfreiem $\mathrm{MeOH}$ $(1 \mathrm{ml})$ gelöst, $\mathrm{HCl}(2 \mathrm{~mL}$, ca. $1.25 \mathrm{M}$ in EtOH) zugegeben und die Mischung $2 \mathrm{~h}$ lang bei RT gerührt. Das Gemisch wurde mit pH 6.2 Puffer $(10 \mathrm{ml})$ verdünnt und anschließend mit $\mathrm{CH}_{2} \mathrm{Cl}_{2}(3 \times 10 \mathrm{ml})$ extrahiert. Die vereinigten organischen Extrakte wurden mit $\mathrm{Na}_{2} \mathrm{SO}_{4}$ entwässert und im Vakuum eingeengt. Reinigung des Rückstands durch Säulenchromatographie ( $\left.\mathrm{SiO}_{2}, 25 \mathrm{~g}, \mathrm{CH}_{2} \mathrm{Cl}_{2} / \mathrm{MeOH}, 30: 1\right)$ ergab $30 \mathrm{mg}(0.14 \mathrm{mmol}, 64 \%)$ des Produkts 216s als farblosen Feststoff.

Schmelzpunkt: $169.5^{\circ} \mathrm{C}$.

DC: $R_{f}=0.40\left(\mathrm{CH}_{2} \mathrm{Cl}_{2} / \mathrm{MeOH}, 30: 1\right)$.

IR: $\tilde{v}=725(\mathrm{~m}), 802(\mathrm{~m}), 1103(\mathrm{~m}), 1211(\mathrm{~s}), 1296(\mathrm{~m}), 1442(\mathrm{~s}), 1674(\mathrm{~s}), 3140(\mathrm{w}) \mathrm{cm}^{-1}$.

${ }^{1}$ H-NMR (400 MHz, DMSO- $\left.d_{6}, 318 \mathrm{~K}\right): \delta=3.93\left(\mathrm{~s}, 3 \mathrm{H}, \mathrm{CH}_{3}\right), 6.72(\mathrm{~d}, J=2.3 \mathrm{~Hz}, 1 \mathrm{H}, \mathrm{CH})$, 7.48 (d, $J=8.8 \mathrm{~Hz}, 1 \mathrm{H}, \mathrm{CH}), 7.74$ (bs, 1H, CH), 8.03 (bd, $J=5.8 \mathrm{~Hz}, 1 \mathrm{H}, \mathrm{CH}), 10.45$ (s, 1H, $\mathrm{OH}), 12.91$ (bs, 1H, NH).

${ }^{13}$ C-NMR (100 MHz, DMSO- $\left.d_{6}, 318 \mathrm{~K}\right): \delta=52.2,102.5,124.7,126.2,129.7,132.1,144.1$, $150.1,154.4,167.7$.

HRMS (ESI): $m / z[\mathrm{M}+\mathrm{H}]^{+}$für $\mathrm{C}_{10} \mathrm{H}_{10} \mathrm{~N}_{3} \mathrm{O}_{3}$ ber.: 220.0717, gef.: 220.0718. 


\section{3-Hydroxy-6-(1H-pyrazol-4-yl)picolinsäuremethylester (216t)}<smiles>COC(=O)c1nc(-c2cn[nH]c2)ccc1O</smiles>

Pyrazol-4-boronsäurepinakolester ${ }^{[314]}$ (54 mg, $0.28 \mathrm{mmol}$ ) wurde nach AV1 umgesetzt. Es wurden $29 \mathrm{mg}(0.13 \mathrm{mmol}, 62 \%)$ des Produkts 216t als beiger Feststoff erhalten.

Schmelzpunkt: $189^{\circ} \mathrm{C}$.

DC: $R_{f}=0.29\left(\mathrm{CH}_{2} \mathrm{Cl}_{2} / \mathrm{MeOH}, 30: 1\right)$.

IR: $\tilde{v}=694(\mathrm{~s}), 1203(\mathrm{~s}), 1373(\mathrm{~m}), 1450(\mathrm{~s}), 1666(\mathrm{~s}), 2924(\mathrm{w}) \mathrm{cm}^{-1}$.

${ }^{1}$ H-NMR $\left(250 \mathrm{MHz}, \mathrm{DMSO}-d_{6}\right): \delta=3.92\left(\mathrm{~s}, 3 \mathrm{H}, \mathrm{CH}_{3}\right), 7.43(\mathrm{~d}, J=8.8 \mathrm{~Hz}, 1 \mathrm{H}, \mathrm{CH}), 7.80(\mathrm{~d}$, $J=8.8 \mathrm{~Hz}, 1 \mathrm{H}, \mathrm{CH}), 8.10(\mathrm{bs}, 2 \mathrm{H}, 2 \times \mathrm{CH}), 10.37$ (s, 1H, OH), 13.02 (bs, 1H, NH).

${ }^{13}$ C-NMR (63 MHz, DMSO- $\left.d_{6}\right): \delta=52.4,121.3,125.2,126.4,132.1,143.8,153.6,168.0$.

HRMS (ESI): $m / z[\mathrm{M}+\mathrm{H}]^{+}$für $\mathrm{C}_{10} \mathrm{H}_{10} \mathrm{~N}_{3} \mathrm{O}_{3}$ ber.: 220.0717, gef.: 220.0716.

\section{3-Hydroxy-6-(chinolin-8-yl)picolinsäuremethylester (216u)}<smiles>COC(=O)c1nc(-c2cccc3cccnc23)ccc1O</smiles>

8-Chinolinylboronsäure (48 mg, $0.28 \mathrm{mmol}$ ) wurde nach AV1 umgesetzt. Es wurden $17.5 \mathrm{mg}$ $(62,4 \mu \mathrm{mol}, 29 \%)$ des Produkts 216u als leicht brauner Feststoff erhalten.

Schmelzpunkt: $198.5^{\circ} \mathrm{C}$.

DC: $R_{f}=0.32($ Heptan/EtOAc, $2: 1)$.

IR: $\tilde{v}=717(\mathrm{~m}), 1103(\mathrm{~m}), 1211(\mathrm{~s}), 1311(\mathrm{~m}), 1365(\mathrm{~m}), 1450(\mathrm{~s}), 1666(\mathrm{~m}), 3047(\mathrm{w})$, $3086(\mathrm{w}) \mathrm{cm}^{-1}$. 
${ }^{1} \mathbf{H}-\mathbf{N M R}\left(300 \mathrm{MHz}, \mathrm{CDCl}_{3}\right): \delta=4.05\left(\mathrm{~s}, 3 \mathrm{H}, \mathrm{CH}_{3}\right), 7.42(\mathrm{dd}, J=8.3,4.2 \mathrm{~Hz}, 1 \mathrm{H}, \mathrm{CH}), 7.49$ $(\mathrm{d}, J=8.8 \mathrm{~Hz}, 1 \mathrm{H}, \mathrm{CH}), 7.66$ (t, $J=7.8 \mathrm{~Hz}, 1 \mathrm{H}, \mathrm{CH}), 7.87(\mathrm{dd}, J=8.2,1.3 \mathrm{~Hz}, 1 \mathrm{H}, \mathrm{CH}), 8.13$ $(\mathrm{dd}, J=7.2,1.4, \mathrm{~Hz}, 1 \mathrm{H}, \mathrm{CH}), 8.21$ (dd, $J=8.3,1.7 \mathrm{~Hz}, 1 \mathrm{H}, \mathrm{CH}), 8.25$ (d, $J=8.8 \mathrm{~Hz}, 1 \mathrm{H}$, $\mathrm{CH}), 8.93(\mathrm{dd}, J=4.2,1.8 \mathrm{~Hz}, 1 \mathrm{H}, \mathrm{CH}), 10.79(\mathrm{~s}, 1 \mathrm{H}, \mathrm{OH})$.

${ }^{13}$ C-NMR $\left(100 \mathrm{MHz}, \mathrm{CDCl}_{3}\right): \delta=53.0,121.1,125.5,126.6,128.6,128.8,129.4,131.1$, 133.4, 136.4, 137.9, 145.9, 149.3, 150.2, 158.1, 170.3 .

HRMS (ESI): $m / z[\mathrm{M}+\mathrm{H}]^{+}$für $\mathrm{C}_{16} \mathrm{H}_{13} \mathrm{~N}_{2} \mathrm{O}_{3}$ ber.: 281.0921, gef.: 281.0921 .

\section{3-Hydroxy-6-(1-trityl-1H-imidazol-4-yl)picolinsäuremethylester (218)}<smiles>COC(=O)c1nc(-c2cn([Tl])cn2)ccc1O</smiles>

6-Brom-3-hydroxypicolinsäuremethylester $\mathbf{( 2 0 4}, 56 \mathrm{mg}, 0.24 \mathrm{mmol})$ wurde zusammen mit 4-(Tributylstannyl)-1-trityl-1H-imidazol ${ }^{[269]}$ (217, $\left.160 \mathrm{mg}, 0.27 \mathrm{mmol}\right)$ und $\mathrm{Pd}\left(\mathrm{PPh}_{3}\right)_{4}(14 \mathrm{mg}$, $5 \mathrm{~mol} \%$ ) in wasserfreiem Toluol (4 mL) in einem Bombenrohr $18 \mathrm{~h}$ lang bei $120{ }^{\circ} \mathrm{C}$ gerührt. Das Gemisch wurde mit EtOAc $(50 \mathrm{~mL})$ verdünnt und mit 50\%iger KF-Lösung $(5 \times 25 \mathrm{~mL})$ gewaschen. Die organische Phase wurde mit $\mathrm{Na}_{2} \mathrm{SO}_{4}$ getrocknet und im Vakuum eingeengt. Mehrmalige Reinigung des Rückstands durch Säulenchromatographie $\left(\mathrm{SiO}_{2}, 40 \mathrm{~g}\right.$, Petrolether/ $\mathrm{CH}_{2} \mathrm{Cl}_{2} /$ Aceton, 3:3:1 $\rightarrow$ 1:1:1; $\mathrm{CH}_{2} \mathrm{Cl}_{2} / \mathrm{MeOH}$, 9:1, Petrolether/ $\mathrm{CH}_{2} \mathrm{Cl}_{2} / \mathrm{EtOAc}$, 1:1:0 $\rightarrow$ 1:1:2) ergab $83 \mathrm{mg}(0.18 \mathrm{mmol}, 75 \%)$ das 3-Hydroxypyridin 218 als leicht gelben Schaum.

DC: $R_{f}=0.50\left(\right.$ Heptan $/ \mathrm{CH}_{2} \mathrm{Cl}_{2} /$ Aceton, $\left.1: 1: 1\right)$.

IR: $\tilde{v}=698(\mathrm{~s}), 744(\mathrm{~s}), 1076(\mathrm{w}), 1172(\mathrm{~m}), 1203(\mathrm{~m}), 1442(\mathrm{~m}), 1554(\mathrm{w}), 1674(\mathrm{~m}) \mathrm{cm}^{-1}$.

${ }^{1} \mathbf{H}-\mathbf{N M R}\left(300 \mathrm{MHz}, \mathrm{CDCl}_{3}\right): \delta=3.97\left(\mathrm{~s}, 3 \mathrm{H}, \mathrm{CH}_{3}\right), 7.16-7.22(\mathrm{~m}, 6 \mathrm{H}), 7.30-7.36(\mathrm{~m}, 9 \mathrm{H})$, $7.40(\mathrm{~d}, J=8.8 \mathrm{HZ}, 1 \mathrm{H}, \mathrm{CH}), 7.45(\mathrm{~d}, J=1.2 \mathrm{~Hz}, 1 \mathrm{H}, \mathrm{CH}), 7.55(\mathrm{~d}, J=1.2 \mathrm{~Hz}, 1 \mathrm{H}, \mathrm{CH}), 8.14$ (d, $J=8.8 \mathrm{~Hz}, 1 \mathrm{H}, \mathrm{CH}), 10.64(\mathrm{~s}, 1 \mathrm{H}, \mathrm{OH})$.

${ }^{13}$ C-NMR $\left(75 \mathrm{MHz}, \mathrm{CDCl}_{3}\right): \delta=52.8,75.7,119.2,126.5,128.1,128.4,128.55,128.60$, $129.8,131.88,131.91,132.0,132.2139 .5,140.0,142.3,145.9,157.5,170.0$. 
HRMS (ESI): $m / z[\mathrm{M}+\mathrm{H}]^{+}$für $\mathrm{C}_{29} \mathrm{H}_{24} \mathrm{~N}_{3} \mathrm{O}_{3}$ ber.: 462.1812, gef.: 462.1807 .

\section{6-Cyano-3-hydroxypicolinsäuremethylester (219)}<smiles>COC(=O)c1nc(C#N)ccc1O</smiles>

Eine Lösung von 6-Brom-3-hydroxypicolinsäuremethylester ${ }^{[267]}(\mathbf{2 0 4}, 3 \mathrm{~g}, 12.9 \mathrm{mmol})$ in wasserfreiem DMF (60 mL) wurde mit CuCN (2.31 g, $25.9 \mathrm{mmol})$ versetzt und $1 \mathrm{~h}$ lang bei $150^{\circ} \mathrm{C}$ gerührt. Nach Abkühlen auf RT wurde das Lösungsmittel im Vakuum entfernt und der Rückstand in $\mathrm{CHCl}_{3}(200 \mathrm{~mL})$ aufgenommen. Die Lösung wurde mit pH 6.2 Puffer $(150 \mathrm{~mL})$, der $\mathrm{Na}_{2}$ EDTA (9.68 g, $\left.26 \mathrm{mmol}\right)$ enthielt, und ges. NaCl-Lsg. (100 ml) gewaschen. Nach Entwässern mit $\mathrm{Na}_{2} \mathrm{SO}_{4}$ und Einengen am Rotationsverdampfer wurde der Rückstand durch Säulenchromatographie $\left(\mathrm{SiO}_{2}, 150 \mathrm{~g}\right.$, Petrolether/EtOAc, 2:1) gereinigt. Es wurden $1.61 \mathrm{~g}$ (9.03 mmol, 70\%) des Nitrils 219 als farbloser Feststoff erhalten.

Schmelzpunkt: $148^{\circ} \mathrm{C}$.

DC: $R_{f}=0.38($ Heptan/EtOAc, 2:1).

IR: $\tilde{v}=667$ (s), $852(\mathrm{~m}), 1099(\mathrm{~m}), 1211$ (s), 1300 (s), 1446 (s), 1678 (s), 2237 (w), $3078(\mathrm{w}) \mathrm{cm}^{-1}$.

${ }^{1} \mathbf{H}-\mathbf{N M R}\left(250 \mathrm{MHz}, \mathrm{CDCl}_{3}\right): \delta=4.08\left(\mathrm{~s}, 3 \mathrm{H}, \mathrm{CH}_{3}\right), 7.47(\mathrm{~d}, J=8.7 \mathrm{~Hz}, 1 \mathrm{H}, \mathrm{CH}), 7.78(\mathrm{~d}$, $J=8.7 \mathrm{~Hz}, 1 \mathrm{H}, \mathrm{CH}), 11.17(\mathrm{~s}, 1 \mathrm{H}, \mathrm{OH})$.

${ }^{13}$ C-NMR (63 MHz, $\left.\mathrm{CDCl}_{3}\right): \delta=53.7,116.4,124.7,127.1,131.6,133.6,160.5,168.6$.

HRMS (ESI): $m / z[\mathrm{M}+\mathrm{H}]^{+}$für $\mathrm{C}_{8} \mathrm{H}_{7} \mathrm{~N}_{2} \mathrm{O}_{3}$ ber.: 179.0451, gef.: 179.0449. 


\section{3-Hydroxy-6-( $N$-hydroxycarbamimidoyl)picolinsäuremthylester (220)}<smiles>COC(=O)c1nc(C(=N)NO)ccc1O</smiles>

Das Nitril 219 (1.54 g, $8.63 \mathrm{mmol})$ wurde in wasserfreiem $\mathrm{MeOH}$ (56 mL) gelöst, mit Hydroxylaminhydrochlorid $(0.90 \mathrm{~g}, 12.95 \mathrm{mmol})$ und $\mathrm{NaHCO}_{3}(1.09 \mathrm{~g}, 12.95 \mathrm{mmol})$ versetzt und die Mischung $2 \mathrm{~h}$ lang unter Rückfluss gerührt. Anschließend wurde das Lösungsmittel im Vakuum entfernt und der Rückstand in $\mathrm{H}_{2} \mathrm{O}(50 \mathrm{ml})$ aufgenommen. Der Feststoff wurde abfiltriert und mit $\mathrm{H}_{2} \mathrm{O}(3 \times 15 \mathrm{~mL})$ gewaschen. Nach Trocknen am Feinvakuum wurden $1.54 \mathrm{~g}$ (7.34 mmol, 85\%) des $N$-Hydroxyamidins 220 als farbloser Feststoff erhalten.

Schmelzpunkt: $210^{\circ} \mathrm{C}$ (Zers.).

DC: $R_{f}=0.44\left(\mathrm{CHCl}_{3} / \mathrm{MeOH}, 9: 1\right)$.

IR: $\tilde{v}=617(\mathrm{~s}), 852(\mathrm{~m}), 948(\mathrm{~s}), 1092(\mathrm{~s}), 1396(\mathrm{~m}), 1654(\mathrm{~m}), 2360(\mathrm{w}), 3070(\mathrm{w}) \mathrm{cm}^{-1}$.

${ }^{1}$ H-NMR $\left(250 \mathrm{MHz}, \mathrm{DMSO}-d_{6}\right): \delta=3.91\left(\mathrm{~s}, 3 \mathrm{H}, \mathrm{CH}_{3}\right), 5.59$ (s, 1H, N-OH), 7.45 (d, $J=8.9 \mathrm{~Hz}, 1 \mathrm{H}, \mathrm{CH}), 7.93(\mathrm{~d}, J=8.9 \mathrm{~Hz}, 1 \mathrm{H}, \mathrm{CH}), 9.87$ (s, 1H, NH), 10.67 (bs, 1H, OH).

${ }^{13}$ C-NMR (63 MHz, DMSO- $\left.d_{6}\right): \delta=52.5,124.7,126.5,131.2,141.3,148.9,155.9,167.2$.

HRMS (ESI): $m / z[\mathrm{M}+\mathrm{H}]^{+}$für $\mathrm{C}_{8} \mathrm{H}_{10} \mathrm{~N}_{3} \mathrm{O}_{4}$ ber.: 212.0666, gef.: 212.0666.

\section{3-Hydroxy-6-(5-methyl-1,2,4-oxadiazol-3-yl)picolinsäure (221)}<smiles>Cc1nc(-c2ccc(O)c(C(=O)O)n2)no1</smiles>

Das $N$-Hydroxyamidin 220 (1.5 g, $7.10 \mathrm{mmol})$ wurde in wasserfreiem Pyridin (23 mL) gelöst, mit Acetylchlorid $(1.20 \mathrm{~mL}, 17.0 \mathrm{mmol})$ versetzt und $3 \mathrm{~h}$ lang bei $115^{\circ} \mathrm{C}$ gerührt. Nach Einengen im Vakuum wurde der Rückstand in THF $(20 \mathrm{~mL})$ und $\mathrm{H}_{2} \mathrm{O}(10 \mathrm{~mL})$ gelöst, mit $\mathrm{LiOH} \times \mathrm{H}_{2} \mathrm{O}(1.19 \mathrm{~g}, 28.4 \mathrm{mmol})$ versetzt und die Mischung $20 \mathrm{~h}$ lang bei RT gerührt. Das THF wurde anschließend am Rotationsverdampfer entfernt und die wässrige Phase mit verd. $\mathrm{H}_{2} \mathrm{SO}_{4}$ auf $\mathrm{pH} 3$ angesäuert. Die wässrige Phase wurde mit $\mathrm{NaCl}$ gesättigt und mit EtOAc 
$(4 \times 50 \mathrm{~mL})$ extrahiert. Die vereinigten organischen Extrakte wurden mit $\mathrm{Na}_{2} \mathrm{SO}_{4}$ entwässert und im Vakuum eingeengt. Umkristallisieren des Rückstands aus $\mathrm{MeOH} / \mathrm{H}_{2} \mathrm{O}$ lieferte $1.26 \mathrm{~g}$ (5.68 mmol, 80\%) der Picolinsäure 221 als leicht gelben Feststoff.

Schmelzpunkt: $225^{\circ} \mathrm{C}$ (Zers.).

DC: $R_{f}=0.14\left(\mathrm{CHCl}_{3} / \mathrm{MeOH} / \mathrm{HCOOH}, 90: 10: 1\right)$.

IR: $\tilde{v}=725(\mathrm{~s}), 1096(\mathrm{~m}), 1242(\mathrm{~s}), 1666(\mathrm{~m}), 1720(\mathrm{~m}), 3387(\mathrm{~b}) \mathrm{cm}^{-1}$.

${ }^{1}$ H-NMR (400 MHz, DMSO- $\left.d_{6}\right): \delta=2.67\left(\mathrm{~s}, 3 \mathrm{H}, \mathrm{CH}_{3}\right), 7.57(\mathrm{~d}, J=8.7 \mathrm{~Hz}, 1 \mathrm{H}, \mathrm{CH}), 8.10(\mathrm{~d}$, $J=8.7 \mathrm{~Hz}, 1 \mathrm{H}, \mathrm{CH})$.

${ }^{13}$ C-NMR (100 MHz, DMSO- $\left.d_{6}\right): \delta=12.0,126.2,128.1,134.0,136.6,157.7,166.9,169.6$, 177.5.

HRMS (ESI): $m / z[\mathrm{M}+\mathrm{H}]^{+}$für $\mathrm{C}_{9} \mathrm{H}_{8} \mathrm{~N}_{3} \mathrm{O}_{4}$ ber.: 222.0509, gef.: 222.0510.

\section{3-Hydroxy-6-((trimethylsilyl)ethinyl)picolinsäuremethylester (222)}

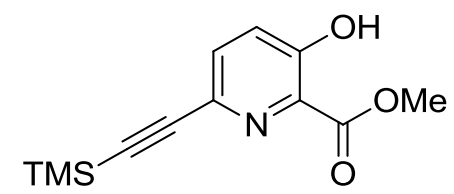

6-Brom-3-hydroxypicolinsäuremethylester ${ }^{[267]} \quad(\mathbf{2 0 4}, \quad 50 \mathrm{mg}, \quad 21.5 \mu \mathrm{mol}) \quad$ wurde $\quad$ in

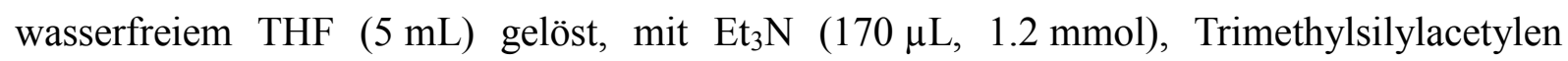
$(100 \mu \mathrm{L}, 0.69 \mathrm{mmol})$ und $\mathrm{CuI}(2.5 \mathrm{mg}, 5$ gew.-\%) versetzt und $15 \mathrm{~min}$ lang bei RT gerührt. Nach Zugabe von $\mathrm{PdCl}_{2}\left(\mathrm{PPh}_{3}\right)_{2}(7.5 \mathrm{mg}, 15$ gew.-\%) wurde das Gemisch $24 \mathrm{~h}$ lang gerührt. Anschließend wurde die Reaktionsmischung im Vakuum eingeengt und der Rückstand durch Säulenchromatographie $\left(\mathrm{SiO}_{2}, 25 \mathrm{~g}\right.$, Petrolether/EtOAc, 5:1) gereinigt. Es wurden $51 \mathrm{mg}$ (20.5 $\mu \mathrm{mol}, 95 \%)$ des TMS-Alkins 222 als braunes Harz erhalten.

DC: $R_{f}=0.51($ Heptan/EtOAc, $4: 1)$.

IR: $\tilde{v}=740(\mathrm{~m}), 840(\mathrm{~s}), 1203(\mathrm{~m}), 1288(\mathrm{w}), 1365(\mathrm{w}), 1450(\mathrm{~m}), 1674(\mathrm{~m}), 2962(\mathrm{w}) \mathrm{cm}^{-1}$.

${ }^{1}$ H-NMR $\left(300 \mathrm{MHz}, \mathrm{CDCl}_{3}\right): \delta=0.23\left(\mathrm{~s}, 9 \mathrm{H}, 3 \times \mathrm{CH}_{3}\right), 4.03\left(\mathrm{~s}, 3 \mathrm{H}, \mathrm{OCH}_{3}\right), 7.30(\mathrm{~d}$, $J=8.7 \mathrm{~Hz}, 1 \mathrm{H}, \mathrm{CH}), 7.55(\mathrm{~d}, J=8.7 \mathrm{~Hz}, 1 \mathrm{H}, \mathrm{CH}), 10.83(\mathrm{~s}, 1 \mathrm{H}, \mathrm{OH})$. 
${ }^{13}$ C-NMR $\left(100 \mathrm{MHz} \mathrm{CDCl}_{3}\right): \delta=-0.3,53.3,94.1,102.6,126.4,129.9,133.6,134.9,158.1$, 169.4 .

Elementaranalyse: Für $\mathrm{C}_{12} \mathrm{H}_{15} \mathrm{NO}_{3} \mathrm{Si}$ ber.: $\mathrm{C}, 57.80$; H, 6.06; N, 5.62; gef.: C, 57.66; H, 6.03; $\mathrm{N}, 5.54$.

\section{6-Ethinyl-3-hydroxypicolinsäuremethylester (223)}

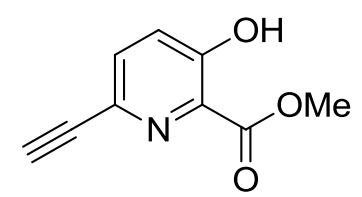

Zu einer Lösung des TMS-Alkins $222(51 \mathrm{mg}, 0.21 \mathrm{mmol})$ in wasserfreiem THF (1 mL) wurden wasserfreies $\mathrm{MeOH}(1 \mathrm{~mL})$ und KF $(36 \mathrm{mg}, 0.62 \mathrm{mmol})$ zugegeben. Die Mischung wurde anschließend $1.5 \mathrm{~h}$ lang bei RT gerührt, dann mit pH 6.2 Puffer (20 mL) verdünnt und mit $\mathrm{CH}_{2} \mathrm{Cl}_{2}(3 \times 10 \mathrm{~mL})$ extrahiert. Die vereinigten organischen Extrakte wurden mit $\mathrm{Na}_{2} \mathrm{SO}_{4}$ entwässert und im Vakuum eingeengt. Reinigung durch Säulenchromatographie $\left(\mathrm{SiO}_{2}, 25 \mathrm{~g}\right.$, Petrolether/EtOAc, 3:1) lieferte $31 \mathrm{mg}(17.6 \mu \mathrm{mol}, 86 \%)$ des Alkins 223 als farblosen Feststoff.

Schmelzpunkt: $169^{\circ} \mathrm{C}$.

DC: $R_{f}=0.24($ Heptan/EtOAc, $4: 1)$.

IR: $\tilde{v}=664(\mathrm{~m}), 725(\mathrm{~s}), 1103(\mathrm{~m}), 1195(\mathrm{~s}), 1442(\mathrm{~s}), 1666(\mathrm{~s}), 2160(\mathrm{w}), 3232(\mathrm{w}) \mathrm{cm}^{-1}$.

${ }^{1}$ H-NMR $\left(300 \mathrm{MHz}, \mathrm{CDCl}_{3}\right): \delta=3.07(\mathrm{~s}, 1 \mathrm{H}, \mathrm{CH}), 4.04\left(\mathrm{~s}, 3 \mathrm{H}, \mathrm{CH}_{3}\right), 7.34(\mathrm{~d}, J=8.7 \mathrm{~Hz}$, $1 \mathrm{H}, \mathrm{CH}), 7.58(\mathrm{~d}, J=8.7 \mathrm{~Hz}, 1 \mathrm{H}, \mathrm{CH}), 10.86(\mathrm{~s}, 1 \mathrm{H}, \mathrm{OH})$.

${ }^{13}$ C-NMR (75 MHz, $\left.\mathrm{CDCl}_{3}\right): \delta=53.3,81.8,126.5,130.2,133.3,133.9,158.4,169.3$.

Elementaranalyse: Für $\mathrm{C}_{9} \mathrm{H}_{7} \mathrm{NO}_{3}$ ber.: C, 61.02; H, 3.98; N, 7.91; gef.: C, 60.89; H, 3.93; N, 7.88 . 
<smiles>COC(=O)c1nc(-c2cnn[nH]2)ccc1O</smiles>

Zu einer Lösung des Alkins 223 (100 mg, $0.56 \mathrm{mmol})$ in wasserfreiem DMF (1.8 mL) und wasserfreiem $\mathrm{MeOH}(0.2 \mathrm{~mL})$ wurden $\mathrm{CuI}(5.4 \mathrm{mg}, 5 \mathrm{~mol} \%)$ und $\mathrm{TMSN}_{3}(112 \mu \mathrm{L}$, $0.85 \mathrm{mmol}$ ) zugegeben und die Mischung auf $100^{\circ} \mathrm{C}$ erhitzt. Nach $18 \mathrm{~h}$ wurde das Lösungsmittel im Vakuum entfernt und der Rückstand durch Säulenchromatographie $\left(\mathrm{SiO}_{2}\right.$, $40 \mathrm{~g}, \mathrm{CH}_{2} \mathrm{Cl}_{2} /$ Petrolether/Aceton, 1:1:1) gereinigt. Es wurden $79 \mathrm{mg}(35.9 \mu \mathrm{mol}, 64 \%)$ des Triazols 224 als gelber Feststoff erhalten.

Schmelzpunkt: $178^{\circ} \mathrm{C}$.

DC: $R_{f}=0.49\left(\mathrm{CH}_{2} \mathrm{Cl}_{2} /\right.$ Heptan/Aceton, $\left.1: 1: 1\right)$.

IR: $\tilde{v}=720(\mathrm{~s}), 817(\mathrm{~m}), 957(\mathrm{~m}), 1219(\mathrm{~m}), 1442(\mathrm{~s}), 1674(\mathrm{~m}), 2654(\mathrm{w}) \mathrm{cm}^{-1}$.

${ }^{1}$ H-NMR (400 MHz, DMSO- $d_{6} /$ TFA 1:1, $\left.343 \mathrm{~K}\right): \delta=3.94\left(\mathrm{~s}, 3 \mathrm{H}, \mathrm{CH}_{3}\right), 7.51(\mathrm{~d}, J=8.7 \mathrm{~Hz}$, 1H, CH), $8.04(\mathrm{~d}, J=8.7 \mathrm{~Hz}, 1 \mathrm{H}, \mathrm{CH}), 8.17(\mathrm{~s}, 1 \mathrm{H}, \mathrm{CH}), \mathrm{NH}-$ und OH-Signale nicht beobachtet.

${ }^{13}$ C-NMR (100 MHz, DMSO-d ${ }_{6} /$ TFA 1:1): $\delta=52.1,125.4,126.4,127.5,132.8,141.3,145.1$, $154.9,167.5$.

HRMS (ESI): $m / z[\mathrm{M}+\mathrm{H}]^{+}$ber. für $\mathrm{C}_{9} \mathrm{H}_{9} \mathrm{~N}_{4} \mathrm{O}_{3}: 221.0669$, gef.: 221.0671 .

\section{6-(Benzo[b]thiophen-2-yl)-3-hydroxypicolinsäure (225a)}

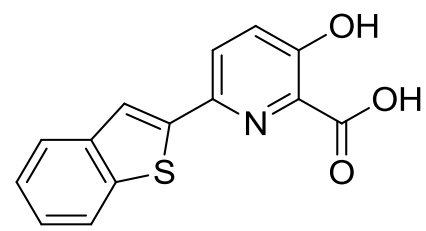

Der Picolinsäuremethylester $216 f(28.5 \mathrm{mg}, 0.10 \mathrm{mmol})$ wurde nach AV2 umgesetzt. Es wurden $22 \mathrm{mg}(81 \mu \mathrm{mol}, 81 \%)$ des Produkts 225a als leicht gelber Feststoff erhalten.

Schmelzpunkt: $193^{\circ} \mathrm{C}$ (Zers.). 
IR: $\tilde{v}=314(\mathrm{~s}), 833(\mathrm{w}), 1095(\mathrm{w}), 1230(\mathrm{~s}), 1392(\mathrm{~s}), 1469(\mathrm{~m}), 3305(\mathrm{w}) \mathrm{cm}^{-1}$.

${ }^{1}$ H-NMR $\left(300 \mathrm{MHz}, \mathrm{CDCl}_{3}\right): \delta=7.37-7.40(\mathrm{~m}, 2 \mathrm{H}, 2 \times \mathrm{CH}), 7.50(\mathrm{~d}, J=8.8 \mathrm{~Hz}, 1 \mathrm{H}, \mathrm{CH})$, $7.75(\mathrm{~s}, 1 \mathrm{H}, \mathrm{CH}), 7.80-7.87(\mathrm{~m}, 2 \mathrm{H}, 2 \times \mathrm{CH}), 8.00(\mathrm{~d}, J=8.8 \mathrm{~Hz}, 1 \mathrm{H}, \mathrm{CH}), 10.39(\mathrm{~s}, 1 \mathrm{H}, \mathrm{OH})$.

${ }^{13}$ C-NMR $\left(75 \mathrm{MHz}, \mathrm{CDCl}_{3}\right): \delta=124.3,124.9,125.6,127.4,127.5,128.0,140.2,140.3$, $141.7,144.0,157.3,168.4$.

HRMS (ESI): $m / z[\mathrm{M}+\mathrm{H}]^{+}$für $\mathrm{C}_{14} \mathrm{H}_{10} \mathrm{NO}_{3} \mathrm{~S}$ ber.: 272.0376, gef.: 272.0385 .

\section{6-(Benzo[b]furan-2'-yl)-3-hydroxypicolinsäure (225b)}

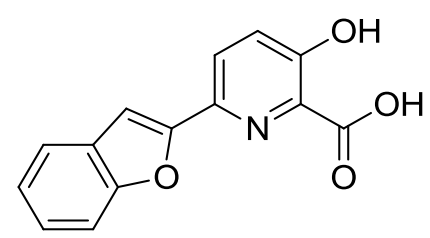

Der Picolinsäuremethylester $\mathbf{2 1 6 g}(27 \mathrm{mg}, 0.10 \mathrm{mmol})$ wurde nach AV2 umgesetzt. Es wurden $20 \mathrm{mg}$ (78 $\mu \mathrm{mol}, 78 \%$ ) des Produkts 225b als farbloser Feststoff erhalten.

Schmelzpunkt: $201{ }^{\circ} \mathrm{C}$ (Zers).

IR: $\tilde{v}=636(\mathrm{~m}), 678(\mathrm{~s}), 806(\mathrm{~s}), 1199(\mathrm{~s}), 1323(\mathrm{~m}), 1427(\mathrm{~m}), 1462(\mathrm{~m}), 1654(\mathrm{~m})$, 3062 (b) $\mathrm{cm}^{-1}$.

${ }^{1}$ H-NMR $\left(300 \mathrm{MHz}\right.$, Dioxan- $\left.d_{6}\right): \delta=7.23-7.35(\mathrm{~m}, 2 \mathrm{H}, 2 \times \mathrm{CH}), 7.51-7.57(\mathrm{~m}, 3 \mathrm{H}, 3 \times \mathrm{CH})$, $7.64(\mathrm{~d}, J=7.5 \mathrm{~Hz}, 1 \mathrm{H}, \mathrm{CH}), 8.08$ (d, $J=8.8 \mathrm{~Hz}, 1 \mathrm{H}, \mathrm{CH}), 10.96$ (bs, 1H, OH).

${ }^{13}$ C-NMR (75 MHz, Dioxan- $\left.d_{6}\right): \delta=104.8,112.1,122.3,124.2,125.9,127.0,127.8,129.8$, $130.9,141.1,155.1,156.0,158.7,170.6$.

HRMS (ESI): $m / z[\mathrm{M}+\mathrm{H}]^{+}$für $\mathrm{C}_{14} \mathrm{H}_{10} \mathrm{NO}_{4}$ ber.: 256.0604, gef.: 256.0605 . 


\section{3-Hydroxy-6-(3'-pyridyl)picolinsäure (225c)}<smiles>O=C(O)c1nc(-c2cccnc2)ccc1O</smiles>

Der Picolinsäuremethylester 216j (50 mg, 0.22 mmol) wurde nach AV2 umgesetzt. Es wurden $40 \mathrm{mg}(0.18 \mathrm{mmol}, 85 \%)$ des Produkts als leicht gelber Feststoff erhalten. Das Produkt 225c wurde isoliert indem das Rohprodukt in einer minimalen Menge konz. $\mathrm{HCl}$ aufgelöst, durch Zugabe von ges. $\mathrm{KHCO}_{3}$-Lsg. wieder ausgefällt, filtriert und mit einer kleinen Menge eiskalter $0.1 \mathrm{~N} \mathrm{HCl}$ gewaschen wurde.

Schmelzpunkt: $240{ }^{\circ} \mathrm{C}$ (Zers).

IR: $\tilde{v}=624(\mathrm{~m}), 1026(\mathrm{w}), 1408(\mathrm{~s}), 1558(\mathrm{~s}), 1739(\mathrm{w}), 2495(\mathrm{~b}), 3356(\mathrm{~b}) \mathrm{cm}^{-1}$.

${ }^{1}$ H-NMR $\left(300 \mathrm{MHz}, \mathrm{D}_{2} \mathrm{O}\right): \delta=5.63-5.76(\mathrm{~m}, 3 \mathrm{H}, 3 \times \mathrm{CH}), 6.36-6.40(\mathrm{~m}, 2 \mathrm{H}, 2 \times \mathrm{CH}), 6.70$ (s, 1H, CH).

${ }^{13}$ C-NMR (75 MHz, $\left.\mathrm{D}_{2} \mathrm{O}\right): \delta=124.1,126.2,128.9,130.5,135.0,138.1,139.3,141.1,145.2$, $157.0,161.8$.

HRMS (ESI): $m / z[\mathrm{M}+\mathrm{H}]^{+}$für $\mathrm{C}_{11} \mathrm{H}_{9} \mathrm{~N}_{2} \mathrm{O}_{3}$ ber.: 217.0608, gef.: 217.0606.

\section{3-Hydroxy-6-(thiophen-2-yl)picolinsäure (225d)}<smiles>O=C(O)c1nc(-c2cccs2)ccc1O</smiles>

Der Picolinsäuremethylester 2161 (23.5 $\mathrm{mg}, 0.10 \mathrm{mmol})$ wurde nach AV2 umgesetzt. Es wurden $13.7 \mathrm{mg}(6.2 \mu \mathrm{mol}, 62 \%)$ des Produkts 225d als beiger Feststoff erhalten.

Schmelzpunkt: $143^{\circ} \mathrm{C}$.

IR: $\tilde{v}=694(\mathrm{~s}), 840(\mathrm{~m}), 1099(\mathrm{~m}), 1219(\mathrm{~s}), 1392(\mathrm{~s}), 1466(\mathrm{~m}), 1716(\mathrm{~s}), 3278(\mathrm{w}) \mathrm{cm}^{-1}$. 
${ }^{1} \mathbf{H}-\mathbf{N M R}\left(300 \mathrm{MHz}, \mathrm{CDCl}_{3}\right): \delta=7.12(\mathrm{dd}, J=4.8,3.9 \mathrm{~Hz}, 1 \mathrm{H}, \mathrm{CH}), 7.41(\mathrm{~d}, J=4.8 \mathrm{~Hz}, 1 \mathrm{H}$, $\mathrm{CH}), 7.46(\mathrm{~d}, J=8.8 \mathrm{~Hz}, 1 \mathrm{H}, \mathrm{CH}), 7.51(\mathrm{~d}, J=3.6 \mathrm{~Hz}, 1 \mathrm{H}, \mathrm{CH}), 7.86(\mathrm{~d}, J=8.8 \mathrm{~Hz}, 1 \mathrm{H}$, $\mathrm{CH}), 10.29$ (bs, 1H, OH).

${ }^{13}$ C-NMR $\left(75 \mathrm{MHz}, \mathrm{CDCl}_{3}\right): \delta=125.0,126.9,127.2,127.8,128.0,128.3,141.9,144.1$, $156.8,168.5$.

HRMS (ESI): $m / z[\mathrm{M}+\mathrm{H}]^{+}$für $\mathrm{C}_{10} \mathrm{H}_{8} \mathrm{NO}_{3} \mathrm{~S}$ ber.: 222.0219, gef.: 222.0219.

\section{3-Hydroxy-6-(thiophen-3-yl)picolinsäure (225e)}<smiles>O=C(O)c1nc(-c2ccsc2)ccc1O</smiles>

Der Picolinsäuremethylester 216m (23.5 mg, $0.10 \mathrm{mmol})$ wurde nach AV2 umgesetzt. Es wurden $14.5 \mathrm{mg}(65 \mu \mathrm{mol}, 65 \%)$ des Produkts 225e als leicht gelber Feststoff erhalten.

Schmelzpunkt: $156.5^{\circ} \mathrm{C}$.

IR: $\tilde{v}=783(\mathrm{~s}), 848(\mathrm{~m}), 1192(\mathrm{~m}), 1215(\mathrm{~s}), 1465(\mathrm{~m}), 1639(\mathrm{~m}), 2924(\mathrm{w}), 3232(\mathrm{w}) \mathrm{cm}^{-1}$.

${ }^{1}$ H-NMR $\left(300 \mathrm{MHz}, \mathrm{MeOH}-d_{4}\right): \delta=7.49(\mathrm{dd}, J=4.8,3.0 \mathrm{~Hz}, 1 \mathrm{H}, \mathrm{CH}), 7.54(\mathrm{~d}, J=8.8 \mathrm{~Hz}$, $1 \mathrm{H}, \mathrm{CH}), 7.72(\mathrm{~d}, J=5.0 \mathrm{~Hz}, 1 \mathrm{H}, \mathrm{CH}), 7.97(\mathrm{~d}, J=8.8 \mathrm{~Hz}, 1 \mathrm{H}, \mathrm{CH}), 8.05(\mathrm{~d}, J=2.9 \mathrm{~Hz}, 1 \mathrm{H}$, $\mathrm{CH})$.

${ }^{13}$ C-NMR $\left(75 \mathrm{MHz}, \mathrm{MeOH}-d_{4}\right): \delta=125.3,127.4,127.7,128.7,129.7,129.9,140.5,145.8$, $158.8,171.1$.

HRMS (ESI): $m / z[\mathrm{M}+\mathrm{H}]^{+}$ber. für $\mathrm{C}_{10} \mathrm{H}_{8} \mathrm{NO}_{3} \mathrm{~S}: 222.0219$, gef.: 222.0225 .

6-(Furan-2-yl)-3-hydroxypicolinsäure (225f)<smiles>O=C(O)c1nc(-c2ccco2)ccc1O</smiles>

Der Picolinsäuremethylester $2160(22 \mathrm{mg}, 0.10 \mathrm{mmol})$ wurde nach AV2 umgesetzt. Es wurden $19.5 \mathrm{mg}(95 \mu \mathrm{mol}, 95 \%)$ des Produkts $225 \mathrm{f}$ als beiger Feststoff erhalten. 
Schmelzpunkt: $174{ }^{\circ} \mathrm{C}$.

IR: $\tilde{v}=682(\mathrm{~m}), 756(\mathrm{~s}), 1222(\mathrm{~s}), 1392(\mathrm{~s}), 1458(\mathrm{~m}), 1716(\mathrm{~s}), 3224(\mathrm{w}) \mathrm{cm}^{-1}$.

${ }^{1} \mathbf{H}-\mathbf{N M R}\left(300 \mathrm{MHz}, \mathrm{CDCl}_{3}\right): \delta=6.55(\mathrm{~d}, J=1.3 \mathrm{~Hz}, 1 \mathrm{H}, \mathrm{CH}), 6.97(\mathrm{~d}, J=3.4 \mathrm{~Hz}, 1 \mathrm{H}, \mathrm{CH})$, 7.48 (d, $J=8.8 \mathrm{~Hz}, 1 \mathrm{H}, \mathrm{CH}), 7.53$ (s, 1H, CH), 7.90 (d, J=8.8 Hz, 1H, CH), 9.43 (bs, 2H, $2 \times \mathrm{OH})$.

${ }^{13}$ C-NMR $\left(75 \mathrm{MHz}, \mathrm{CDCl}_{3}\right): \delta=108.6,112.2,126.5,127.5,128.0,140.7,143.6,151.4$, $156.7,168.5$.

HRMS (ESI): $m / z[\mathrm{M}+\mathrm{H}]^{+}$für $\mathrm{C}_{10} \mathrm{H}_{8} \mathrm{NO}_{4}$ ber.: 206.0448, gef.: 206.0446.

\section{6-Cyano-3-hydroxypicolinsäure (225g)}<smiles>N#Cc1ccc(O)c(C(=O)O)n1</smiles>

6-Cyano-3-hydroxypicolinsäuremethylester (219, $50 \mathrm{mg}, 281 \mu \mathrm{mol})$ wurde nach AV2 umgesetzt. Es wurden $45.5 \mathrm{mg}$ (278 mmol, 99\%) der Picolinsäure 225g als farbloser Feststoff erhalten.

Schmelzpunkt: $190^{\circ} \mathrm{C}$ (Zers.).

DC: $R_{f}=0.18\left(\mathrm{CH}_{2} \mathrm{Cl}_{2} / \mathrm{MeOH}, 10: 1\right)$.

IR: $\tilde{v}=732(\mathrm{w}), 848(\mathrm{w}), 1203(\mathrm{~m}), 1458(\mathrm{~m}), 1674(\mathrm{~m}) \mathrm{cm}^{-1}$.

${ }^{1}$ H-NMR $\left(300 \mathrm{MHz}, \mathrm{DMSO}-d_{6}\right) \delta=7.56(\mathrm{~d}, J=8.7 \mathrm{~Hz}, 1 \mathrm{H}, \mathrm{CH}), 8.04(\mathrm{~d}, J=8.7 \mathrm{~Hz}, 1 \mathrm{H}$, $\mathrm{CH}), 11.46(\mathrm{bs}, 2 \mathrm{H}, 2 \times \mathrm{OH})$.

${ }^{13}$ C-NMR (63 MHz, DMSO- $\left.d_{6}\right): \delta=117.3,122.2,126.2,133.2,136.2,158.6,168.1$.

HRMS (ESI): $m / z\left[\mathrm{M}-\mathrm{H}^{+}\right]^{-}$für $\mathrm{C}_{7} \mathrm{H}_{3} \mathrm{~N}_{2} \mathrm{O}_{3}$ ber.: 163.0149, gef.: 163.0143 . 


\section{4,6-Dibrom-3-methoxymethoxy-picolinsäuremethylester (229)}<smiles>COC(=O)c1nc(Br)cc(Br)c1OC</smiles>

Eine Lösung von 4,6-Dibrom-3-hydroxypicolinsäuremethylester ${ }^{[278]}$ (228, $\left.4.10 \mathrm{~g}, 13.2 \mathrm{mmol}\right)$ in wasserfreiem $\mathrm{CH}_{2} \mathrm{Cl}_{2}(105 \mathrm{~mL})$ wurde bei $0^{\circ} \mathrm{C}$ mit $\mathrm{EtNi} \operatorname{Pr}_{2}(6.78 \mathrm{~mL}, 39.6 \mathrm{mmol})$ und MOMCl (Achtung!, $2.01 \mathrm{~mL}, 26.4 \mathrm{mmol}$ ) versetzt. Das Gemisch wurde langsam aufgetaut und $20 \mathrm{~h}$ lang gerührt. Nach Zugabe von ges. $\mathrm{NaHCO}_{3}$-Lösung $(150 \mathrm{~mL})$ wurde mit $\mathrm{CH}_{2} \mathrm{Cl}_{2}$ $(3 \times 75 \mathrm{~mL})$ extrahiert und die vereinigten organischen Extrakte mit $\mathrm{Na}_{2} \mathrm{SO}_{4}$ entwässert. Das Lösungsmittel wurde im Vakuum entfernt und der Rückstand durch Säulenchromatographie $\left(\mathrm{SiO}_{2}, 250 \mathrm{~g}\right.$, Petrolether/EtOAc, 2:1) gereinigt. Es wurden $4.07 \mathrm{~g}(11.5 \mathrm{mmol}, 87 \%)$ des MOM-Ethers 229 als farbloser Feststoff erhalten.

Schmelzpunkt: $66.5^{\circ} \mathrm{C}$.

DC: $R_{f}=0.56($ Heptan/EtOAc, $2: 1)$.

IR: $\tilde{v}=712$ (s), 803 (s), 863 (s), 914 (s), $1071(\mathrm{~m}), 1208$ (s), 1423 (m), 1546 (w), 1717 (m), $2837(\mathrm{w}), 2943(\mathrm{w}), 2962(\mathrm{w}) \mathrm{cm}^{-1}$.

${ }^{1} \mathbf{H}-\mathbf{N M R}\left(400 \mathrm{MHz}, \mathrm{CDCl}_{3}\right): \delta=3.59\left(\mathrm{~s}, 3 \mathrm{H}, \mathrm{CH}_{3}\right), 3.96\left(\mathrm{~s}, 3 \mathrm{H}, \mathrm{CH}_{3}\right), 5.15\left(2 \mathrm{H}, \mathrm{CH}_{2}\right), 7.85$ (s, 1H, CH).

${ }^{13}$ C-NMR (100 MHz, $\left.\mathrm{CDCl}_{3}\right): \delta=53.2,58.2,101.1,131.5,134.7,135.2,144.8,150.5,163.4$.

HRMS (ESI): $m / z[\mathrm{M}+\mathrm{H}]^{+}$für $\mathrm{C}_{9} \mathrm{H}_{10} \mathrm{Br}_{2} \mathrm{NO}_{4}$ ber.: 353.8951 , gef.: 353.8956 .

\section{4-Brom-3-hydroxypicolinsäuremethylester (230)}<smiles>COC(=O)c1nccc(Br)c1O</smiles>

In Analogie zu Verdelet et al. ${ }^{[278]}$ wurde der MOM-Ether 229 (271 mg, $0.76 \mathrm{mmol}$ ) zusammen mit Natriumformiat $(58 \mathrm{mg}, 0.85 \mathrm{mmol})$ in entgastem DMF $(6 \mathrm{~mL})$ suspendiert und mit 
$\mathrm{Pd}\left(\mathrm{PPh}_{3}\right)_{4}(44 \mathrm{mg}, 5 \mathrm{~mol} \%)$ versetzt. Das Gemisch wurde auf $80{ }^{\circ} \mathrm{C}$ erhitzt und $20 \mathrm{~h}$ lang gerührt. Das Lösungsmittel wurde danach im Vakuum entfernt und der Rückstand durch Säulenchromatographie $\left(\mathrm{SiO}_{2}, 40 \mathrm{~g}\right.$, Petrolether- EtOAc, 2:1) gereinigt. Die Fraktionen mit einem $R_{f}=0.38$ (Heptan/EtOAc, 2:1) wurden vereinigt und eingeengt. Der erhaltene Feststoff wurde in wasserfreiem $\mathrm{CH}_{2} \mathrm{Cl}_{2}(3 \mathrm{ml})$ gelöst und mit TFA $(1 \mathrm{~mL})$ versetzt. Das Gemisch wurde 20 min lang bei RT gerührt, mit Toluol $(3 \mathrm{~mL})$ verdünnt und im Vakuum eingeengt. Nach Säulenchromatographie des Rückstands $\left(\mathrm{SiO}_{2}, \quad 25 \mathrm{~g}\right.$, Petrolether/ $\mathrm{CH}_{2} \mathrm{Cl}_{2} /$ Aceton/HCOOH, 110:110:10:1) wurden $46 \mathrm{mg}(0.17 \mathrm{mmol}, 22 \%)$ des Hydroxypyridins $\mathbf{2 3 0}$ als farbloser Feststoff erhalten.

Schmelzpunkt: $97^{\circ} \mathrm{C}$.

DC: $R_{f}=0.45\left(\mathrm{Heptan} / \mathrm{CH}_{2} \mathrm{Cl}_{2} /\right.$ Aceton/HCOOH, 100:100:10:1).

IR: $\tilde{v}=710$ (s), 856 (s), 1087 (s), 1165 (s), 1242 (s), 1350 (s), 1411 (s), 1558 (s), 1674 (s), 1913 (b), 2322 (w), 3109 (b) $\mathrm{cm}^{-1}$.

${ }^{1}$ H-NMR $\left(300 \mathrm{MHz}, \mathrm{CDCl}_{3}\right): \delta=4.05\left(\mathrm{~s}, 3 \mathrm{H}, \mathrm{CH}_{3}\right), 7.68$ (d, J=4.8 Hz, 1H, CH), 8.06 (d, $J=4.7 \mathrm{~Hz}, 1 \mathrm{H}, \mathrm{CH}), 11.28(\mathrm{~s}, 1 \mathrm{H}, \mathrm{OH})$.

${ }^{13}$ C-NMR (75 MHz, $\left.\mathrm{CDCl}_{3}\right): \delta=53.6,122.4,140.4,133.1,141.2,156.2,169.5$.

HRMS (ESI): $m / z[\mathrm{M}+\mathrm{H}]^{+}$für $\mathrm{C}_{7} \mathrm{H}_{7} \mathrm{BrNO}_{3}$ ber.: 231.9604, gef.: 231.9608 .

\section{4-Brom-3-hydroxypicolinsäure (231)}<smiles>O=C(O)c1nccc(Br)c1O</smiles>

Eine Lösung des Methylesters 230 (70 mg, $0.30 \mathrm{mmol})$ in THF (4 mL) und $\mathrm{H}_{2} \mathrm{O}(2 \mathrm{ml})$ wurde mit $\mathrm{LiOH} \times \mathrm{H}_{2} \mathrm{O}(26 \mathrm{mg}, 0.62 \mathrm{mmol})$ versetzt und $24 \mathrm{~h}$ lang bei RT gerührt. Das Gemisch wurde anschließend mit $\mathrm{AcOH}(0.5 \mathrm{~mL})$ versetzt und im Vakuum eingeengt. Der Rückstand wurde in $\mathrm{HCl}(4 \mathrm{~mL}, 0.1 \mathrm{M})$ gelöst, die wässrige Phase mit $\mathrm{NaCl}$ gesättigt und mit $\mathrm{CHCl}_{3}$ $20 \mathrm{~h}$ lang in einem Perforator kontinuierlich extrahiert. Die organische Phase wurde mit $\mathrm{Na}_{2} \mathrm{SO}_{4}$ entwässert und das Lösungsmittel am Rotationsverdampfer entfernt. Nach Trocknen 
des Rückstands am Hochvakuum wurden $57 \mathrm{mg}$ (0.26 mmol, 87\%) der Picolinsäure 231 als farbloser Feststoff erhalten.

Schmelzpunkt: $221.5-222.0^{\circ} \mathrm{C}$ (Zers.).

IR: $\tilde{v}=610$ (s), 980 (s), 1080 (s), 1473 (s), 1589 (b), 1674 (b), 1975 (b), 3055 (b), $3101(\mathrm{~b}) \mathrm{cm}^{-1}$.

${ }^{1}$ H-NMR $\left(300 \mathrm{MHz}, \mathrm{DMSO}-d_{6}\right): \delta=7.89(\mathrm{~d}, J=5.0 \mathrm{~Hz}, 1 \mathrm{H}, \mathrm{CH}), 8.15(\mathrm{~d}, J=5.0 \mathrm{~Hz}, 1 \mathrm{H}$, $\mathrm{CH})$.

${ }^{13}$ C-NMR (75 MHz, DMSO- $\left.d_{6}\right): \delta=127.6,130.4,131.5,132.5,160.3,165.1$.

HRMS (ESI): $m / z[\mathrm{M}+\mathrm{H}]^{+}$für $\mathrm{C}_{6} \mathrm{H}_{5} \mathrm{BrNO}_{3}$ ber.: 217.9447, gef.: 217.9449 .

3-Methoxymethoxypicolinsäuremethylester $(101)^{[321]}$<smiles>COC(=O)c1ncccc1OC</smiles>

Eine Lösung von 3-Hydroxypicolinsäuremethylester ${ }^{[278]}$ (100, $\left.500 \mathrm{mg}, 3.26 \mathrm{mmol}\right)$ in wasserfreiem $\mathrm{CH}_{2} \mathrm{Cl}_{2}(25 \mathrm{~mL})$ wurde auf $0^{\circ} \mathrm{C}$ gekühlt und nacheinander mit $\mathrm{EtN} \mathrm{Pr}_{2}$ (1.75 mL, $10.1 \mathrm{mmol}$ ) und Methoxymethylchlorid (Achtung!, $545 \mu \mathrm{L}, 7.17 \mathrm{mmol}$ ) versetzt. Nach 15 min Rühren bei $0{ }^{\circ} \mathrm{C}$ wurde das Kühlbad entfernt und die Mischung $3 \mathrm{~h}$ lang bei RT gerührt. Die Reaktionsmischung wurde mit pH 3 Puffer $(50 \mathrm{~mL})$ verdünnt und mit $\mathrm{CH}_{2} \mathrm{Cl}_{2}$ $(3 \times 20 \mathrm{~mL})$ extrahiert. Die vereinigten organischen Extrakte wurden mit $\mathrm{Na}_{2} \mathrm{SO}_{4}$ entwässert und im Vakuum eingeengt. Reinigung des Rückstands durch Säulenchromatographie $\left(\mathrm{SiO}_{2}\right.$, $40 \mathrm{~g}$, Petrolether/EtOAc, 1:1) ergab $629 \mathrm{mg}$ (3.19 mmol, 98\%) des MOM-Ethers 101 als farbloses Öl.

DC: $R_{f}=0.24($ Heptan/EtOAc, $1: 1)$.

IR: $\tilde{v}=663(\mathrm{w}), 802(\mathrm{~m}), 964$ (s), 1080 (s), 1099 (s), 1199 (s), 1300 (s), 1450 (m), 1732 (s), $2954(\mathrm{w}) \mathrm{cm}^{-1}$. 
${ }^{1} \mathbf{H}-\mathbf{N M R}\left(300 \mathrm{MHz}, \mathrm{CDCl}_{3}\right): \delta=3.50\left(\mathrm{~s}, 3 \mathrm{H}, \mathrm{CH}_{3}\right), 3.96\left(\mathrm{~s}, 3 \mathrm{H}, \mathrm{CH}_{3}\right), 5.26\left(\mathrm{~s}, 2 \mathrm{H}, \mathrm{CH}_{2}\right)$, $7.37(\mathrm{dd}, J=8.6,4.6 \mathrm{~Hz}, 1 \mathrm{H}, \mathrm{CH}), 7.59(\mathrm{dd}, J=8.6,1.1 \mathrm{~Hz}, 1 \mathrm{H}, \mathrm{CH}), 8.31(\mathrm{dd}, J=4.5$, $1.2 \mathrm{~Hz}, 1 \mathrm{H}, \mathrm{CH})$.

${ }^{13}$ C-NMR (75 MHz, $\left.\mathrm{CDCl}_{3}\right): \delta=52.6,56.5,94.9,124.1,126.9,149.6,142.3,153.1,165.1$.

\section{3-Methoxymethoxy-5-phenylpicolinsäuremethylester (233)}<smiles>COC(=O)c1ncc(-c2ccccc2)cc1OC</smiles>

Eine Lösung von $\mathrm{B}_{2} \operatorname{pin}_{2}(25 \mathrm{mg}, 98.4 \mu \mathrm{mol}), \operatorname{Ir}(\mathrm{OMe})(1,5-\mathrm{COD})_{2}(8.3 \mathrm{mg}, 2.5 \mathrm{~mol} \%)$ und 4,4'-Di-tert-butyl-2,2'-dipyridyl (6.7 mg, $5 \mathrm{~mol} \%)$ in THF $(0.5 \mathrm{~mL})$ wurde $30 \mathrm{~min}$ lang bei RT gerührt. Danach wurde weiteres $\mathrm{B}_{2} \operatorname{pin}_{2}(126 \mathrm{mg}, 0.50 \mathrm{mmol})$ und der MOM-Ether 101 (99 mg, $0.50 \mathrm{mmol}$ ) zugegeben. Das Gemisch wurde $20 \mathrm{~h}$ lang bei $50{ }^{\circ} \mathrm{C}$ gerührt, danach mit $\mathrm{Et}_{2} \mathrm{O}$ verdünnt und im Vakuum eingeengt. Der Rückstand wurde anschließend zwei Mal durch eine dünne $\mathrm{SiO}_{2}$-Schicht filtriert (Petrolether $/ \mathrm{CH}_{2} \mathrm{Cl}_{2} / \mathrm{Et}_{2} \mathrm{O}, 1: 1: 1$ ). Nach Einengen im Vakuum wurde der Boronsäureester als gelbes Öl erhalten, welches direkt weiter umgesetzt wurde.

Das Öl wurde in wasserfreiem DMF (5 mL) gelöst, mit Iodbenzol $(224 \mu \mathrm{L}, 1.99 \mathrm{mmol})$, $\mathrm{K}_{2} \mathrm{CO}_{3}(138 \mathrm{mg}, 1 \mathrm{mmol})$ und $\mathrm{Pd}\left(\mathrm{PPh}_{3}\right)_{4}(29 \mathrm{mg}, 5 \mathrm{~mol} \%)$ versetzt und $23 \mathrm{~h}$ lang bei $50^{\circ} \mathrm{C}$ gerührt. Nach Entfernen der Lösungsmittel im Vakuum wurde der Rückstand in pH 7 Puffer $(25 \mathrm{~mL})$ aufgenommen, mit $\mathrm{CHCl}_{3}(3 \times 10 \mathrm{~mL})$ extrahiert und die vereinigten organischen Extrakte mit $\mathrm{Na}_{2} \mathrm{SO}_{4}$ entwässert. Das Lösungsmittel wurde im Vakuum entfernt und der Rückstand durch Säulenchromatographie $\left(\mathrm{SiO}_{2}, 25 \mathrm{~g}\right.$, Petrolether $/ \mathrm{CH}_{2} \mathrm{Cl}_{2} /$ Aceton, 10:10:1) gereinigt. Es wurden $27 \mathrm{mg}(99 \mu \mathrm{mol}, 20 \%)$ des Esters 233 als leicht brauner Feststoff erhalten.

Schmelzpunkt: $59{ }^{\circ} \mathrm{C}$.

DC: $R_{f}=0.51\left(\right.$ Heptan/ $\mathrm{CH}_{2} \mathrm{Cl}_{2} /$ Aceton, 10:10:1).

IR: $\tilde{v}=662(\mathrm{~m}), 727(\mathrm{~s}), 740(\mathrm{~s}), 854(\mathrm{~m}), 1101(\mathrm{~m}), 1198(\mathrm{~s}), 1294(\mathrm{~s}), 1438(\mathrm{~s}), 1667(\mathrm{~m})$, $2961(\mathrm{w}), 3092(\mathrm{~b}), 3237(\mathrm{~m}) \mathrm{cm}^{-1}$. 
${ }^{1} \mathbf{H}-\mathbf{N M R}\left(400 \mathrm{MHz}, \mathrm{CDCl}_{3}\right): \delta=5.55\left(\mathrm{~s}, 3 \mathrm{H}, \mathrm{CH}_{3}\right), 4.00\left(\mathrm{~s}, 3 \mathrm{H}, \mathrm{CH}_{3}\right), 5.35\left(\mathrm{~s}, 2 \mathrm{H}, \mathrm{CH}_{2}\right)$, 7.42-7.51 (m, 3H, $3 \times \mathrm{CH}), 7.59-7.61(\mathrm{~m}, 2 \mathrm{H}, 2 \times \mathrm{CH}), 7.78(\mathrm{~d}, J=1.8 \mathrm{~Hz}, 1 \mathrm{H}, \mathrm{CH}), 8.56(\mathrm{~d}$, $J=1.8 \mathrm{~Hz}, 1 \mathrm{H}, \mathrm{CH})$.

${ }^{13}$ C-NMR $\left(100 \mathrm{MHz} \mathrm{CDCl}_{3}\right): \delta=52.6,56.6,95.1,122.4,127.4,128.9,129.2,136.5,137.6$, $140.5,140.9,153.6,164.9$.

HRMS (ESI): $m / z[\mathrm{M}+\mathrm{H}]^{+}$für $\mathrm{C}_{15} \mathrm{H}_{16} \mathrm{NO}_{4}$ ber.: 274.1074, gef.: 274.1078.

\subsection{Synthese von Bis-Carboxamid-Liganden}

\section{$N, N^{\prime}-(1,2-P h e n y l e n) b i s\left(3^{6}-h y d r o x y p i c o l i n a m i d\right)(102)$}

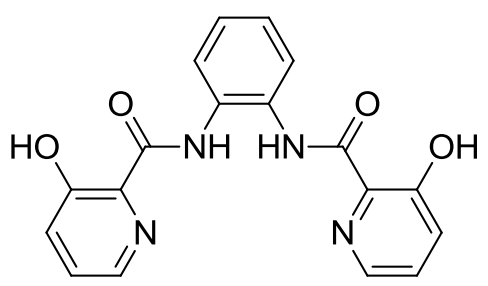

Eine Lösung von $o$-Phenylendiamin (239, $50 \mathrm{mg}, 0.46 \mathrm{mmol})$ und 3-Hydroxypicolinsäure (238, $135 \mathrm{mg}, 0.97 \mathrm{mmol})$ in wasserfreiem THF $(5 \mathrm{~mL})$ wurde auf $0{ }^{\circ} \mathrm{C}$ gekühlt, mit $\mathrm{EtN} \mathrm{Pr}_{2}$ (500 $\mu \mathrm{L}, 2.77 \mathrm{mmol})$ und PyAOP (1.01 g, $1.94 \mathrm{mmol})$ versetzt. Das Kühlbad wurde entfernt und die Mischung $4 \mathrm{~h}$ lang bei RT gerührt. Nach Zugabe von pH 3 Puffer (15 mL) wurde mit $\mathrm{CH}_{2} \mathrm{Cl}_{2}(3 \times 20 \mathrm{ml})$ extrahiert und die vereinigten organischen Extrakte mit $\mathrm{H}_{2} \mathrm{O}(20 \mathrm{ml})$ gewaschen. Die Lösung wurde mit $\mathrm{Na}_{2} \mathrm{SO}_{4}$ entwässert und im Vakuum eingeengt. Reinigung des Rückstands durch Säulenchromatographie $\left(\mathrm{SiO}_{2}, 25 \mathrm{~g}, \mathrm{CHCl}_{3} / \mathrm{EtOAc} / \mathrm{HCOOH}, 10: 5: 1\right)$ ergab 136 mg (0.39 mmol, 84\%) des Bis-Amids 102 als farblosen Feststoff.

Schmelzpunkt: $181.5^{\circ} \mathrm{C}$.

DC: $R_{f}=0.70\left(\mathrm{CHCl}_{3} / \mathrm{EtOAc} / \mathrm{HCOOH}, 10: 5: 1\right)$.

IR: $\tilde{v}=628(\mathrm{~m}), 694(\mathrm{~m}), 748(\mathrm{~s}), 1184(\mathrm{~m}), 1288(\mathrm{~m}), 1446(\mathrm{~m}), 1519(\mathrm{~m}), 1662(\mathrm{~m}), 3213$ (w), $3329(\mathrm{w}) \mathrm{cm}^{-1}$.

${ }^{1}$ H-NMR $\left(300 \mathrm{MHz}, \mathrm{CDCl}_{3}\right): \delta=7.33-.740(\mathrm{~m}, 6 \mathrm{H}, 6 \times \mathrm{CH}), 7.77(\mathrm{dd}, J=5.7,3.6 \mathrm{~Hz}, 2 \mathrm{H}$, $2 \times \mathrm{CH}), 8.08(\mathrm{~d}, J=2.0 \mathrm{~Hz}, 2 \mathrm{H}, 2 \times \mathrm{CH}), 10.25(\mathrm{~s}, 2 \mathrm{H}, 2 \times \mathrm{NH}), 11.73(\mathrm{~s}, 2 \mathrm{H}, 2 \times \mathrm{OH})$. 
${ }^{13}$ C-NMR (75 MHz, $\left.\mathrm{CDCl}_{3}\right): \delta=125.1,129.4,126.8,129.1,129.4,139.9,158.2,167.5$.

HRMS (ESI): $m / z[\mathrm{M}+\mathrm{H}]^{+}$für $\mathrm{C}_{18} \mathrm{H}_{15} \mathrm{~N}_{4} \mathrm{O}_{4}$ ber.: 351.1088 , gef.: 351.1091 .

3-Methoxypicolinsäure (241) ${ }^{[322]}$<smiles>COc1cccnc1C(=O)O</smiles>

3-Methoxypicolinsäuremethylester ${ }^{[308]}(\mathbf{2 4 0}, 1.5 \mathrm{~g}, 8.97 \mathrm{mmol})$ wurde in THF (18 mL) und $\mathrm{H}_{2} \mathrm{O}(9 \mathrm{~mL})$ gelöst, mit $\mathrm{LiOH} \times \mathrm{H}_{2} \mathrm{O}(753 \mathrm{mg}, 17.9 \mathrm{mmol})$ versetzt und $3 \mathrm{~h}$ lang bei RT gerührt. Das Lösungsmittel wurde im Vakuum entfernt und der Rückstand in $0.1 \mathrm{M} \mathrm{HCl}$ gelöst, die Lösung auf pH 2 angesäuert und mit festem $\mathrm{NaCl}$ gesättigt. Das Gemisch wurde $20 \mathrm{~h}$ lang mit $\mathrm{CHCl}_{3}$ mit einem Perforator kontinuierlich extrahiert, die organische Phase mit $\mathrm{Na}_{2} \mathrm{SO}_{4}$ entwässert und das Lösungsmittel am Rotationsverdampfer entfernt. Es wurden 1.17 g (7.62 mmol, 85\%) 3-Methoxy-picolinsäure (241) als farbloser Feststoff erhalten.

Schmelzpunkt: $149-150{ }^{\circ} \mathrm{C}$.

Alle relevanten analytischen Daten stimmen mit den veröffentlichten Werten überein. ${ }^{[322]}$

\section{$N, N^{\prime}$-(1,2-Phenylen)bis(3'-methoxypicolinamid) (104)}

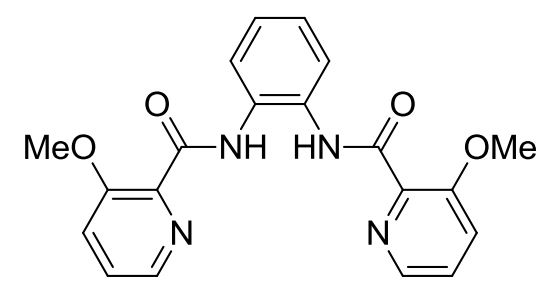

In Analogie zur Darstellung von 102 wurde $o$-Phenylendiamin (239, $390 \mathrm{mg}, 3.61 \mathrm{mmol}) \mathrm{mit}$ 3-Methoxypicolinsäure (241, 1.09 g, $7.22 \mathrm{mmol})$ umgesetzt. Nach Säulenchromatographie $\left(\mathrm{SiO}_{2}, 250 \mathrm{~g}, \mathrm{CHCl}_{3} / \mathrm{MeOH} / \mathrm{HCOOH}, 100: 5: 1\right)$ und Umkristallisieren aus $\mathrm{MeOH}$, wurden $493 \mathrm{mg}$ (2.60 mmol, 36\%) des Bis-Amids 104 als farbloser Feststoff erhalten.

Schmelzpunkt: $149-150{ }^{\circ} \mathrm{C}$.

DC: $R_{f}=0.47\left(\mathrm{CHCl}_{3} / \mathrm{MeOH} / \mathrm{HCOOH}, 100: 5: 1\right)$. 
IR: $\tilde{v}=741(\mathrm{~s}), 802(\mathrm{~m}), 1080$ (m), 1180 (m), 1242 (m), 1284 (s), 1421 (s), 1454 (s), 1485 (s), $1689(\mathrm{~s}), 3240(\mathrm{w}), 3356(\mathrm{w}) \mathrm{cm}^{-1}$.

${ }^{1} \mathbf{H}-\mathbf{N M R}\left(300 \mathrm{MHz}, \mathrm{CDCl}_{3}\right): \delta=3.95\left(\mathrm{~s}, 6 \mathrm{H}, 2 \times \mathrm{CH}_{3}\right), 7.24-7.29(\mathrm{~m}, 2 \mathrm{H}, 2 \times \mathrm{CH}), 7.40-$ $7.47(\mathrm{~m}, 4 \mathrm{H}, 4 \times \mathrm{CH}), 7.92(\mathrm{dd}, J=5.8,3.6 \mathrm{~Hz}, 2 \mathrm{H}, 2 \times \mathrm{CH}), 8.20(\mathrm{bs}, 2 \mathrm{H}, 2 \times \mathrm{CH}), 9.96(\mathrm{~s}$, $2 \mathrm{H}, 2 \times \mathrm{NH})$.

${ }^{13}$ C-NMR $\left(75 \mathrm{MHz}\right.$, DMSO- $\left.d_{6}\right): \delta=55.8,120.9,124.8,125.3,127.0,130.6,140.0,141.0$, 154.4, 163.3 .

HRMS (ESI): $m / z[\mathrm{M}+\mathrm{H}]^{+}$für $\mathrm{C}_{20} \mathrm{H}_{19} \mathrm{~N}_{4} \mathrm{O}_{4}$ ber.: 379.1401 , gef.: 379.1401 .

\section{6-Fluor-3-methoxymethoxypicolinsäuremthylester (243)}<smiles>COC(=O)c1nc(F)ccc1OC</smiles>

Eine Lösung des MOM-Ethers $101(1 \mathrm{~g}, 5.07 \mathrm{mmol})$ in wasserfreiem MeCN (101 mL) wurde mit $\mathrm{AgF}_{2}(3.7 \mathrm{~g}, 25.4 \mathrm{mmol})$ versetzt und $18 \mathrm{~h}$ lang bei RT gerührt. Die Mischung wurde durch Kieselguhr filtriert und der Filterkuchen mit EtOAc $(3 \times 100 \mathrm{~mL})$ gewaschen. Die organische Phase wurde mit ges. $\mathrm{NaHCO}_{3}$-Lsg., Wasser und ges. NaCl-Lsg. (je $100 \mathrm{~mL}$ ) gewaschen und anschließend mit $\mathrm{MgSO}_{4}$ entwässert. Die Lösung wurde im Vakuum eingeengt und der Rückstand durch Säulenchromatographie $\left(\mathrm{SiO}_{2}, 80 \mathrm{~g}\right.$, Petrolether - EtOAc, 1:1) gereinigt. Es wurden $574 \mathrm{mg}$ (2.66 mmol, 53\%) des fluorierten MOM-Ethers 243 als farbloses Öl zusammen mit nicht umgesetztem Ausgangsmaterial 101 (323 mg, 32\%) erhalten.

DC: $R_{f}=0.46($ Heptan$/$ EtOAc, $1: 1) ; R_{f}($ Ausgangsmaterial $)=0.24($ Heptan $/$ EtOAc, $1: 1)$.

IR: $\tilde{v}=667(\mathrm{w}), 725(\mathrm{w}), 921(\mathrm{~m}), 968$ (s), 1076 (s), 1153 (s), 1226 (s), 1462 (s), 1732 (s), $2954(\mathrm{w}) \mathrm{cm}^{-1}$.

${ }^{1} \mathbf{H}-\mathbf{N M R}\left(300 \mathrm{MHz}, \mathrm{CDCl}_{3}\right): \delta=3.52\left(\mathrm{~s}, 3 \mathrm{H}, \mathrm{CH}_{3}\right), 3.96\left(\mathrm{~s}, 3 \mathrm{H}, \mathrm{CH}_{3}\right), 5.24\left(\mathrm{~s}, 2 \mathrm{H}, \mathrm{CH}_{2}\right)$, $7.06(\mathrm{dd}, J=9.0,3.9 \mathrm{~Hz}, 1 \mathrm{H}, \mathrm{CH}), 7.75(\mathrm{dd}, J=9.0,6.2 \mathrm{~Hz}, 1 \mathrm{H}, \mathrm{CH})$.

${ }^{13}$ C-NMR (75 MHz, $\left.\mathrm{CDCl}_{3}\right): \delta=52.8,56.6,95.9,113.2(\mathrm{~d}, J=39.5 \mathrm{~Hz}), 130.9$ (d, $J=8.2 \mathrm{~Hz}), 135.8(\mathrm{~d}, J=13.4 \mathrm{~Hz}), 151.3(\mathrm{~d}, J=3.7 \mathrm{~Hz}), 156.6(\mathrm{~d}, J=237.7 \mathrm{~Hz}), 163.8$. 
HRMS (ESI): $m / z[\mathrm{M}+\mathrm{Na}]^{+}$für $\mathrm{C}_{9} \mathrm{H}_{10} \mathrm{FNNaO}_{4}$ ber.: 238.0486, gef.: 238.0488.

\section{6-Fluor-3-methoxypicolinsäuremethylester (242)}<smiles>COC(=O)c1nc(F)ccc1OC</smiles>

3-Methoxypicolinsäuremethylester ${ }^{[308]}(\mathbf{2 4 0}, 1.77 \mathrm{~g}, 10.6 \mathrm{mmol})$ wurde analog zur Darstellung von 243 mit $\mathrm{AgF}_{2}$ (4.60 g, $\left.31.8 \mathrm{mmol}\right)$ umgesetzt. Es wurden $1.33 \mathrm{~g}$ (7.18 mmol, 68\%) des fluorierten Pyridins 242 als farbloser Feststoff, zusammen mit $272 \mathrm{mg}$ (1.63 mmol, 15\%) nicht umgesetzten Ausgangsmaterial (240) erhalten.

Schmelzpunkt: $130^{\circ} \mathrm{C}$.

DC: $R_{f}=0.21($ Heptan/EtOAc, $2: 1)$.

IR: $\tilde{v}=667(\mathrm{~m}), 748(\mathrm{~m}), 837(\mathrm{~m}), 1007$ (s), $1096(\mathrm{~s}), 1215$ (s), 1431 (s), 1496 (s), 1728 (m) $\mathrm{cm}^{-1}$.

${ }^{1} \mathbf{H}-\mathbf{N M R}\left(300 \mathrm{MHz}, \mathrm{CDCl}_{3}\right): \delta=3.89\left(\mathrm{~s}, 3 \mathrm{H}, \mathrm{CH}_{3}\right), 3.99\left(\mathrm{~s}, 3 \mathrm{H}, \mathrm{CH}_{3}\right), 7.06(\mathrm{dd}, J=9.0$, $3.9 \mathrm{~Hz}, 1 \mathrm{H}, \mathrm{CH}), 7.49$ (dd, $J=9.0,6.0 \mathrm{~Hz}, 1 \mathrm{H}, \mathrm{CH})$.

${ }^{13}$ C-NMR $\left(75 \mathrm{MHz}, \mathrm{CDCl}_{3}\right): \delta=52.6,56.9,113.5(\mathrm{~d}, J=40.2 \mathrm{~Hz}), 126.2(\mathrm{~d}, J=7.5 \mathrm{~Hz})$, $134.2(\mathrm{~d}, J=13.4 \mathrm{~Hz}), 153.7(\mathrm{~d}, J=3.7 \mathrm{~Hz}), 155.6(\mathrm{~d}, J=236.2 \mathrm{~Hz}), 163.8$.

HRMS (ESI): $m / z[\mathrm{M}+\mathrm{H}]^{+}$für $\mathrm{C}_{8} \mathrm{H}_{9} \mathrm{FNO}_{3}$ ber.: 186.0561, gef.: 186.0562 .

6-Fluor-3-hydroxypicolinsäuremethylester (244)<smiles>COC(=O)c1nc(F)ccc1O</smiles>

Eine Lösung des MOM-Ethers $243(1.64 \mathrm{~g}, 7.62 \mathrm{mmol})$ in wasserfreiem $\mathrm{CH}_{2} \mathrm{Cl}_{2}(30 \mathrm{~mL})$ wurde mit TFA (10 mL) versetzt und die Mischung 20 min lang bei RT gerührt. Das Gemisch wurde anschließend mit Toluol $(100 \mathrm{~mL})$ verdünnt und im Vakuum zur Trockne eingeengt. 
Nach Säulenchromatographie $\left(\mathrm{SiO}_{2}, 150 \mathrm{~g}, \mathrm{CH}_{2} \mathrm{Cl}_{2} /\right.$ Petrolether/Aceton, 5:5:1) wurden $1.26 \mathrm{~g}$ (7.31 mmol, 96\%) des Phenols 244 als farbloser Feststoff erhalten.

Schmelzpunkt: $68-69^{\circ} \mathrm{C}$.

DC: $R_{f}=0.66\left(\mathrm{CH}_{2} \mathrm{Cl}_{2} /\right.$ Heptan/Aceton, 5:5:1).

IR: $\tilde{v}=671(\mathrm{~m}), 710(\mathrm{~m}), 778(\mathrm{~m}), 849(\mathrm{~m}), 1091(\mathrm{~s}), 1180(\mathrm{~s}), 1203(\mathrm{~s}), 1450(\mathrm{~s}), 1682(\mathrm{~m})$, $3248(\mathrm{w}) \mathrm{cm}^{-1}$.

${ }^{1} \mathbf{H}-\mathbf{N M R}\left(300 \mathrm{MHz}, \mathrm{CDCl}_{3}\right): \delta=4.03\left(\mathrm{~s}, 3 \mathrm{H}, \mathrm{CH}_{3}\right), 7.11(\mathrm{dd}, J=8.9,3.4 \mathrm{~Hz}, 1 \mathrm{H}, \mathrm{CH}), 7.49$ (dd, $J=8.9,6.3 \mathrm{~Hz}, 1 \mathrm{H}, \mathrm{CH}), 10.56$ (s, 1H, OH).

${ }^{13}$ C-NMR (75 MHz, $\left.\mathrm{CDCl}_{3}\right): \delta=53.2,117.3(\mathrm{~d}, J=41 \mathrm{~Hz}), 124.9(\mathrm{~d}, J=14.2 \mathrm{~Hz}), 132.2(\mathrm{~d}$, $J=7.5 \mathrm{~Hz}), 155.0(\mathrm{~d}, J=236.2 \mathrm{~Hz}), 156.9$ (d, $J=2.2 \mathrm{~Hz}), 169.0$.

HRMS (ESI): $m / z[\mathrm{M}+\mathrm{H}]^{+}$für $\mathrm{C}_{7} \mathrm{H}_{7} \mathrm{FNO}_{3}$ ber.: 172.0404, gef.: 172.0403 .

6-Fluor-3-hydroxypicolinsäure (246)<smiles>O=C(O)c1nc(F)ccc1O</smiles>

Der Methylester 244 (178 mg, $1.04 \mathrm{mmol})$ wurde in 1,4-Dioxan $(53 \mathrm{~mL})$ und $\mathrm{H}_{2} \mathrm{O}(27 \mathrm{~mL})$ gelöst, mit $\mathrm{KHCO}_{3}(416 \mathrm{mg}, 4.16 \mathrm{mmol})$ versetzt und das Gemisch $5 \mathrm{~h}$ lang bei $60{ }^{\circ} \mathrm{C}$ gerührt. Die Mischung wurde am Rotationsverdampfer eingeengt und der Rückstand in einer gerade ausreichenden Menge 0.1 M HCl (30-40 mL) gelöst und die Lösung mit festem $\mathrm{NaCl}$ gesättigt. Nach Extraktion mit EtOAc $(4 \times 50 \mathrm{~mL})$ wurden die vereinigten organischen Extrakte mit $\mathrm{MgSO}_{4}$ entwässert und im Vakuum eingeengt. Es wurden $157 \mathrm{mg}(1.00 \mathrm{mmol}$, 96\%) der Picolinsäure 246 als farbloser Feststoff erhalten.

Schmelzpunkt: $131-132.5^{\circ} \mathrm{C}$.

IR: 663 (s), 763 (m), 856 (m), 1207 (s), 1427 (s), 1454 (s), 1655 (m), $3086(\mathrm{w}) \mathrm{cm}^{-1}$.

${ }^{1} \mathbf{H}-\mathbf{N M R}\left(300 \mathrm{MHz}, \mathrm{CDCl}_{3}\right): \delta=4.41(\mathrm{bs}, 2 \mathrm{H}, 2 \times \mathrm{OH}), 7.10(\mathrm{dd}, J=8.9,3.0 \mathrm{~Hz}, 1 \mathrm{H}, \mathrm{CH})$, $7.48(\mathrm{dd}, J=8.8,6.4 \mathrm{~Hz}, 1 \mathrm{H}, \mathrm{CH})$. 
${ }^{13}$ C-NMR (75 MHz, $\left.\mathrm{CDCl}_{3}\right): \delta=117.3(\mathrm{~d}, J=43.2 \mathrm{~Hz}), 124.9(\mathrm{~d}, J=13.4 \mathrm{~Hz}), 132.3(\mathrm{~d}$, $J=7.4 \mathrm{~Hz}), 154.7$ (d, $J=238.4 \mathrm{~Hz}), 156.7$ (d, $J=2.2 \mathrm{~Hz}), 169.7$.

Elementaranalyse: Für $\mathrm{C}_{28} \mathrm{H}_{27} \mathrm{NO}_{4}$ ber.: C, 45.87; H, 2.57; N, 8.92; gef. C, 45.88; H, 2.57; N, 8.57 .

\section{6-Fluor-3-methoxypicolinsäure (245)}<smiles>COc1ccc(F)nc1C(=O)O</smiles>

Eine Lösung des Methylesters $242(500 \mathrm{mg}, 2.70 \mathrm{mmol})$ in $\mathrm{CH}_{2} \mathrm{Cl}_{2}(22 \mathrm{~mL})$ und $\mathrm{MeOH}$ $(10 \mathrm{~mL})$ wurde bei $0{ }^{\circ} \mathrm{C}$ mit einer Lösung aus 2,2,2-Trifluorethanol $(10 \mathrm{~mL})$ und $5 \mathrm{M} \mathrm{NaOH}$ $(2.7 \mathrm{~mL})$ tropfenweise versetzt. Das Gemisch wurde anschließend $20 \mathrm{~h}$ lang bei RT gerührt, mit pH 3 Puffer $(20 \mathrm{~mL})$ verdünnt und mit verd. $\mathrm{HCl}$ auf $\mathrm{pH} 2$ angesäuert. Die Mischung wurde mit $\mathrm{CHCl}_{3}(4 \times 20 \mathrm{~mL})$ extrahiert und die vereinigten organischen Extrakte mit $\mathrm{Na}_{2} \mathrm{SO}_{4}$ entwässert. Nach Entfernen der Lösungsmittel im Vakuum wurden $424 \mathrm{mg}$ (2.48 mmol, 92\%) der Picolinsäure 245 als leicht gelber Feststoff erhalten.

Schmelzpunkt: $155^{\circ} \mathrm{C}$.

IR: $\tilde{v}=748(\mathrm{~m}), 833(\mathrm{~m}), 1003(\mathrm{~m}), 1099(\mathrm{~m}), 1234(\mathrm{~s}), 1473(\mathrm{~m}), 1686(\mathrm{~m}) \mathrm{cm}^{-1}$.

${ }^{1}$ H-NMR $\left(300 \mathrm{MHz}, \mathrm{DMSO}-d_{6}\right): \delta=3.85\left(\mathrm{~s}, 3 \mathrm{H}, \mathrm{CH}_{3}\right), 7.33(\mathrm{dd}, J=9.0,3.5 \mathrm{~Hz}, 1 \mathrm{H}, \mathrm{CH})$, $7.84(\mathrm{dd}, J=9.0,6.3 \mathrm{~Hz}, 1 \mathrm{H}, \mathrm{CH})$.

${ }^{13}$ C-NMR (75 MHz, DMSO- $\left.d_{6}\right): \delta=56.9,112.4(\mathrm{~d}, J=39.5 \mathrm{~Hz}), 127.4(\mathrm{~d}, J=8.2 \mathrm{~Hz}), 136.6$ (d, $J=14.2 \mathrm{~Hz}), 151.9(\mathrm{~d}, J=3.7 \mathrm{~Hz}), 155.4(\mathrm{~d}, J=231.0 \mathrm{~Hz}), 165.1$.

HRMS (ESI): $m / z[\mathrm{M}+\mathrm{H}]^{+}$für $\mathrm{C}_{7} \mathrm{H}_{7} \mathrm{FNO}_{3}$ ber.: 172.0404, gef.: 172.0404 . 


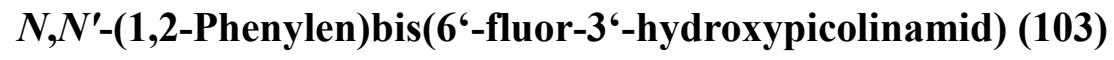

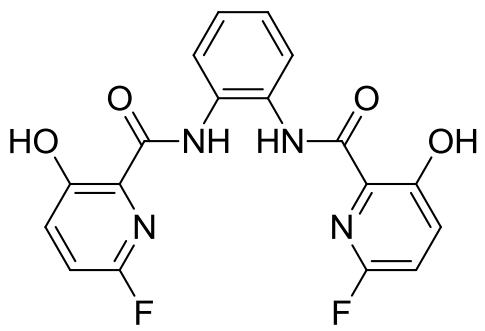

Eine Lösung von $o$-Phenylendiamin (239, $271 \mathrm{mg}, 2.58 \mathrm{mmol})$ und PyAOP (2.96 g, $5.68 \mathrm{mmol})$ in wasserfreiem THF $(16 \mathrm{ml})$ wurde auf $0{ }^{\circ} \mathrm{C}$ gekühlt und eine Lösung von 6Fluor-3-hydroxypicolinsäure $(\mathbf{2 4 6}, 811 \mathrm{mg}, 5.16 \mathrm{mmol})$ in wasserfreiem THF (40 mL) wurde langsam zugetropft. Nach vollständiger Zugabe wurde das Gemisch langsam aufgetaut und $18 \mathrm{~h}$ lang bei RT gerührt. Nach Zugabe von pH 3 Puffer $(150 \mathrm{~mL})$ wurde mit $\mathrm{CH}_{2} \mathrm{Cl}_{2}$ $(3 \times 75 \mathrm{~mL})$ extrahiert, die vereinigten organischen Extrakte mit $\mathrm{H}_{2} \mathrm{O}(75 \mathrm{~mL})$ gewaschen und mit $\mathrm{Na}_{2} \mathrm{SO}_{4}$ entwässert. Die Lösungsmittel wurden im Vakuum entfernt und der Rückstand durch Säulenchromatographie $\left(\mathrm{SiO}_{2}, 250 \mathrm{~g}, \mathrm{CHCl}_{3} / \mathrm{EtOAc}, 40: 1\right)$ gereinigt. Es wurden $580 \mathrm{mg}$ (1.49 mmol, 58\%) des Bis-Amids 103 als farbloser Feststoff erhalten.

Schmelzpunkt: $232^{\circ} \mathrm{C}$.

DC: $R_{f}=0.90\left(\mathrm{CHCl}_{3} / \mathrm{EtOAc}, 40: 1\right)$.

IR: $\tilde{v}=667(\mathrm{~s}), 756(\mathrm{~m}), 795(\mathrm{~m}), 968(\mathrm{~m}), 1165(\mathrm{~m}), 1249(\mathrm{~m}), 1427(\mathrm{~m}), 1450$ (s), 1512 (m), $1651(\mathrm{~m}), 3317(\mathrm{w}) \mathrm{cm}^{-1}$.

${ }^{1}$ H-NMR $\left(300 \mathrm{MHz}, \mathrm{CDCl}_{3}\right): \delta=7.08(\mathrm{dd}, J=8.9,3.1 \mathrm{~Hz}, 2 \mathrm{H}, 2 \times \mathrm{CH}), 7.39(\mathrm{dd}, J=5.9$, $3.5 \mathrm{~Hz}, 2 \mathrm{H}, 2 \times \mathrm{CH}), 7.51(\mathrm{dd}, J=8.8,6.4 \mathrm{~Hz}, 2 \mathrm{H}, 2 \times \mathrm{CH}), 7.75(\mathrm{dd}, J=5.9,3.6 \mathrm{~Hz}, 2 \mathrm{H}, 2 \times$ $\mathrm{CH}), 9.86(\mathrm{~s}, 2 \mathrm{H}, 2 \times \mathrm{NH}), 11.59(\mathrm{~s}, 2 \mathrm{H}, 2 \times \mathrm{OH})$.

${ }^{13}$ C-NMR (75 MHz, DMSO- $\left.d_{6}\right): \delta=116.9$ (d, $\left.J=41.0 \mathrm{~Hz}\right), 126.0,126.15,126.18,126.26$, 126.5, 129.9, 133.1 (d, $J=8.2 \mathrm{~Hz}), 154.0$ (d, $J=234.7 \mathrm{~Hz}), 155.7$ (d, $J=1.5 \mathrm{~Hz}), 166.4$.

HRMS (ESI): $m / z[\mathrm{M}+\mathrm{H}]^{+}$für $\mathrm{C}_{18} \mathrm{H}_{13} \mathrm{~F}_{2} \mathrm{~N}_{4} \mathrm{O}_{4}$ ber.: 387.0899, gef.: 387,0907. 


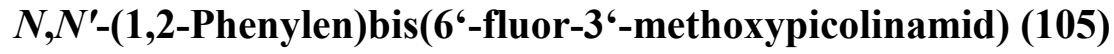

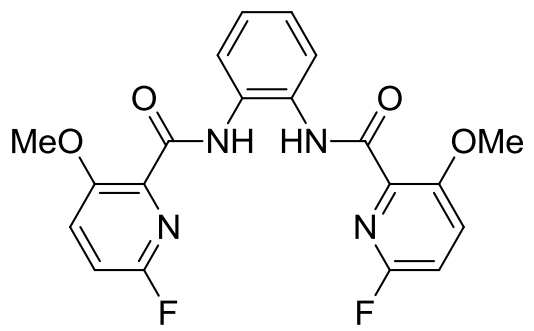

In Analogie zur Darstellung von 103 wurde $o$-Phenylendiamin (239, $218 \mathrm{mg}, 2.02 \mathrm{mmol}) \mathrm{mit}$ 6-Fluor-3-methoxypicolinsäure $\mathbf{( 2 4 5}, 687 \mathrm{mg}, 4.02 \mathrm{mmol})$ umgesetzt. Nach Säulenchromatographie $\left(\mathrm{SiO}_{2}, 250 \mathrm{~g}, \mathrm{CHCl}_{3} / \mathrm{MeOH} / \mathrm{HCOOH}, 150: 5: 1\right)$ und Umkristallisieren aus MeOH wurden 509 mg (1.22 mmol, 61\%) des Bis-Amids 105 als farbloser Feststoff erhalten.

Schmelzpunkt: $185^{\circ} \mathrm{C}$.

DC: $R_{f}=0.60\left(\mathrm{CHCl}_{3} / \mathrm{MeOH} / \mathrm{HCOOH}, 100: 5: 1\right)$.

IR: $\tilde{v}=756$ (s), 833 (m), 1076 (s), 1257 (s), 1435 (s), 1450 (s), 1485 (s), 1686 (m), $3356(\mathrm{w}) \mathrm{cm}^{-1}$.

${ }^{1} \mathbf{H}-\mathbf{N M R}\left(300 \mathrm{MHz}, \mathrm{CDCl}_{3}\right): \delta=3.97\left(\mathrm{~s}, 6 \mathrm{H}, 2 \times \mathrm{CH}_{3}\right), 7.09(\mathrm{dd}, J=8.9,3.7 \mathrm{~Hz}, 2 \mathrm{H}$, $2 \times \mathrm{CH}), 7.25-7.28(\mathrm{~m}, 2 \mathrm{H}, 2 \times \mathrm{CH}), 7.56(\mathrm{dd}, J=8.8,6.1 \mathrm{~Hz}, 2 \mathrm{H}, 2 \times \mathrm{CH}), 7.85(\mathrm{dd}, J=5.7$, $3.7 \mathrm{~Hz}, 2 \mathrm{H}, 2 \times \mathrm{CH}), 9.63(\mathrm{~s}, 2 \mathrm{H}, 2 \times \mathrm{NH})$.

${ }^{13}$ C-NMR (75 MHz, $\left.\mathrm{CDCl}_{3}\right): \delta=57.2,113.5,114.1,124.8,126.2,127.3,127.4,130.1,134.5$, 134.6, 154.36, 154.40, 156.6, 161.3.

HRMS (ESI): $m / z[\mathrm{M}+\mathrm{H}]^{+}$für $\mathrm{C}_{20} \mathrm{H}_{17} \mathrm{~F}_{2} \mathrm{~N}_{4} \mathrm{O}_{4}$ ber.: 415.1212, gef.: 415.1217 . 



\section{Abkürzungsverzeichnis}

$[\alpha] \quad$ Spezifische optische Drehung

)) Ultraschall

Ac Acetyl

All Allyl

Alloc Allyloxycarbonyl

Äq. $\quad$ Äquivalent

Bn Benzyl

ber. berechnet

Boc tert-Butyloxycarbonyl

brsm bezogen auf zurückgewonnenes Startmaterial

d Tag

DBU 1,8-Diazabicyclo[5.4.0]undec-7-en

DC Dünnschichtchromatographie

DCE 1,2-Dichlorethan

DEAD Diethylazodicarboxylat

DEPBT 3-(Diethoxyphosphoryloxy)-1,2,3-benzotriazin-4(3H)-on

Dha Dehdroalanin

Dhb Dehydrobutyrin

DIAD Di-iso-propylazodicarboxylat

DMA $\quad N, N$-Dimethylacetamid

DMAP 4-N,N-Dimethylaminopyridin

DME 1,2-Dimethoxyethan

DMF $\quad N, N$-Dimethylformamid

DMSO Dimethylsulfoxid

DNA Desoxyribonukleinsäure

Dpm Diphenylmethyl

dppf 1,1'-Bis(diphenylphosphino)ferrocen

dtbpy 4,4'-Di-tert-butyl-2,2'-dipyridyl

EDCI 1-Ethyl-3-(3-dimethylaminopropyl)carbodiimid Hydrochlorid

EF Elongationsfaktor

ESI Elektronensprayionisation

Et Ethyl 


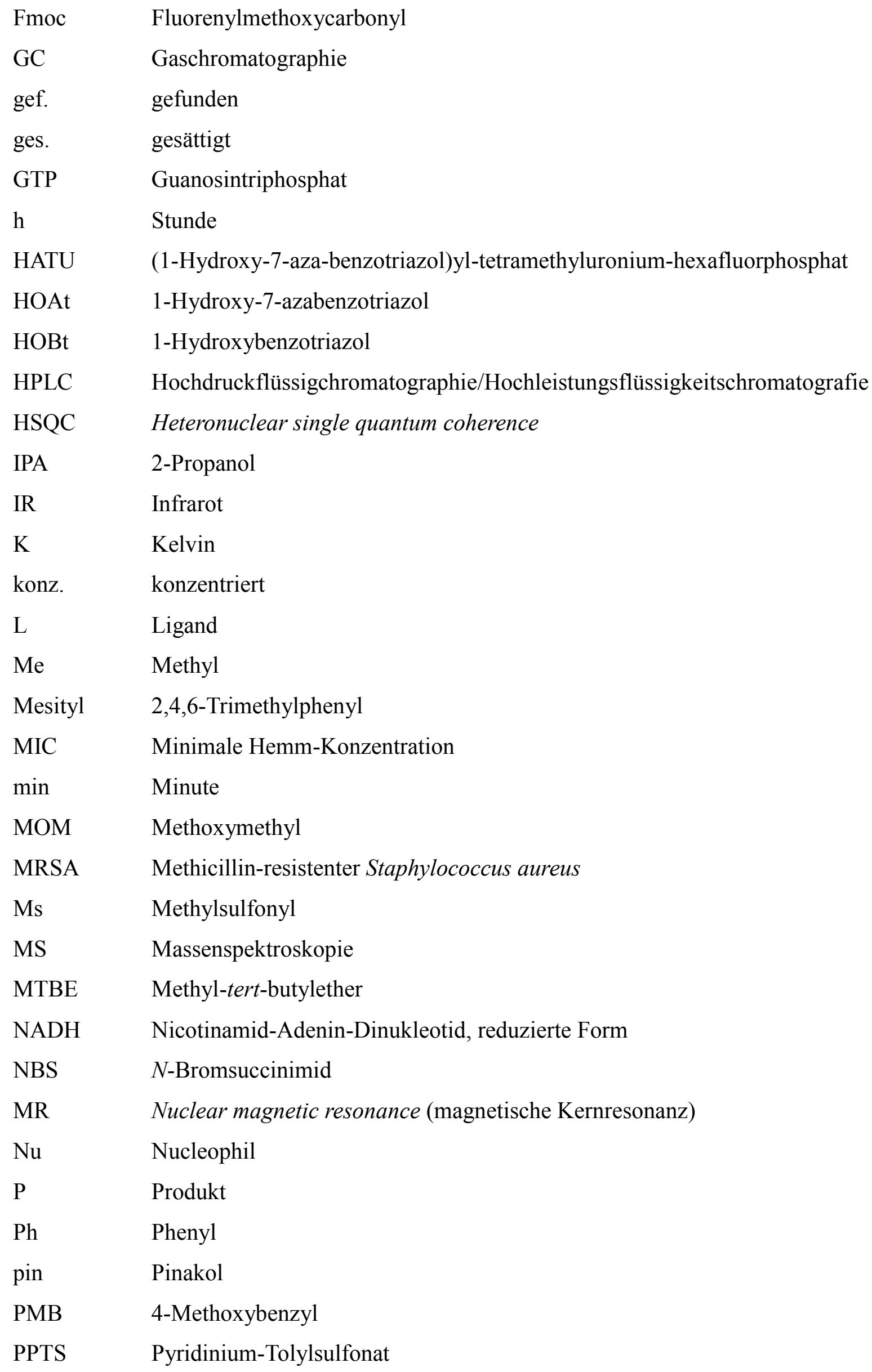


PyAOP (7-Azabenzotriazol-1-yl-oxy)-tris(pyrrolidino)phosphonium Hexafluorophosphat

PyBOP (Benzotriazol-1-yl-oxy)-tris(pyrrolidino)phosphonium Hexafluorophosphat

PyDOP [(3,4-Dihydro-4-oxo-1,2,3-benzotriazin-3-yl)-oxy]-tris(pyrrolidino)phosphonium Hexafluorophosphat

R Substituent

$\mathrm{R}_{f} \quad$ Retentionsfaktor

RNA Ribonukleinsäure

$\mathrm{R} t \quad$ Retentionszeit

RT Raumtemperatur

SAR Sttuktur-Aktivitäts-beziehung

SM Startmaterial

t Zeit

T Temperatur

TBAF Tetrabutylammoniumfluorid

TBAOH Tetrabutylammoniumhydroxid

TBS tert-Butyldimetylsilyl

$t \mathrm{Bu} \quad$ tert-Butyl

Tce 2,2,2-Trichloethyl

TES Triethylsilyl

Tf Trifluoromethansulfonyl

TFA Trifluoressigsäure

TFAA Trifluoressigsäureanhydrid

TFE Trifluorethanol

THF Tetrahydrofuran

THP Tetrahydropyranyl

Thr Threonin

TIPS Tri-iso-propylsilyl

TMP 2,2,6,6-Tetramethylpiperidin

TMS Trimethylsilyl

$\operatorname{Tr} \quad$ Triphenylmethyl

Ts $\quad p$-Toluensulfonyl

UV Ultraviolet 
9 Abkürzungsverzeichnis

Vol Volumen 


\section{Literaturverzeichnis}

[1] K. C. Nicolaou, Isr. J. Chem. 2011, 51, 312-313.

[2] K. C. Nicolaou, S. A. Snyder, Proc. Natl. Acad. Sci. U.S.A. 2004, 101, 11929-11936.

[3] R. F. Service, Science 1999, 285, 184.

[4] C. Djerassi, Science 1999, 285, 835.

[5] K. C. Nicolaou, D. Vourloumis, N. Winssinger, P. S. Baran, Angew. Chem. 2000, 112, 46-126; Angew. Chem. Int. Ed. 2000, 39, 44-122.

[6] K. Sanderson, Nature 2007, 448, 630-631.

[7] A. Fürstner, Angew. Chem. 2014, 126, 8-9; Angew. Chem. Int. Ed. 2014, 53, 8-9.

[8] P. Ball, Nature 2015, 528, 327-329.

[9] L. H. Briggs, H. T. Openshaw, R. Robinson, J. Chem. Soc. 1946, 903-908.

[10] R. Robinson, Experientia 1946, 2, 28-29.

[11] R. B. Woodward, M. P. Cava, W. D. Ollis, A. Hunger, H. U. Daeniker, K. Schenker, J. Am. Chem. Soc. 1954, 76, 4749-4751.

[12] K. Roth, Chem. unserer Zeit 2011, 45, 202-218.

[13] K. C. Nicolaou, E. J. Sorensen, Classics in Total Synthesis, VCH, Weinheim, 1996.

[14] D. Lefranc, M. A. Ciufolini, Angew. Chem. 2009, 121, 4262-4265; Angew. Chem. Int. Ed. 2009, 48, 4198-4201.

[15] M. Matveenko, G. Liang, E. M. W. Lauterwasser, E. Zubía, D. Trauner, J. Am. Chem. Soc. 2012, 134, 9291-9295.

[16] R. W. Hoffmann, Angew. Chem. 2013, 125, 133-140; Angew. Chem. Int. Ed. 2013, 52, 123-130.

[17] J. Hong, Chem. Eur. J. 2014, 20, 10204-10212.

[18] D. J. Newman, G. M. Cragg, J. Nat. Prod. 2016, 79, 629-661.

[19] J.-Y. Wach, K. Gademann, Synlett 2012, 163-170.

[20] T. D. Aicher, K. R. Buszek, F. G. Fang, C. J. Forsyth, S. H. Jung, Y. Kishi, M. C. Matelich, P. M. Scola, D. M. Spero, S. K. Yoon, J. Am. Chem. Soc. 1992, 114, 31623164. 
[21] A. W. G. Burgett, Q. Li, Q. Wei, P. G. Harran, Angew. Chem. 2003, 115, 5111-5116; Angew. Chem. Int. Ed. 2003, 42, 4961-4966.

[22] G. Wang, L. Shang, A. W. G. Burgett, P. G. Harran, X. Wang, Proc. Natl. Acad. Sci. U.S.A. 2007, 104, 2068-2073.

[23] P. Nagorny, N. Sane, B. Fasching, B. Aussedat, S. J. Danishefsky, Angew. Chem. 2012, 124, 999-1003; Angew. Chem. Int. Ed. 2012, 51, 975-979.

[24] P. Wang, S. Dong, J. A. Brailsford, K. Iyer, S. D. Townsend, Q. Zhang, R. C. Hendrickson, J. Shieh, M. A. S. Moore, S. J. Danishefsky, Angew. Chem. 2012, 124, 11744-11752; Angew. Chem. Int. Ed. 2012, 51, 11576-11584.

[25] Rhône-Poulenc S. A., FR 1392453, 1961.

[26] M. C. Bagley, J. W. Dale, E. A. Merritt, X. Xiong, Chem. Rev. 2005, 105, 685-714.

[27] R. A. Hughes, C. J. Moody, Angew. Chem. 2007, 119, 8076-8101; Angew. Chem. Int. Ed. 2007, 46, 7930-7954.

[28] K. C. Nicolaou, J. S. Chen, D. J. Edmonds, A. A. Estrada, Angew. Chem. 2009, 121, 670-732; Angew. Chem. Int. Ed. 2009, 48, 660-719.

[29] X. Just-Baringo, F. Albericio, M. Álvarez, Mar. Drugs 2014, 12, 317-351.

[30] F. Benazet, M. Cartier, J. Florent, C. Godard, G. Jung, J. Lunel, D. Mancy, C. Pascal, J. Renaut, P. Tarridec, J. Theilleux, R. Tissier, M. Dubost, L. Ninet, Experientia 1980, $36,414-416$.

[31] N. M. Haste, W. Thienphrapa, D. N. Tran, S. Loesgen, P. Sun, S.-J. Nam, P. R. Jensen, W. Fenical, G. Sakoulas, V. Nizet, M. E. Hensler, J. Antibiot. 2012, 65, 593-598.

[32] J. M. Harms, D. N. Wilson, F. Schluenzen, S. R. Connell, T. Stachelhaus, Z. Zaborowska, C. M. T. Spahn, P. Fucini, Mol. Cell 2008, 30, 26-38.

[33] S. Baumann, S. Schoof, M. Bolten, C. Haering, M. Takagi, K. Shin-ya, H.-D. Arndt, J. Am. Chem. Soc. 2010, 132, 6973-6981.

[34] X. Zhang, M. Fen, X. Shi, L. Bai, P. Zhou, Appl. Microbiol. Biotechnol. 2008, 78, 991995.

[35] F. Benazet, J. R. Cartier, Poult. Sci. 1980, 59, 1405-1415.

[36] M. Riedrich, Dissertation, TU Dortmund, 2009, DOI: 10.17877/DE290R-8231.

[37] J.-Y. Lu, Dissertation, TU Dortmund, 2009, DOI: 10.17877/DE290R-8748.

[38] T. L. Su, Br. J. Exp. Pathol. 1948, 29, 473-481.

[39] R. Donovick, J. F. Pagano, H. A. Stout, M. J. Weinstein, Antibiot. Annu. 1955-1956, 3, 554-559. 
[40] J. D. Dutcher, J. Vandeputte, Antibiot. Annu. 1955-1956, 3, 560-561.

[41] W. P. Jambor, B. A. Steinberg, L. O. Suydam, Antibiot. Annu. 1955-1956, 3, 562-563.

[42] S. M. Dennis, T. G. Nagaraja, A. D. Dayton, Res. Vet. Sci. 1986, 41, 251-256.

[43] Q. Zhang, W. Liu, Nat. Prod. Rep. 2013, 30, 218-226.

[44] X. Just-Baringo, F. Albericio, M. Álvarez, Angew. Chem. 2014, 126, 6720-6735; Angew. Chem. Int. Ed. 2014, 53, 6602-6616.

[45] M. S. Puar, T. M. Chan, V. Hegde, M. Patel, P. Bartner, K. J. Ng, B. N. Pramanik, R. D. MacFarlane, J. Antibiot. 1998, 51, 221-224.

[46] K. L. Constantine, L. Mueller, S. Huang, S. Abid, K. S. Lam, W. Li, J. E. Leet, J. Am. Chem. Soc. 2002, 124, 7284-7285.

[47] W. Li, J. E. Leet, H. A. Ax, D. R. Gustavson, D. M. Brown, L. Turner, K. Brown, J. Clark, H. Yang, J. Fung-Tomc, K. S. Lam, J. Antibiot. 2003, 56, 226-231.

[48] J. E. Leet, W. Li, H. A. Ax, J. A. Matson, S. Huang, R. Huang, J. L. Cantone, D. Drexler, R. A. Dalterio, K. S. Lam, J. Antibiot. 2003, 56, 232-242.

[49] H. Jayasuriya, K. Herath, J. G. Ondeyka, C. Zhang, D. L. Zink, M. Brower, F. P. Gailliot, J. Greene, G. Birdsall, J. Venugopal, M. Ushio, B. Burgess, G. Russotti, A. Walker, M. Hesse, A. Seeley, B. Junker, N. Connors, O. Salazar, O. Genilloud, K. Liu, P. Masurekar, J. F. Barrett, S. B. Singh, J. Antibiot. 2007, 60, 554-564.

[50] C. Zhang, K. Herath, H. Jayasuriya, J. G. Ondeyka, D. L. Zink, J. Occi, G. Birdsall, J. Venugopal, M. Ushio, B. Burgess, P. Masurekar, J. F. Barrett, S. B. Singh, J. Nat. Prod. 2009, 72, 841-847.

[51] C. Zhang, J. Occi, P. Masurekar, J. F. Barrett, D. L. Zink, S. Smith, R. Onishi, S. Ha, O. Salazar, O. Genilloud, A. Basilio, F. Vicente, C. Gill, E. J. Hickey, K. Dorso, M. Motyl, S. B. Singh, J. Am. Chem. Soc. 2008, 130, 12102-12110.

[52] K. C. Nicolaou, M. Zak, S. Rahimipour, A. A. Estrada, S. H. Lee, A. O'Brate, P. Giannakakou, M. R. Ghadiri, J. Am. Chem. Soc. 2005, 127, 15042-15044.

[53] S. K. Radhakrishnan, U. G. Rhat, D. E. Hughes, I. C. Wang, R. H. Costa, A. L. Gartel, Cancer Res. 2006, 66, 9731-9735.

[54] U. G. Bhat, P. A. Zipfel, D. S. Tyler, A. L. Gartel, Cell Cycle 2008, 7, 1851-1855.

[55] B. D. Bowling, N. Doudican, P. Manga, S. J. Orlow, Cancer Chemother. Pharmacol. 2008, 63, 37-43.

[56] J. M.-M. Kwok, S. S. Myatt, C. M. Marson, R. C. Coombes, D. Constantinidou, E. W.F. Lam, Mol. Cancer Ther. 2008, 7, 2022-2032.

[57] U. G. Bhat, M. Halasi, A. L. Gartel, PLoS ONE 2009, 4, e5592. 
[58] N. S. Hegde, D. A. Sanders, R. Rodriguez, S. Balasubramanian, Nat. Chem. 2011, 3, $725-731$.

[59] L. Jiang, X. Wu, P. Wang, T. Wen, C. Yu, L. Wei, H. Chen, J. Cancer Res. Clin. Onc. 2015, 141, 971-981.

[60] J. Y. Wang, X. H. Jia, H. Y. Xing, Y. J. Li, W. W. Fan, N. Li, S. Y. Xie, Mol. Med. Rep. 2015, 12, 1457-1464.

[61] B. Clough, M. Strath, P. Preiser, P. Denny, I. Wilson, FEBS Lett. 1997, 406, 123-125.

[62] G. A. McConkey, M. J. Rogers, T. F. McCutchan, J. Bio. Chem. 1997, 272, 2046-2049.

[63] B. Clough, K. Rangachari, M. Strath, P. R. Preiser, R. J. M. Iain Wilson, Protist 1999, 150, 189-195.

[64] M. Sullivan, J. Li, S. Kumar, M. J. Rogers, T. F. McCutchan, Mol. Biochem. Parasitol. 2000, 109, 17-23.

[65] S. Chaubey, A. Kumar, D. Singh, S. Habib, Mol. Microbio. 2005, 56, 81-89.

[66] S. Schoof, G. Pradel, M. N. Aminake, B. Ellinger, S. Baumann, M. Potowski, Y. Najajreh, M. Kirschner, H.-D. Arndt, Angew. Chem. 2010, 122, 3389-3393; Angew. Chem. Int. Ed. 2010, 49, 3317-3321.

[67] M. Ueno, S. Furukawa, F. Abe, M. Ushioda, K. Fujine, S. Johki, H. Hatori, H. Ueda, J. Antibiot. 2004, 57, 590-596.

[68] M. Aoki, T. Ohtsuka, M. Yamada, Y. Ohba, H. Yoshizaki, H. Yasuno, T. Sano, J. Watanabe, K. Yokose, H. Seto, J. Antibiot. 1991, 44, 582-588.

[69] M. Hashimoto, T. Murakami, K. Funahashi, T. Tokunaga, K.-i. Nihei, T. Okuno, T. Kimura, H. Naoki, H. Himeno, Bioorg. Med. Chem. 2006, 14, 8259-8270.

[70] C. L. Cox, J. I. Tietz, K. Sokolowski, J. O. Melby, J. R. Doroghazi, D. A. Mitchell, ACS Chem. Bio. 2014, 9, 2014-2022.

[71] N. Mizuhara, M. Kuroda, A. Ogita, T. Tanaka, Y. Usuki, K.-i. Fujita, Bioorg. Med. Chem. 2011, 19, 5300-5310.

[72] Y. Xing, D. E. Draper, Biochemistry 1996, 35, 1581-1588.

[73] M. V. Rodnina, A. Savelsbergh, V. I. Katunin, W. Wintermeyer, Nature 1997, 385, 3741.

[74] M. V. Rodnina, A. Savelsbergh, N. B. Matassova, V. I. Katunin, Y. P. Semenkov, W. Wintermeyer, Proc. Natl. Acad. Sci. U.S.A. 1999, 96, 9586-9590.

[75] G. Lentzen, R. Klinck, N. Matassova, F. Aboul-ela, A. I. H. Murchie, Chem. Biol. 2003, 10, 769-778. 
[76] S. Baumann, S. Schoof, S. D. Harkal, H.-D. Arndt, J. Am. Chem. Soc. 2008, 130, 5664-5666.

[77] J. D. Walter, M. Hunter, M. Cobb, G. Traeger, P. C. Spiegel, Nucleic Acids Res. 2012, 40, 360-370.

[78] M. J. LaMarche, J. A. Leeds, A. Amaral, J. T. Brewer, S. M. Bushell, G. Deng, J. M. Dewhurst, J. Ding, J. Dzink-Fox, G. Gamber, A. Jain, K. Lee, L. Lee, T. Lister, D. McKenney, S. Mullin, C. Osborne, D. Palestrant, M. A. Patane, E. M. Rann, M. Sachdeva, J. Shao, S. Tiamfook, A. Trzasko, L. Whitehead, A. Yifru, D. Yu, W. Yan, Q. Zhu, J. Med. Chem. 2012, 55, 2376-2387.

[79] E. Selva, N. Montanini, S. Stella, A. Soffientini, L. Gastaldo, M. Denaro, J. Antibiot. 1997, 50, 22-26.

[80] S. E. Heffron, F. Jurnak, Biochemistry 2000, 39, 37-45.

[81] A. Parmeggiani, I. M. Krab, S. Okamura, R. C. Nielsen, J. Nyborg, P. Nissen, Biochemistry 2006, 45, 6846-6857.

[82] A. Parmeggiani, P. Nissen, FEBS Letters 2006, 580, 4576-4581.

[83] F. Reusser, Biochemistry 1969, 8, 3303-3308.

[84] J. M. Liesch, K. L. Rinehart, J. Am. Chem. Soc. 1977, 99, 1645-1646.

[85] R. C. M. Lau, K. L. Rinehart, J. Antibiot. 1994, 47, 1466-1472.

[86] S. Kodani, A. Ninomiya, Asian J. Chem. 2013, 25, 490-492.

[87] B. S. Yun, H. Seto, Biosci. Biotech. Biochem. 1995, 59, 876-880.

[88] J. Thompson, E. Cundliffe, M. J. R. Stark, J. Gen. Microbio. 1982, 128, 875-884.

[89] T. Murakami, T. G. Holt, C. J. Thompson, J. Bacteriol. 1989, 171, 1459-1466.

[90] D. J. Holmes, J. L. Caso, C. J. Thompson, EMBO J. 1993, 12, 3183-3191.

[91] C. L. Myers, J. Harris, J. C. K. Yeung, J. F. Honek, ChemBioChem 2014, 15, 681-687.

[92] J. Habazettl, M. Allan, P. R. Jensen, H.-J. Sass, C. J. Thompson, S. Grzesiek, Proc. Natl. Acad. Sci. U.S.A. 2014, 111, E5498-E5507.

[93] B. S. Yun, T. Hidaka, K. Furihata, H. Seto, J. Antibiot. 1994, 47, 510-514.

[94] B. S. Yun, T. Hidaka, K. Furihata, H. Seto, J. Antibiot. 1994, 47, 969-975.

[95] B. S. Yun, T. Hidaka, K. Furihata, H. Seto, J. Antibiot. 1994, 47, 1541-1545.

[96] B.-S. Yun, T. Hidaka, K. Furihata, H. Seto, Tetrahedron 1994, 50, 11659-11664. 
[97] C.-g. Shin, K. Okumura, M. Shigekuni, Y. Nakamura, Chem. Lett. 1998, 27, 139-140.

[98] K. Okumura, Y. Nakamura, C.-g. Shin, Bull. Chem. Soc. Jpn. 1999, 72, 1561-1569.

[99] K. Okumura, T. Suzuki, Y. Nakamura, C.-g. Shin, Bull. Chem. Soc. Jpn. 1999, 72, 2483-2490.

[100] M. A. Ciufolini, Y.-C. Shen, Org. Lett. 1999, 1, 1843-1846.

[101] C. J. Moody, M. C. Bagley, Chem. Commun. 1998, 2049-2050.

[102] M. C. Bagley, K. E. Bashford, C. L. Hesketh, C. J. Moody, J. Am. Chem. Soc. 2000, 122, 3301-3313.

[103] R. A. Hughes, S. P. Thompson, L. Alcaraz, C. J. Moody, Chem. Commun. 2004, 946948.

[104] R. A. Hughes, S. P. Thompson, L. Alcaraz, C. J. Moody, J. Am. Chem. Soc 2005, 127, 15644-15651.

[105] K. C. Nicolaou, B. Zou, D. H. Dethe, D. B. Li, D. Y. K. Chen, Angew. Chem. 2006, 118, 7950-7956; Angew. Chem. Int Ed. 2006, 45, 7786-7792.

[106] K. C. Nicolaou, D. H. Dethe, G. Y. C. Leung, B. Zou, D. Y. K. Chen, Chem. Asian J. 2008, 3, 413-429.

[107] H. M. Müller, O. Delgado, T. Bach, Angew. Chem. 2007, 119, 4855-4858; Angew. Chem. Int. Ed. 2007, 46, 4771-4774.

[108] O. Delgado, H. M. Müller, T. Bach, Chem. Eur. J. 2008, 14, 2322-2339.

[109] K. C. Nicolaou, D. H. Dethe, D. Y. K. Chen, Chem. Comm. 2008, 2632-2634.

[110] C. Ammer, T. Bach, Chem. Eur. J. 2010, 16, 14083-14093.

[111] V. S. Aulakh, M. A. Ciufolini, J. Am. Chem. Soc. 2011, 133, 5900-5904.

[112] X. Just-Baringo, P. Bruno, L. K. Ottesen, L. M. Cañedo, F. Albericio, M. Álvarez, Angew. Chem. 2013, 125, 7972-7975; Angew. Chem. Int Ed. 2013, 52, 7818-7821.

[113] K. C. Nicolaou, B. S. Safina, M. Zak, A. A. Estrada, S. H. Lee, Angew. Chem. 2004, 116, 5197-5202; Angew. Chem. Int. Ed. 2004, 43, 5087-5092.

[114] K. C. Nicolaou, M. Zak, B. S. Safina, S. H. Lee, A. A. Estrada, Angew. Chem. 2004, 116, 5202-5207; Angew. Chem. Int. Ed. 2004, 43, 5092-5097.

[115] K. C. Nicolaou, B. S. Safina, M. Zak, S. H. Lee, M. Nevalainen, M. Bella, A. A. Estrada, C. Funke, F. J. Zécri, S. Bulat, J. Am. Chem. Soc. 2005, 127, 11159-11175.

[116] K. C. Nicolaou, M. Zak, B. S. Safina, A. A. Estrada, S. H. Lee, M. Nevalainen, J. Am. Chem. Soc. 2005, 127, 11176-11183. 
[117] T. Mori, S. Higashibayashi, T. Goto, M. Kohno, Y. Satouchi, K. Shinko, K. Suzuki, S. Suzuki, H. Tohmiya, K. Hashimoto, M. Nakata, Tetrahedron Lett. 2007, 48, 13311335.

[118] T. Mori, S. Higashibayashi, T. Goto, M. Kohno, Y. Satouchi, K. Shinko, K. Suzuki, S. Suzuki, H. Tohmiya, K. Hashimoto, M. Nakata, Chem. Asian J. 2008, 3, 984-1012.

[119] T. Mori, S. Higashibayashi, T. Goto, M. Kohno, Y. Satouchi, K. Shinko, K. Suzuki, S. Suzuki, H. Tohmiya, K. Hashimoto, M. Nakata, Chem. Asian J. 2008, 3, 1013-1025.

[120] K. P. Wojtas, M. Riedrich, J.-Y. Lu, P. Winter, T. Winkler, S. Walter, H.-D. Arndt, Angew. Chem. 2016, 128, 9924-9928; Angew. Chem. Int. Ed. 2016, 55, 9772-9976.

[121] L. M. Canedo Hernandez, F. Romero Millan, A. Fernandez Medarde, R. I. Fernandez Chimeno, J. C. Hidalgo Villar, WO 2012062906A1, 2012.

[122] S. Stella, N. Montanini, F. Lemonnier, P. Ferrari, L. Colombo, F. Marinelli, P. Landini, I. Ciciliato, B. P. Goldstein, E. Selva, M. Denaro, J. Antibiot. 1995, 48, 780-786.

[123] P. Ferrari, L. Colombo, S. Stella, E. Selva, L. F. Zerilli, J. Antibiot. 1995, 48, 13041311.

[124] M. Sakai, K. Hashimoto, H. Shirahama, Heterocycles 1997, 44, 319-324.

[125] K. Hashimoto, M. Sakai, T. Okuno, H. Shirahama, Chem. Commun. 1996, 1139-1140.

[126] N. M. Okeley, Y. Zhu, W. A. van der Donk, Org. Lett. 2000, 2, 3603-3606.

[127] S.-L. You, H. Razavi, J. W. Kelly, Angew. Chem. 2003, 115, 87-89; Angew. Chem. Int. Ed. 2003, 42, 83-85.

[128] P. Bruno, S. Peña, X. Just-Baringo, F. Albericio, M. Álvarez, Org. Lett. 2011, 13, 4648-4651.

[129] X. Just-Baringo, P. Bruno, F. Albericio, M. Álvarez, Tetrahedron Lett. 2011, 52, 54355437.

[130] P. Wipf, C. P. Miller, J. Org. Chem. 1993, 58, 3604-3606.

[131] K. C. Nicolaou, A. A. Estrada, M. Zak, S. H. Lee, B. S. Safina, Angew. Chem. 2005, 117, 1402-1406; Angew. Chem. Int. Ed. 2005, 44, 1378-1382.

[132] X. Just-Baringo, P. Bruno, C. Pitart, J. Vila, F. Albericio, M. Álvarez, J. Med. Chem. 2014, 57, 4185-4195.

[133] H. Depaire, J.-P. Thomas, A. Brun, G. Lukacs, Tetrahedron Lett. 1977, 18, 1395-1396.

[134] H. Depaire, J.-P. Thomas, A. Brun, A. Olesker, G. Lukacs, Tetrahedron Lett. 1977, 18, 1403-1406. 
[135] D. R. Houck, L. C. Chen, P. J. Keller, J. M. Beale, H. G. Floss, J. Am. Chem. Soc. 1987, 109, 1250-1252.

[136] D. R. Houck, L. C. Chen, P. J. Keller, J. M. Beale, H. G. Floss, J. Am. Chem. Soc. 1988, $110,5800-5806$.

[137] U. Mocek, A. R. Knaggs, R. Tsuchiya, T. Nguyen, J. M. Beale, H. G. Floss, J. Am. Chem. Soc. 1993, 115, 7557-7568.

[138] T. Prange, A. Ducruix, C. Pascard, J. Lunel, Nature 1977, 265, 189-190.

[139] C. Pascard, A. Ducruix, J. Lunel, T. Prange, J. Am. Chem. Soc. 1977, 99, 6418-6423.

[140] R. Sinha Roy, A. M. Gehring, J. C. Milne, P. J. Belshaw, C. T. Walsh, Nat. Prod. Rep. 1999, 16, 249-263.

[141] P. G. Arnison, M. J. Bibb, G. Bierbaum, A. A. Bowers, T. S. Bugni, G. Bulaj, J. A. Camarero, D. J. Campopiano, G. L. Challis, J. Clardy, P. D. Cotter, D. J. Craik, M. Dawson, E. Dittmann, S. Donadio, P. C. Dorrestein, K.-D. Entian, M. A. Fischbach, J. S. Garavelli, U. Goransson, C. W. Gruber, D. H. Haft, T. K. Hemscheidt, C. Hertweck, C. Hill, A. R. Horswill, M. Jaspars, W. L. Kelly, J. P. Klinman, O. P. Kuipers, A. J. Link, W. Liu, M. A. Marahiel, D. A. Mitchell, G. N. Moll, B. S. Moore, R. Muller, S. K. Nair, I. F. Nes, G. E. Norris, B. M. Olivera, H. Onaka, M. L. Patchett, J. Piel, M. J. T. Reaney, S. Rebuffat, R. P. Ross, H.-G. Sahl, E. W. Schmidt, M. E. Selsted, K. Severinov, B. Shen, K. Sivonen, L. Smith, T. Stein, R. D. Sussmuth, J. R. Tagg, G.-L. Tang, A. W. Truman, J. C. Vederas, C. T. Walsh, J. D. Walton, S. C. Wenzel, J. M. Willey, W. A. van der Donk, Nat. Prod. Rep. 2013, 30, 108-160.

[142] B. W. Bycroft, M. S. Gowland, J. Chem. Soc., Chem. Commun. 1978, 256-258.

[143] W. Liu, Y. Xue, M. Ma, S. Wang, N. Liu, Y. Chen, ChemBioChem 2013, 14, 15441547.

[144] Y. Yu, L. Duan, Q. Zhang, R. Liao, Y. Ding, H. Pan, E. Wendt-Pienkowski, G. Tang, B. Shen, W. Liu, ACS Chem. Biol. 2009, 4, 855-864.

[145] Q. Zhang, Y. Li, D. Chen, Y. Yu, L. Duan, B. Shen, W. Liu, Nat. Chem. Biol. 2011, 7, 154-160.

[146] G. Sicoli, J.-M. Mouesca, L. Zeppieri, P. Amara, L. Martin, A. L. Barra, J. C. Fontecilla-Camps, S. Gambarelli, Y. Nicolet, Science 2016, 351, 1320-1323.

[147] K. C. Nicolaou, S. H. Lee, A. A. Estrada, M. Zak, Angew. Chem. 2005, 117, 38023806; Angew. Chem. Int. Ed. 2005, 44, 3736-3740.

[148] K. C. Nicolaou, A. A. Estrada, G. C. Freestone, S. H. Lee, X. Alvarez-Mico, Tetrahedron 2007, 63, 6088-6114.

[149] K. Umemura, H. Noda, J. Yoshimura, A. Konn, Y. Yonezawa, C.-g. Shin, Tetrahedron Lett. 1997, 38, 3539-3542. 
[150] M. Iwakawa, Y. Kobayashi, S.-i. Ikuta, J. Yoshimura, Chem. Lett. 1982, 11, 19751978.

[151] K. Umemura, T. Tate, M. Yamaura, J. Yoshimura, Y. Yonezawa, C.-g. Shin, Synthesis 1995, 1995, 1423-1426.

[152] K. Koerber-Plé, G. Massiot, Synlett 1994, 1994, 759-760.

[153] C.-g. Shin, Y. Yamada, K. Hayashi, Y. Yonezawa, K. Umemura, T. Tanji, J. Yoshimura, Heterocycles 1996, 43, 891-898.

[154] D. J. Bentley, J. Fairhurst, P. T. Gallagher, A. K. Manteuffel, C. J. Moody, J. L. Pinder, Org. Biomol. Chem. 2004, 2, 701-708.

[155] T. Belhadj, A. Nowicki, C. J. Moody, Synlett 2006, 2006, 3033-3036.

[156] M. C. Kimber, C. J. Moody, Chem. Commun. 2008, 591-593.

[157] H.-J. Hwang, M. A. Ciufolini, J. Org. Chem. 2015, 80, 4184-4188.

[158] A. Reissert, Chem. Ber. 1897, 30, 1030-1053.

[159] J. M. Herbert, Tetrahedron Lett. 2004, 45, 817-819.

[160] M. Riedrich, S. Harkal, H.-D. Arndt, Angew. Chem. 2007, 119, 2755-2758; Angew. Chem. Int. Ed. 2007, 46, 2701-2703.

[161] J.-Y. Lu, M. Riedrich, M. Mikyna, H.-D. Arndt, Angew. Chem. 2009, 121, 8281-8284; Angew. Chem. Int. Ed. 2009, 48, 8137-8140.

[162] P. Loos, C. Ronco, M. Riedrich, H.-D. Arndt, Eur. J. Org. Chem. 2013, 2013, 32903315 .

[163] J.-Y. Lu, H.-D. Arndt, J. Org. Chem. 2007, 72, 4205-4212.

[164] P. B. Alper, S.-C. Hung, C.-H. Wong, Tetrahedron Lett. 1996, 37, 6029-6032.

[165] P. T. Nyffeler, C.-H. Liang, K. M. Koeller, C.-H. Wong, J. Am. Chem. Soc. 2002, 124, 10773-10778.

[166] P. A. Grieco, S. Gilman, M. Nishizawa, J. Org. Chem. 1976, 41, 1485-1486.

[167] K. Nakamura, T. Isaka, H. Toshima, M. Kodaka, Tetrahedron Lett. 2004, 45, 72217224.

[168] K.-i. Tanaka, H. Sawanishi, Tetrahedron 1998, 54, 10029-10042.

[169] K.-i. Tanaka, H. Sawanishi, Tetrahedron Asym. 1998, 9, 71-77.

[170] M. D. Fletcher, T. E. Hurst, T. J. Miles, C. J. Moody, Tetrahedron 2006, 62, 54545463. 
[171] C. W. Holzapfel, G. R. Pettit, J. Org. Chem. 1985, 50, 2323-2327.

[172] R. C. Kelly, I. Gebhard, N. Wicnienski, J. Org. Chem. 1986, 51, 4590-4594.

[173] M. W. Bredenkamp, C. W. Holzapfel, W. J. van Zyl, Synth. Commun. 1990, 20, 22352249.

[174] H. Li, X. Jiang, Y.-h. Ye, C. Fan, T. Romoff, M. Goodman, Org. Lett. 1999, 1, 91-94.

[175] J.-Y. Lu, M. Riedrich, K. P. Wojtas, H.-D. Arndt, Synthesis 2013, 45, 1300-1311.

[176] M. A. Ciufolini, Y. C. Shen, J. Org. Chem. 1997, 62, 3804-3805.

[177] C.-g. Shin, A. Okabe, A. Ito, A. Ito, Y. Yonezawa, Bull. Chem. Soc. Jpn. 2002, 75, 1583-1596.

[178] V. S. Aulakh, M. A. Ciufolini, J. Org. Chem. 2009, 74, 5750-5753.

[179] Y. Peng, C. Ji, Y. Chen, C. Huang, Y. Jiang, Synth. Commun. 2004, 34, 4325-4330.

[180] K. Maeda, H. Kosaka, Y. Okami, H. Umezawa, J. Antibiot. 1953, 6, 140.

[181] M. Kuroya, B. Takahashi, Y. Hinuma, T. Yashima, K. Watanabe, S. Hamada, J. Antibiot. 1954, 7, 58-59.

[182] G. Koyama, Y. Iitaka, K. Maeda, H. Umezawa, Tetrahedron Lett. 1967, 8, 3587-3590.

[183] N. Miyaura, A. Suzuki, J. Chem. Soc., Chem. Commun. 1979, 866-867.

[184] M. A. Larsen, J. F. Hartwig, J. Am. Chem. Soc. 2014, 136, 4287-4299.

[185] J. J. Li, C-H Bond Activation in Organic Synthesis, CRC Press, Boca Raton, 2015.

[186] P. Winter, Bachelorarbeit, TU Dortmund, 2007.

[187] M. Riedrich, persönliche Mitteilungen, Laborjournale.

[188] H. Böhme, E. Mundlos, W. Lehners, O.-E. Herboth, Chem. Ber. 1957, 90, 2008-2013.

[189] K. C. Nicolaou, A. A. Estrada, S. H. Lee, G. C. Freestone, Angew. Chem. 2006, 118, 5490-5494; Angew. Chem. Int. Ed. 2006, 45, 5364-5368.

[190] R. L. Augustine, Heterogeneous Catalysis for the Synthetic Chemist, Marcel Dekker Inc., New York, 1996.

[191] K. Tangdenpaisal, W. Phakhodee, S. Ruchirawat, P. Ploypradith, Tetrahedron 2013, 69, 933-941.

[192] D. Chen, C. Xu, J. Deng, C. Jiang, X. Wen, L. Kong, J. Zhang, H. Sun, Tetrahedron 2014, 70, 1975-1983. 
[193] M. Saudi, A. van Aerschot, Molecules 2013, 18, 8524.

[194] R. G. Hiskey, E. L. Smithwick, J. Am. Chem. Soc. 1967, 89, 437-441.

[195] K. Biggadike, D. C. Humber, B. Laundon, A. G. Long, M. V. J. Ramsay, Tetrahedron 1985, 41, 2025-2031.

[196] E. F. De Medeiros, J. M. Herbert, R. J. K. Taylor, J. Chem. Soc. Perkin Trans. 1 1991, 2725-2730.

[197] D. A. Pearson, M. Blanchette, M. L. Baker, C. A. Guindon, Tetrahedron Lett. 1989, $30,2739-2742$.

[198] M. M. Bowersnemia, M. M. Joullie, Heterocycles 1983, 20, 817-828.

[199] J. A. Gomez-Vidal, R. B. Silverman, Org. Lett. 2001, 3, 2481-2484.

[200] X. Zhang, A. C. Schmitt, W. Jiang, Tetrahedron Lett. 2001, 42, 5335-5338.

[201] T. Kiho, M. Nakayama, K. Yasuda, S. Miyakoshi, M. Inukai, H. Kogen, Bioorg. Med. Chem. 2004, 12, 337-361.

[202] S.-M. Yu, W.-X. Hong, Y. Wu, C.-L. Zhong, Z.-J. Yao, Org. Lett. 2010, 12, 1124-1127.

[203] E. J. Corey, N. H. Andersen, R. M. Carlson, J. Paust, E. Vedejs, I. Vlattas, R. E. K. Winter, J. Am. Chem. Soc. 1968, 90, 3245-3247.

[204] M. J. Miller, J. Org. Chem. 1980, 45, 3131-3132.

[205] À. López-Macià, J. C. Jiménez, M. Royo, E. Giralt, F. Albericio, J. Am. Chem. Soc. 2001, 123, 11398-11401.

[206] M. Royo, Jose C. Jiménez, A. López-Macià, E. Giralt, F. Albericio, Eur. J. Org. Chem. 2001, 2001, 45-48.

[207] J. C. Jiménez, N. Bayó, B. Chavarria, À. López-Macrà, M. Royo, E. Nicolas, E. Giralt, F. Albericio, Lett. Pept. Sci. 2002, 9, 135-141.

[208] N. Bayó, J. C. Jiménez, L. Rivas, E. Nicolás, F. Albericio, Chem. Eur. J. 2003, 9, 1096-1103.

[209] H. Sai, T. Ogiku, H. Ohmizu, Synthesis 2003, 2003, 0201-0204.

[210] E. J. Corey, H. Cho, C. Rücker, D. H. Hua, Tetrahedron Lett. 1981, 22, 3455-3458.

[211] M. Sakaitani, Y. Ohfune, Tetrahedron Lett. 1985, 26, 5543-5546.

[212] M. Sakaitani, Y. Ohfune, J. Org. Chem. 1990, 55, 870-876.

[213] P. F. Kruse, N. Geurkink, K. L. Grist, J. Am. Chem. Soc. 1954, 76, 5796-5797. 
[214] D. B. Dess, J. C. Martin, J. Org. Chem. 1983, 48, 4155-4156.

[215] I. Raztsou, Masterarbeit, Friedrich-Schiller-Universität, Jena, 2016.

[216] M. Barber, J. H. Jones, M. J. Witty, J. Chem. Soc. Perkin Trans. 1 1979, 2425-2428.

[217] R. B. Woodward, K. Heusler, J. Gosteli, P. Naegeli, W. Oppolzer, R. Ramage, S. Ranganathan, H. Vorbrüggen, J. Am. Chem. Soc. 1966, 88, 852-853.

[218] D. S. Kemp, R. I. Carey, J. Org. Chem. 1989, 54, 3640-3646.

[219] K. Schank, Chem. Ber. 1969, 102, 383-387.

[220] H. Wojciechowska, R. Pawłowicz, R. Andruszkiewicz, J. Grzybowska, Tetrahedron Lett. 1978, 19, 4063-4064.

[221] H. Ogura, O. Sato, K. Takeda, Tetrahedron Lett. 1981, 22, 4817-4818.

[222] R. Andruszkiewicz, A. Czerwiński, Synthesis 1982, 1982, 968-969.

[223] M. E. Fox, I. C. Lennon, G. Meek, Tetrahedron Lett. 2002, 43, 2899-2902.

[224] L. A. Carpino, J. Xia, A. El-Faham, J. Org. Chem. 2004, 69, 54-61.

[225] T. Hoeeg-Jensen, C. E. Olsen, A. Holm, J. Org. Chem. 1994, 59, 1257-1263.

[226] T. D. Nelson, R. D. Crouch, Synthesis 1996, 1996, 1031-1069.

[227] R. D. Crouch, Tetrahedron 2004, 60, 5833-5871.

[228] R. D. Crouch, Tetrahedron 2013, 69, 2383-2417.

[229] G. Rousseau, B. Breit, Angew. Chem. 2011, 123, 2498-2543; Angew. Chem. Int. Ed. 2011, 50, 2450-2494.

[230] H. B. Henbest, R. A. L. Wilson, J. Chem. Soc. 1957, 1958-1965.

[231] S. Winstein, J. Sonnenberg, J. Am. Chem. Soc. 1961, 83, 3235-3244.

[232] X. Zhang, K. N. Houk, Acc. Chem. Res. 2005, 38, 379-385.

[233] A. H. Hoveyda, D. A. Evans, G. C. Fu, Chem. Rev. 1993, 93, 1307-1370.

[234] Y.-Q. Zhang, N. Funken, P. Winterscheid, A. Gansäuer, Angew. Chem. 2015, 127, 7035-7038; Angew. Chem. Int. Ed. 2015, 54, 6931-6934.

[235] H. Gilman, R. L. Bebb, J. Am. Chem. Soc. 1939, 61, 109-112.

[236] G. Wittig, G. Fuhrmann, Chem. Ber. 1940, 73, 1197-1218.

[237] V. Snieckus, Chem. Rev. 1990, 90, 879-933. 
[238] M. Schlosser, Angew. Chem. 2005, 117, 380-398; Angew. Chem. Int. Ed. 2005, 44, 376-393.

[239] S. Florio, A. Salomone, Synthesis 2016, 48, 1993-2008.

[240] N. Tezuka, K. Shimojo, K. Hirano, S. Komagawa, K. Yoshida, C. Wang, K. Miyamoto, T. Saito, R. Takita, M. Uchiyama, J. Am. Chem. Soc. 2016, 138, 91669171.

[241] D. Krahn, Diplomarbeit, TU Dortmund, 2009.

[242] P. C. Sadek, C. J. Koester, L. D. Bowers, J. Chromatogr. Sci. 1987, 25, 489-493.

[243] A. Schneegans, J. E. Gerock, Zeit. Anal. Chem. 1893, 32, 363-363.

[244] H. Goldschmidt, V. Scholz, Chem. Ber. 1907, 40, 624-641.

[245] L. Pekkarinen, E. Tommila, Acta Chem. Scand. 1959, 13, 1019-1030.

[246] M. L. Bender, F. J. Kezdy, B. Zerner, J. Am. Chem. Soc. 1963, 85, 3017-3024.

[247] F. M. Menger, J. H. Smith, J. Am. Chem. Soc. 1969, 91, 5346-5349.

[248] M. N. Khan, Int. J. Chem. Kin. 1987, 19, 415-434.

[249] M. Niyaz Khan, A. A. Audu, J. Phys. Org. Chem. 1992, 5, 129-141.

[250] G. T. Bourne, S. W. Golding, R. P. McGeary, W. D. F. Meutermans, A. Jones, G. R. Marshall, P. F. Alewood, M. L. Smythe, J. Org. Chem. 2001, 66, 7706-7713.

[251] R. Shen, C. T. Lin, E. J. Bowman, B. J. Bowman, J. A. Porco, Org. Lett. 2002, 4, 3103-3106.

[252] R. Shen, C. T. Lin, E. J. Bowman, B. J. Bowman, J. A. Porco, J. Am. Chem. Soc. 2003, $125,7889-7901$.

[253] S. Hanessian, R. R. Vakiti, S. Dorich, S. Banerjee, F. Lecomte, J. R. DelValle, J. Zhang, B. Deschênes-Simard, Angew. Chem. 2011, 123, 3559-3562; Angew. Chem. Int. Ed. 2011, 50, 3497-3500.

[254] R. J. Sharpe, J. T. Malinowski, J. S. Johnson, J. Am. Chem. Soc. 2013, 135, 1799017998.

[255] A. Albert, J. N. Phillips, J. Chem. Soc. 1956, 1294-1304.

[256] F. E. Scully, J. Hoigné, Chemosphere 1987, 16, 681-694.

[257] C. Melander, D. A. Horne, J. Org. Chem. 1997, 62, 9295-9297.

[258] H. Ogawara, K. Maeda, G. Koyama, H. Naganawa, H. Umezawa, Chem. Pharm. Bull. 1968, 16, 679-687. 
[259] T. A. Mukhtar, G. D. Wright, Chem. Rev. 2005, 105, 529-542.

[260] T. T. Huang, Y. M. Wang, J. Yin, Y. H. Du, M. F. Tao, J. Xu, W. Q. Chen, S. J. Lin, Z. X. Deng, J. Biol. Chem. 2011, 286, 20648-20657.

[261] M. Kinoshita, M. Nakata, K. Takarada, K. Tatsuta, Tetrahedron Lett. 1989, 30, 74197422.

[262] R. C. Hartkoorn, C. Sala, J. Neres, F. Pojer, S. Magnet, R. Mukherjee, S. Uplekar, S. Boy-Röttger, K. H. Altmann, S. T. Cole, EMBO Mol. Med. 2012, 4, 1032-1042.

[263] K. Takayama, C. Wang, G. S. Besra, Clinic. Microbiol. Rev. 2005, 18, 81-101.

[264] R. C. Hartkoorn, F. Pojer, J. A. Read, H. Gingell, J. Neres, O. P. Horlacher, K.-H. Altmann, S. T. Cole, Nat. Chem. Biol. 2014, 10, 96-98.

[265] O. P. Horlacher, Dissertation, ETH Zürich, 2014, DOI: 10.3929/ethz-a-010186540.

[266] H.-D. Arndt, K.-H. Altmann et al., in Vorbereitung.

[267] J. Renou, M. Loiodice, M. Arboleas, R. Baati, L. Jean, F. Nachon, P.-Y. Renard, Chem. Commun. 2014, 50, 3947-3950.

[268] A.-L. Gérard, A. Bouillon, C. Mahatsekake, V. Collot, S. Rault, Tetrahedron Lett. 2006, 47, 4665-4669.

[269] M. C. Jetter, A. B. Reitz, Synthesis 1998, 829-831.

[270] M. Tamura, Y. Ise, Y. Okajima, N. Nishiwaki, M. Ariga, Synthesis 2006, 3453-3461.

[271] M. E. Voss, C. M. Beer, S. A. Mitchell, P. A. Blomgren, P. E. Zhichkin, Tetrahedron 2008, 64, 645-651.

[272] J. Roppe, N. D. Smith, D. Huang, L. Tehrani, B. Wang, J. Anderson, J. Brodkin, J. Chung, X. Jiang, C. King, B. Munoz, M. A. Varney, P. Prasit, N. D. P. Cosford, J. Med. Chem. 2004, 47, 4645-4648.

[273] Z. P. Demko, K. B. Sharpless, J. Org. Chem. 2001, 66, 7945-7950.

[274] C. R. Rice, S. Worl, J. C. Jeffrey, R. L. Paul, M. D. Ward, J. Chem. Soc., Dalton Trans. 2001, 550-559.

[275] J. A. Joule, K. Mills, Heterocyclic Chemistry, Wiley, West Sussex, 2010.

[276] K. Sonogashira, Y. Tohda, N. Hagihara, Tetrahedron Lett. 1975, 16, 4467-4470.

[277] T. Jin, S. Kamijo, Y. Yamamoto, Eur. J. Org. Chem. 2004, 3789-3791.

[278] T. Verdelet, G. Mercey, N. Correa, L. Jean, P.-Y. Renard, Tetrahedron 2011, 67, 87578762 . 
[279] M. E. Logan, M. E. Oinen, Organometallics 2006, 25, 1052-1054.

[280] F. Paul, J. Patt, J. F. Hartwig, J. Am. Chem. Soc. 1994, 116, 5969-5970.

[281] J. J. Hirner, M. J. Zacuto, Tetrahedron Lett. 2009, 50, 4989-4993.

[282] G. Chelucci, S. Figus, J. Mol. Catal. A: Chem. 2014, 393, 191-209.

[283] D. F. Fischer, R. Sarpong, J. Am. Chem. Soc. 2010, 132, 5926-5927.

[284] L. Zhao, C. Tsukano, E. Kwon, Y. Takemoto, M. Hirama, Angew. Chem. 2013, 125, 1766-1769; Angew. Chem. Int. Ed. 2013, 52, 1722-1725.

[285] J. N. Newton, D. F. Fischer, R. Sarpong, Angew. Chem. 2013, 125, 1770-1774; Angew. Chem. Int. Ed. 2013, 52, 1726-1730.

[286] H. Serizawa, K. Aikawa, K. Mikami, Chem. Eur. J. 2013, 19, 17692-17697.

[287] D. C. Pryde, L. H. Jones, D. P. Gervais, D. R. Stead, D. C. Blakemore, M. D. Selby, A. D. Brown, J. W. Coe, M. Badland, D. M. Beal, R. Glen, Y. Wharton, G. J. Miller, P. White, N. L. Zhang, M. Benoit, K. Robertson, J. R. Merson, H. L. Davis, M. J. McCluskie, Plos One 2013, 8, 1-16.

[288] I. A. I. Mkhalid, J. H. Barnard, T. B. Marder, J. M. Murphy, J. F. Hartwig, Chem. Rev. 2010, $110,890-931$.

[289] J. F. Hartwig, Acc. Chem. Res. 2012, 45, 864-873.

[290] J. Yamaguchi, A. D. Yamaguchi, K. Itami, Angew. Chem. 2012, 124, 9092-9142; Angew. Chem. Int. Ed. 2012, 51, 8960-9009.

[291] A. S. Voisin, A. Bouillon, J.-C. Lancelot, S. Rault, Tetrahedron 2005, 61, 1417-1421.

[292] K. Amaike, K. Itami, J. Yamaguchi, Chem. Eur. J. 2016, 22, 4384-4388.

[293] C. Napoli, L. J. Ignarro, Ann. Rev. Pharmacol. Toxicol. 2003, 43, 97-123.

[294] B. E. Mann, R. Motterlini, Chem. Commun. 2007, 4197-4208.

[295] U. Schatzschneider, Eur. J. Inorg. Chem. 2010, 2010, 1451-1467.

[296] R. Motterlini, L. E. Otterbein, Nat. Rev. Drug. Discov. 2010, 9, 728-743.

[297] G. Gasser, N. Metzler-Nolte, Curr. Opin. Chem. Bio. 2012, 16, 84-91.

[298] S. H. Heinemann, T. Hoshi, M. Westerhausen, A. Schiller, Chem. Commun. 2014, 50, 3644-3660.

[299] J. Marhenke, K. Trevino, C. Works, Coordin. Chem. Rev. 2016, 306, Part 2, 533-543. 
[300] P. G. Wang, M. Xian, X. Tang, X. Wu, Z. Wen, T. Cai, A. J. Janczuk, Chem. Rev. 2002, 102, 1091-1134.

[301] B. J. Heilman, M. A. Gonzalez, P. K. Mascharak, in Progress in Inorganic Chemistry Vol. 58, John Wiley \& Sons, Inc., 2014, 185-224.

[302] N. L. Fry, B. J. Heilman, P. K. Mascharak, Inorg. Chem. 2011, 50, 317-324.

[303] N. L. Fry, M. J. Rose, D. L. Rogow, C. Nyitray, M. Kaur, P. K. Mascharak, Inorg. Chem. 2010, 49, 1487-1495.

[304] A. K. Patra, M. J. Rose, K. A. Murphy, M. M. Olmstead, P. K. Mascharak, Inorg. Chem. 2004, 43, 4487-4495.

[305] M. J. Rose, N. L. Fry, R. Marlow, L. Hinck, P. K. Mascharak, J. Am. Chem. Soc. 2008, $130,8834-8846$.

[306] M. J. Rose, M. M. Olmstead, P. K. Mascharak, J. Am. Chem. Soc. 2007, 129, 53425343.

[307] A. M. Smith, M. C. Mancini, S. Nie, Nat. Nanotech. 2009, 4, 710-711.

[308] K. G. McLure, P. R. Young, US 20130281396, 2013.

[309] P. S. Fier, J. F. Hartwig, Science 2013, 342, 956-960.

[310] P. S. Fier, J. F. Hartwig, J. Am. Chem. Soc. 2014, 136, 10139-10147.

[311] K. P. Wojtas, J.-Y. Lu, D. Krahn, H.-D. Arndt, Chem. Asian J. 2016, 11, 2859-2862.

[312] R. K. Boeckman, Jr., P. Shao, J. J. Mullins, Org. Synth. 2000, 77, 141-152.

[313] D. W. Robbins, J. F. Hartwig, Org. Lett. 2012, 14, 4266-4269.

[314] S. M. Preshlock, D. L. Plattner, P. E. Maligres, S. W. Krska, R. E. Maleczka, M. R. Smith, Angew. Chem. 2013, 125, 13153-13157; Angew. Chem. Int. Ed. 2013, 52, 12915-12919.

[315] J. Takagi, K. Sato, J. F. Hartwig, T. Ishiyama, N. Miyaura, Tetrahedron Lett. 2002, 43, $5649-5651$.

[316] G. Hobbs, C. Frazer, D. J. Gardner, J. Cullum, S. Oliver, App. Microbiol. Biotechnol. 1989, 31, 272-277.

[317] M. D. Shepherd, M. K. Kharel, M. A. Bosserman, J. Rohr, in Current Protocols in Microbiology, John Wiley \& Sons, Inc., 2005.

[318] M. J. Amoroso, D. Schubert, P. Mitscherlich, P. Schumann, E. Kothe, J. Basic Microbiol. 2000, 40, 295-301. 
[319] A. W. J. Logan, J. S. Parker, M. S. Hallside, J. W. Burton, Org. Lett. 2012, 14, $2940-$ 2943.

[320] L. Feliu, W. Ajana, J. A. Joule, F. LopezCalahorra, M. Alvarez, Heterocycles 1997, 45, 1299-1308.

[321] R. Boulahjar, A. Ouach, C. Matteo, S. Bourg, M. Ravache, R. 1. Guével, S. Marionneau, T. Oullier, O. Lozach, L. Meijer, C. Guguen-Guillouzo, S. Lazar, M. Akssira, Y. Troin, G. Guillaumet, S. Routier, J. Med. Chem. 2012, 55, 9589-9606.

[322] E. V. Brown, M. B. Shambhu, J. Org. Chem. 1971, 36, 2002-2004. 

11 Analytische Daten 


\subsection{Daten zu den Einkristall-Röntgenstrukturanalysen}

Tabelle 11.1:Kristallographische Daten und Details zur Verfeinerung der EinkristallRöntgenstrukturanalyse von 156.

\begin{tabular}{|c|c|}
\hline Verbindung & 156 \\
\hline Formel & $\mathrm{C}_{14} \mathrm{H}_{12} \mathrm{~N}_{2} \mathrm{O}_{6} \mathrm{~S}$ \\
\hline Molekulargewicht $\left[\mathrm{g} \cdot \mathrm{mol}^{-1}\right]$ & 336.32 \\
\hline Wellenlänge $\lambda[\AA]$ & 0.71073 \\
\hline Temperatur $T[\mathrm{~K}]$ & $133(2)$ \\
\hline Kristallsystem & Monoklin \\
\hline Kristallgröße [mm] & $0.06 \times 0.06 \times 0.04$ \\
\hline Raumgruppe & $\mathrm{P} 2_{1}$ \\
\hline Zellparameter $a[\AA]$ & $9.9314(4)$ \\
\hline$b[\AA]$ & $6.7256(2)$ \\
\hline$c[\AA]$ & $11.6925(5)$ \\
\hline$\alpha\left[^{\circ}\right]$ & 90 \\
\hline$\beta\left[^{\circ}\right]$ & $106.475(2)$ \\
\hline$\gamma\left[^{\circ}\right]$ & 90 \\
\hline Volumen $V\left[\AA^{3}\right]$ & $748.93(5)$ \\
\hline Formeleinheiten $Z$ pro Elementarzelle & 2 \\
\hline Dichte $\rho_{\text {calcd. }}\left[\mathrm{g} \cdot \mathrm{cm}^{-3}\right]$ & 1.491 \\
\hline Absorptionskoeffizient $\mu\left[\mathrm{mm}^{-1}\right]$ & 0.250 \\
\hline$F(000)$ & 348 \\
\hline$\theta$-Bereich $\left[^{\circ}\right]$ & $2.38-27.48$ \\
\hline Index-Bereich & $\begin{aligned}-12 & \leq h \leq 12 \\
-8 & \leq k \leq 8 \\
-15 & \leq l \leq 13\end{aligned}$ \\
\hline Gemessene Reflexe & 4400 \\
\hline Vollständigkeit $\theta_{\max }[\%]$ & 98.9 \\
\hline Unabhängige Reflexe $\left(R_{\text {int }}\right)$ & $3193\left(R_{\text {int }}=0.0199\right)$ \\
\hline Verfeinerungsparameter/Fixierungen & $256 / 1$ \\
\hline Absorptionskorrektur & Keine \\
\hline $\begin{array}{l}\text { Gewichteter Zuverlässigkeitsfaktor } w R_{2}{ }^{(a)} \\
\text { (alle Daten, gegen } F^{2} \text { ) }\end{array}$ & 0.0844 \\
\hline Zuverlässigkeitsfaktor $R_{1}[I>2 \sigma(I)]^{(\mathrm{a})}$ & 0.0342 \\
\hline Gütefaktor $S^{(\mathrm{b})}$ & 1.085 \\
\hline Restelektronendichte $\left[\mathrm{e} \cdot \AA^{-3}\right]$ & $0.276 /-0.190$ \\
\hline Interne Messnummer & FO4763 \\
\hline
\end{tabular}

(a) $R_{1}=\frac{\sum|| F_{\mathrm{o}}|-| F_{\mathrm{c}}||}{\sum\left|F_{\mathrm{o}}\right|}, w R_{2}=\sqrt{\frac{\sum\left[w\left(F_{\mathrm{o}}^{2}-F_{\mathrm{c}}^{2}\right)^{2}\right]}{\sum\left[w\left(F_{\mathrm{o}}^{2}\right)^{2}\right]}}$ mit $w^{-1}=\sigma^{2}\left(F_{\mathrm{o}}^{2}\right)+(a P)^{2} ;$ (b) $S=\sqrt{\frac{\sum\left[w\left(F_{\mathrm{o}}^{2}-F_{\mathrm{c}}^{2}\right)^{2}\right]}{N_{\mathrm{o}}-N_{\mathrm{p}}}}$ 
Tabelle 11.2: Kristallographische Daten und Details zur Verfeinerung der Einkristall-Röntgenstrukturanalyse von 66 .

\begin{tabular}{|c|c|}
\hline Verbindung & 66 \\
\hline Formel & $\mathrm{C}_{14} \mathrm{H}_{12} \mathrm{~N}_{2} \mathrm{O}_{6} \mathrm{~S}$ \\
\hline Molekulargewicht $\left[\mathrm{g} \cdot \mathrm{mol}^{-1}\right]$ & 336.32 \\
\hline Wellenlänge $\lambda[\AA]$ & 0.71073 \\
\hline Temperatur $T[\mathrm{~K}]$ & $133(2)$ \\
\hline Kristallsystem & Monoklin \\
\hline Kristallgröße [mm] & $0.056 \times 0.02 \times 0.02$ \\
\hline Raumgruppe & $\mathrm{P} 2{ }_{1} / \mathrm{c}$ \\
\hline Zellparameter $a[\AA]$ & $17.3568(9)$ \\
\hline$b[\AA]$ & $4.7355(3)$ \\
\hline$c[\AA]$ & $17.1697(9)$ \\
\hline$\alpha\left[^{\circ}\right]$ & 90 \\
\hline$\beta\left[^{\circ}\right]$ & $91.352(3)$ \\
\hline$\gamma\left[{ }^{\circ}\right]$ & 90 \\
\hline Volumen $V\left[\AA^{3}\right]$ & $1410.84(14)$ \\
\hline Formeleinheiten $Z$ pro Elementarzelle & 4 \\
\hline Dichte $\rho_{\text {calcd. }}\left[\mathrm{g} \cdot \mathrm{cm}^{-3}\right]$ & 1.583 \\
\hline Absorptionskoeffizient $\mu\left[\mathrm{mm}^{-1}\right]$ & 0.265 \\
\hline$F(000)$ & 696 \\
\hline$\theta$-Bereich $\left[{ }^{\circ}\right]$ & $2.35-25.68$ \\
\hline Index-Bereich & $\begin{array}{c}-21 \leq h \leq 21 \\
-3 \leq k \leq 5 \\
-20 \leq l \leq 20\end{array}$ \\
\hline Gemessene Reflexe & 11098 \\
\hline Vollständigkeit $\theta_{\max }[\%]$ & 99.8 \\
\hline Unabhängige Reflexe $\left(R_{\text {int }}\right)$ & $2677\left(R_{\mathrm{int}}=0.0095\right)$ \\
\hline Verfeinerungsparameter & 215 \\
\hline Absorptionskorrektur & semi-empirisch \\
\hline $\begin{array}{l}\text { Gewichteter Zuverlässigkeitsfaktor } w R_{2}^{(a)} \\
\left(\text { alle Daten, gegen } F_{0}^{2} \text { ) }\right.\end{array}$ & 0.1954 \\
\hline Zuverlässigkeitsfaktor $R_{1}[I>2 \sigma(I)]^{(\mathrm{a})}$ & 0.0800 \\
\hline Gütefaktor $S^{(\mathrm{b})}$ & 1.104 \\
\hline Restelektronendichte $\left[\mathrm{e} \cdot \AA^{-3}\right]$ & $0.514 /-0.497$ \\
\hline Interne Messnummer & FO5595 \\
\hline
\end{tabular}

(a) $R_{1}=\frac{\sum|| F_{\mathrm{o}}|-| F_{\mathrm{c}}||}{\sum\left|F_{\mathrm{o}}\right|}, w R_{2}=\sqrt{\frac{\sum\left[w\left(F_{\mathrm{o}}^{2}-F_{\mathrm{c}}^{2}\right)^{2}\right]}{\sum\left[w\left(F_{\mathrm{o}}^{2}\right)^{2}\right]}}$ mit $w^{-1}=\sigma^{2}\left(F_{\mathrm{o}}^{2}\right)+(a P)^{2} ;$ (b) $S=\sqrt{\frac{\sum\left[w\left(F_{\mathrm{o}}^{2}-F_{\mathrm{c}}^{2}\right)^{2}\right]}{N_{\mathrm{o}}-N_{\mathrm{p}}}}$ 


\subsection{Spektren zur Totalsynthese von Nosiheptid}

PW10B.020.001.1r.esp<smiles>C=C(C(=O)OCC)c1c(COC2CCCCO2)cccc1[N+](=O)[O-]</smiles>
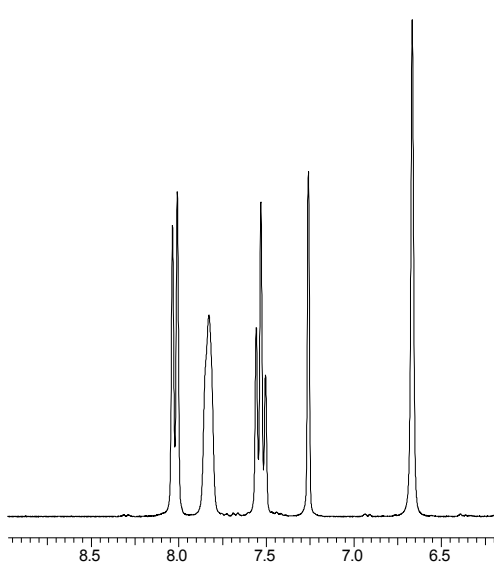

$\mathrm{NO}_{2}$

$$
\text { ( }
$$

Abbildung 11.1: ${ }^{1} \mathrm{H}-\mathrm{NMR}-$ Spektrum des $\alpha$-Ketoesters 118 (300 MHz, $\mathrm{CDCl}_{3}$ ).

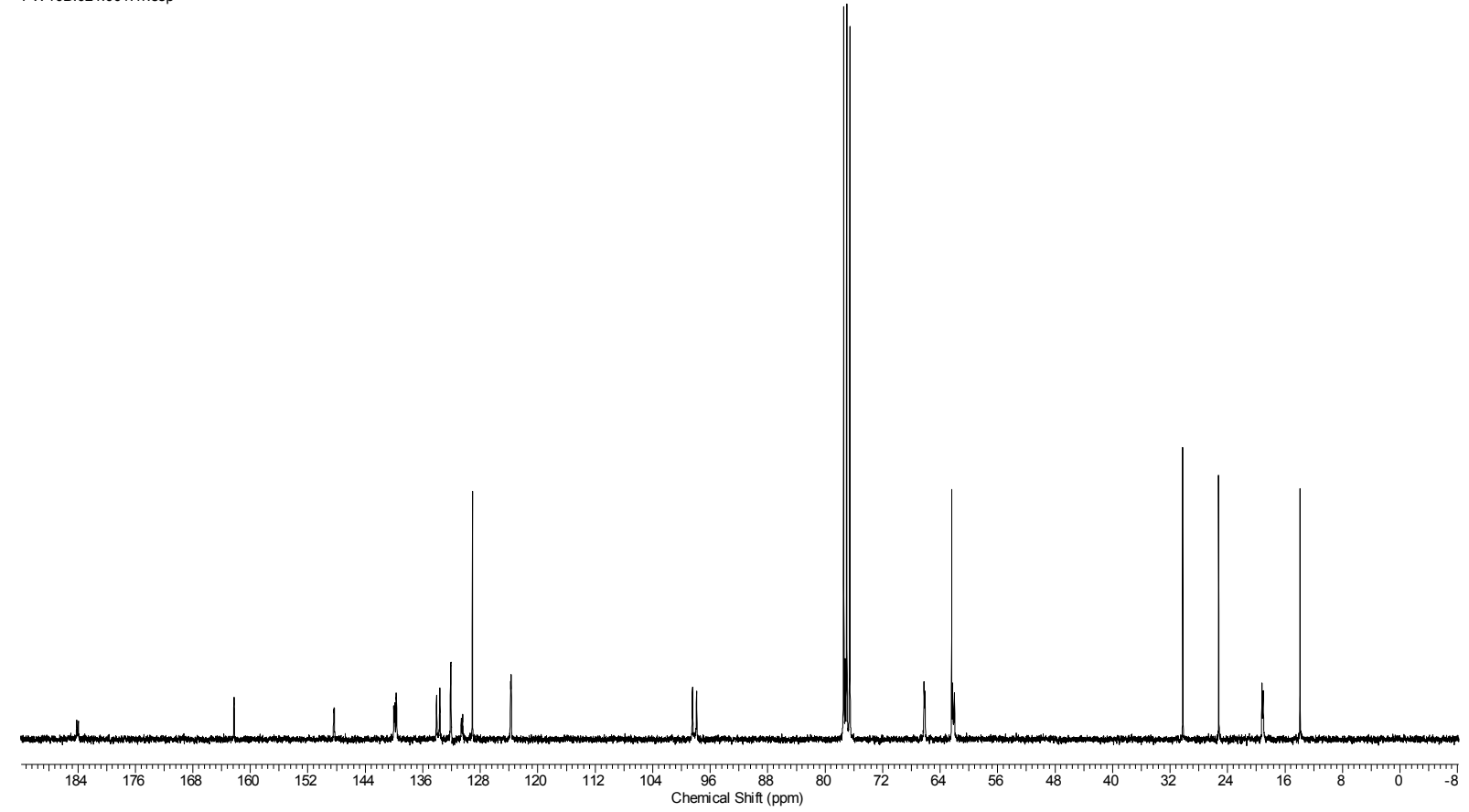

Abbildung 11.2: ${ }^{13} \mathrm{C}-\mathrm{NMR}-$ Spektrum des $\alpha$-Ketoesters $118\left(75 \mathrm{MHz}, \mathrm{CDCl}_{3}\right)$. 
PW74_neu.010.001.1r.esp<smiles>CCCC(=O)OCc1cccc2[nH]c(C(=O)OC(c3ccccc3)c3ccccc3)c(C)c12</smiles>
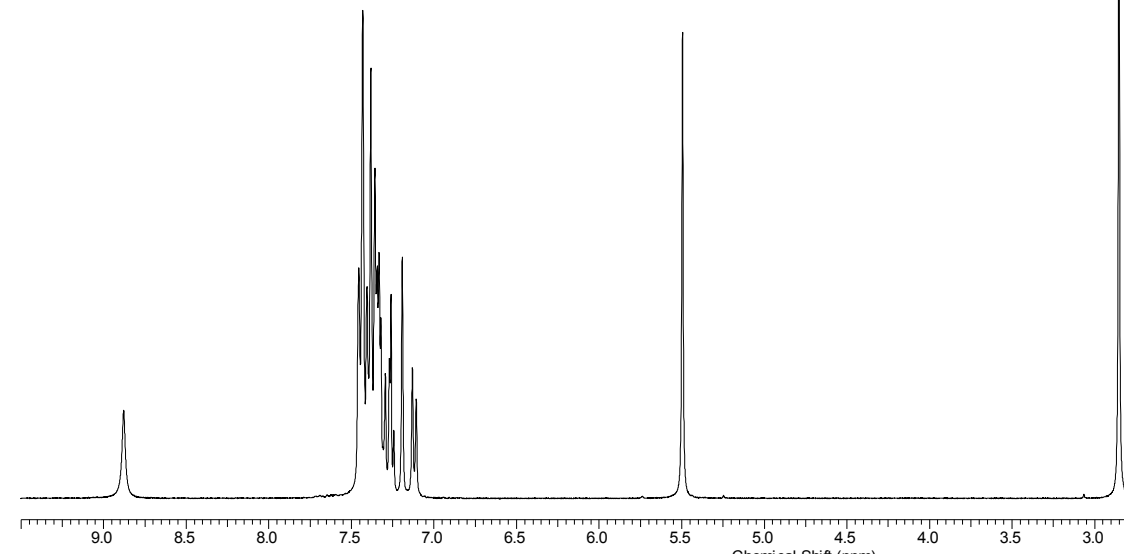

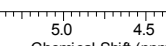

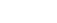

Abbildung 11.3: ${ }^{1} \mathrm{H}-\mathrm{NMR}-S p e k t r u m$ des Buttersäureesters $132\left(300 \mathrm{MHz}, \mathrm{CDCl}_{3}\right)$.

PW74_neu.011.001.1r.esp

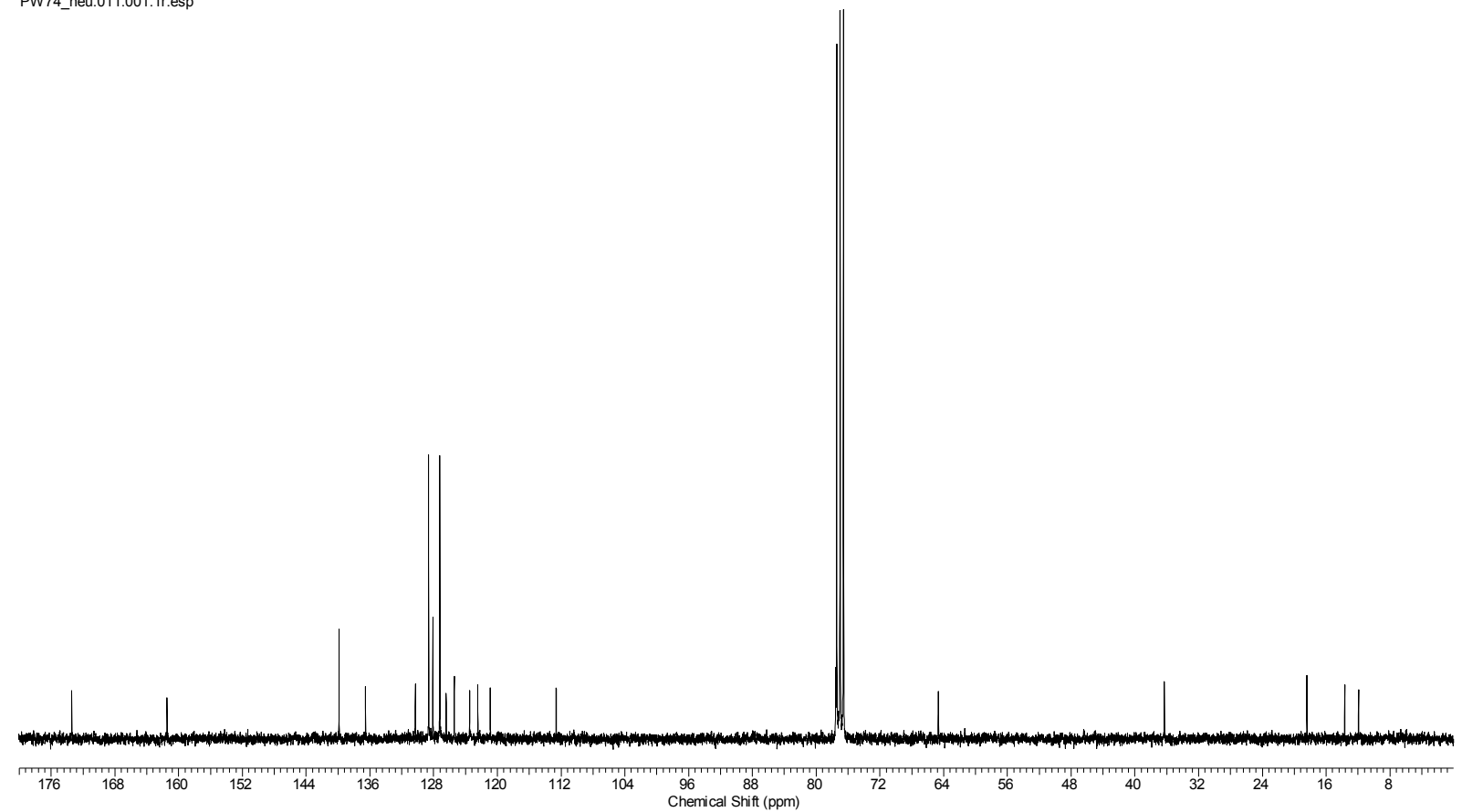

Abbildung 11.4: ${ }^{13} \mathrm{C}-\mathrm{NMR}-$ Spektrum des Buttersäureesters $132\left(75 \mathrm{MHz}, \mathrm{CDCl}_{3}\right)$. 


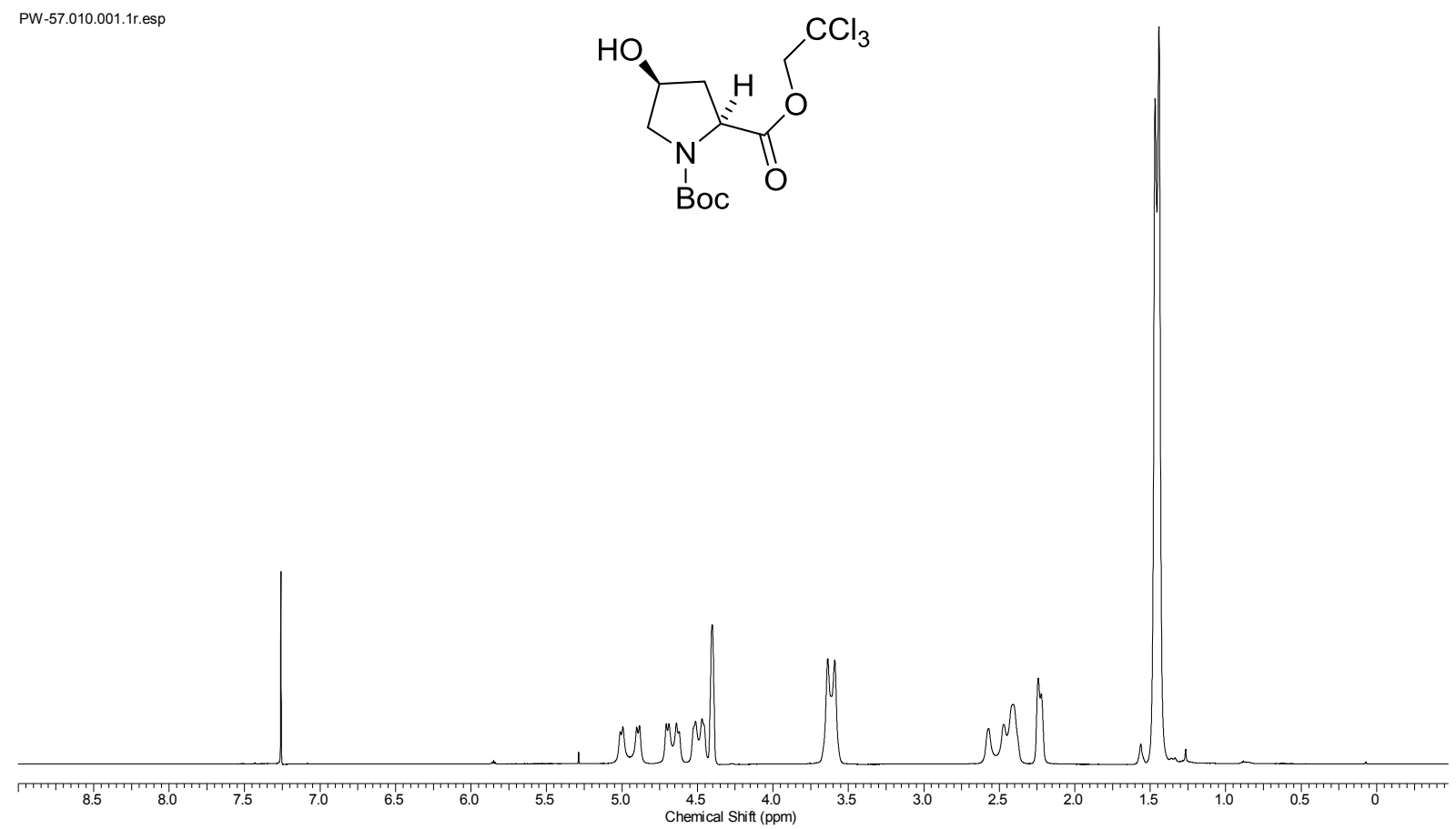

Abbildung 11.5: ${ }^{1} \mathrm{H}-\mathrm{NMR}-S p e k t r u m$ des 4-Hydroxypyrrolidins $136\left(600 \mathrm{MHz}, \mathrm{CDCl}_{3}, 323 \mathrm{~K}\right)$.

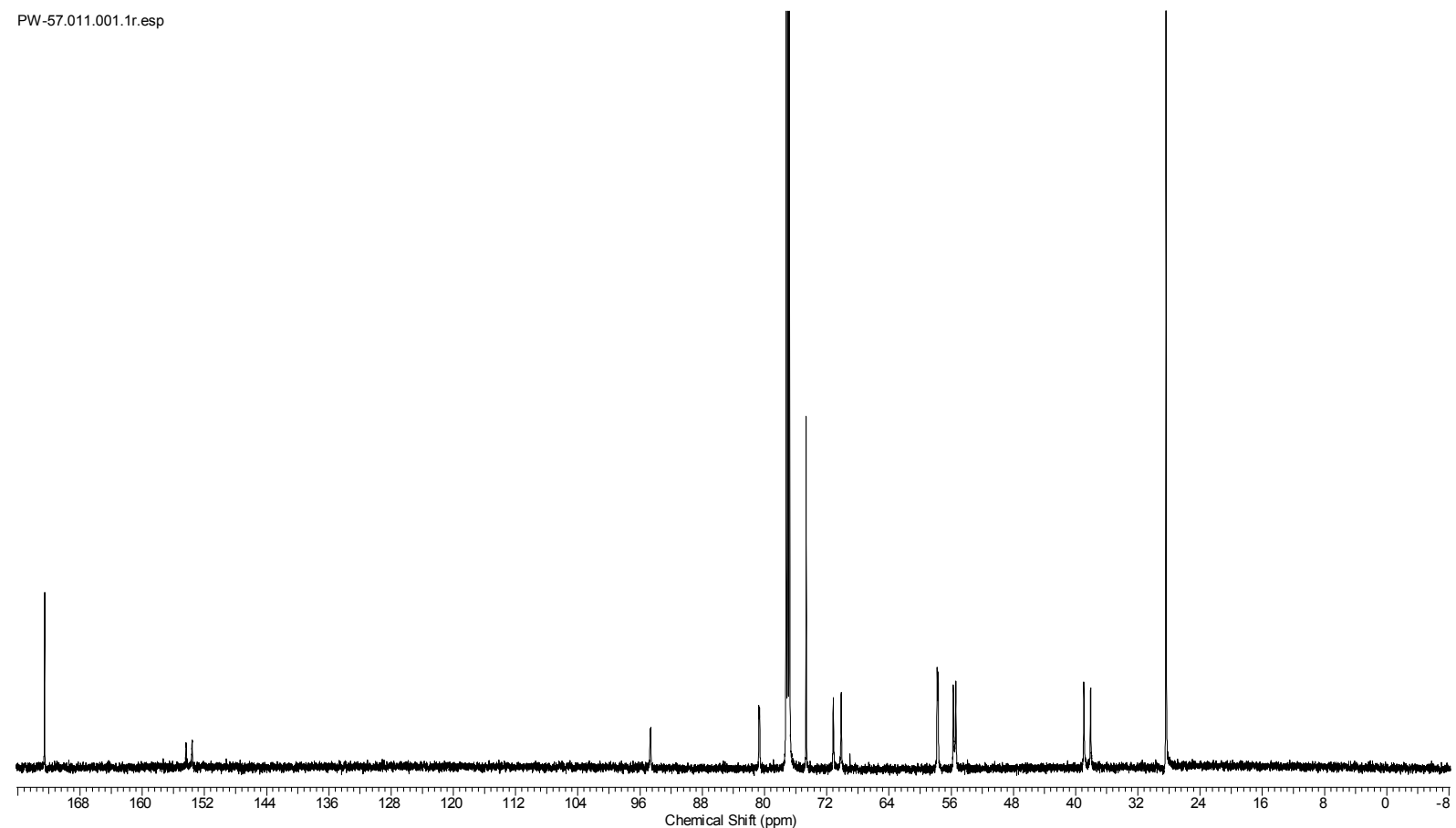

Abbildung 11.6: ${ }^{13} \mathrm{C}-\mathrm{NMR}-$ Spektrum des 4-Hydroxypyrrolidins $136\left(150 \mathrm{MHz}, \mathrm{CDCl}_{3}, 323 \mathrm{~K}\right)$. 


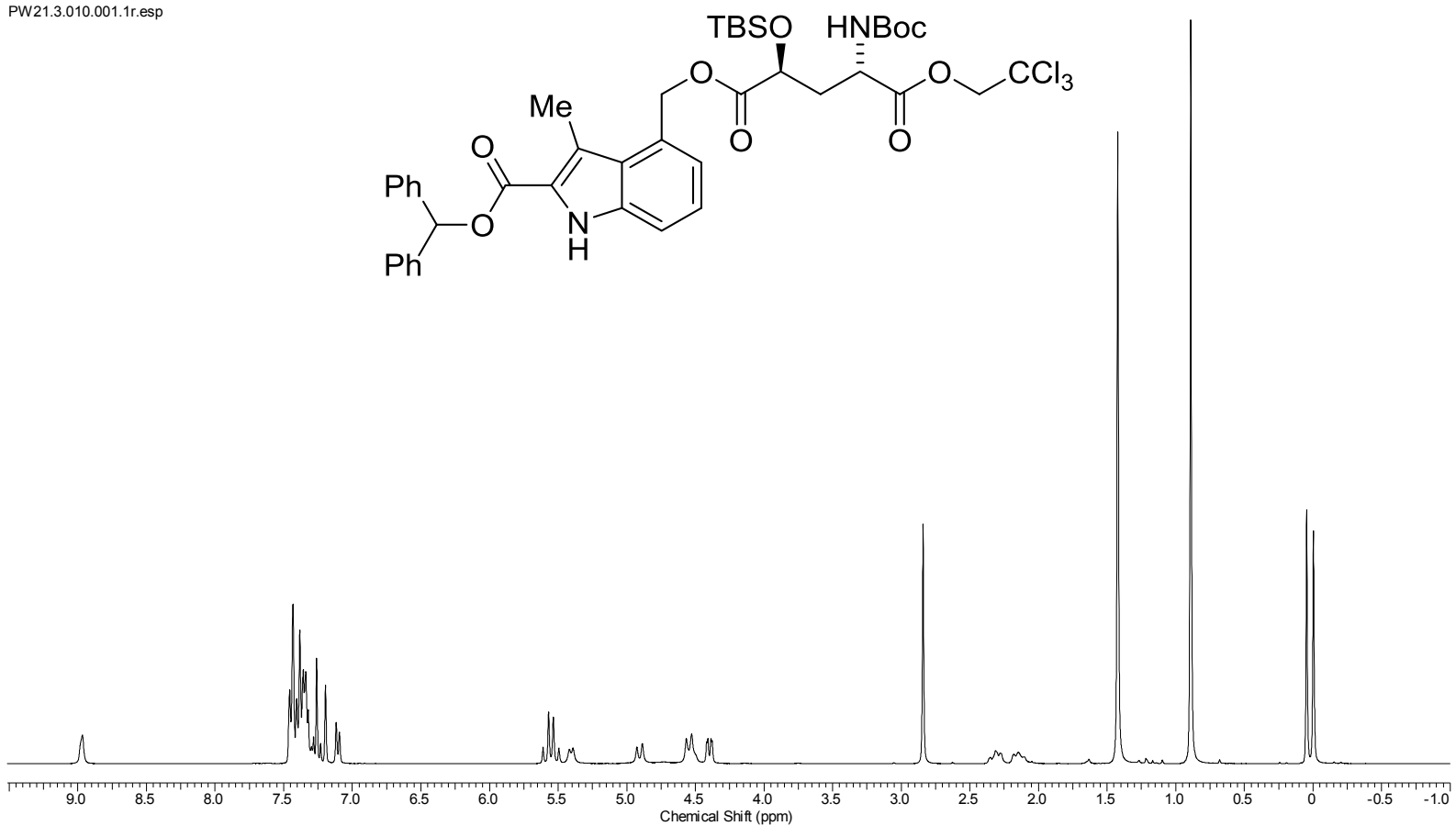

Abbildung 11.7: ${ }^{1} \mathrm{H}-\mathrm{NMR}-$ Spektrum des Triesters $137\left(300 \mathrm{MHz}, \mathrm{CDCl}_{3}\right)$.

PW21.3.011.001.1r.esp

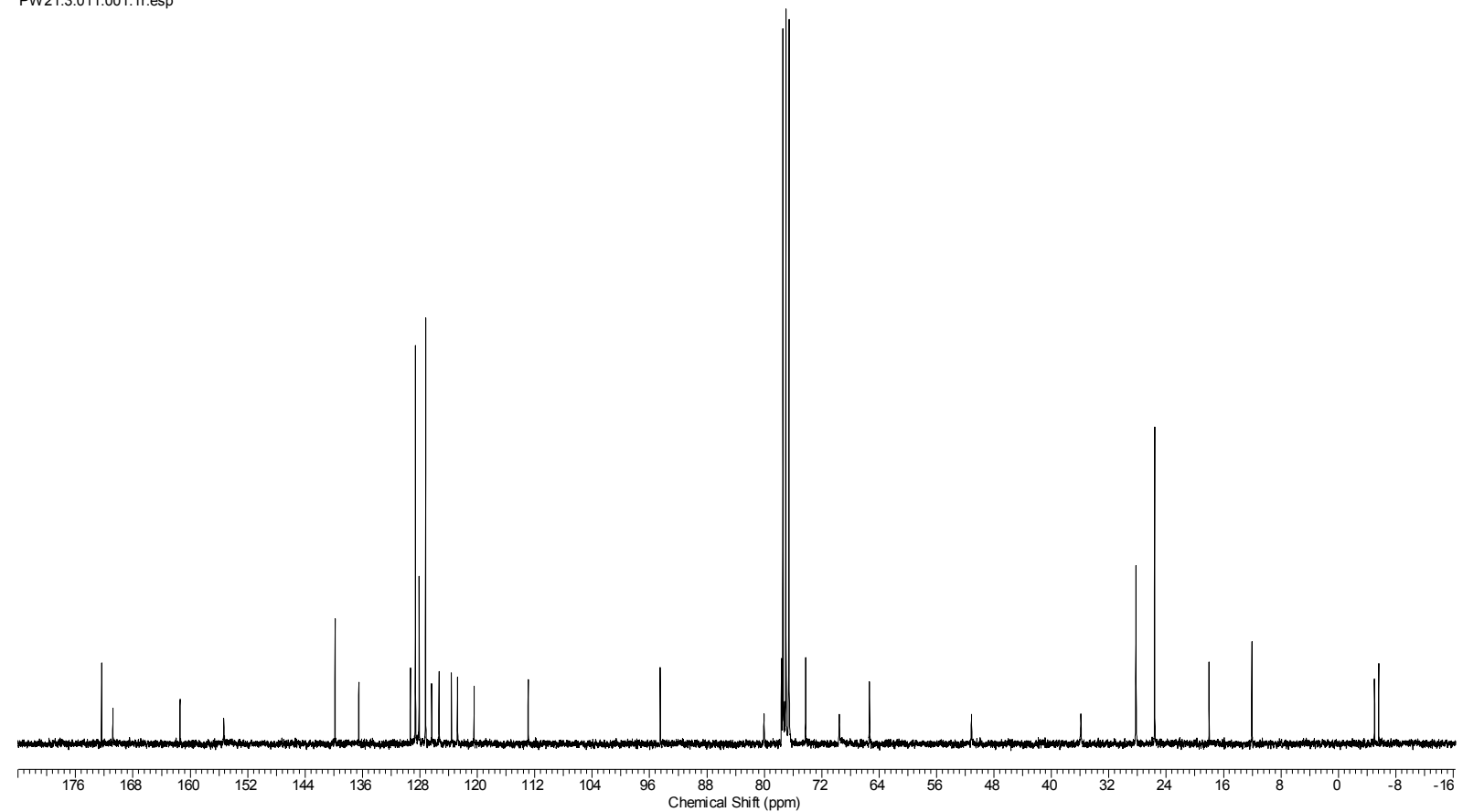

Abbildung 11.8: ${ }^{13} \mathrm{C}-\mathrm{NMR}-$ Spektrum des Triesters $137\left(75 \mathrm{MHz}, \mathrm{CDCl}_{3}\right)$. 
<smiles>Cc1c(C(=O)OC(c2ccccc2)c2ccccc2)[nH]c2cccc(COC(=O)[C@@H](C[C@@H](OC(C)(C)C)C(=O)O)C(=O)OC(C)(C)C)c12</smiles>

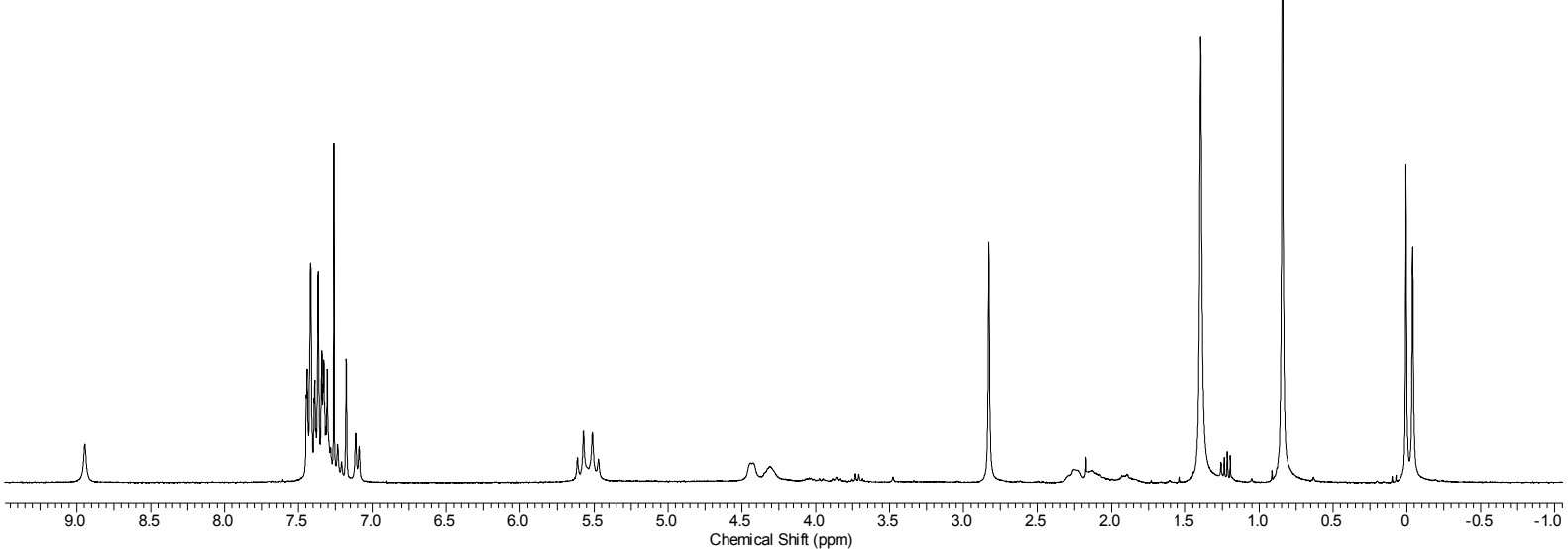

Abbildung 11.9: ${ }^{1} \mathrm{H}-\mathrm{NMR}-$ Spektrum des Glutaminsäure-Derivats $138\left(300 \mathrm{MHz}, \mathrm{CDCl}_{3}\right)$.

PW24.5 new.011.001.1r.esp

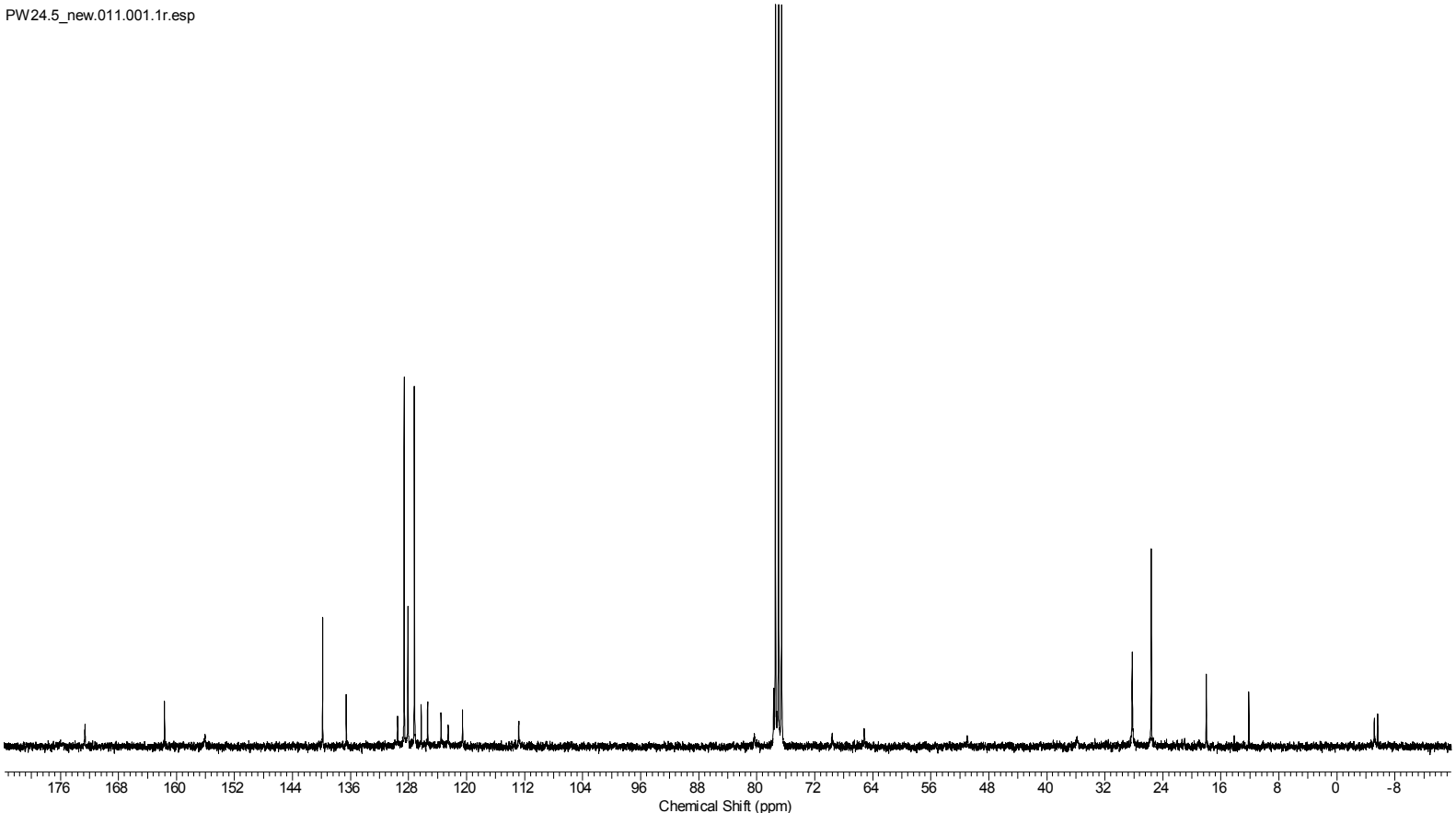

Abbildung 11.10: ${ }^{13} \mathrm{C}-\mathrm{NMR}$-Spektrum des Glutaminsäure-Derivats $138\left(75 \mathrm{MHz}, \mathrm{CDCl}_{3}\right)$. 


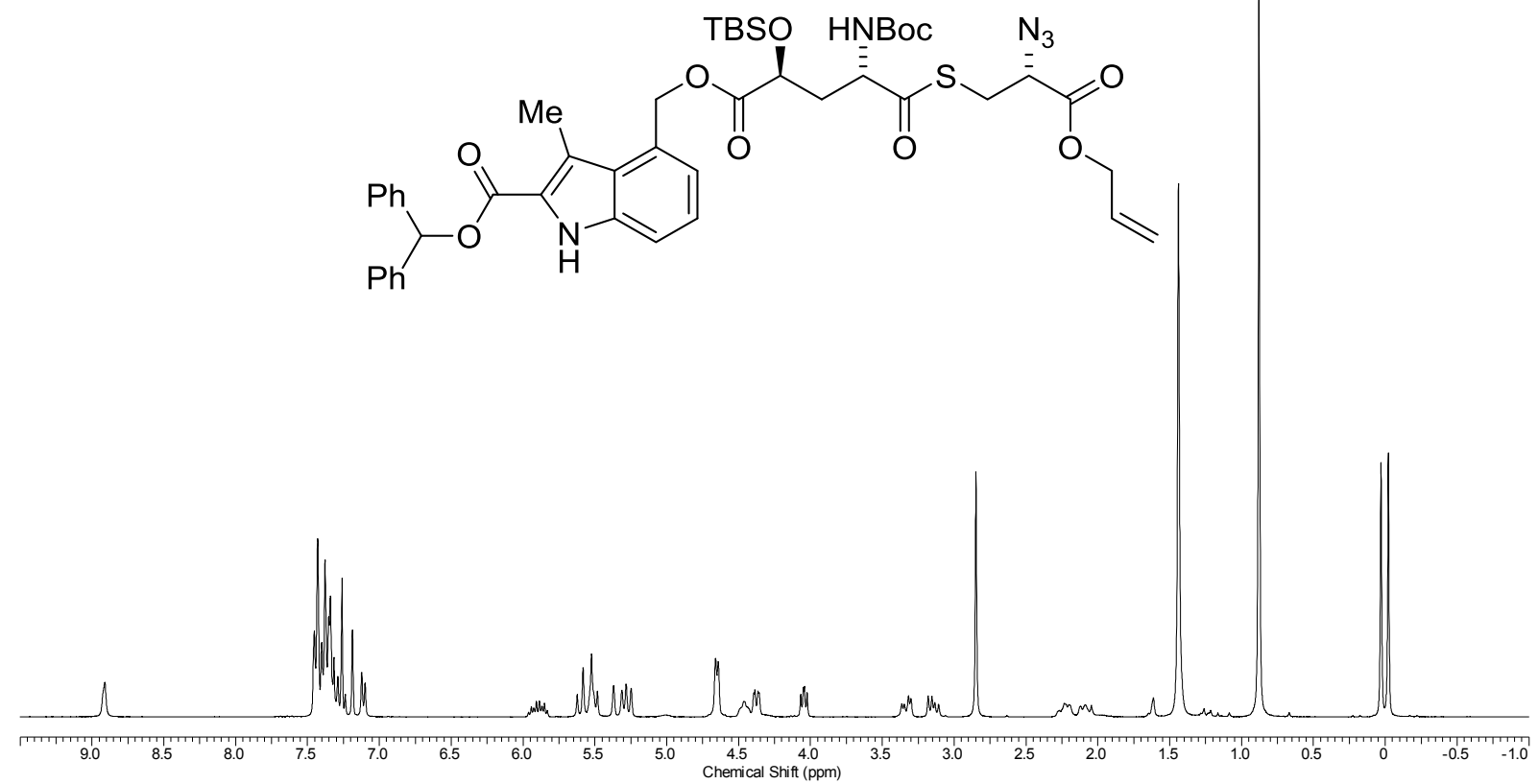

Abbildung 11.11: ${ }^{1} \mathrm{H}-\mathrm{NMR}-$ Spektrum des Thioesters $139\left(300 \mathrm{MHz}, \mathrm{CDCl}_{3}\right)$.

PW27.011.001.1r.esp

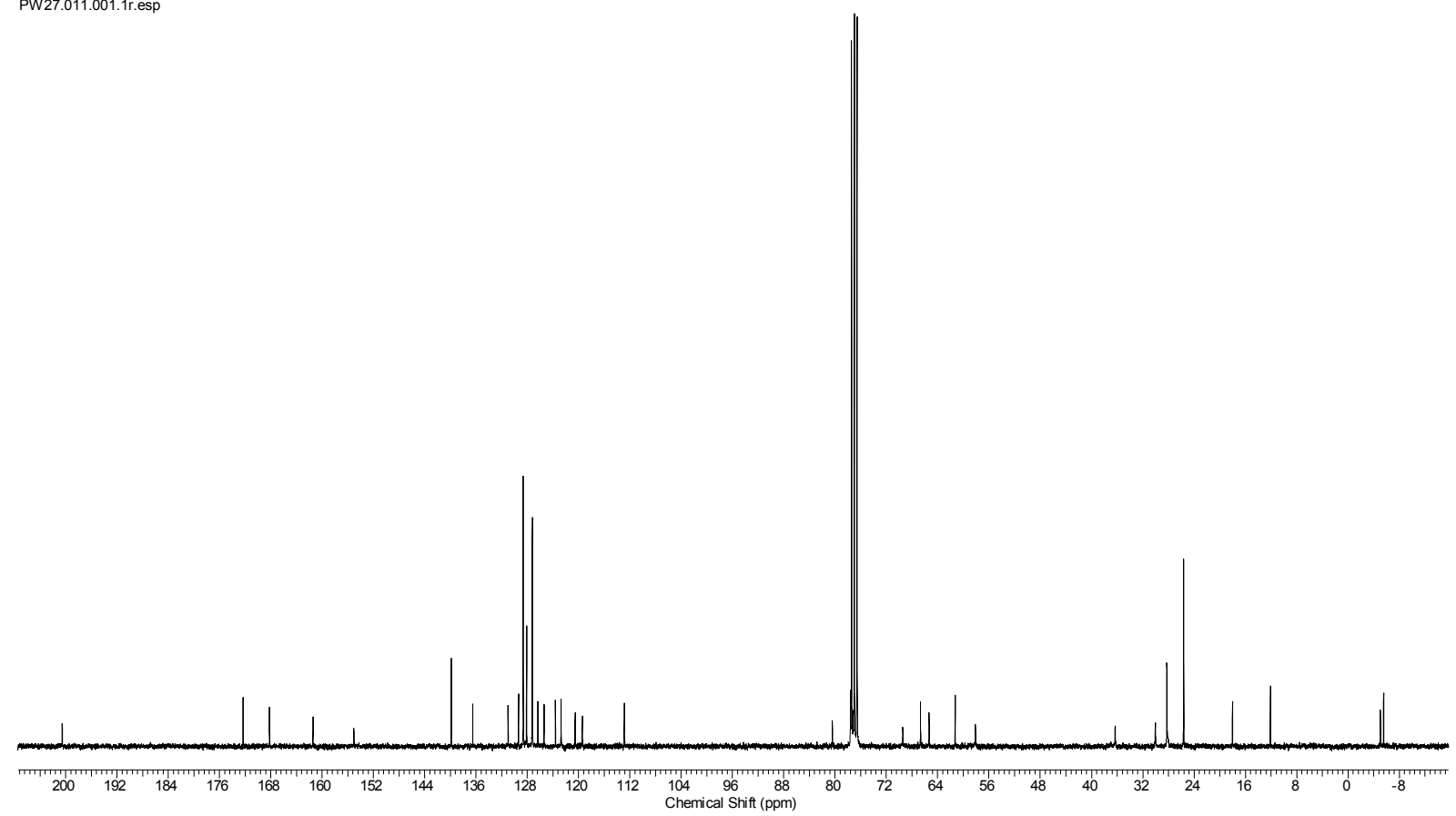

Abbildung 11.12: ${ }^{13} \mathrm{C}-\mathrm{NMR}-$ Spektrum des Thioesters $139\left(75 \mathrm{MHz}, \mathrm{CDCl}_{3}\right)$. 

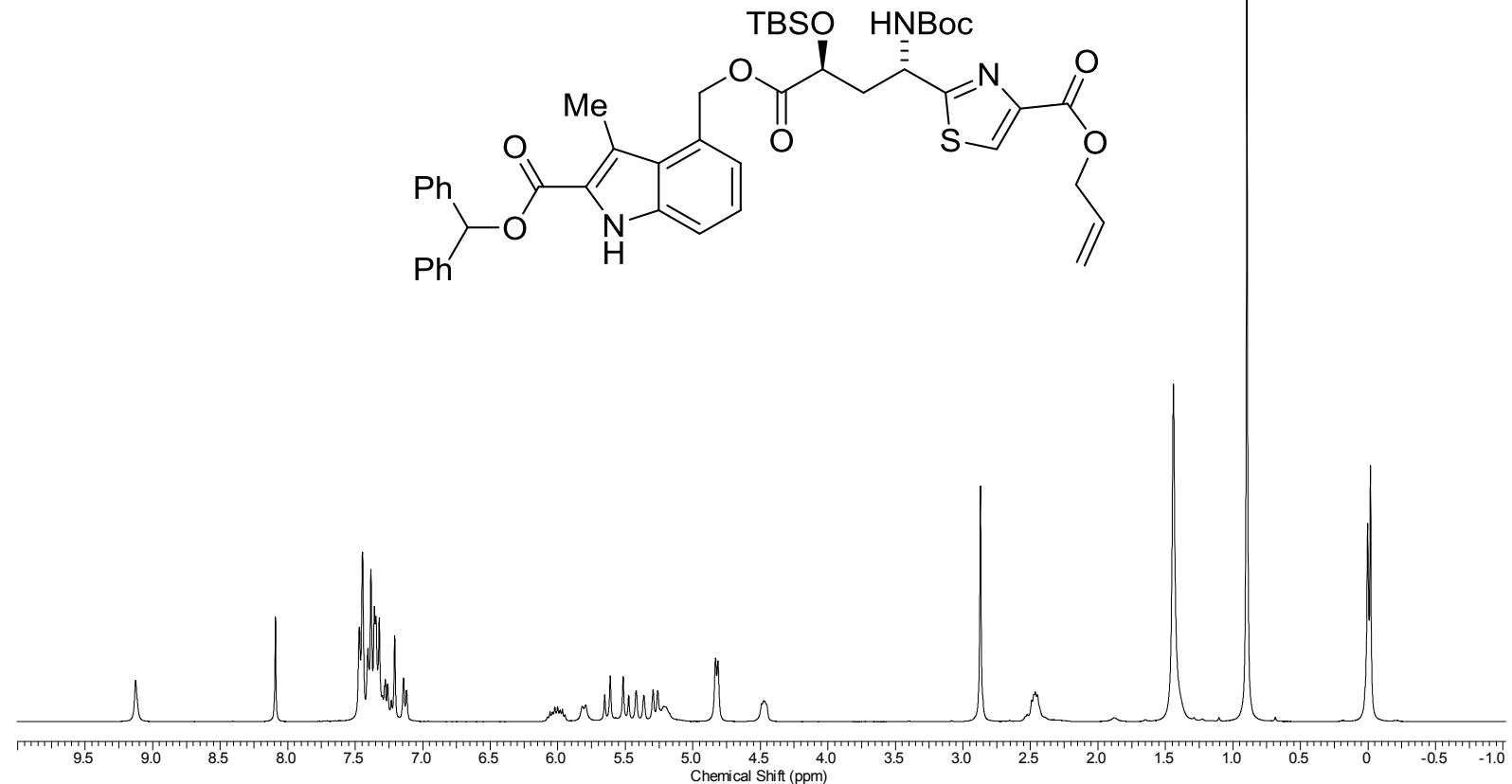

Abbildung 11.13: ${ }^{1} \mathrm{H}-\mathrm{NMR}-$ Spektrum des Thiazols $95\left(300 \mathrm{MHz}, \mathrm{CDCl}_{3}\right)$.

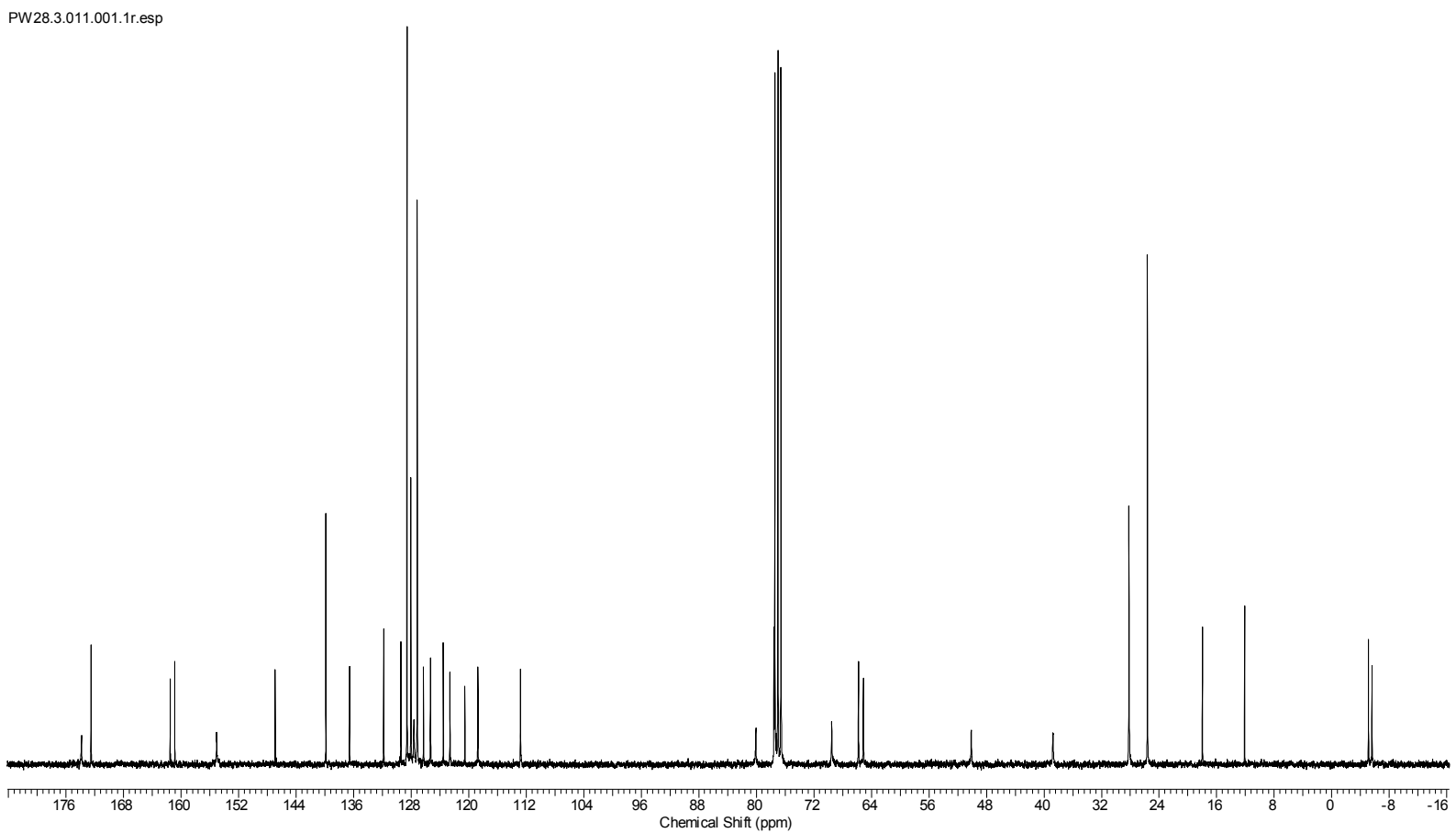

Abbildung 11.14: ${ }^{13} \mathrm{C}$-NMR-Spektrum des Thioesters 95 (75 MHz, $\left.\mathrm{CDCl}_{3}\right)$. 


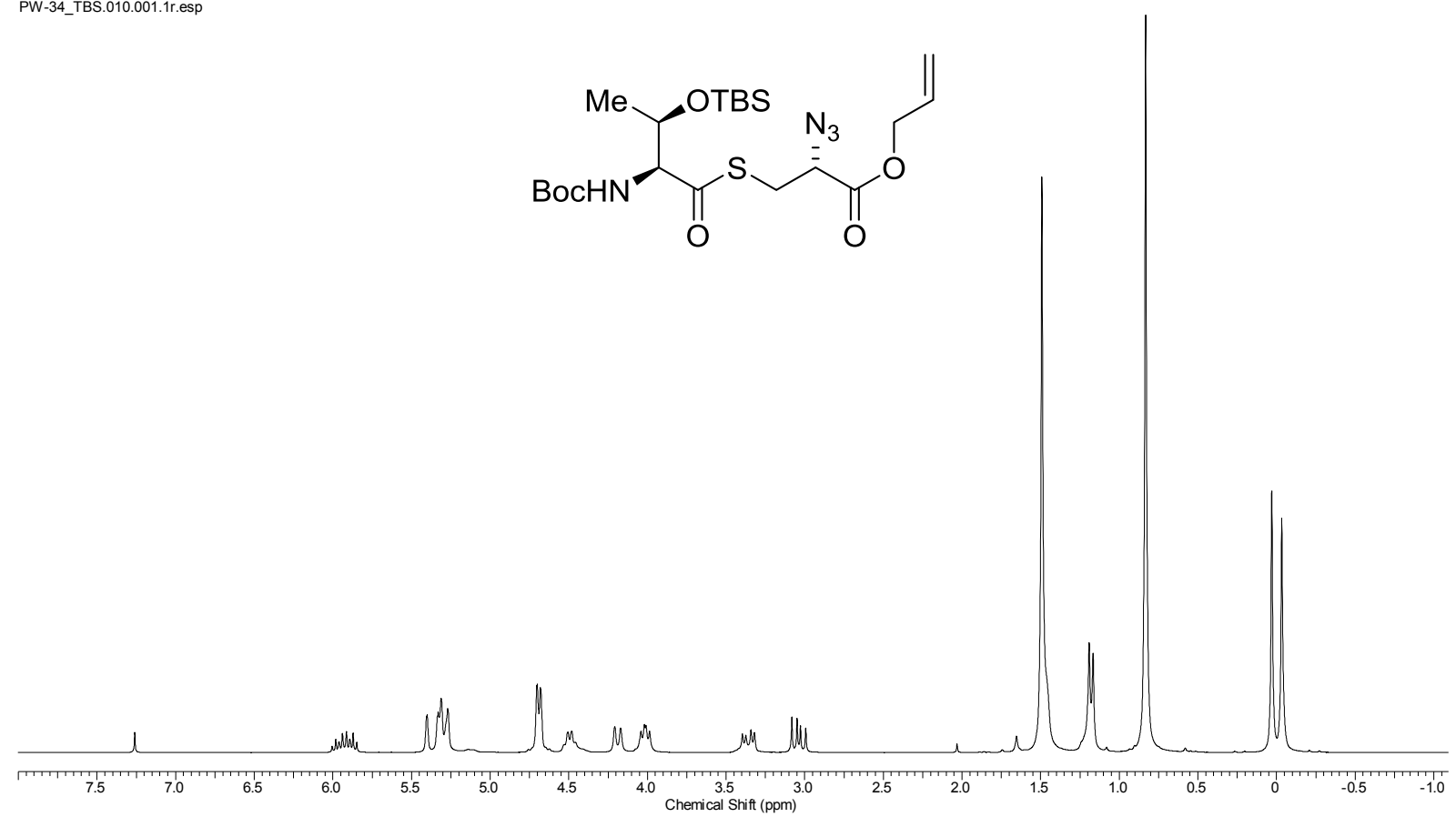

Abbildung 11.15: ${ }^{1} \mathrm{H}-\mathrm{NMR}-$ Spektrum des Thioesters $141\left(250 \mathrm{MHz}, \mathrm{CDCl}_{3}\right)$.

PW-34_TBS.011.001.1r.esp

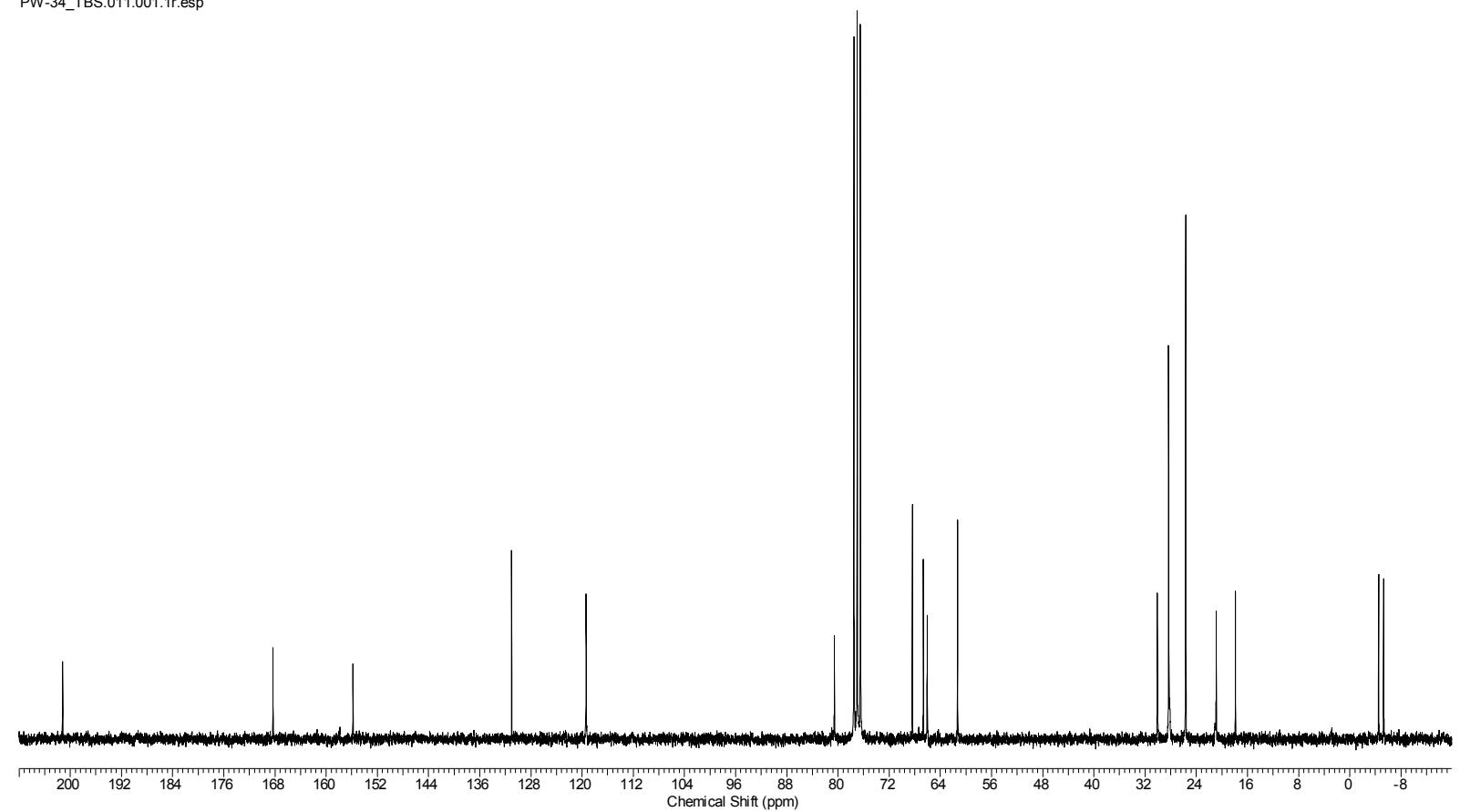

Abbildung 11.16: ${ }^{13} \mathrm{C}-\mathrm{NMR}-$ Spektrum des Thioesters $141\left(63 \mathrm{MHz}, \mathrm{CDCl}_{3}\right)$. 
<smiles>C=CCOC(=O)c1csc(C(NC(=O)OC(C)(C)C)C(C)[OH2+])n1</smiles>

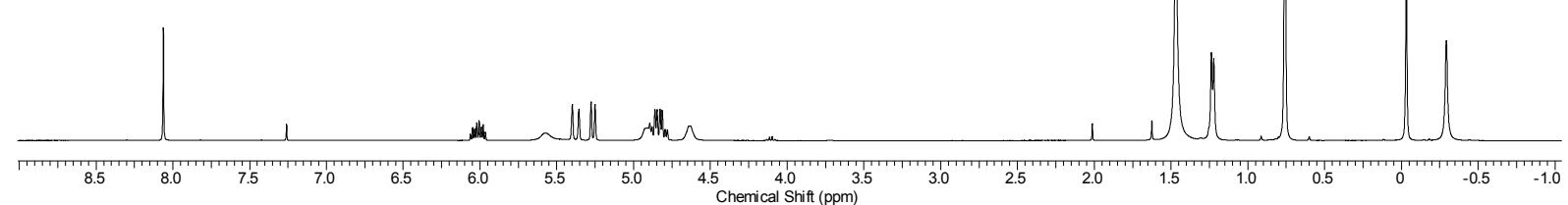

Abbildung 11.17: ${ }^{1} \mathrm{H}-\mathrm{NMR}-S p e k t r u m$ des Thiazols 142 (400 MHz, $\mathrm{CDCl}_{3}$ ).

PW43_TBS.011.001.1r.esp

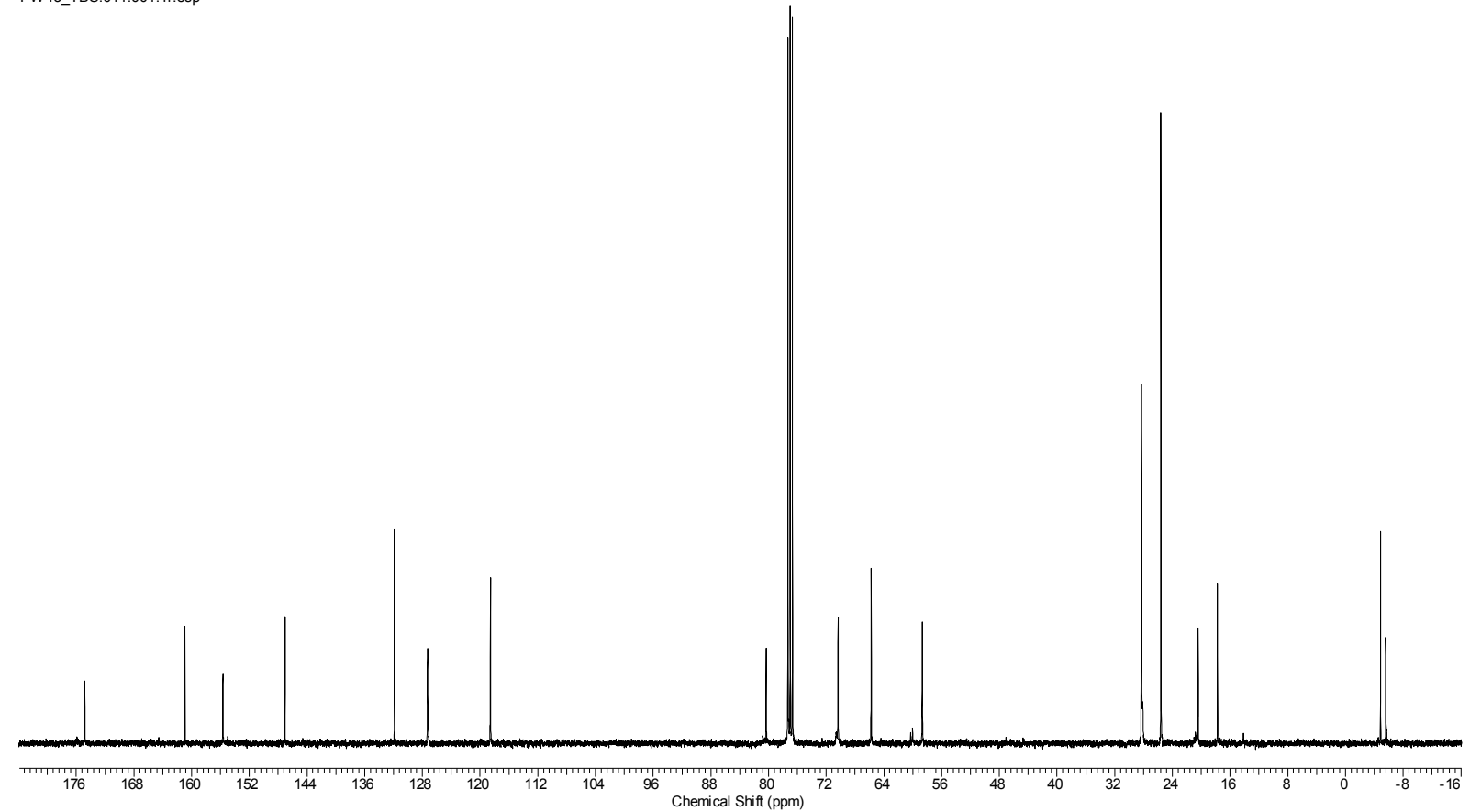

Abbildung 11.18: ${ }^{13} \mathrm{C}-\mathrm{NMR}-$ Spektrum des Thiazols $142\left(100 \mathrm{MHz}, \mathrm{CDCl}_{3}\right)$. 


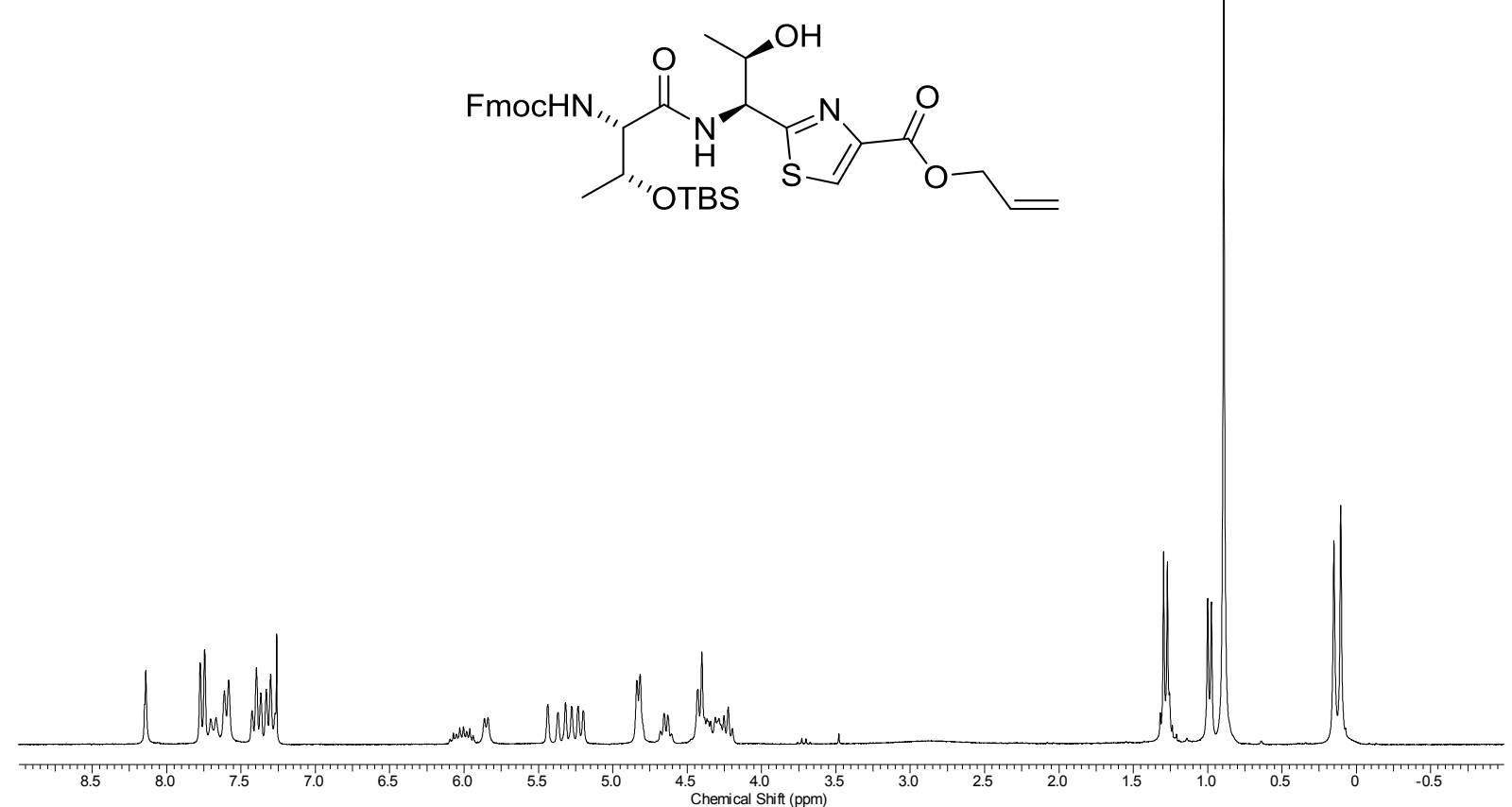

Abbildung 11.19: ${ }^{1} \mathrm{H}-\mathrm{NMR}-S p e k t r u m$ des Thiazols $144\left(250 \mathrm{MHz}, \mathrm{CDCl}_{3}\right)$.

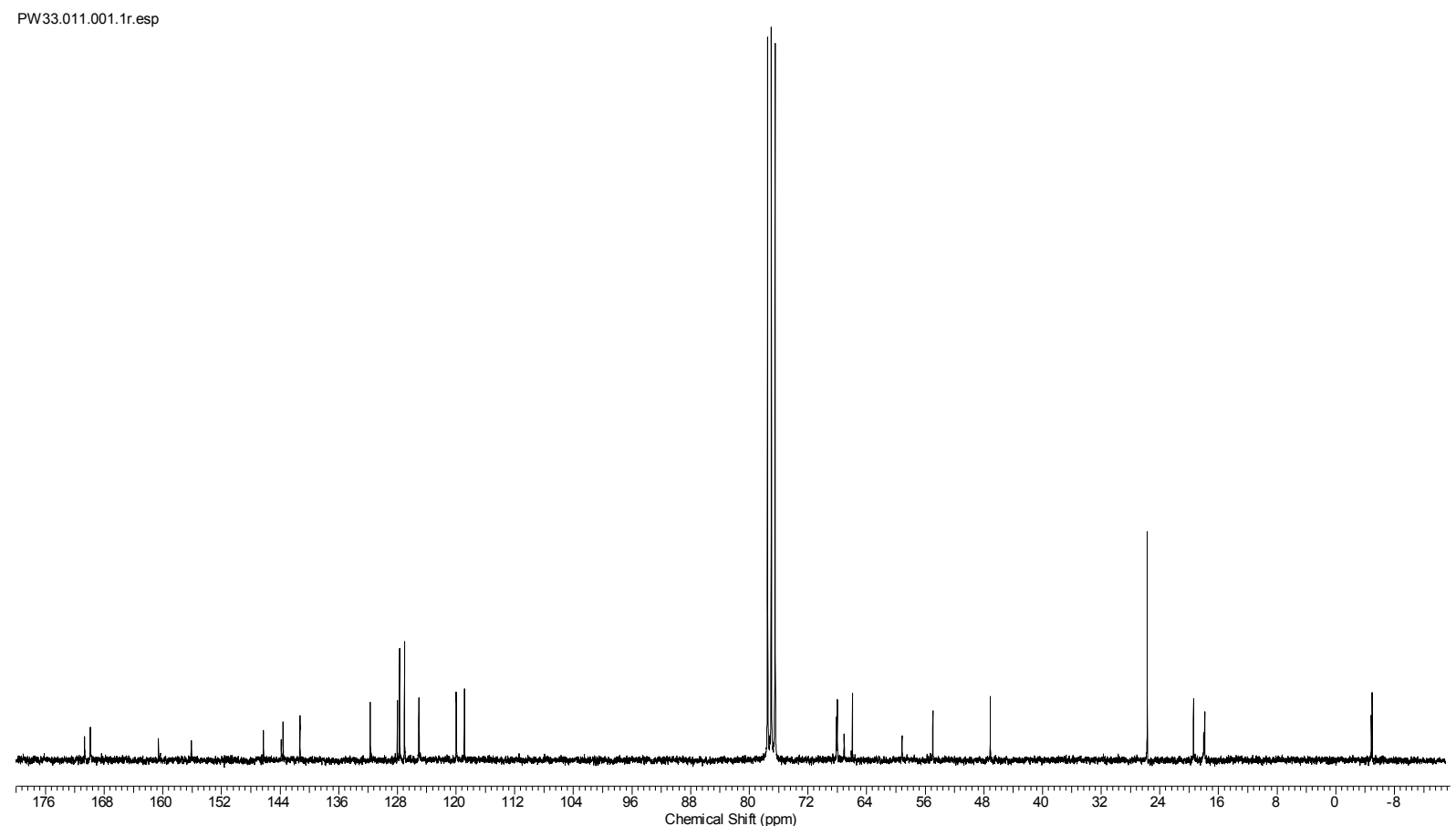

Abbildung 11.20: ${ }^{13} \mathrm{C}-\mathrm{NMR}-$ Spektrum des Thiazols 144 (63 MHz, $\mathrm{CDCl}_{3}$ ). 


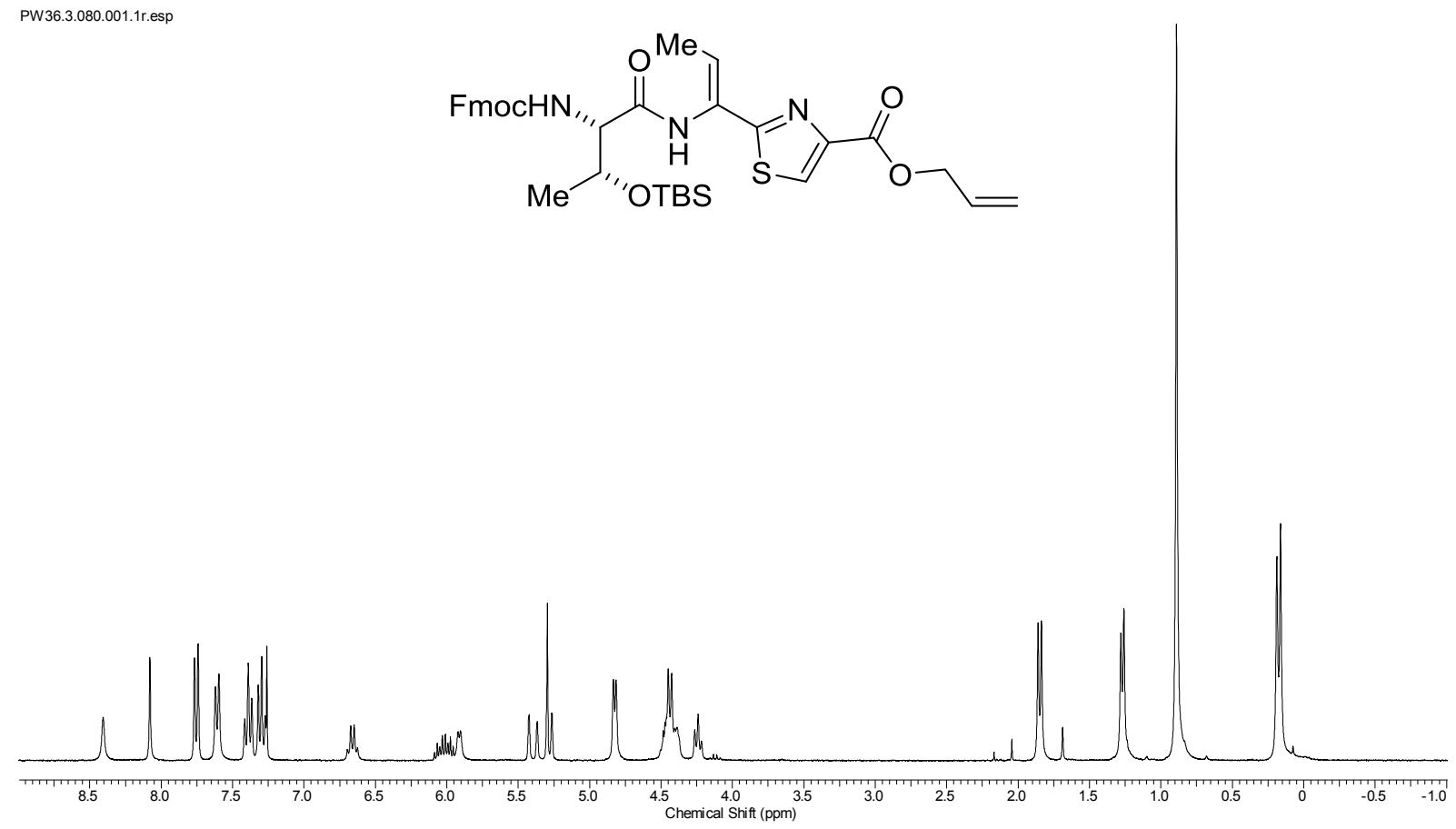

Abbildung 11.21: ${ }^{1} \mathrm{H}-\mathrm{NMR}-$ Spektrum des Thiazols $145\left(300 \mathrm{MHz}, \mathrm{CDCl}_{3}\right)$.

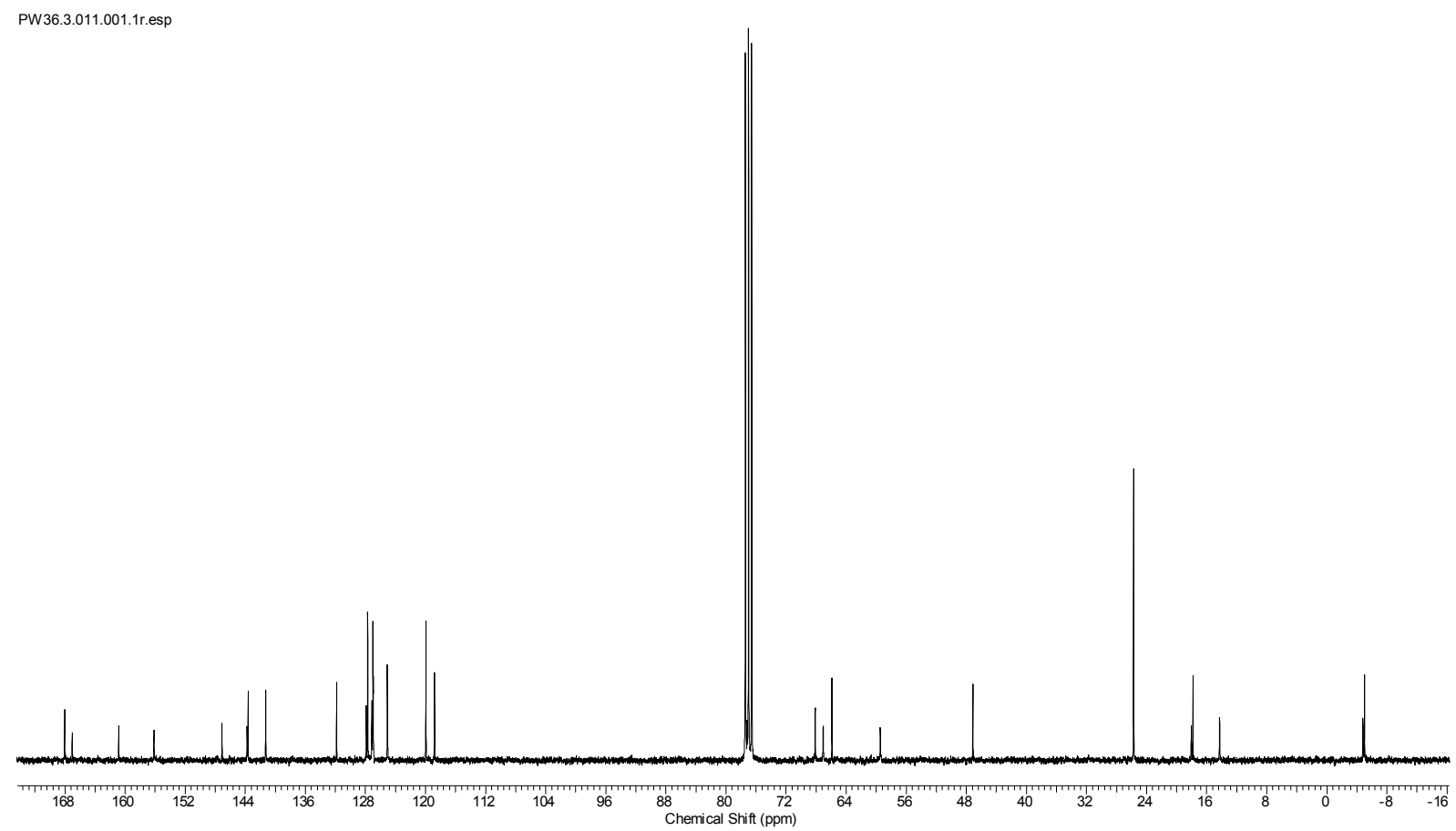

Abbildung 11.22: ${ }^{13} \mathrm{C}-\mathrm{NMR}-$ Spektrum des Thiazols $145\left(75 \mathrm{MHz}, \mathrm{CDCl}_{3}\right)$. 


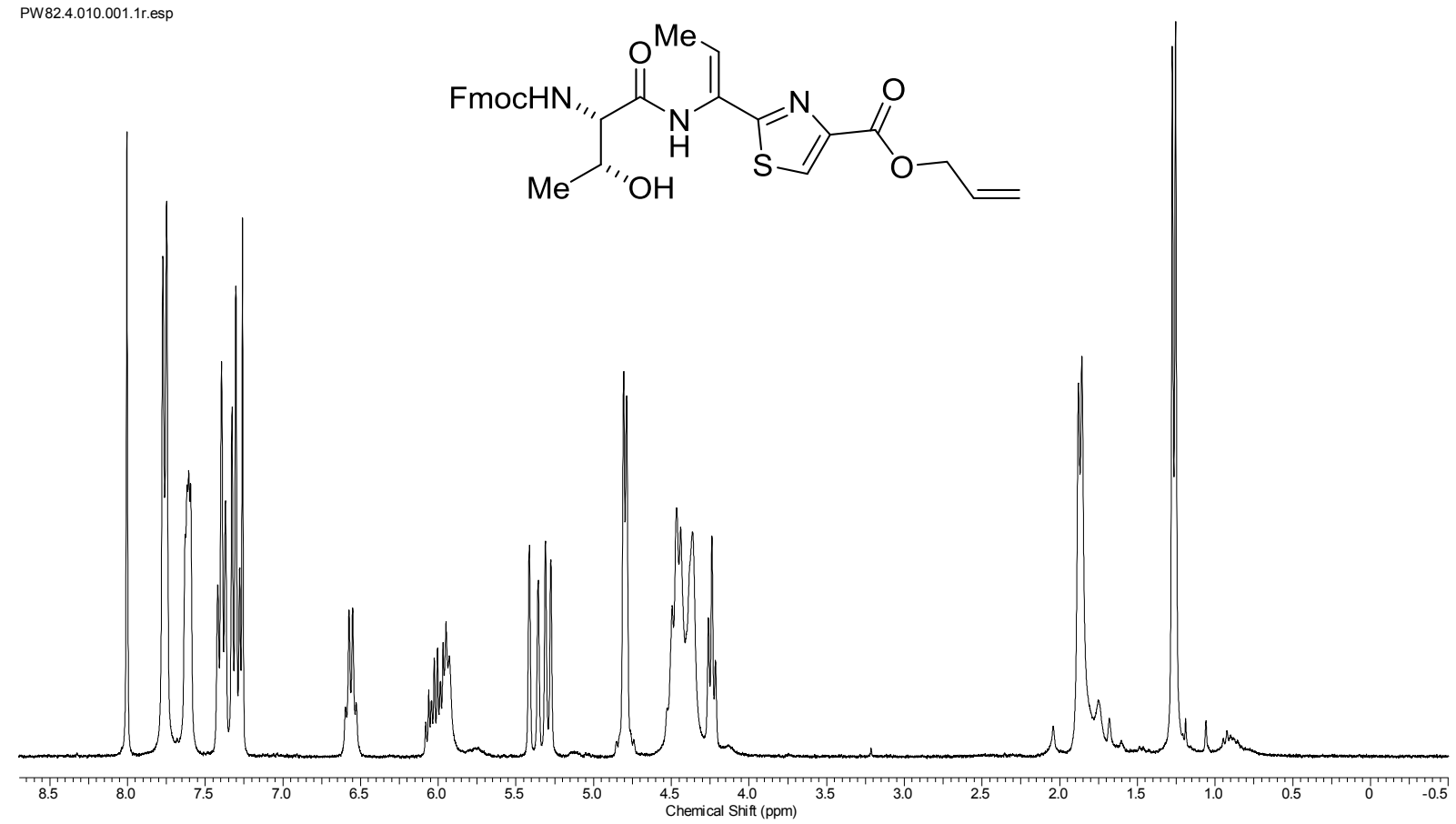

Abbildung 11.23: ${ }^{1} \mathrm{H}-\mathrm{NMR}-S p e k t r u m$ des Thiazols $146\left(300 \mathrm{MHz}, \mathrm{CDCl}_{3}\right)$.

PW82.4.011.001.1r.esp

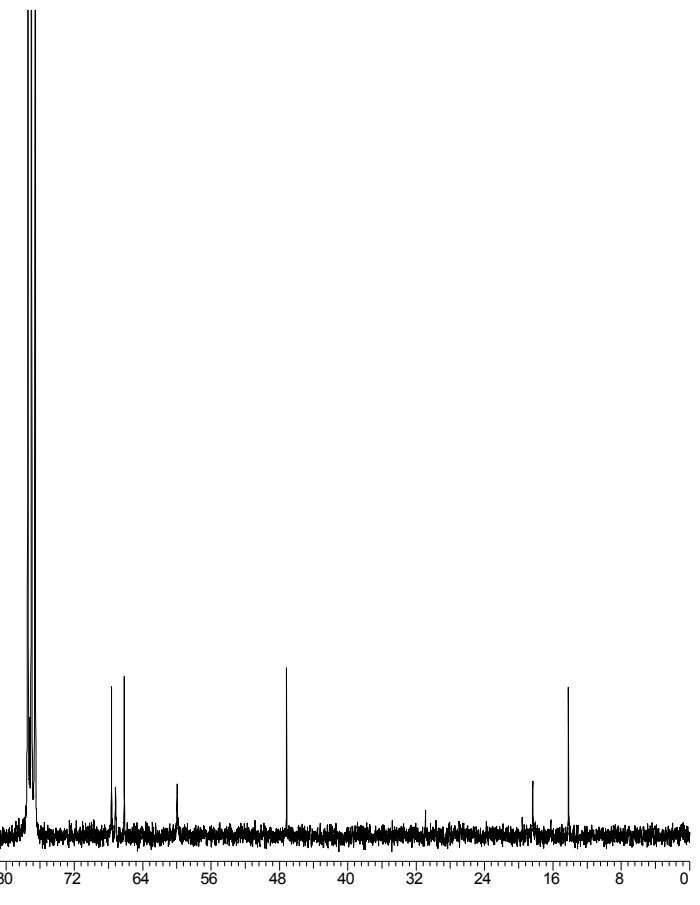

Abbildung 11.24: ${ }^{13} \mathrm{C}-\mathrm{NMR}-$ Spektrum des Thiazols $146\left(75 \mathrm{MHz}, \mathrm{CDCl}_{3}\right)$. 


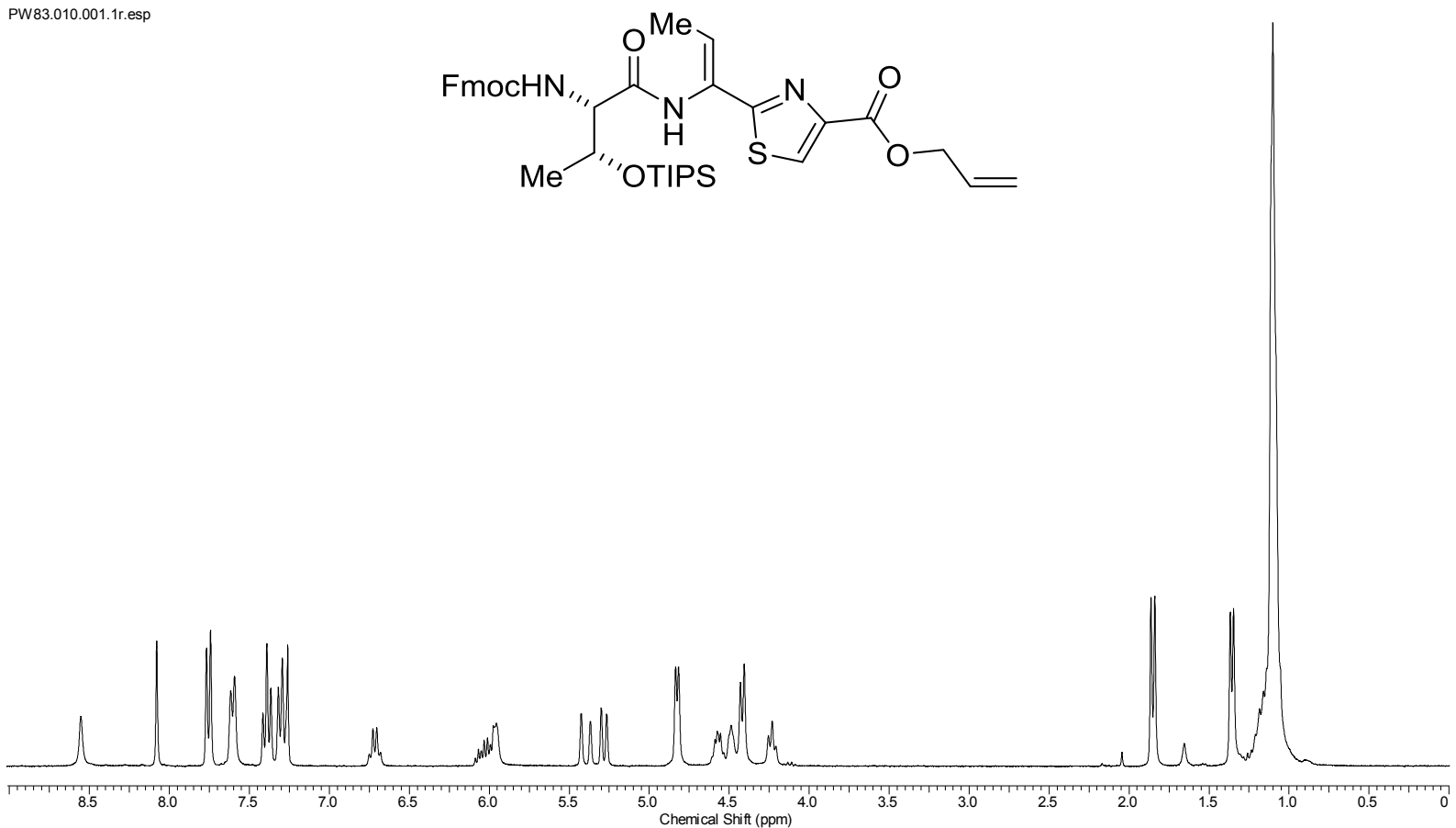

Abbildung 11.25: ${ }^{1} \mathrm{H}-\mathrm{NMR}-S p e k t r u m$ des Thiazols $147\left(300 \mathrm{MHz}, \mathrm{CDCl}_{3}\right)$.

PW83.014.001.1r.esp

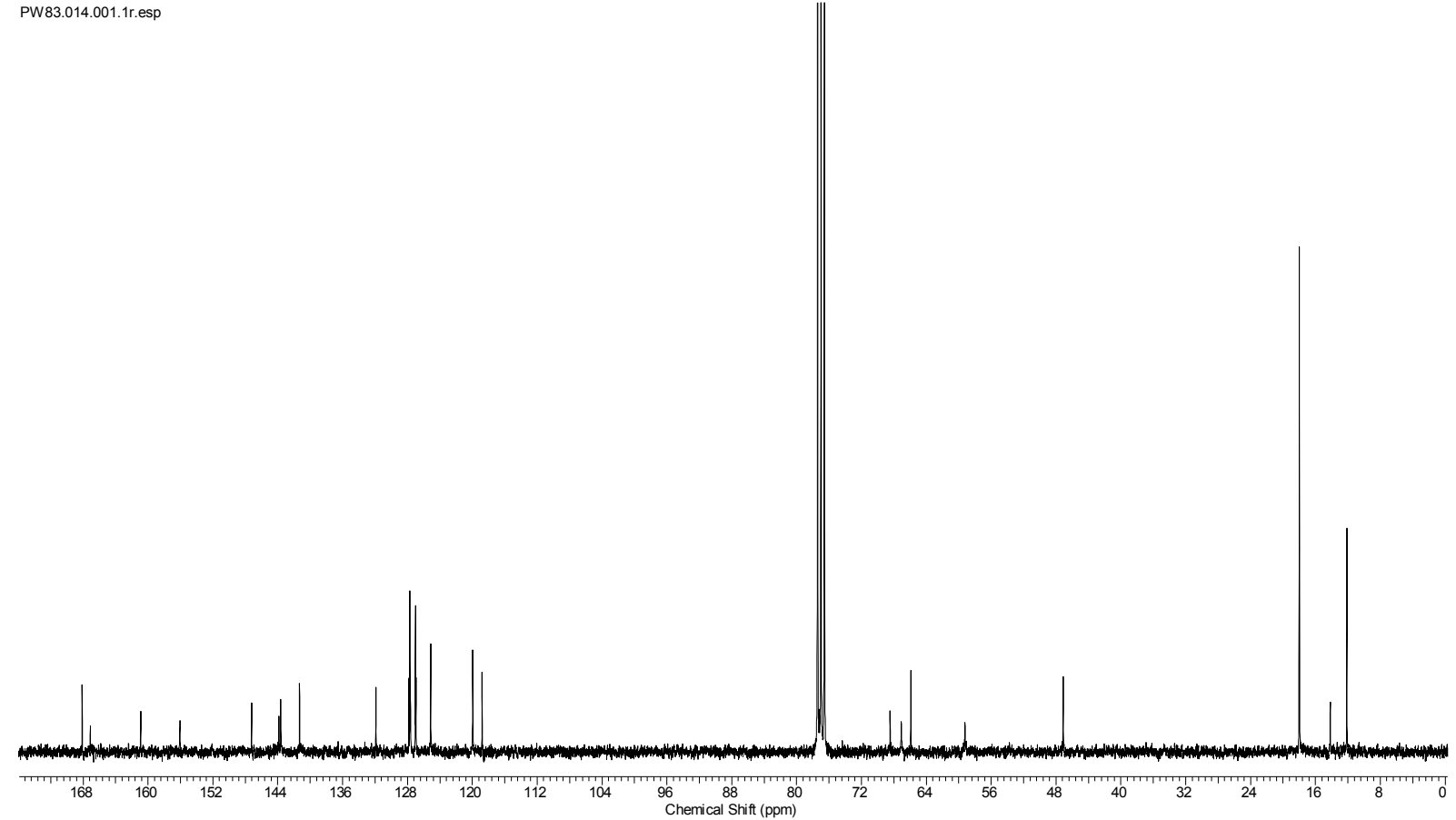

Abbildung 11.26: ${ }^{13} \mathrm{C}-\mathrm{NMR}-$ Spektrum des Thiazols $147\left(75 \mathrm{MHz}, \mathrm{CDCl}_{3}\right)$. 


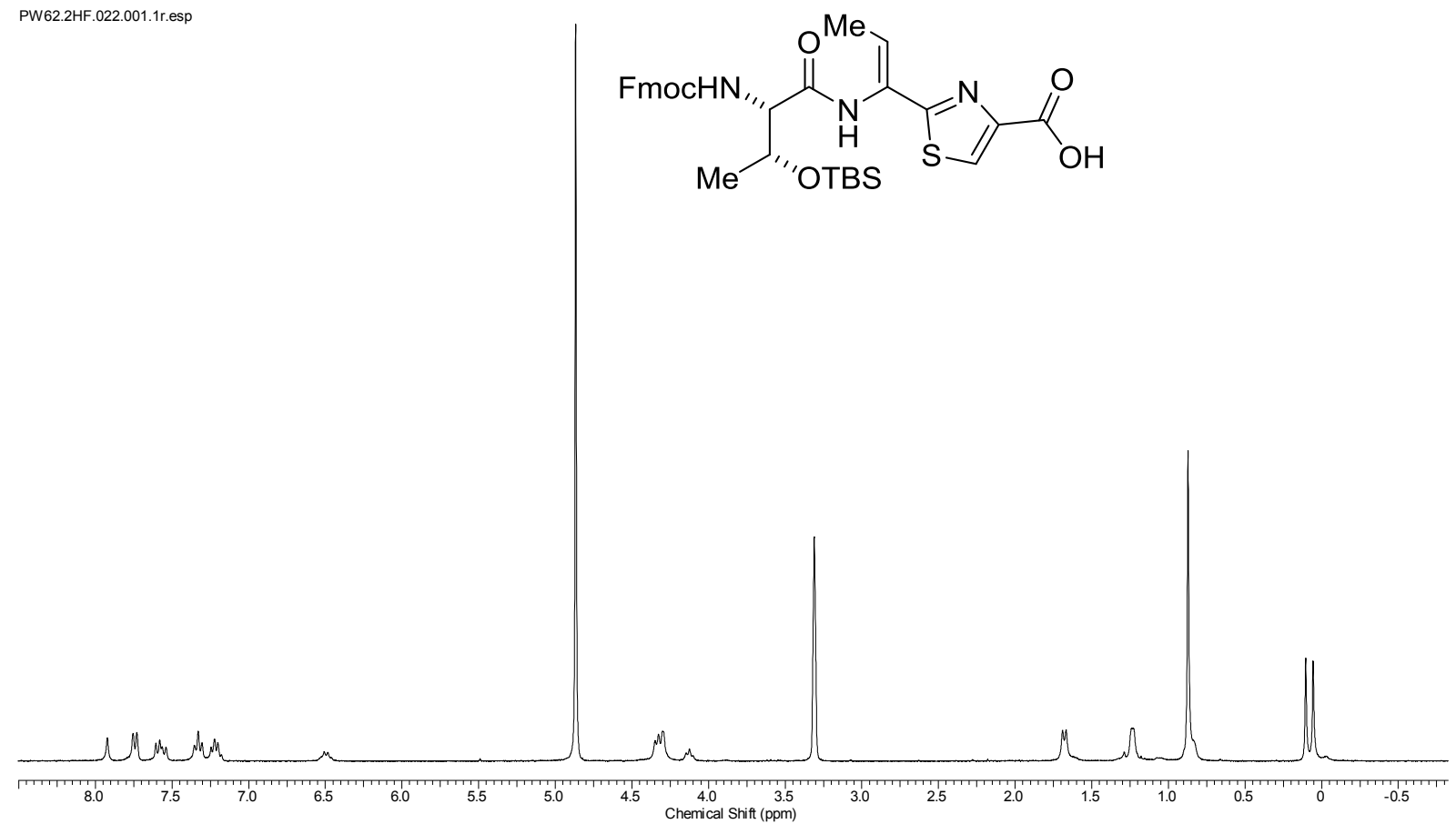

Abbildung 11.27: ${ }^{1} \mathrm{H}-\mathrm{NMR}-S p e k t r u m$ der Thiazolcarbonsäure $110\left(300 \mathrm{MHz}, \mathrm{MeOH}-d_{4}\right)$.

PW62.2HF.025.001.1r.esp

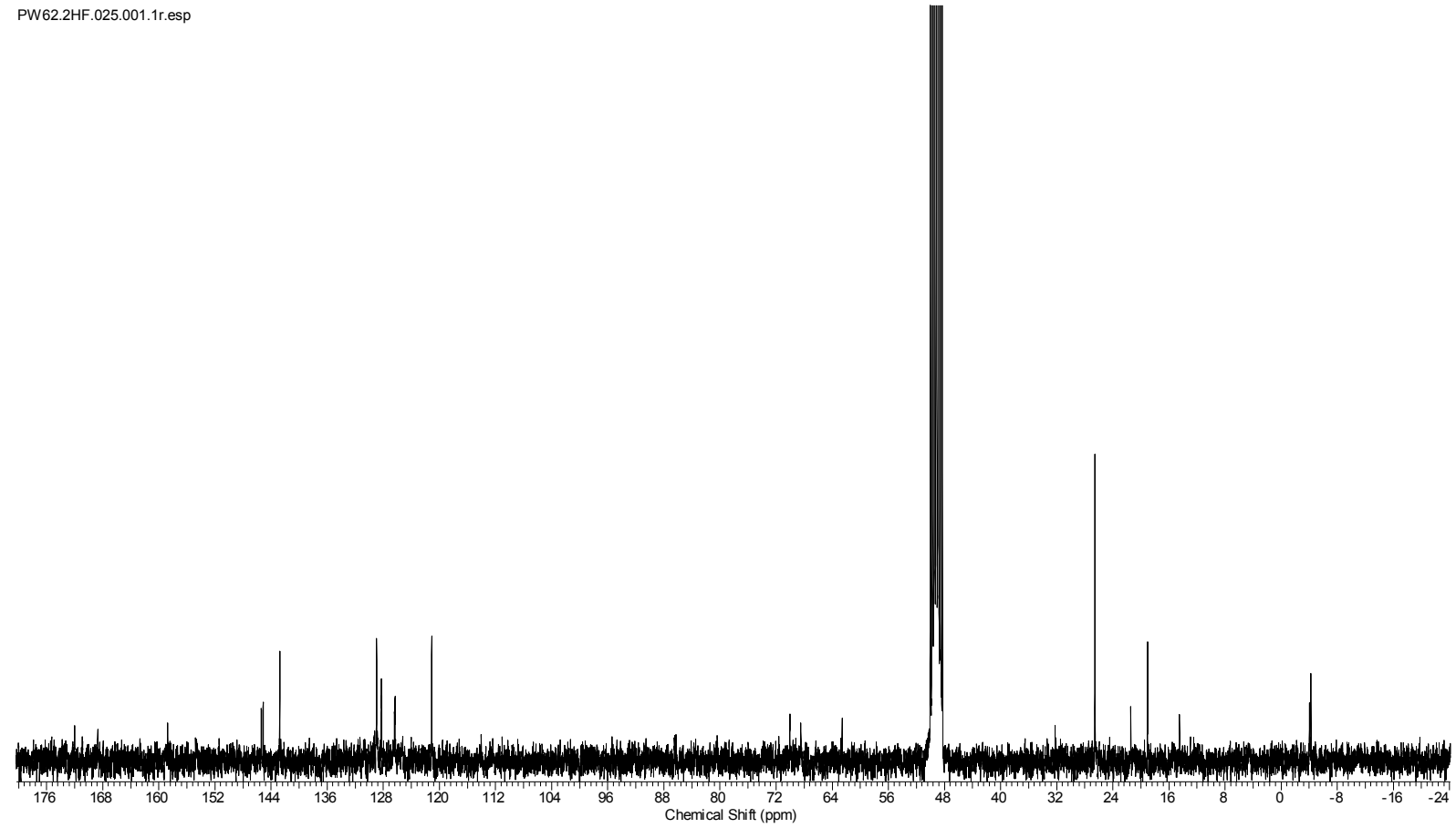

Abbildung 11.28: ${ }^{13} \mathrm{C}-\mathrm{NMR}-$ Spektrum der Thiazolcarbonsäure $110\left(75 \mathrm{MHz}, \mathrm{MeOH}-d_{4}\right)$. 
<smiles>C/C=C(\NC(=O)[C@@H](NC(F)F)[C@H](C)OC)c1nc(C(=O)O)cs1</smiles>

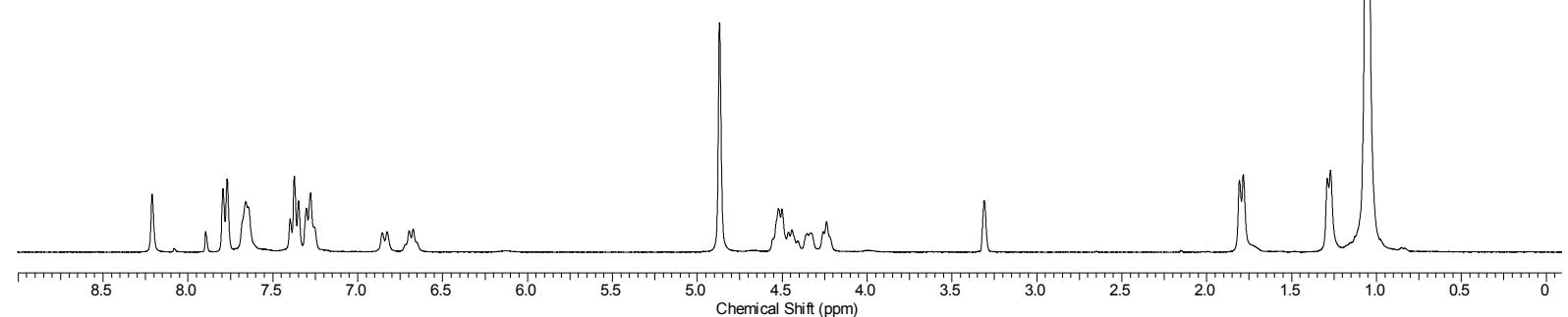

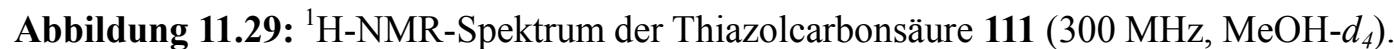

PW84.3.011.001.1r.esp

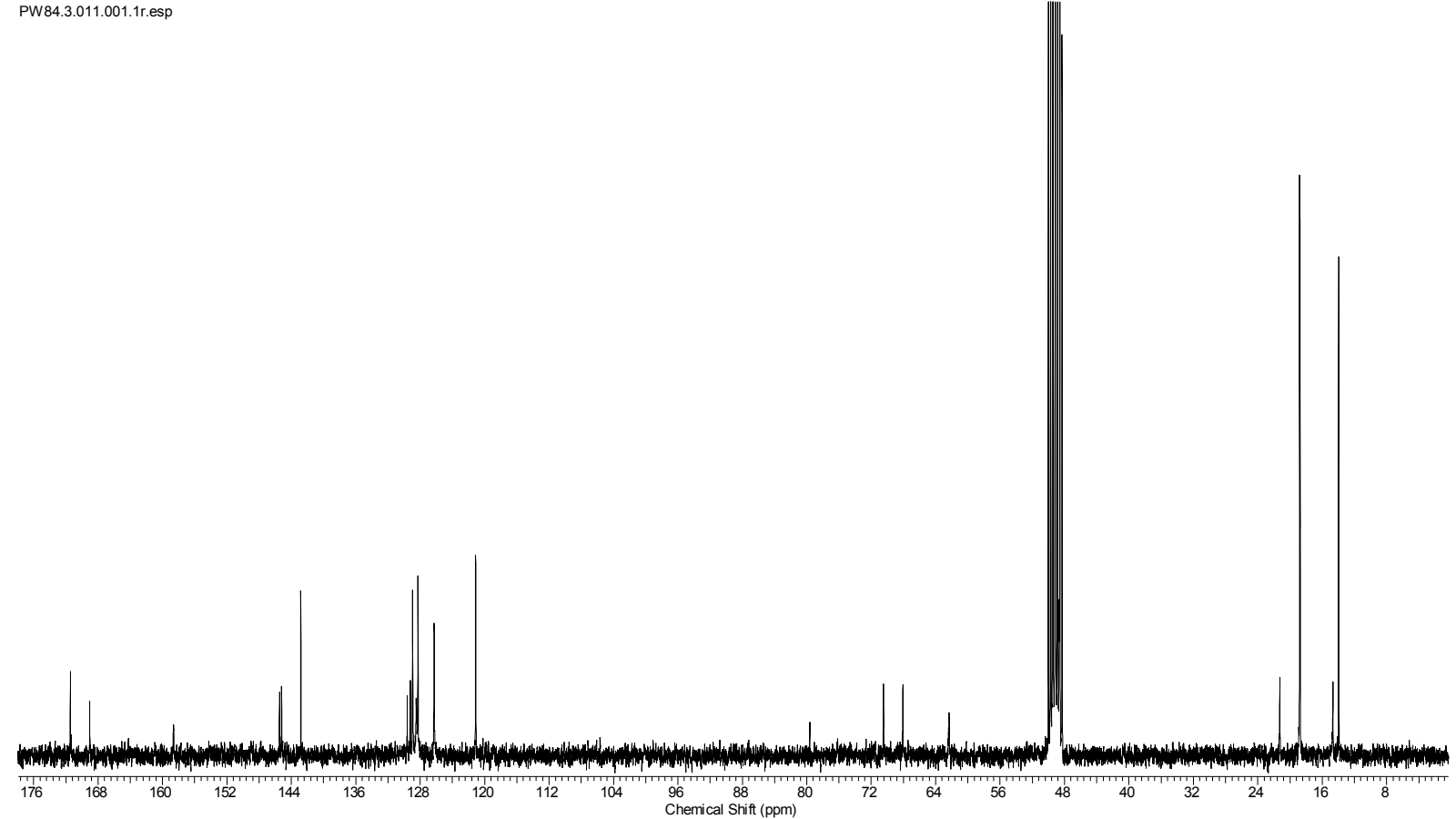

Abbildung 11.30: ${ }^{13} \mathrm{C}-\mathrm{NMR}-$ Spektrum der Thiazolcarbonsäure $111\left(75 \mathrm{MHz}, \mathrm{MeOH}-d_{4}\right)$. 


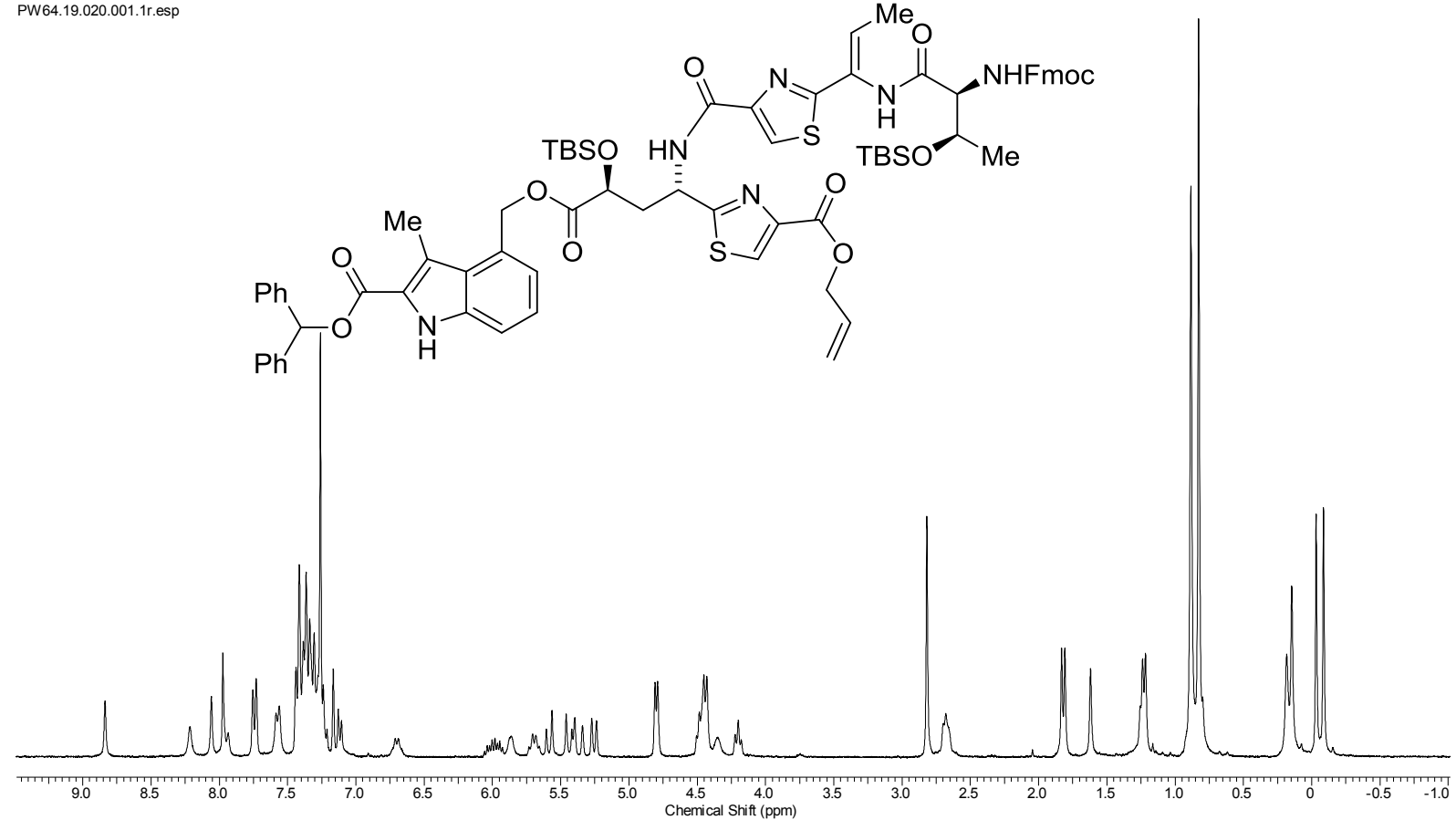

Abbildung 11.31: ${ }^{1} \mathrm{H}-\mathrm{NMR}-S p e k t r u m$ des Bis-Thiazols 149 (300 MHz, $\left.\mathrm{CDCl}_{3}\right)$.

PW64.19.021.001.1r.esp

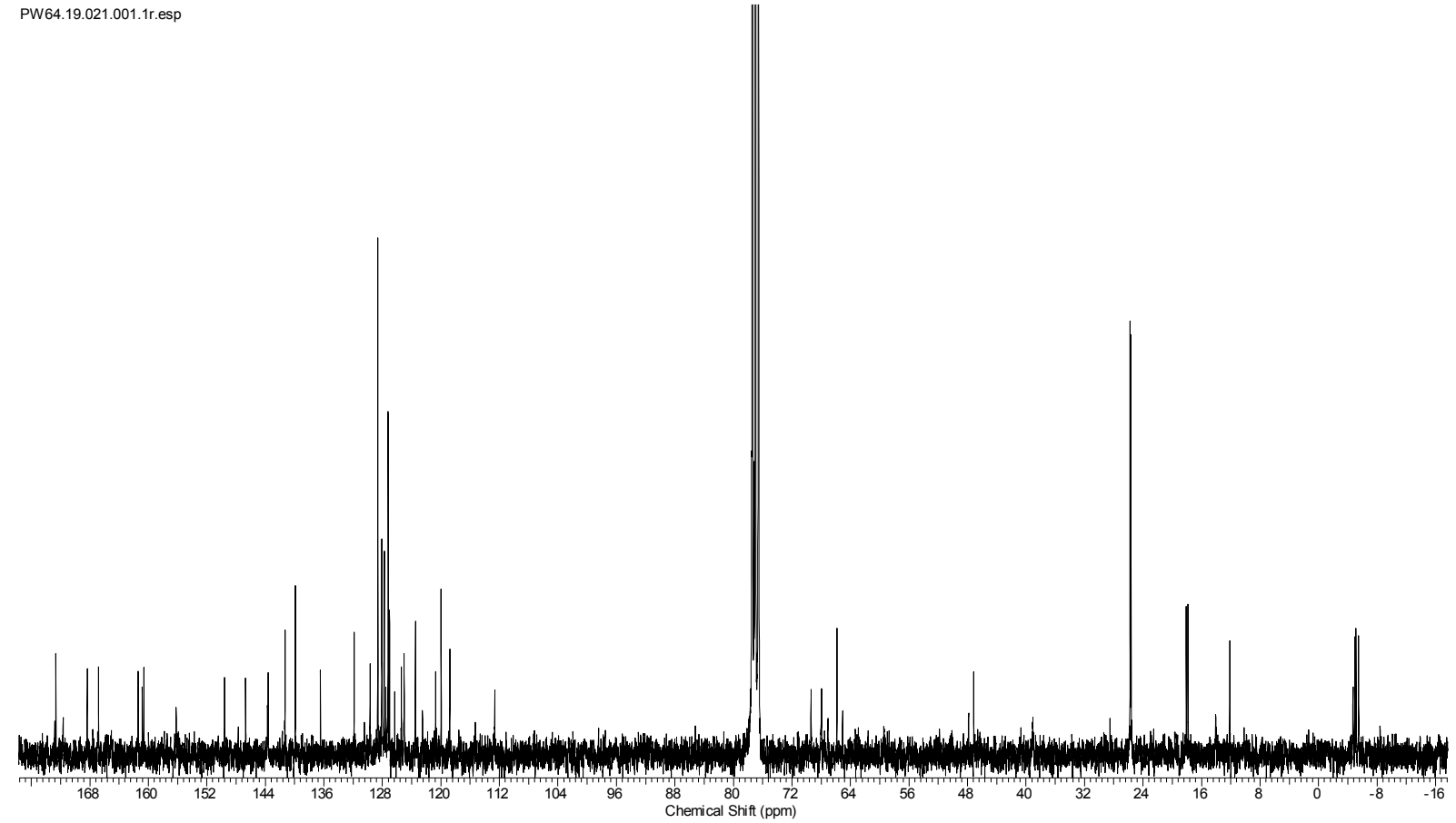

Abbildung 11.32: ${ }^{13} \mathrm{C}-\mathrm{NMR}-$ Spektrum des Bis-Thiazols $149\left(75 \mathrm{MHz}, \mathrm{CDCl}_{3}\right)$. 


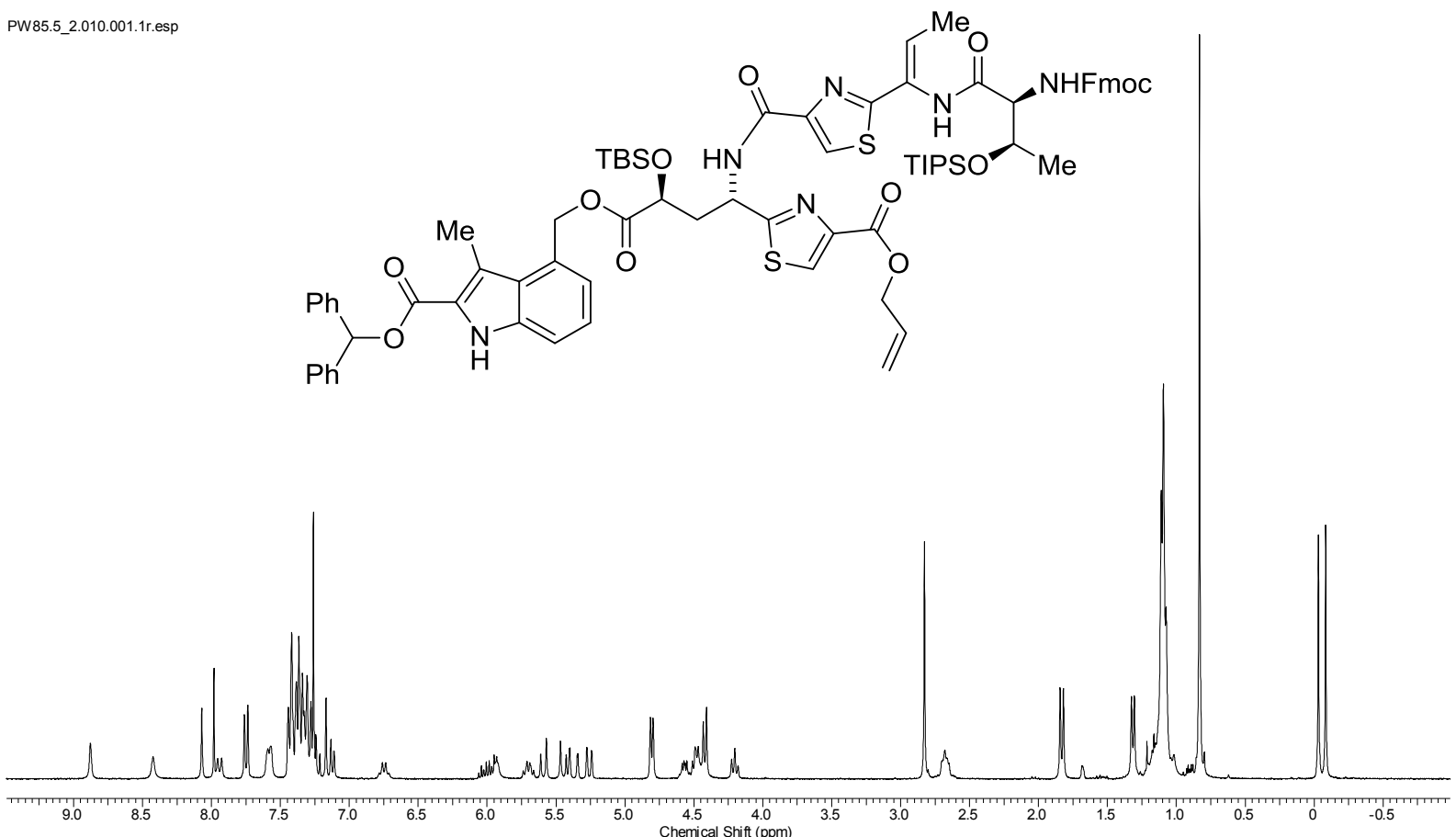

Abbildung 11.33: ${ }^{1} \mathrm{H}-\mathrm{NMR}-S p e k t r u m$ des Bis-Thiazols $150\left(300 \mathrm{MHz}, \mathrm{CDCl}_{3}\right)$.

PW85_4.014.001.1r.esp

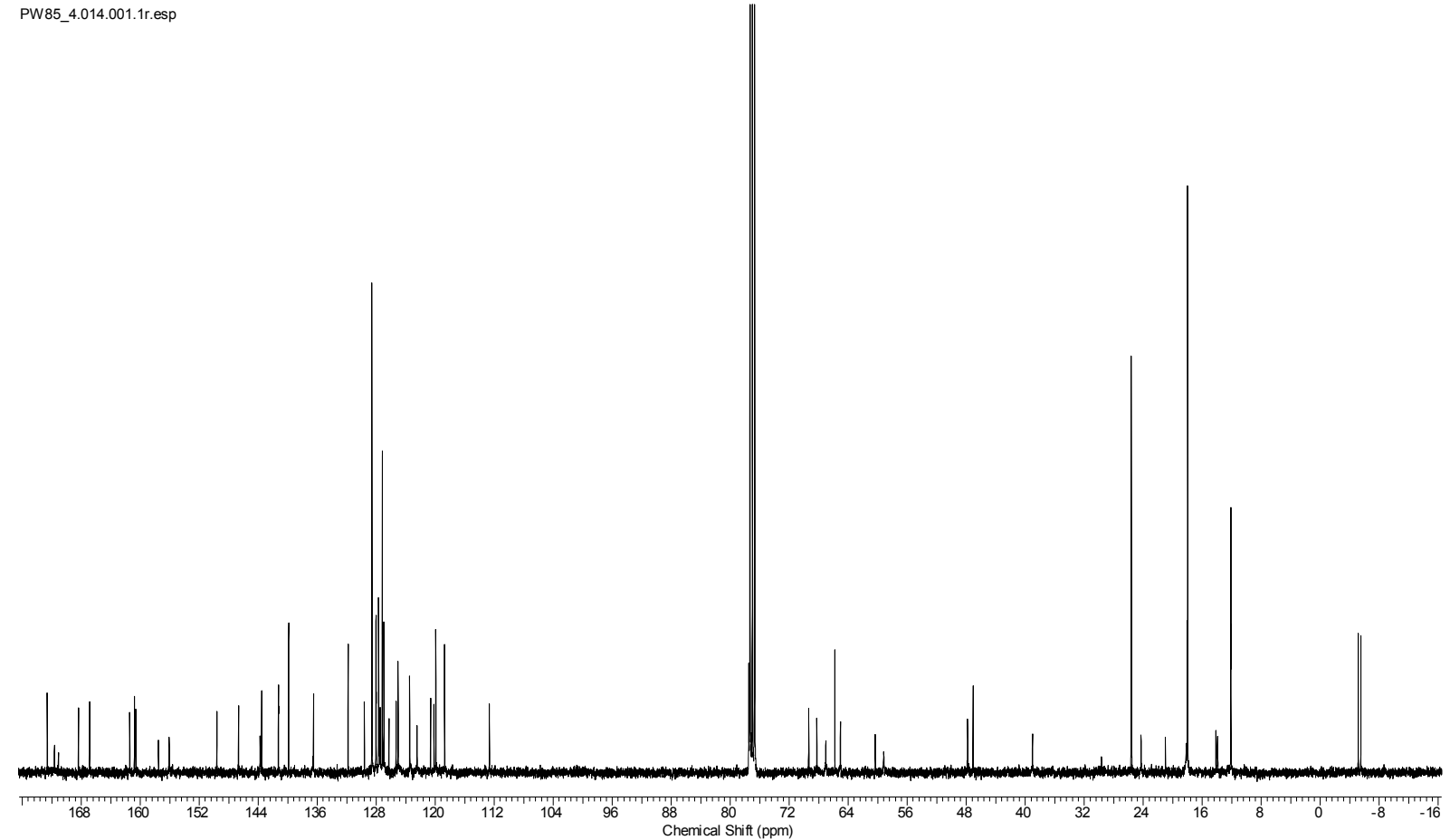

Abbildung 11.34: ${ }^{13} \mathrm{C}-\mathrm{NMR}-$ Spektrum des Bis-Thiazols $150\left(100 \mathrm{MHz}, \mathrm{CDCl}_{3}\right)$. 

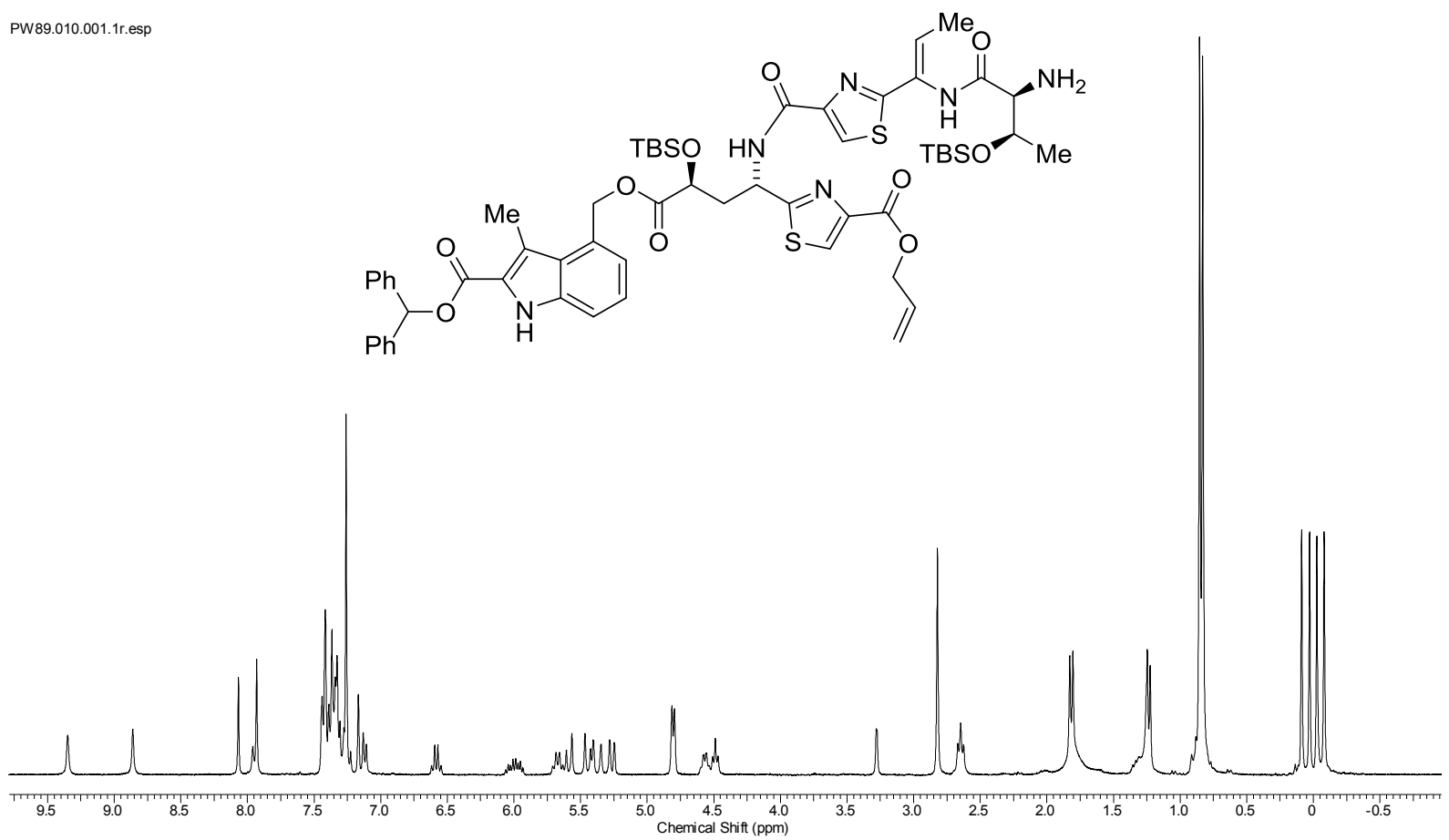

Abbildung 11.35: ${ }^{1} \mathrm{H}-\mathrm{NMR}-$ Spektrum des Bis-Thiazolamins $93\left(300 \mathrm{MHz}, \mathrm{CDCl}_{3}\right)$.

PW89.011.001.1r.esp

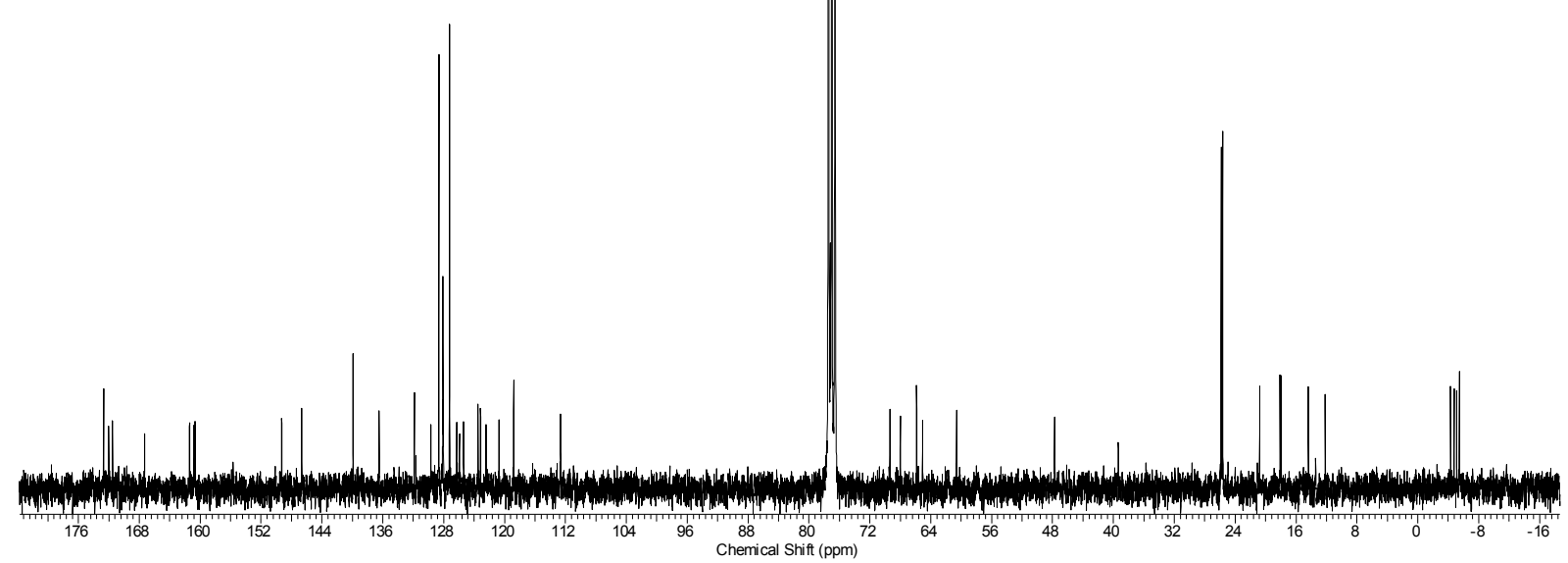

Abbildung 11.36: ${ }^{13} \mathrm{C}-\mathrm{NMR}-$ Spektrum des Bis-Thiazolamins 93 (75 MHz, $\mathrm{CDCl}_{3}$ ). 


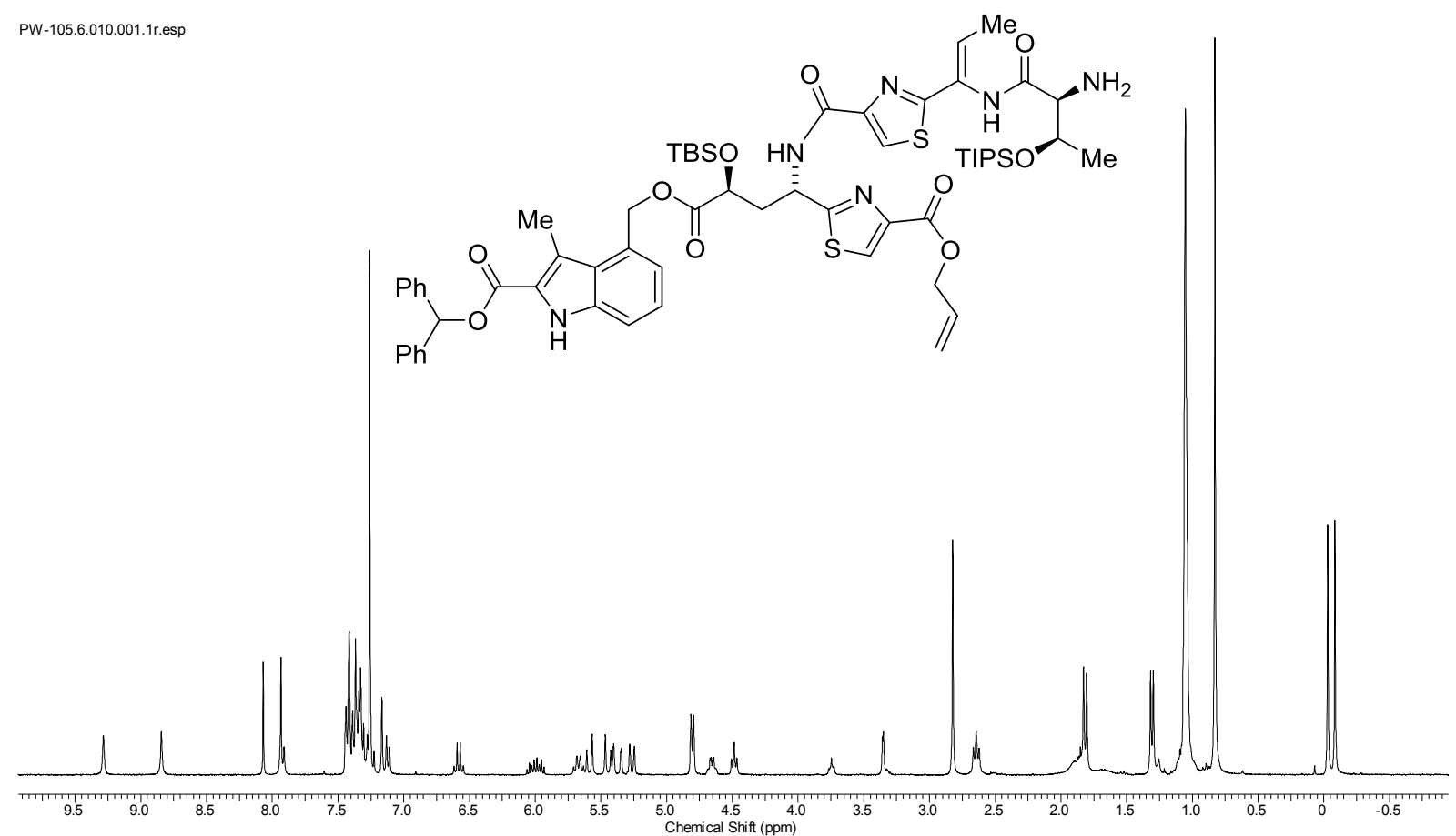

Abbildung 11.37: ${ }^{1} \mathrm{H}-\mathrm{NMR}-$ Spektrum des Bis-Thiazolamins $94\left(300 \mathrm{MHz}, \mathrm{CDCl}_{3}\right)$.

PW-105.6.013.001.1r.esp

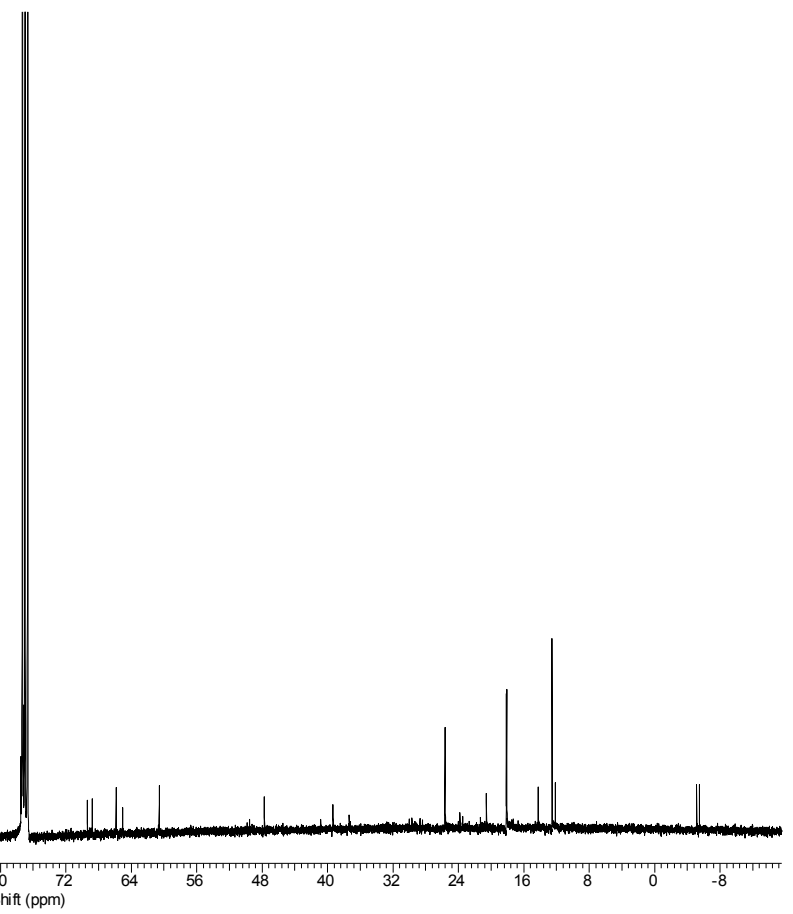

Abbildung 11.38: ${ }^{13} \mathrm{C}-\mathrm{NMR}-$ Spektrum des Bis-Thiazolamins $94\left(100 \mathrm{MHz}, \mathrm{CDCl}_{3}\right)$. 


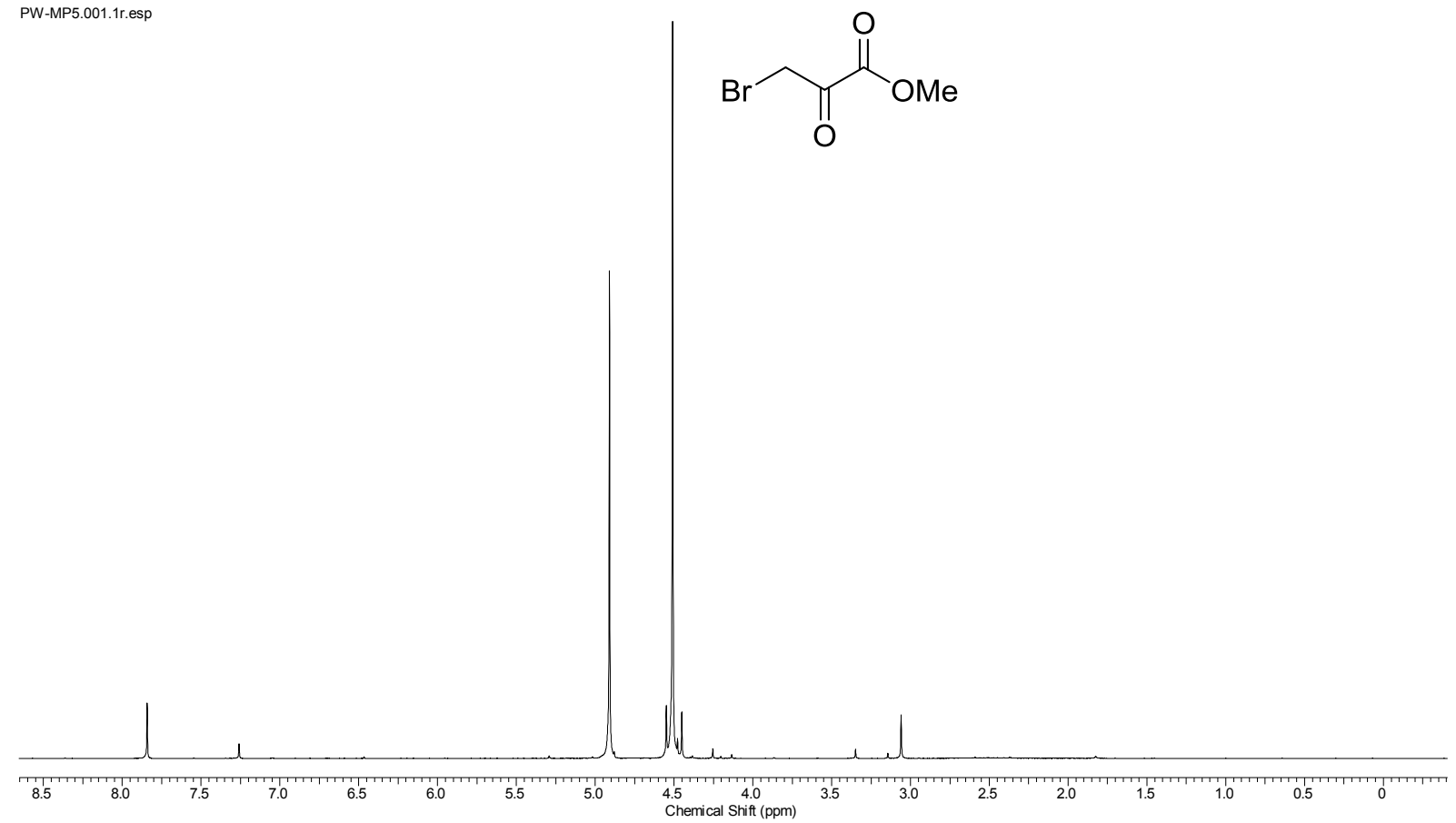

Abbildung 11.39: ${ }^{1} \mathrm{H}-\mathrm{NMR}-S p e k t r u m$ des Methylesters $152\left(250 \mathrm{MHz}, \mathrm{CDCl}_{3}\right)$.

PW5-Me.040.001.1r.esp

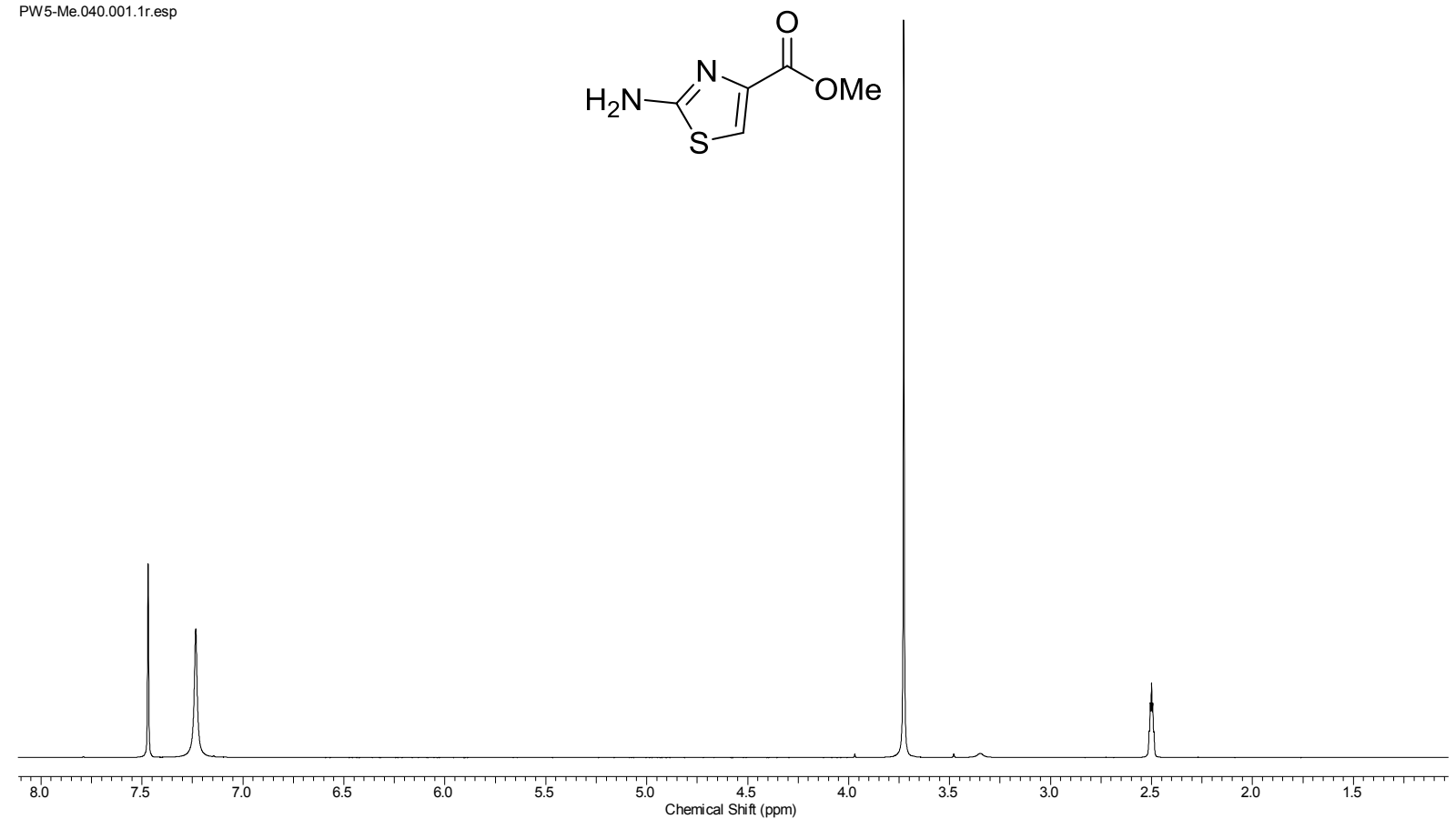

Abbildung 11.40: ${ }^{1} \mathrm{H}-\mathrm{NMR}-$ Spektrum des Thiazols 153 (300 MHz, DMSO- $\left.d_{6}\right)$. 


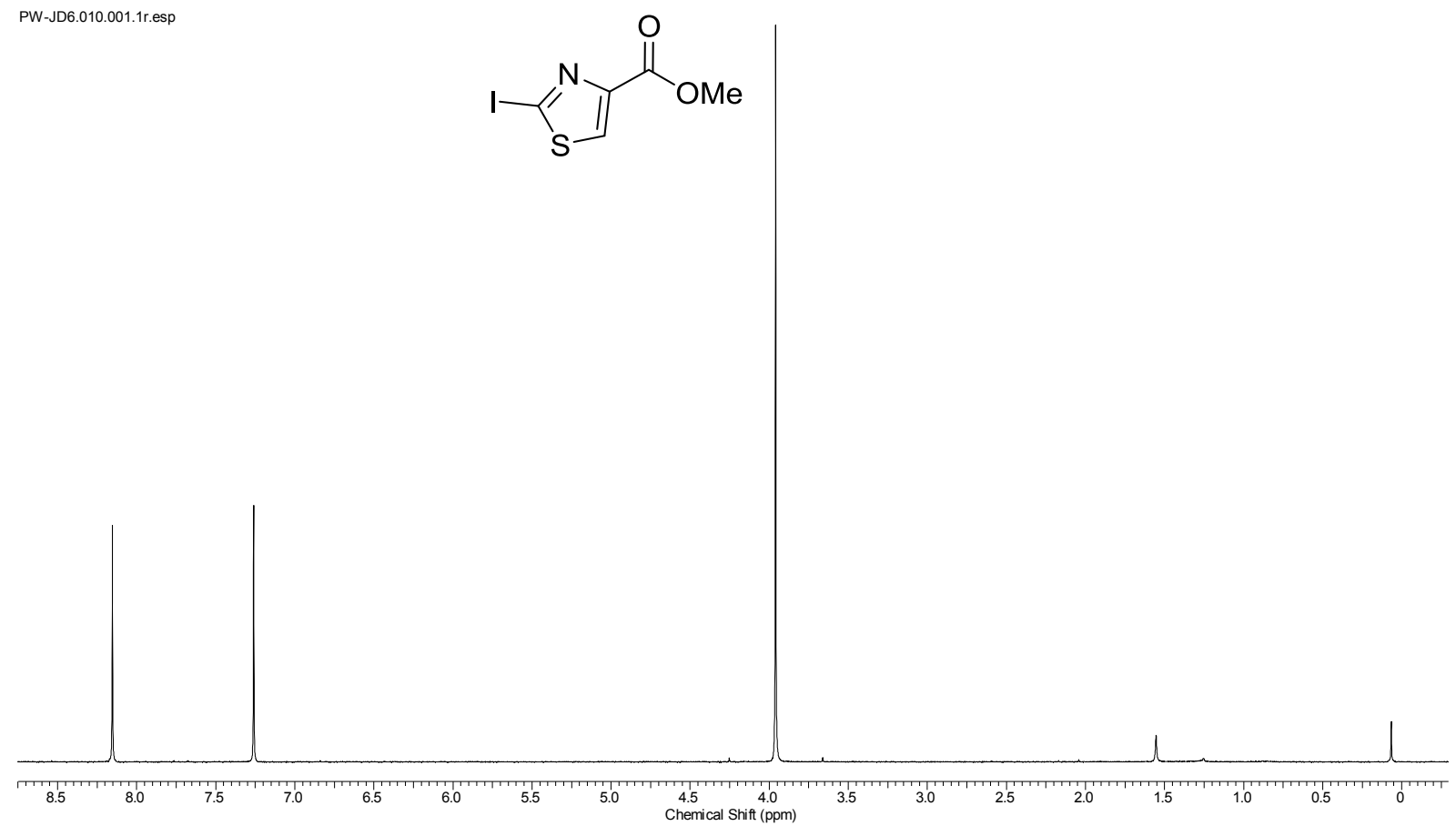

Abbildung 11.41: ${ }^{1} \mathrm{H}-\mathrm{NMR}-S p e k t r u m$ des Thiazols $154\left(250 \mathrm{MHz}, \mathrm{CDCl}_{3}\right)$.

PW15.080.001.1r.esp<smiles>COC(=O)c1csc(C#CC(C)O)n1</smiles>

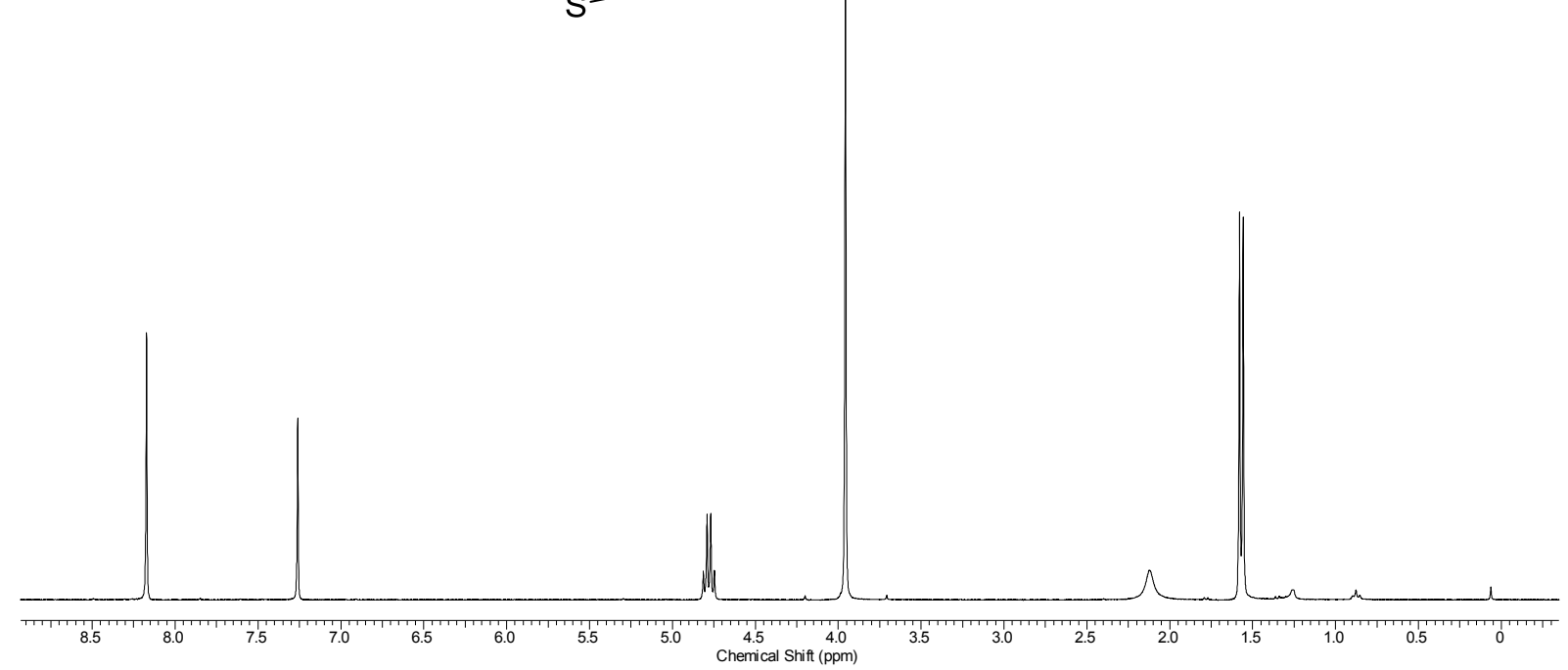

Abbildung 11.42: ${ }^{1} \mathrm{H}-\mathrm{NMR}-$ Spektrum des Propargylalkohols $155\left(300 \mathrm{MHz}, \mathrm{CDCl}_{3}\right)$. 


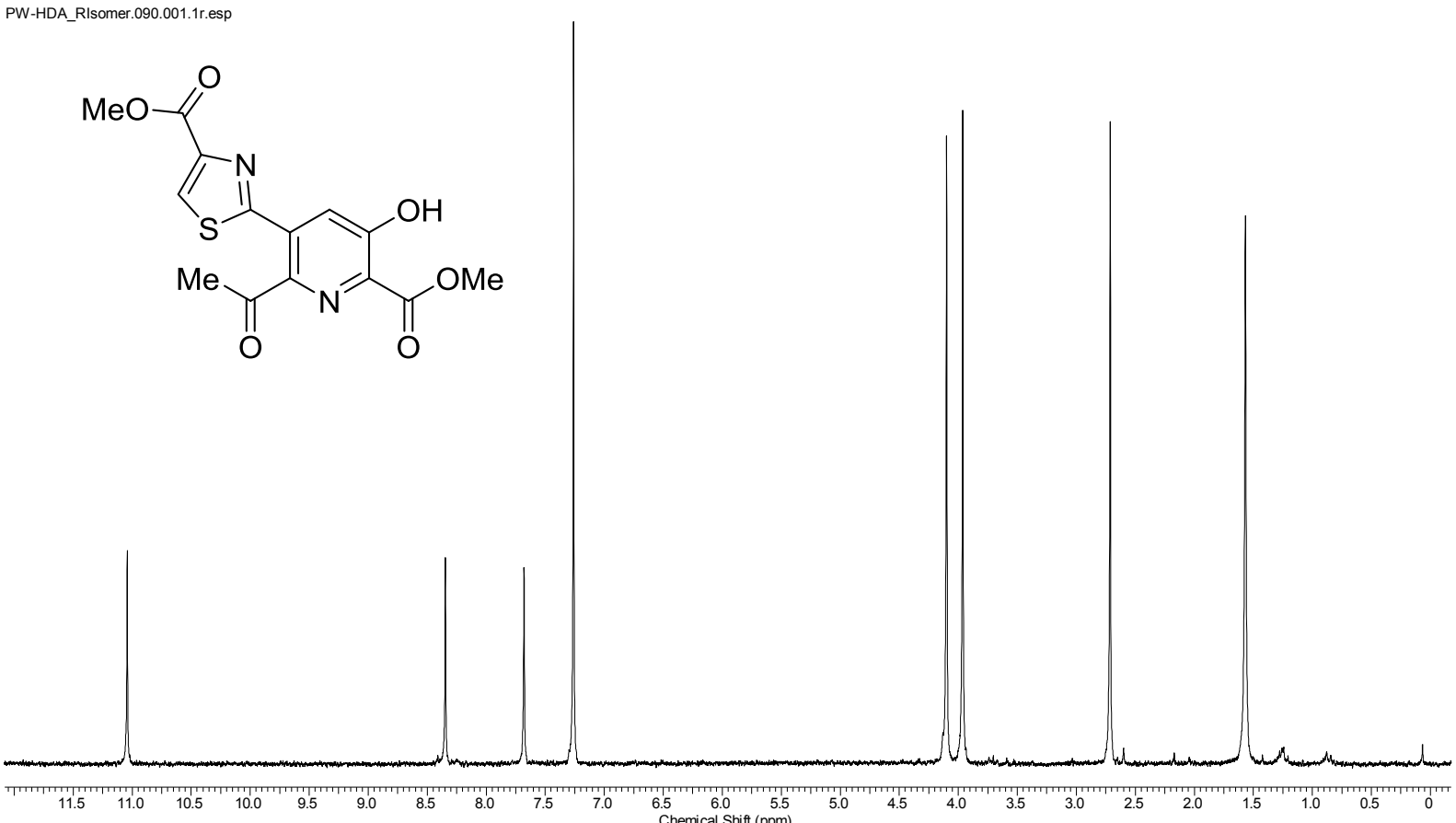

Abbildung 11.43: ${ }^{1} \mathrm{H}-\mathrm{NMR}-$ Spektrum des 3-Hydroxypyridins $46\left(250 \mathrm{MHz}, \mathrm{CDCl}_{3}\right)$.<smiles>COC(=O)c1csc(-c2nc(C(=O)OC)c(C(C)=O)cc2O)n1</smiles>

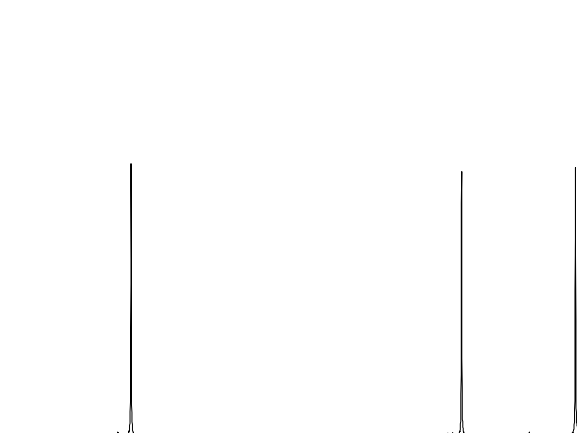

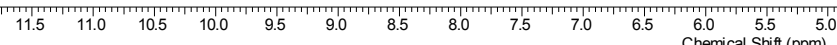

Abbildung 11.44: ${ }^{1} \mathrm{H}-\mathrm{NMR}-\mathrm{Spektrum}$ des 3-Hydroxypyridins $66\left(250 \mathrm{MHz}, \mathrm{CDCl}_{3}\right)$. 


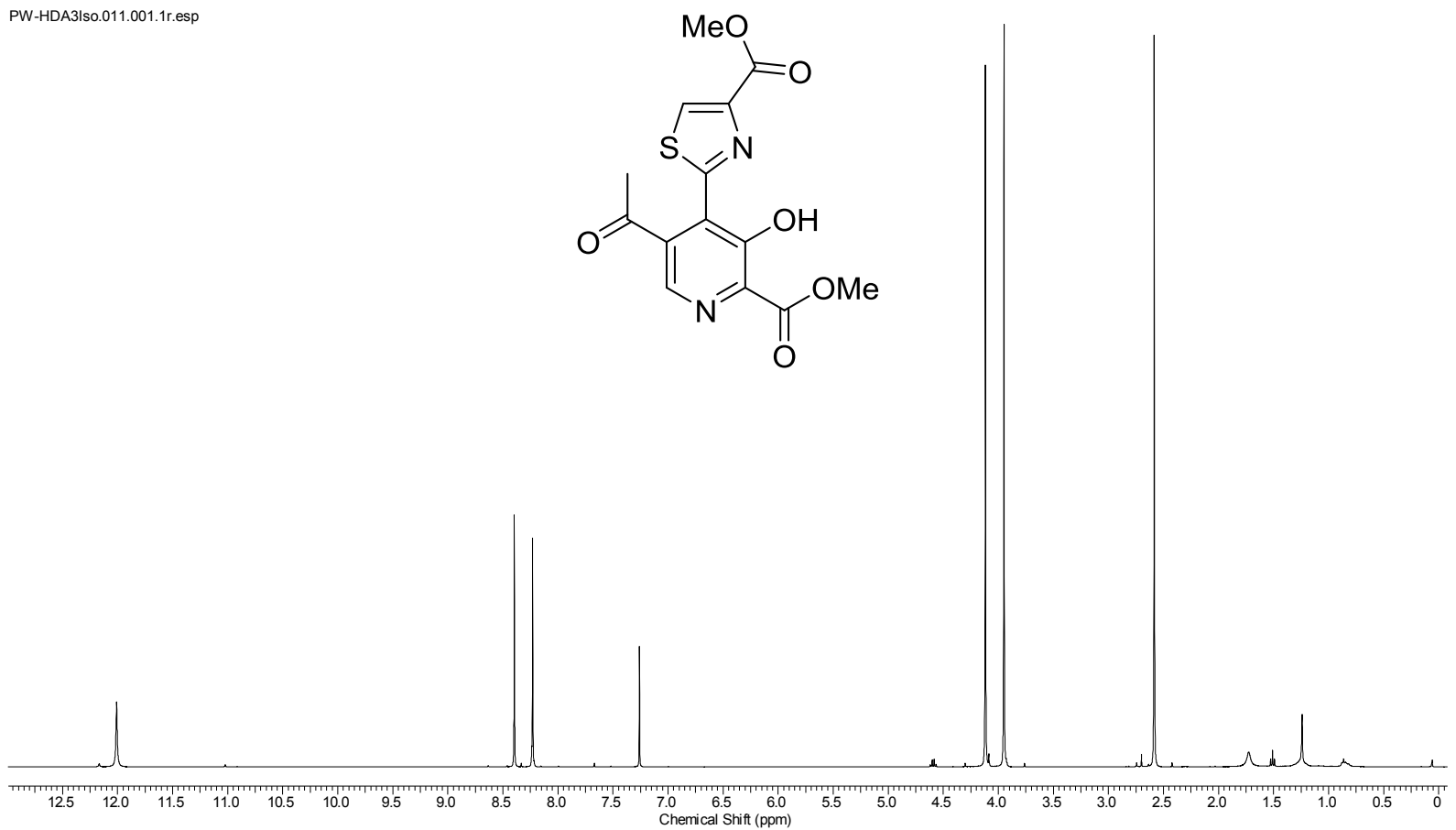

Abbildung 11.45: ${ }^{1} \mathrm{H}-\mathrm{NMR}-$ Spektrum des 3-Hydroxypyridins $156\left(400 \mathrm{MHz}, \mathrm{CDCl}_{3}\right)$.

PW-HDA3Iso.010.001.1r.esp

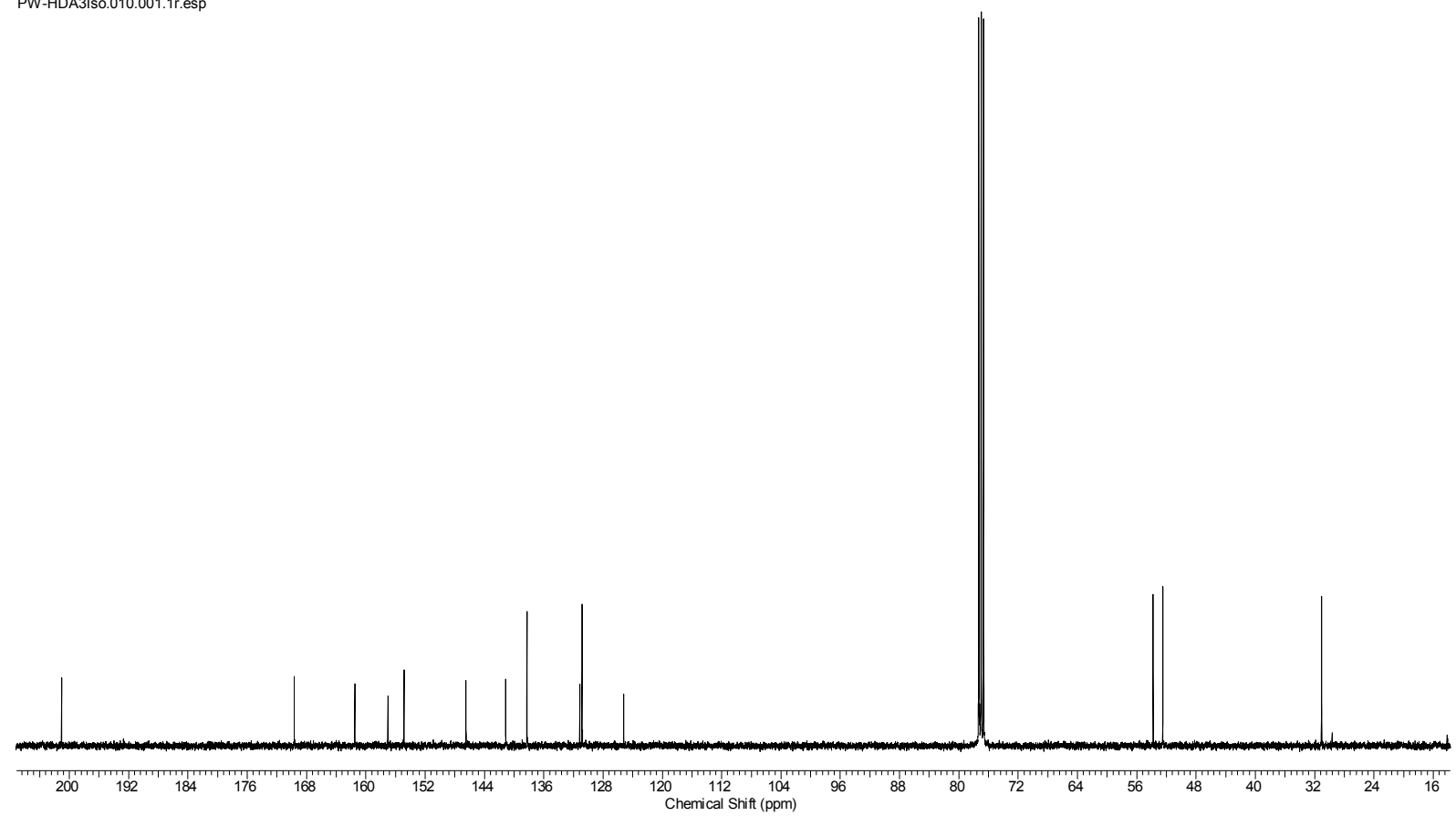

Abbildung 11.46: ${ }^{13} \mathrm{C}-\mathrm{NMR}-$ Spektrum des 3-Hydroxypyridins $156\left(100 \mathrm{MHz}, \mathrm{CDCl}_{3}\right)$. 
PW-47.012.001.1r.esp
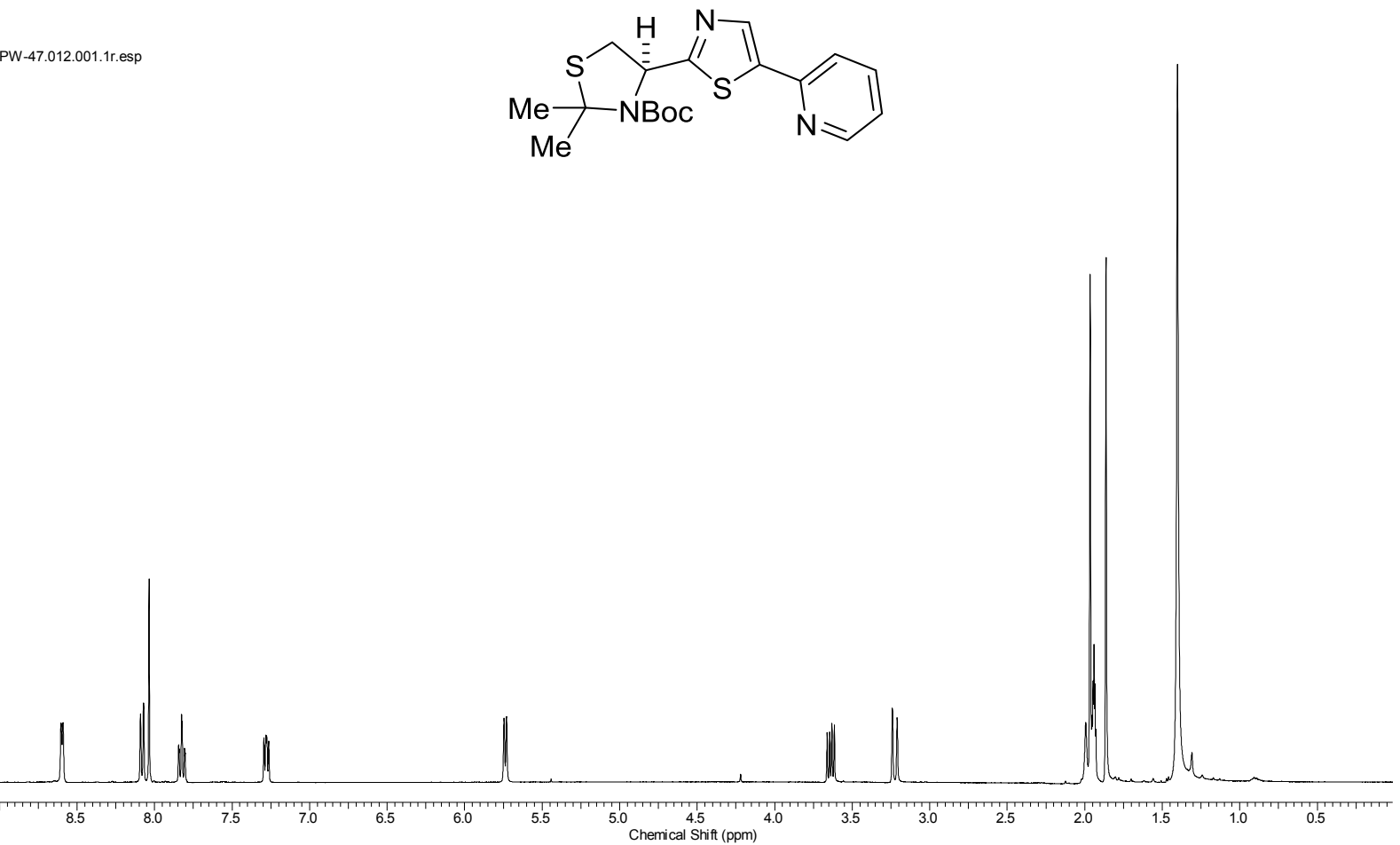

Abbildung 11.47: ${ }^{1} \mathrm{H}-\mathrm{NMR}-\mathrm{Spektrum}$ des Thiazols $162\left(400 \mathrm{MHz}, \mathrm{MeCN}-\mathrm{d}_{3}, 343 \mathrm{~K}\right)$.

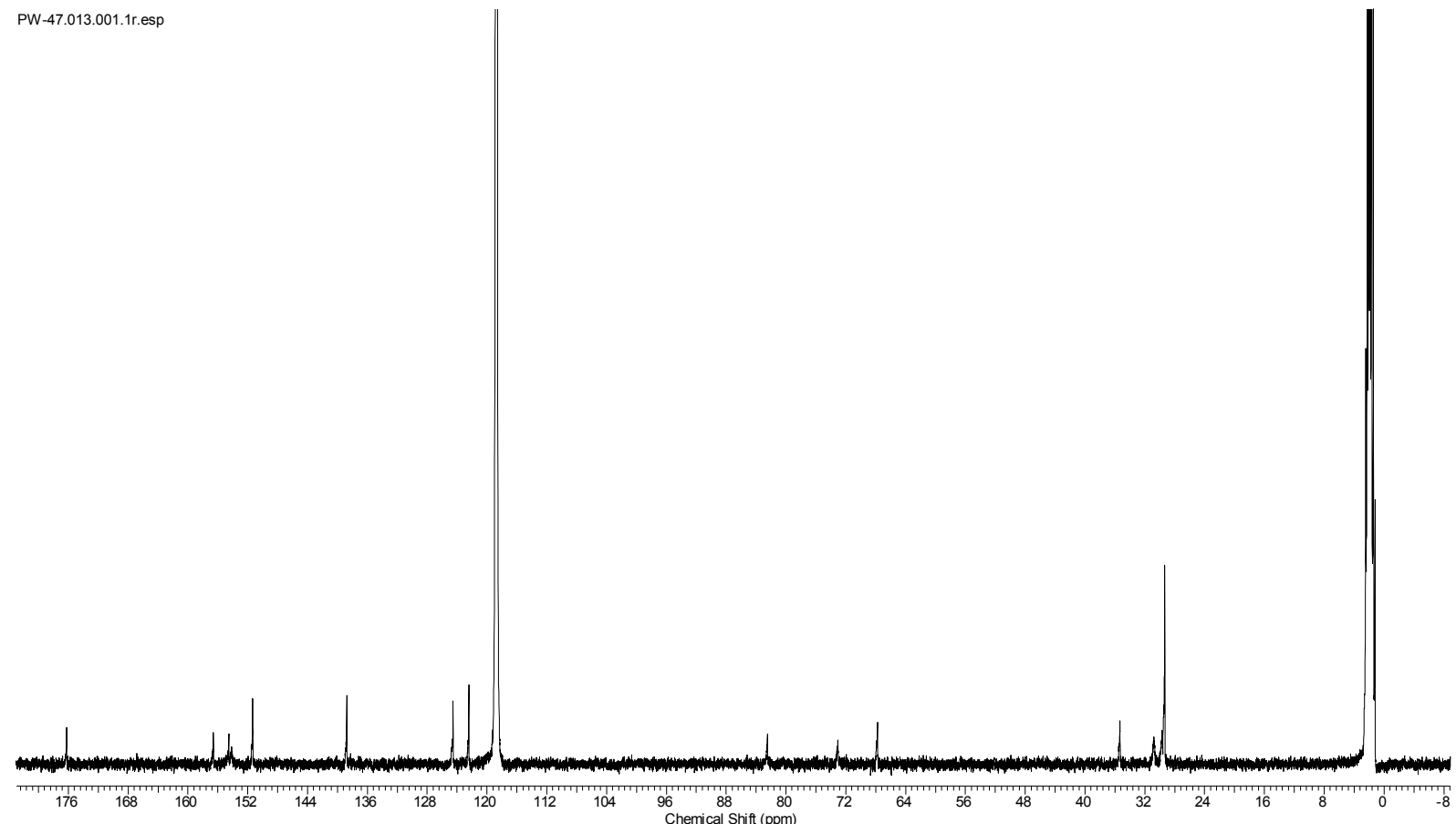

Abbildung 11.48: ${ }^{13} \mathrm{C}-\mathrm{NMR}-$ Spektrum des Thiazols $162\left(100 \mathrm{MHz}, \mathrm{MeCN}-\mathrm{d}_{3}, 343 \mathrm{~K}\right)$. 


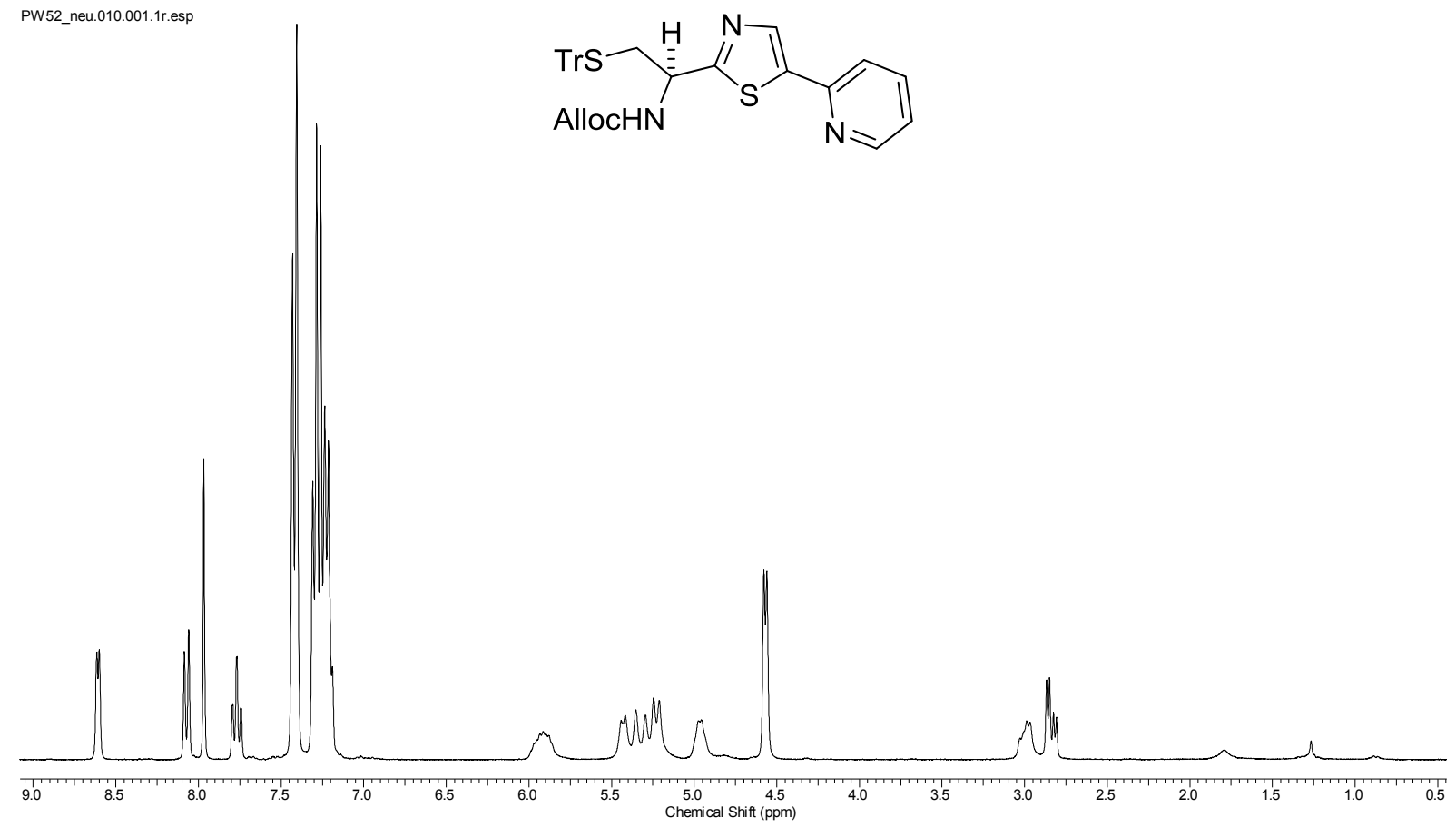

Abbildung 11.49: ${ }^{1} \mathrm{H}-\mathrm{NMR}-$ Spektrum des Thiazols $163\left(300 \mathrm{MHz}, \mathrm{CDCl}_{3}\right)$.

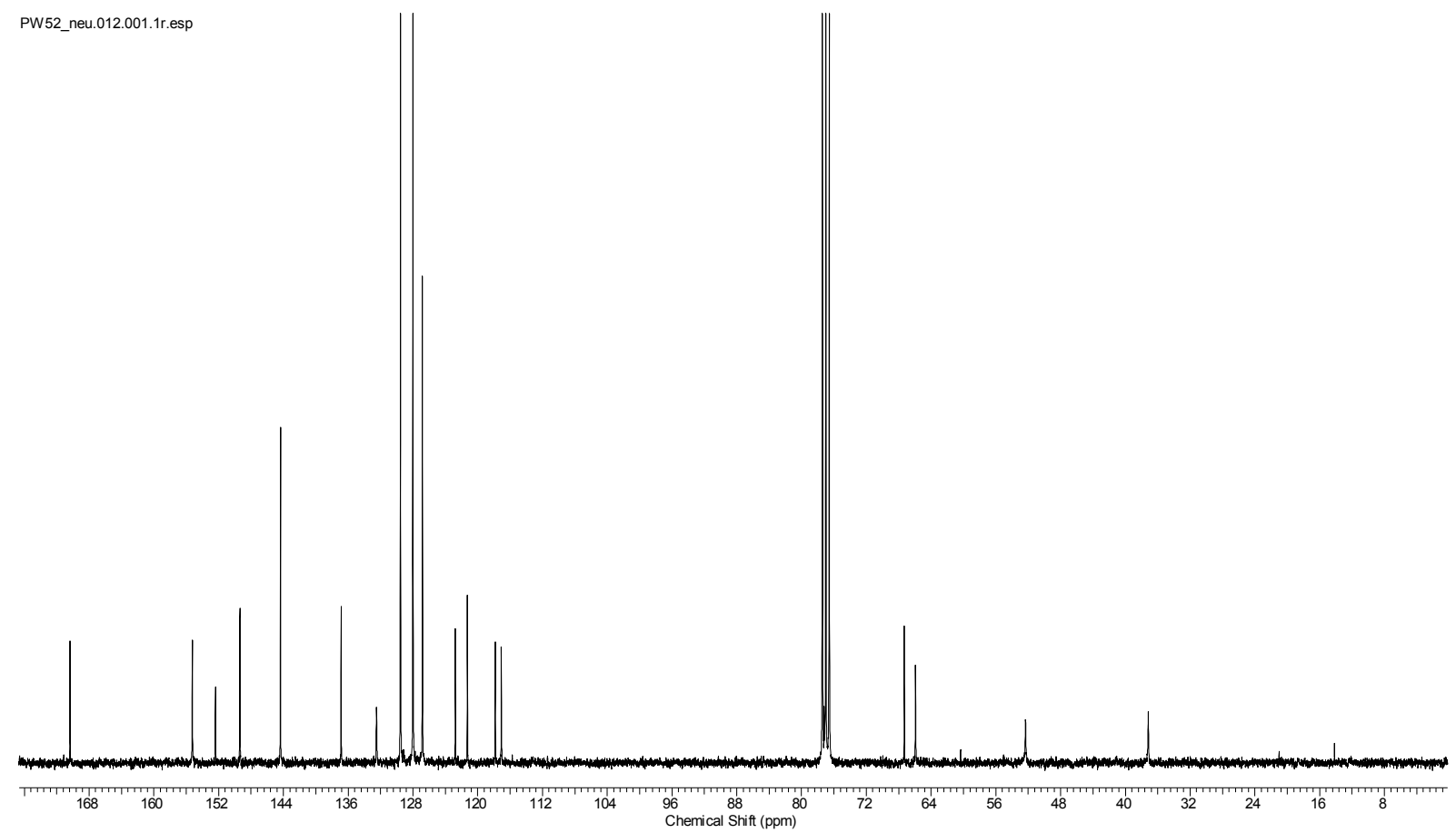

Abbildung 11.50: ${ }^{13} \mathrm{C}-\mathrm{NMR}-$ Spektrum des Thiazols 163 (75 MHz, $\left.\mathrm{CDCl}_{3}\right)$. 


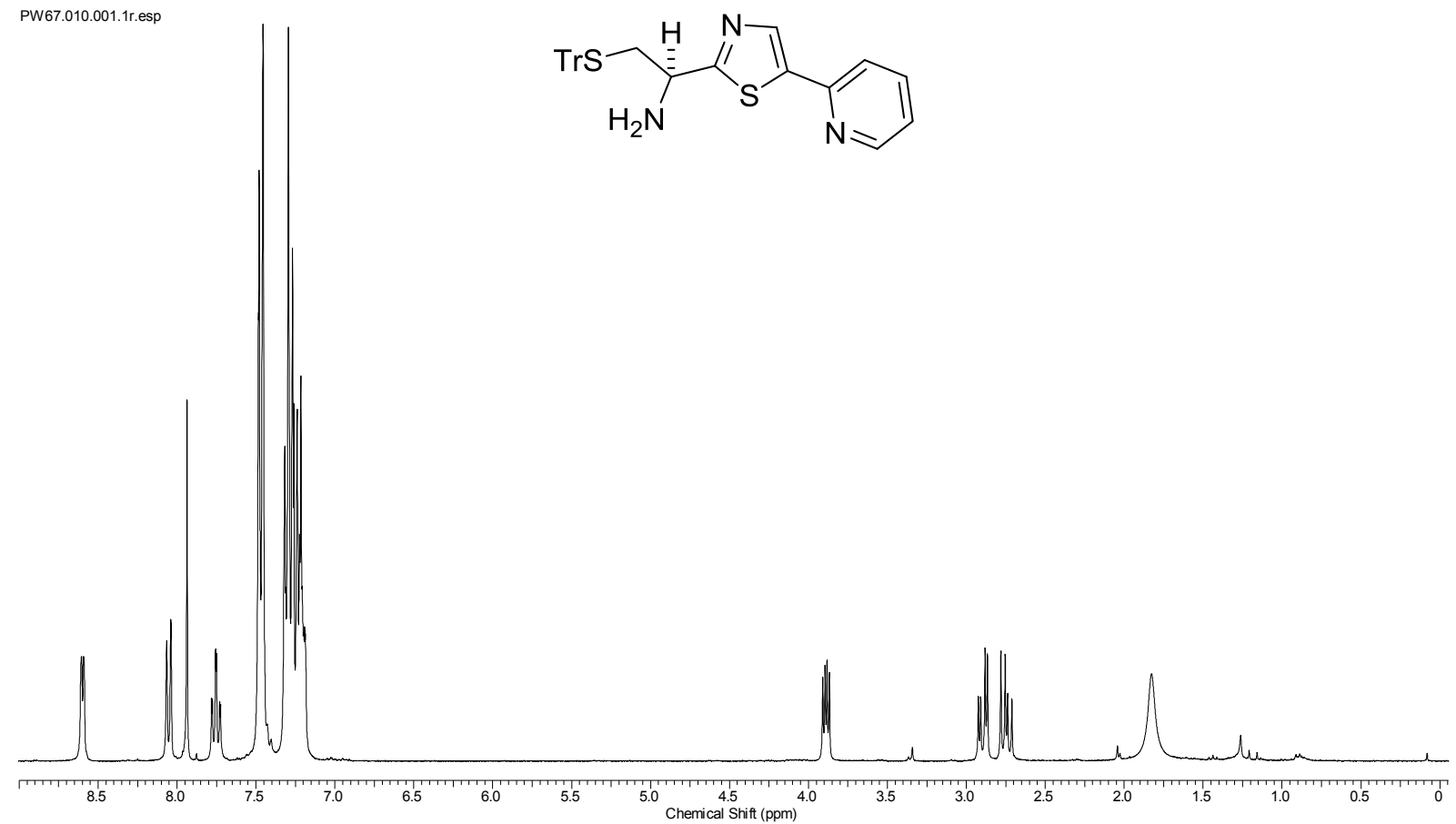

Abbildung 11.51: ${ }^{1} \mathrm{H}-\mathrm{NMR}-$ Spektrum des Thiazolamins $164\left(300 \mathrm{MHz}, \mathrm{CDCl}_{3}\right)$.

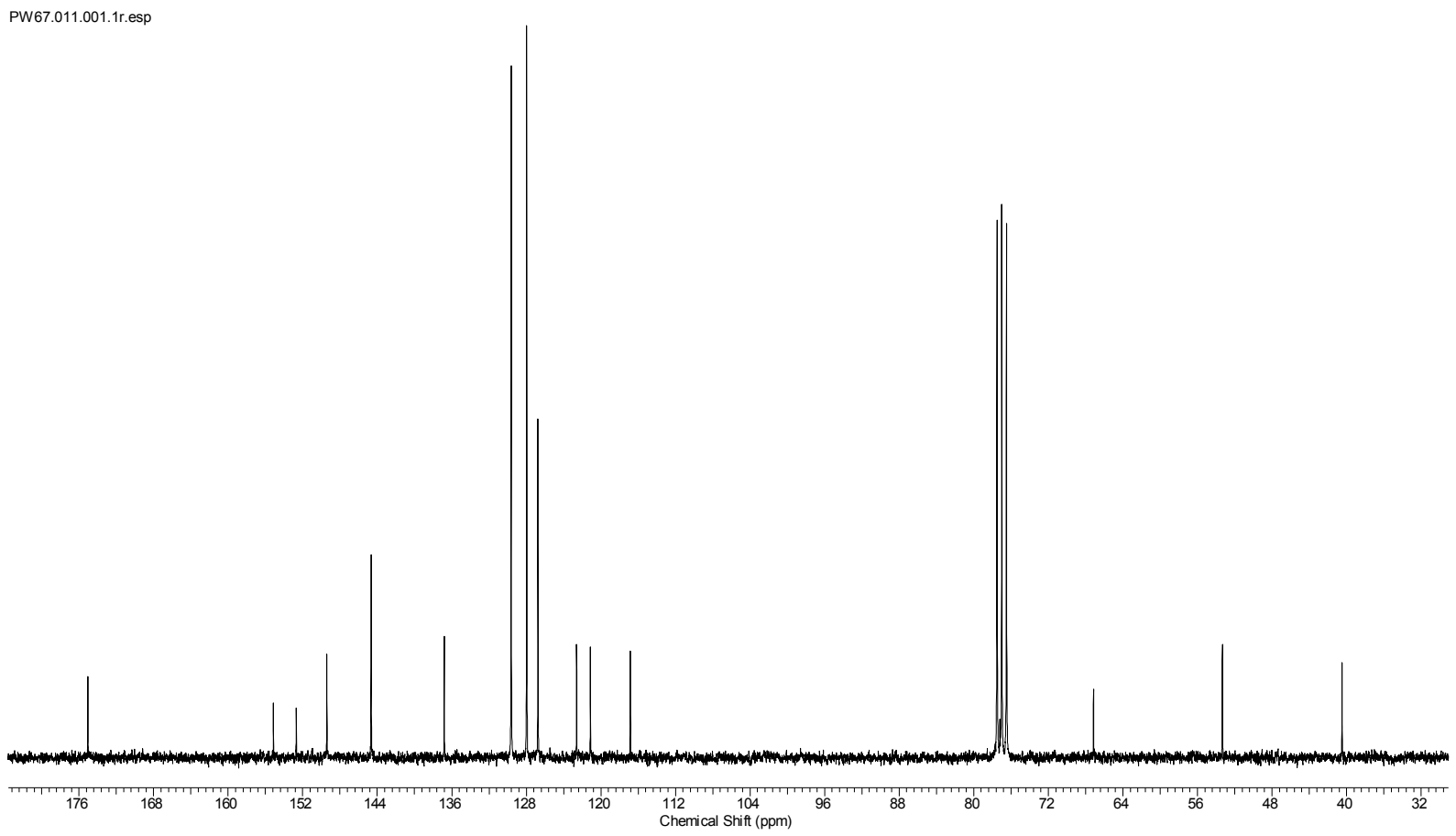

Abbildung 11.52: ${ }^{13} \mathrm{C}-\mathrm{NMR}-$ Spektrum des Thiazolamins $164\left(63 \mathrm{MHz}, \mathrm{CDCl}_{3}\right)$. 


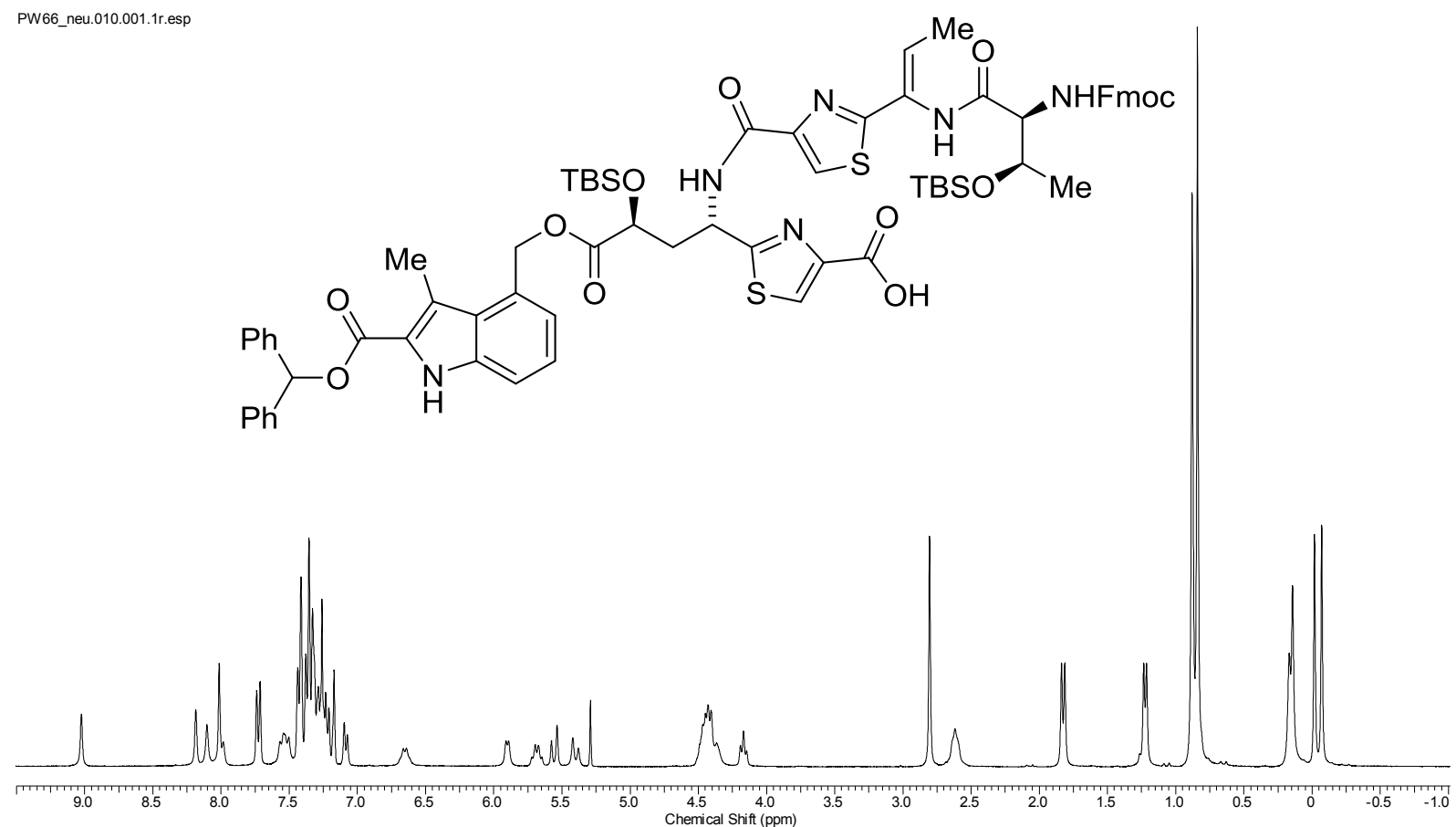

Abbildung 11.53: ${ }^{1} \mathrm{H}-\mathrm{NMR}-$ Spektrum der Bis-Thiazolcarbonsäure $159\left(300 \mathrm{MHz}, \mathrm{CDCl}_{3}\right)$.

PW66_neu.011.001.1r.esp

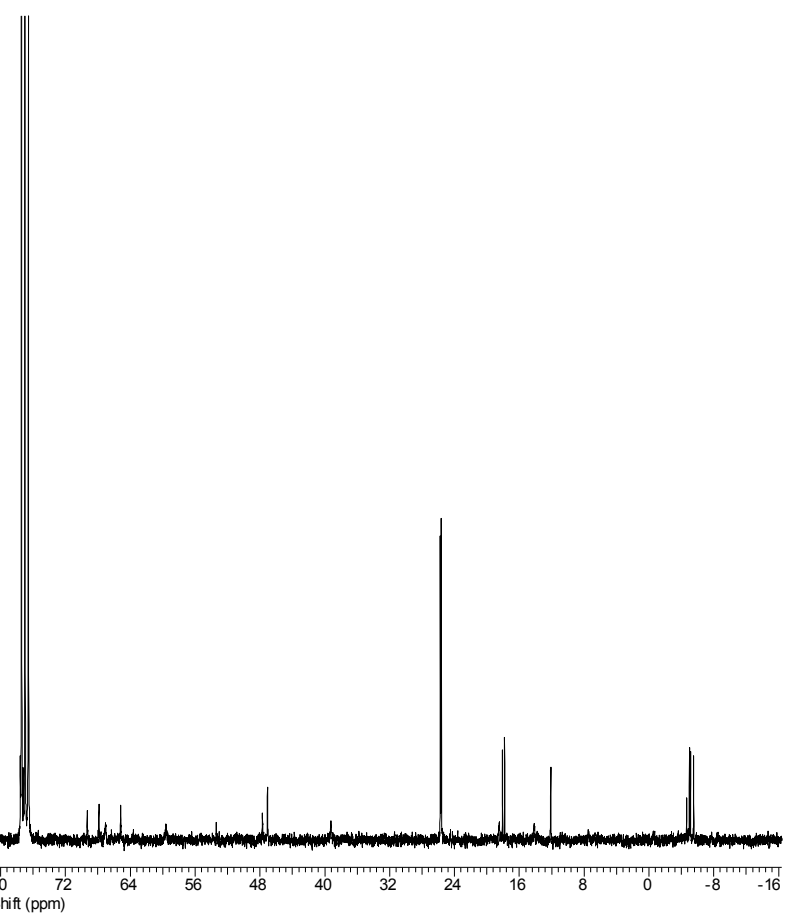

Abbildung 11.54: ${ }^{13} \mathrm{C}-\mathrm{NMR}-$ Spektrum der Bis-Thiazolcarbonsäure $159\left(75 \mathrm{MHz}, \mathrm{CDCl}_{3}\right)$. 


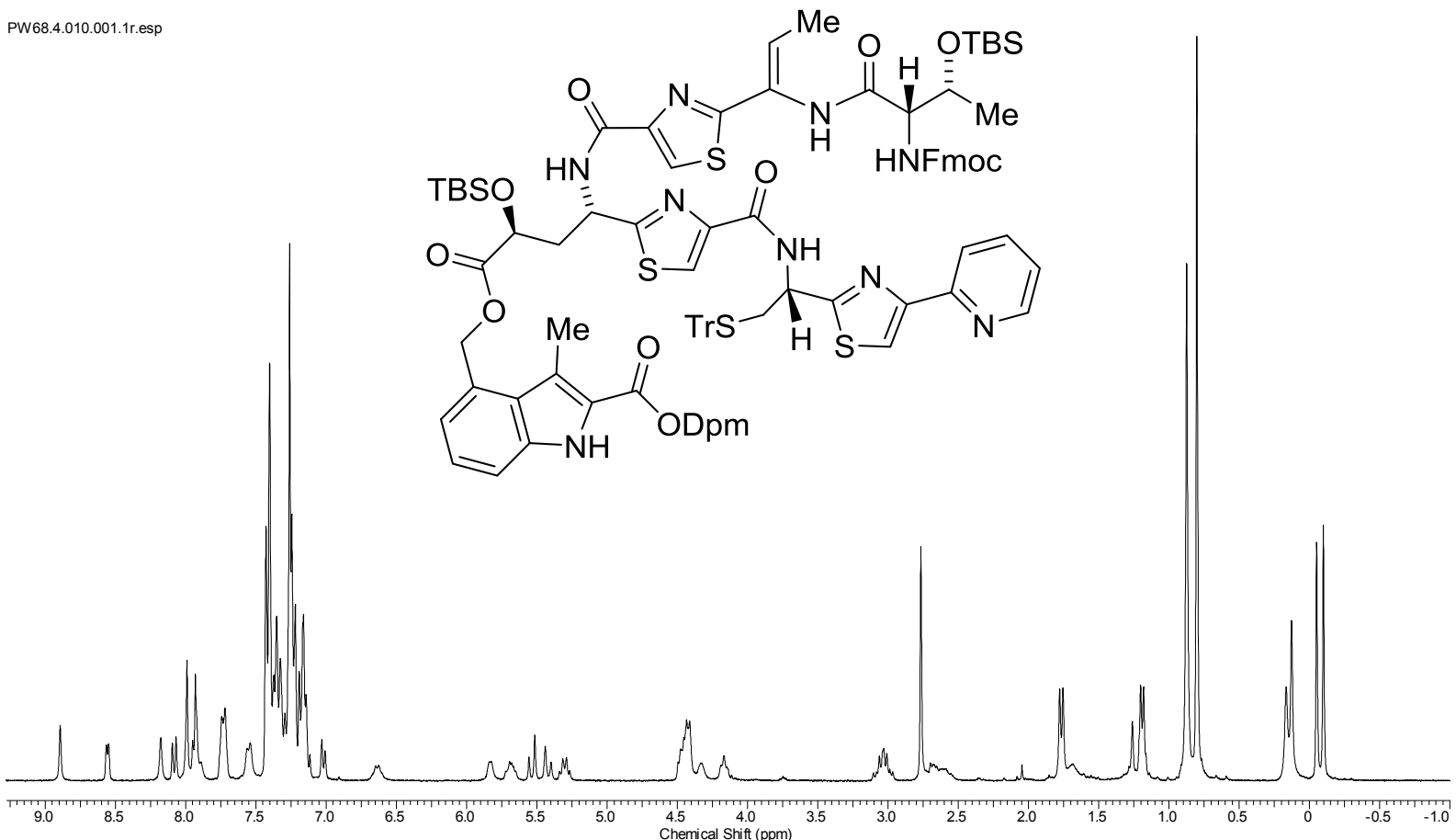

Abbildung 11.55: ${ }^{1} \mathrm{H}-\mathrm{NMR}-\mathrm{Spektrum}$ des B-Ring-Testsystems 113 (300 MHz, $\left.\mathrm{CDCl}_{3}\right)$.

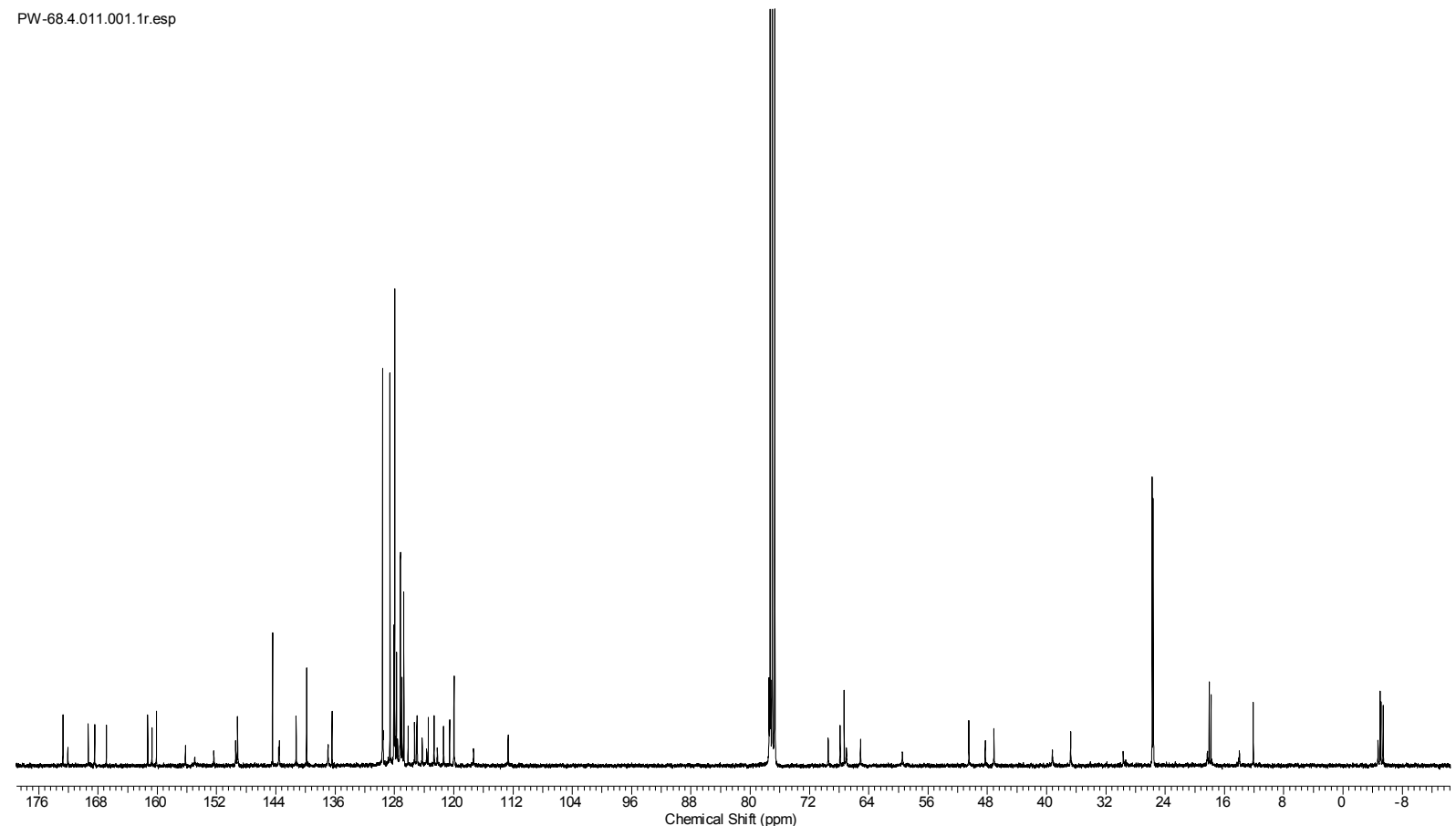

Abbildung 11.56: ${ }^{13} \mathrm{C}-\mathrm{NMR}-$ Spektrum des B-Ring-Testsystems $113\left(100 \mathrm{MHz}, \mathrm{CDCl}_{3}\right)$. 


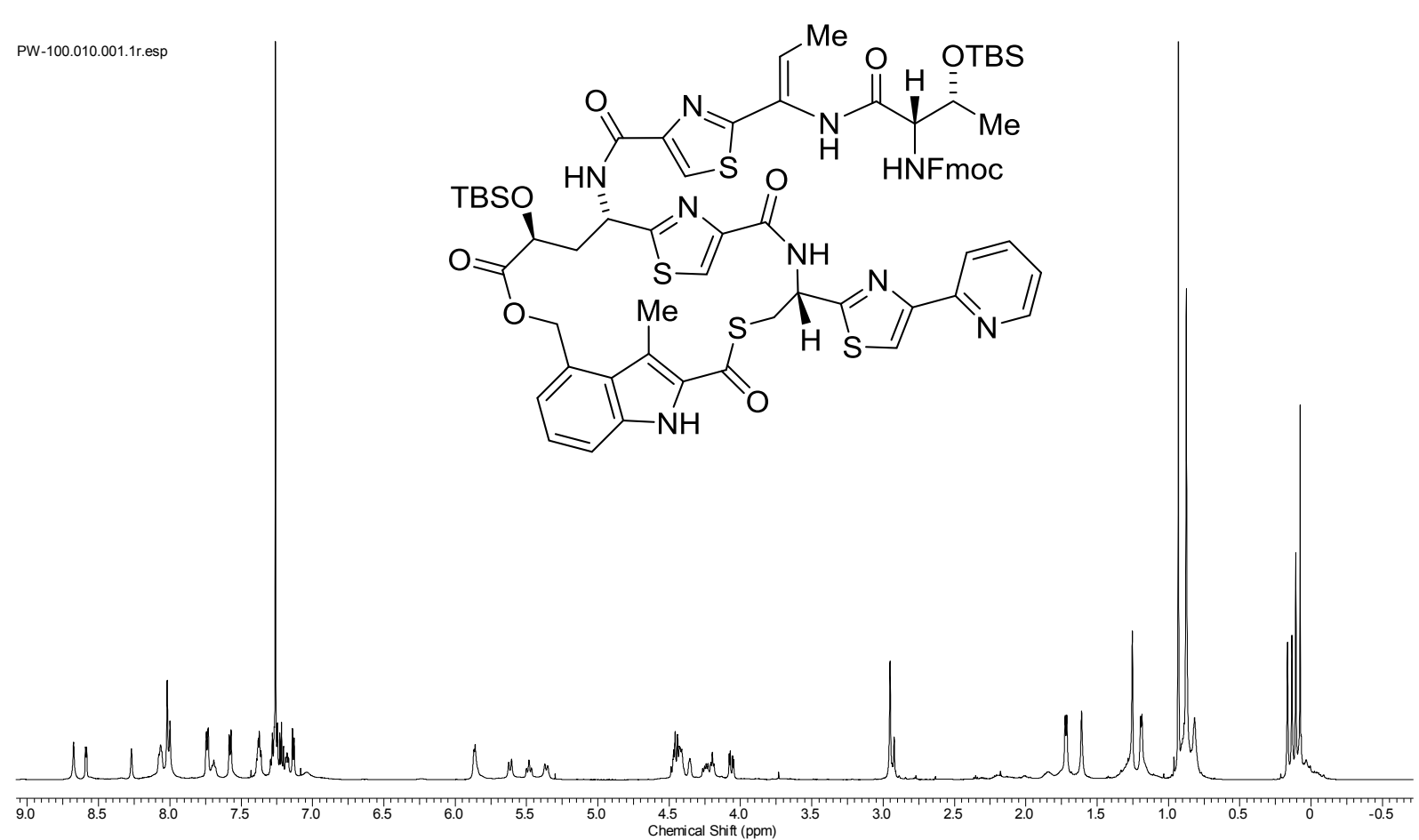

Abbildung 11.57: ${ }^{1} \mathrm{H}-\mathrm{NMR}-S p e k t r u m$ des erweiterten Nosiheptid-B-Rings $165\left(600 \mathrm{MHz}, \mathrm{CDCl}_{3}\right)$.

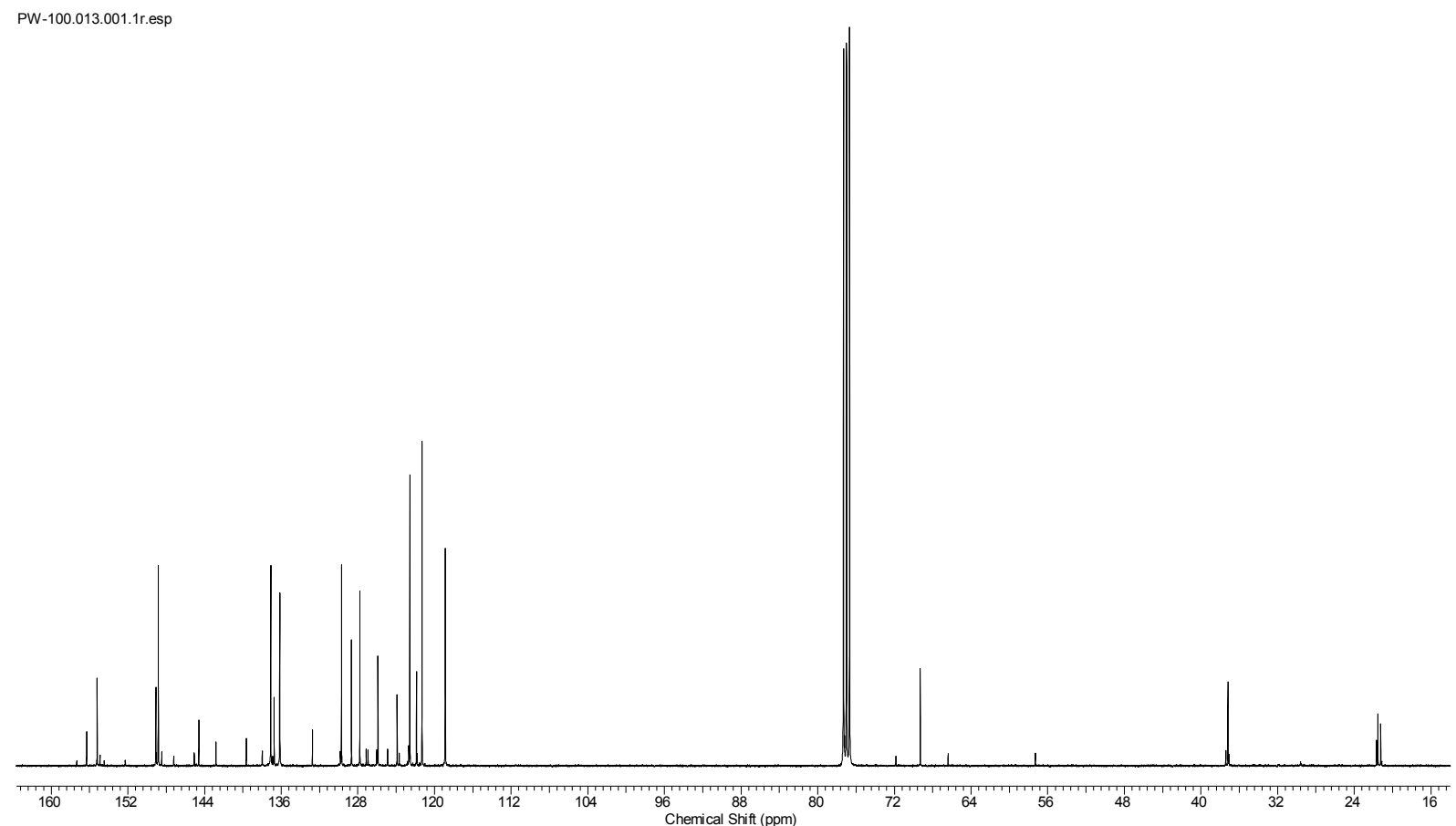

Abbildung 11.58: ${ }^{13} \mathrm{C}-\mathrm{NMR}-$ Spektrum des erweiterten Nosiheptid-B-Rings 165 (100 MHz, $\left.\mathrm{CDCl}_{3}\right)$. 


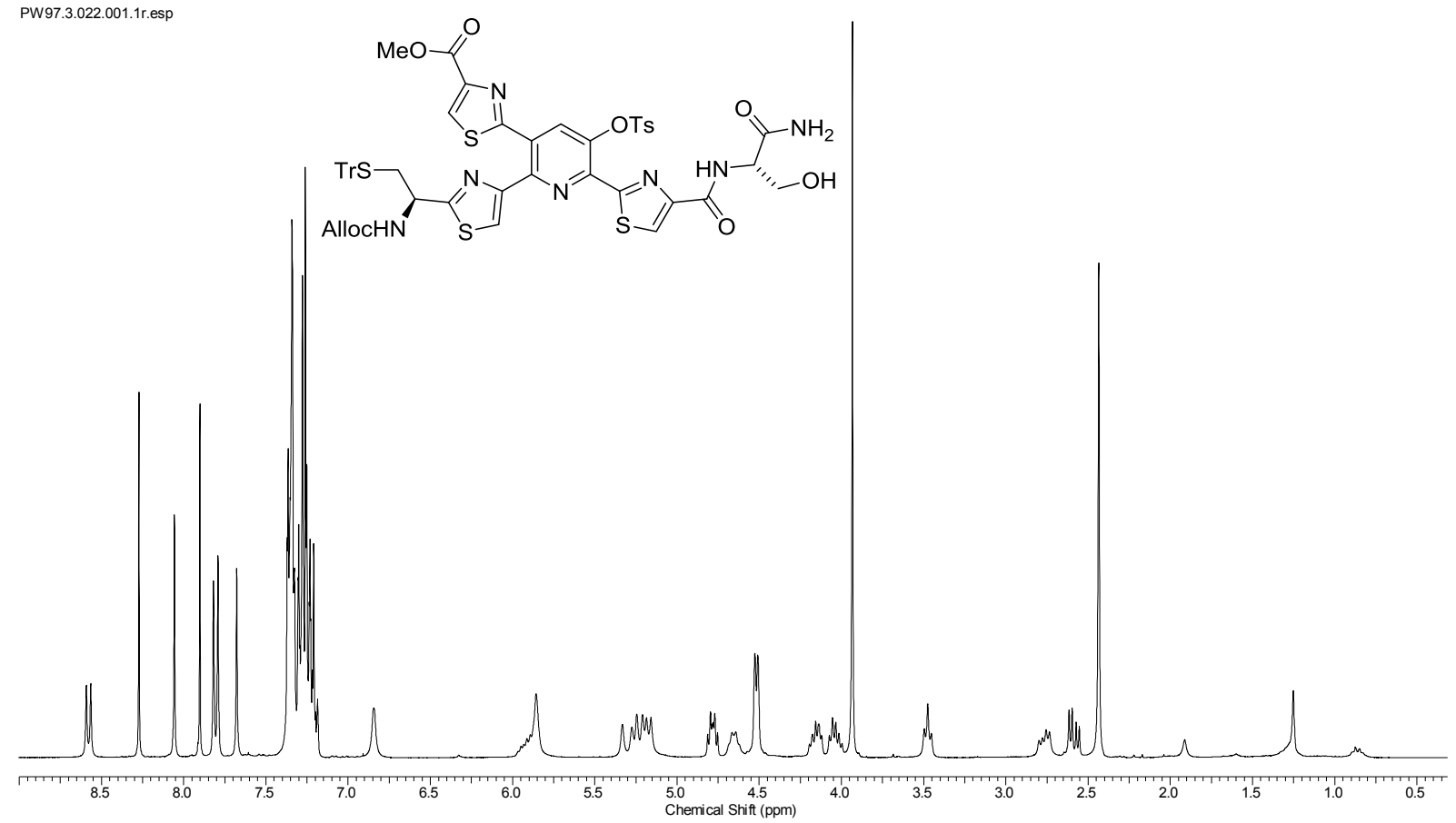

Abbildung 11.59: ${ }^{1} \mathrm{H}-\mathrm{NMR}-S p e k t r u m$ des Serinyl-Alkohols $166\left(300 \mathrm{MHz}, \mathrm{CDCl}_{3}\right)$.

PW97.3.010.001.1r.esp

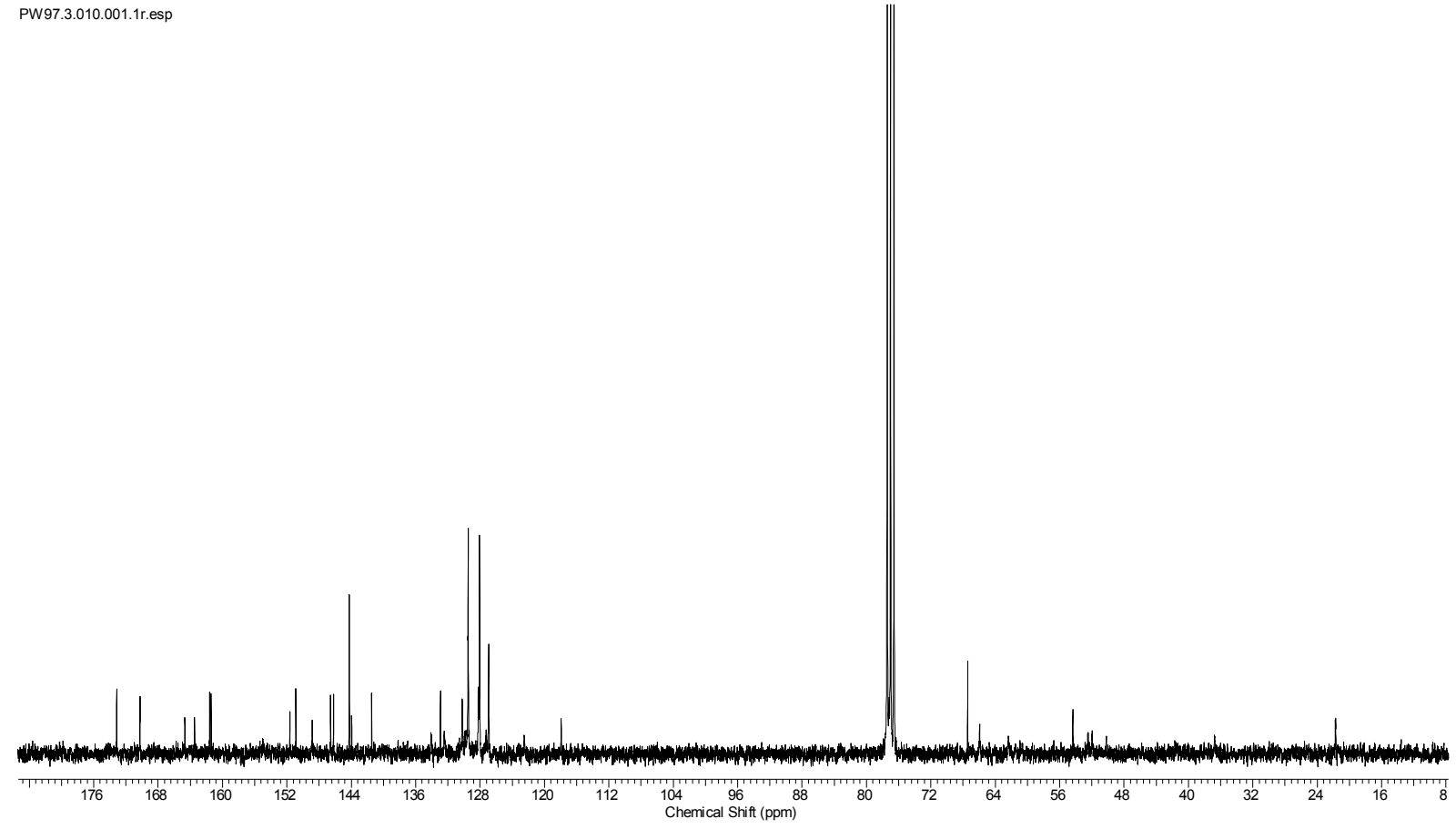

Abbildung 11.60: ${ }^{13} \mathrm{C}-\mathrm{NMR}-$ Spektrum des Serinyl-Alkohols $166\left(75 \mathrm{MHz}, \mathrm{CDCl}_{3}\right)$. 


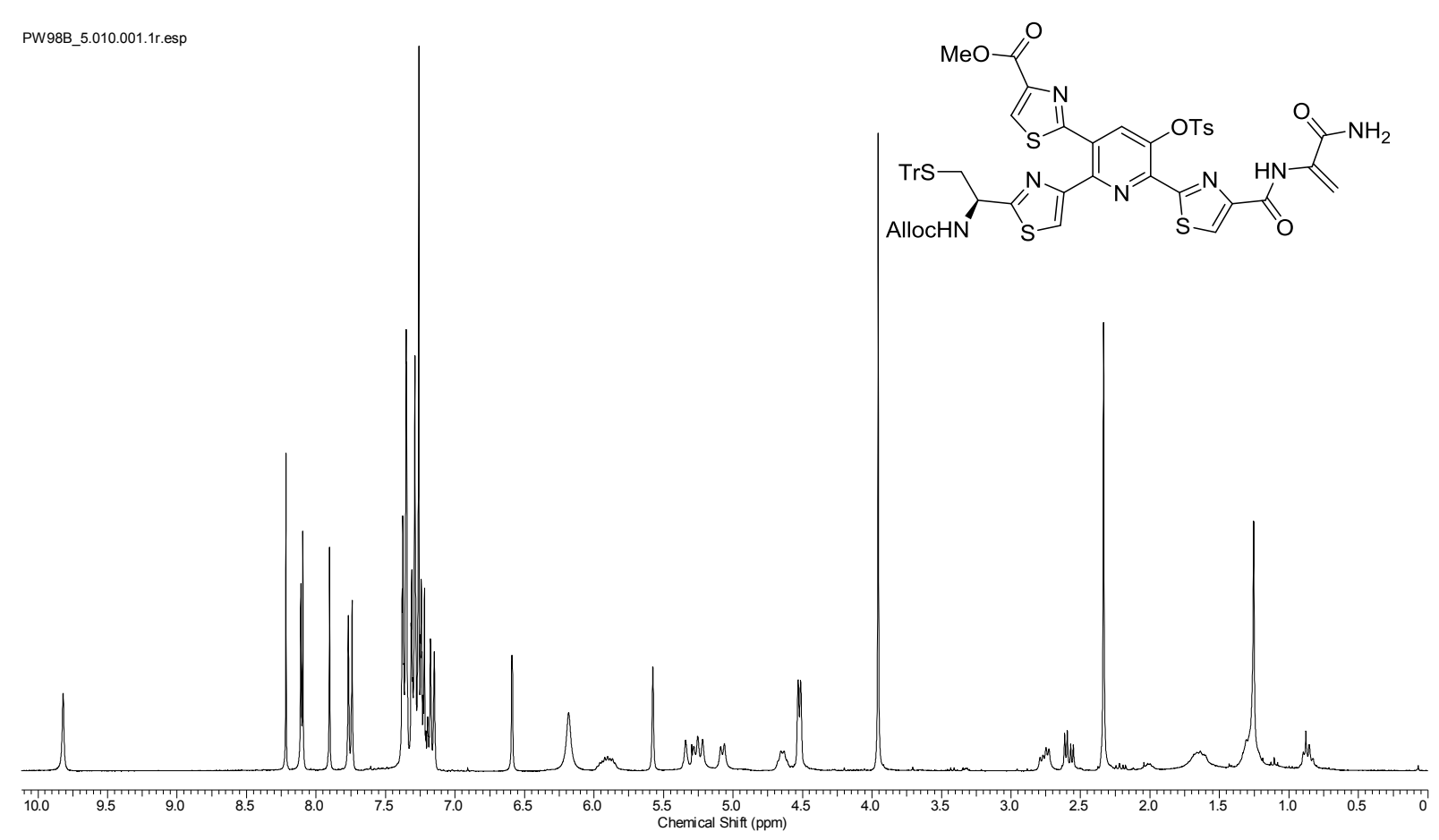

Abbildung 11.61: ${ }^{1} \mathrm{H}-\mathrm{NMR}-$ Spektrum des Dehydroalanin-Derivats $167\left(300 \mathrm{MHz}, \mathrm{CDCl}_{3}\right)$.

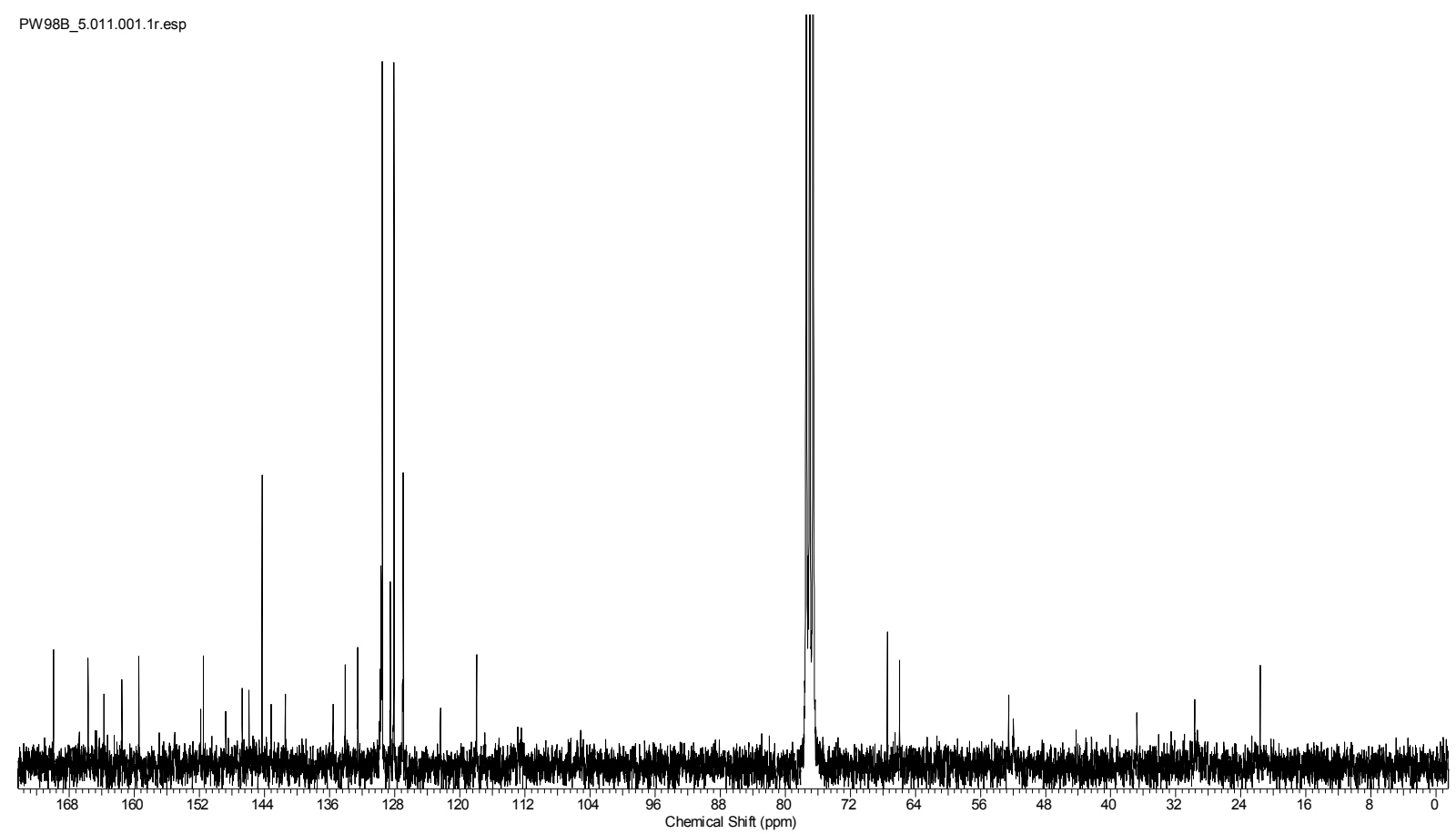

Abbildung 11.62: ${ }^{13} \mathrm{C}-\mathrm{NMR}-$ Spektrum des Dehydroalanin-Derivats $167\left(75 \mathrm{MHz}, \mathrm{CDCl}_{3}\right)$. 


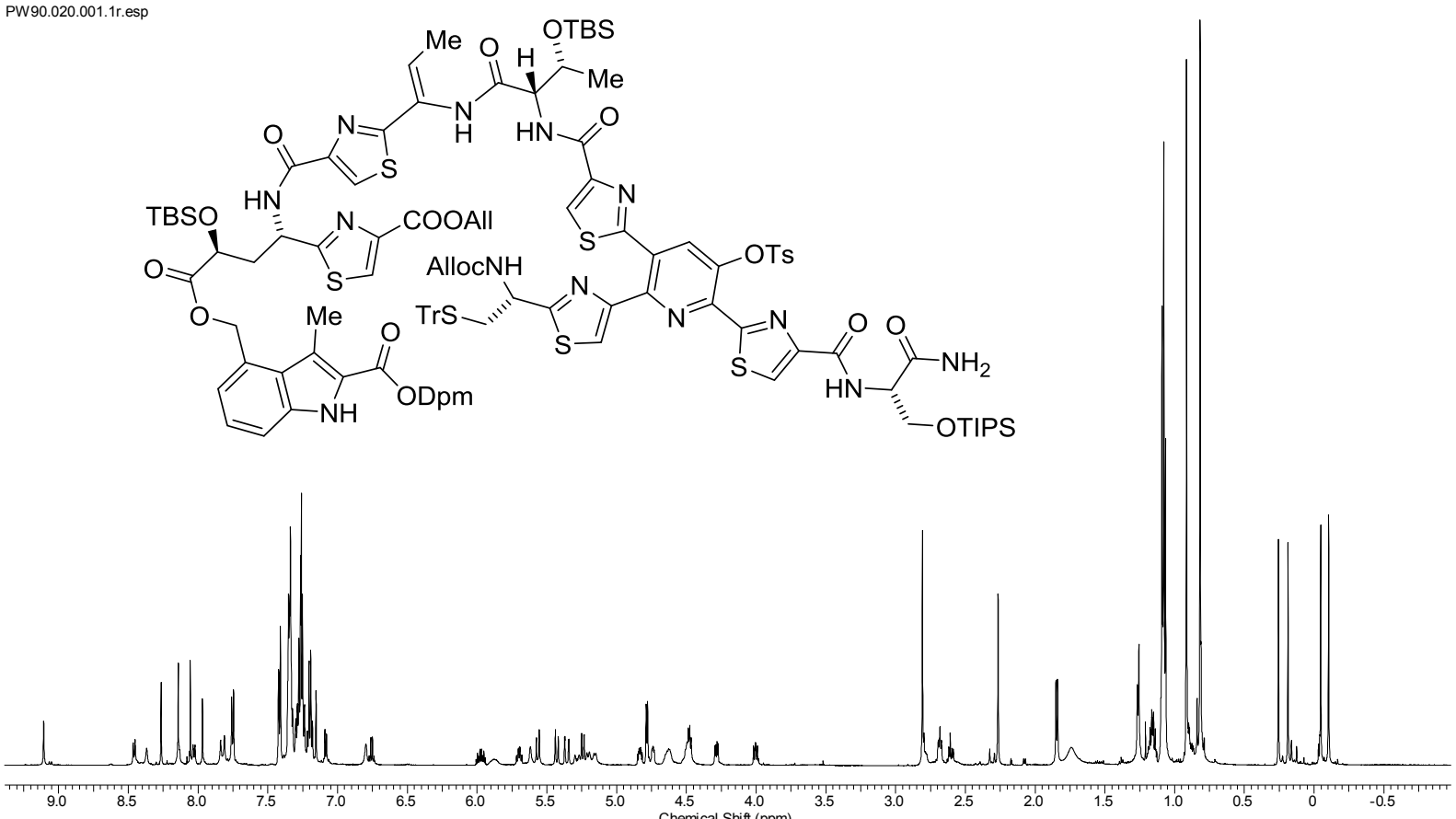

Abbildung 11.63: ${ }^{1} \mathrm{H}-\mathrm{NMR}-$ Spektrum des linearen Vorläufers $114\left(600 \mathrm{MHz}, \mathrm{CDCl}_{3}\right)$.

PW90.022.001.1r.esp

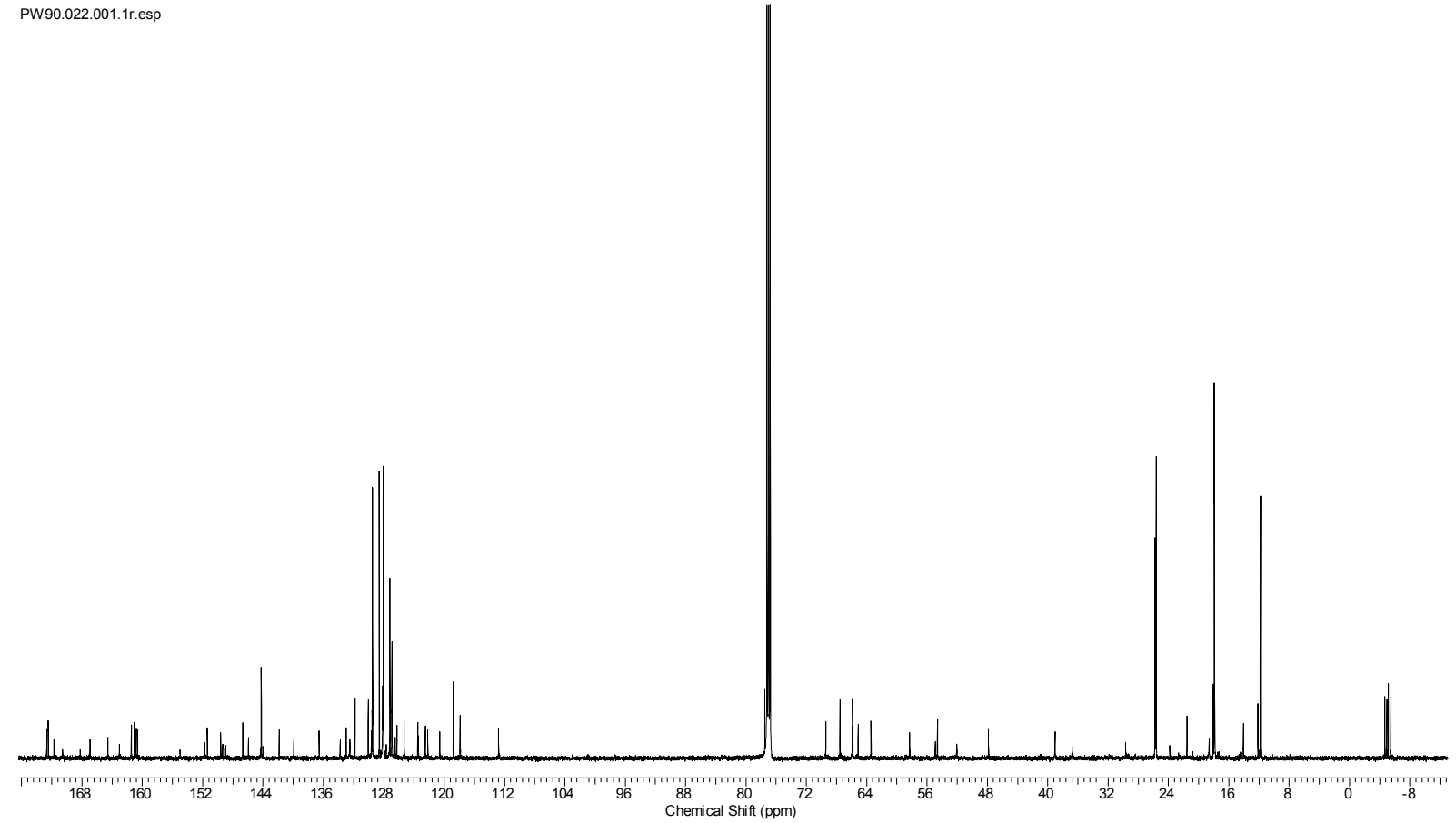

Abbildung 11.64: ${ }^{13} \mathrm{C}-\mathrm{NMR}-$ Spektrum des linearen Vorläufers $114\left(150 \mathrm{MHz}, \mathrm{CDCl}_{3}\right)$. 


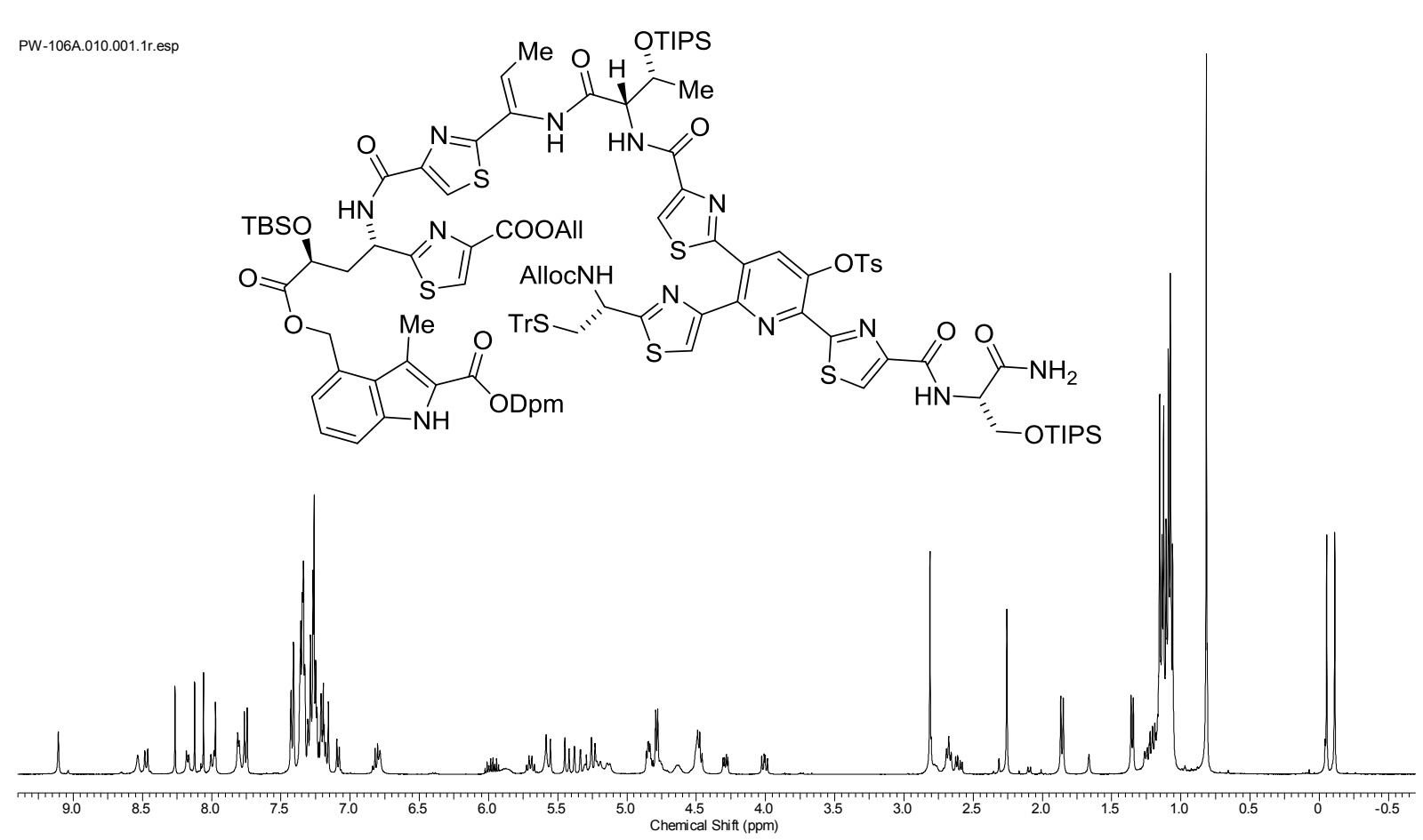

Abbildung 11.65: ${ }^{1} \mathrm{H}-\mathrm{NMR}-$ Spektrum des linearen Vorläufers 115 (400 MHz, $\mathrm{CDCl}_{3}$ ).

PW-106A.021.001.1r.esp

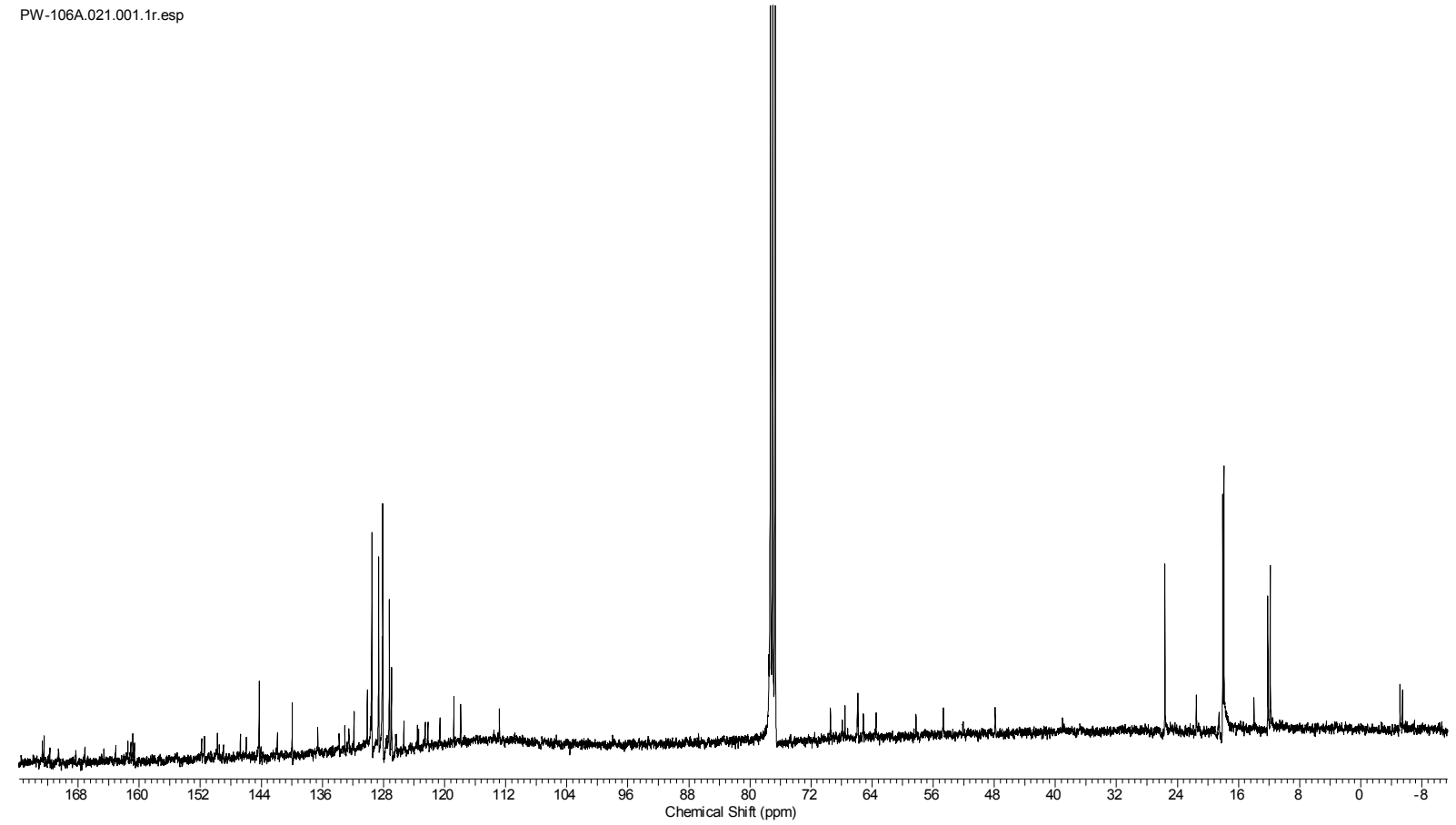

Abbildung 11.66: ${ }^{13} \mathrm{C}-\mathrm{NMR}-$ Spektrum des linearen Vorläufers $115\left(100 \mathrm{MHz}, \mathrm{CDCl}_{3}\right)$. 


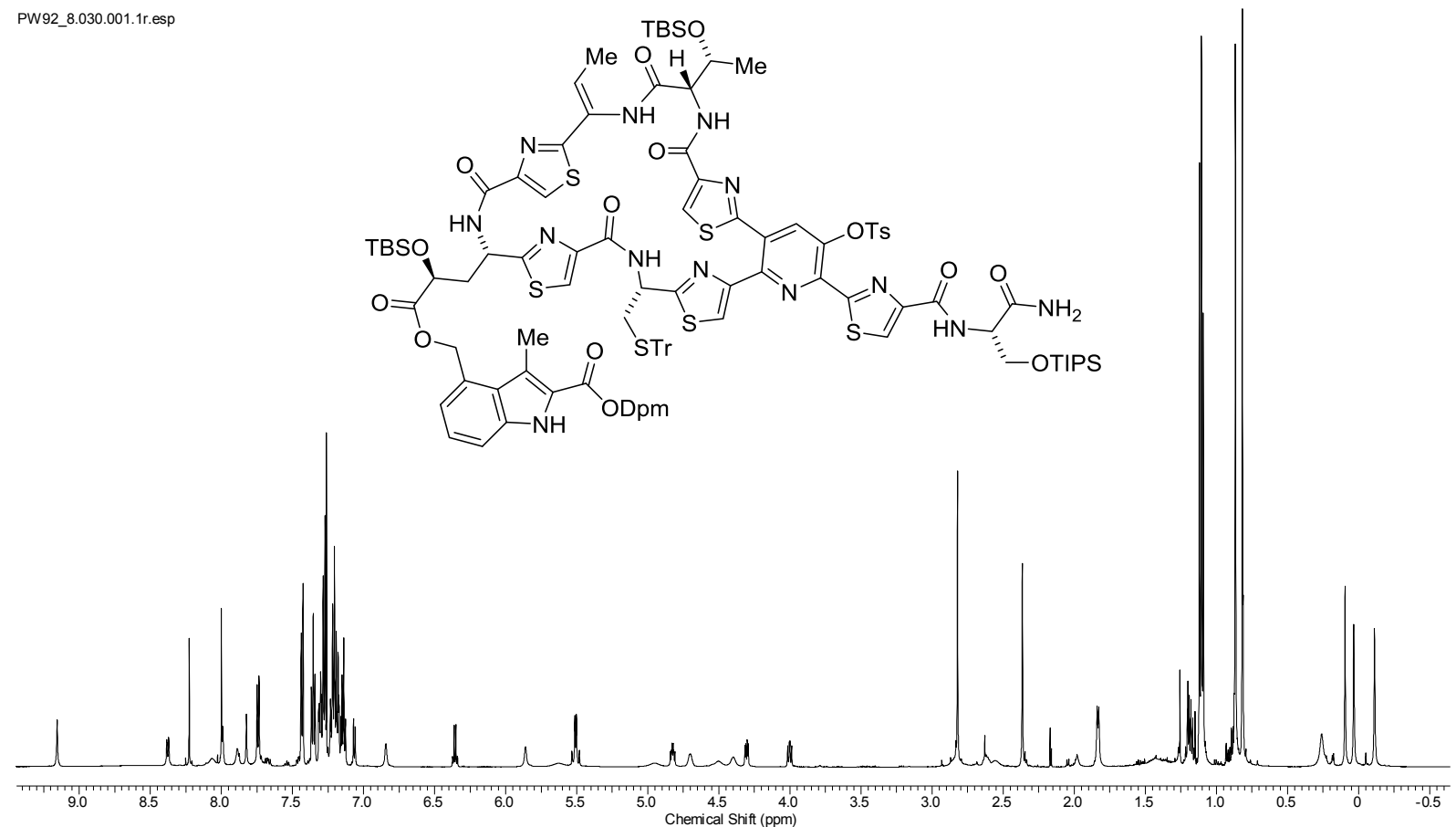

Abbildung 11.67: ${ }^{1} \mathrm{H}-\mathrm{NMR}-$ Spektrum des Makrolactams $175\left(600 \mathrm{MHz}, \mathrm{CDCl}_{3}\right)$.

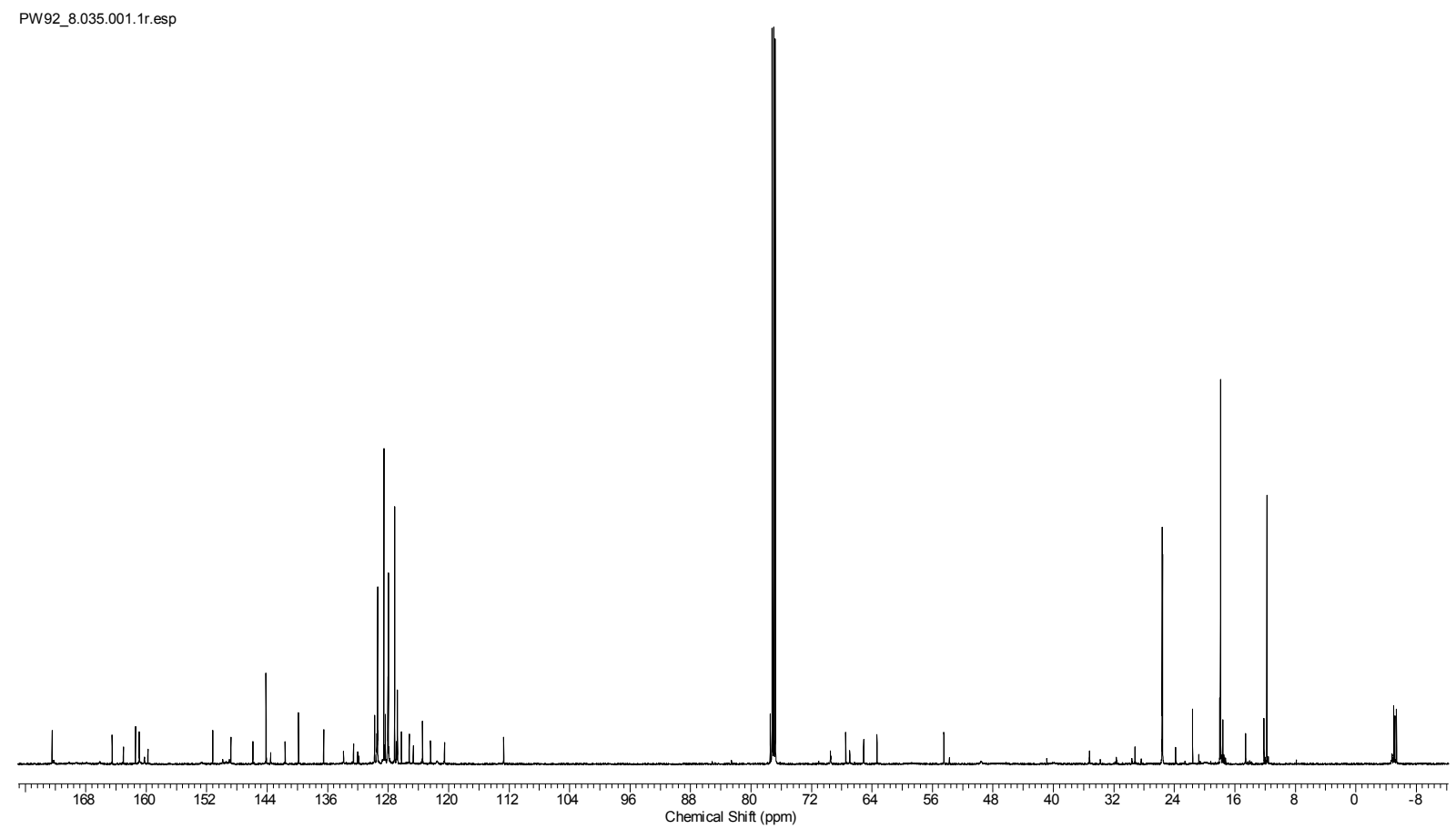

Abbildung 11.68: ${ }^{13} \mathrm{C}-\mathrm{NMR}-$ Spektrum des Makrolactams $175\left(150 \mathrm{MHz}, \mathrm{CDCl}_{3}\right)$. 
11.2 Spektren zur Totalsynthese von Nosiheptid

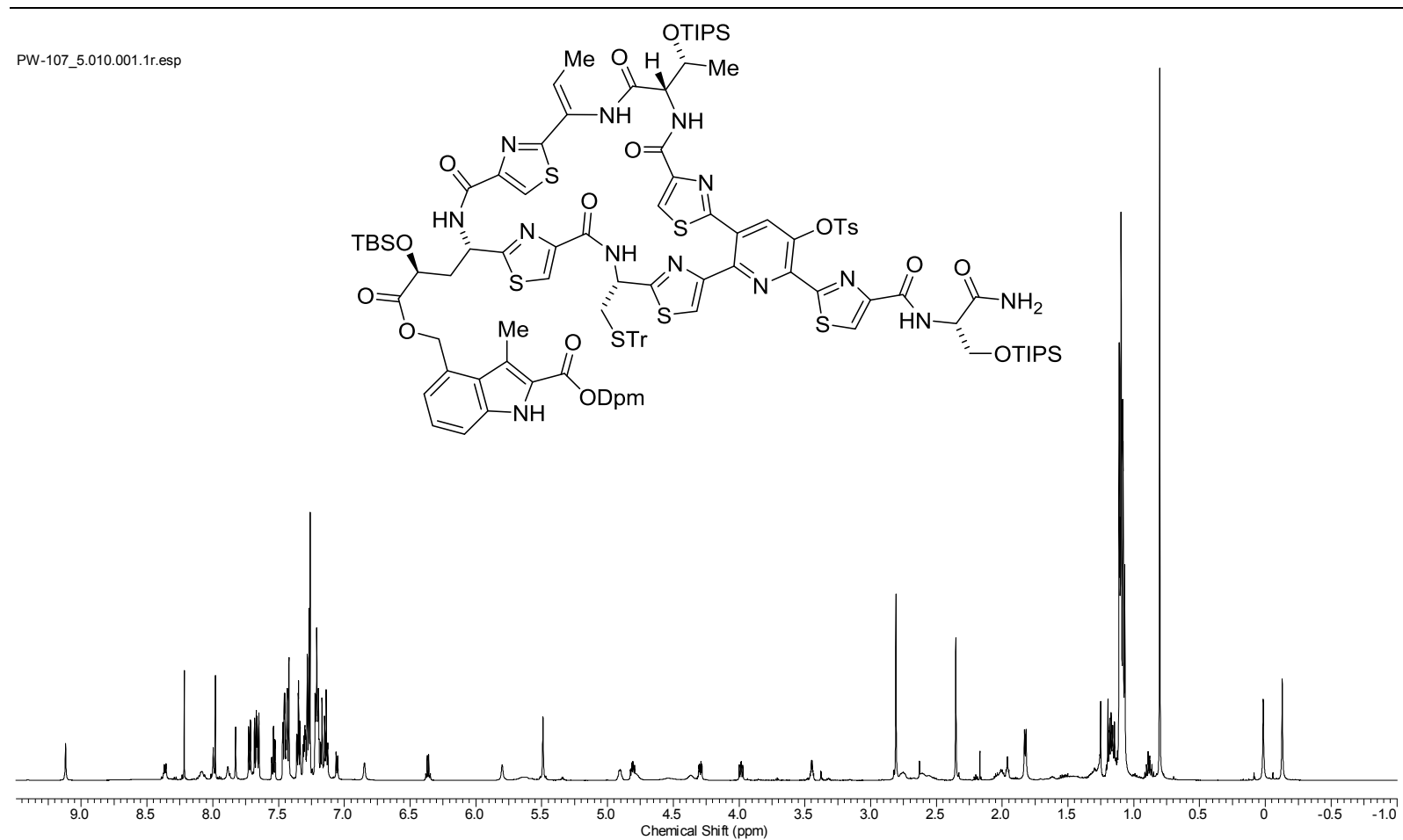

Abbildung 11.69: ${ }^{1} \mathrm{H}-\mathrm{NMR}-$ Spektrum des Makrolactams $176\left(600 \mathrm{MHz}, \mathrm{CDCl}_{3}\right)$.

PW-107_5.011.001.1r.esp

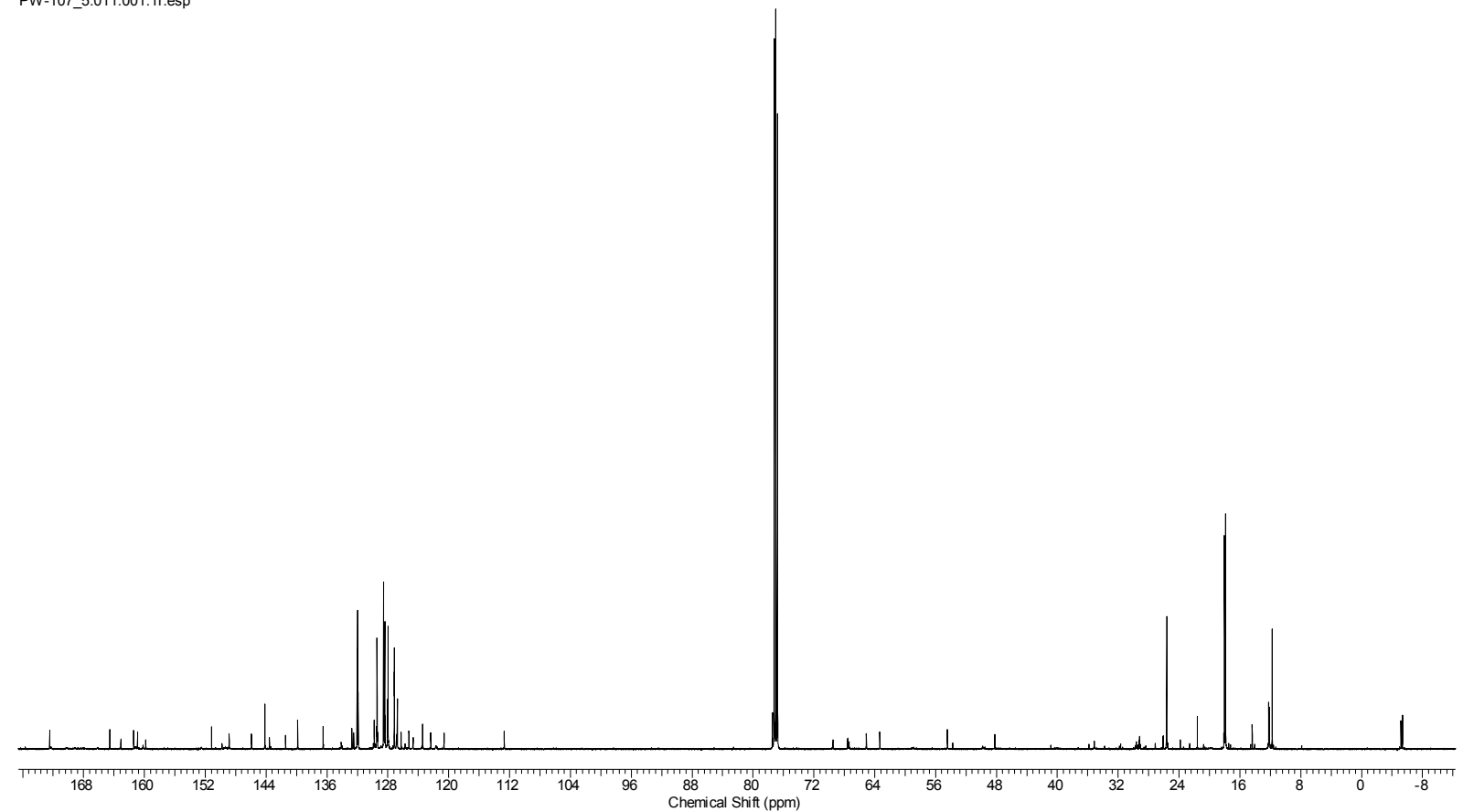

Abbildung 11.70: ${ }^{13} \mathrm{C}-\mathrm{NMR}$-Spektrum des Makrolactams $176\left(150 \mathrm{MHz}, \mathrm{CDCl}_{3}\right)$. 


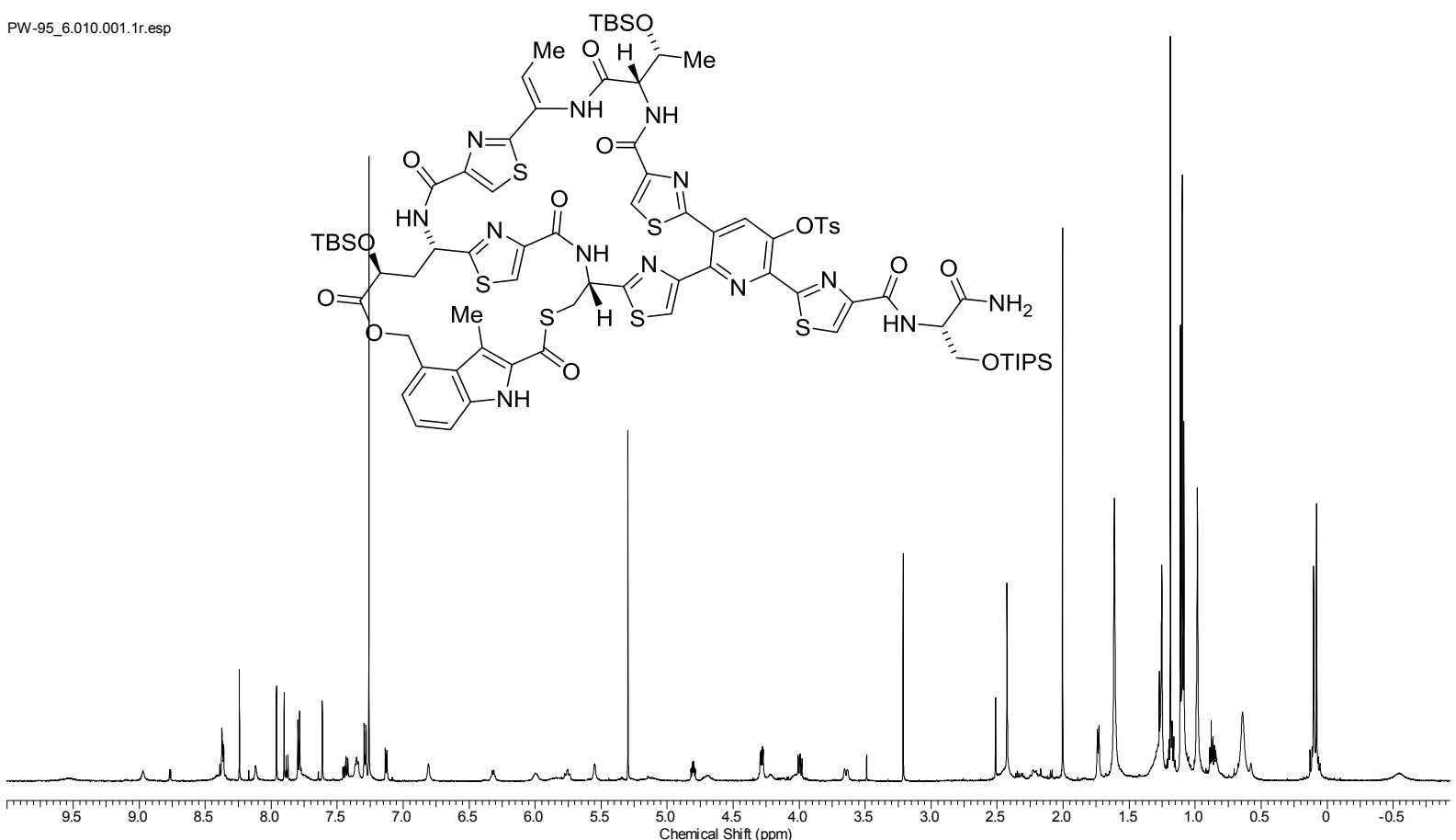

Abbildung 11.71: ${ }^{1} \mathrm{H}-\mathrm{NMR}-S p e k t r u m$ des Makrothiolactons $116\left(600 \mathrm{MHz}, \mathrm{CDCl}_{3}\right)$.

PW-95_6.015.001.1r.esp

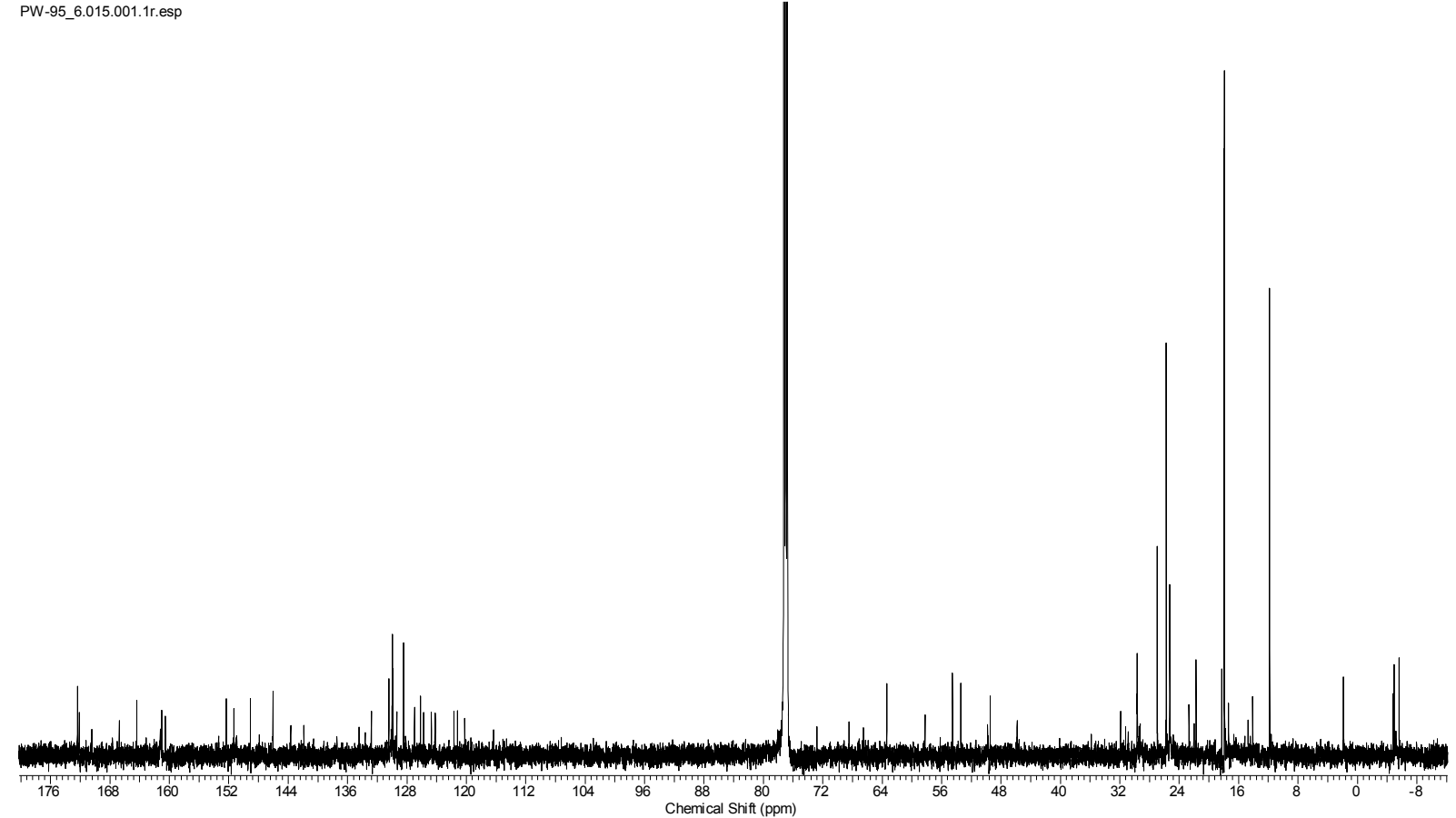

Abbildung 11.72: ${ }^{13} \mathrm{C}-\mathrm{NMR}-$ Spektrum des Makrothiolactons $116\left(150 \mathrm{MHz}, \mathrm{CDCl}_{3}\right)$. 


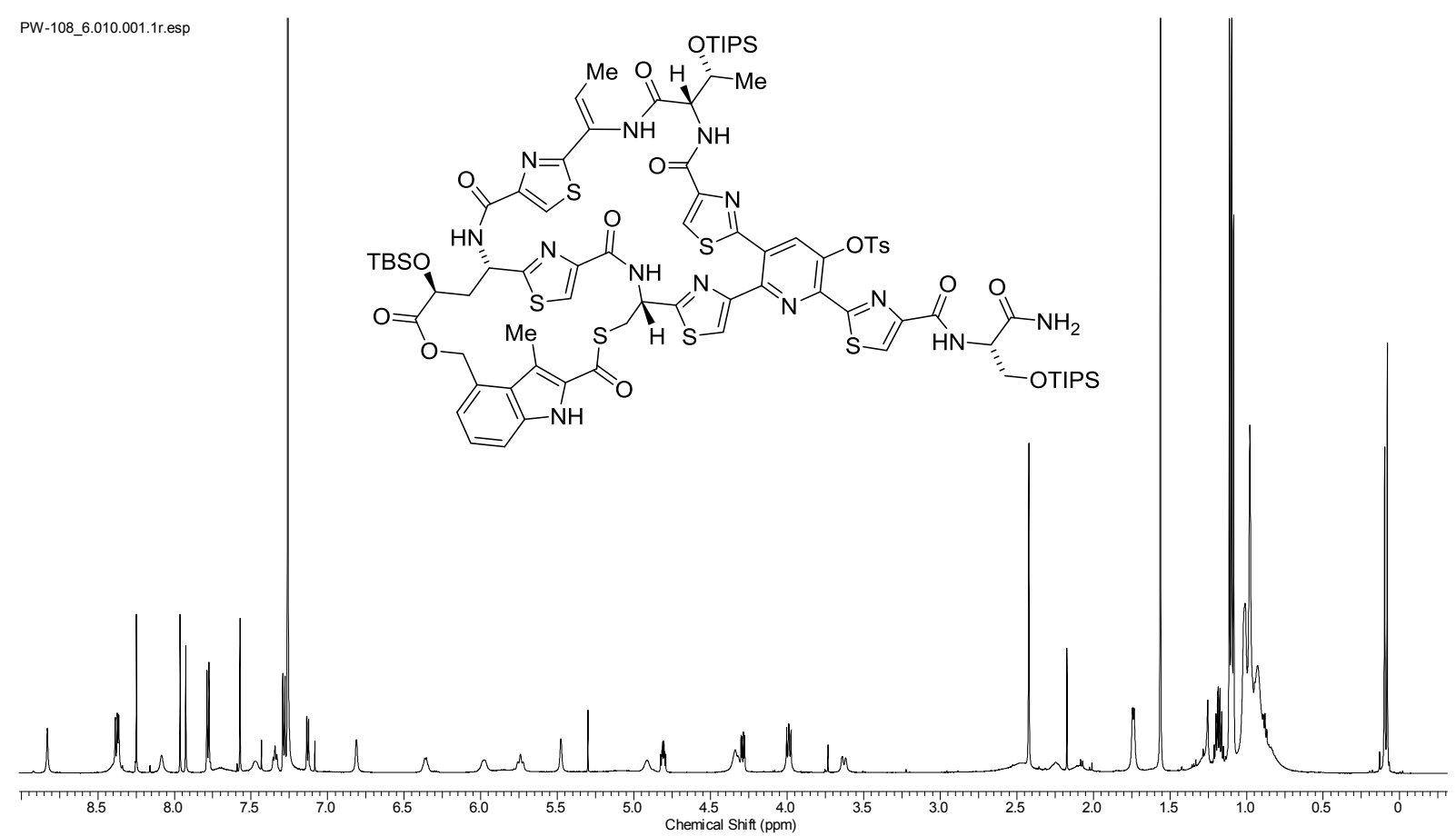

Abbildung 11.73: ${ }^{1} \mathrm{H}-\mathrm{NMR}-S p e k t r u m$ des Makrothiolactons $117\left(600 \mathrm{MHz}, \mathrm{CDCl}_{3}\right)$.

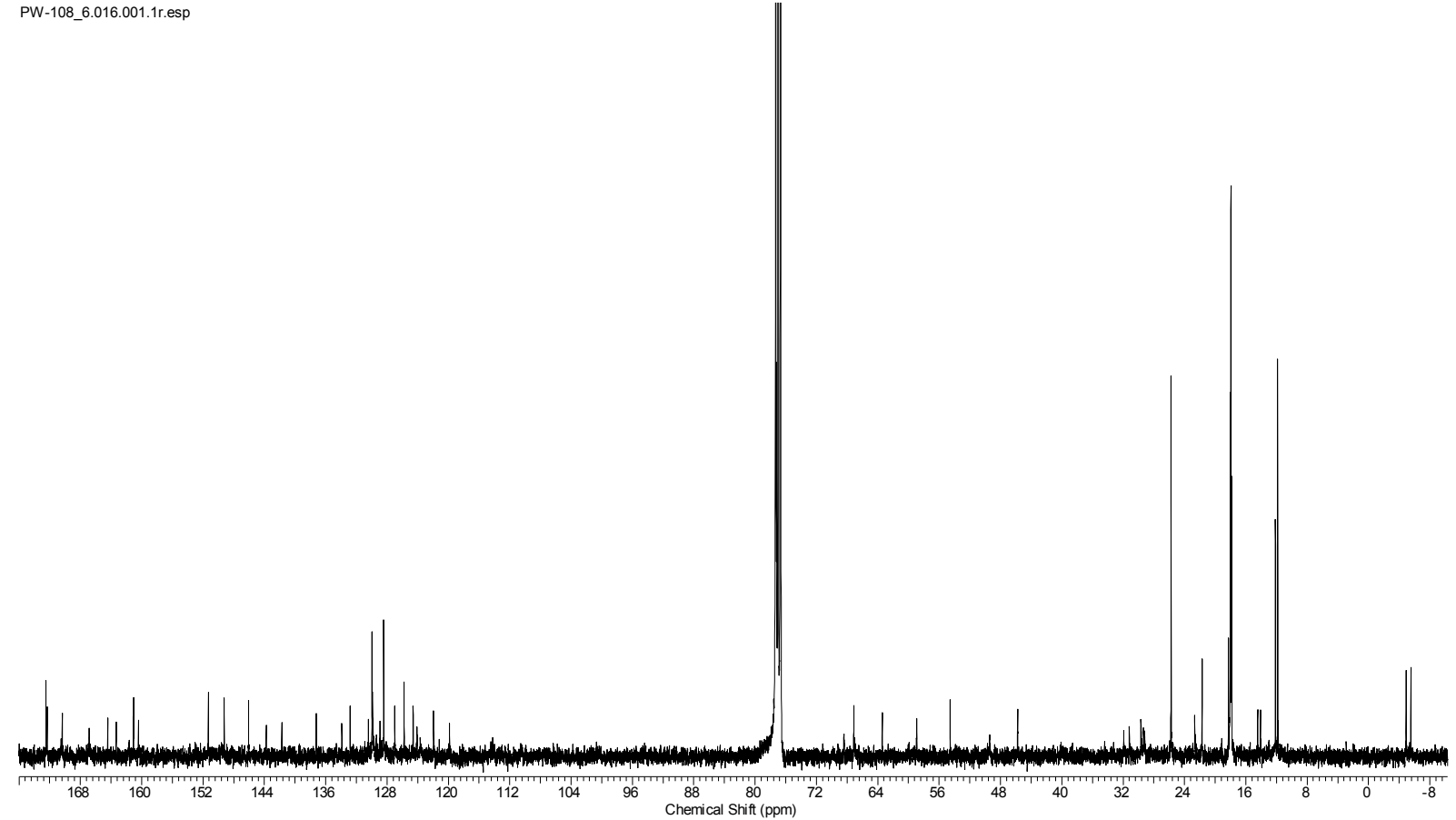

Abbildung 11.74: ${ }^{13} \mathrm{C}-\mathrm{NMR}-$ Spektrum des Makrothiolactons $117\left(100 \mathrm{MHz}, \mathrm{CDCl}_{3}\right)$. 

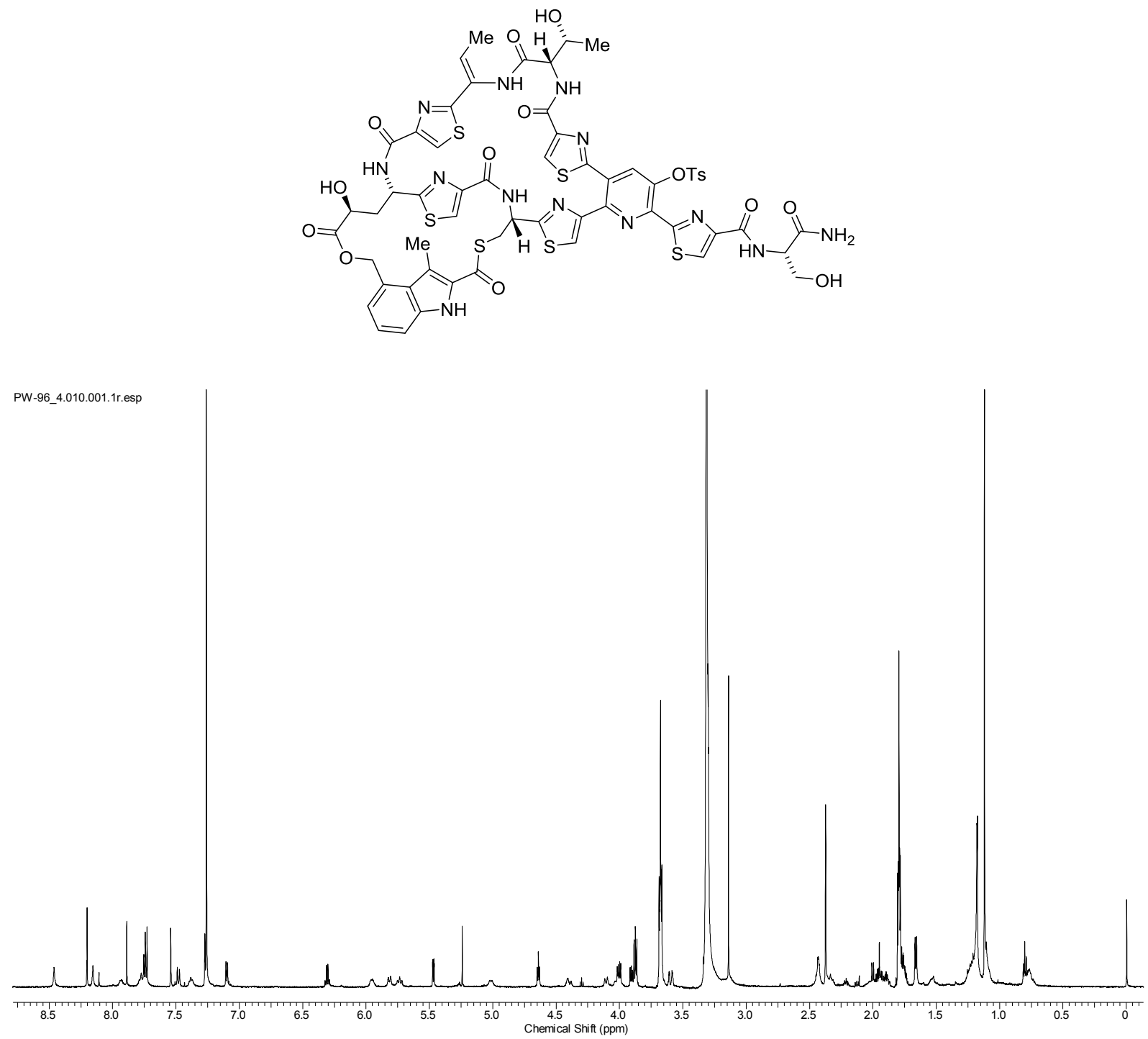

Abbildung 11.75: ${ }^{1} \mathrm{H}-\mathrm{NMR}-$ Spektrum von Ts-Hydrato-Nosiheptid $177\left(600 \mathrm{MHz}, \mathrm{CDCl}_{3} / \mathrm{MeOH}^{-} d_{4}\right.$, 9:1). 
11.2 Spektren zur Totalsynthese von Nosiheptid

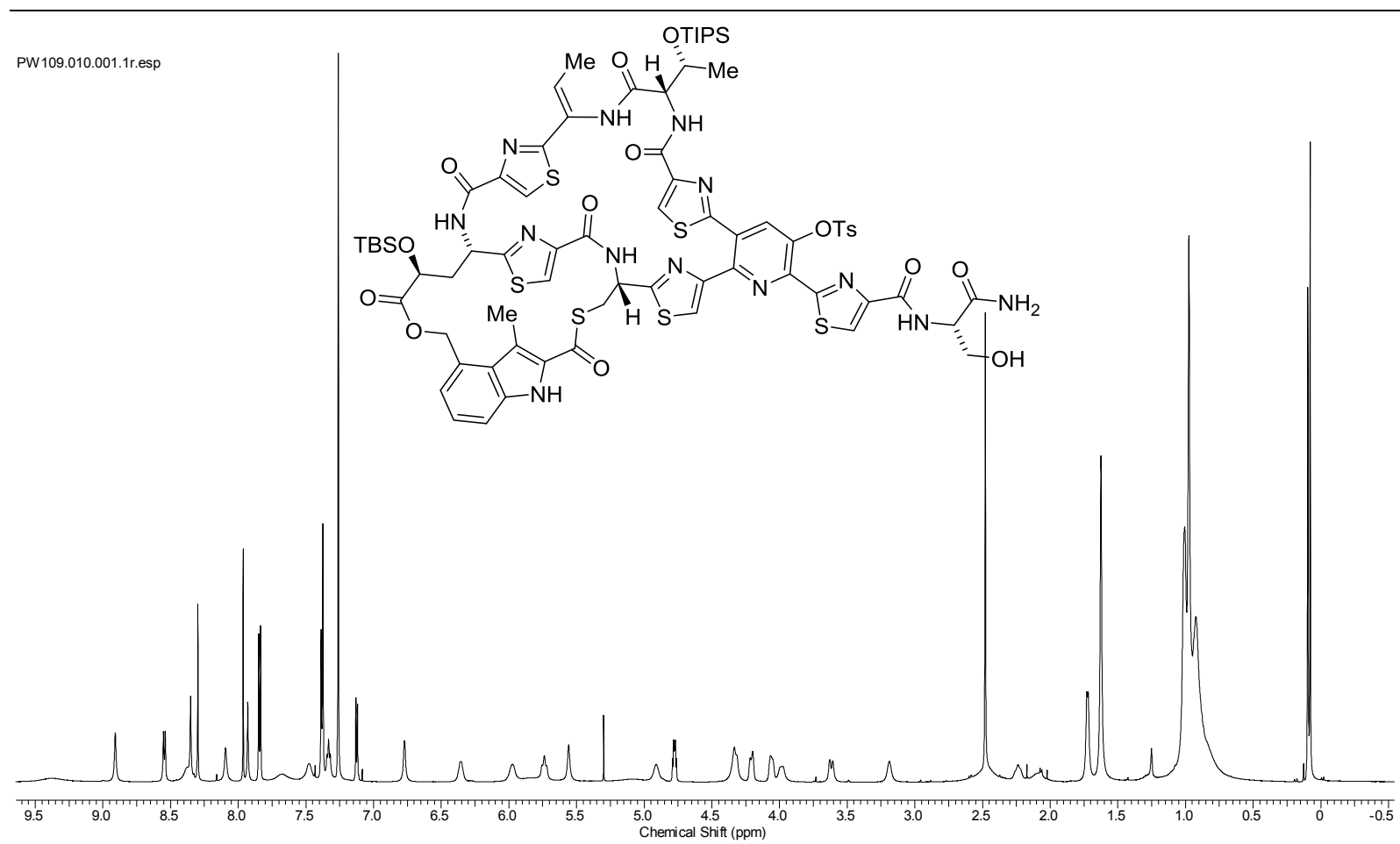

Abbildung 11.76: ${ }^{1} \mathrm{H}-\mathrm{NMR}-S p e k t r u m$ des Serinyl-Alkohols $179\left(600 \mathrm{MHz}, \mathrm{CDCl}_{3}\right)$.

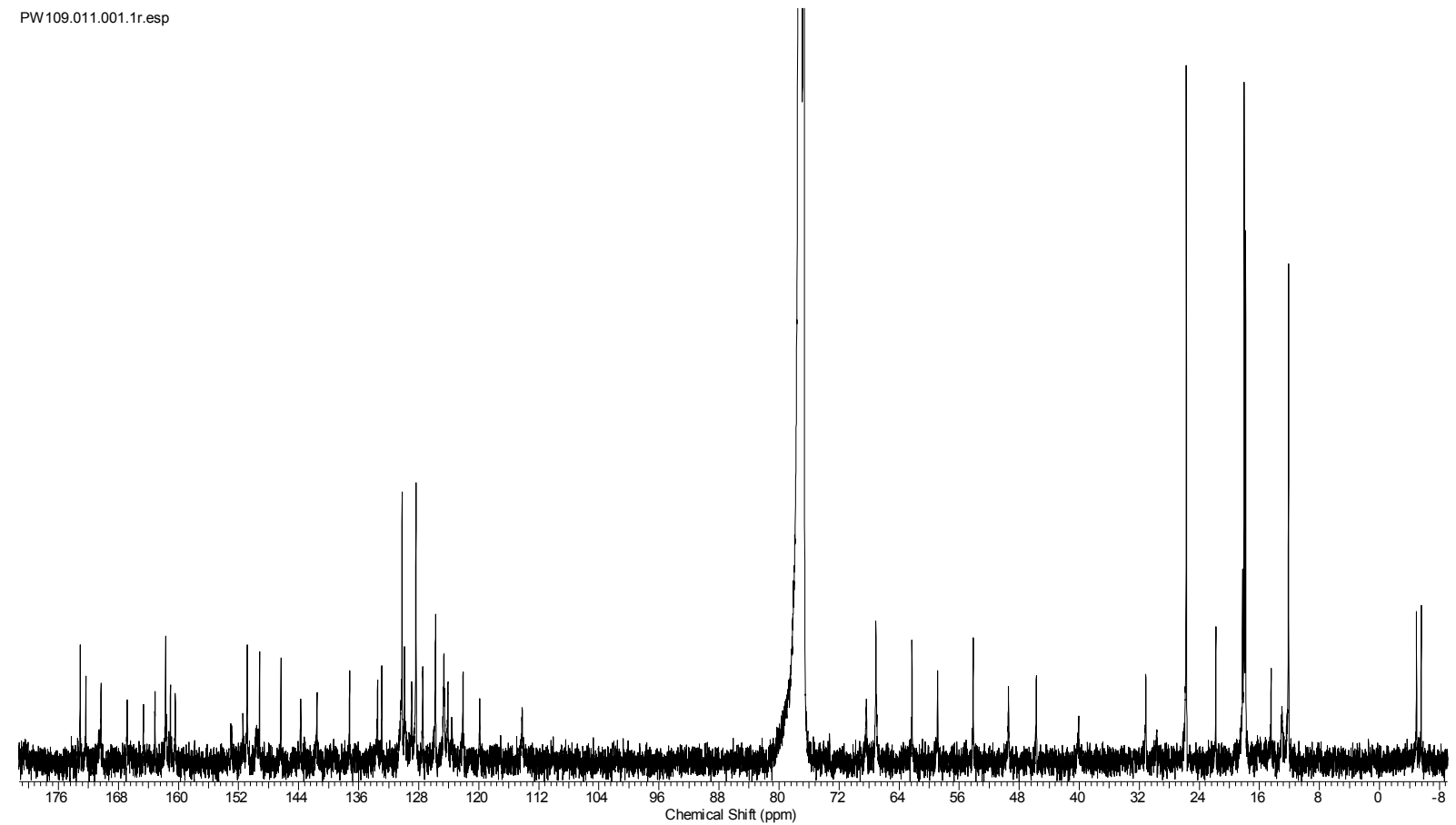

Abbildung 11.77: ${ }^{13} \mathrm{C}-\mathrm{NMR}-S p e k t r u m$ des Serinyl-Alkohols $179\left(100 \mathrm{MHz}, \mathrm{CDCl}_{3}\right)$. 

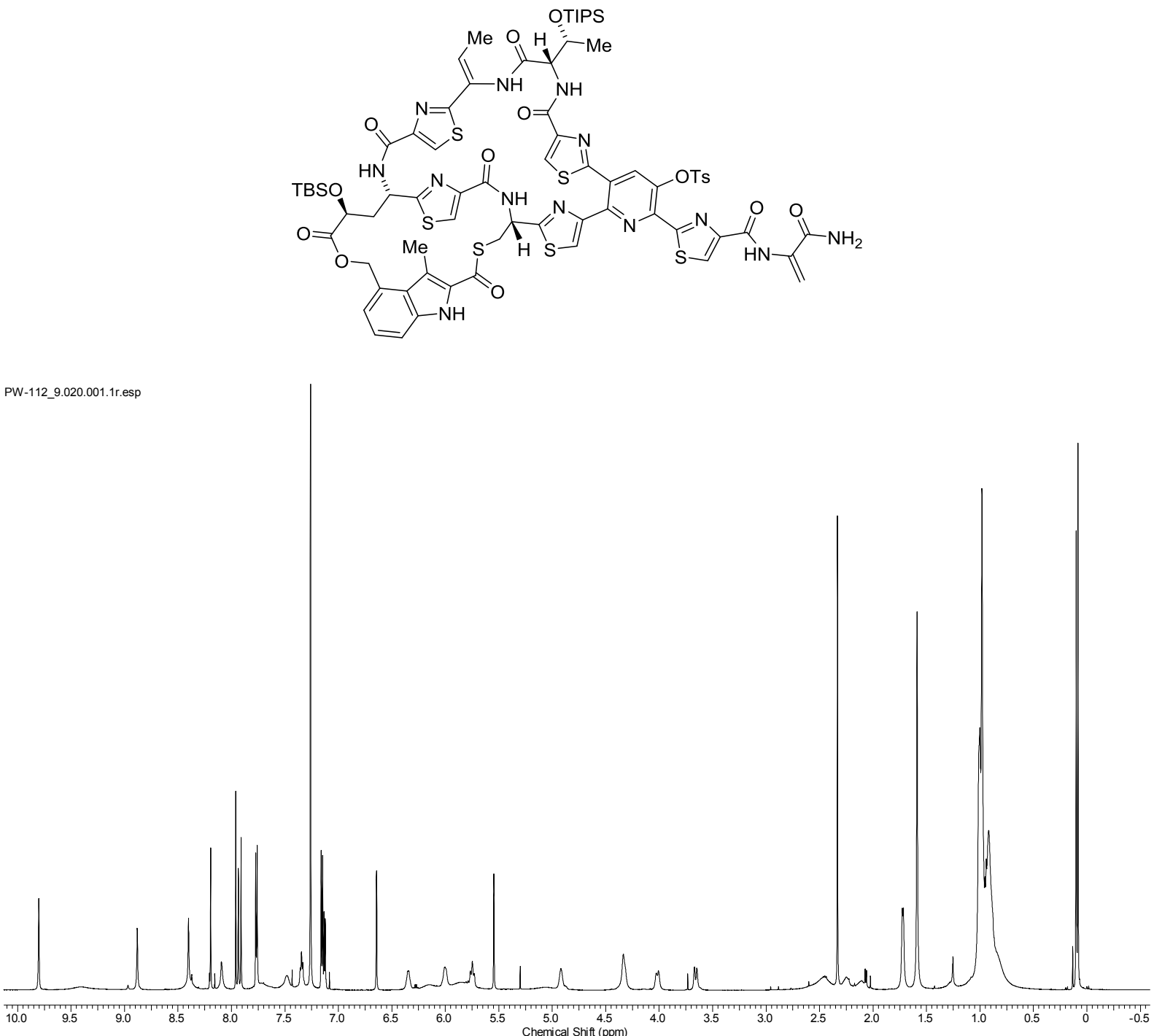

Abbildung 11.78: ${ }^{1} \mathrm{H}-\mathrm{NMR}-S p e k t r u m$ des Dehydroalanins $180\left(600 \mathrm{MHz}, \mathrm{CDCl}_{3}\right)$. 


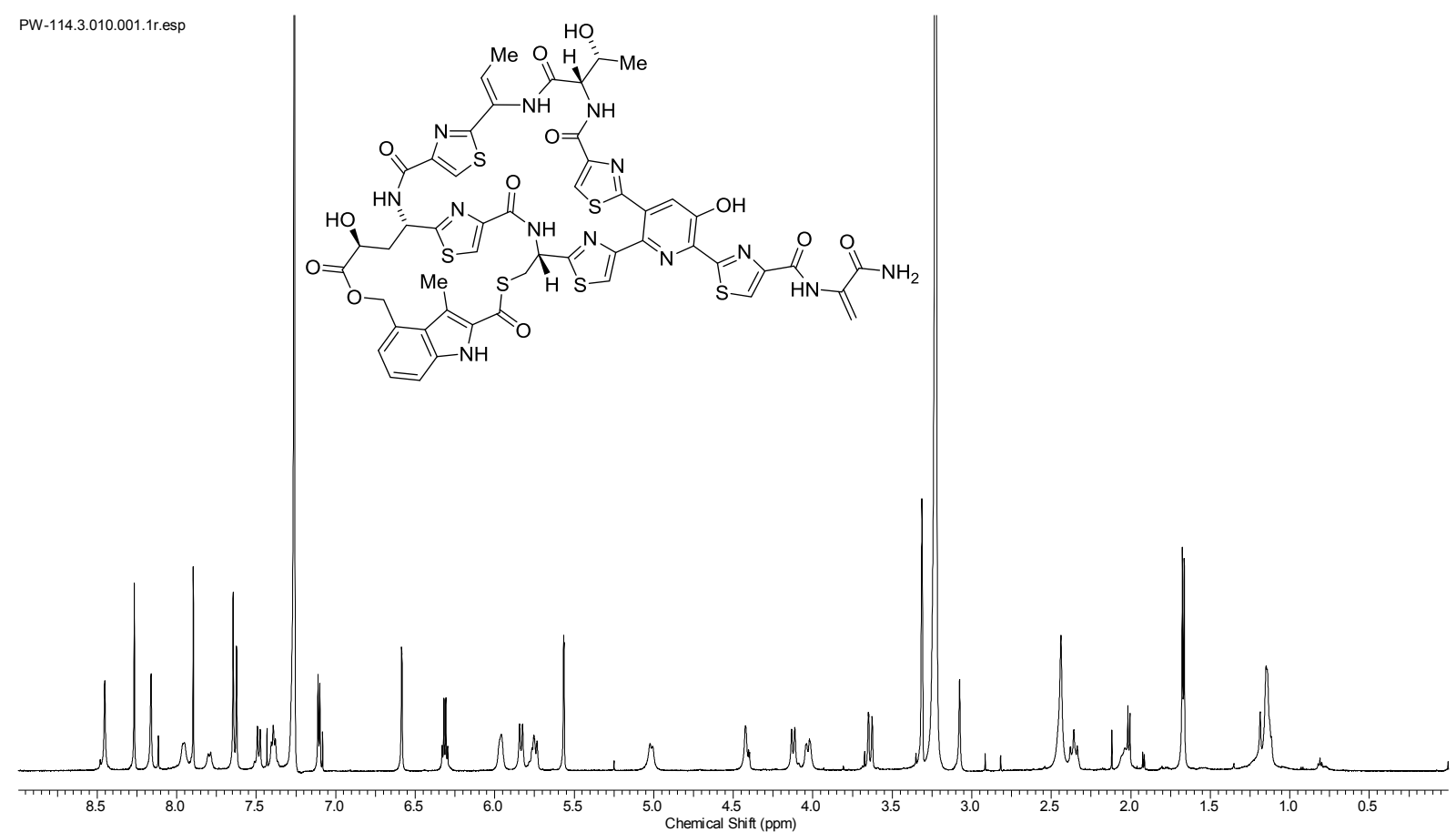

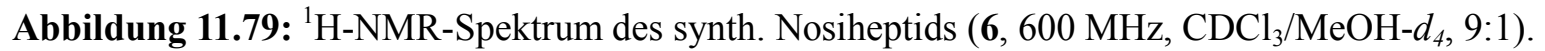

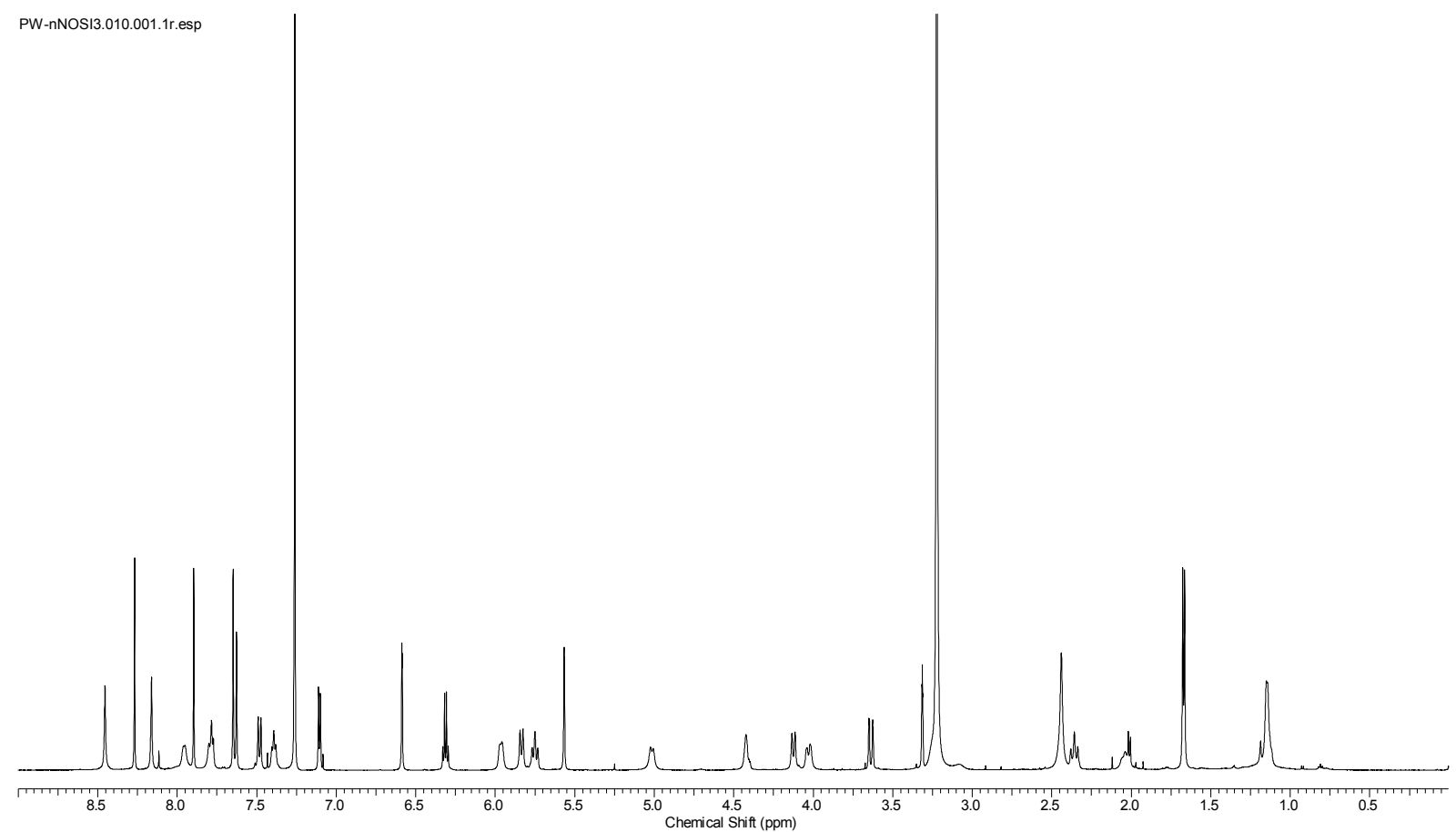

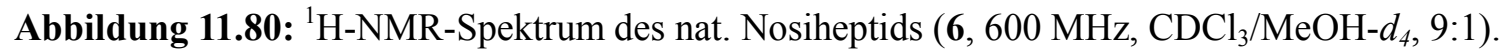




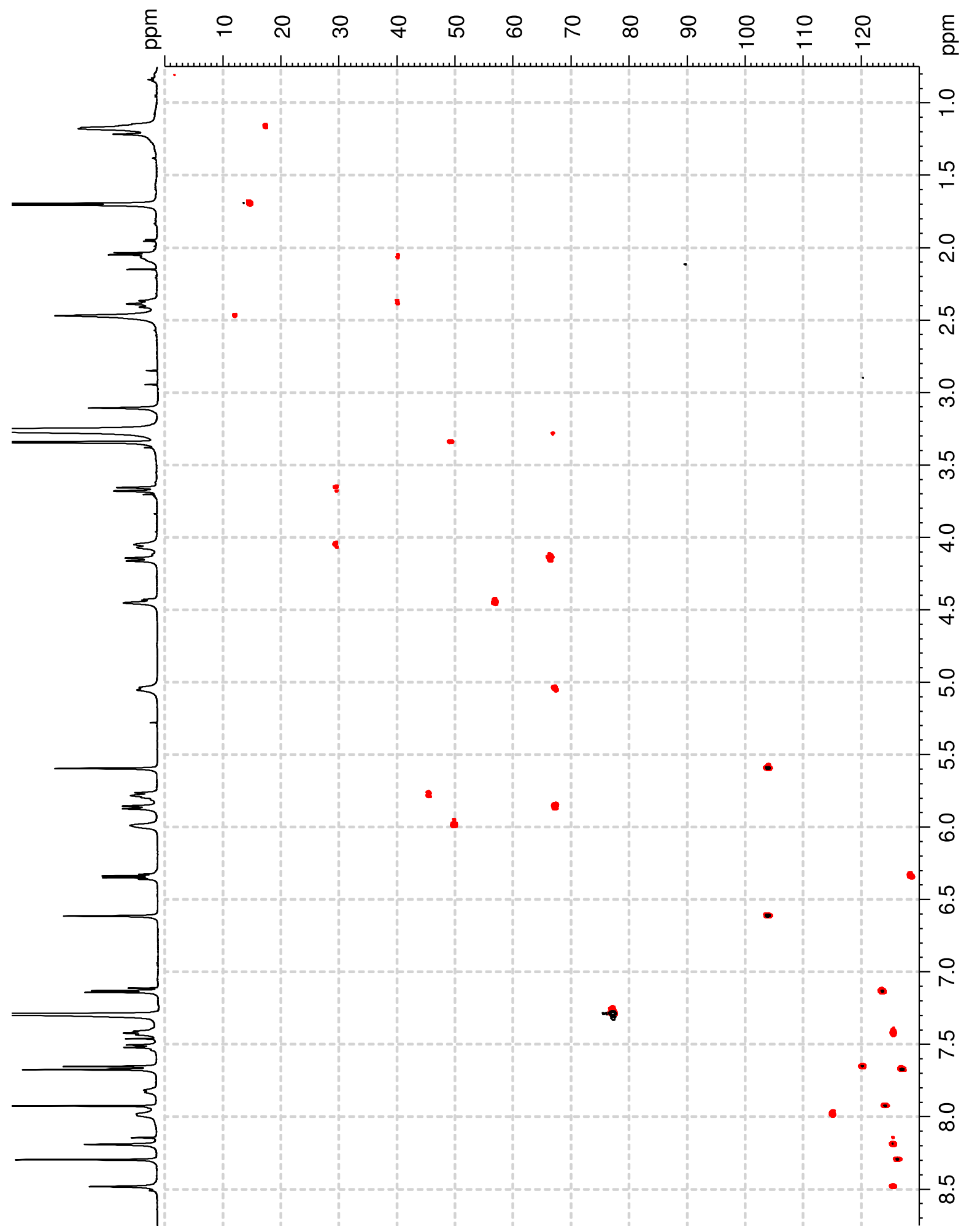

Abbildung 11.81: ${ }^{1} \mathrm{H}-{ }^{13} \mathrm{C}-\mathrm{HSQC}$ des syn. Nosiheptids (6, $\left.600 \mathrm{MHz}, \mathrm{CDCl}_{3} / \mathrm{MeOH}-d_{4}, 9: 1\right)$. 


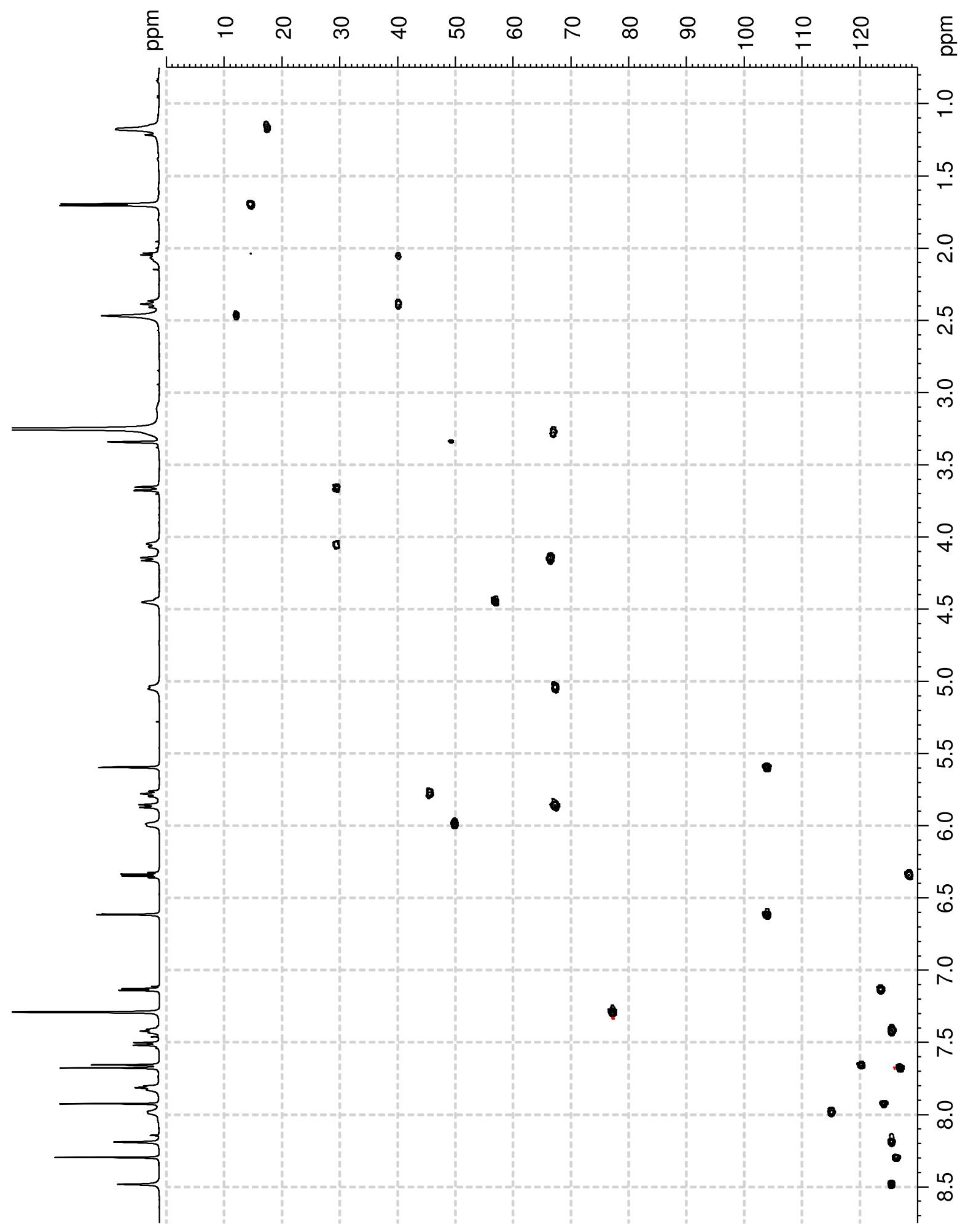

Abbildung 11.82: ${ }^{1} \mathrm{H}_{-}{ }^{13} \mathrm{C}-\mathrm{HSQC}$ des nat. Nosiheptids (6, $\left.600 \mathrm{MHz}, \mathrm{CDCl}_{3} / \mathrm{MeOH}-d_{4}, 9: 1\right)$. 


\subsection{Spektren zu Nachbargruppen-assistierten Umsetzungen}

KPW-48.010.001.1r.esp<smiles>COC(=O)c1cc(OC)c(C(=O)OC)nc1C(=O)OC</smiles>
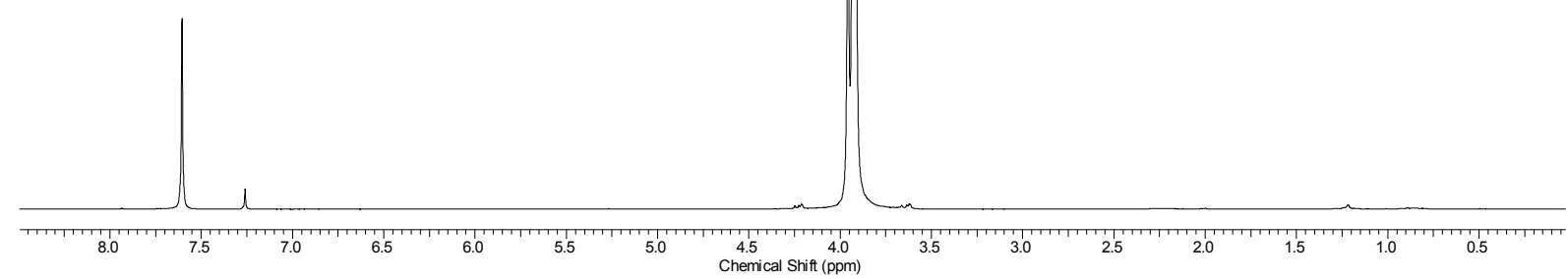

Abbildung 11.83: ${ }^{1} \mathrm{H}-\mathrm{NMR}-$ Spektrum des Methylethers $98\left(250 \mathrm{MHz}, \mathrm{CDCl}_{3}\right)$.

KPW-16_4.010.001.1r.esp<smiles>CCOC(=O)c1nc(C(=O)OC)c(C(=O)OC)cc1O</smiles>

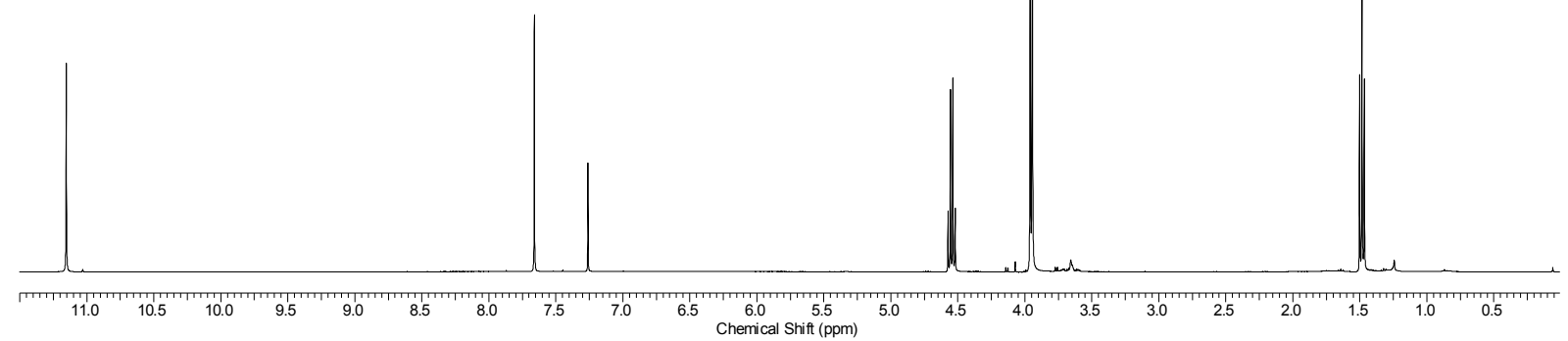

Abbildung 11.84: ${ }^{1} \mathrm{H}-\mathrm{NMR}-$ Spektrum des Ethylesters $196\left(400 \mathrm{MHz}, \mathrm{CDCl}_{3}\right)$. 


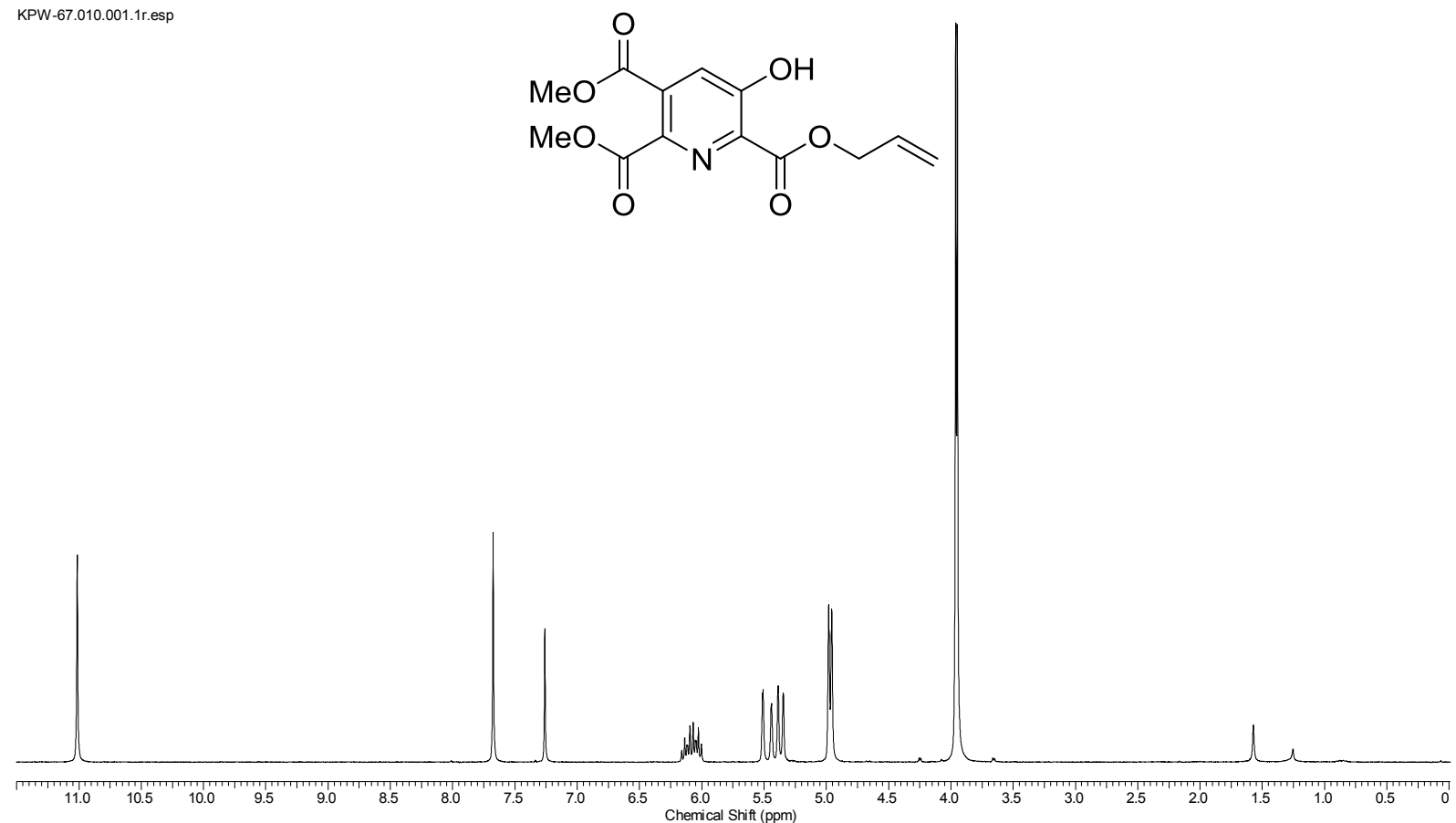

Abbildung 11.85: ${ }^{1} \mathrm{H}-\mathrm{NMR}-$ Spektrum des Allylesters $197\left(250 \mathrm{MHz}, \mathrm{CDCl}_{3}\right)$.

KPW-70.010.001.1r.esp<smiles>COC(=O)c1cc(O)c(C(=O)OC(C)C)nc1C(=O)OC</smiles>

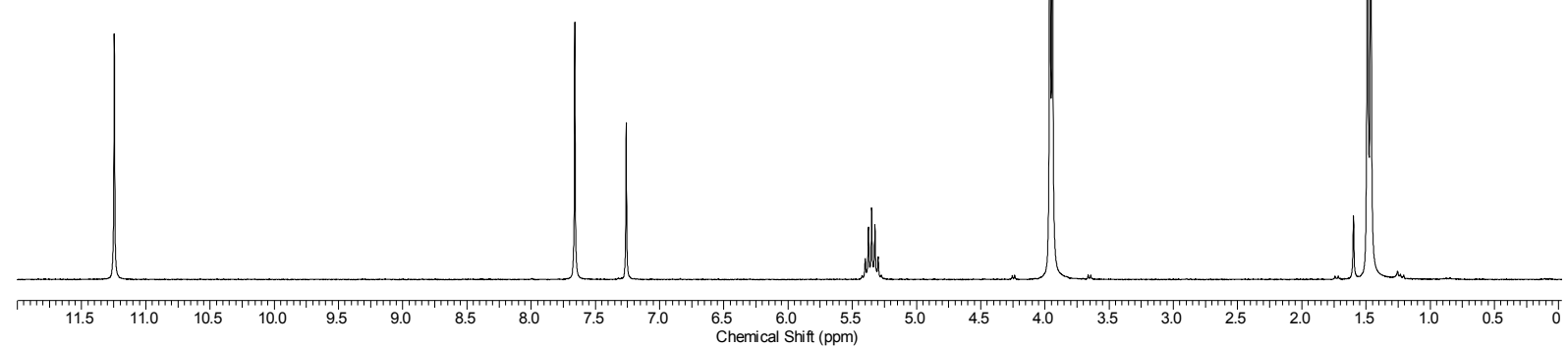

Abbildung 11.86: ${ }^{1} \mathrm{H}-\mathrm{NMR}-$ Spektrum des 2-Propylesters $198\left(250 \mathrm{MHz}, \mathrm{CDCl}_{3}\right)$. 


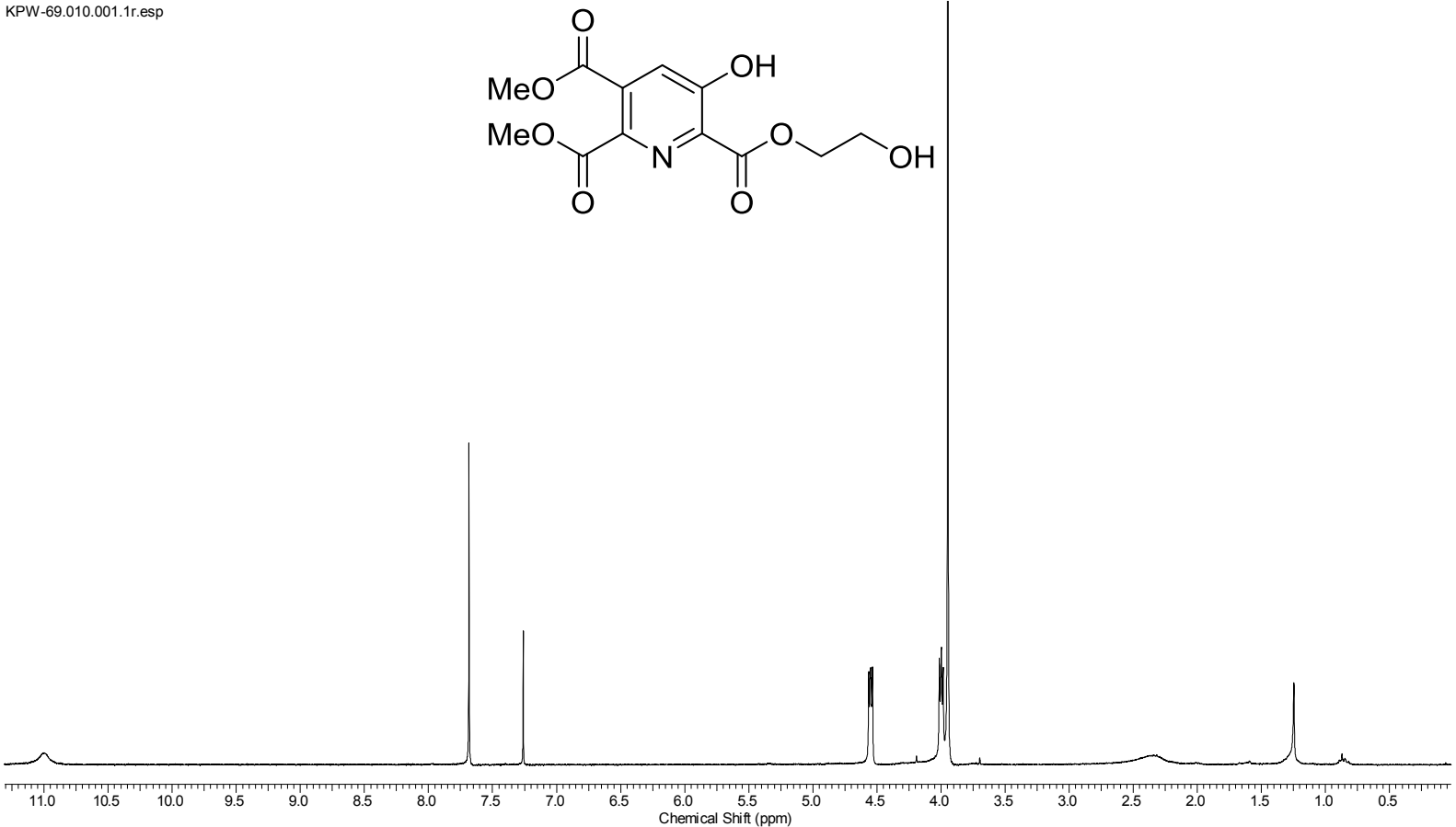

Abbildung 11.87: ${ }^{1} \mathrm{H}-\mathrm{NMR}-$ Spektrum des Glycolesters $199\left(300 \mathrm{MHz}, \mathrm{CDCl}_{3}\right)$.

KPW-66.010.001.1r.esp<smiles>COC(=O)c1cc(O)c(C(=O)OCc2ccccc2)nc1C(=O)OC</smiles>

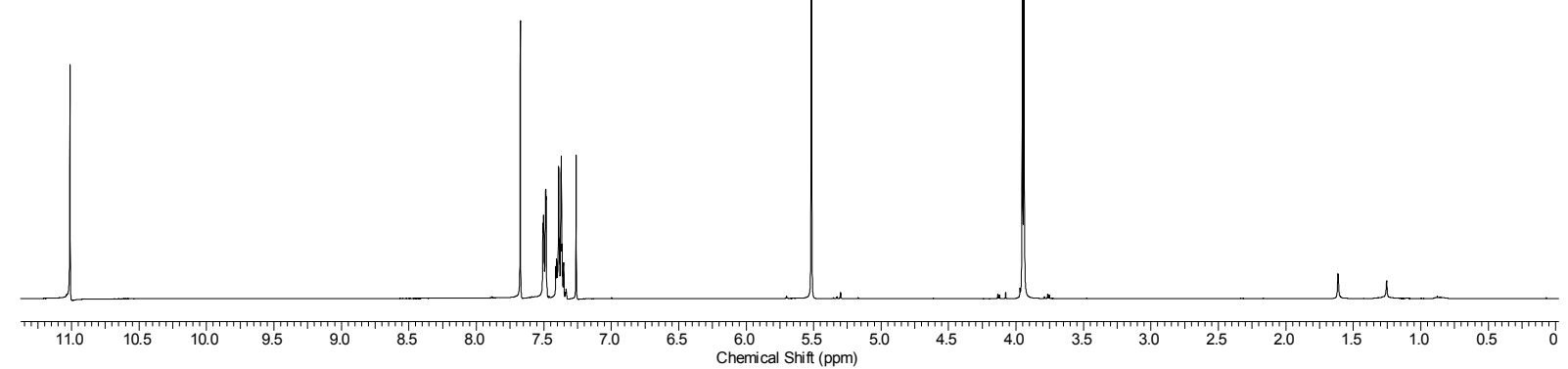

Abbildung 11.88: ${ }^{1} \mathrm{H}-\mathrm{NMR}-S p e k t r u m$ des Benzylesters $200\left(400 \mathrm{MHz}, \mathrm{CDCl}_{3}\right)$. 


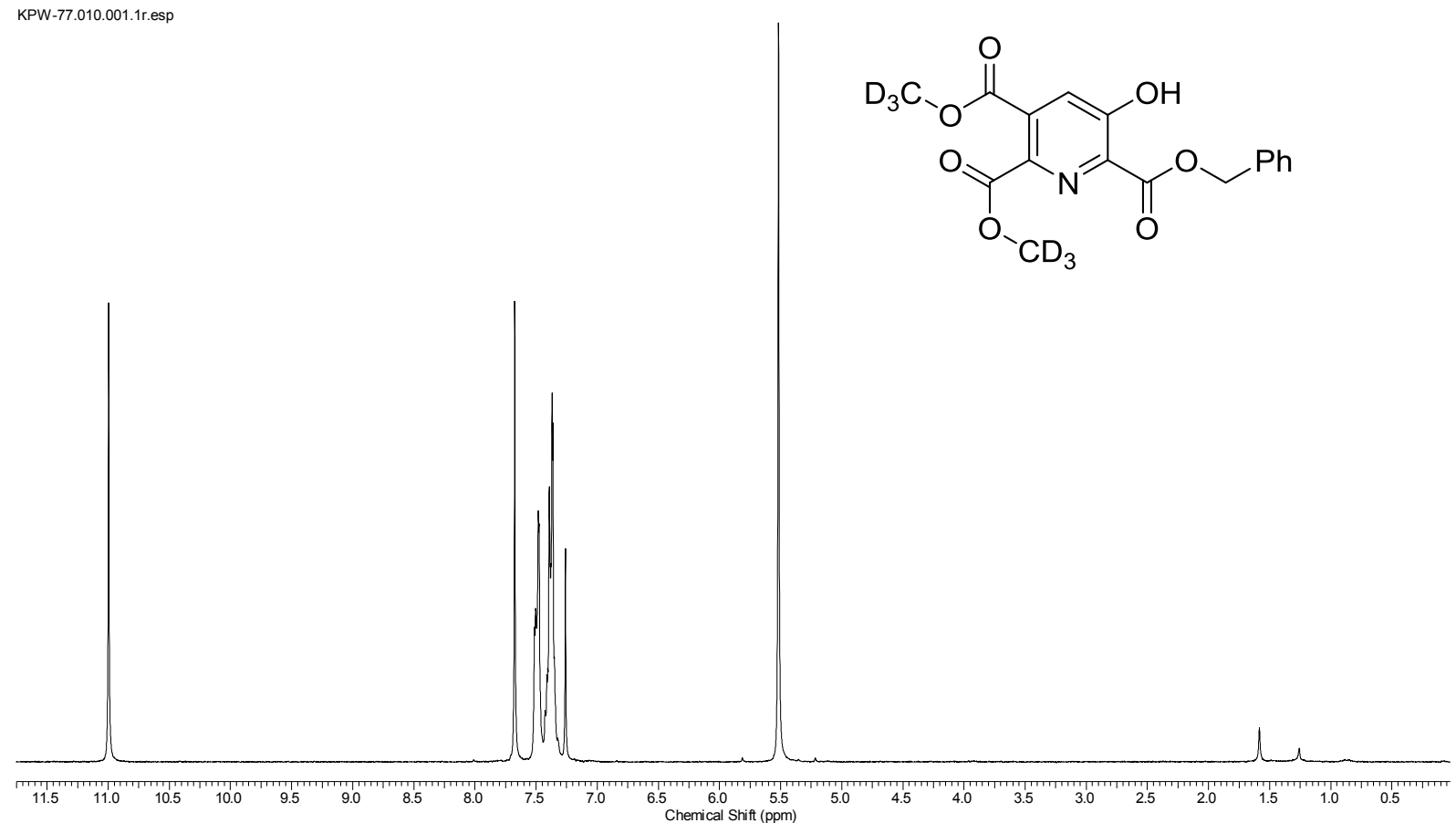

Abbildung 11.89: ${ }^{1} \mathrm{H}-\mathrm{NMR}-$ Spektrum des Benzylesters $201\left(250 \mathrm{MHz}, \mathrm{CDCl}_{3}\right)$.

KPW-50.010.001.1r.esp<smiles>CCOC(=O)c1nc(C(=O)OCC)c(C(=O)OC)cc1OC</smiles>

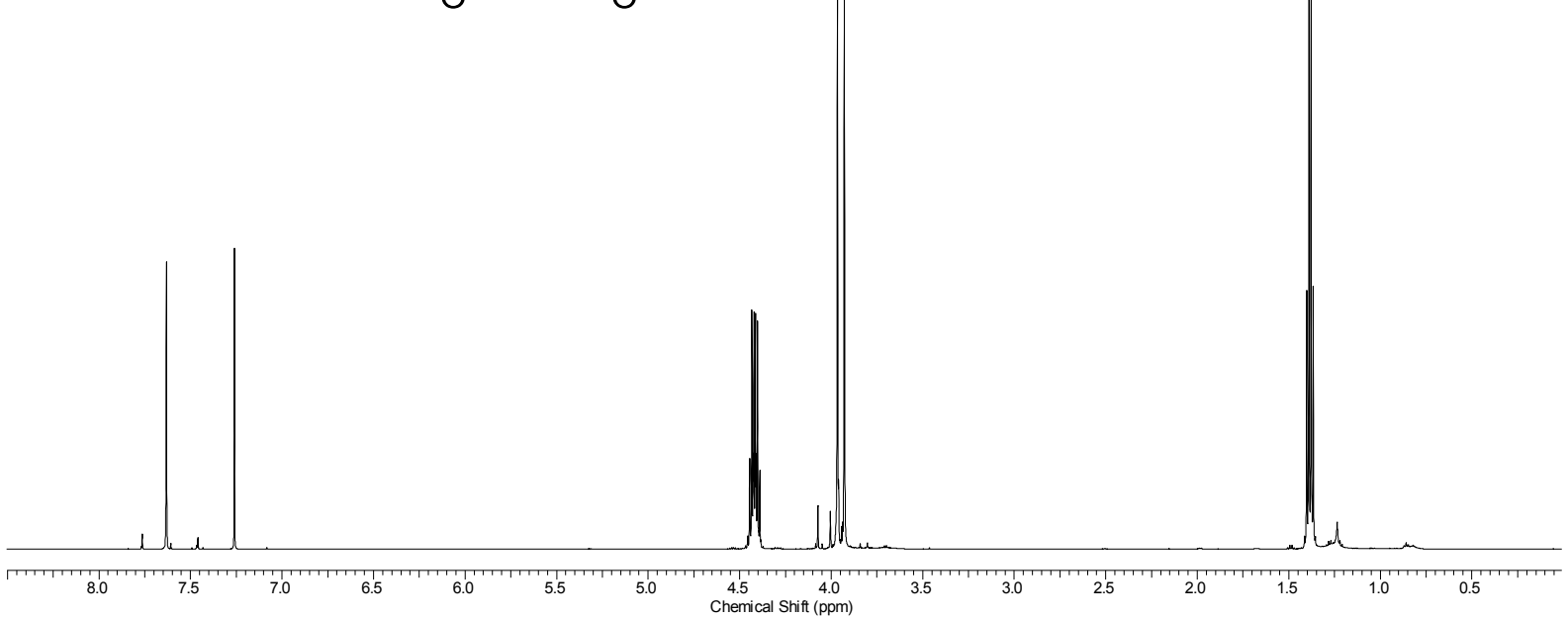

Abbildung 11.90: ${ }^{1} \mathrm{H}-\mathrm{NMR}-$ Spektrum des Diethylesters 203 (600 MHz, $\mathrm{CDCl}_{3}$ ). 
KPW_95.010.001.1r.esp<smiles>CCCNC(=O)c1nc(Br)ccc1O</smiles>

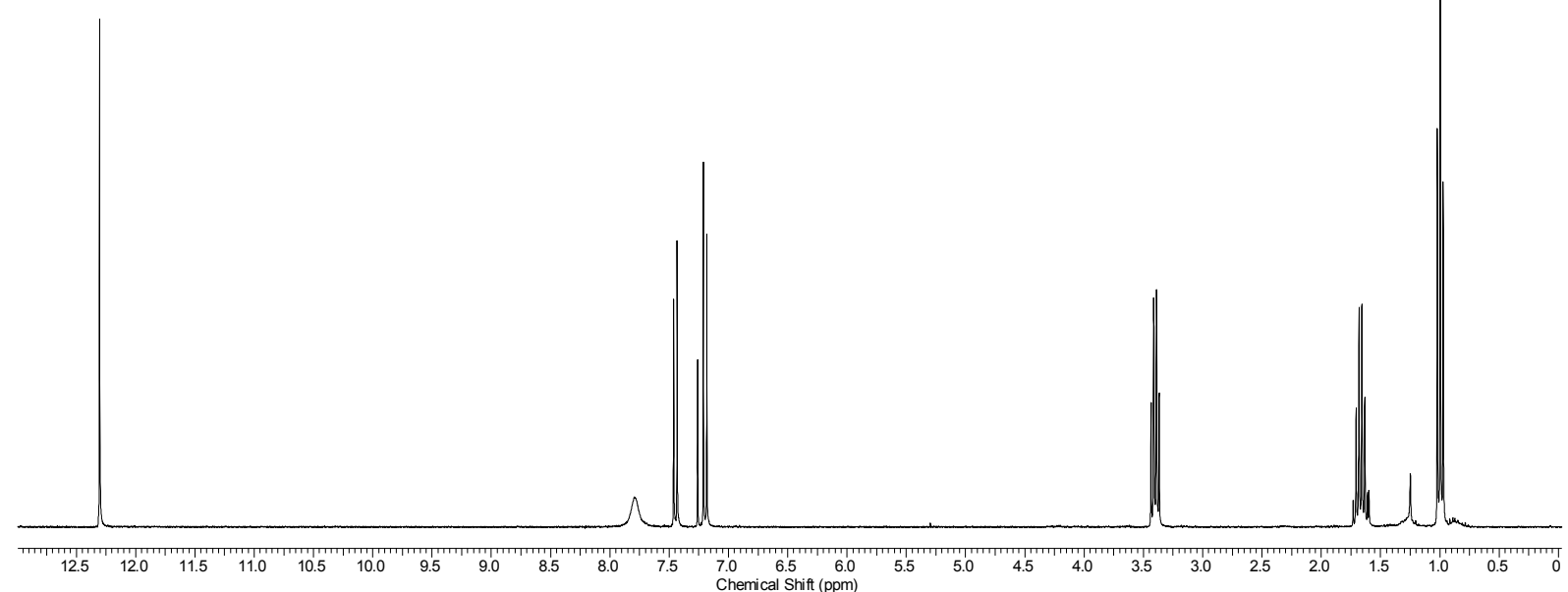

Abbildung 11.91: ${ }^{1} \mathrm{H}-\mathrm{NMR}-$ Spektrum des $n$-Propylamids $205\left(300 \mathrm{MHz}, \mathrm{CDCl}_{3}\right)$.<smiles>Cc1ccc(S(=O)(=O)n2cnc(C=O)c2)cc1</smiles>
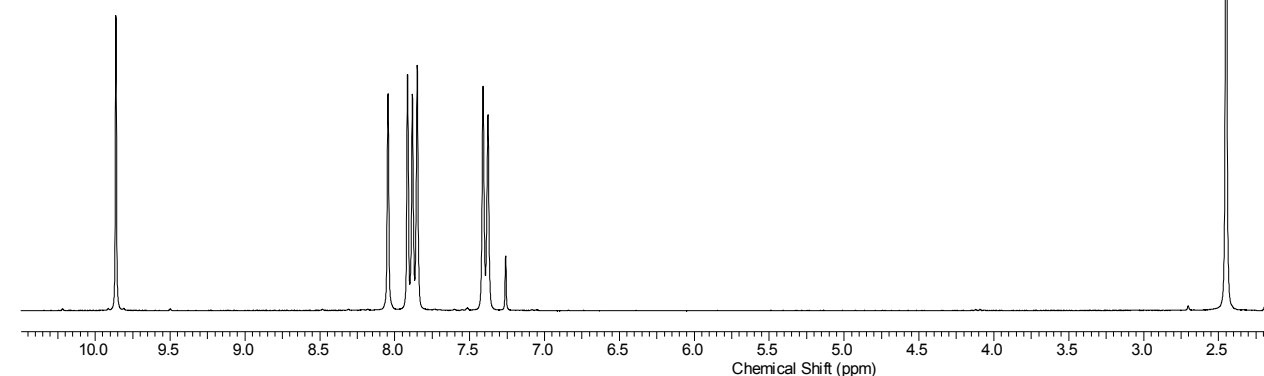

Abbildung 11.92: ${ }^{1} \mathrm{H}-\mathrm{NMR}-\mathrm{Spektrum}$ des Aldehyds $207\left(250 \mathrm{MHz}, \mathrm{CDCl}_{3}\right)$. 


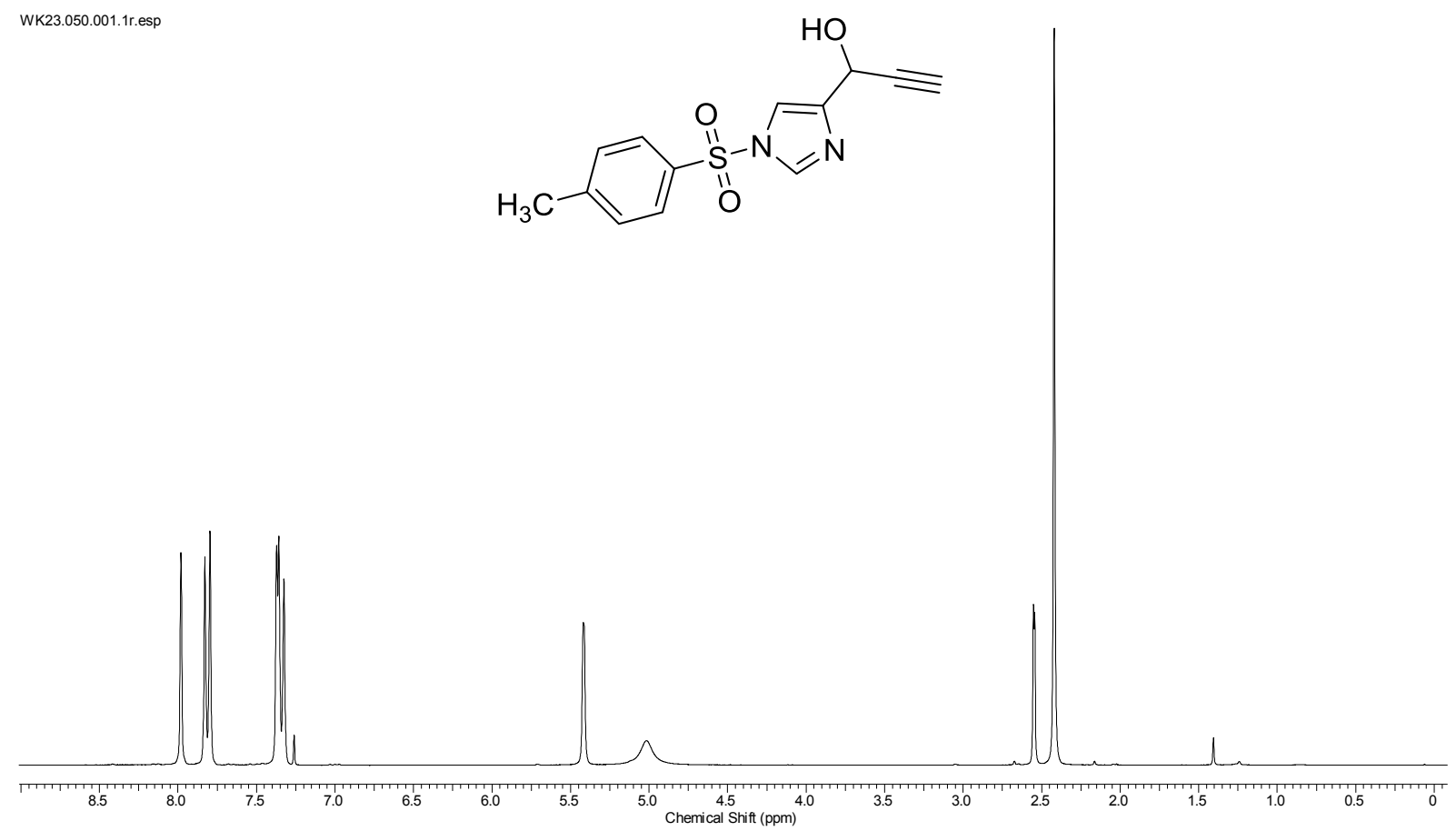

Abbildung 11.93: ${ }^{1} \mathrm{H}-\mathrm{NMR}-$ Spektrum des Propargylalkohols $208\left(250 \mathrm{MHz}, \mathrm{CDCl}_{3}\right)$.

WK25.030.001.1r.esp<smiles>C#CC(=O)c1cn(S(=O)(=O)c2ccc(C)cc2)cn1</smiles>

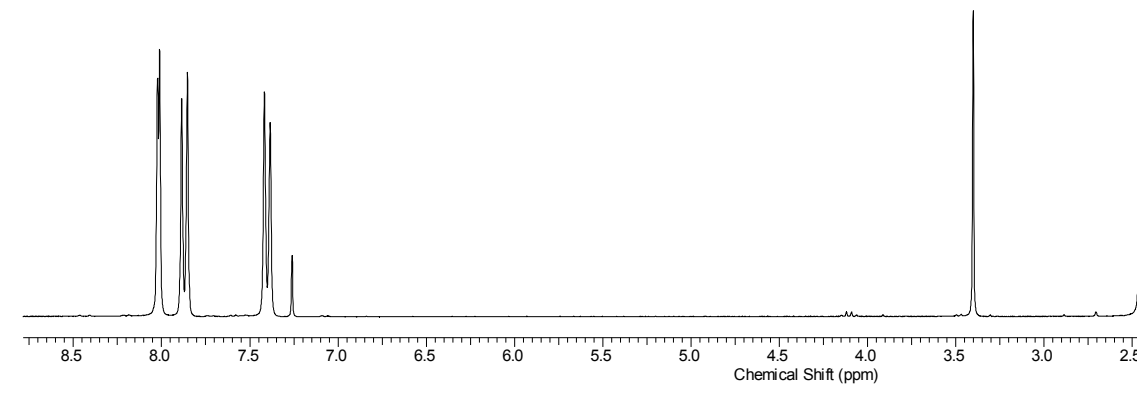

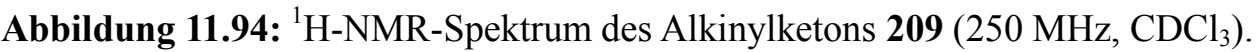




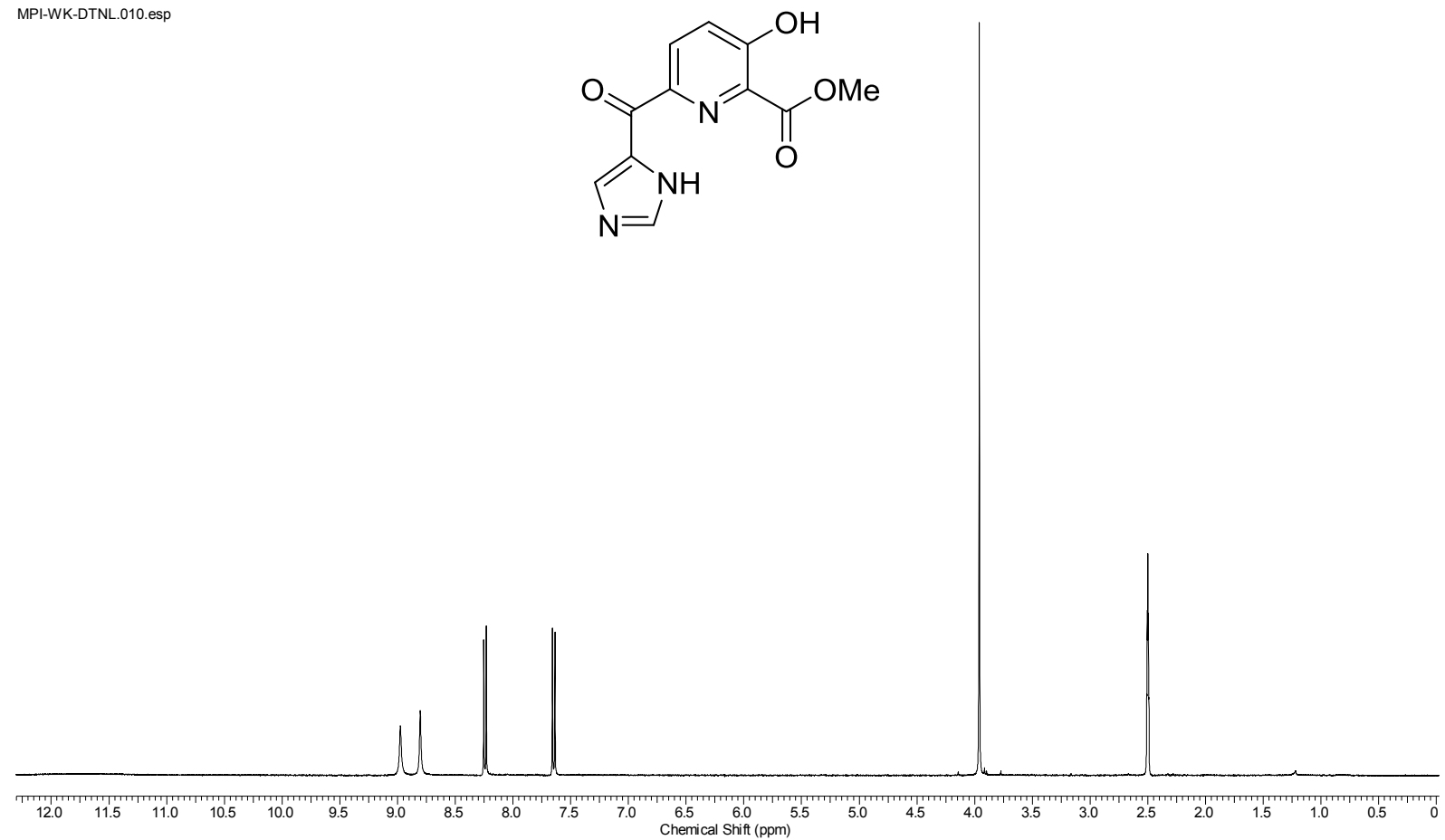

Abbildung 11.95: ${ }^{1} \mathrm{H}-\mathrm{NMR}-S p e k t r u m$ des 3-Hydroxypyridins $210\left(400 \mathrm{MHz}\right.$, DMSO- $\left.d_{6}\right)$.

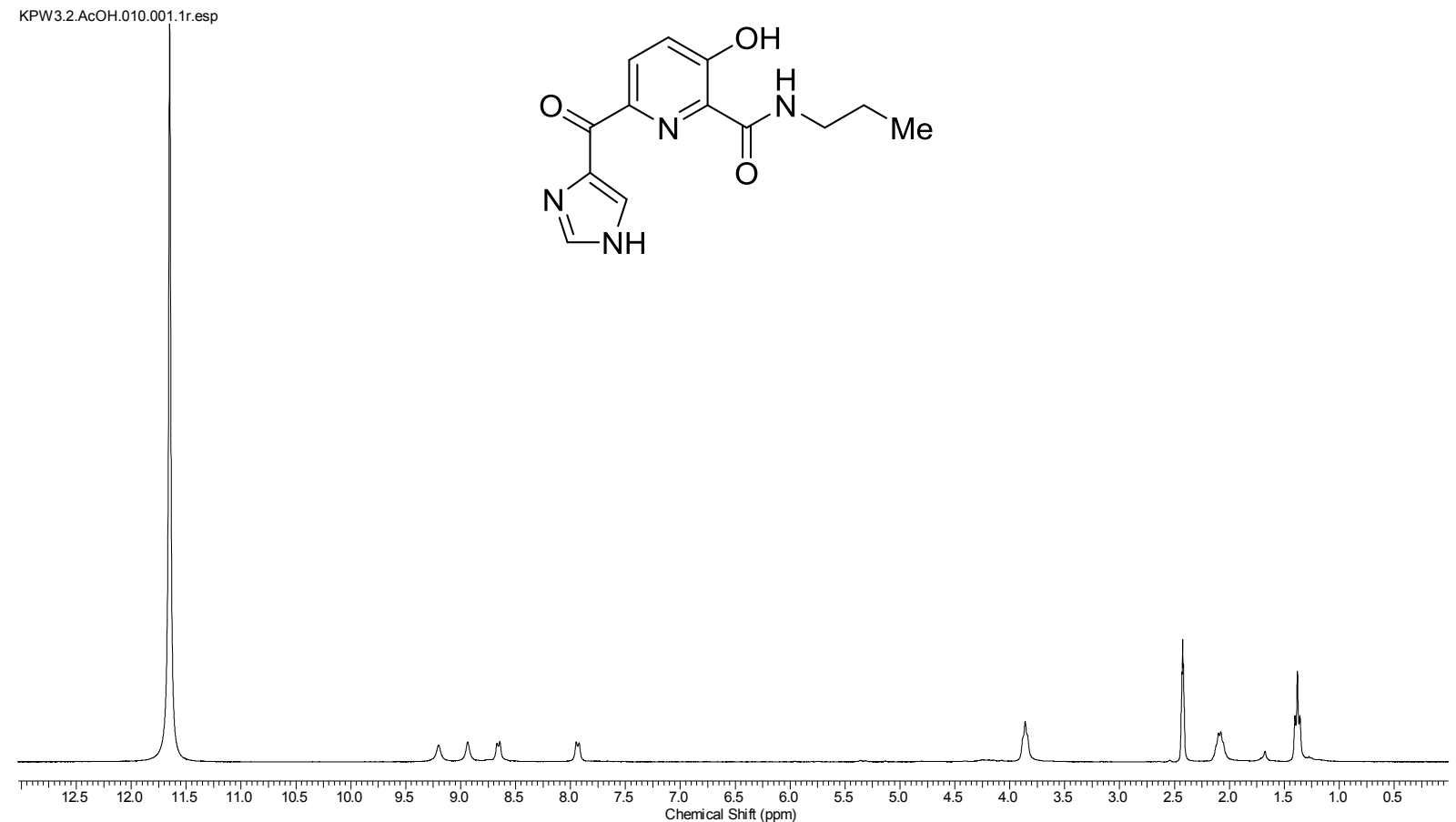

Abbildung 11.96: ${ }^{1} \mathrm{H}-\mathrm{NMR}-$ Spektrum des $n$-Propylamids $211\left(300 \mathrm{MHz}, \mathrm{AcOH}-d_{4}\right)$. 


\subsection{Spektren zur Synthese substituierter 3-Hydroxypicolinate}

YC3.2.010.001.1r.esp

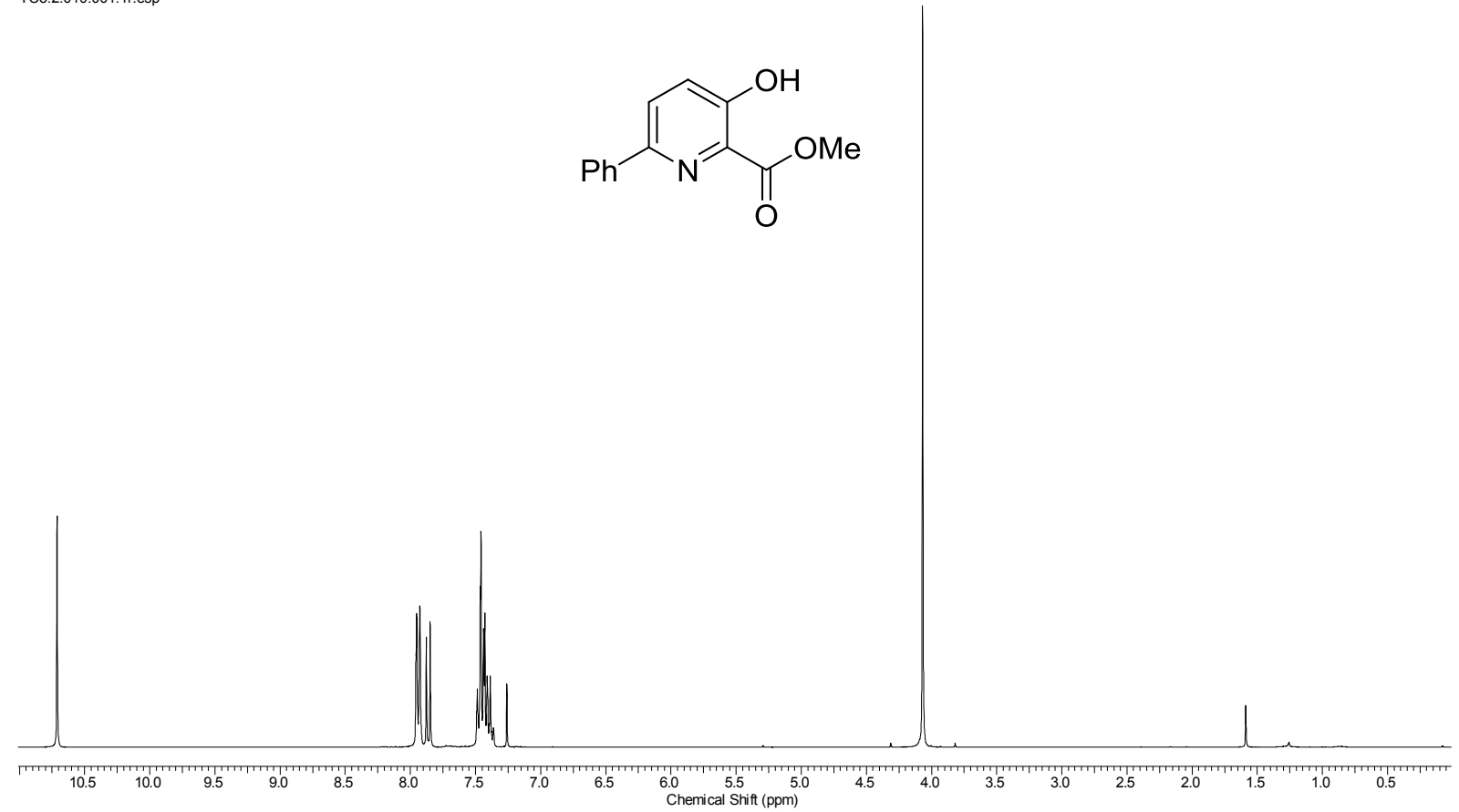

Abbildung 11.98: ${ }^{1} \mathrm{H}-\mathrm{NMR}-S p e k t r u m$ des 3-Hydroxypyridins $216 a\left(300 \mathrm{MHz}, \mathrm{CDCl}_{3}\right)$.

YC10.010.001.1r.esp

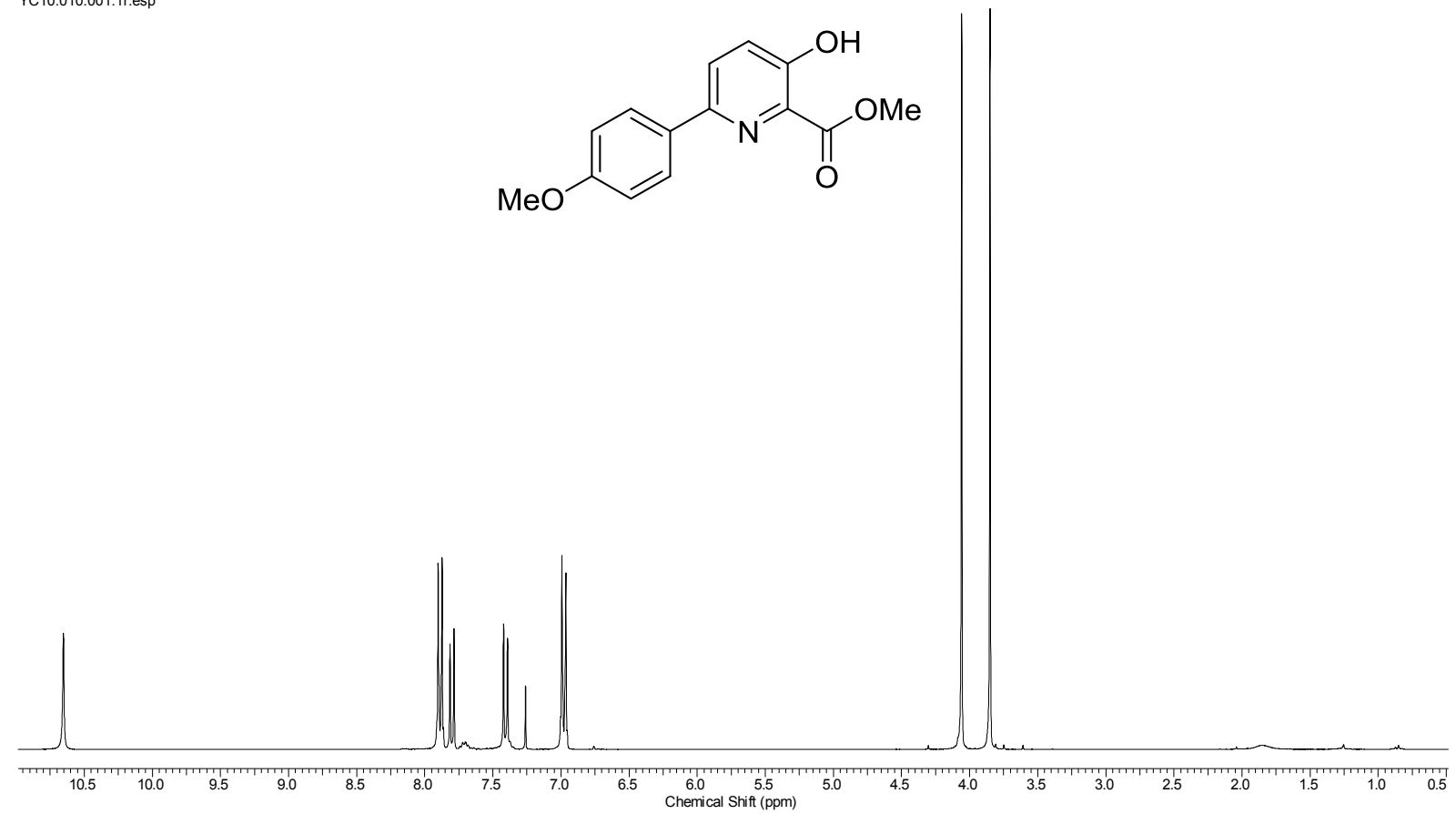

Abbildung 11.99: ${ }^{1} \mathrm{H}-\mathrm{NMR}-$ Spektrum des 3-Hydroxypyridins $216 \mathbf{b}\left(300 \mathrm{MHz}, \mathrm{CDCl}_{3}\right)$. 


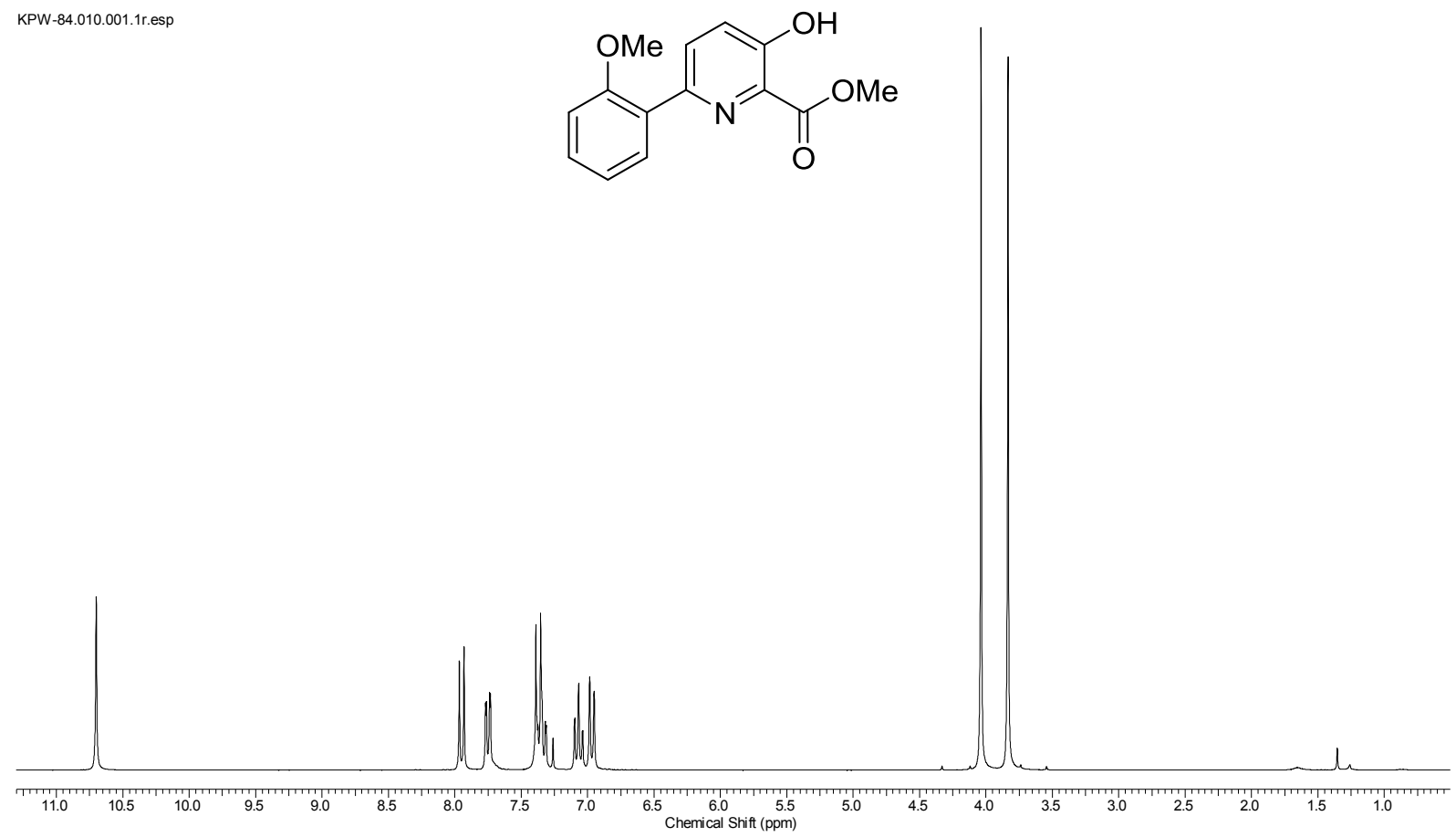

Abbildung 11.100: ${ }^{1} \mathrm{H}-\mathrm{NMR}-$ Spektrum des 3-Hydroxypyridins 216c (400 MHz, $\left.\mathrm{CDCl}_{3}\right)$.

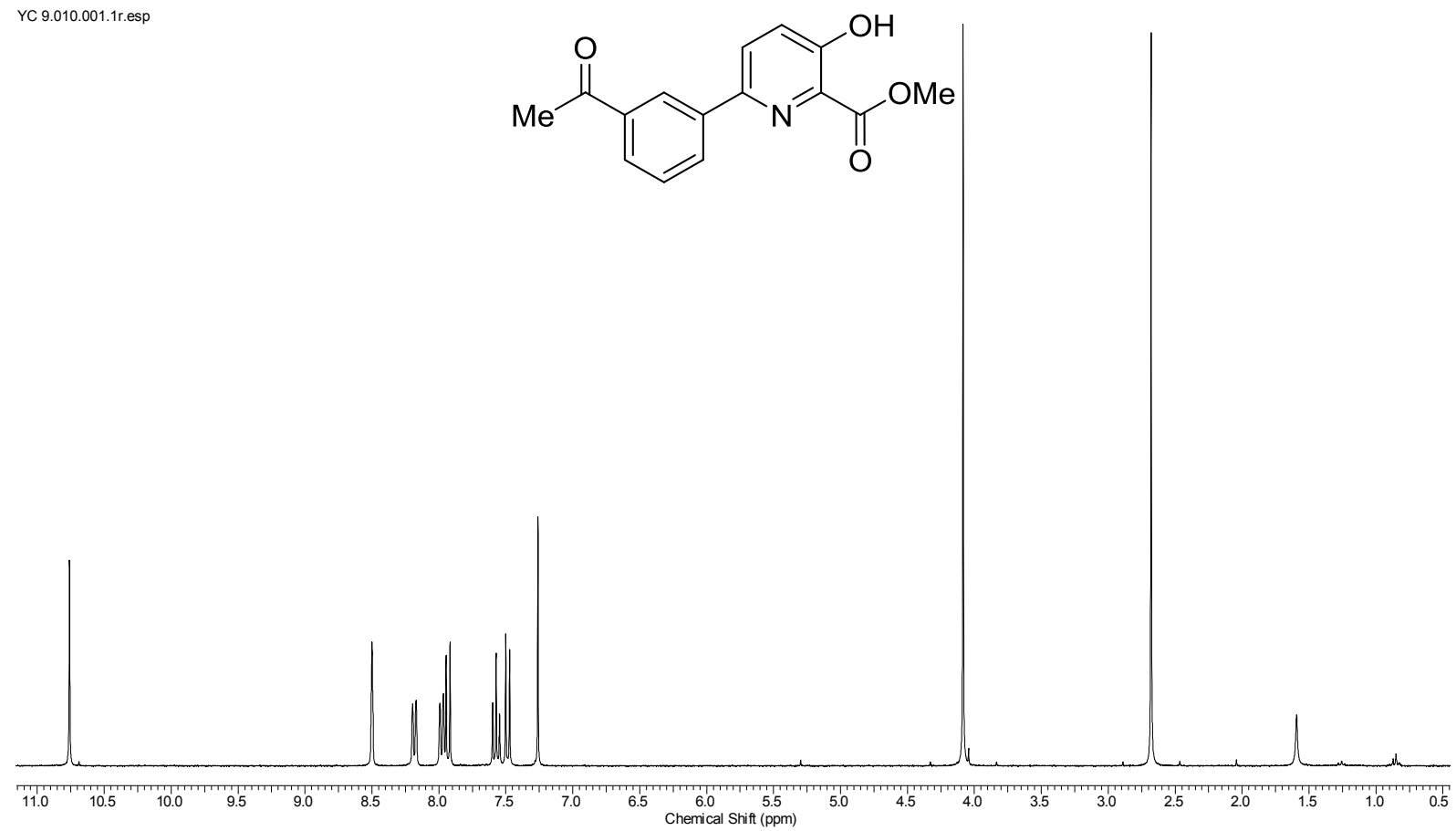

Abbildung 11.101: ${ }^{1} \mathrm{H}-\mathrm{NMR}-$ Spektrum des 3-Hydroxypyridins 216d (300 MHz, $\left.\mathrm{CDCl}_{3}\right)$. 


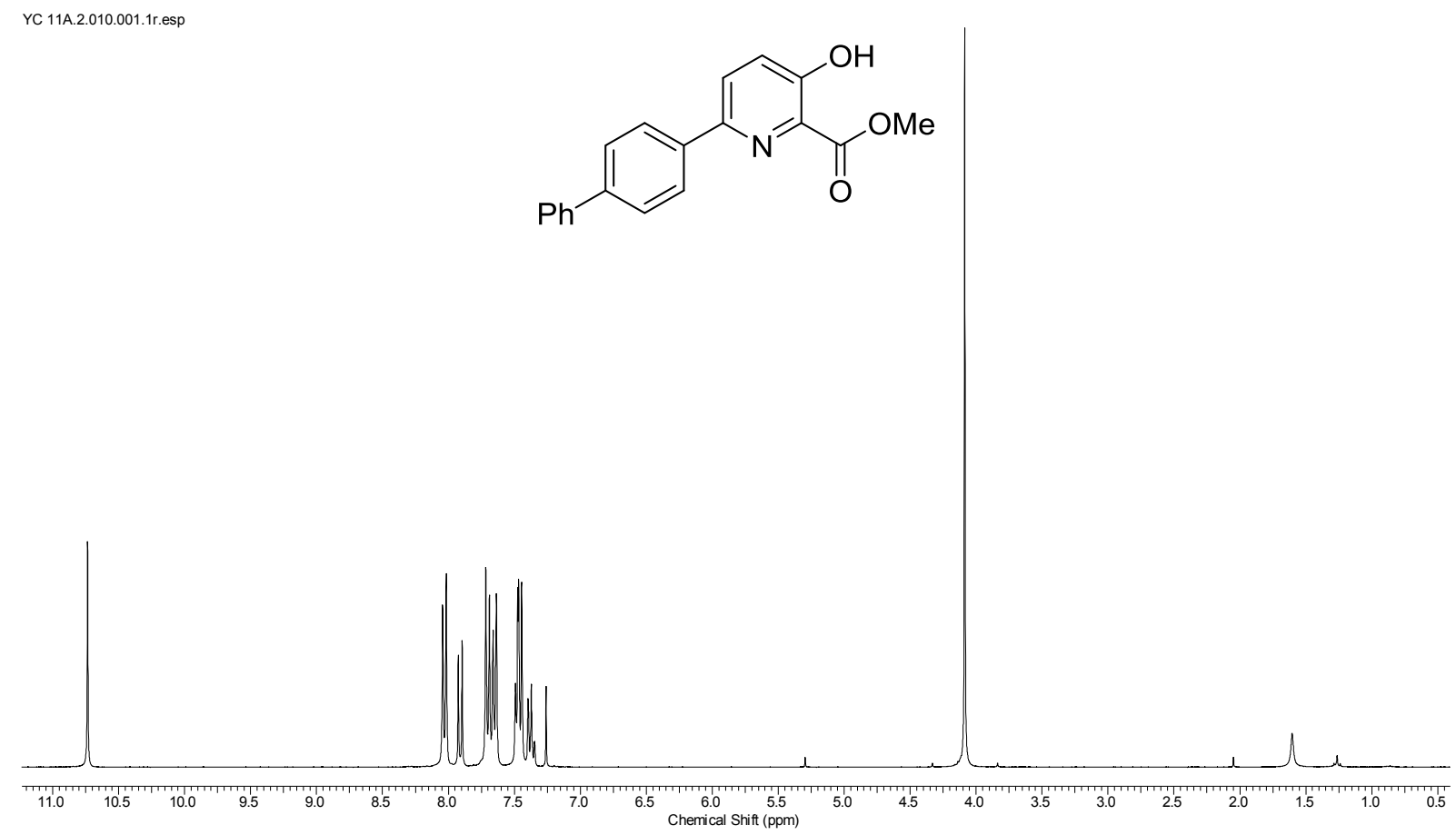

Abbildung 11.102: ${ }^{1} \mathrm{H}-\mathrm{NMR}-$ Spektrum des 3-Hydroxypyridins 216e (300 MHz, $\left.\mathrm{CDCl}_{3}\right)$.

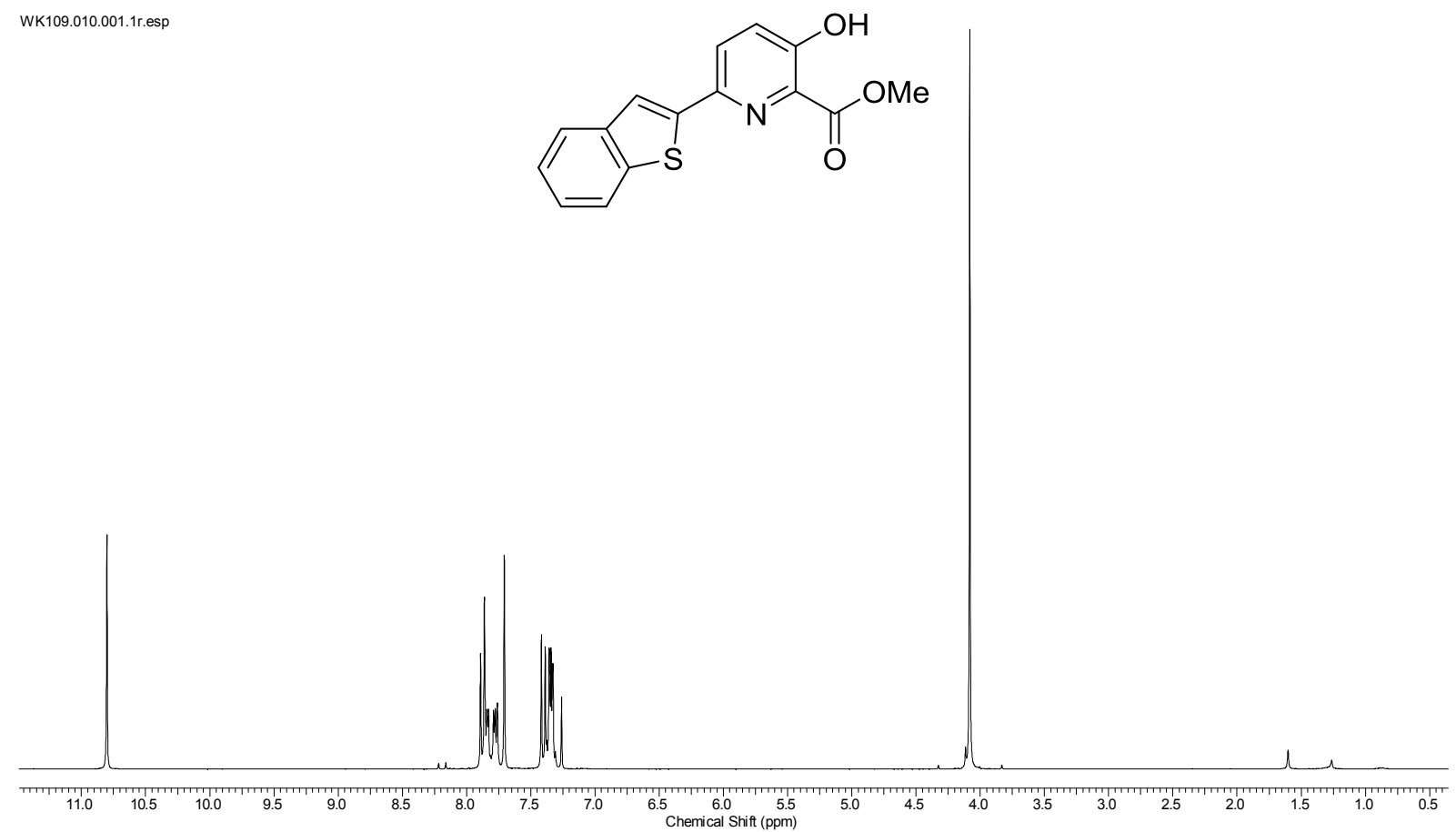

Abbildung 11.103: ${ }^{1} \mathrm{H}-\mathrm{NMR}-$ Spektrum des 3-Hydroxypyridins $216 \mathbf{f}\left(300 \mathrm{MHz}, \mathrm{CDCl}_{3}\right)$. 


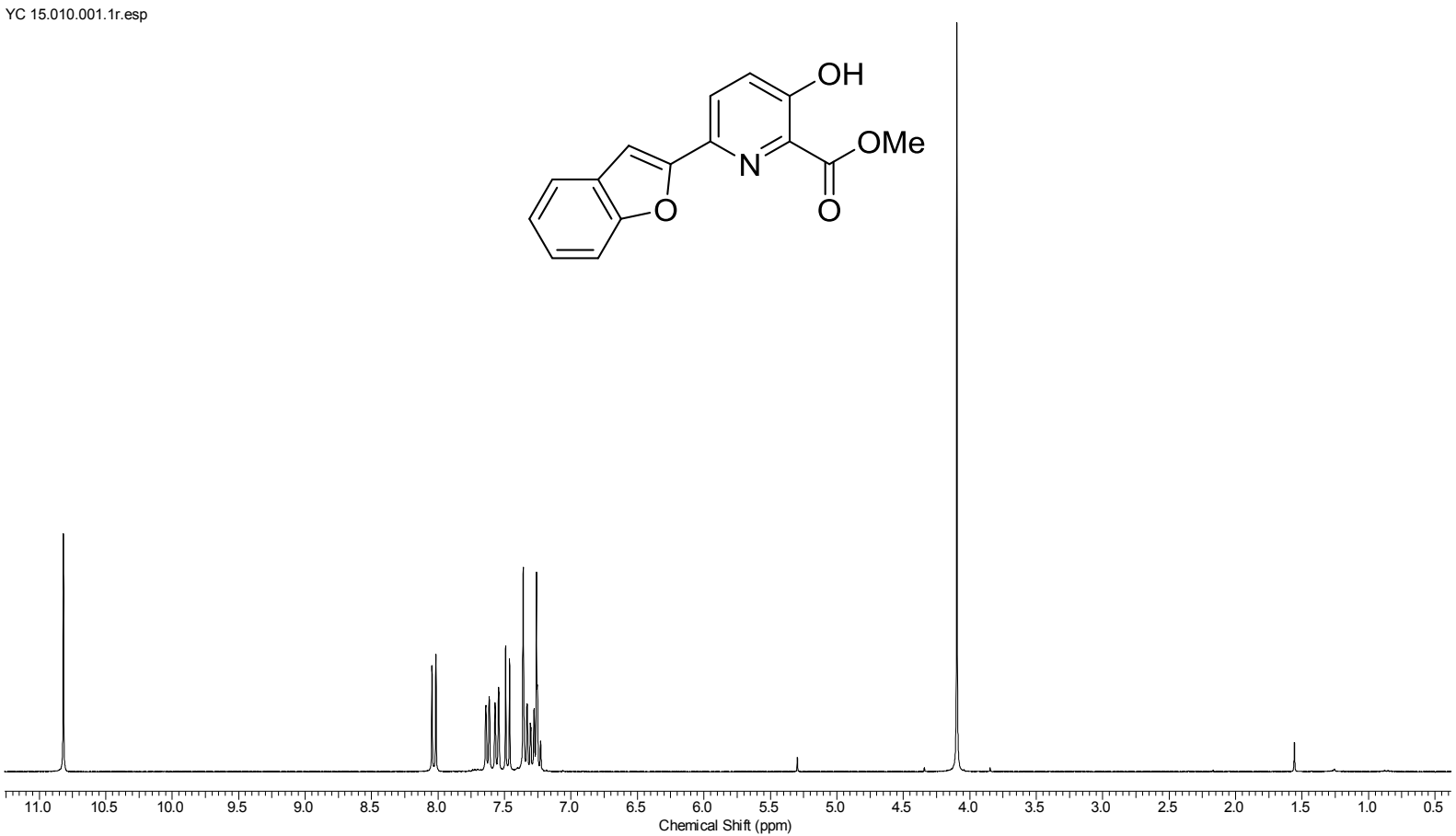

Abbildung 11.104: ${ }^{1} \mathrm{H}-\mathrm{NMR}-$ Spektrum des 3-Hydroxypyridins $216 \mathrm{~g}\left(300 \mathrm{MHz}, \mathrm{CDCl}_{3}\right.$ ).

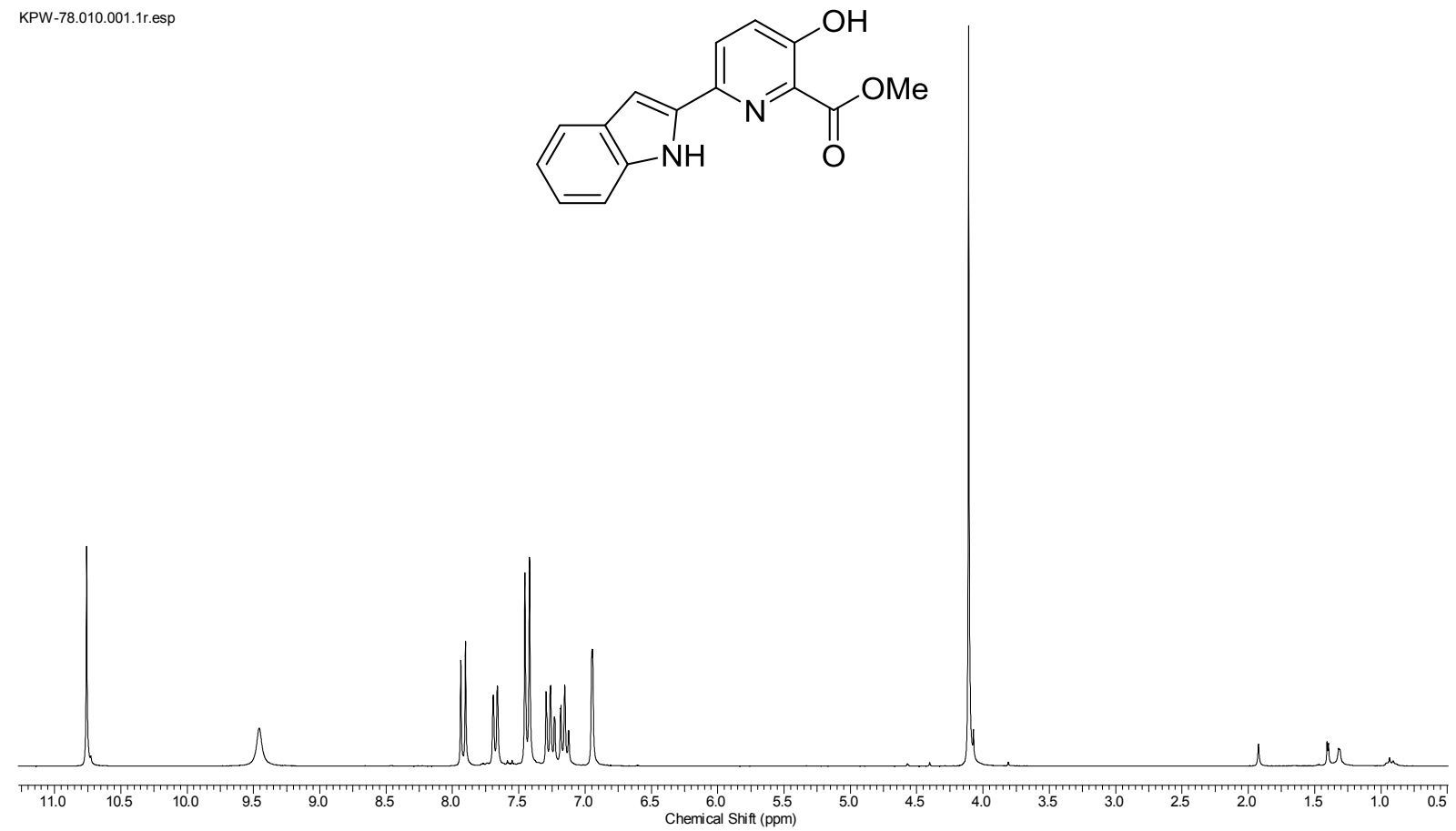

Abbildung 11.105: ${ }^{1} \mathrm{H}-\mathrm{NMR}-$ Spektrum des 3-Hydroxypyridins $216 \mathbf{h}\left(250 \mathrm{MHz}, \mathrm{CDCl}_{3}\right)$. 


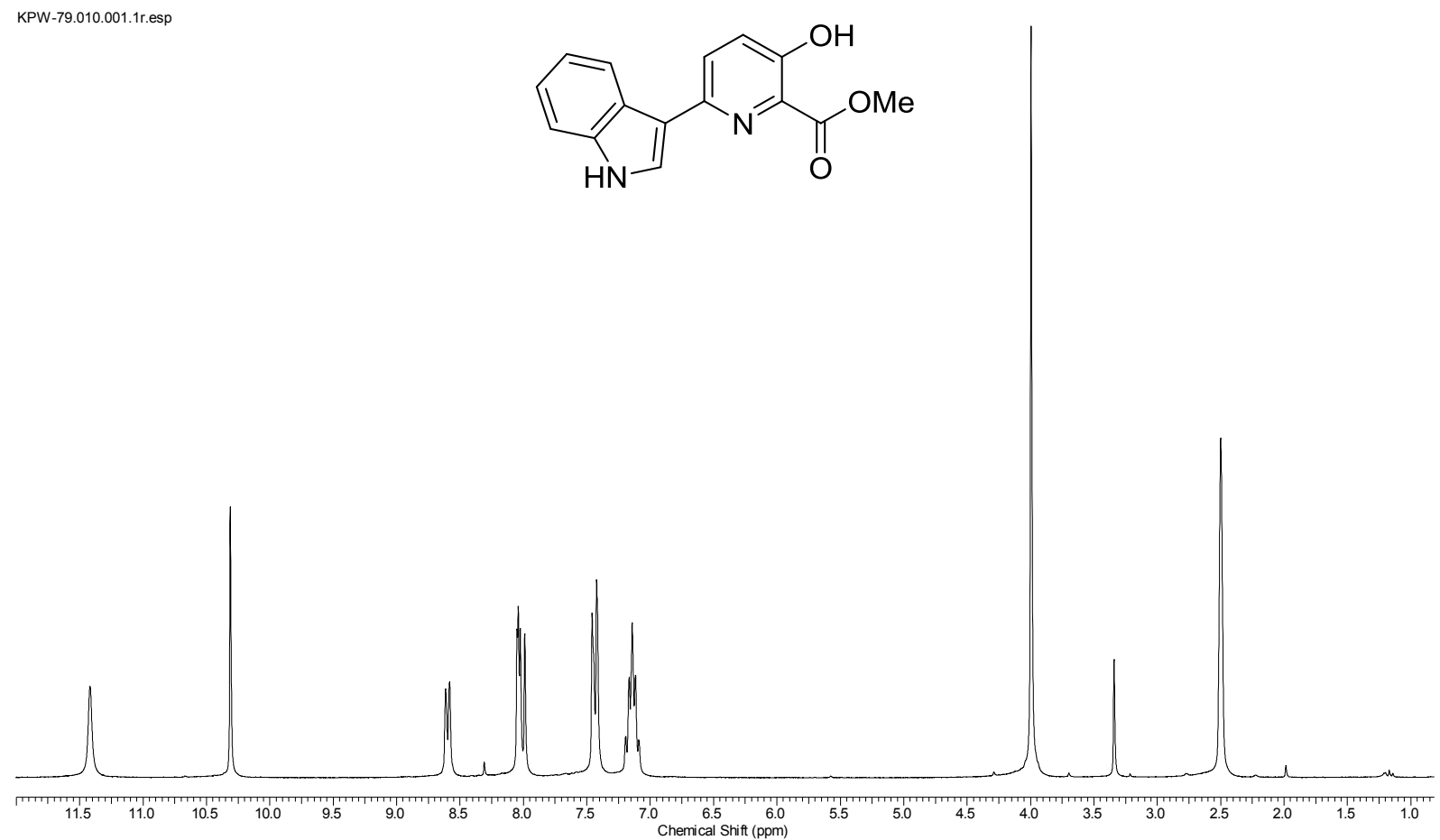

Abbildung 11.106: ${ }^{1} \mathrm{H}-\mathrm{NMR}-$ Spektrum des 3-Hydroxypyridins $216 \mathbf{i}\left(250 \mathrm{MHz}\right.$, DMSO- $\left.d_{6}\right)$.

YC1.010.001.1r.esp<smiles>COC(=O)c1nc(-c2cccnc2)ccc1O</smiles>

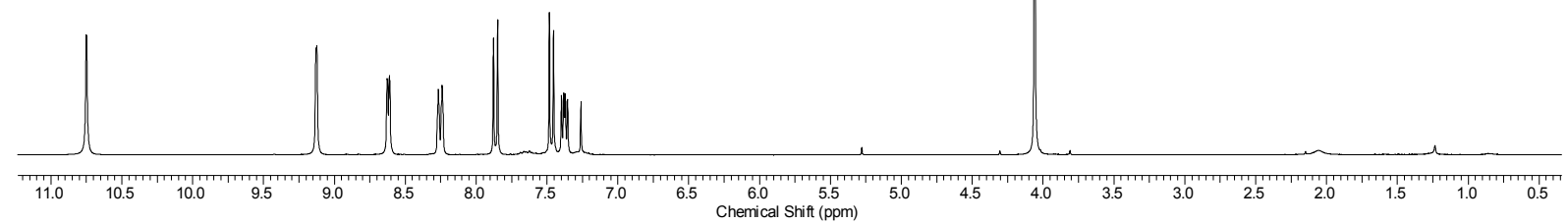

Abbildung 11.107: ${ }^{1} \mathrm{H}-\mathrm{NMR}-$ Spektrum des 3-Hydroxypyridins 216j (300 MHz, $\mathrm{CDCl}_{3}$ ). 


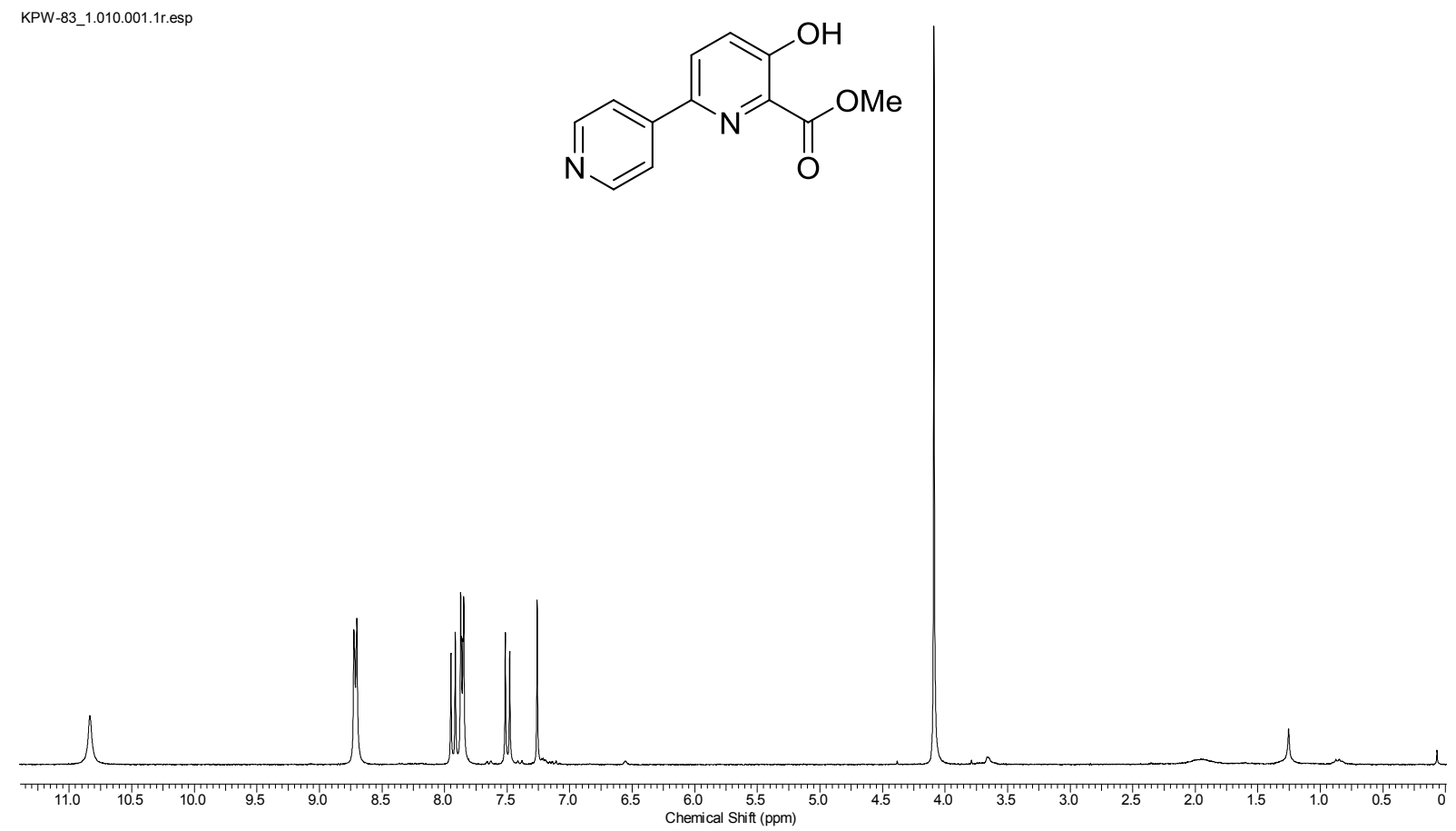

Abbildung 11.108: ${ }^{1} \mathrm{H}-\mathrm{NMR}-$ Spektrum des 3-Hydroxypyridins 216k (300 MHz, $\left.\mathrm{CDCl}_{3}\right)$.

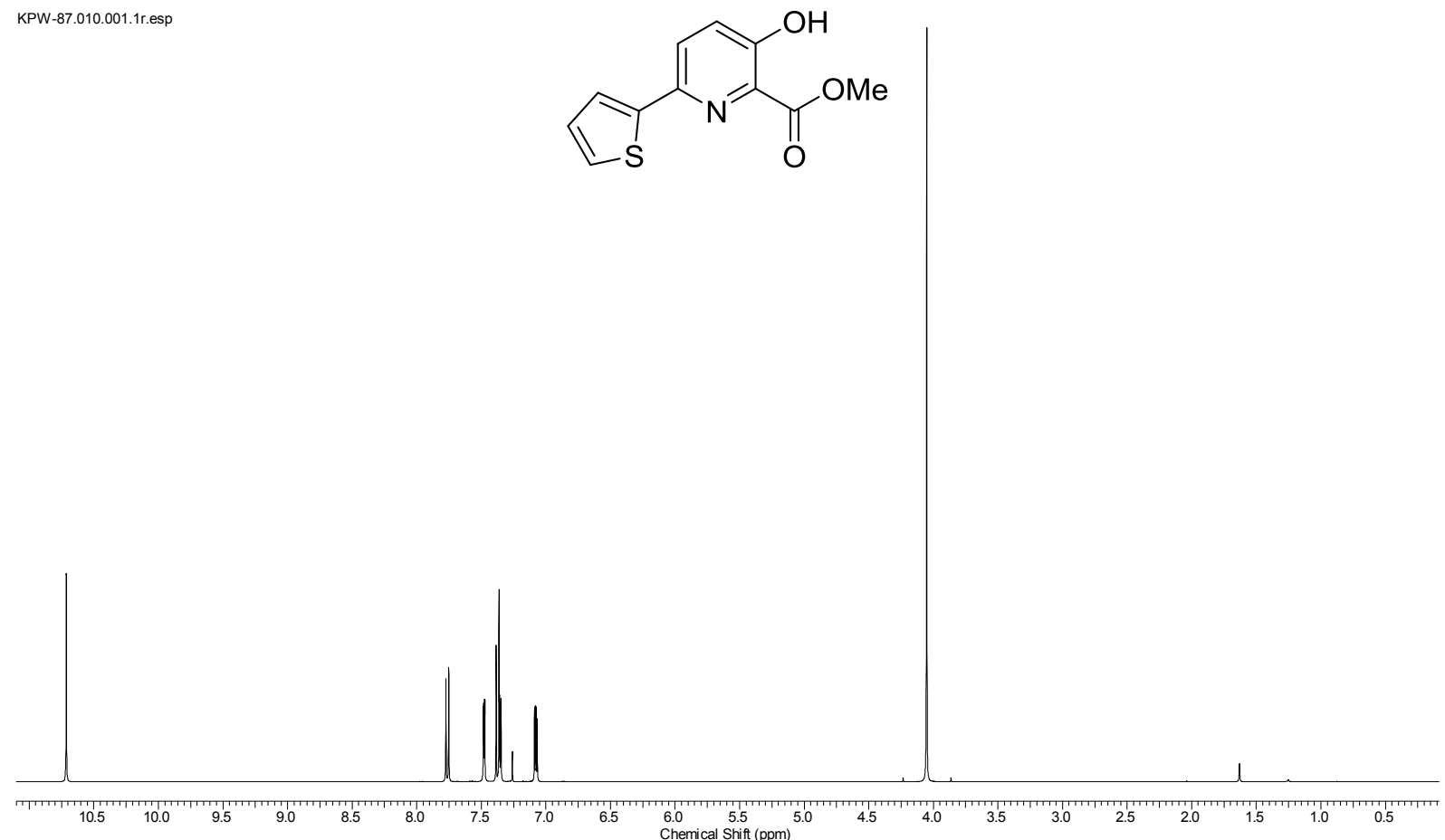

Abbildung 11.109: ${ }^{1} \mathrm{H}-\mathrm{NMR}-$ Spektrum des 3-Hydroxypyridins 2161 (400 MHz, $\mathrm{CDCl}_{3}$ ). 


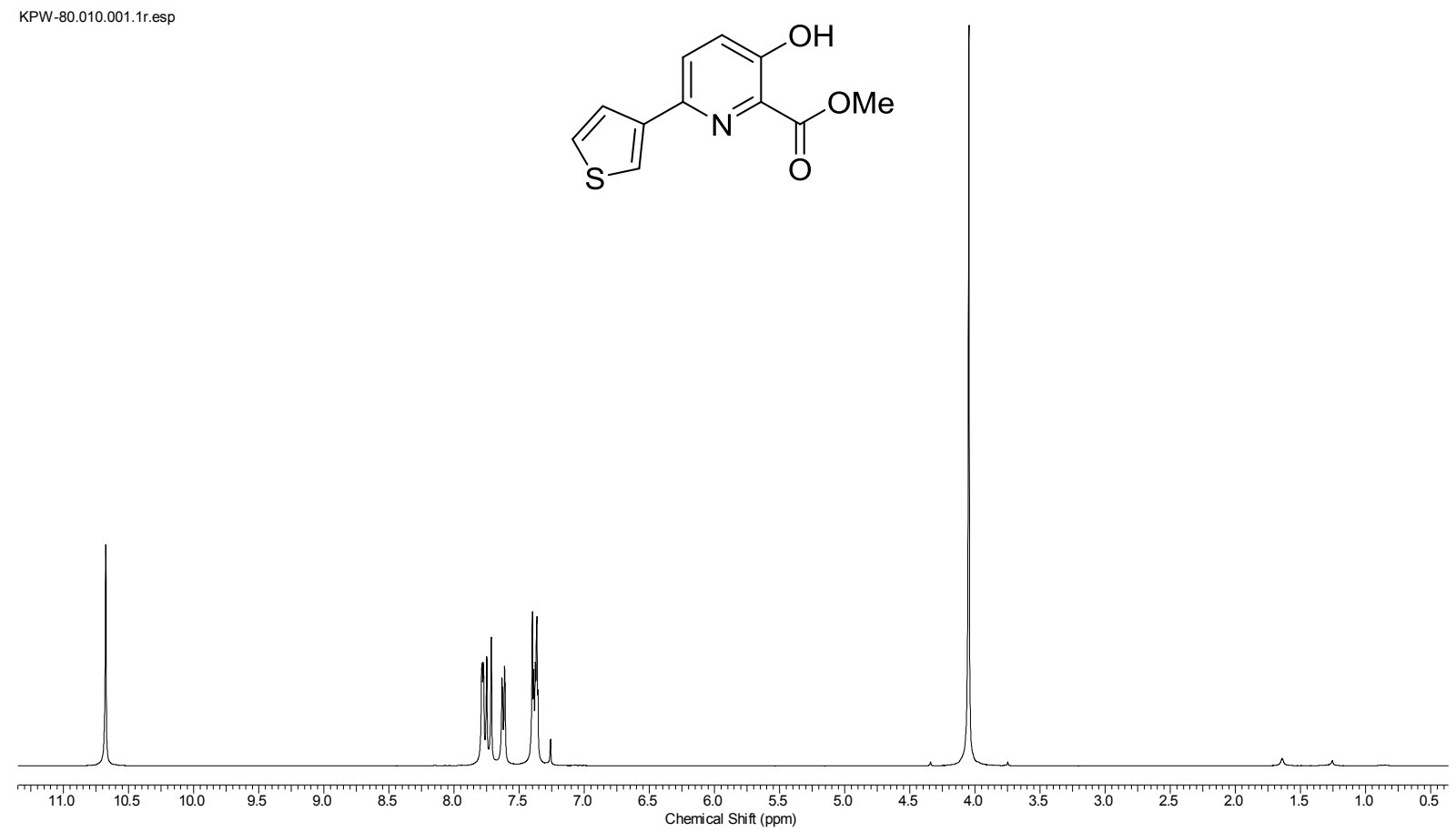

Abbildung 11.110: ${ }^{1} \mathrm{H}-\mathrm{NMR}-$ Spektrum des 3-Hydroxypyridins $216 \mathbf{m}\left(250 \mathrm{MHz}, \mathrm{CDCl}_{3}\right)$.

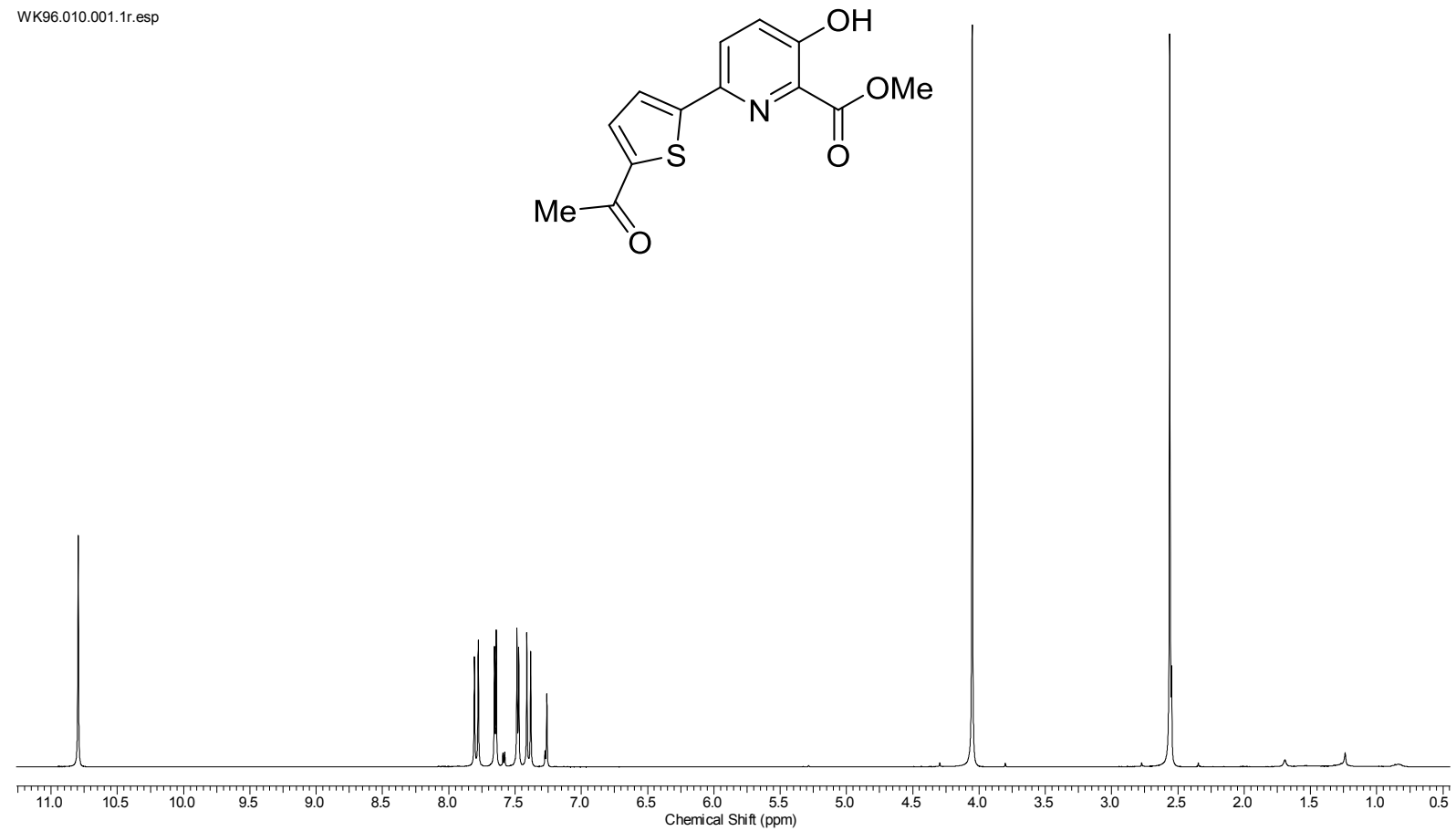

Abbildung 11.111: ${ }^{1} \mathrm{H}-\mathrm{NMR}-$ Spektrum des 3-Hydroxypyridins 216n (300 MHz, $\left.\mathrm{CDCl}_{3}\right)$. 


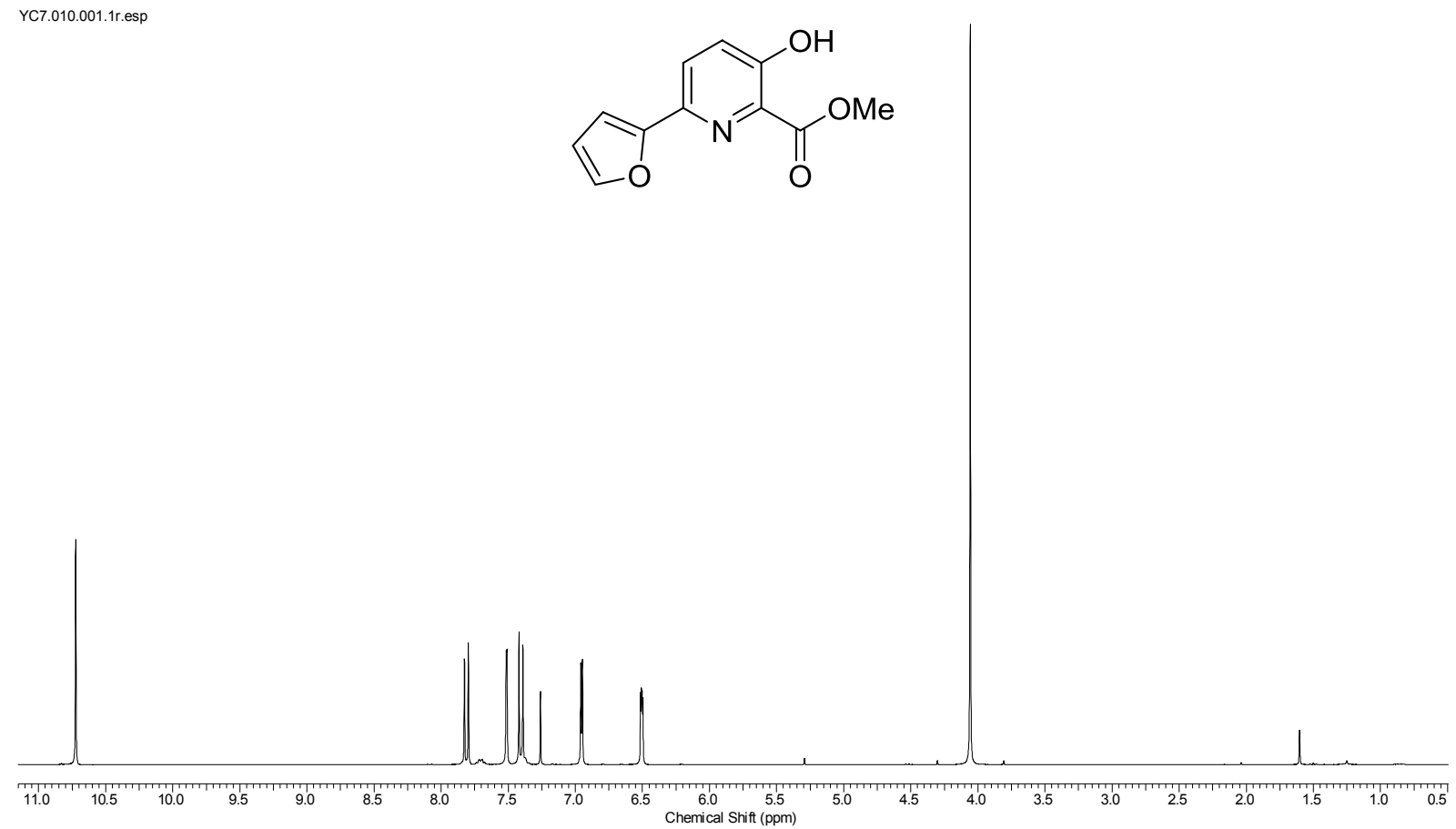

Abbildung 11.112: ${ }^{1} \mathrm{H}-\mathrm{NMR}-$ Spektrum des 3-Hydroxypyridins $2160\left(300 \mathrm{MHz}, \mathrm{CDCl}_{3}\right)$.

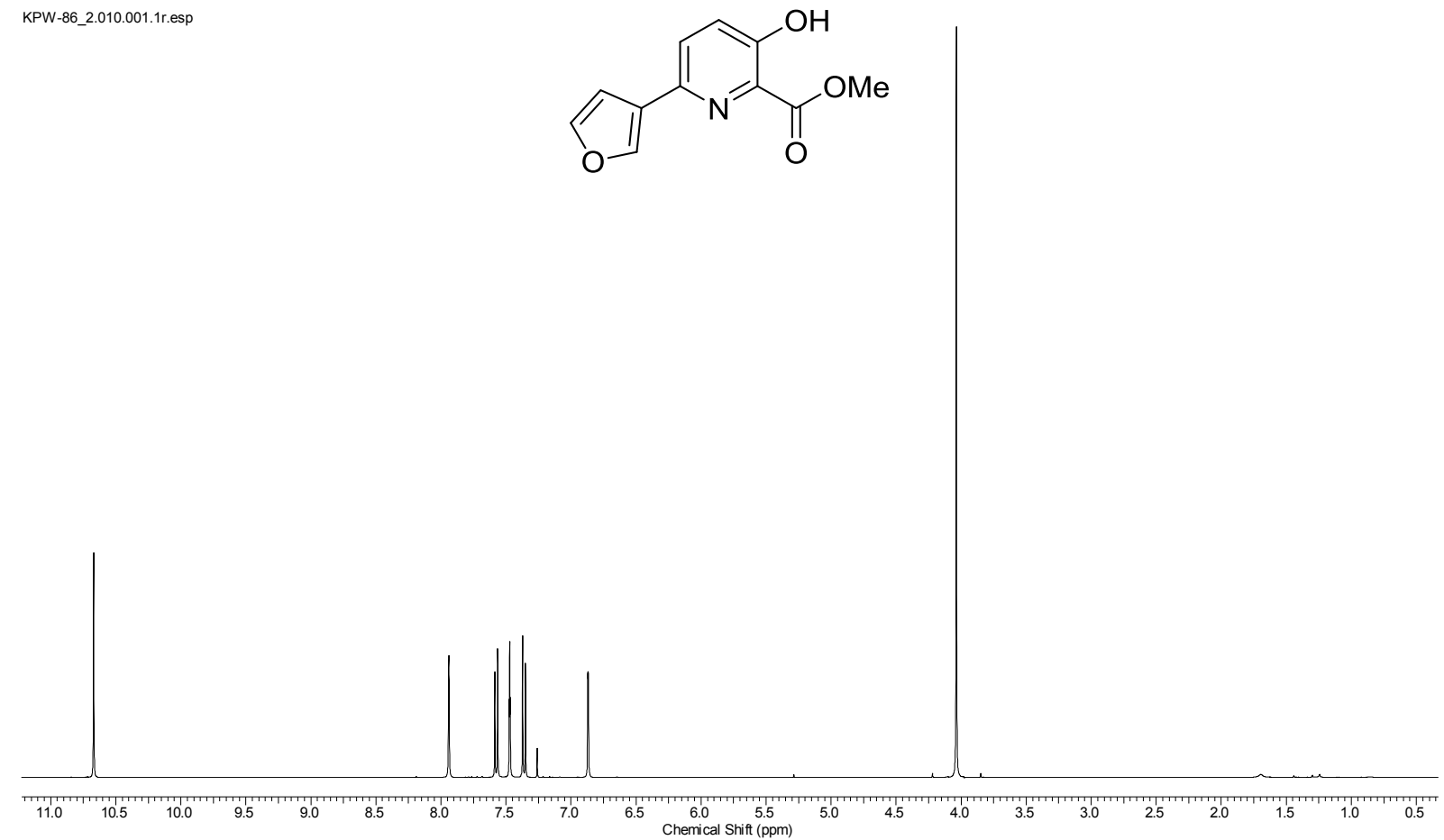

Abbildung 11.113: ${ }^{1} \mathrm{H}-\mathrm{NMR}-$ Spektrum des 3-Hydroxypyridins 216p (400 MHz, $\mathrm{CDCl}_{3}$ ). 


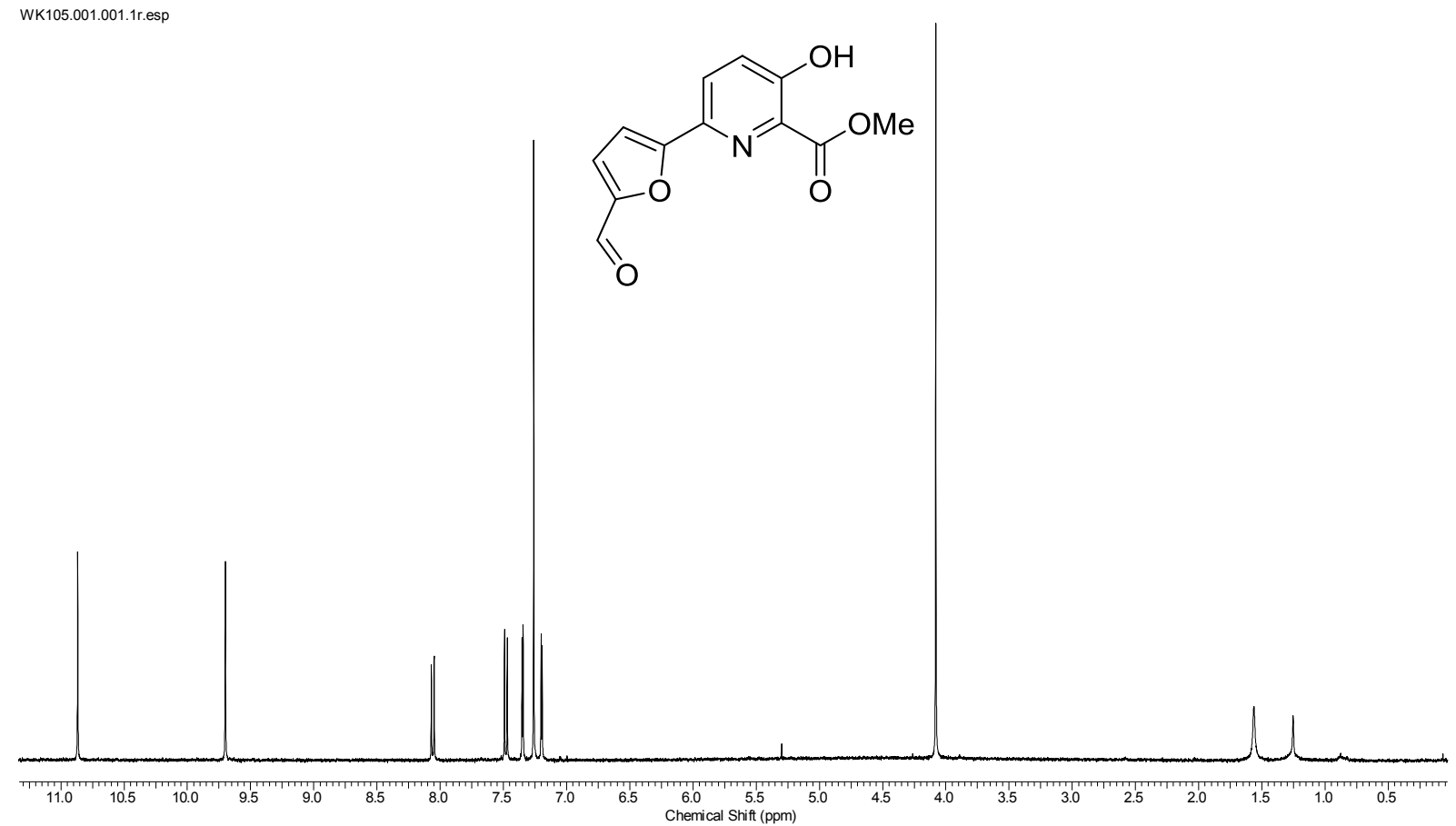

Abbildung 11.114: ${ }^{1} \mathrm{H}-\mathrm{NMR}-$ Spektrum des 3-Hydroxypyridins 216q (400 MHz, $\left.\mathrm{CDCl}_{3}\right)$.

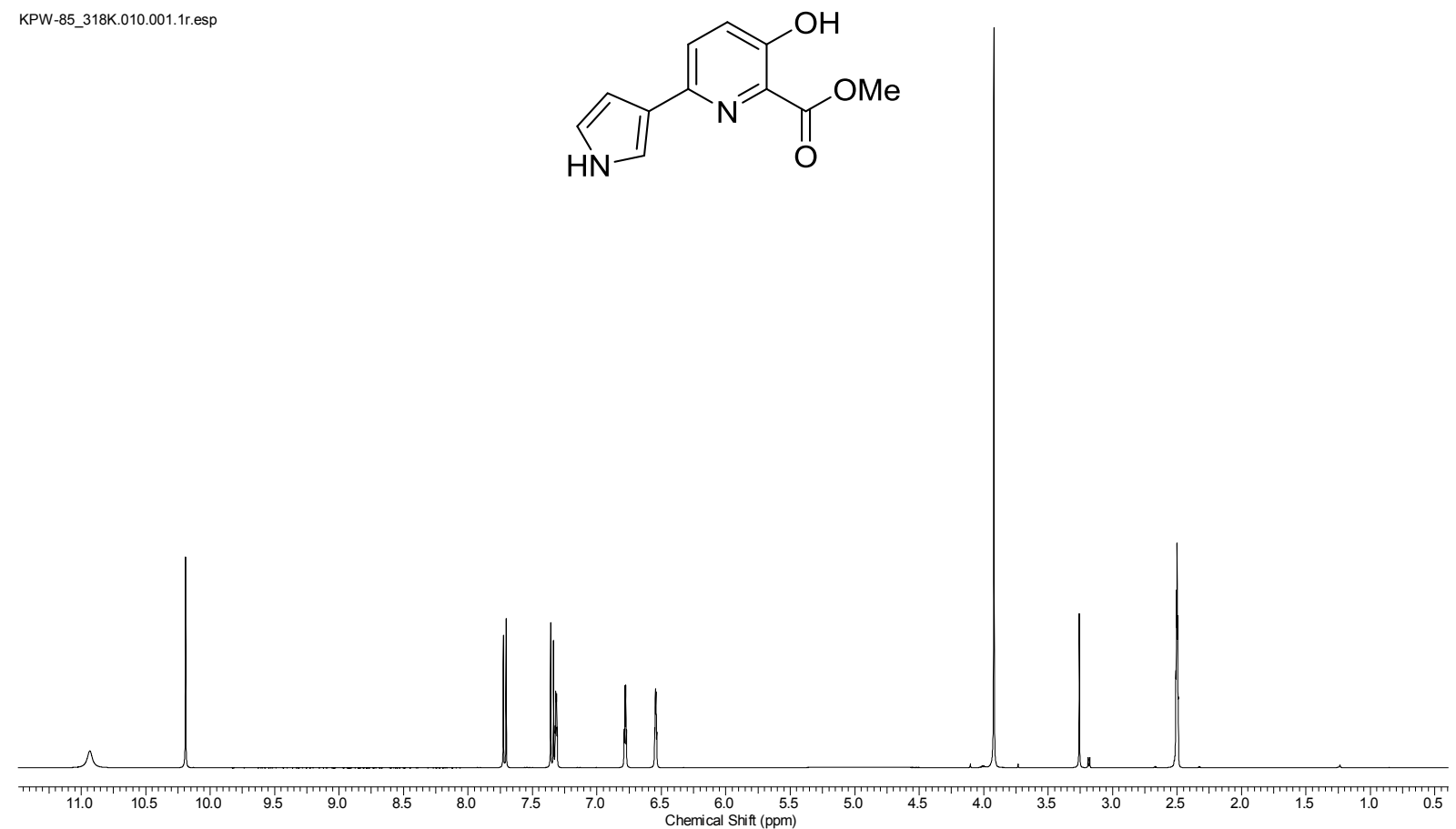

Abbildung 11.115: ${ }^{1} \mathrm{H}-\mathrm{NMR}-S p e k t r u m$ des 3-Hydroxypyridins 216r (400 MHz, DMSO- $\left.d_{6}, 318 \mathrm{~K}\right)$. 


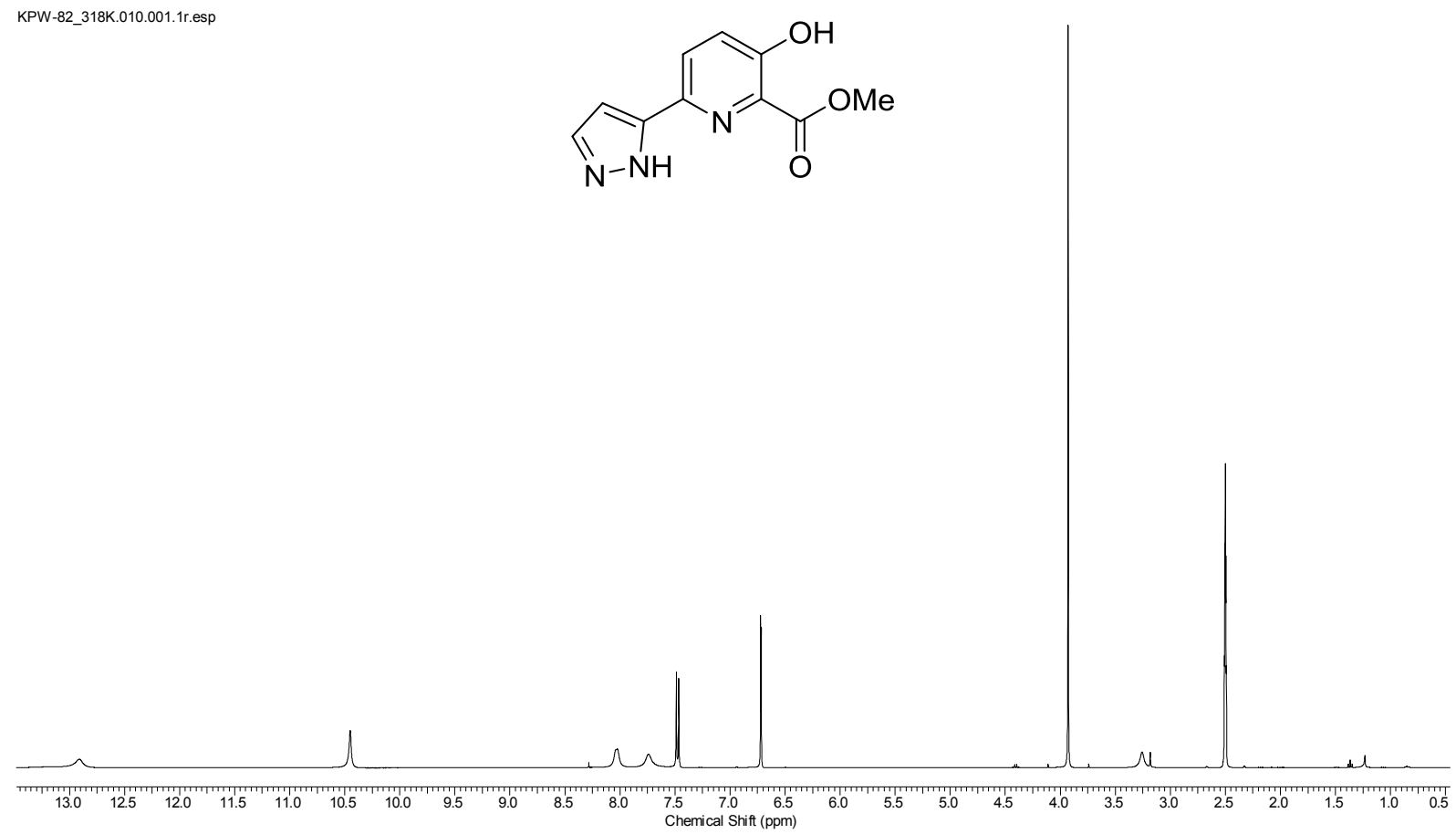

Abbildung 11.116: ${ }^{1} \mathrm{H}-\mathrm{NMR}-$ Spektrum des 3-Hydroxypyridins $216 \mathrm{~s}\left(400 \mathrm{MHz}\right.$, DMSO- $\left.d_{6}, 318 \mathrm{~K}\right)$.

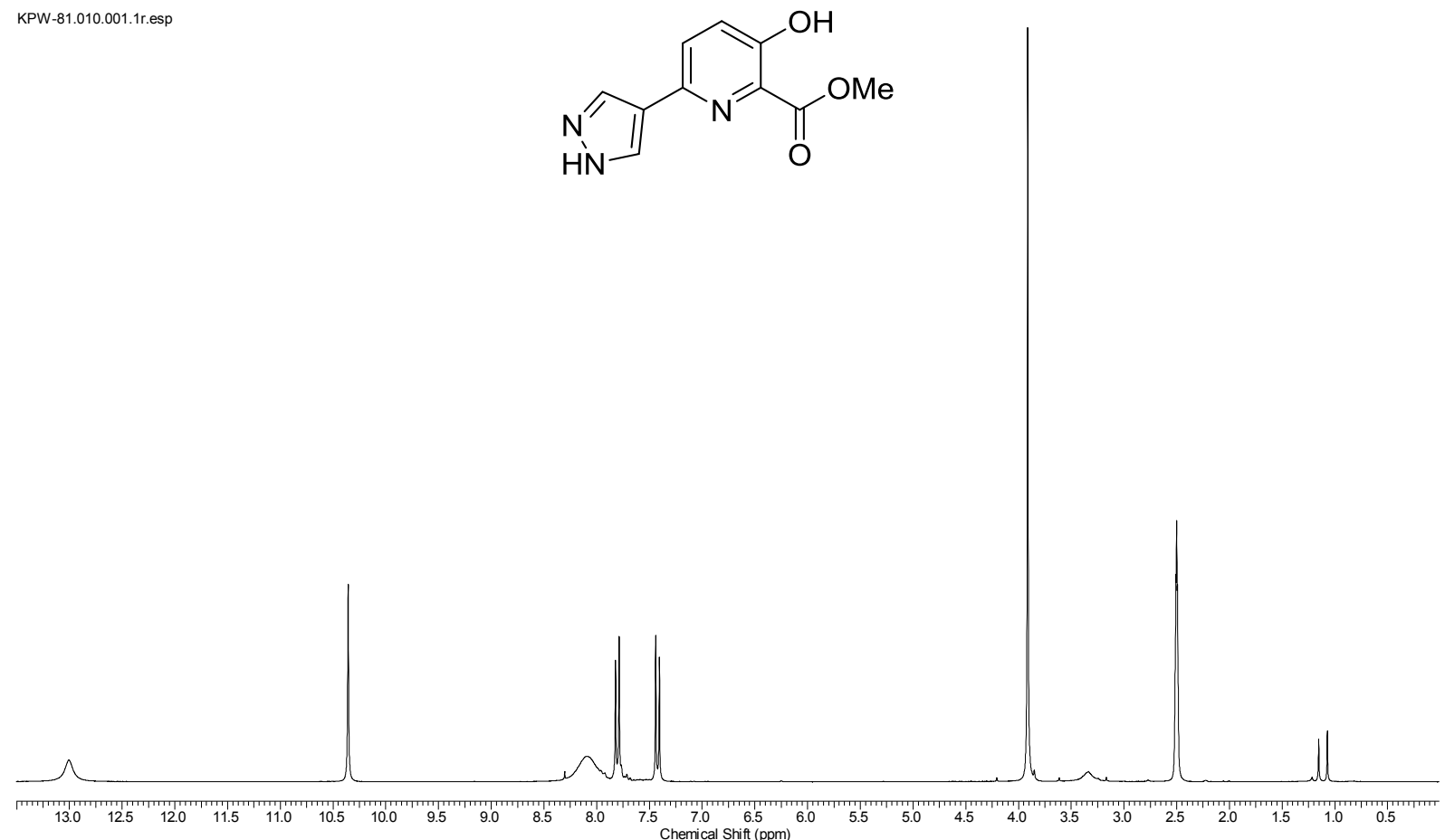

Abbildung 11.117: ${ }^{1} \mathrm{H}-\mathrm{NMR}-\mathrm{Spektrum}$ des 3-Hydroxypyridins 216s (250 MHz, DMSO- $\left.d_{6}\right)$. 


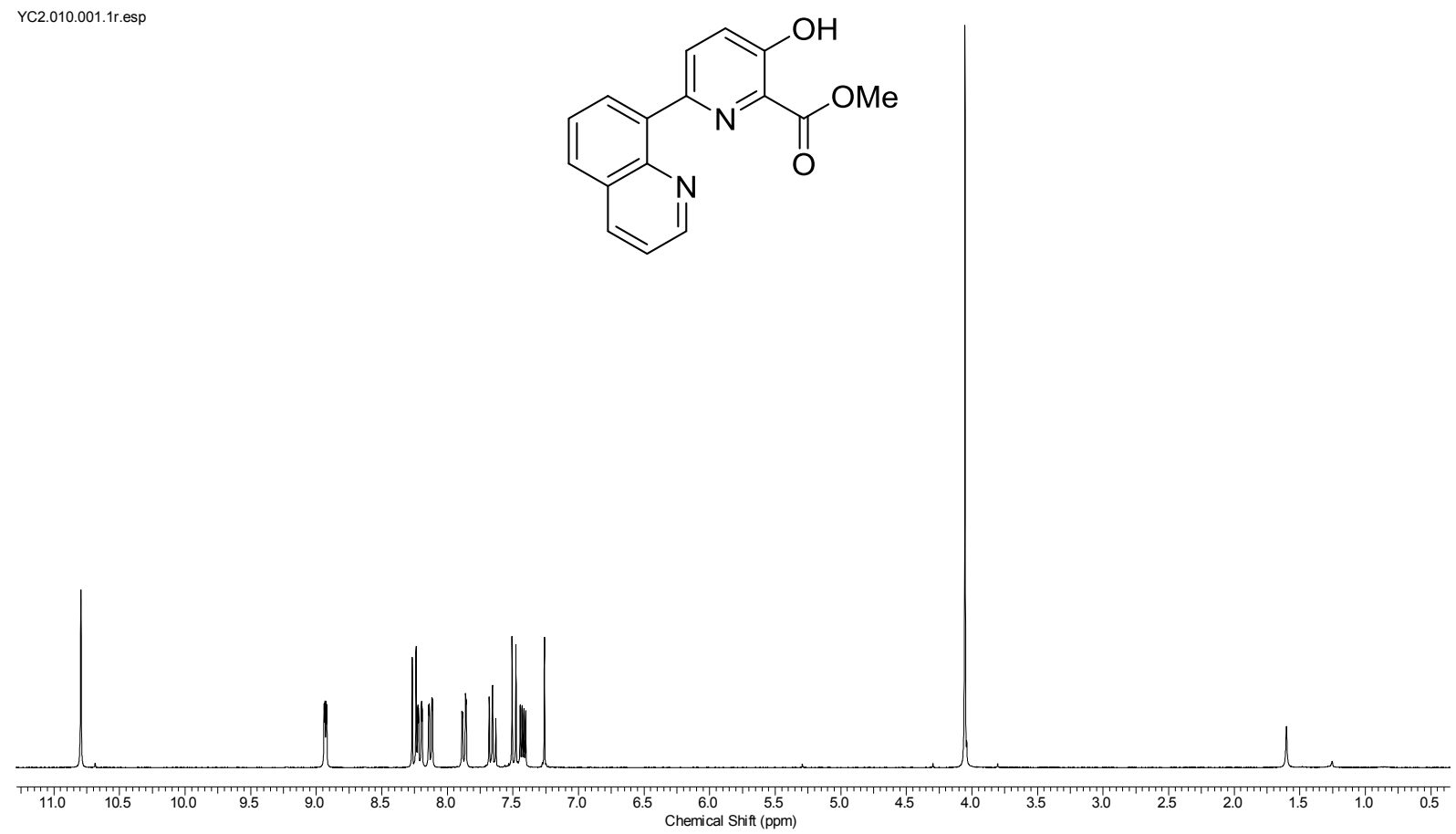

Abbildung 11.118: ${ }^{1} \mathrm{H}-\mathrm{NMR}-$ Spektrum des 3-Hydroxypyridins 216t (300 MHz, $\left.\mathrm{CDCl}_{3}\right)$.

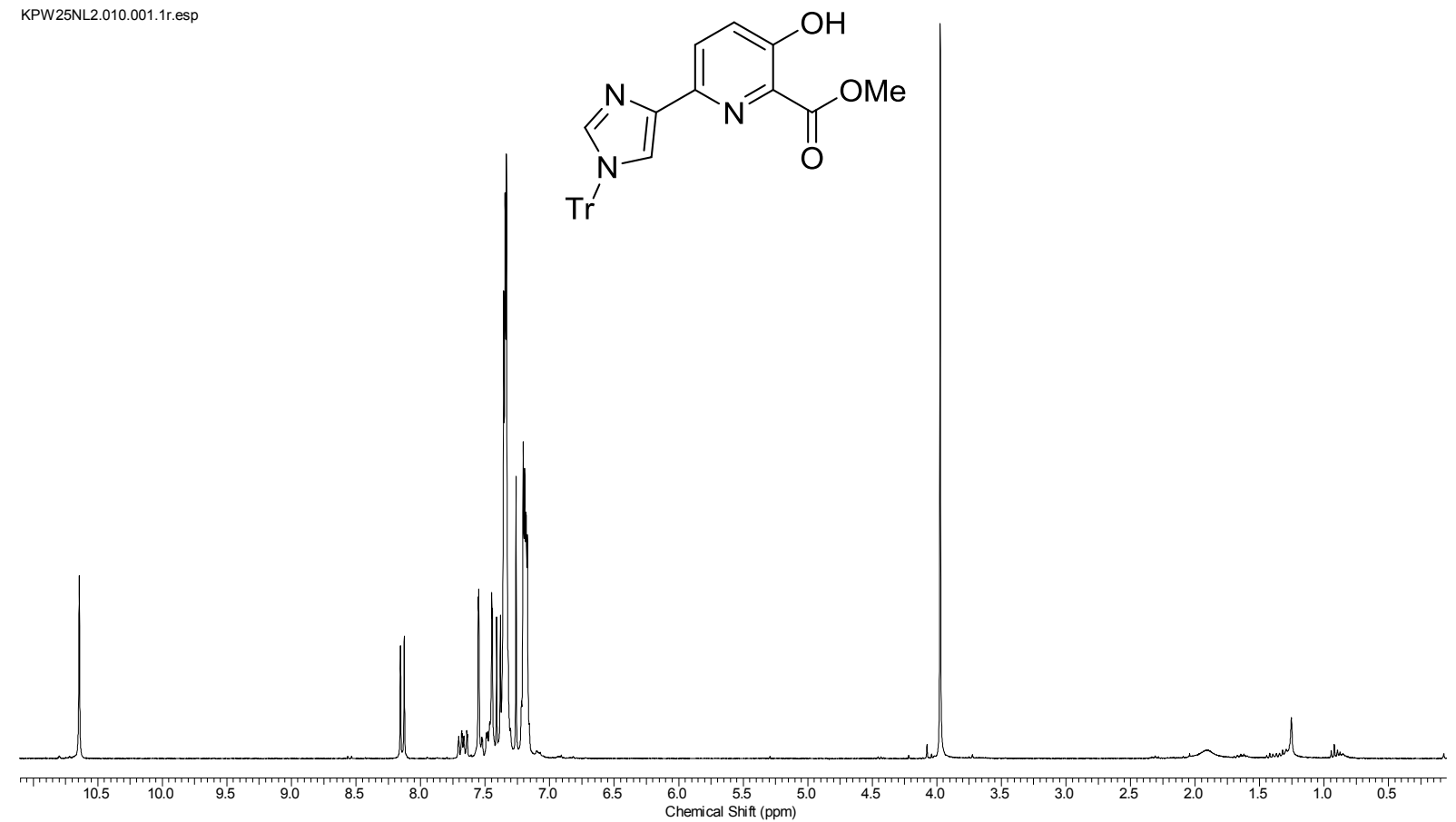

Abbildung 11.119: ${ }^{1} \mathrm{H}-\mathrm{NMR}-S p e k t r u m$ des 3-Hydroxypyridins 218 (300 MHz, $\left.\mathrm{CDCl}_{3}\right)$. 


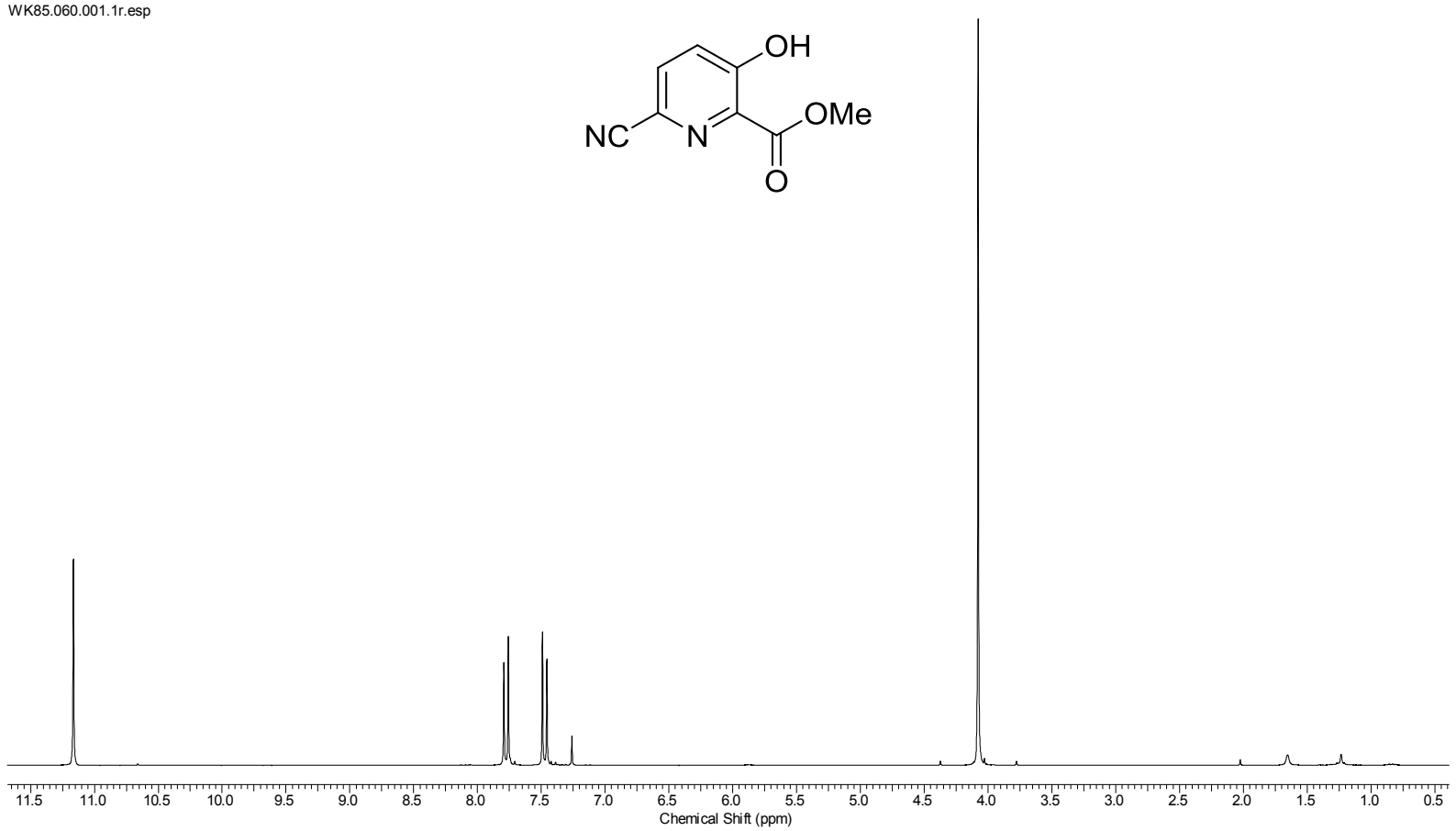

Abbildung 11.120: ${ }^{1} \mathrm{H}-\mathrm{NMR}-\mathrm{Spektrum}$ des Nitrils 219(250 $\left.\mathrm{MHz}, \mathrm{CDCl}_{3}\right)$.

WK87.160.001.1r.esp<smiles>COC(=O)c1nc(C(=N)NO)ccc1O</smiles>

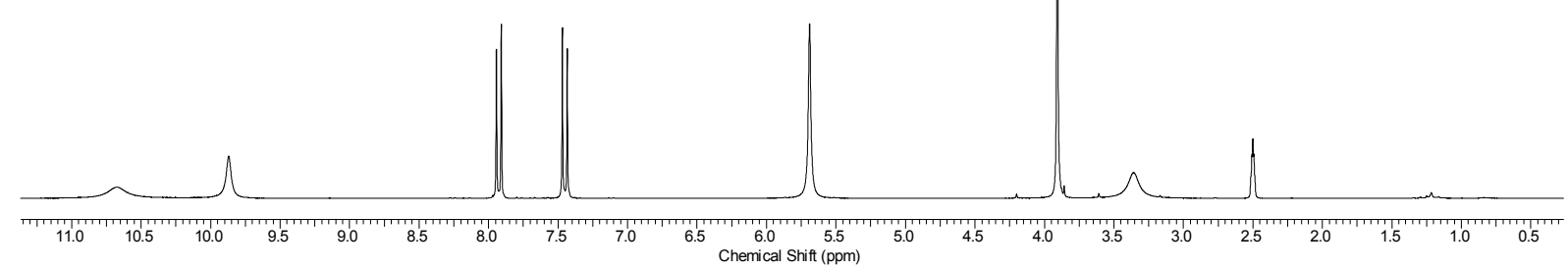

Abbildung 11.121: ${ }^{1} \mathrm{H}-\mathrm{NMR}-\mathrm{Spektrum}$ des $N$-Hydroxyamidins $220\left(250 \mathrm{MHz}\right.$, DMSO- $\left.d_{6}\right)$. 
WK93.110.001.1r.esp<smiles>Cc1nc(-c2ccc(O)c(C(=O)O)n2)no1</smiles>

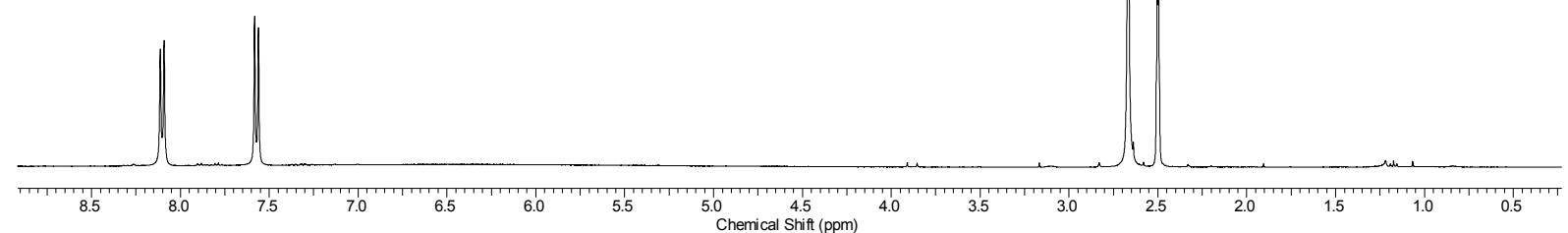

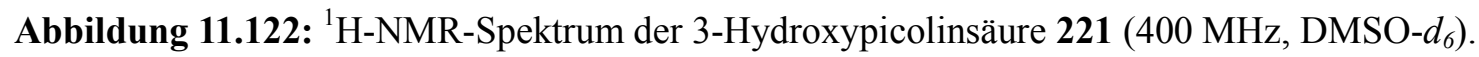

KPW89.010.001.1r.esp<smiles>COC(=O)c1nc(C#CSC(C)(C)C)ccc1O</smiles>

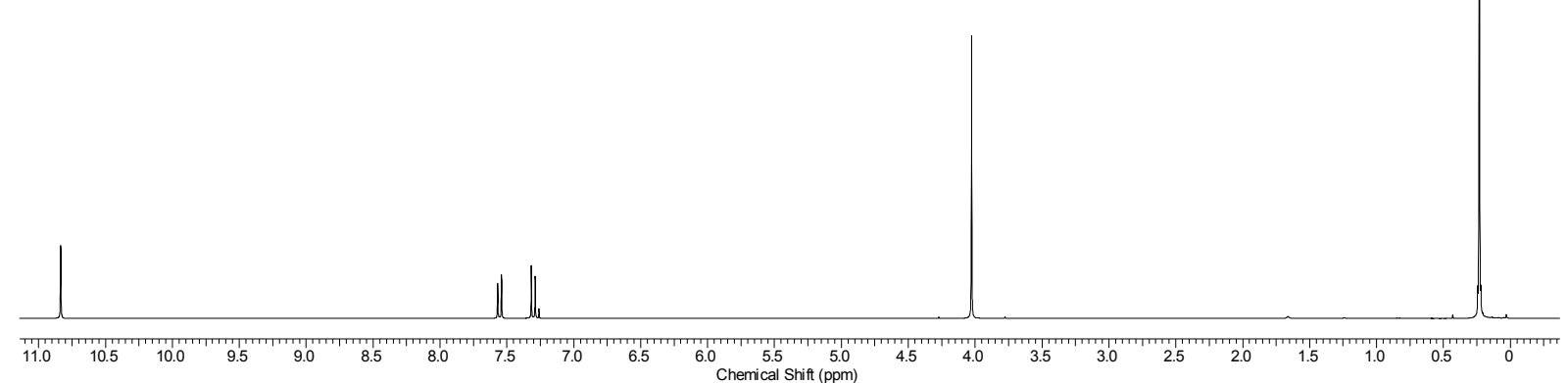

Abbildung 11.123: ${ }^{1} \mathrm{H}-\mathrm{NMR}-$ Spektrum des TMS-Alkins $222\left(300 \mathrm{MHz}, \mathrm{CDCl}_{3}\right)$. 


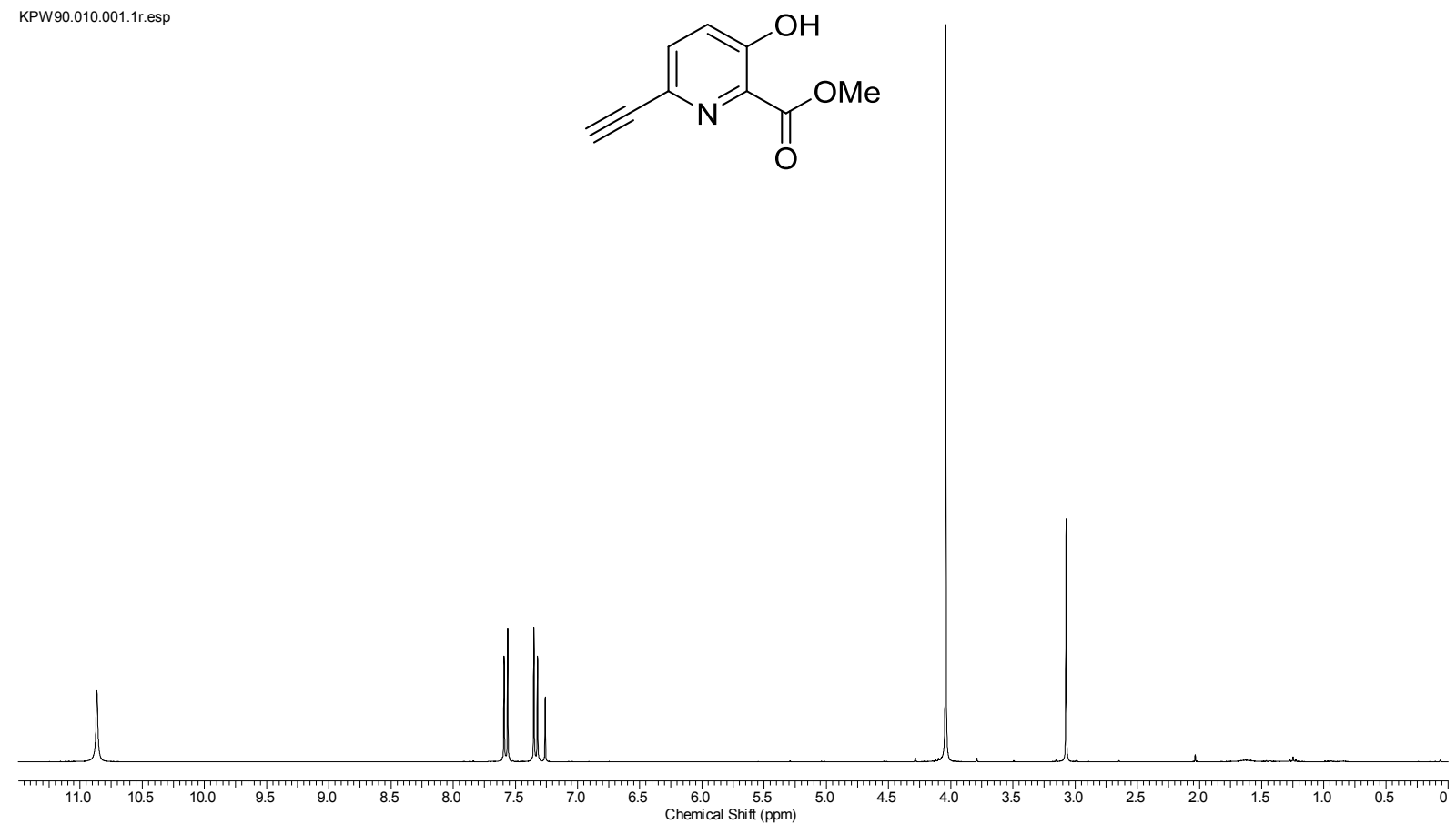

Abbildung 11.124: ${ }^{1} \mathrm{H}-\mathrm{NMR}-$ Spektrum des Alkins 223 (300 MHz, $\left.\mathrm{CDCl}_{3}\right)$.

YC_3HP_tria_tfa.014.001.1r.esp<smiles>COC(=O)c1nc(-c2cnn[nH]2)ccc1O</smiles>

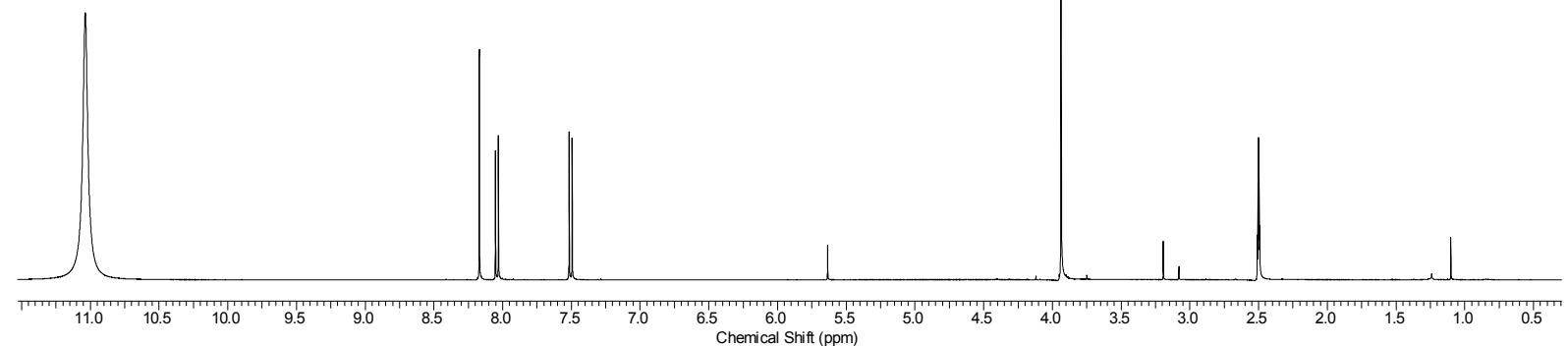

Abbildung 11.125: ${ }^{1} \mathrm{H}-\mathrm{NMR}-$ Spektrum des 3-Hydroxypyridins $224\left(300 \mathrm{MHz}\right.$, DMSO- $d_{6} / \mathrm{TFA}, 1: 1$, $343 \mathrm{~K})$. 


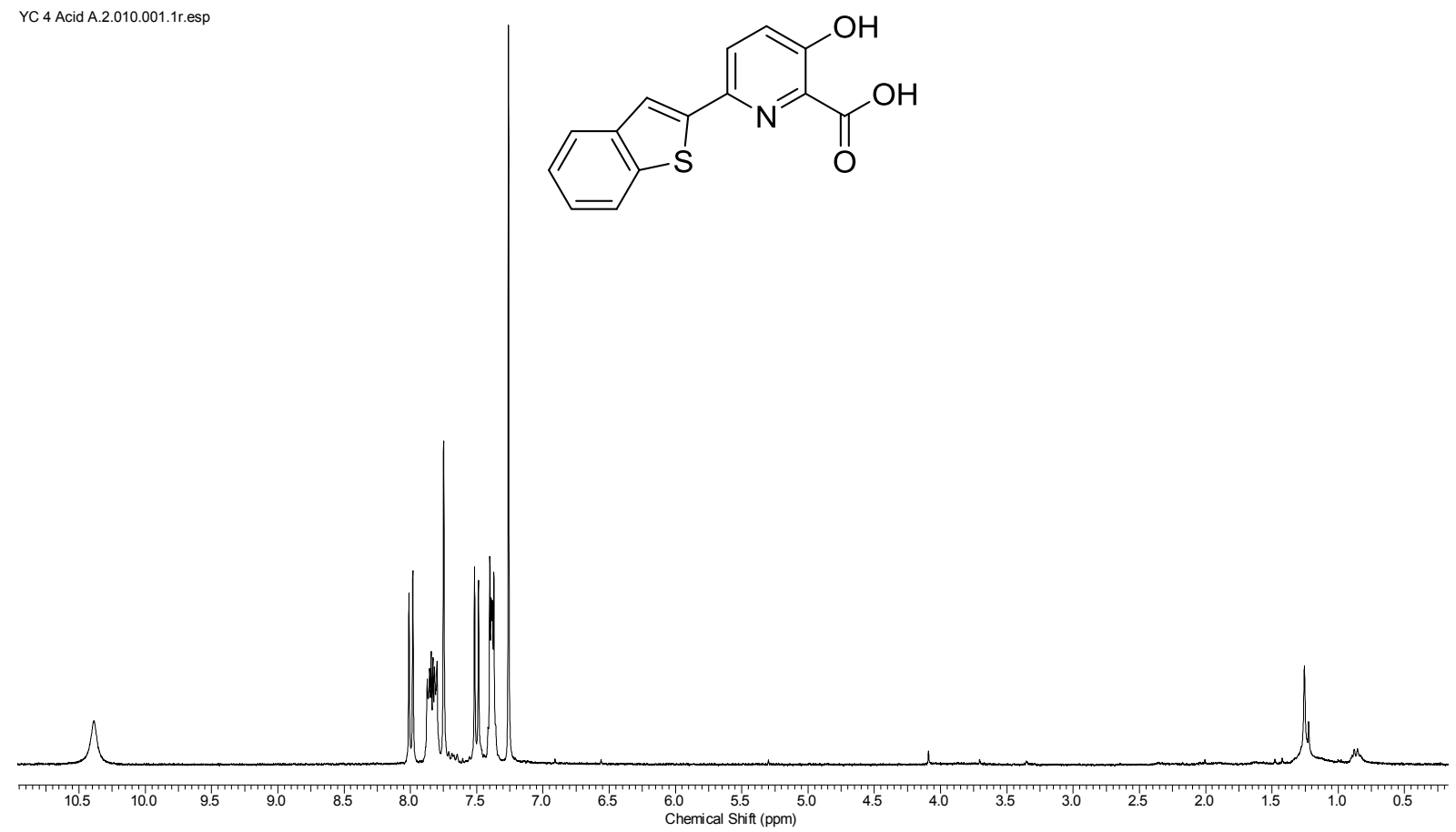

Abbildung 11.126: ${ }^{1} \mathrm{H}-\mathrm{NMR}-$ Spektrum der 3-Hydroxypicolinsäure 225a (300 MHz, $\left.\mathrm{CDCl}_{3}\right)$.

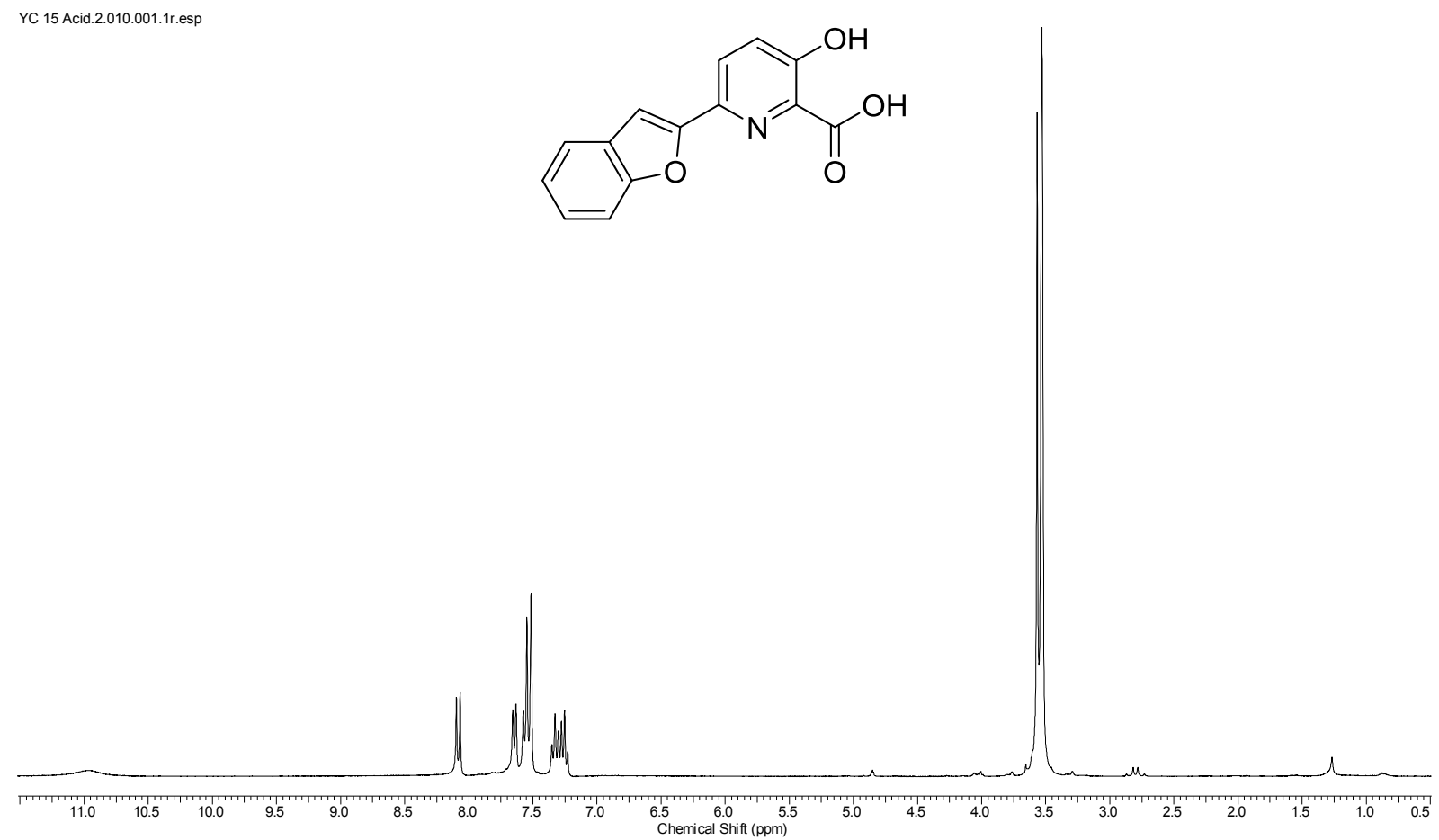

Abbildung 11.127: ${ }^{1} \mathrm{H}-\mathrm{NMR}-$ Spektrum der 3-Hydroxypicolinsäure 225b (300 MHz, 1,4-Dioxan- $\left.d_{8}\right)$. 


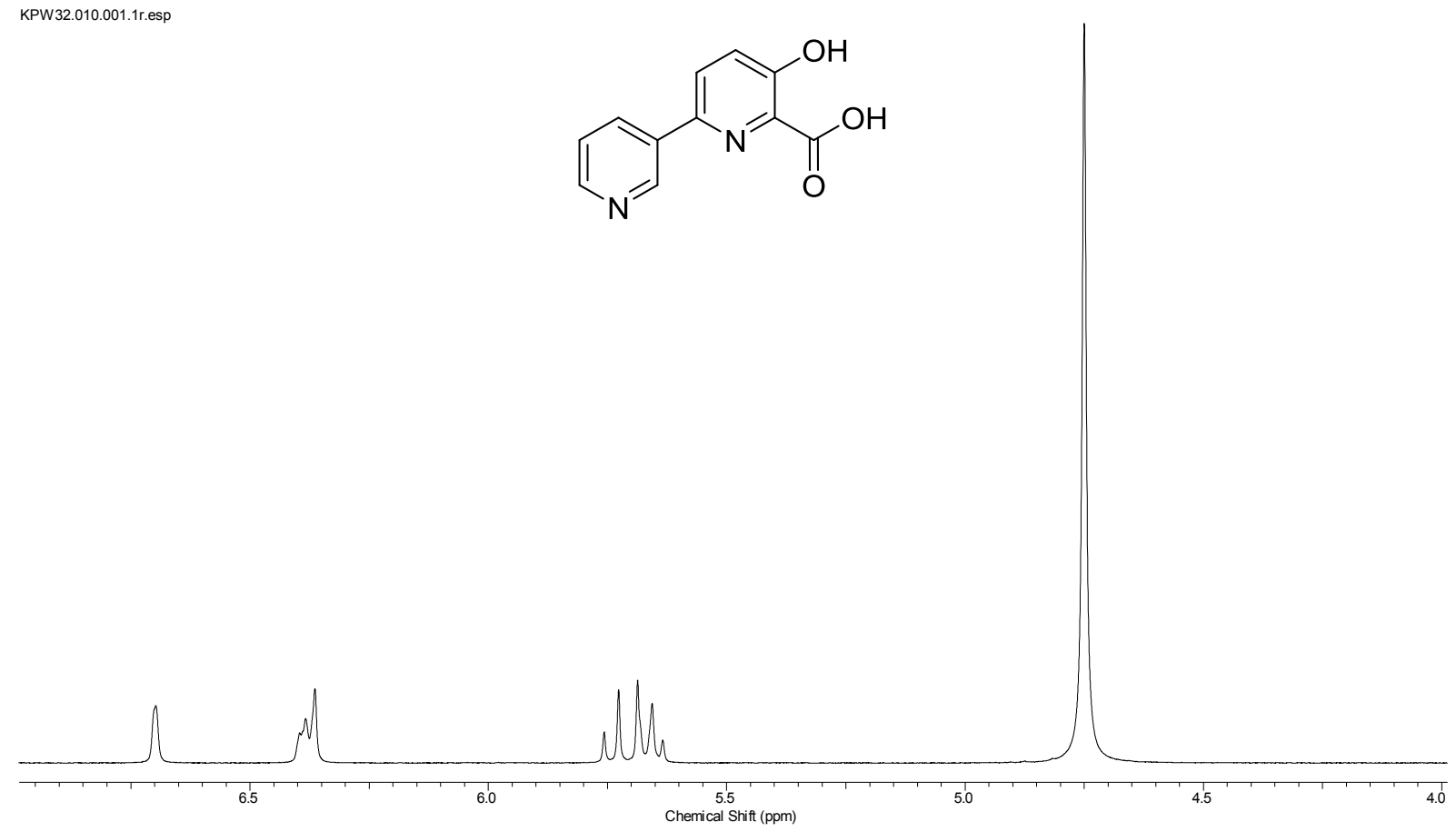

Abbildung 11.128: ${ }^{1} \mathrm{H}-\mathrm{NMR}-$ Spektrum der 3-Hydroxypicolinsäure 225c $\left(300 \mathrm{MHz}, \mathrm{D}_{2} \mathrm{O}\right)$.

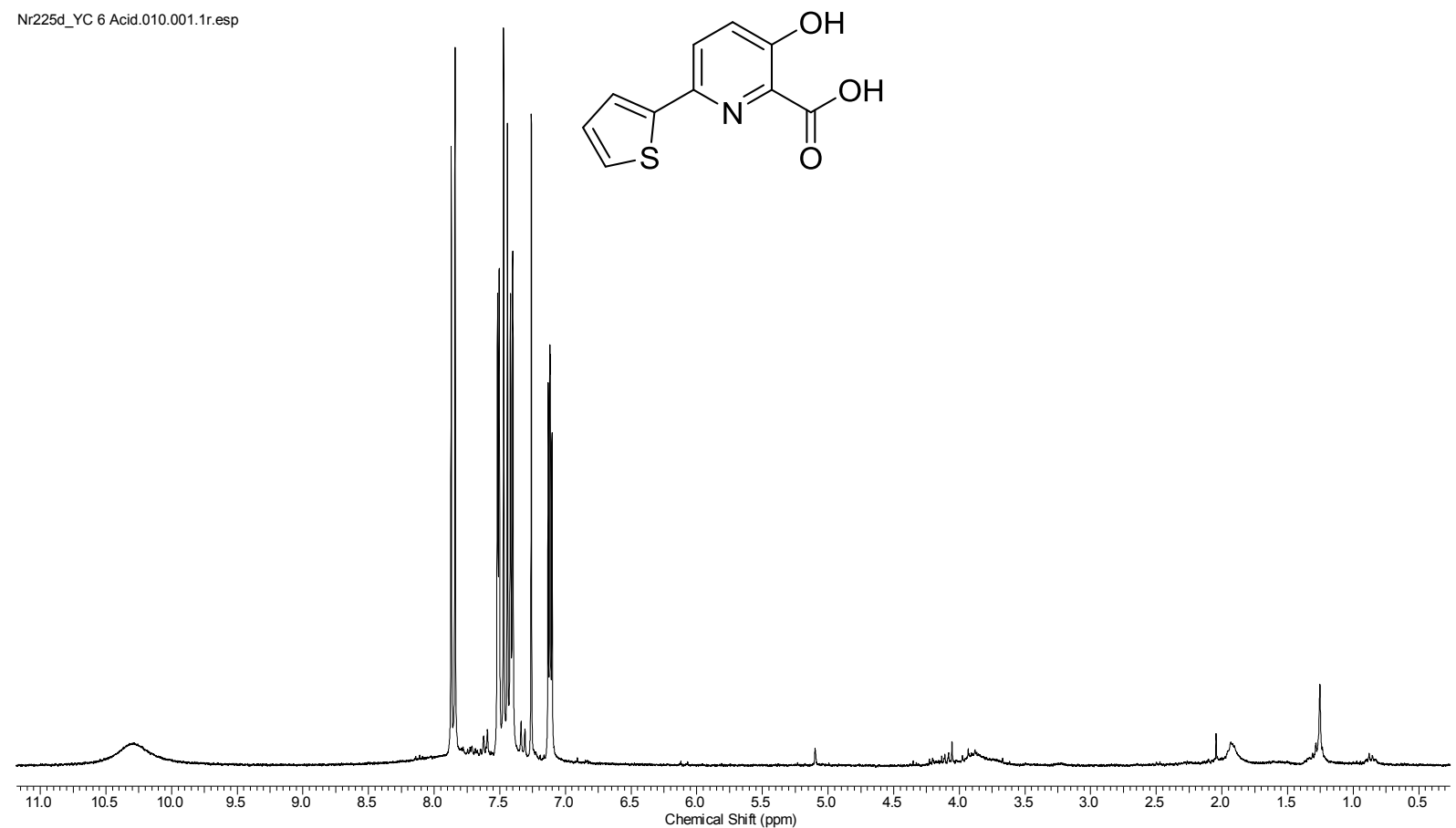

Abbildung 11.129: ${ }^{1} \mathrm{H}-\mathrm{NMR}-S p e k t r u m$ der 3-Hydroxypicolinsäure $225 \mathrm{e}\left(300 \mathrm{MHz}, \mathrm{CDCl}_{3}\right)$. 


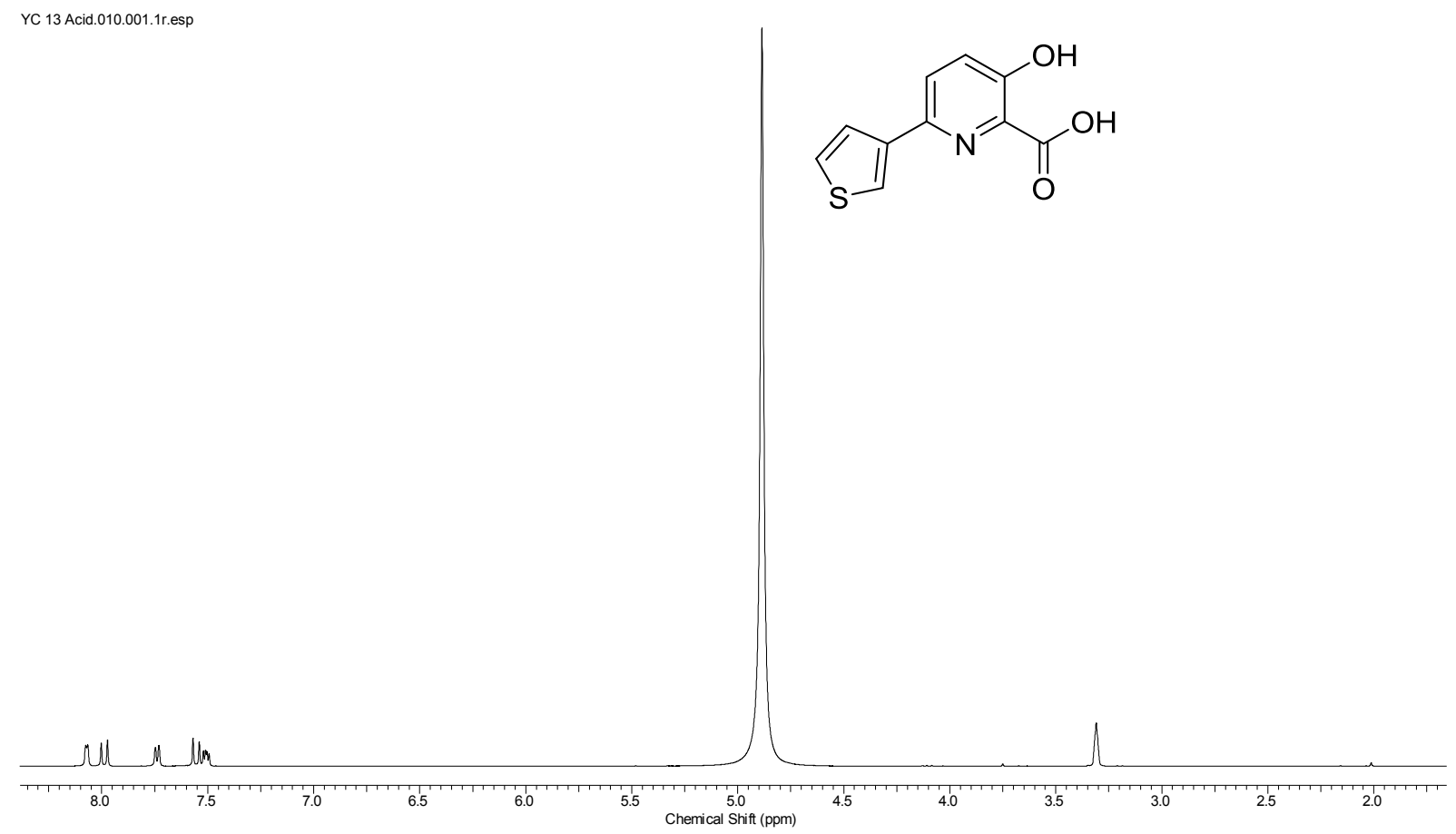

Abbildung 11.130: ${ }^{1} \mathrm{H}-\mathrm{NMR}-$ Spektrum der 3-Hydroxypicolinsäure 225e (300 MHz, MeOH- $\left.d_{4}\right)$.

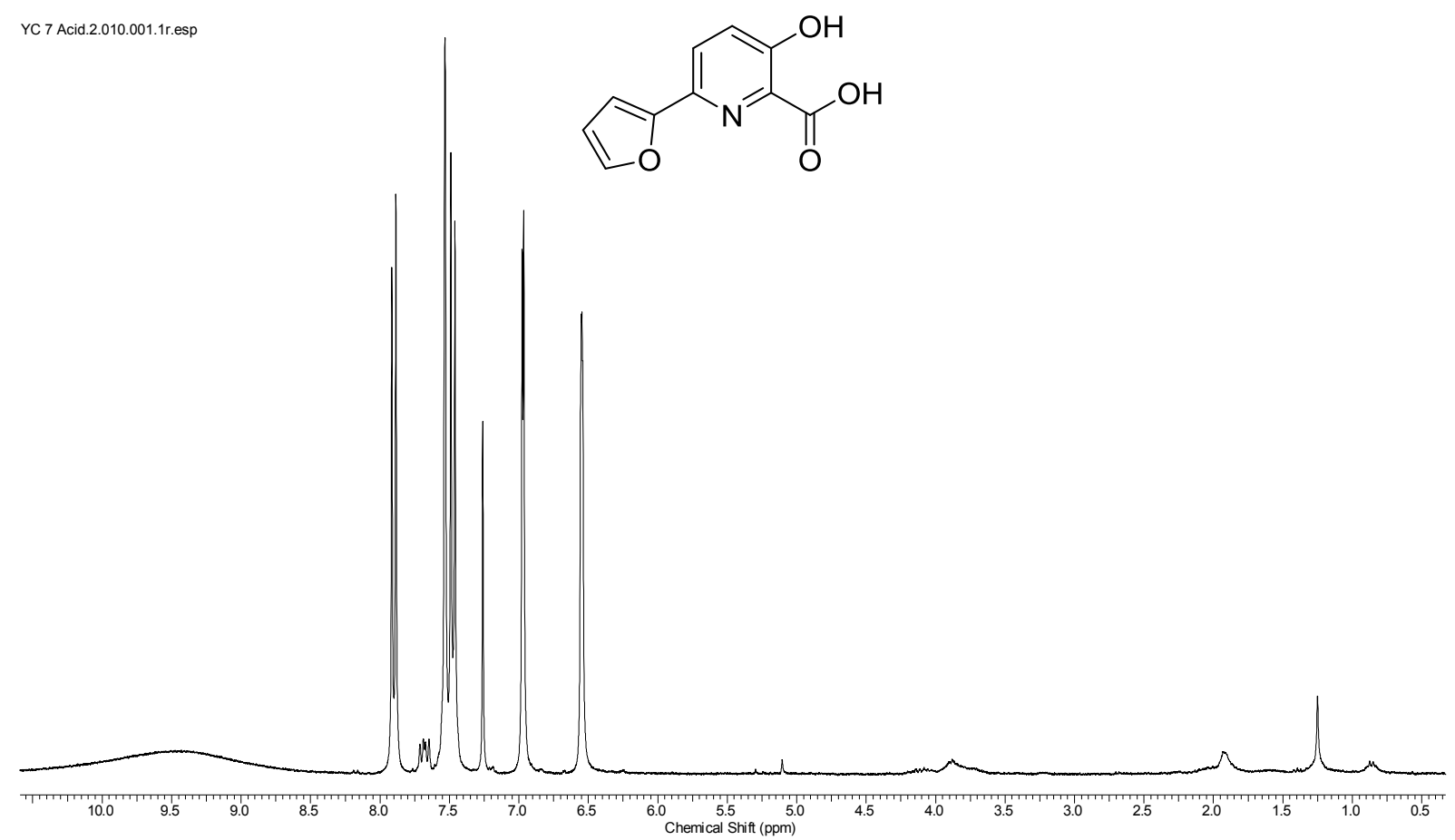

Abbildung 11.131: ${ }^{1} \mathrm{H}-\mathrm{NMR}-$ Spektrum der 3-Hydroxypicolinsäure $225 f\left(300 \mathrm{MHz}, \mathrm{CDCl}_{3}\right)$. 


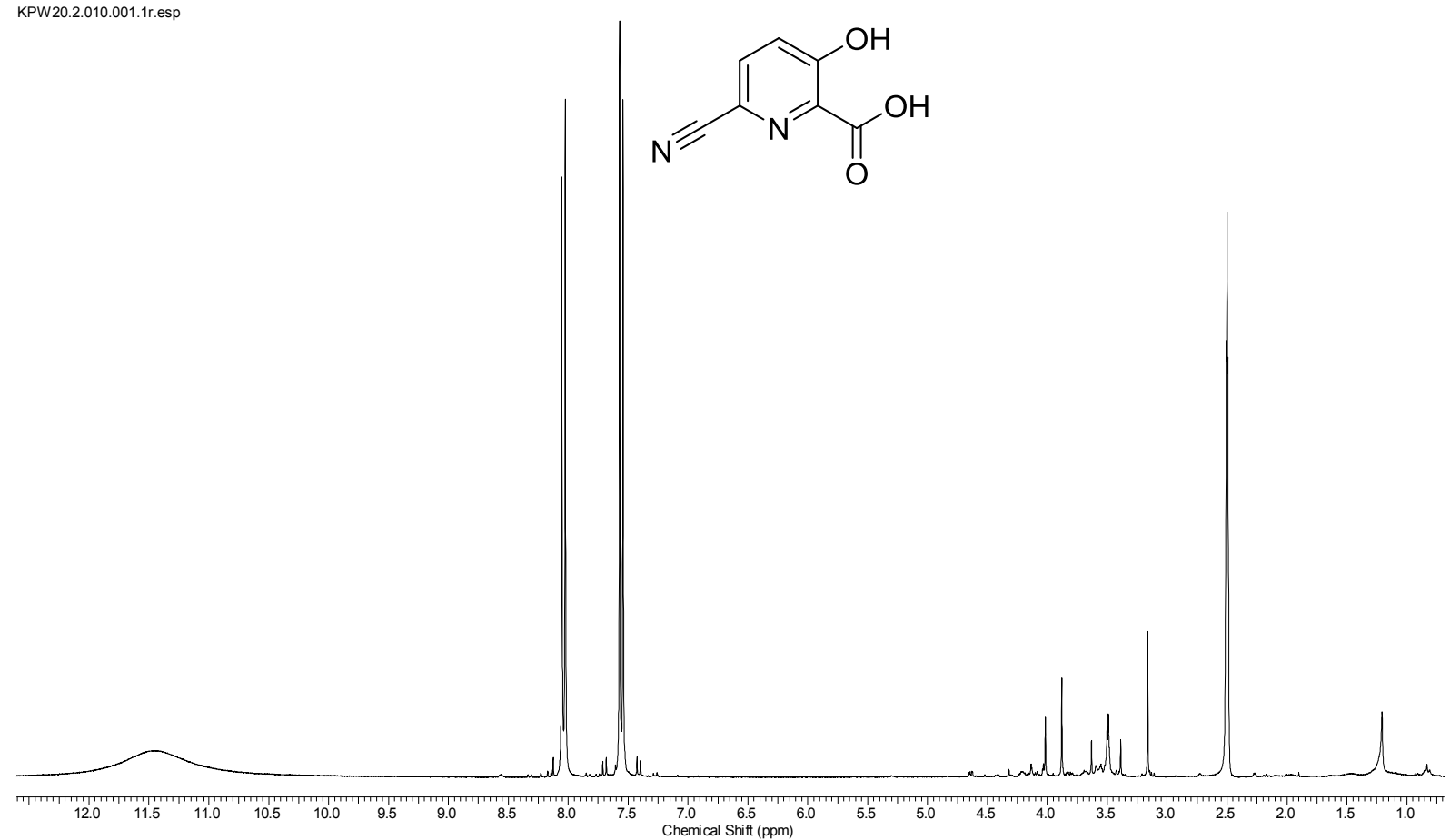

Abbildung 11.132: ${ }^{1} \mathrm{H}-\mathrm{NMR}-$ Spektrum der 3-Hydroxypicolinsäure 225g (300 MHz, DMSO- $\left.d_{6}\right)$.

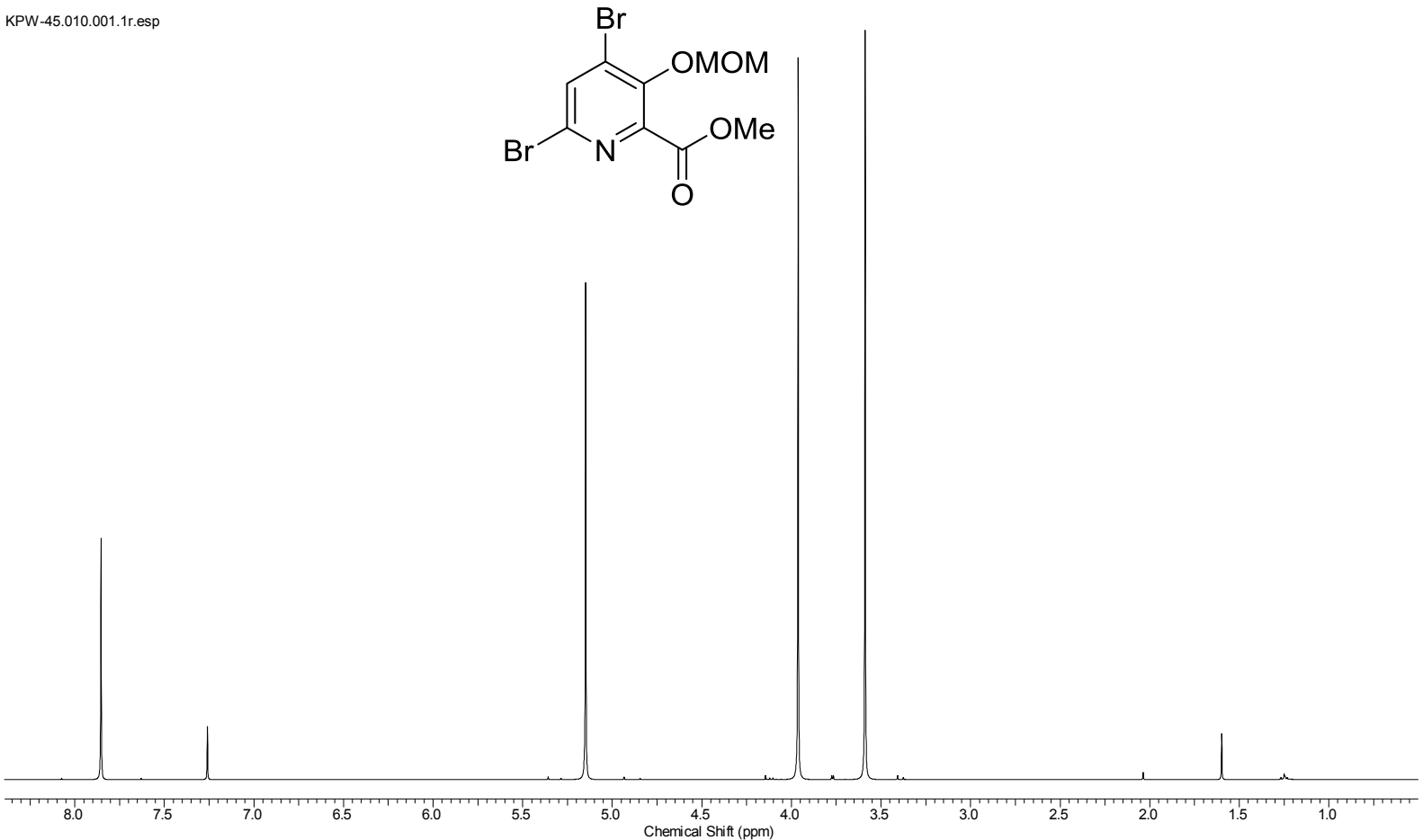

Abbildung 11.133: ${ }^{1} \mathrm{H}-\mathrm{NMR}-$ Spektrum der MOM-Ethers $229\left(400 \mathrm{MHz}, \mathrm{CDCl}_{3}\right)$. 


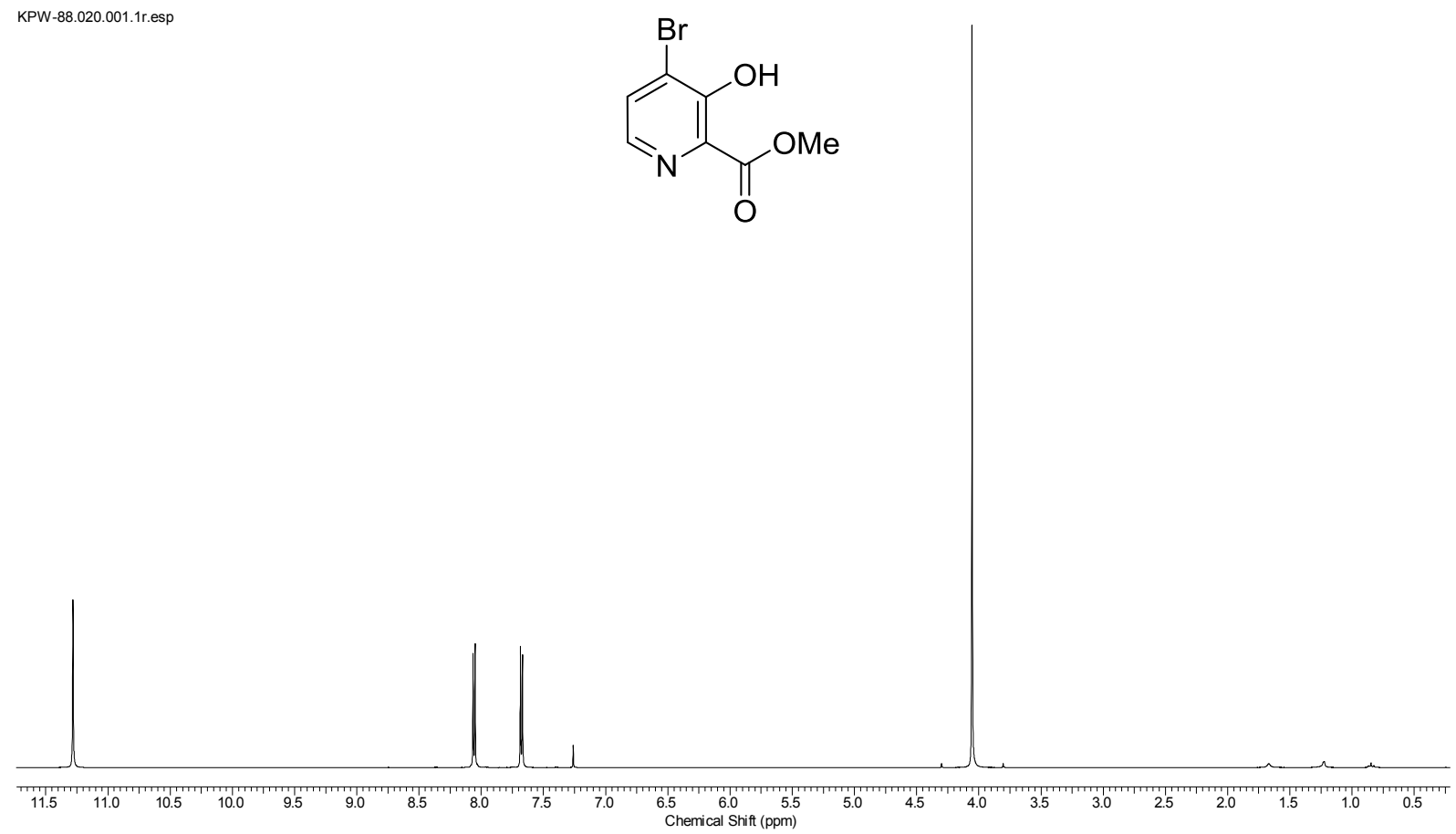

Abbildung 11.134: ${ }^{1} \mathrm{H}-\mathrm{NMR}-$ Spektrum des 3-Hydroxypyridins $230\left(300 \mathrm{MHz}, \mathrm{CDCl}_{3}\right)$.

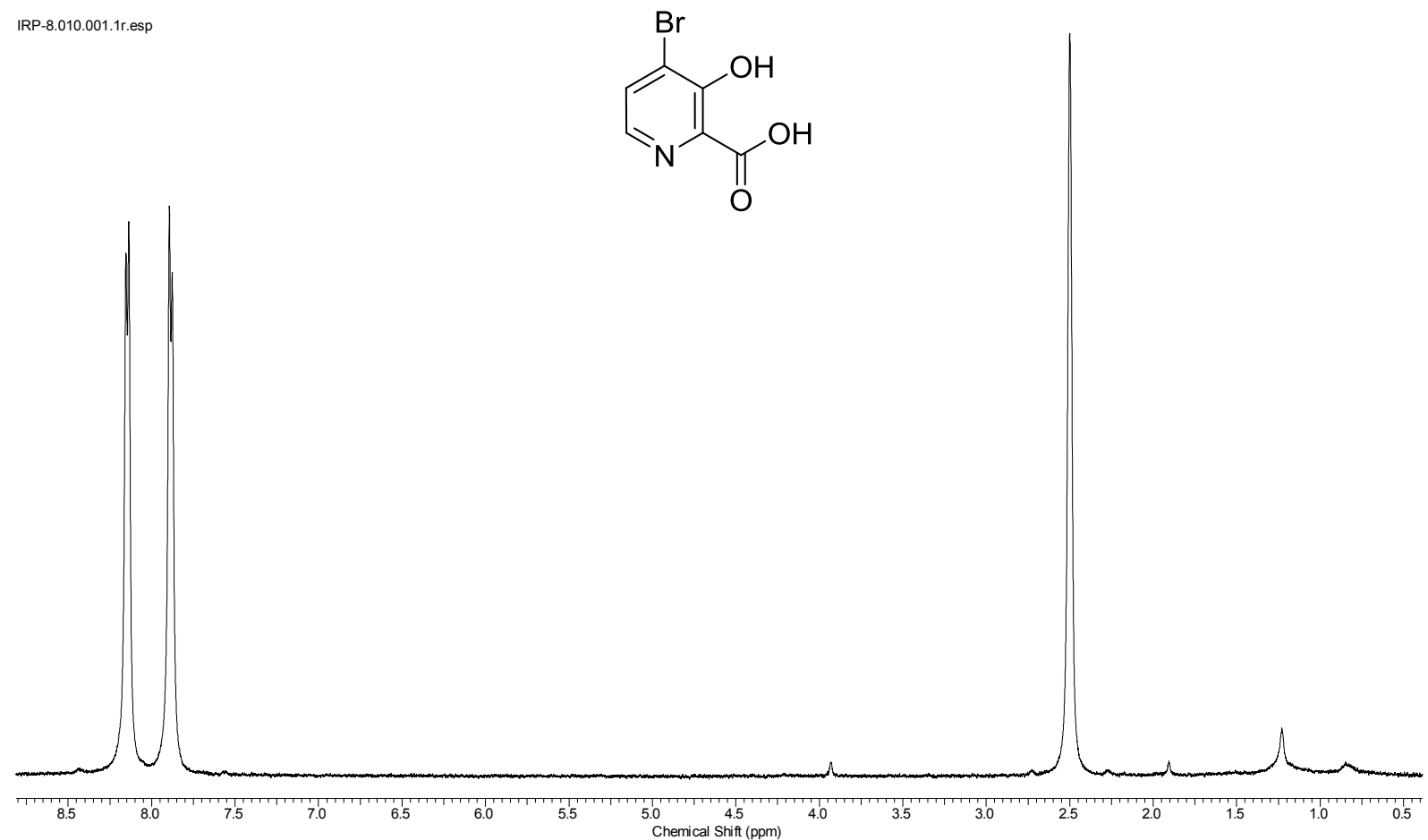

Abbildung 11.135: ${ }^{1} \mathrm{H}-\mathrm{NMR}-$ Spektrum der 3-Hydroxypicolinsäure 231 (300 MHz, DMSO- $\left.d_{6}\right)$. 


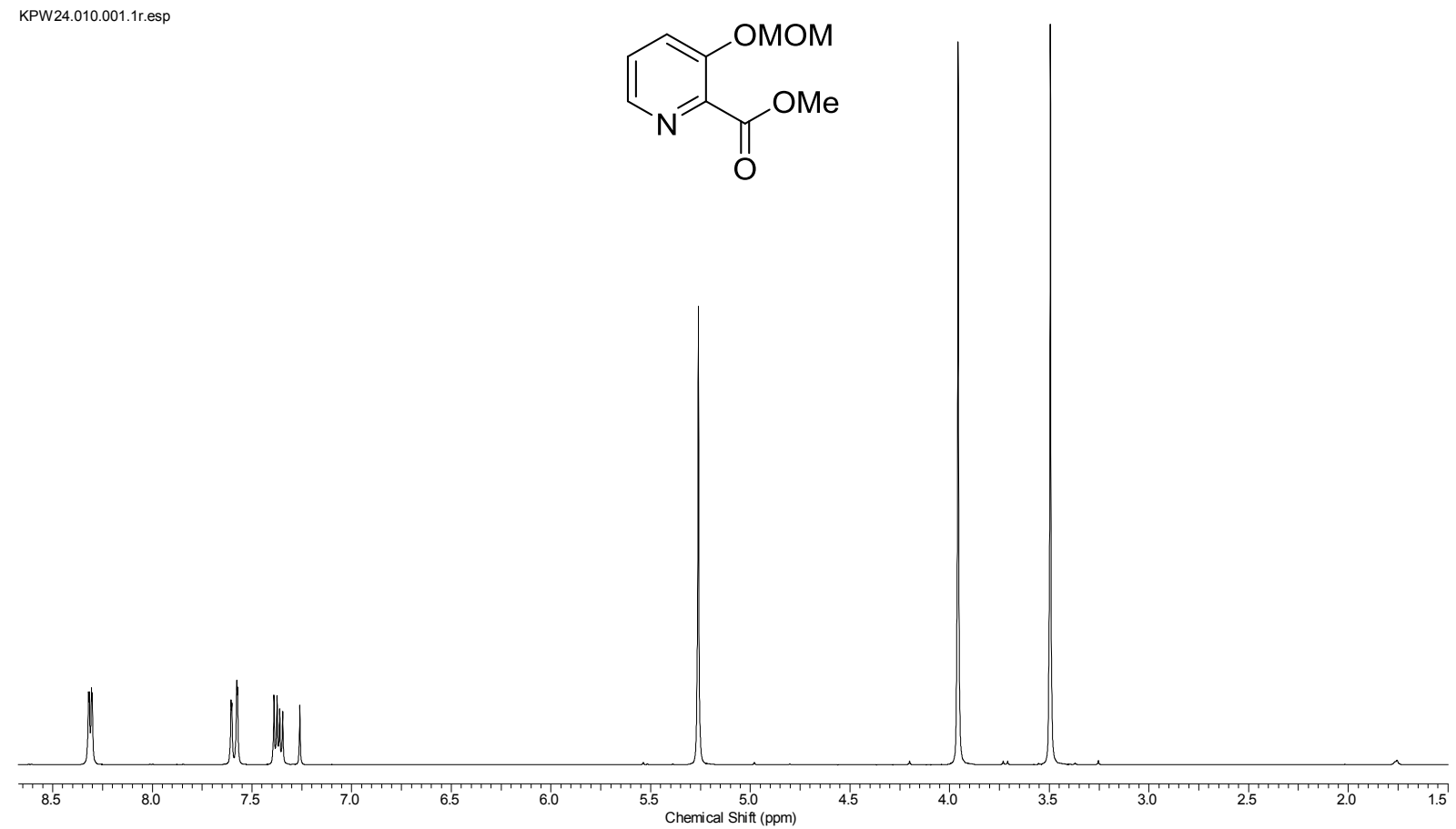

Abbildung 11.136: ${ }^{1} \mathrm{H}-\mathrm{NMR}-$ Spektrum des MOM-Ethers $101\left(300 \mathrm{MHz}, \mathrm{CDCl}_{3}\right)$.

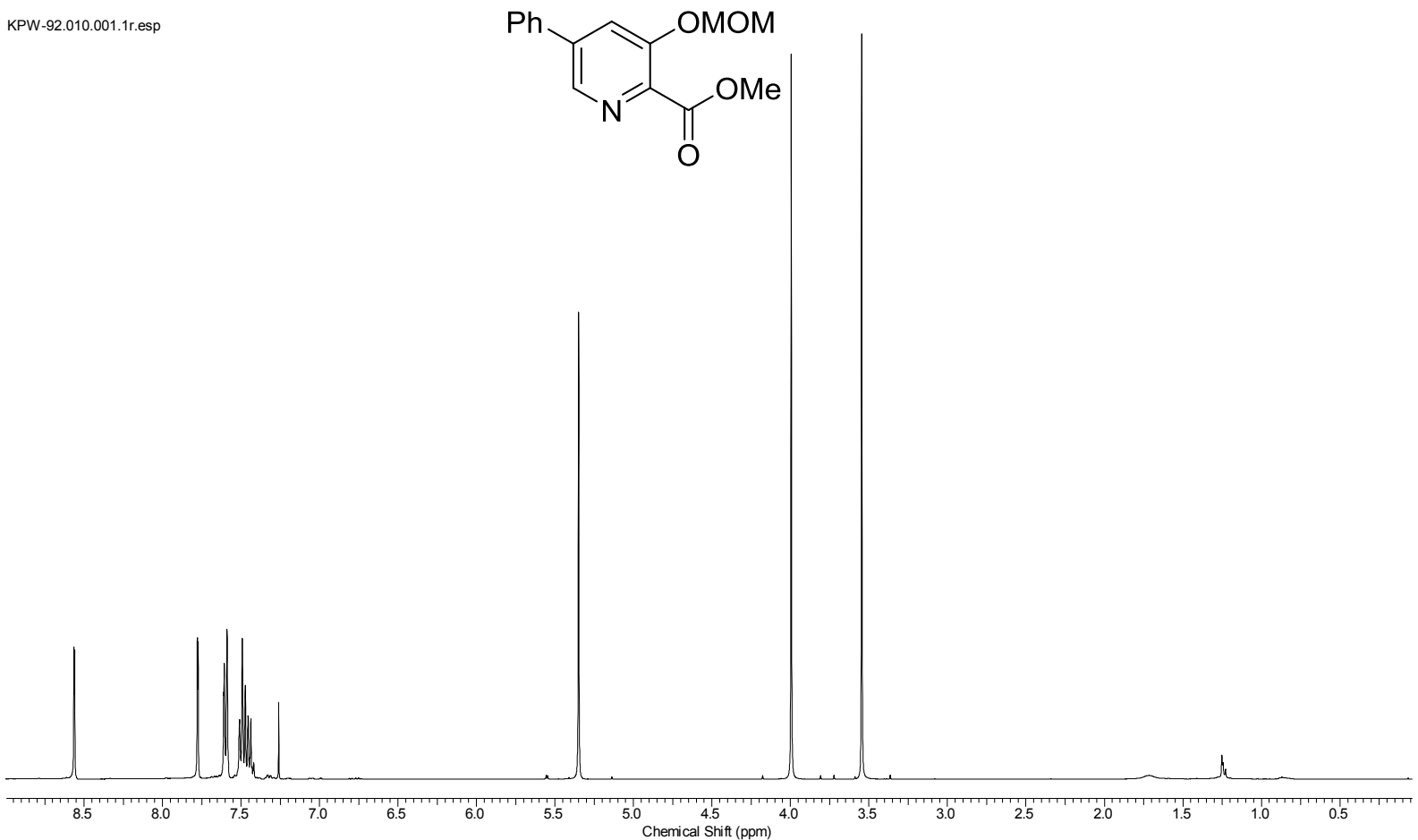

Abbildung 11.137: ${ }^{1} \mathrm{H}-\mathrm{NMR}-$ Spektrum des MOM-Ethers 233 (400 MHz, $\mathrm{CDCl}_{3}$ ). 


\subsection{Spektren zur Synthese von Bis-Carboxamid-Liganden}

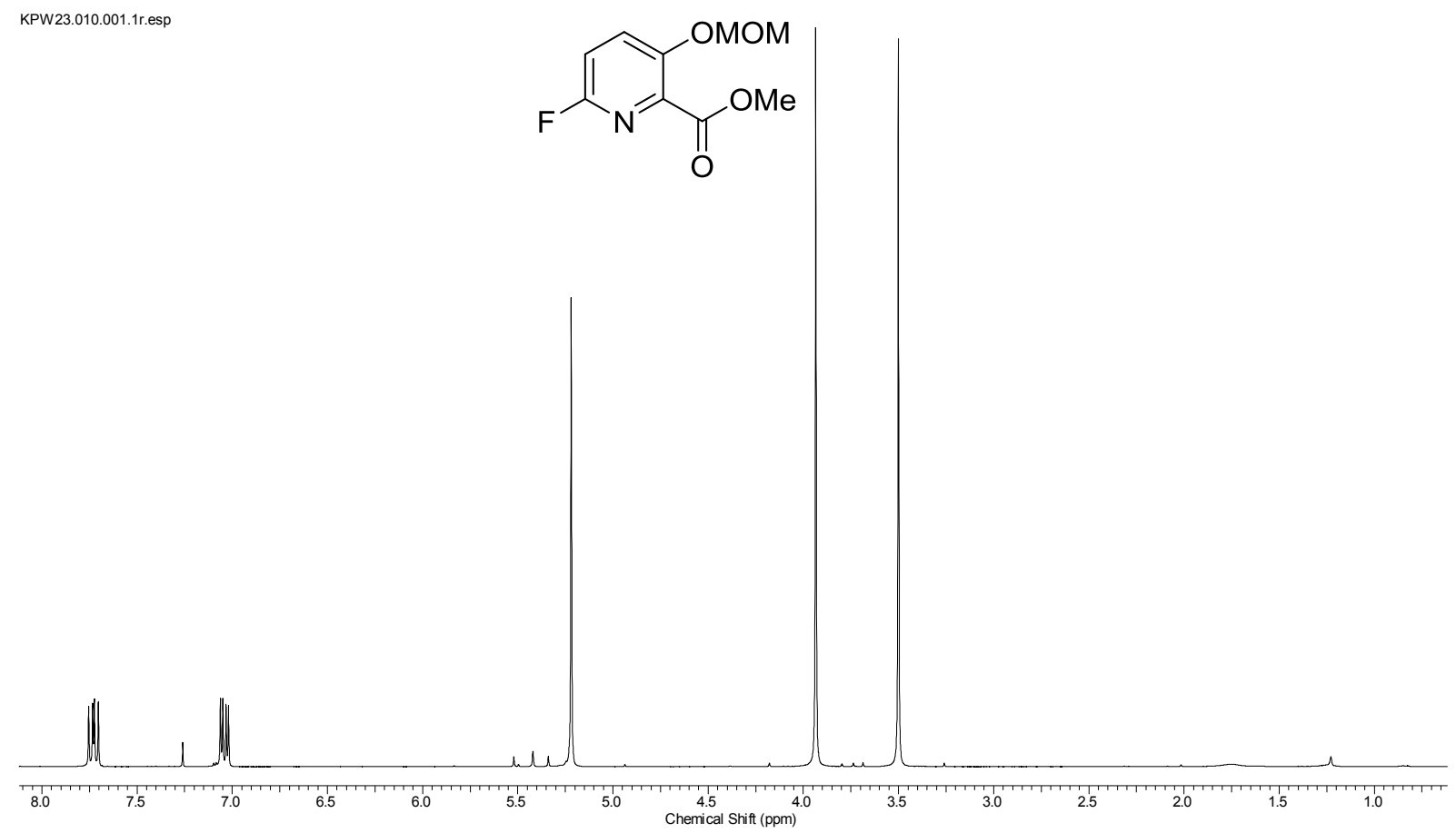

Abbildung 11.138: ${ }^{1} \mathrm{H}-\mathrm{NMR}-$ Spektrum des MOM-Ethers 243 (300 MHz, $\left.\mathrm{CDCl}_{3}\right)$.

KPW26.010.001.1r.esp<smiles>COC(=O)c1nc(F)ccc1O</smiles>

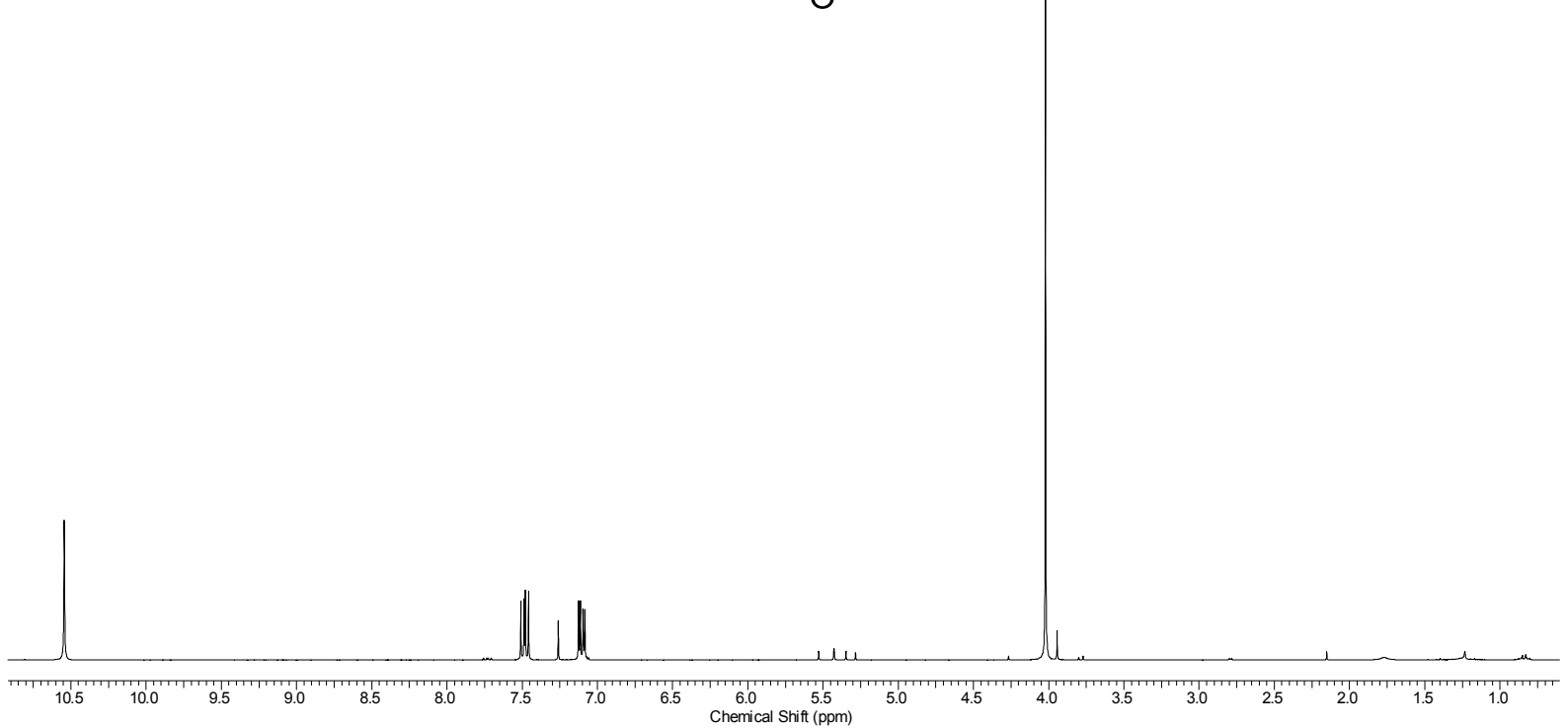

Abbildung 11.139: ${ }^{1} \mathrm{H}-\mathrm{NMR}-$ Spektrum des 3-Hydroxypyridins $244\left(300 \mathrm{MHz}, \mathrm{CDCl}_{3}\right)$. 


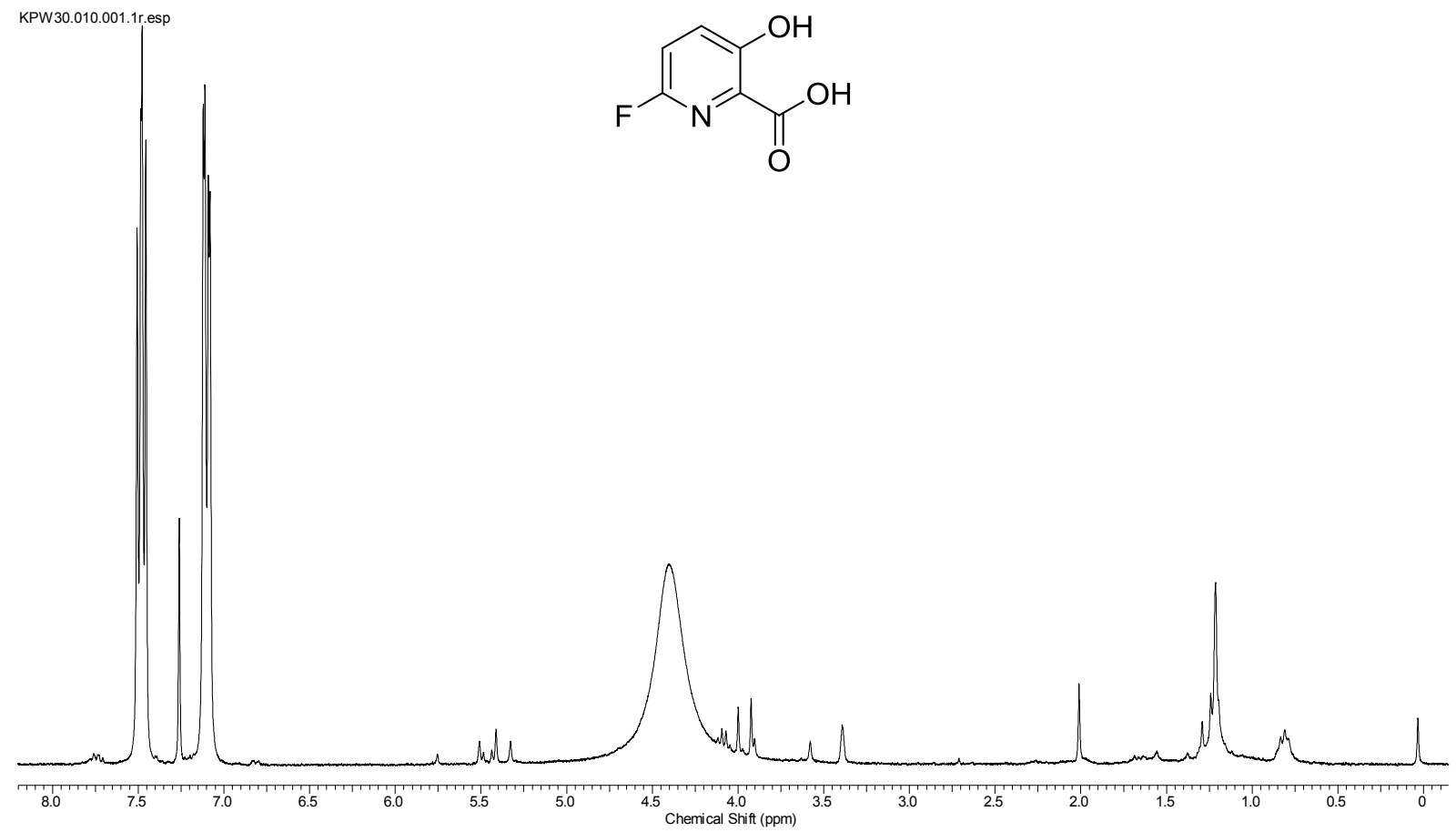

Abbildung 11.140: ${ }^{1} \mathrm{H}-\mathrm{NMR}-$ Spektrum der 3-Hydroxypicolinsäure 246 (300 MHz, $\mathrm{CDCl}_{3}$ ).

MS_15.010.001.1r.esp<smiles>COc1cccnc1C(=O)O</smiles>

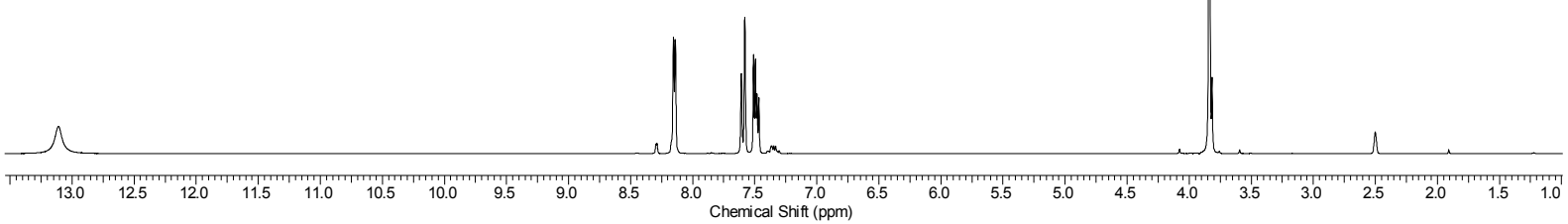

Abbildung 11.141: ${ }^{1} \mathrm{H}-\mathrm{NMR}-S p e k t r u m$ von 3-Methoxypicolinsäure (241, $\left.300 \mathrm{MHz}, \mathrm{DMSO}-d_{6}\right)$. 


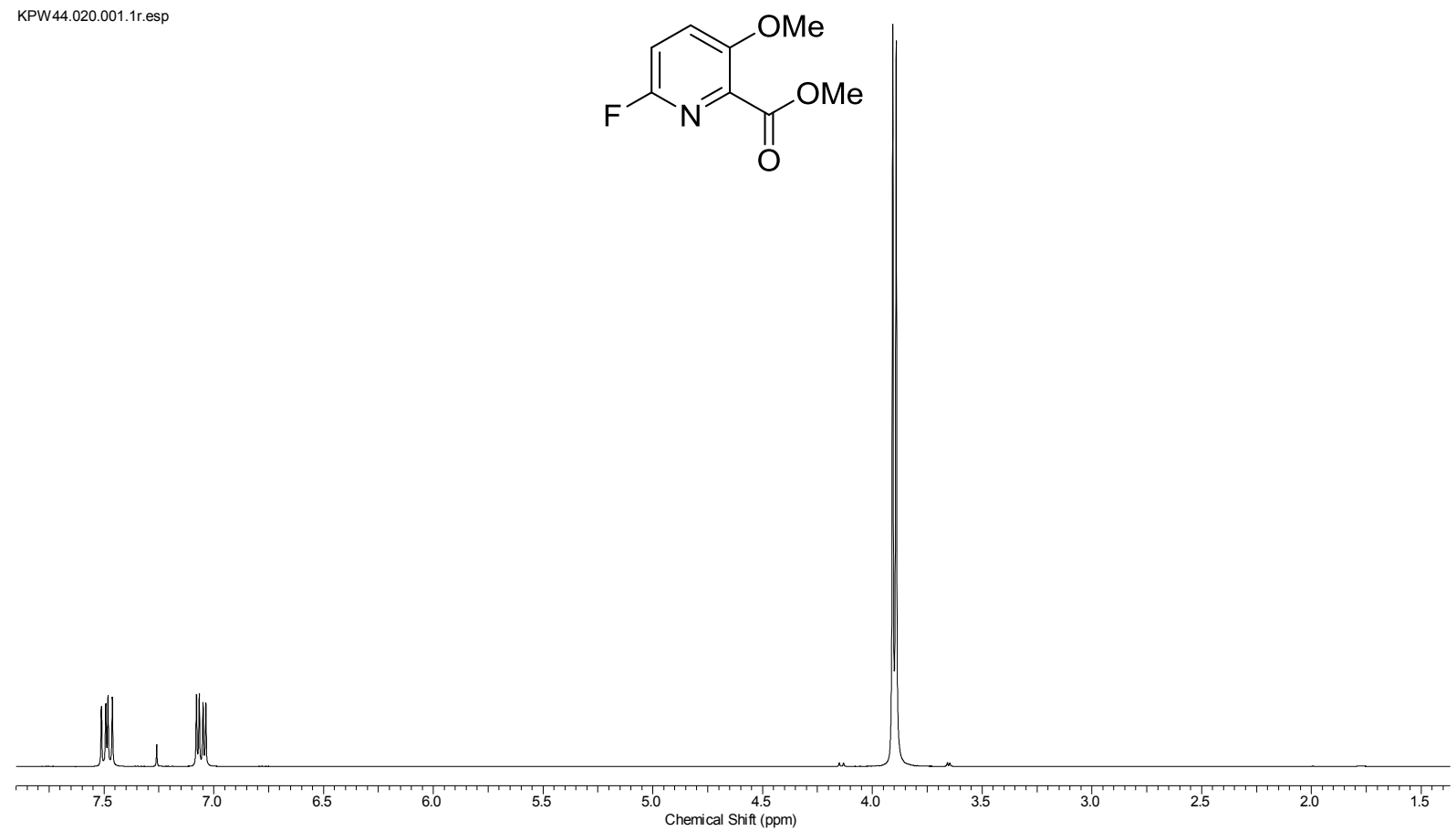

Abbildung 11.142: ${ }^{1} \mathrm{H}-\mathrm{NMR}-$ Spektrum des Pyridins $242\left(300 \mathrm{MHz}, \mathrm{CDCl}_{3}\right)$.

KPW-MS18.010.001.1r.esp<smiles>COc1ccc(F)nc1C(=O)O</smiles>
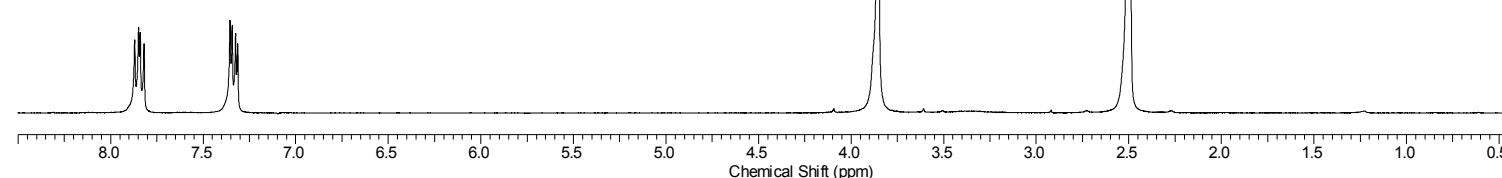

Abbildung 11.143: ${ }^{1} \mathrm{H}-\mathrm{NMR}-$ Spektrum der Picolinsäure 245 (300 MHz, DMSO- $d_{6}$ ). 


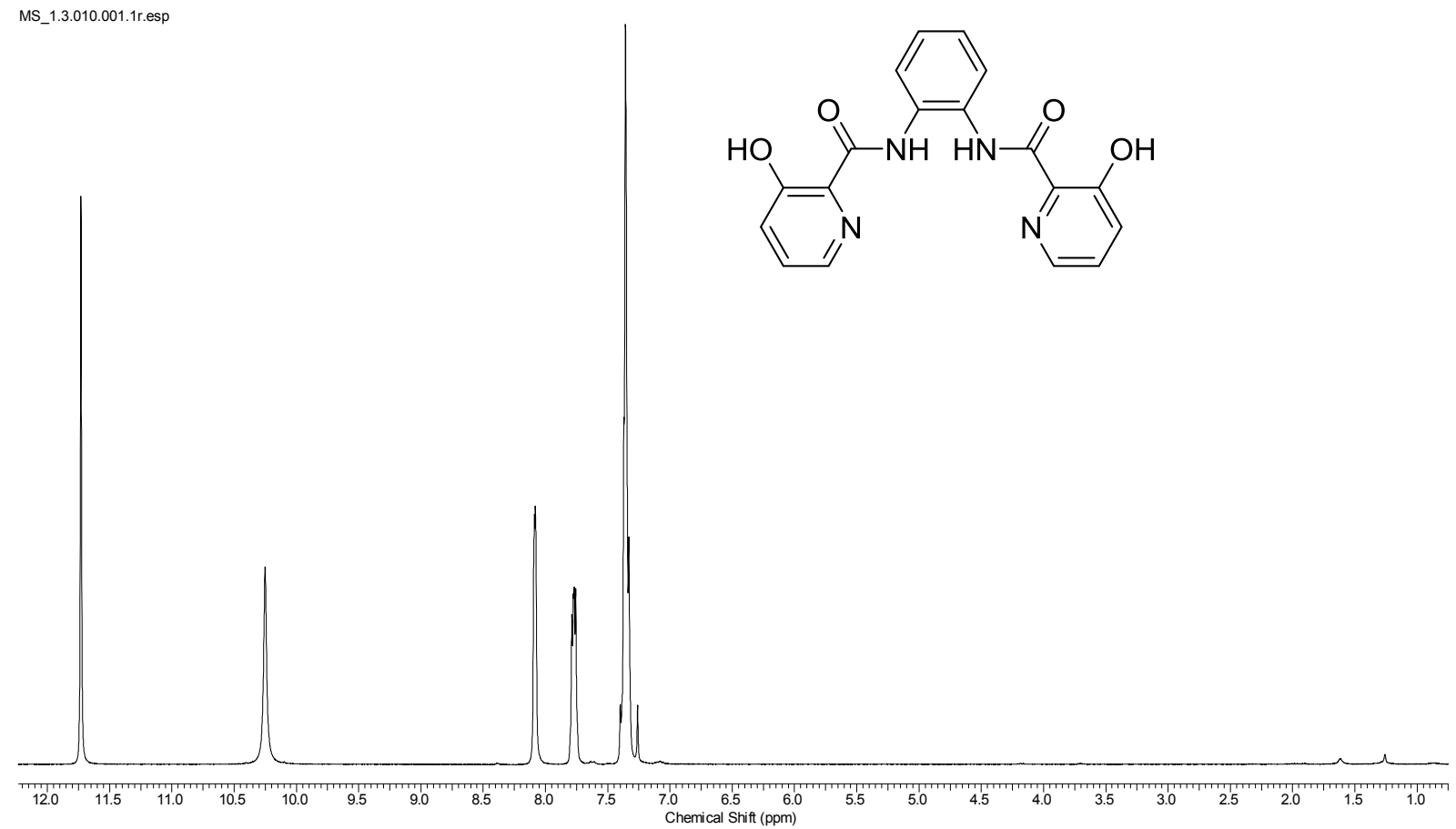

Abbildung 11.144: ${ }^{1} \mathrm{H}-\mathrm{NMR}-$ Spektrum des Liganden $102\left(300 \mathrm{MHz}, \mathrm{CDCl}_{3}\right)$.

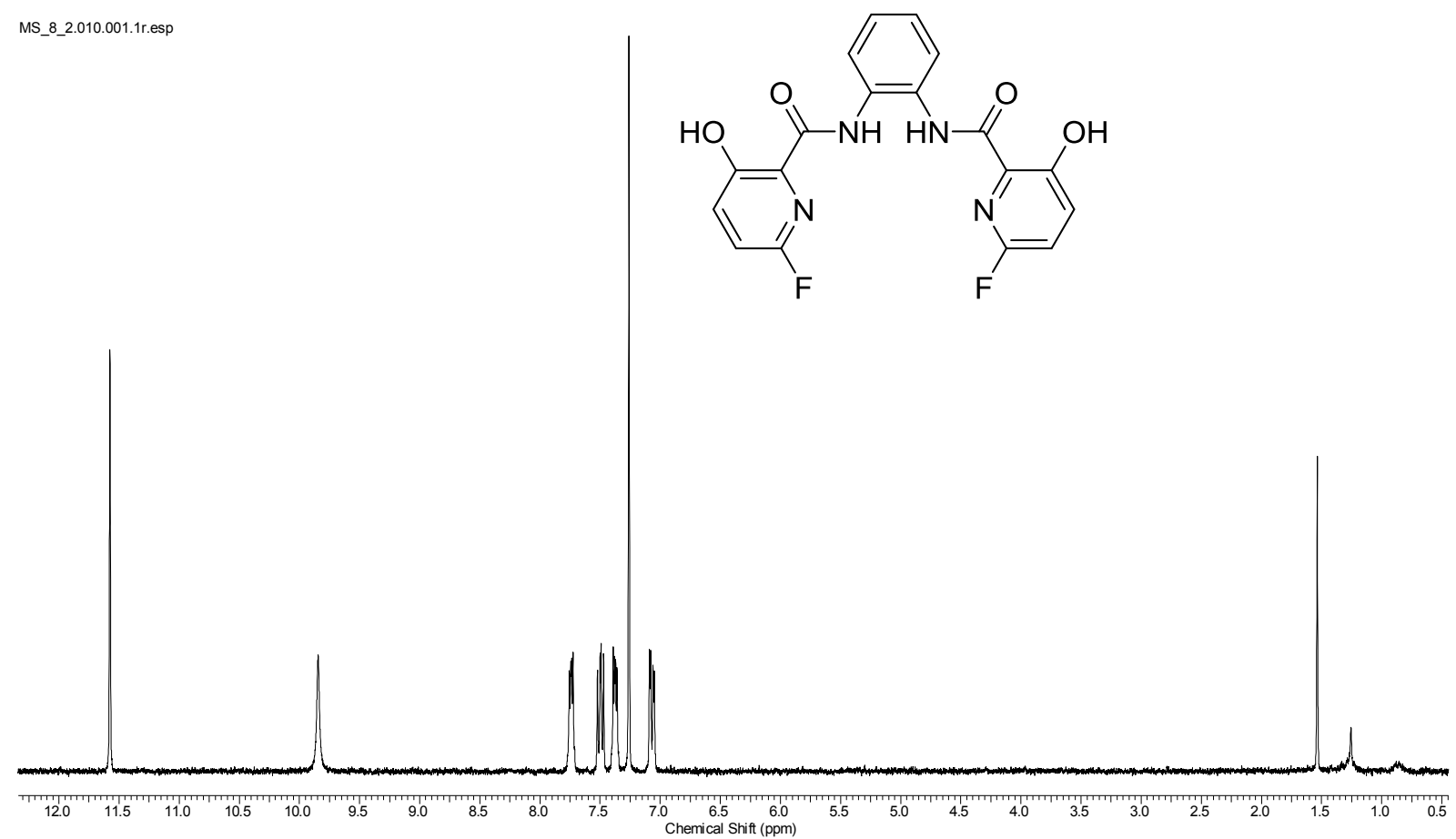

Abbildung 11.145: ${ }^{1} \mathrm{H}-\mathrm{NMR}-$ Spektrum des Liganden 103 (300 MHz, $\mathrm{CDCl}_{3}$ ). 


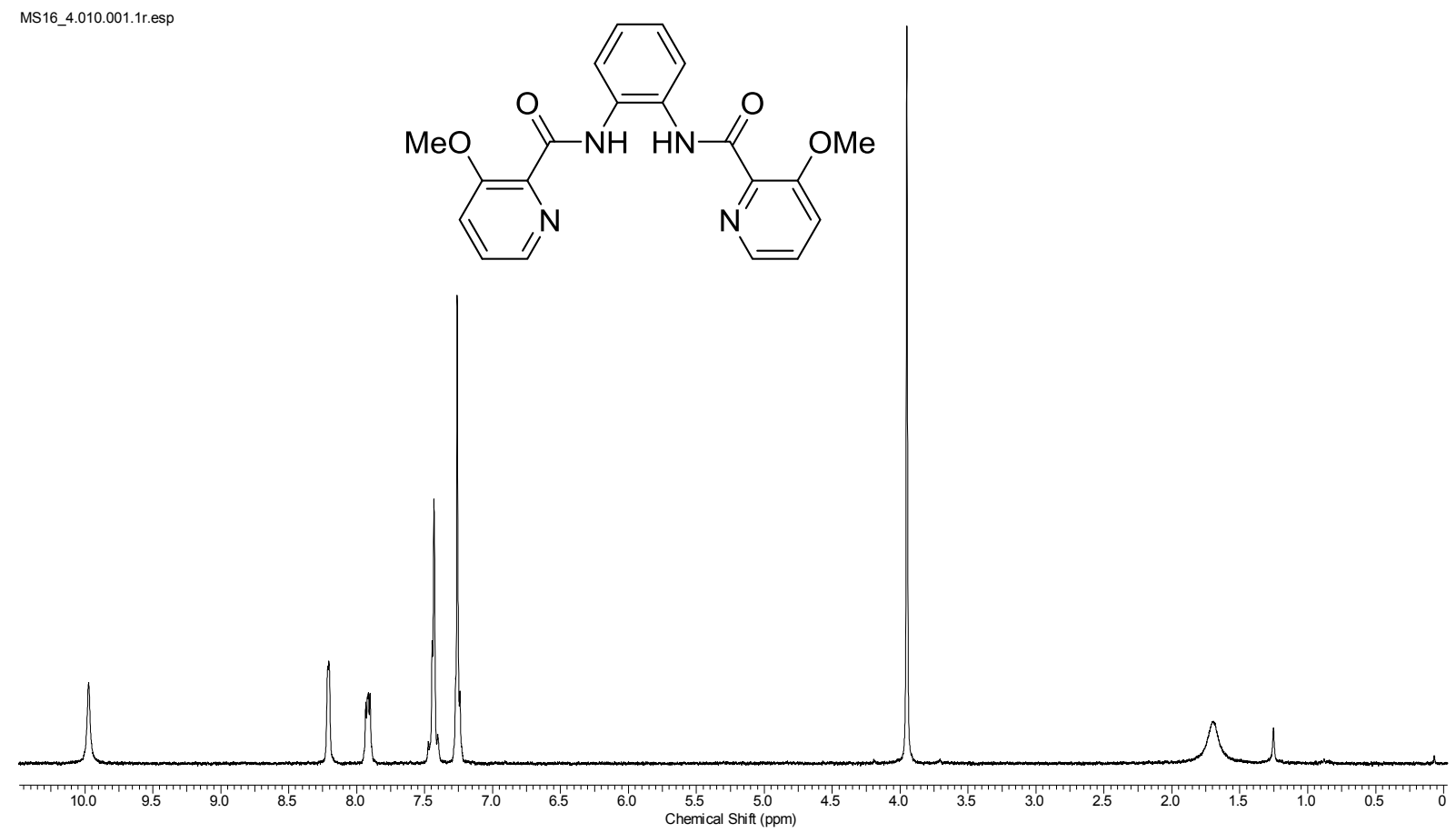

Abbildung 11.146: ${ }^{1} \mathrm{H}-\mathrm{NMR}-$ Spektrum des Liganden $104\left(300 \mathrm{MHz}, \mathrm{CDCl}_{3}\right)$.

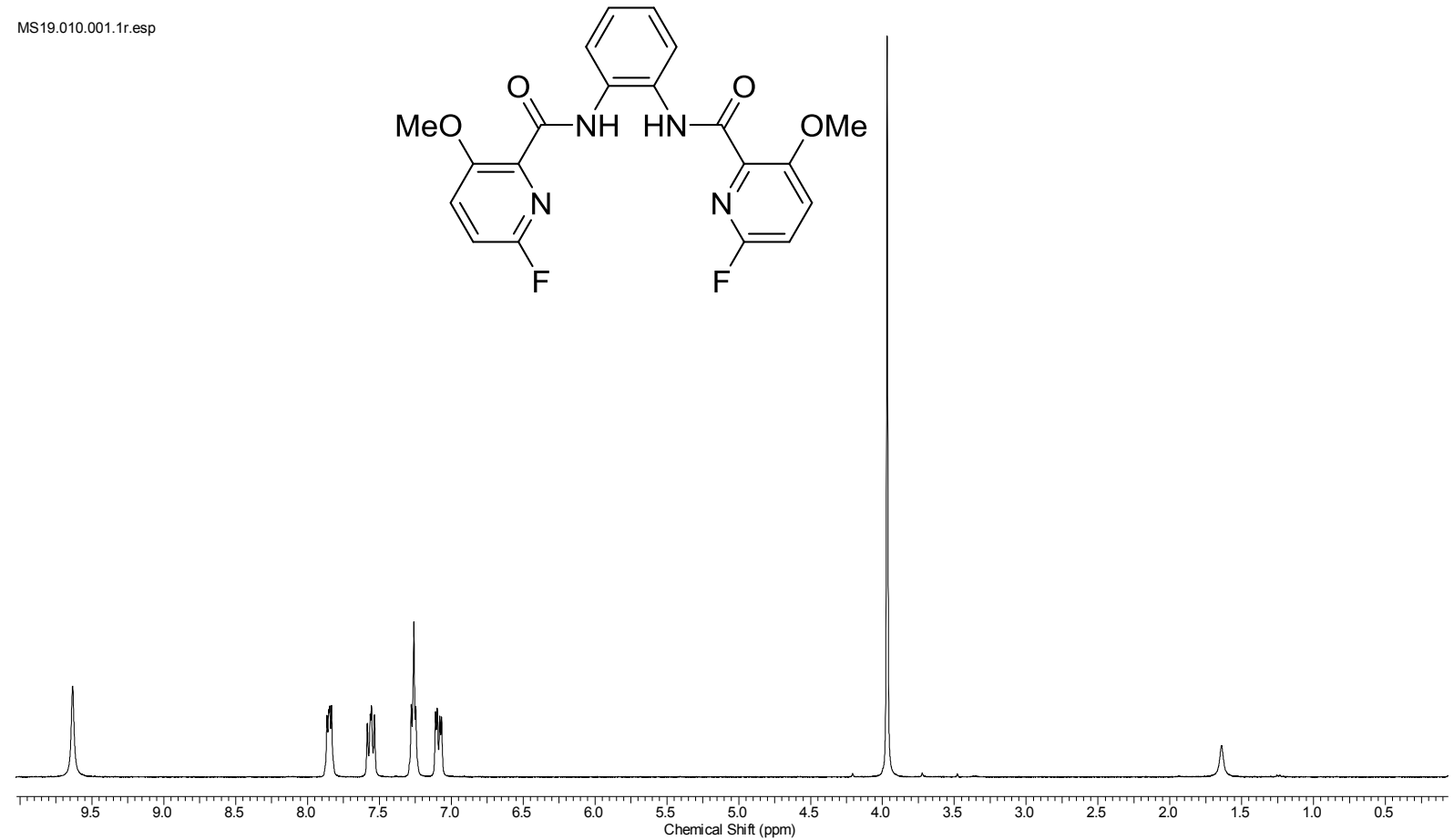

Abbildung 11.147: ${ }^{1} \mathrm{H}-\mathrm{NMR}-$ Spektrum des Liganden $105\left(300 \mathrm{MHz}, \mathrm{CDCl}_{3}\right.$ ). 


\subsection{Spektren zu semisynthetischen Arbeiten an Nosiheptid}

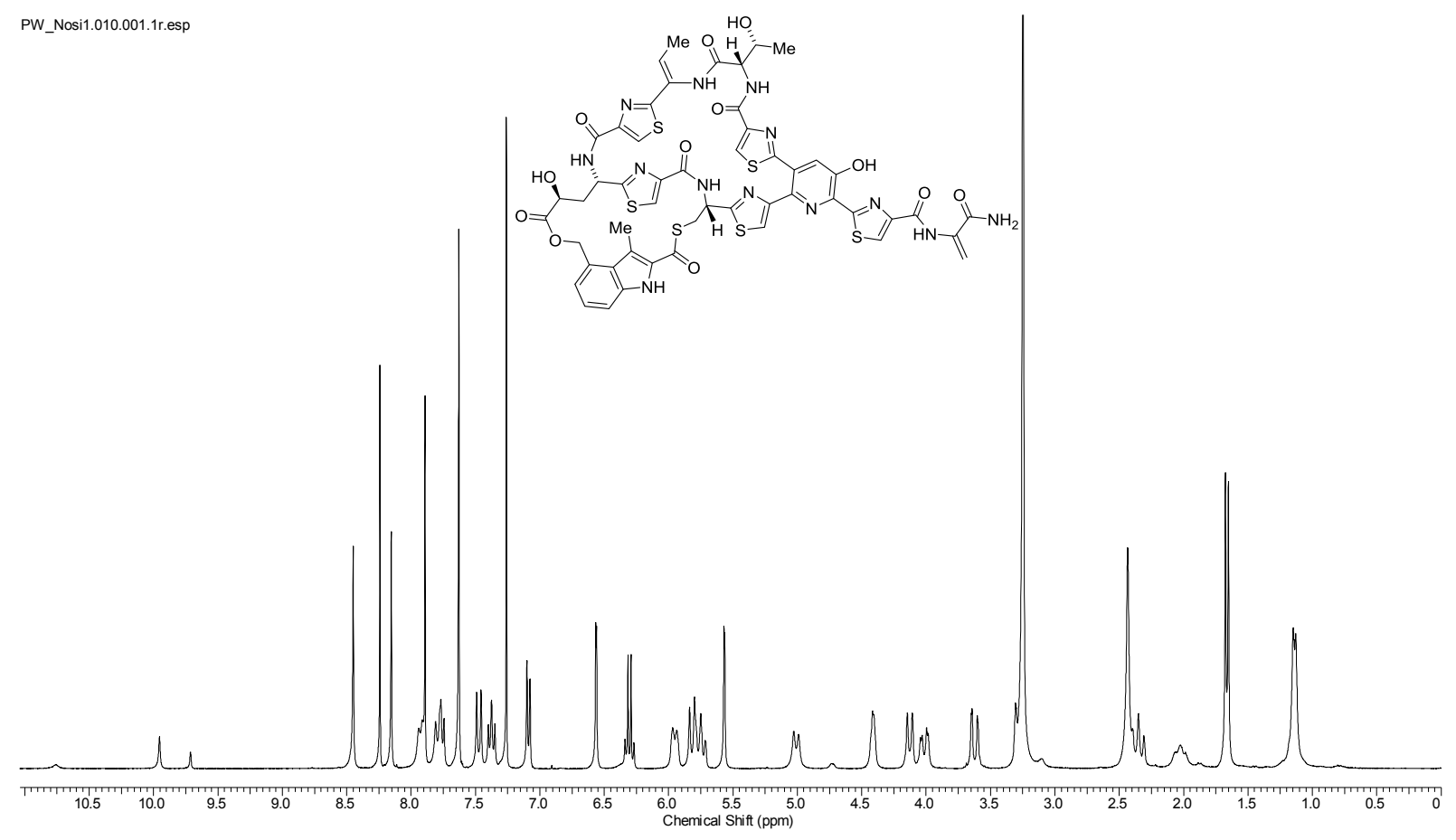

Abbildung 11.148: ${ }^{1} \mathrm{H}-\mathrm{NMR}-\mathrm{Spektrum}$ des nat. Nosiheptids (6, $\left.300 \mathrm{MHz}, \mathrm{CDCl}_{3} / \mathrm{MeOH}-d_{4}, 9: 1\right)$. 


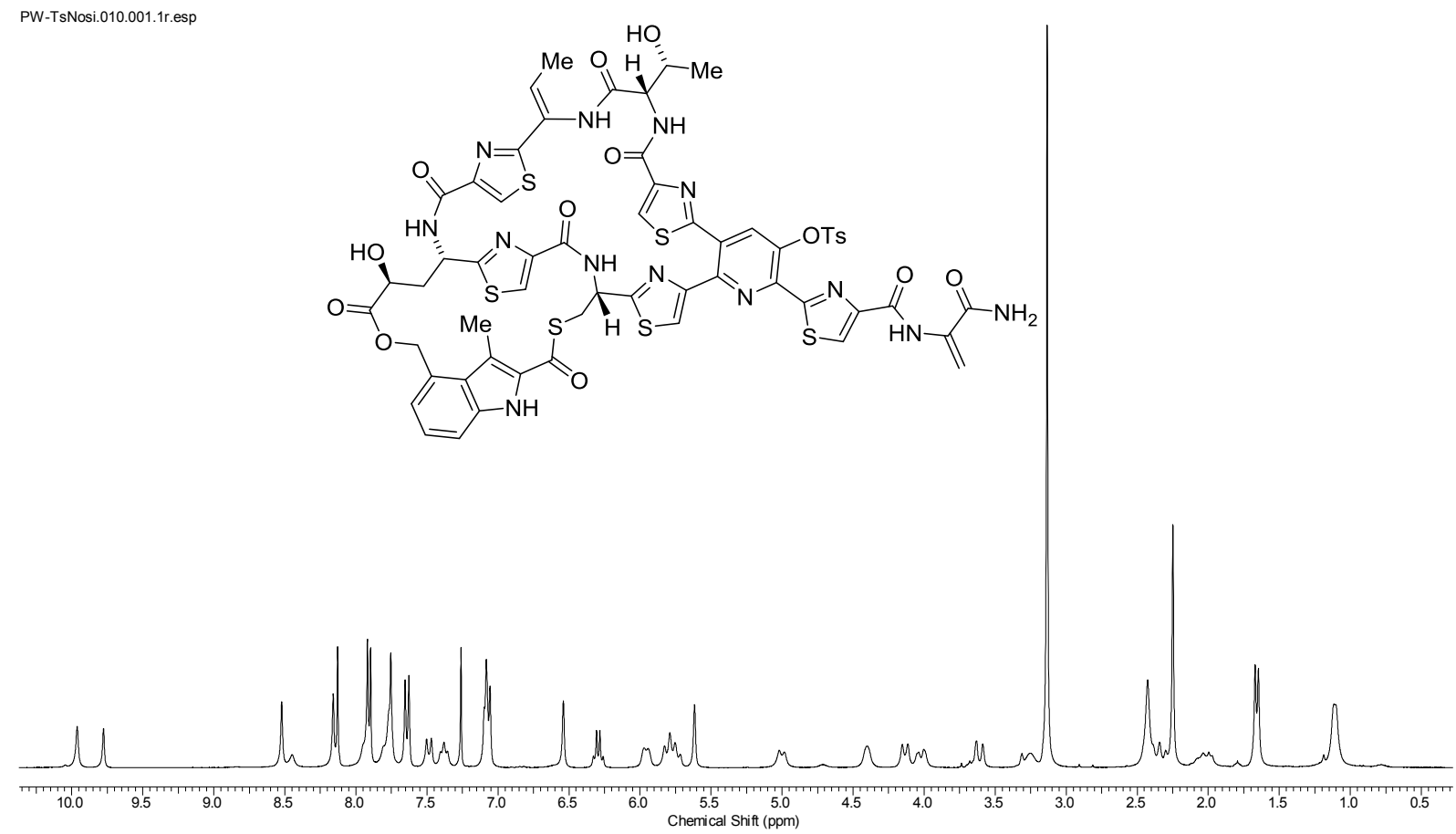

Abbildung 11.149: ${ }^{1} \mathrm{H}-\mathrm{NMR}-\mathrm{Spektrum}$ von Tosyl-Nosiheptid (169, $\left.300 \mathrm{MHz}, \mathrm{CDCl}_{3} / \mathrm{MeOH}-d_{4}, 9: 1\right)$.

PW-TsNosi.011.001.1r.esp

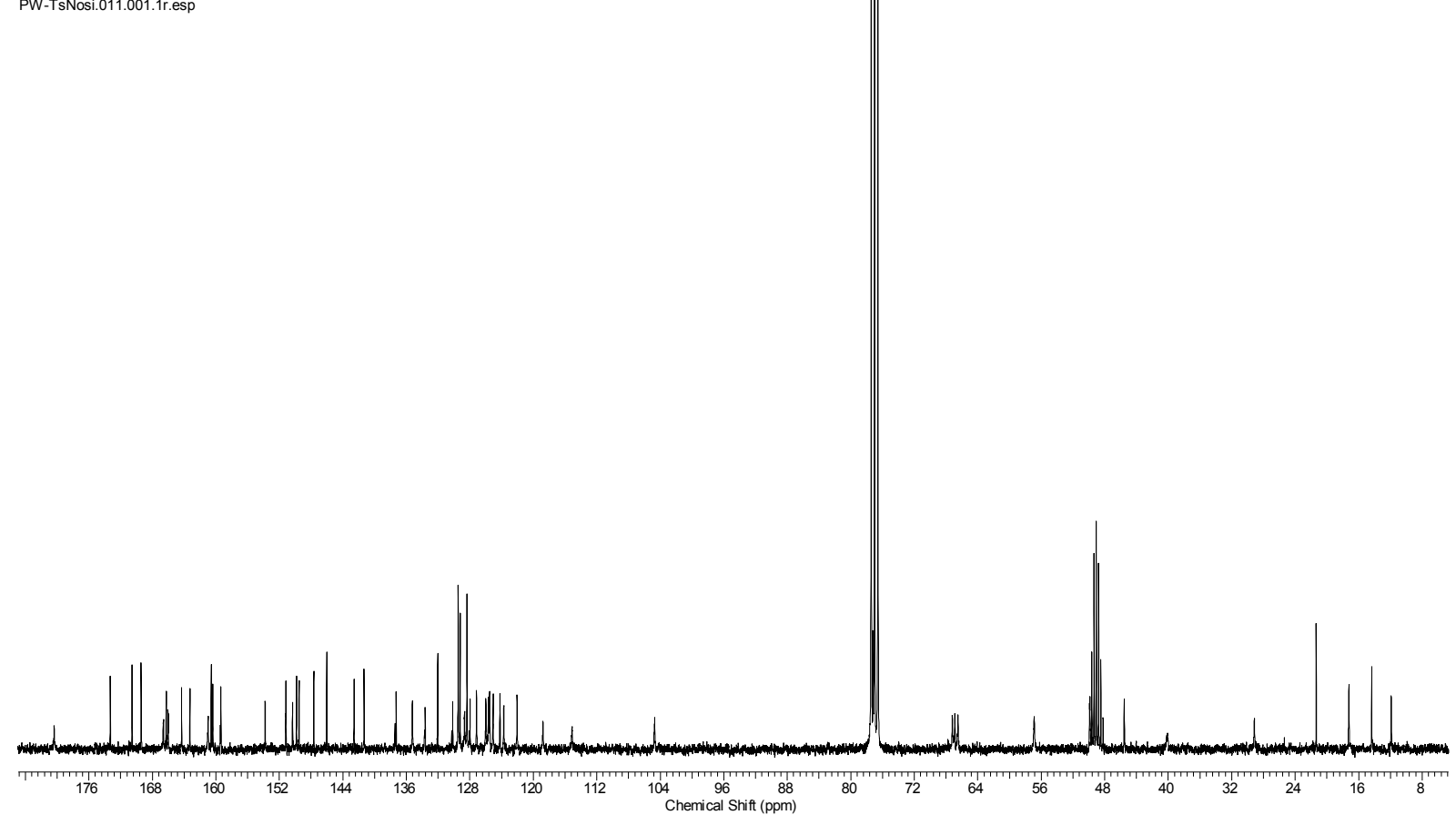

Abbildung 11.150: ${ }^{13} \mathrm{C}-\mathrm{NMR}-S p e k t r u m$ von Tosyl-Nosiheptid (169, $\left.75 \mathrm{MHz}, \mathrm{CDCl}_{3} / \mathrm{MeOH}-\mathrm{d}_{4}, 9: 1\right)$. 


\section{Danksagung}

Ich bedanke mich bei Prof. Dr. Hans-Dieter Arndt für die interessante und herausfordernde Themenstellung und die Bereitstellung eines exzellenten Arbeitsumfeldes. Sowohl sein uneingeschränkter Rückhalt in allen fachlichen Belangen als auch das entgegengebrachte Vertrauen ermöglichten eine große wissenschaftliche Freiheit.

Prof. Dr. Georg Pohnert danke ich für die bereitwillige Übernahme des Zweitgutachtens.

Allen Mitgliedern des AK Arndt und AK Vilotijević, insbesondere meinen Laborkollegen Dr. Kirtikumar Jadhav, Robert Freund, Veselin Nasufović und Andrea Schramm möchte ich für die gute Zusammenarbeit und ausgezeichnete Arbeitsatmosphäre danken. Meinen Bürokollegen Sabri Chiha und Sebastian Schwenk danke ich für eine motivierende Zusammenarbeit und ihre große Hilfsbereitschaft.

Bei der Synthese von Intermediaten für die Totalsynthese von Nosiheptid haben mich viele Studenten unterstützt. Mein Dank gilt Z. Artexte-Alonso, M. Brensing, D. Buff, J. Dahlke und N. Didukh. Ihar Raztsou und Andrea Schramm möchte ich besonders für die Unterstützung bei der Synthese des 3-Hydroxypyridin-Kerns und den damit verbundenen, gefühlt unendlich vielen, Säulenchromatographien danken. Andrea Schramm danke ich darüber hinaus für die Kristallisation der 3-Hydroxypyiridin-Isomere.

Lisa-Maria Semmrau und Sophia Walter danke ich für die Unterstützung bei der Durchführung der Hemmhoftests.

Bedanken möchte ich mich auch bei Dr. Christian Janßen für eine hilfreiche Diskussion bezüglich der Indolfragment-Synthese und Vorschlägen zur Wahl eines geeigneten Katalysators.

Meinem Schweizer Kollegen Thomas Winkler danke ich herzlichst für die Bereitstellung des Nosiheptid-Fermentationskuchens, den zahlreichen Diskussionen bezüglich der Eigenschaften dieses mittlerweile liebgewonnen Moleküls und steter Hilfe bei der Auswertung von NMRSpektren.

Den studentischen Hilfskräften X. Ai, Y. Cong und M. Sauer möchte ich für Ihre Unterstützung bei der Synthese der substituierten 3-Hydroxypicolinate sowie der Biscarboxamid-Liganden danken. 
Zahlreichen Mitgliedern des AK Schubert, AK Beckert und AK Heinze danke ich für die entgegenkommende Bereitstellung von Geräten sowie diverser Chemikalien.

Ein großer Dank gilt dem NMR-Team-Mitgliedern Dr. P. Bellstedt, Dr. W. Günther, F. Pielenz, B. Rambach und G. Sentis für einen hervorragenden und uneingeschränkten Service.

Chantale Martin und Dr. Lydia Seyfarth danke ich für die Messung zahlreicher HRMSProben, vor allem für die Messungen nach Dienstschluss.

Dr. Helmar Görls danke ich für die Durchführung der Röntgenkristallstrukturanalysen.

Ich bedanke mich herzlich für die Unterstützung durch die wissenschaftlichen Angestellten Dr. C. Ronco, Dr. D. Weiß und H. Zdon, sowie durch Sylke Kaiser.

Ein besonderer Dank geht an Dr. Lydia Seyfarth und Angelika Güther, die durch ihren unermüdlichen Einsatz stets alle Hürden im Laboralltag beiseite geräumt und eine reibungslose Infrastruktur aufrechterhalten haben.

Jun.-Prof. Rene Königs danke ich für die Durchsicht des vorliegenden Manuskripts.

Nicht zuletzt möchte ich mich herzlich bei all jenen Personen bedanken, die mich im privaten Umfeld unterstützten. Ganz speziell danke ich meinen Eltern, Brüdern und Philipp für ihre unbegrenzte Zuwendung. Diese Hilfe lässt sich nicht ermessen.

Liebe Alexa, vielen, vielen Dank für deine ständige Ermunterung, unermesslich viel Geduld und deine bedingungslose Liebe. 


\section{Selbständigkeitserklärung}

Ich erkläre,

dass ich die vorliegende Arbeit selbstständig und unter Verwendung der angegeben Hilfsmittel, persönlichen Mitteilungen (siehe Danksagung) und Quellen angefertigt habe.

Weimar, den . Dezember 2016

K. Philip Wojtas 